UC-NRLF

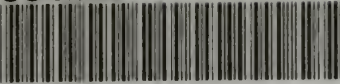

B $4 \quad 094 \quad 850$ 


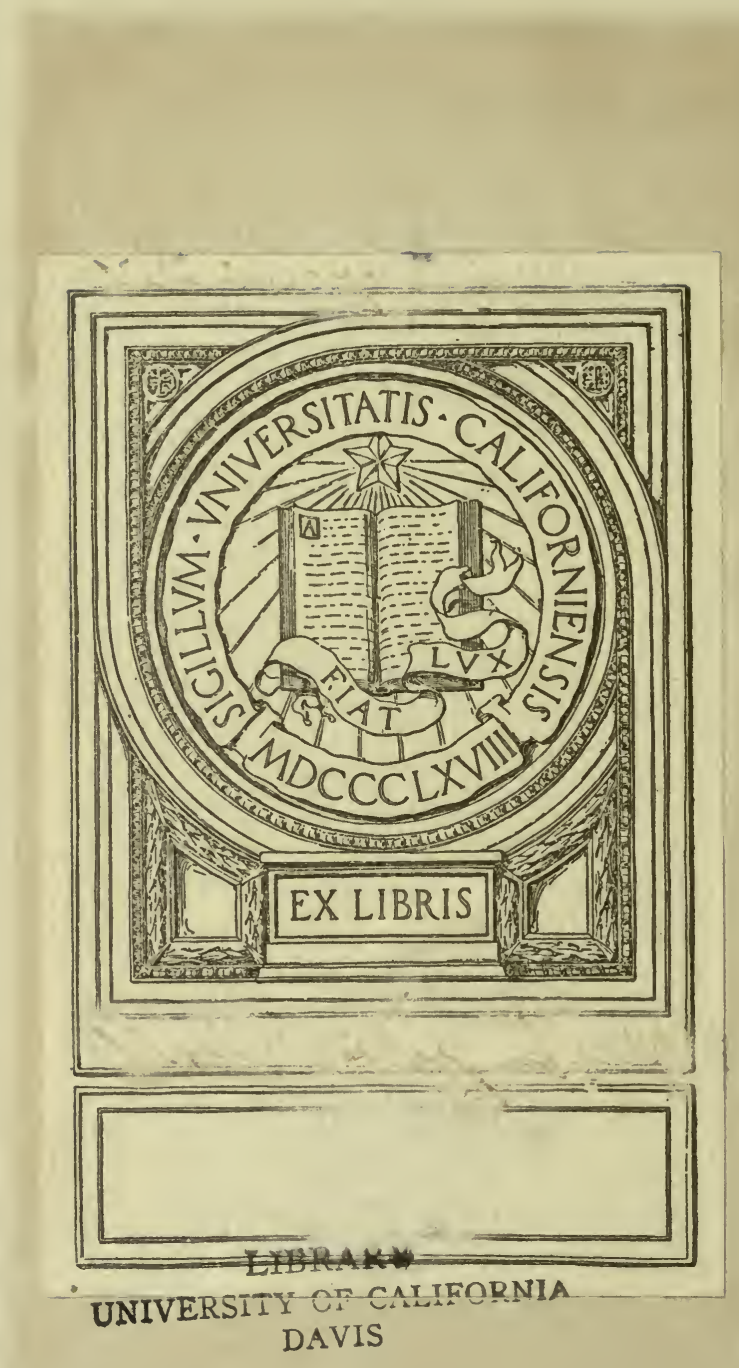


UNIVERSITY OF CALIFORNIA LIBRARY

THIS BOOK IS DUE ON THE LAST DATE STAMPED BELOW

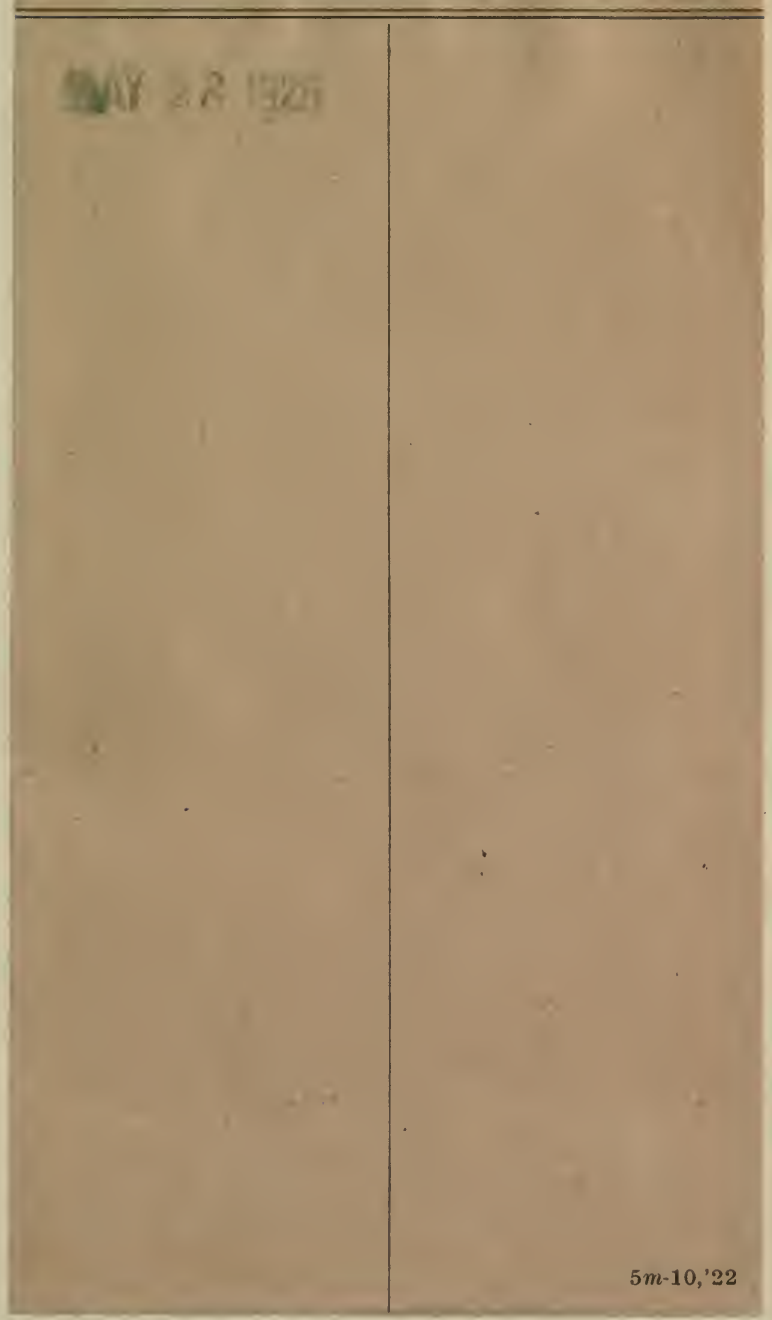


Digitized by the Internet Archive in 2007 with funding from Microsoft Corporation 


\section{ANIMAL PARASITES AND \\ HUMAN DISEASE}

BY

ASA C. ChANDleR, M.S., Pн.D.

INSTRUCTOR IN BIOLOGY, RICE INSTITUTE, HOUSTON, TEXAS

SECOND EDITION, REVISED

NEW YORK

JOHN WILEY \& SONS, INC.

LONDON: CHAPMAN \& HALL, LiMiTED

1922 
Copyright, 1918, 1922

BY

Asa C. Chandler 
To

MY MOTHER

WHOSE SELF-DENYING LOVE AND UNFAILING

DEVOTION MADE MY SCIENTIFIC

EDUCATION POSSIBLE

623 



\section{PREFACE}

IT is the belief of the writer that one of the most pressing needs of the present time is the education of the people as a whole in the subjects of vital importance with which this book deals, and an increased interest in this field of scientific work. Scientists are the leaders of the world, and should constantly endeavor to keep a little ahead of the lay population who follow them. It is, however, important that the leaders should not only blaze the trail, but should make it sufficiently easy to find so that the followers may not fall too far behind. In the intense fascination of exploring the trail, and the eager impulse to press on to newer and ever newer fields, the scientist is in danger of forgetting the handicaps of his followers, and of leaving them hopelessly in the rear. Popular ignorance of many important facts of parasitology and preventive medicine, even facts which have been common bases of operation for scientists for many years, is deplorable. To a large extent, however, the scientists themselves are to blame, for in their enthusiasm for discovery they have forgotten to make it possible for the laity to reap the benefits of their investigations. There is even a tendency to belittle the efforts of those workers who devote their energies toward assisting the general public to keep in touch with scientific progress. A book or paper which collects the work of others, models it into a connected whole, and makes readily available what before was widely scattered and accessible only to a skilled "library-prowler," is stigmatized by the term "mere compilation." It is the firm belief of the writer that this is not only unjust but unwise. No less mental and physical energy, if not perhaps even more, is necessary for efficient " mere compilation" than for the addition of new facts to scientific knowledge, and the value to civilization, which must be the ultimate criterion by which all scientific work is judged, must be equally as great, if not greater. The value of connecting related facts is twofold: it helps to keep the world in general somewhere nearly abreast of the times, and it is a distinct aid to further 
progress. Having the courage of his convictions along these lines, the writer has spent much time which he might otherwise have spent on original research in the compilation and popularization of the subject matter of this book.

It is the aim of this volume to present the important facts of parasitology, as related to human disease, in such a manner as to make it readable and useful not primarily to the parasitologist, but to the public health and immigration service officers; to the physicians who are concerned with something more than their local practice; to teachers of hygiene, domestic science or other subjects in which health and preventive medicine are important; to college and high school students; to the traveler; and to the farmer or merchant who is interested in the progress of science and civilization. It is the hope of the author that this book may not only be a means of making available for the laity facts which may and probably will be of direct importance to them at one time or another, but that it may also be instrumental in arousing the interest of more students in this branch of science, to the ultimate end of enlisting a larger number in the ranks of its workers.

No attempt has been made in the following pages to give detailed descriptions of parasites, or to go further into their classification than seemed necessary to give a correct conception of them. Likewise discussions of correct scientific names and synonymy have been entirely omitted, since, important and interesting as they may be to a parasitologist, they are of no interest to the lay reader. An attempt has been made to use scientific names which are most generally accepted as correct, except that in cases of disagreement between American and European usage, the American name has been used. In cases where some other name than that adopted in this book has been or is still in common use, it is given in parenthesis to afford a clue to the literature associated with it.

The endeavor to avoid repetition in the discussion of certain parasites in one chapter, and of their transmitting agents in another, has often presented difficulties, since some facts might equally well be included in either place. As far as possible these facts have been given in the place where the author has felt that they would most often be sought, but mistakes have undoubtedly been made, and furthermore what one reader would 
search for under " malaria," for instance, another would seek under "mosquitoes," and vice versa. For this reason frequent cross-references are given.

As far as has seemed advisable, without too greatly encumbering the text with round-about phrases, scientific terms have been omitted or if used have been explained. It is difficult to keep constantly before one the unfamiliarity with even everyday scientific terms of many readers for whom this book is intended, but an earnest attempt to do so has been made.

In the text the author has purposely refrained from citing references and from mentioning more than a few names of investigators. It obviously would be impossible to give references, or even to mention more than a small per cent of the thousands of contributors to the material here assembled without making the text cumbersome and unreadable, especially for the readers for whom the book is especially prepared. Only a few of the leading figures in the history of each group of parasites have been mentioned; other citations would have meant a more or less arbitrary selection of a few from among many, which must inevitably result in injustice.

For similar reasons no bibliography is given. Instead, the author has prepared a list of "Sources of Information" which includes the names of all the leading periodicals in which important articles on parasitology have appeared or are likely to appear, and a list of books which cover all or a portion of the field of parasitology in a comprehensive manner. In these books will be found bibliographies; most of the references cited in these bibliographies will be found in the magazines or papers listed in "Sources of Information" and this list will aid anyone interested in pursuing the subject farther to keep in touch with the new work which is constantly appearing. The author has felt that more real value would attach to such a list than to a list perhaps 50 times as long and yet inevitably incomplete, containing exact references to particular articles.

The illustrations, with two or three exceptions, have been drawn by the author either from specimens or from illustrations of other authors. Pen and ink drawings have been used consistently in place of photographs since it is believed that such drawings, if carefully done, are far more valuable for scientific purposes than are photographs. The trained eye is able by 
voluntary concentration on certain parts, and inattention to others, to see much more than can a camera, which has no such power of adjustment. A pen and ink drawing can, therefore, represent more accurately what can be seen by the eye than can a photograph. The author has received valuable advice regarding the illustrations from Mr. A. J. Stover, scientific illustrator at the Oregon Agricultural College, and wishes to take this opportunity to express appreciation for it.

Deep appreciation is felt for the invariable willingness with which authors, editors and publishers of scientific papers and books have given permission to copy illustrations. Special mention should be made, however, of the generosity of Sir Patrick Manson and of the American publishers of his "Tropical Diseases," Wm. Wood and Co.; of Dr. A. Alcock, author of "Entomology for Medical Officers"; of Professor Wm. A. Riley and Dr. Johannsen, authors of "A Manual of Medical Entomology," and of Dr. A. W. Sellards, who, in the absence of Dr. Strong, lent photographs taken in Peru by the Harvard School of Tropical Medicine. The illustrations taken from the journal Parasitology have been especially numerous, and mention should, therefore, be made of the unreserved permission to use them given by the editor, Professor G. H. F. Nuttall. Many illustrations of worms have been taken from the work of two of the real pioneers in the study of helminthology, Professor Karl Leuckart of the University of Leipzig, under whom many of the present parasitologists were trained, and Professor Arthur Looss of the University of Cairo in Egypt. It is a high tribute to the work of Professor Leuckart that many illustrations published by him in the first comprehensive work on the animal parasites of man, in 1863, are still the best available ones and will be found reproduced in the majority of modern works on the subject.

Particular appreciation is felt for the assistance received from three publications which contain reviews of current literature in particular phases of medical zoölogy, namely, the Tropical Diseases Bulletin, which reviews practically all current work on protozoan parasites and helminthology, the Review of Applied Entomology, Series B, containing abstracts of nearly all work on medical and veterinary entomology, and the Journal of the American Medical Association, which gives references to all 
articles in the leading medical journals of all countries, and reviews many of them. Any of these periodicals will be lent by the Association library, at the average cost of postage, to any member of the Association. These three publications, on account of their scope and thoroughness, are of inestimable value to anyone who attempts to keep pace with the progress of the medical sciences. There are, however, few if any of the journals or books listed under "Sources of Information" which have not been drawn upon either for illustrations or information or both. All of these, collectively, have made this book possible, and to them, and to the workers who contribute to them, are due, therefore, not only the thanks of the author but also the thanks of everyone who may profit in any way by this book.

The writer is very deeply indebted to the authorities who have been kind enough to read the manuscript, and who have freely given the benefit of helpful suggestions and criticisms. Professor Gary N. Calkins, Professor of Protozoölogy at Columbia University, Dr. B. H. Ransom, Zoölogist of the U. S. Bureau of Animal Industry, and Dr. L. O. Howard, Chief of the U. S. Bureau of Entomology, have helped materially to round off the rough corners, and fill in the chinks, of the sections on Protozoa, "worms," and arthropods, respectively.

Hearty thanks is also due my wife, Belle Clarke Chandler, for the invaluable assistance she has given by her constant and efficient coöperation in the editorial part of the work. 



\section{TABLE OF CONTENTS}

Chap.

I. Introduction........................... $1-11$

II. Parasites in General. .................. 12-25

\section{PART I. PROTOZOA}

III. Introduction to Protozon. . . . . . . . . . . . . . . . 26-37

IV. SpIRochetes.................. 38-73D

Relapsing Fever. . . . . . . . . . . . . . . . . 42-48

Syphilis............................ 48-62

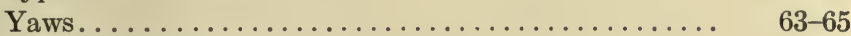

Infectious Jaundice. . . . . . . . . . . 65 . 69

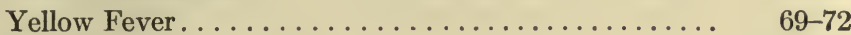

Rat-bite Fever.................. 73-73A

Other Spirochæte Diseases............. 73A-73D

V. Leishman Bodies and Leishmaniasis ........... 74-92

Kala-azar. . . . . . . . . . . . . . . . . . . $77-82$

Infantile Kala-azar. . . . . . . . . . . . . . . $82-84$

Oriental Sore..................... 84-88

Espundia...................... 89-92

VI. Trypanosomes and Sleeping Sickness .......... 93-114

Sleeping Sickness . . . . . . . . . . . . . . . . 98

Chagas' Disease..................... 108-114

VII. Intestinal Flagellates and Ciliates $\ldots \ldots \ldots \ldots \ldots$ 115-127

Bi-flagellate Protozoa. ................. 117-118

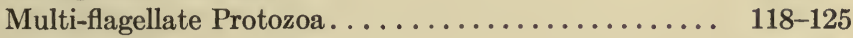

Ciliates........................ 126-127

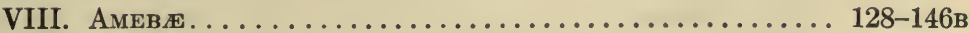

Intestinal Amœbæ................... 132-142

Mouth Amœbæ..................... 142-146в

IX. Malaria ......................... 147-167

X. Other Sporozoa, and Obscure or Invisible Parasites 168-194 Coccidians............................ 170-173

Rhinosporidium ..................... 173-174

Sarcosporidia..................... 174-176 
Char.

Oroya Fever 176-181

Dengue. $182-184$

Phlebotomus Fever

184-185

Rickettsia-like Organisms

185-191

Chlamydozoa.

191-194

\section{PART II. "WORMS"}

XI. Introduction to the "Worms" ............ 196-206

XII. The Flukes. . . . . . . . . . . . . . . . . . . . 207-230

Blood Flukes........................ 213-220

Lung Flukes....................... 220-224

Liver Flukes. . ..................... 224-228

Intestinal Flukes..................... 228-230

XIII. The Tapeworms...................... 231-253

Family Tæniidæ..................... 239-245

Family Dibothriocephalidæ............... 245-247

Larval Tapeworms in Man.............. 247-253

XIV. Hookworms. . . . . . . . . . . . . . . . . . . . 254-269

XV. Other Intestinal Roundworms............ 270-285

XVI. Trichina Worms...................... 286-297

XVII. Filaria and their Allies . . . . . . . . . . . . . . . . 298-314

Filaria bancrofti..................... 299-307

Other Species of Filariæ............... 307-314

XVIII. LeEches. . . . . . . . . . . . . . . . . . . . . 315-321

\section{PART III. ARTHROPODS}

XIX. Introduction to Arthropod. . . . . . . . . . . . . 322-330

XX. The Mites........................... 331-351

Harvest Mites. . . . . . . . . . . . . . . . . 333-337

Other Occasionally Parasitic Species........... 337-341

Itch Mites. . . . . . . . . . . . . . . . . . . . . 342-346

Hair-follicle Mites...................... 346-348

Tongue-Worms...................... 348-351

XXI. Trcks. . . . . . . . . . . . . . . . . . . . . . . 352-369

Ticks and Disease. ................... 359-363

Other Troublesome Ticks................ 364-369 
Chap.

XXII. Bedbugs and their Allies.

Bedbugs

371-379

Other Parasitic Bugs.

$379-383$

Remedies and Prevention.................. 383

Fumigation . . . . . . . . . . . . . . . . . . . . 3836

XXIII. LICE . . . . . . . . . . . . . . . . . . . . 387-403

XXIV. Fleas. . . . . . . . . . . . . . . . . . . . . 404-423

XXV. Mosquitoes.......................... 424-462

Mosquitoes and Malaria................ 438-443

Mosquitoes and Yellow Fever.............. 443-448

Mosquitoes and Dengue................ 448-449

Mosquitoes and Filaria.................... 449-451

Mosquitoes and Dermatobia................ 451-453

Mosquito Bites and Remedies for Them........ 453-455

Control and Extermination............... 455-462

XXVI. Other Blood-sucking Flies............... 463-508

Phlebotomus Flies. . . . . . . . . . . . . . . . . . 466-473

True Midges (Chironomidæ)................ 473-477

Blackflies or Buffalo Gnats................... 478-484

Gadflies (Tabanidæ) . . . . . . . . . . . . . . . . . 484-490

Tsetse Flies. . . . . . . . . . . . . . . . . . . 490-504

Stable-Flies, Stomoxys, and Their Allies......... 504-508

XXVII. Fly Maggots and Myiasis................. 509-528

Blood-sucking Maggots................... 511-513

Maggots under the Skin ................ 513-519

Myiasis of Wounds and of Natural Cavities of the Body 519-523

Myiasis of the Intestine................. 523-528

Sources of Information $\ldots \ldots \ldots \ldots \ldots \ldots \ldots \ldots \ldots \ldots \ldots$ 529-533 



\section{ANIMAL PARASITES AND HUMAN DISEASE}

\section{CHAPTER I \\ INTRODUCTION}

ONE of the most appalling realizations with which every student of nature is brought face to face is the universal and unceasing struggle for existence which goes on during the life of every living organism, from the time of its conception until death. We like to think of nature's beauties; to admire her outward appearance of peacefulness; to set her up as an example for human emulation. Yet under her seeming calm there is going on everywhere - in every pool, in every meadow, in every forest - murder, pillage, starvation and suffering.

Man often considers himself exempt from this interminable struggle for existence. His superior intelligence has given him an insuperable advantage over the wild beasts which might otherwise prey upon him; his inventive genius defies the attacks of climate and the elements; his altruism, which is perhaps his greatest attribute, protects, to a great extent, the weak and poorly endowed individuals from the quick elimination which is the inevitable lot of the unfit in every other species of animal on the earth. Exempt as we are, to a certain extent, from these phases of the struggle for existence, we have not yet freed ourselves from two other phases of it, war and disease. We have some reason for hoping that after the present world-wide conflagration of war has burned itself out and its ashes, the flesh and bones of its countless victims, have disintegrated and disappeared from view, we may be able to free ourselves from the probability of ever again taking part in or witnessing such a spectacle. That the helpless bondage in which we were once held by disease will never again be our lot, we can say with more assurance. 
One by one the diseases which formerly held the world in terror, or made parts of it practically uninhabitable, are falling before the onslaught of modern science. The vast majority of human and animal diseases are now known to be caused by organisms which live as parasites within the body. In all but a few cases these organisms are now definitely known, their habits understood, their means of transmission and multiplication worked out. What such knowledge means to the human race can hardly be overestimated. In the 14 th century Europe was swept by an epidemic of plague which destroyed probably one-fourth of her entire population - something like $25,000,000$ people. That a similar epidemic would have swept over the United States in the present century had it not been for modern scientific knowledge of the cause and means of transmission of plague, which made it possible to nip the epidemic in the bud in San Francisco and New Orleans, is reasonable to believe. In the latter part of the 19th century the French attempt to build a canal at Panama failed dismally after a stupendous loss of life from yellow fever and malaria. Shipload after shipload of laborers, engineers, nurses and doctors were sent to the great "white-man's graveyard," the majority to succumb in a few weeks or months to these diseases, at that time uncontrollable. In the early part of the 20th century, by exterminating malaria and yellow fever on the Canal Zone, through the application of the knowledge which had been gained in the intervening years, the Americans made possible the building of the Panama Canal. In an incredibly short time this zone was transformed from a veritable pest hole to one of the healthiest places in the world, and incidentally the "conquest of the tropics," previously looked upon as a more or less hopeless task, was shown to be not only possible but profitable. To quote another example, throughout the history of the world typhus fever has hovered like a death dragon over nearly every army camp ever assembled, and has followed in the wake of war to add the last touch of horror and desperation to the inhabitants of the countries involved. In the present unprecedented war only those countries which have not kept abreast of the times in the application of scientific knowledge have suffered seriously from this terrible scourge. Were it not for the application of modern knowledge the horrors of the present war would have been even more awful than they are now. 
A decade or two ago a child's reader contained the following lines:

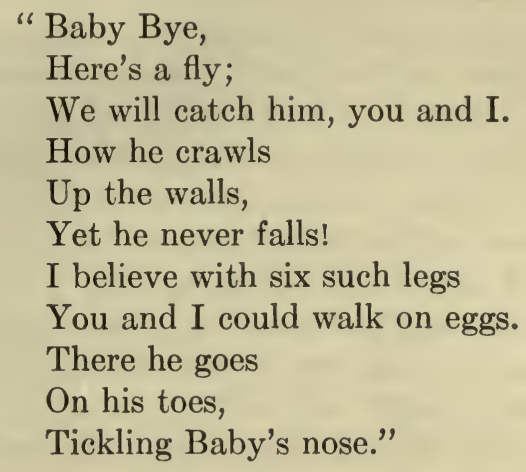

What a contrast to this attitude toward the housefly are our present-day fly-swatting campaigns, our crusades against possible breeding places of flies, and our education of the public by slogans, placards, lectures, magazine articles and books regarding the filthy habits and disease-carrying propensities of this selfsame housefly!

But let us not think for a moment that the battle is won. Not only are there some diseases which still baffle our attempts to cure them or to control them, or even to understand their nature, but those which we already know how to control are by no means subdued. Plague continues to take a toll of life in India amounting to at least several hundreds of thousands a year; malaria even today destroys directly or indirectly millions of people every year and more or less completely incapacitates many millions more; syphilis is yet one of the principal causes of insanity, paralysis, still-births and barrenness in the civilized world, and is estimated to exist in 10 per cent of the population of the United States, i.e., in about 10,000,000 people; hookworms still infect and render more or less imperfect over half a billion people in the world; - and these are all diseases the causes of which are known, the means of transmission recognized, methods of prevention understood, and the cure of which, with the exception of plague, is entirely possible.

It is evident that the crying need of the present time is not so much additions to our knowledge of the cause, control and prevention of diseases, much as this is to be hoped for, as it is 
the efficient application of what we already know. Popular ignorance of diseases, even such common ones as malaria and syphilis, is nothing short of appalling. This ignorance is by no means confined to the poorly educated masses; it is widespread among educated, college-bred people, and, piteous as it may seem, is characteristic of many professional men, among them even physicians bearing good reputation. There are a number of causes for this unfortunate condition. Many physicians of the old school have been so busy or so unprogressive that they have never attempted to add to or modify the knowledge they had when they first took up the medical profession 20 or 30 years ago; people with erroneous or distorted views of things publish their ideas in newspapers or magazines as authoritative statements, and thus unmeaningly mislead the enormous number of people who swallow such newspaper articles without even a flicker of hesitation; quack doctors, those hellish buzzards who prey upon the innate gullibility of the greater part of the human race, willfully mislead and scatter at random the seeds of misinformation which have held back the progress of sanitation and health to a pitiful extent and have borne as their fruit sorrow, misery and suffering; and, finally, such is the conservativeness, or rather imperviousness, of our species that a new idea requires, often, not decades but centuries to penetrate thoroughly the popular mind. It is nearly 60 years since Darwin brought the theory of evolution into serious consideration and showed the folly of belief in special creation, yet it is no exaggeration to say that a very large majority of people at the present time do not believe in evolution. It is 250 years since the idea that living organisms do not spontaneously spring into existence from non-living matter was first promulgated, and nearly 60 years since the last vestige of possibility was torn from the theory of spontaneous generation, yet even today the prevalence of such beliefs as that "horse-hair snakes" develop out of horse hairs in water is nothing short of astonishing. It is 120 years since Jenner proved the efficacy of vaccination against smallpox, yet there exist at the present time numerous anti-vaccination societies whose sole purpose is to denounce vaccination as an impractical and illogical proceeding. How can we expect popular belief in the mosquito transmission of malaria which was demonstrated only 20 years ago! 
The importance of the study of parasites in connection with human disease to every community in the world is becoming more and more obvious, even to those relatively free from parasitic diseases. There are those who think that such diseases as kala-azar, sleeping sickness, Oriental fluke infections and many other local or "tropical" diseases are of no vital importance except to inhabitants of the countries directly influenced or to travelers through these countries. That the importance of such parasitic diseases is far greater than this is obvious from the fact that, with modern facilities for communication and with the extent of foreign immigration at the present time, there is no part of the world so remote that the things which affect it may not also affect any other part of the world if conditions are suitable.

There is probably no common exotic infection which is not repeatedly brought into the United States through immigration ports, especially in ports where the most thorough medical inspection of immigrants is not made. In the port of San Francisco alone over 50 per cent of 6428 Orientals whose fæces were examined in the course of a little over two years were infected with hookworms, each one capable of starting a new center of infection in a previously free community. According to Dr. Billings of the U. S. Immigration Service, during the "Hindu Invasion" of the Pacific Coast of the United States in 1911, about 90 per cent of all arriving Hindus were found to be infected with hookworms. It is unfortunate that even at the present time a considerable proportion of arriving Orientals cannot, under the immigration law, be subjected to medical examination.

The possibility or probability of other diseases becoming established in places not before troubled by them is a subject of vital importance to any community or nation. Some of them have already become established in places which were formerly free. The fact that acquaintance with these exotic diseases is lacking in the new territory, their nature not understood, means of curing them unfamiliar, and means of prevention of spread unknown, often results in much needless suffering and loss of life. Furthermore, many infections are much wider in distri-. bution than has formerly been supposed. To cite one example, amebic dysentery, and liver abscess which is often the sequel to 
it, occurs over the greater part of the United States, yet not only are the people unfamiliar with the disease and its cause, but most physicians are unacquainted with it and do not know how to diagnose or treat it.

The history of modern medicine, so far as infectious diseases are concerned, is nothing more nor less than the history of parasitology in its broad sense, including bacterial and fungous parasites as well as animal parasites. Previous to the beginnings of our knowledge of the existence of microscopic parasites, and of the effects produced by them, nearly all diseases were interpreted as visitations from angry deities, as the work of demons or as the effect of supernatural causes. Such ideas are still prevalent in those parts of the world where bacteriology and parasitology have not yet penetrated.

With the exception of the superficial acquaintance which the ancients had with external parasites and a few parasitic worms, parasitology began about the middle of the 16th century when Fracastorio, an Italian, published his belief that disease was due to invisible organisms multiplying within the body. With the invention of the microscope by the Dutch lens-grinder, Leeuwenhoek, actual observation of microscopic organisms became possible, and this famous pioneer in science observed, in 1675, " animalculæ" in rain-water, putrid infusions, saliva, and diarrheal excretions, and made illustrations of them. Based on these scanty observations; the idea that all diseases were caused by these "animalculæ" became rampant during the succeeding century. In 1762 Plenciz, a physician of Vienna, apparently with the tongue of a prophet, expressed the idea that all infectious diseases were caused by living organisms, that there was a special "germ" for each disease, that the incubation period of diseases was due to the time necessary for the infecting organisms to multiply, and that the organisms might be conveyed through the air as well as by direct or indirect contact. In the 18th and 19th centuries there was much controversy as to the origin of "germs" and the possibility of their spontaneous generation from putrefying matter. A belief in the origin of living organisms only from pre-existing organisms was first expressed by the Italian Redi, in 1668, but scientific proof of it came much later. Experiments by Spallanzani in 1769, Schulze in 1836, Schwann in 1839, Schroeder and von Dusch in 
1854, and finally Pasteur in 1860 removed one by one the last straws to which the sinking theory of spontaneous generation was still clinging.

With the exception of tapeworms and some intestinal roundworms, one of the first worm parasites to be discovered in man was Trichinella, in its larval stage in the muscles, this discovery being made by Peacock in 1828 . The hookworm was discovered by Dubini in Italy in 1838; the blood fluke and the dwarf tapeworm by Bilharz in Egypt in 1851; Filaria (larvæ) by Demarquay in 1863; the Chinese human liver fluke by MacConnell in India and MacGregor in Mauritius in 1874; the adult Filaria by Bancroft in 1876 . The first parasitic protozoan to be discovered and recognized as such was the ciliate, Balantidium coli, a cause of dysentery, discovered by Malinsten in 1856. The spirochæte of relapsing fever was discovered by Obermeier in 1873; the dysentery ameba by Lösch in 1875; the malaria parasite by Laveran in 1880; the sleeping sickness trypanosome by Forde and Dutton in 1901; the Leishman bodies of kala-azar by Leishman, and independently by Donovan, in 1903; the spirochæte of syphilis by Schaudinn in 1905.

Knowledge of the complicated life histories characteristic of many parasites practically began with Zenker's demonstration of the life cycle of Trichinella in 1860 and Leuckart's experimental proof of the strange life cycle of the beef tapeworm in 1861 . In 1874 Weinland discovered the snail in which the liver fluke develops, though the relation of flukes to molluses had been previously suspected. In 1879 the epoch-making discovery of the rôle of the mosquito in the development of filarial worms was made by Manson and the science of Medical Entomology was born. This discovery has been so far reaching in its results and it has revolutionized preventive medicine to such an extent that it may justly be looked upon as marking the beginning of a new era in the history of preventive medicine, comparable with the discovery of the germ causation of disease. One of the first and certainly the greatest outcome of the discovery was the discovery by Ross in 1898 of the relation between mosquitoes and malaria. Other important discoveries concerning life histories and modes of infection quickly followed. The transmission of trypanosome diseases by tsetse flies was discovered by Bruce in 1893; the relation of mosquitoes to yellow fever by the American 
Yellow Fever Commission in 1900, and to dengue by Graham in 1902; the relation of ticks to African relapsing fever by Dutton and Todd, and independently by Koch, in 1905; the relation of ticks to spotted fever by Ricketts in 1906; the relation of lice to typhus by Nicolle and his fellow workers in Algeria in 1909, and independently by Ricketts and Wilder and by Anderson and Goldberger in Mexico in the same year (published in 1910); the relation of cone-noses to a South American trypanosome disease by Chagas in 1909; the life history of blood flukes by Leiper in 1914 and 1915; and the rôle of crabs as second intermediate hosts of lung flukes by Nakagawa in 1916 .

The evolution of knowledge concerning the treatment of parasitic diseases has proceeded along two distinct lines, one, destruction of the parasites by drugs which are more or less specifically poisonous to them, the other by vaccination or immunization. One of the first specific drugs known was quinine for malaria. The use of cinchona bark from which quinine in its various forms is manufactured is said to have originated with the Indians of Ecuador, and to have been introduced into Europe by Spaniards in 1642. The sulphate of quinine, which is the form of the drug in commonest use now, was first used in 1840 . In 1880 Bozzolo, an Italian, first introduced thymol as a remedy for intestinal worms, especially hookworm, and this has been considered a standard and almost specific cure for hookworm until within the last two years, when oil of Chenopodium has been substituted for it to a large extent. The next specific drug of great importance to be discovered was salvarsan for spirochætes, discovered by Ehrlich in 1905. In the same year atoxyl, one of the most efficient remedies yet discovered for trypanosome diseases, was discovered by Thomas. Emetin was discovered to be a specific remedy for amebic dysentery by Rogers in 1913 as the result of Vedder's work with ipecac, from which emetin is manufactured. In 1914 tartar emetic, previously used as an alternative for arsenic compounds (chiefly atoxyl) against trypanosomes, was discovered by Vianna to be a wonderfully efficient specific remedy for the severe South American leishmaniasis, and was subsequently found to be specific for all Leishmania diseases.

Treatment and prevention of disease by immunization has experienced a wonderful development in the past 35 or 40 years. 
Some of the phenomena of natural acquired immunity were of course familiar even to the ancients, and people have practiced for centuries exposing themselves to diseases at convenient times in order to acquire subsequent immunity. Jenner in 1797 devised vaccination with cowpox to give immunity to smallpox. It was not until 1880 and 1881 that Pasteur discovered the possibility of producing immunity by inoculation of germs artificially rendered harmless or relatively harmless, or by the inoculation of the strained excretions of the bacteria as they exist in pure cultures.

From Pasteur's epoch-making discoveries has arisen in the last 25 years the science of immunology. Although up to the present time the successful use of vaccinations or inoculations for cure of, or protection from, disease germs has been applied chiefly to bacterial diseases, the same principles of immunity apply to diseases caused by animal parasites and we may confidently expect in the not distant future a great extension of this relatively new field of medicine to diseases caused by animal parasites.) It has already been applied successfully to some spirochæte diseases, and to some trypanosome diseases. The difficulty of growing many animal parasites in cultures has largely held back progress along this line, and it is only recently that much advancement has been made. Only a few years ago culturability and non-culturability were believed to be distinguishing characteristics between bacteria and Protozoa. Although methods for growing pure cultures of bacteria artificially were devised and used by Pasteur in 1858, and greatly improved by Robert Koch 15 or 20 years later, it was not until 1903 that the artificial cultivation of trypanosomes was accomplished by two American workers, Novy and MacNeal. In 1905 Rogers in India succeded in cultivating the Leishman bodies of kala-azar, and thus established their relationship to certain flagellated parasites of invertebrate animals. Since then other investigators have succeeded in the cultivation of other parasitic protozoans, the latest important accomplishment along this line being the successful cultivation of spirochætes by Noguchi in 1910-12, and of malarial parasites by Bass and Johns in 1913. As yet no pathogenic amebæ have been successfully cultured, probably due to their dependence on the presence and action of certain kinds of bacteria. 
Even more important, if anything, than ability to grow parasites on artificial cultures in order to experiment with them, is ability to transplant them into animals which can be experimented on. Only by wholesale animal experimentation, carried on patiently and persistently, for years sometimes, could many of the great medical victories of the past 25 years have been won. To quote from MacNeal, "The importance of experimentation upon animals in the development of our knowledge concerning disease-producing microörganisms can hardly be over-estimated. ... Only in this way (by the use of animals in considerable numbers) has it been possible to discover the causal relation of bacteria to disease, and the way in which diseases are transmitted. ... The inoculation of animals also provides accurately controlled material for studying the course and termination of the disease as well as the gross or microscopic lesions produced by it." One can hardly help feeling bitter against those well-meaning but misguided individuals who publicly denounce and endeavor to minimize the unselfish and tireless labors of scientists who have made possible the alleviation and prevention of so much human misery and suffering. To quote from Dr. W. W. Keen in speaking of the results of Dr. Flexner's experiments on monkeys and guinea-pigs with one of the most deadly human diseases, cerebrospinal meningitis: " which was the more cruel, Dr. Flexner and his assistants who operated on 25 monkeys and 100 guinea-pigs with the pure and holy purpose of finding an antidote to a deadly disease and with the result of saving hundreds, and in the future thousands on thousands of human lives; or the women who were 'fanned into fury ' in their opposition to all experiments on living animals at the Rockefeller Institute ' no matter how great the anticipated benefit?'

"If these misguided women had had their way, they would have nailed up the doors of the Rockefeller Institute, would have prevented these experiments on one hundred and twenty-five animals, and by doing so would have ruthlessly condemned to death for all future time five hundred human beings in every one thousand attacked by cerebrospinal meningitis!

"If your son or daughter falls ill with the disease, to whom will you turn for help - to Flexner or to the anti-vivisectionists?" 
It is inconceivable that any anti-vivisectionist, if bitten by a rabid dog, would not hasten to be given a Pasteur treatment to prevent the horrible death from hydrophobia which would otherwise almost inevitably result, or if stricken with syphilis would not submit to treatment with salvarsan in order to prevent the probable ruin not only of his own life, but also of the lives of his life-mate and of his unborn children. There is little thought then of the blood of the monkeys, guinea-pigs, or other animals with which the God of Knowledge was paid to make such treatments possible!

The discoveries mentioned in this brief résumé of the history of parasitic diseases are but a few of the more conspicuous milestones on the path of progress of modern medicine as related to animal parasites. They may be likened to the posts of a fence, while the hundreds of other discoveries, less striking in themselves, perhaps, but nevertheless necessary, correspond to the pickets. The posts are useless without the pickets as are the pickets without the posts. There is not one of the great outstanding discoveries in the field of parasitology and preventive medicine which could have been made without the aid of the less illustrious accomplishments of many other scientists. Our present ability to cope with and control disease is due not alone to the great work of such men as Manson, Laveran, Ross, Pasteur, Koch, Reed, Schaudinn and Ricketts, but also to the careful, pains-taking work of thousands of other investigators, who, often without any semblance of the honor and recognition which they deserve, and perhaps even under the stigma of public denunciation, work for the joy of the working and feel amply repaid if they add a few pickets to the fence of scientific progress. 


\section{CHAPTER II}

\section{PARASITES IN GENERAL}

Definition. - According to the Standard Dictionary, a parasite is a living organism, either animal or plant, that lives on or in some other organism from which it derives its nourishment for the whole or part of its existence. In the following pages only those parasites which belong to the animal kingdom are taken into consideration. The vegetable parasites, chiefly bacteria and fungi, are dealt with only incidentally.

It is often difficult to draw a sharp line between parasites and predatory animals; a panther is unquestionably a predatory animal, and a tapeworm is unquestionably a parasite, but a mosquito or horsefly might well belong in either category. It is usual to look upon an organism as a parasite when it habitually preys upon other organisms which are superior to it in size and strength. In accordance with this view all animals which habitually prey upon man, other than a few which occasionally attack and overcome him by superior physical prowess, may be considered as parasites and are so treated here.

The state of dependence of an inferior on a superior organism probably arose very soon after life began to differentiate in the world. It would be difficult, if not impossible, to explain step by step the details of the process of evolution by which some of the highly specialized parasites reached their present condition. In some cases parasitism has probably grown out of a harmless association of different kinds of organisms, one of the members of the association, by virtue, perhaps, of characteristics already possessed, developing the power of living at the expense of the other, and ultimately becoming more and more dependent upon it.

Kinds of Parasites. - There are all kinds and degrees of parasitism. There are facultative parasites which may be parasitic or free-living at will, and obligatory parasites which must live on or in some other organism during all or part of their lives, and which perish if prevented from doing so. There are inter- 
mittent parasites which visit and leave their hosts at intervals. Some, as mosquitoes, visit their hosts only long enough to get a meal, others, as certain lice, leave their hosts only for the purpose of moulting and laying eggs, and still others, as the cattle tick, Margaropus annulatus, never leave except to lay eggs. There are parasites which pass only part of their life cycles as parasites; botflies, for instance, are parasitic only as larvæ, hookworms only as adults. Some organisms live parasitically in two or more different animals, often of widely different species, in the course of their life histories. Such, for instance, are the filarial worms and numerous protozoan parasites, which begin life in a vertebrate animal, continue it in an insect, and finish it in a vertebrate again; the tapeworms, which begin life in certain vertebrates and finish it in other individuals of the same or different species; the flukes, which begin life as free-living embryos, continue it through two or more asexual generations in particular species of snails, become again free-living or else parasitize second intermediate hosts such as crabs or fishes, and finally gain admittance to their ultimate vertebrate hosts. There are permanent parasites which live their whole lives, from the time of hatching to death, in a single host, but in which the eggs, or the corresponding cysts in the case of Protozoa, must be transferred to a new host before a second generation can develop. Such are many intestinal protozoans and round worms. The final degree of parasitism is reached, perhaps, in those parasites which live not only their whole lives, but generation after generation on a single host, becoming transferred from host to host only by direct contact. Such are the scab mites and many species of lice. There is every gradation among all the types of parasites mentioned above, and a complete classification of parasites according to mode of life would contain almost as many types as there are kinds of parasites.

It is sometimes convenient to classify parasites according to whether they are external or internal. External parasites, as the name implies, are those which live on the surface of the body of their hosts, sucking blood or feeding upon hair, feathers, skin or secretions of the skin. Internal parasites live inside the body, in the digestive tract or other cavities of the body, in the organs, in the blood, in the tissues, or even within the cells. No sharp line of demarcation can be drawn between external and internal 
parasites since inhabitants of the mouth and nasal cavities and such worms and mites as burrow just under the surface of the skin might be placed in either category.

Effects of Parasitism on Parasites. - The effect of parasitism is felt by both parasite and host. There is a sort of mutual adaptation between the two which is developed in proportion to the time that the relationship of host and parasite has existed. It is obviously to the disadvantage of internal parasites to cause the death of their host, for in so doing they destroy themselves. It is likewise to the disadvantage of external parasites, not so much to cause the death of their host, as to produce such pain or irritation as to lead to their own destruction at the hands of the irritated host. It is interesting to note, for instance, that insects which depend to a large extent on man for food have less painful bites than do insects which only occasionally or accidentally bite human beings. Together with a softening down of the effects of the parasite on the host, there is a concomitant increase in the tolerance of the host to the parasite. It is a wellestablished fact that a disease introduced into a place where it is not endemic, i.e., does not normally exist, is more destructive than in places where it is endemic. The variations in susceptibility to parasites are directly connected with the subject of immunity, which will be discussed later. An organism and the parasites which are particularly adapted to live with it may, in a way, be looked upon as a sort of compound organism. Those parasites which live part of their life in vertebrate animals and part in other parasites of these animals, as lice, ticks and biting flies, are absolutely dependent for their existence on the relationships of the vertebrates and their parasites, and form a sort of third party to the association.

Aside from the toning down of their effects on the host, parasites are often very highly modified in structure to meet the demands of their particular environment. As a group, parasites have little need for sense organs and seldom have them as highly developed as do related free-living animals. Fixed parasites do not need, and do not have, well-developed organs of locomotion, if, indeed, they possess any at all. Intestinal parasites do not need highly organized digestive tracts, and the tapeworms and spinyheaded worms have lost this portion of their anatomy completely. On the other hand, parasites must be specialized, often to a very 
high degree, to adhere to or to make their way about in their particular host, or the particular part of the host, in which they find suitable conditions for existence. Examples of specializations of external parasites are the compressed bodies of fleas, permitting them to glide readily between the hairs of their hosts; the backward-projecting spines of fleas, which are of much assistance in forcing a path through dense hair by preventing any back-sliding; the clasping talons on the claws of lice; the barbed probosces of ticks; and the tactile hairs of mites. In these same parasites can be observed marked degenerations in the loss of eyes and other sense organs, absence of wings, and, in some cases, reduction of legs. Internal parasites are even more peculiar combinations of degeneration and specialization. They possess all sorts of hooks, barbs, suckers and boring apparatus, yet they have practically no sense organs or special organs of locomotion, a very simple nervous system, and sometimes, as said before, a complete absence of the digestive tube.

Still more remarkable are the specializations of parasites, in their reproduction and life history, to insure, as far as possible, a safe transfer to new hosts for the succeeding generations. Every structure, every function, every instinct of many of these parasites is modified, to a certain extent, for the sole purpose of reproduction. A fluke does not eat to live, it eats only to reproduce. The complexity to which the development of the reproductive systems may go is almost incredible. In some adult tapeworms not only does every segment bear complete male and female reproductive systems, but it bears two sets of each. The number of eggs produced by many parasitic worms may run well into the hundreds of thousands. The complexity of the life history is no less remarkable. Not only are free-living stages interposed, and intermediate hosts made to serve as transmitting agents, but often asexual multiplications, sometimes to the extent of several generations, are passed through during the course of these remarkable experiences.

Effects of Parasites on Hosts. - The effects of parasites on their hosts are almost as numerous and as varied as are the kinds of parasites, and vary besides with the susceptibility of the individual concerned, his physical condition, and complication with other infections. In general it may be said that a parasite damages its host in one or more of three ways: (1) by robbing it of food 
which has not yet been assimilated and utilized, (2) by mechanically injuring its tissues or organs, (3) by the formation of excretions or "toxins," which act as poisons.

The first method of damage is of least importance, though it is obvious that the amount of food abstracted by some parasites, e.g., large tapeworms which may reach a length of several yards and grow at the rate of several feet a month, must be considerable.

Much more serious are the various kinds of mechanical injury to tissues or organs. This damage is done by the blood-sucking parasites, such as hookworms, flukes, leeches and blood-sucking arthropods and the tissue-devouring forms, such as dysenteric amebæ, malaria parasites, lung flukes, and fly maggots, which may not only devour the cells of the body, but may also cause hemorrhages, give portals of entry for other infections, and perforate the intestine. Here also belong the obstructing parasites, which by their presence block bloodvessels, as do subtertian malaria parasites or blood flukes; stop up lymph vessels, as do adult filarial worms; or partially or completely close up such ducts as the bile duct and pancreatic duct, as do liver flukes. There are also parasites which damage and inflame the tissues by boring through them, as does Trichinella, the guinea-worm, itch mites and fly maggots.

The third type of injury, by excretion of toxic substances, is done to some extent by practically all parasites. In external parasites the damage is usually done by an excretion, usually of the salivary glands, which prevents the coagulation of blood, and tends to inflame the tissues with which it comes in contact. In the case of internal parasites the toxic substances are probably in most cases the waste products of the parasites, voided into or absorbed by the blood or neighboring tissues. In many cases these toxins have specific actions on particular tissues or organs, so that parasites in one part of the body may do their chief damage to an entirely different part. Intestinal worms, for instance, may produce considerably greater derangements of the blood or of the nervous system than of the intestine; the trypanosome of Chagas' disease produces, by means of toxins, specific effects on the thyroid gland and gives rise to the symptoms which result from interference with the gland, even though the parasites may not be located in the gland itself; the bite of certain ticks along the line of the spinal cord or on the middle 
line of the cranium produces a specific effect on the motor nerves, causing paralysis, presumably through the action of salivary secretions or of the excretion from the coxal glands; the amebæ of pyorrhea, or the bacteria associated with them, which infect the teeth and gums give rise to such symptoms as rheumatic pains in the joints, anemia and a disturbance of digestion. In fact it may be said that a very large number of diseases or abnormal conditions which were once attributed to purely physical causes, such as imperfections in the organization of the body, or which have been accepted merely as common derangements of the human machine for which no direct cause could be found, have been traced to the effect of particular parasites located, perhaps, in some unsuspected part of the body. We are daily widening the scope of this phase of pathology, and this is one of the main reasons for the present important position of parasitology among the medical sciences.

Modes of Infection and Transmission. - The portals of entry and means of transmission of parasites is a question of the most vital importance from the standpoint of preventive medicine. In the past few decades wonderful strides in our knowledge along these lines have been made, but there is much yet to be found out.

With a very few exceptions animal parasites do not exist in air and dust as do many vegetable parasites, although some spirochætes, coughed from the lungs or throat, may infect other individuals by being breathed in, and the granules formed by some of these spirochætes may be blown about with dust and thus infect in the manner of many bacteria.

Many parasites may be spread by direct or indirect contact with infected parts, e.g., the spirochætes of syphilis and yaws, the mouth amebæ, the parasites of Oriental sore, itch mites and, of course, free-moving external parasites. The parasites of the digestive system and of other internal organs gain entrance in one of two ways. They may bore directly through the skin as larvæ, e.g., hookworm. More commonly they enter the mouth as cysts or eggs, e.g., dysentery amebæ and Ascaris; as larvæ, e.g., pinworm; or as adults, e.g., leeches. Access to the mouth is gained in many different ways, but chiefly with impure water, with unwashed vegetables fertilized with " night soil," or with food contaminated by dust, flies or unclean hands. The para- 
sites of the blood or lymphatic systems usually rely on biting arthropods (insects, ticks and mites) to transmit them from host to host, and it is in this capacity, i.e., as transmitters and intermediate hosts of blood parasites, that parasitic arthropods are of such vast importance (see p. 322).

Geographic Distribution. - The geographic distribution and dispersal of parasites is another subject which has received much fruitful attention in recent years. Parasites, like other organisms, are dependent upon certain physical conditions of their environment in order to thrive. One of the most important limitations on the dispersal of a parasitic disease is the distribution of suitable hosts. Some parasites can live with apparently equal vigor in a large number of hosts, others are confined to a few or to one only. A double limitation is placed on parasites which require two hosts in order to complete their life history; they obviously cannot exist beyond the territory where both hosts exist together. The local as well as geographic distribution of the hosts is, of course, effective in limiting the distribution of the parasites. In the case of human parasites, the alternate host is practically the only limiting factor. The geographic distribution of human sleeping sickness is coincident with the distribution of certain species of tsetse flies; the distribution of yellow fever nowhere extends beyond the range of a certain species of mosquito; Rocky Mountain spotted fever is geographically limited by the distribution of a certain species of tick. The accidental or gradual extension of the range of one of these intermediate hosts is likely to be followed by an extension of the disease carried by it. It sometimes happens that a strain of a certain parasite establishes itself in a new host, thus often greatly extending the territory which it affects, and this is a possibility which must always be remembered and watched for. The trypanosome of Rhodesian sleeping sickness, for instance, is very possibly a race of Trypanosoma brucei, which is common in domesticated and wild game animals in a large portion of Africa. Some slight alteration in the nature of the parasite has made it possible for it to affect human beings and thus give rise to a new disease. A somewhat different situation is presented in the case of Rocky Mountain spotted fever, the parasite of which has not yet been discovered. In nature apparently only one species of tick acts as an intermediate host, 
though experimentally other ticks may become infective. There is obviously a constant possibility of the establishment of the disease in other species of ticks and thus of greatly widening the area affected.

Temperature is an important limiting factor for those parasites which can be directly influenced by it, as external parasites and those which are free-living during a part of their existence. In Mexico, for instance, human lice are entirely absent from the hot coastal plains, though abundant on the high central plateau. Hookworms, which are free-living in their young stages, are confined to a broad strip around the tropical and warm temperate portions of the world, and occur outside these limits only in short-lived epidemics during the warm part of the year. Such parasites as bots and screw-worms are equally exposed to the influence of climate, since they are free-living in the adult stage.

Some parasites are limited by other environmental conditions. In the case of such intermittent external parasites as mosquitoes, biting flies and cone-noses it is obvious that not only temperature and humidity, but also the presence of suitable breeding places and of suitable haunts during resting times must be necessary for their continued existence. Again, the local distribution of hookworms is determined, to a large degree, at least, by the nature of the soil. These worms abound where sandy soil occurs, but are rare or absent where there is only limey or clayey soil.

Natural Immunity. - As has already been pointed out, when a parasite is introduced into a region where it was previously unknown, or, what amounts to the same thing, new hosts are introduced into its territory, its ravages are usually worse than in places where it has been endemic for a long time. The hosts and parasites of a given region come to a point of equilibrium. The host becomes largely immune to the effects of the parasite, and yet harbors it in sufficient numbers to form a reservoir for it, and thereby acts as a "carrier." In some cases a total or partial immunity is built up in youth, when the power of resistance to parasitic invasion is usually high; in other cases it is the result of a long struggle extending through many generations. A good example of immunity acquired in youth is found in the case of yellow fever, and of partial immunity, gained through many generations of adaptation, in the case of hookworms in negroes. The terrible destruction wrought by sleeping sickness 
when introduced into virgin territory in Uganda is a good example of the results which may come from such an introduction: in districts where sleeping sickness has existed for a long time the death rate from it is often only two or three per thousand, whereas in one district in Uganda the population was reduced from 300,000 to 100,000 in about seven years. It is not improbable that the extinction of many of the striking types of animals which dominated the earth in past geologic ages may have been brought about by the sudden appearance of or exposure to new and deadly parasites; only those forms of life which were able to resist the onslaught of the parasites remained to continue the course of evolution.

This leads us to a consideration of the remarkable facts of immunity. The power of the blood of vertebrate animals to react against invading organisms or poisons by producing substances which will destroy them is one of the most wonderful adaptations in all the realm of nature. Though the details of the reaction are still unknown, the chemical substances concerned still undetermined, and many of the influencing factors not yet understood, yet the progress in our knowledge of the mechanism of acquired immunity has taken great strides since Pasteur first placed the development of immunity on a scientific basis not quite 40 years ago. There are several ways in which the body may react against parasites. One method is by the activity of the large free-moving white blood corpuscles, which actually capture and devour the parasites after the manner of predaceous protozoans. This, of course, can be done only in case of very small parasites, such as bacteria and Leishman bodies. Apparently the parasites first must be rendered digestible to the white blood corpuscles by the presence of an accessory substance in the blood, known as an opsonin. This substance may be thought of as acting like a sugar coating on a bitter pill, though its effect is more analogous to that of cooking on starch, i.e. increased digestibility. Opsonins are normally present in the blood but increase as a reaction to the presence of parasites. The degree of development of opsonins in the blood, and consequent power of the white blood cells to capture and digest parasites, is known as the opsonic index.

Sometimes a number of cells work together to form a capsule around larger parasites, thus walling them in and limiting the 
sphere of their activity; this occurs in the case of Trichinella, filarial worms, larval tapeworms, fly maggots, etc. It occasionally happens that enzymes are developed which enable the surrounding cells slowly to digest the parasites thus imprisoned.

More important than these physical methods by which the body is able to combat parasites are the biological or chemical methods. One of the most wonderful adaptations in the animal kingdom is the ability of tissues, chiefly the blood and lymph of vertebrate animals, to react against invading cells, whether they be bacteria, Protozoa, blood corpuscles of unrelated animals, or other foreign cells, by producing substances known as antibodies which dissolve these cells, or cause them to agglutinate, i.e., clump together and lose any motile power they may have. The living body is also able to destroy the poisonous action of the excretions or toxins of parasites by the development of protective substances known as anti-toxins which form some sort of a chemical union with the toxins. Other poisonous products are rendered harmless by the formation of "precipitins" which have the special property of uniting with the toxic substances to produce insoluble and consequently harmless precipitates. Precipitins are found to react against any foreign protein, of parasitic origin or otherwise, introduced into the body. There is some question as to whether immunity reactions against animal parasites are exactly comparable with those against bacteria, but the difference is probably a matter of degree and not of fundamental nature. The higher organization of Protozoa and of other animal parasites enables some of them to react against the destructive influence of the serum by encysting, or by forming spores, and thus they are able to continue their existence in spite of the development of immunity, though in very limited numbers and with limited activity. The result is immunity without sterilization; in other words, although the body becomes more or less completely immune as far as suffering from the effects of the parasite is concerned, yet the parasites, limited in number and activity, still exist within it, and such a host becomes an "immune carrier." With few exceptions protozoan diseases are contrasted with bacterial diseases in this respect. The gradual development of anti-toxins, precipitins, etc., probably accounts to a large extent for the relative immunity which is 
developed against the effects of intestinal worms as well as against blood and tissue parasites.

Artificial Immunity. - In every case the reaction of the body against parasites invading it is due to the presence of some particular substance in the parasite which stimulates the body to react against it. This substance, whatever it may be, is called an antigen. The possibility of acquiring immunity without being subjected to the disease lies in the fact that the antigen is also present in parasites which have been weakened, by one of several methods, to such an extent as to be powerless to cause the usual symptoms. It may also be present in the dead parasites or even in the strained excretions from parasites, as obtained from pure cultures. Vaccinations, in the broad sense, are inoculations into the system of weakened or dead parasites or of their products. The body reacts against the harmless antigen thus injected and antibodies are built up just as if the disease had been actually passed through. Antibodies persist throughout life in the case of some parasites, for several years in others, and for only a short time in still others. When the efficacy of the naturally or artificially acquired immunity is gone, as determined by experimentation, a new vaccination must be submitted to in order to obtain protection. Thus yellow fever immunity, which, however, cannot be artificially produced, normally persists through life; smallpox immunity, as acquired by vaccination, for a number of years; and artificially acquired typhoid immunity for about three years.

Still another method of inducing immunity is possible. By rendering some susceptible animal very highly immune to a particular parasite by repeated inoculations of virulent germs, its serum becomes so charged with antibodies and so powerful in its action against the particular parasite involved, that a very small quantity of such serum injected into another animal or man is sufficient to give a "passive" immunity - passive because the second animal has taken no active part in the formation of antibodies. Such immune serum has been found of value in the prevention and cure of certain spirochæte diseases as well as a number of bacterial diseases. It has the advantage of causing no discomfort during the development of the immunity, but usually is of shorter duration than "active" immunity. 
The principles of artificial immunity, as remarked before, have only recently become understood, but the science of immunology is yearly becoming extended. As this paper is being written, experiments with preventive and curative inoculations against typhus fever and against infantile paralysis are being worked out and there is reason to hope that before another year dawns these two terrible diseases may be added to the already considerable list of diseases which can be prevented by artificially produced immunity. Smallpox, rabies, cholera and diphtheria are some of the more important diseases whose guns have been unloaded by this means. While, as remarked before, comparatively little advance has been made in the application of the principles of immunity to animal parasites, yet there seems to be hope that in the coming years many diseases caused by Protozoa and worms may be conquered by further knowledge of immunology.

Anaphylaxis. - Mention should be made of the phenomenon of anaphylaxis, commonly defined as an exaggerated susceptibility to the poisonous effect of foreign substances in the blood, and to account for which many different explanations have been proposed. Based on extensive experimental work, Novy and De Kruif have recently (1917) offered a new and revolutionary explanation which is bound to be of far-reaching significance. According to these workers, normal circulating blood must be presumed to contain a substance, termed the "poison matrix," comparable in a general way with the substance in the blood known as fibrinogen. The latter substance, under certain conditions or in the presence of certain reagents, is transformed into fibrin, which forms a network of fibers in the meshes of which the blood corpuscles are caught, and by means of which the clotting of blood is effected. The same reaction which leads to the coagulation of blood also transforms the poison matrix into an actively poisonous substance or "anaphylatoxin," which produces the symptoms commonly known as anaphylaxis. Furthermore, it is shown that the transformation of the poison matrix into anaphylatoxin is induced or accelerated by the addition of almost any foreign substance to the blood, e.g., bacteria, trypanosomes, tissue cells, agar, peptone, starch, various salts, and even distilled water. In other words "the circulating blood, through a variety of agents, may be changed from a beneficial and harm- 
less to an injurious and poisonous state. The foreign substance is merely the trigger which, so to speak, ignites or explodes the charge contained within the blood vessels." In the case of socalled "specific anaphylaxis," in which anaphylactic poisoning results from the injection of particular kinds of organisms or toxins, as, for instance, the shock that results from typhoid vaccination of a person already immune to typhoid, the specific action is due to the production of ordinary anaphylatoxin by the interaction of an antibody, already developed, with the antigen which produced it. To cite another example, it has been shown that injection of the ground bodies of ox warbles into cattle which have been infested by even small numbers of these maggots produces an anaphylactic shock. According to the theory of Novy and De Kruif this would be explained as follows: the presence of warbles in the cattle causes the production of antibodies in the blood. Injection of warbles places large quantities of the antigen in the blood. Interaction of antibody and antigen produces a substance which transforms the poison matrix always present in the blood into anaphylatoxin, and the latter produces the symptoms of poisoning. The theory has recently been advanced that the severe effects of the bites of some bloodsucking arthropods, such as ticks, mites and blackflies, in which the first attacks are much milder than the later ones, may be in the nature of anaphylactic reactions. According to the theory of Novy and De Kruif, these effects would be produced by the formation of anaphylatoxin as the result of an interaction of an antigen in the arthropod's salivary secretions with an antibody already formed in the bitten individual.

Novy and De Kruif point out the possibility that substances inducing the formation of anaphylatoxin may be produced in a normal individual by some peculiarity of diet, exposure, obscure infections, etc., and while the amount of poison thus produced may not be sufficient to cause an acute anaphylactic shock, it may be sufficient to cause a subacute or chronic form of poisoning, leading to anemia, cachexia, etc. The significant statement is also made, and is apparently well supported, that a considerable part of the toxic effects of infectious diseases is in all probability due to the formation of anaphylatoxin. The socalled "endotoxins" supposed to be liberated in the blood by the disintegration of bacteria and other parasites possibly do 
not exist as specifically toxic substances. They may be substances which induce the formation of anaphylatoxin.

Should further investigation indicate that much of the toxic effects in various infectious diseases are really produced by a single substance, anaphylatoxin, the treatment of such diseases will be revolutionized. In addition to the giving of drugs to destroy the infecting organisms, an attempt must be made to find an agent to destroy anaphylatoxin in the blood and to prevent its further formation. Novy and De Kruif have shown that, in test tubes at least, alkali not only destroys but also prevents the further formation of this poison, and they suggest the use of alkalis in the treatment of conditions in which anaphylaxis may be playing a part. Already striking results have been obtained in severe cases in which anaphylactic poisoning was believed to exist, by the simple administration of sodium bicarbonate or sodium acetate in doses of from three to five grams dissolved in about a half a glass of water, given at intervals of half an hour to an hour. The object of this is to raise the alkalinity of the blood to a maximum level and to keep it there during the time anaphylatoxin is being formed. If confirmed by further investigation, these facts must be looked upon as among the most important discoveries in the entire history of medicine, and how far reaching their effects may be cannot now be even guessed. 


\section{PART I - PROTOZOA}

\section{CHAPTER III \\ INTRODUCTION TO PROTOZOA}

Place of Protozoa in the Animal Kingdom. - It is usual for zoölogists at the present time to divide the entire Animal Kingdom into two great sub-kingdoms, the Protozoa and the Metazoa. These groups are very unequal as regards number of species. The Metazoa include all the animals with which the majority of people are familiar, from the simple sponges and jellyfishes, through the worms, molluses, and the vast horde of insects and their allies, to the highly organized vertebrate animals, including man himself. The Protozoa, on the other hand, include only microscopic or almost microscopic animals, the very existence of which is absolutely unknown to the average lay person. Although some Protozoa are readily visible to the naked eye there are others, such as the yellow fever organism, which are too small to be seen even under the highest power of the microscope. There is no question but that in point of numbers of individuals the Protozoa exceed the other animals, millions to one; a pint jar of stagnant water may contain many billions of these minute animals. About 10,000 species of Protozoa have been described, but it is probable that there are thousands more which are not yet known to science.

The distinction between the Protozoa and Metazoa is based on a characteristic which is of the most fundamental nature. The Protozoa are animals which perform all the essential functions of life within the compass of a single cell. The Metazoa, on the other hand, are many-celled animals, with specialized cells set apart to perform particular functions. A protozoan cell, even though sometimes living in a colony of individuals which are all bound together, can live its life and reproduce its kind quite independently of any other cells, having in itself the powers of digestion, respiration, excretion and secretion, sensi- 
bility, motility and reproduction. Most metazoan cells, on the other hand, are so specialized for particular functions that, if separated from the other cells with which they are associated in the body, they die almost immediately.

The very fact of evolution makes it difficult to draw a sharp and fast line between two groups of organisms, even between such fundamentally different groups as the Protozoa and Metazoa. There are always border line exceptions which make the work of the systematic zoölogist at once difficult and interesting. In the case in hand there are colonial Protozoa in which all of the cells are not exactly alike, but have at least the beginnings of specialization. Some protozoans, such as the intestinal flagellate Giardia (or Lamblia), are composed, as adults, of essentially two cells instead of one. Such animals have been placed by some authors in a distinct order to which the name Diplozoa (double animals) has been applied. On the other hand, in the lowest metazoans, the sponges, there is only very limited specialization of the cells, while in the little-known animals which are designated as "Mesozoa" there is even less differentiation.

The distinction between Protozoa and Bacteria, though involving the distinction between animals and plants, is much more difficult. As we descend the evolutionary scale of plants and animals the usual distinctions between them disappear and it becomes difficult if not impossible definitely to place certain species in either the plant or animal kingdom. The possession of a distinct nucleus of some kind and some type of sexual reproduction are the characteristics which usually distinguish the Protozoa from the less highly organized Bacteria. Often, however, it is difficult to discover sexual phenomena, or to interpret them with safety, and the presence or absence of a nucleus is sometimes equally difficult to determine. In such cases peculiarities in life cycle, chemical reactions, staining properties and the like are resorted to as distinguishing characteristics. Most biologists are now inclined to group all of the single-celled animals and plants, including Bacteria, into one great group known as the Protista, a suggestion first made by Ernest Haeckel. The existence of such groups of organisms as the Spirochætes and the Piroplasmata, occupying intermediate positions between Protozoa and Bacteria, and of such groups as the chlorophyllbearing flagellates, occupying an intermediate position between 
protozoans and green algæ, makes such a group as the Protista appear both natural and convenient.

Structure. - A protozoan, in its simplest form, conforms to the usual definition of a cell - a bit of protoplasm containing

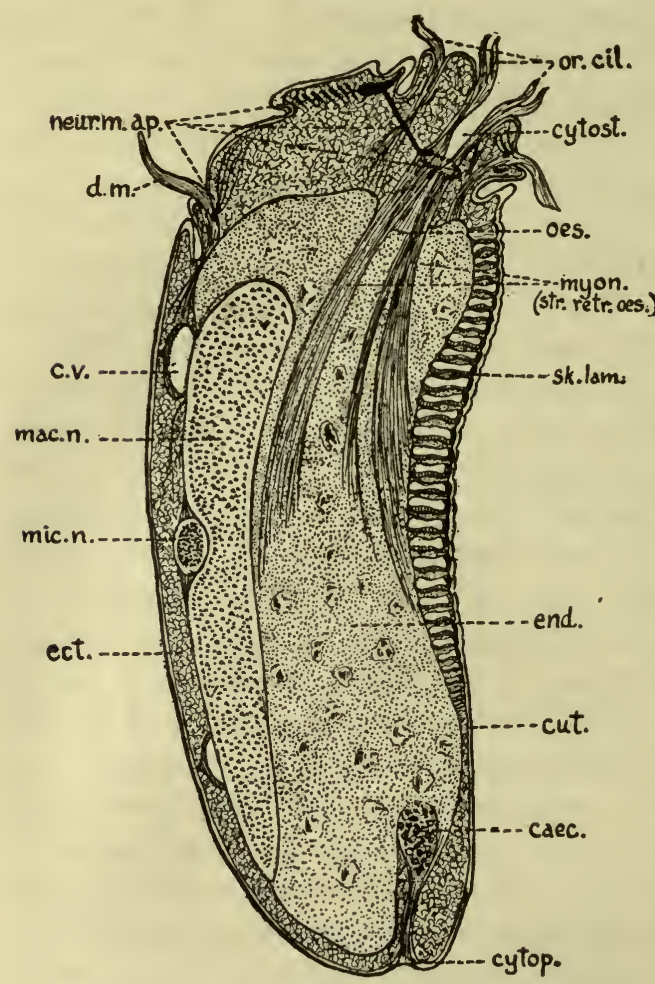

Fig. 1. A complex ciliate, Diplodinium ecaudatum, showing highly developed organelles; cæc., cæcum or rectal canal; cut., cuticle; c.v., contractile vacuole; cytop., cytopyge or cell anus; cytost., cytostome or cell mouth; d.m., dorsal membranelle; ect., ectoplasm; end., endoplasm; mac. n., macronucleus; mic. n., micronucleus; myon. (str. retr. œs.), myonemes, strands for retracting œsophagus; œs., œsophagus; or. cil., oral cilia; sk. lam., skeletal laminæ. $\times 750$. (After Sharpe.) there is no nucleus as such, though the essential substance of
the nucleus, chromatin, is always present, but in scattered par-
ticles.
The protoplasm of a protozoan is usually more or less clearly there is no nucleus as such, though the essential substance of
the nucleus, chromatin, is always present, but in scattered par-
ticles.
The protoplasm of a protozoan is ustally more or less clearly there is no nucleus as such, though the essential substance of
the nucleus, chromatin, is always present, but in scattered par-
ticles.
The protoplasm of a protozoan is usually more or less clearly there is no nucleus as such, though the essential substance of
the nucleus, chromatin, is always present, but in scattered par-
ticles.
The protoplasm of a protozoan is ustally more or less clearly a nucleus. S o m etimes there are two or more similar nuclei and in the majority of ciliates there are two nuclei which differ from each other both inform and function, a large " macronucleus" which is associated with the ordinary vegetative processes of the cell, and a small "micronucleus" which apparently is concerned only with sexual reproduction. In some protozoans nuclear material is extruded from the nucleusitself into the protoplasm outside where it floats about in the form of minute particles or granules known as chromidia, the latter sometimes having the power, under certain circumstances, of forming new nuclei. In some Protozoa 
divisible into an outer and inner zone, the ectoplasm and endoplasm, respectively (Fig. 1). There is no fundamental difference between these two layers of protoplasm, merely a difference in density. The ectoplasm is the less fluid and comparatively clear, while the endoplasm is more fluid and somewhat granular. The clearness of the differentiation between ectoplasm and endoplasm is sometimes useful in distinguishing species of protozoans, especially amebæ. The ectoplasm differs from the endoplasm
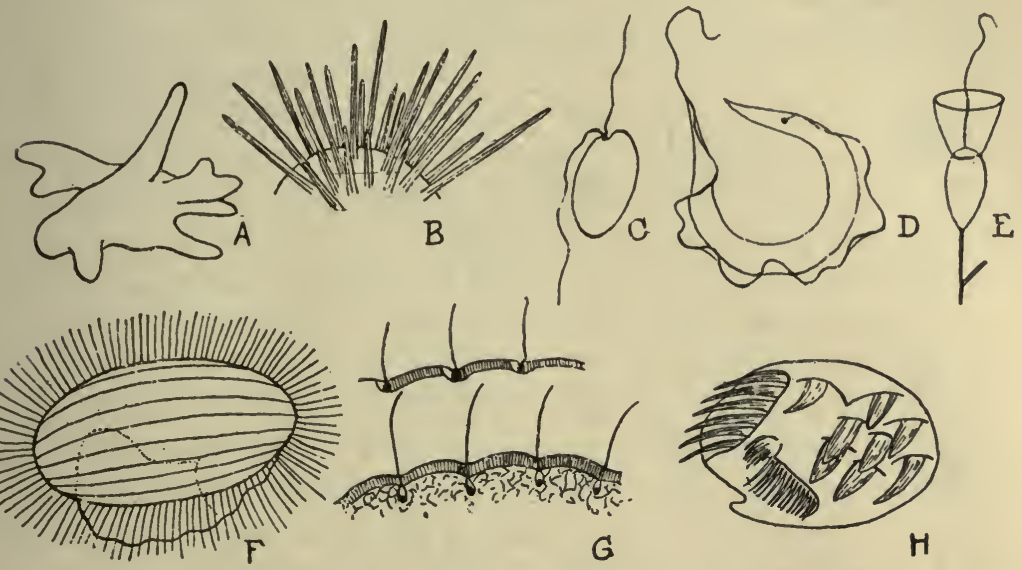

Fig. 2. Types of organs of locomotion in Protozoa; $A, A m c e b$ with pseudopodia; $B$, a heliozoan with "axopodia"; $C$, Bodo with free flagella; D, Trypanosoma with flagellum attached to undulating membrane; $E$, Choanoflagellate with flagellum and "collar"; F, Pleuronema with cilia and undulating membrane formed of fused cilia; $G$, modes of insertion of cilia; $H, A s p i d i s c a$ with cirri. (Figs. $F$ to $H$ from Calkins.)

in function as well as in appearance. The ectoplasm may be likened to the body wall and appendages of higher animals while the endoplasm may be compared with the viscera or internal organs. The endoplasm digests food and has the power of secretion and excretion, while the ectoplasm produces the various organelles for locomotion, food getting, oxygen absorption and special senses. The term "organelle" is used in place of " organ" for structures which are only parts of a single cell.

Organelles. - The organelles contained in a protozoan's body may be many and varied. Those connected with movement or locomotion differ in different groups and form the chief characteristic on which the usual classification into Sarcodina, Flagellata, 
Ciliata and Sporozoa has been based. The simplest type of movement is by means of simple outflowings of the body protoplasm known as pseudopodia (Fig. 2A). This is the common type of movement in one of the four great classes of Protozoa, the Sarcodina. In the Flagellata the organelles for locomotion are long lashlike outgrowths known as flagella (Fig. 2C), from one to eight or more in number. These originate from a particle of deep-staining material which is called the blepharoplast or "centrosome." In many parasitic flagellates there is another deep-staining body, of very variable size and form, known as the
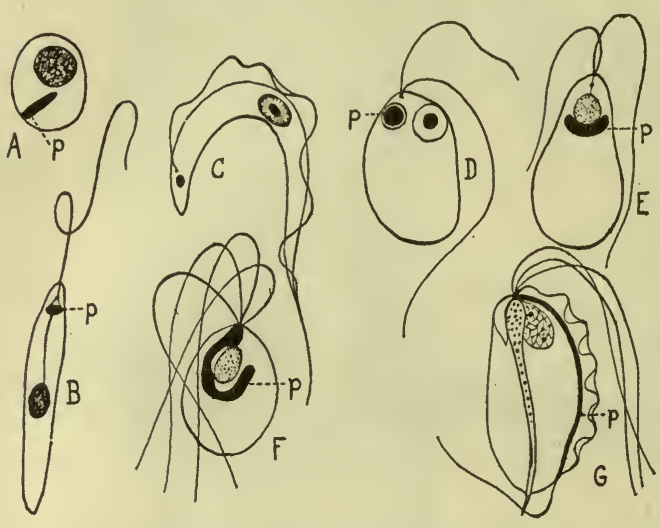
parabasal body (also called by some authors the kineto-nucleus or blepharoplast). Various types of parabasal bodies are shown in Fig. 3. This body usually arises from the basal granule and often remains connected with it, apparently being associated with

FIG. 3. Types of parabasal bodies (p). A, Leish - the function of locomania; B, Herpetomonas; C, Trypanosoma; D, Prowa- motion. From the
zekia cruzi; E, Prowazekia lacerta; F, Polymas; G, Trichomonas augusta.

fact that it seldom occurs except in parasitic forms it is possibly a special adaptation to the peculiar environment encountered by such animals. By some protozoologists the parabasal body has been looked upon as a second nucleus with the special function of control over the locomotor activities of the animal, and it has been thought to originate by direct division from the main nucleus, but there is no conclusive evidence for this view. As a result of the idea that the parabasal body is of nuclear nature some workers have separated those protozoans which possess a distinct " kineto-nucleus" from those which lack it, creating the order "Binucleata" for them.

In the Ciliata the organs of locomotion are in the form of cilia (Fig. 2F), hairlike outgrowths which are shorter and more numerous than flagella and different from them in motion. 
Each cilium arises from a tiny deep-staining dot or basal granule (Fig. 2G), which, however, is probably not homologous with the blepharoplast of the flagellates.

Various modifications of the organelles of locomotion occur, e.g., the undulating membrane of many flagellates (Fig. 2D), formed by a delicate membrane connecting a flagellum with the body; the "collar" of the choanoflagellates (Fig. 2E); the membranelles and cirri of ciliates (Fig. $2 \mathrm{~F}$ and $\mathrm{H}$ ), formed by the fusion of rows or groups of cilia; and the axopodia (Fig. 2B) of some Sarcodina formed by the development of supporting rods in pseudopodia, thus making a permanent structure. Of quite a different nature, but none the less organelles of movement, are the myonemes (Fig. 1, myo.), found in many Protozoa, and corresponding to the muscle fibers of Metazoa. They enable the animals to twist and bend their bodies. The myonemes are extremely delicate contractile fibers which run in various directions in the ectoplasm of the animal; they occur most commonly in flagellates and ciliates. In some protozoans structures have been described which show every evidence of being highly organized neuromotor apparatus, i.e., a definitely arranged and organized substance having a nervous control over the contractile fibers or myonemes (Fig. 1, mot.).

Organelles for food-taking occur chiefly in the flagellates and ciliates. Such protozoans may have a "cytostome" or cell mouth for the ingestion of food (Fig. 1), and a "cytopyge" or cell anus for the elimination of waste matter. They may also have a delicate membranous pharynx (Fig. 1, cytost.) for leading the food material into the endoplasm, and food vacuoles into which the food is accumulated and in which it is circulated inside the body. In some protozoans, namely the Suctoria, a much modified group of ciliates, there are developed sucking tentacles or the absorption of food. In others there are tiny capsules in the ectoplasm containing minute threads which can be shot forth when stimulated, and used either to overpower prey or for protection from enemies. For the excretion of waste products of the body there is often present one or more contractile vacuoles (Fig. 1, c.v.), little cavities in the protoplasm of the body which expand with water containing urea and other waste matters conducted to them by tiny radiating canals, and which periodically contract, forcing their contents 
outside of the cell, sometimes through a definite excretory pore. Sense organs in the form of pigment spots sensitive to light, and outgrowths sensitive to chemical substances, giving, perhaps, a sensation comparable with taste, are present in some species, especially in free-living ones. Various organelles serving the function of a skeleton may be developed in the form of a tough cuticle, a chitinous, calcareous or siliceous shell, a chitinous supporting rod or " axostyle" (Fig. 30, axo), or even a complicated internal skeleton of calcareous material. While no protozoan possesses all of these organelles, many possess a considerable number of them and exhibit a degree of complexity and organization almost incredible in a single-celled animal which is barely, if at all, visible to the naked eye.

Physiology and Reproduction. - In their physiology and manner of life the Protozoa differ among themselves almost as much as do the Metazoa. Some ingest solid food through a cytostome or by wrapping themselves around it, others possess chlorophyll and are nourished in a typical plant manner, and still others absorb nutriment by osmosis from the fluids or tissues in which they live. Acid substances corresponding to the gastric juice and alkaline substances simulating the intestinal juices may be present in the protozoan body, often localized in definite regions, and acting upon the food as it circulates in the food vacuoles. The waste material either is voided through a cytopyge or is left behind by a simple flowing away of the protoplasm. Body excretions are collected by the contractile vacuoles and voided by them, or they are simply passed through the body wall by osmosis.

The multiplication or reproduction of protozoans is of two quite distinct types, an asexual multiplication, more or less comparable with the multiplication of cells in a metazoan body, and sexual reproduction, comparable with a similar phenomenon in the higher animals. Several common asexual methods of multiplication occur amongst protozoans, namely, simple fission, or division into two more or less equal parts; budding, or separation of one or more small parts from the parent cell; and multiple fission or sporulation, a breaking up into a number of individuals or spores. Multiplication by one of these asexual methods may go on with great rapidity for a long time, but sooner or later some process at least remotely resembling sexual reproduction usually 
occurs. While such a process has not been observed in many protozoans, it presumably occurs in all under certain conditions.

The analogy between a protozoan life cycle and a metazoan life cycle has become understood only in recent years. As a result of the painstaking experiments of Calkins and other protozoölogists, it is now usual to compare the entire life cycle of a protozoan animal from one sexual reproduction to the next, including all the intervening asexual generations, resulting perhaps in millions of individuals, with the life cycle of a single metazoan. According to this view the asexual reproduction, as remarked above, is comparable with the multiplication of cells in a metazoan body, except that, instead of all the cells resulting from such multiplication remaining together and becoming specialized for particular functions, they separate and live as independent individuals. Just as the cells of a metazoan body grow old after a variable length of time and lose their youthful vitality and reproductive power, so the protozoan cells, after a variable number of multiplications, gradually lose their vitality and reproductive power. In the metazoan certain cells have the power of renewing their waning vitality by union with a cell of the opposite sex (sexual reproduction), thus beginning the cycle again. In the protozoan the sexual phenomena which have been observed are believed to have the same significance, and there is evidence that at least in some Protozoa the sexual power may be confined to certain individuals which would then be comparable with the sex cells of the metazoans. Calkins' experiments led him to believe that in Paramocium, a common ciliated protozoan on which he experimented particularly, old age and death were inevitable after a variable number of asexual generations without sexual reproduction. It has recently been shown, however, that when conditions of life are perfect, Paramœcium may continue to multiply asexually for an indefinite time. Periodically, however, a complete reorganization of the cells occurs which apparently has an effect similar to that produced by sexual reproduction, the animals having renewed vitality for many generations. This remarkable process, named "endomixis," is strikingly analogous to parthenogenesis (development of unfertilized eggs) in higher animals. Another analogy is that under unfavorable or adverse conditions sexual reproduction replaces endomixis, just as in such animals as 
rotifers and small crustaceans it replaces parthenogenesis, though either endomixis or parthenogenesis apparently may continue indefinitely with conditions favorable.

Another phenomenon which is often, though not always, associated with sexual reproduction is encystment, i.e., the development of an impervious enclosing capsule in which the delicate protozoan cell is able to resist extremely adverse environmental conditions, such as very high or low temperatures, drouth, presence of injurious substances, lack of oxygen, etc. The degree of protection afforded by encystment can be judged from the fact that encysted amebæ exist in considerable numbers on the sun-baked sands of Egypt. Encystment may take place whenever environmental conditions become unfavorable, or as a normal stage of existence following sexual reproduction, thus being comparable with the impervious shelled eggs of many higher animals, or sometimes as a step preliminary to some form of asexual reproduction. Nearly or quite all parasitic protozoans which are not transmitted by an intermediate host adapt themselves for passive transfer from one host to another by encystment.

A full understanding of the significance and limitations of the sexual and asexual phases of the life histories of parasitic Protozoa is of great importance, since means of control and prevention often hinge on these points. In many species of protozoan parasites a different host is required for the sexual portion of the life "isistory than that utilized for asexual reproduction, though this is not true, in general, of the intestinal parasites. Some species, although normally utilizing a second host for the sexual reproduction, are apparently able at times to pass from host to host without the intervention of an intermediate host of different species. This is true, for instance, of the sleeping sickness trypanosome, $T$. gambiense, which is normally transmitted by a tsetse fly, Glossina palpalis, as an intermediate host, but which is thought to be capable of direct transmission by sexual intercourse as well. It is interesting to note also that, according to observations made by Gonder on trypanosomes (quoted by Nuttall), characters such as immunity to certain drugs, acquired by protozoans and maintained through thousands if asexual generations in vertebrate hosts, may be blotted out at a stroke in the invertebrate host by the sexual process which presumably occurs there. The great significance of this is 
evident: one of the difficulties connected with drug treatment of some protozoan diseases is the power of the protozoans to become immune to the drug when given in doses which are not destructive to the host; if such immunity is lost during transmission by an intermediate host there is no danger of an immune race of the parasite becoming permanently established.

Classification. - It is little wonder that such a varied assemblage of single-celled animals as constitutes the group Protozoa should be difficult to classify. It is obvious that these simple animals may be profoundly modified by their environment and such modifications can actually be seen in the course of the life history of many. The changes in form undergone by a trypanosome, for instance, under different environmental conditions and at different periods in the life history are represented in Fig. 18.

It has been the custom among zoölogists to divide the Protozoa into four classes, based principally upon the nature of the organs of locomotion. These classes in brief are as follows: Sarcodina, including forms with pseudopodia; Flagellata, including forms with flagella; Ciliata, including forms with cilia; and Sporozoa, a heterogeneous assemblage of parasitic protozoans which as adults have no organs of locomotion, and which reproduce by breaking up into spores. Though other classifications have been attempted, the above system is the one generally used. It is probable that it is not in all respects a natural classification, and that changes in it will be made with increasing knowledge of Protozoa. A few examples of the difficulties connected, with this classification may be pointed out. There are protozoans, as Craigia, which are typical Sarcodina during part of their life cycle and typical flagellates during another part, and some, such as certain soil amebæ, which readily change from one phase to another under the influence of varying environmental conditions; there are others, as Mastigamoba, which exhibit at once typical pseudopodia and a whip-like organ which can only be regarded as a flagellum; there are species having organs in every way intermediate between flagella and cilia; the Sporozoa contain some species, such as the malaria parasites, Plasmodium, which during a part of their life have typical pseudopodia and suggest relationship with the Sarcodina, others which show striking affinities to the Flagellata, and still others which possess coiled projectile threads in polar capsules, 
resembling the nematocysts of jellyfishes. Some of the latter have recently been elevated to the rank of a separate class, Cnidosporidia, by the German parasitologist, Braun. Many other difficulties in connection with the classification of the Protozoa as outlined above could be cited, but since no more acceptable classification has yet been proposed this classification is followed here.

The class Sarcodina consists in the main of free-living forms occurring in the ocean, fresh water and soil. Many of the marine forms are furnished with calcareous shells which are largely instrumental in building up chalk deposits. The majority of the parasitic species belong to the genus Endamøba.

The class Flagellata contains some of the most primitive as well as some very highly specialized kinds of animals. Many of the free-living forms possess chlorophyll and are included by botanists in the Plant Kingdom. There could be little question about their vegetable nature were it not for the fact that there is every gradation between those which are typical plants in form and function and those which are equally typical animals in every respect. The parasitic species are all of distinctly animal nature, some ingesting and devouring solid food, others absorbing food by osmosis. With the flagellates were once included, also, the spirochætes on account of a supposed relationship with the trypanosomes, but this theory has long since been exploded, and the spirochretes are now usually looked upon as only distantly related to the flagellates.

The class Ciliata is least important of the four classes of Protozoa from the parasitologist's point of view. There is only one species of ciliate, Balantidium coli, which is common and widespread enough and pathogenic enough in its effects to deserve serious consideration as a human parasite. A few other intestinal ciliates have been discovered in man but they are of little importance.

The class Sporozoa contains parasitic forms exclusively, but fortunately man is peculiarly exempt from the attacks of all but a few species. Among the few, however, are included the malarial parasites, which rank among the first of pathogenic organisms as regards significance to the human race as a whole. It is possible that the undiscovered parasites of such diseases as Rocky Mountain spotted fever, yellow fever and dengue, and 
the parasites of obscure nature associated with smallpox, rabies and other important diseases may prove to be members of this group.

Importance. - Taken as a whole the Protozoa must be looked upon as a group of organisms of prime importance as human parasites. Although Leeuwenhoek discovered the existence of Protozoa nearly 250 years ago, the first parasitic species, Balantidium coli, was discovered by Malmsten in 1856, only 61 years ago. At the present time a large proportion of medical practice and disease prevention in tropical countries, and a considerable proportion in all countries, depends on our knowledge of the habits and life history of parasitic Protozoa, nearly all of which has been gained in the last 35 years, and much of it in the last 15 years. Almost daily new discoveries in connection with disease-causing Protozoa are being made; there are few branches of scientific research which offer a brighter or more promising field of endeavor for students at the present time than the investigation of pathogenic Protozoa. 


\section{CHAPTER IV}

\section{SPIROCHETES}

General Account. - On the vague unsettled borderline between Bacteria and Protozoa there is a group of organisms which are waging a frightful war against human life and health. These organisms, commonly known as spirochætes, when first discovered were supposed to be of bacterial nature. Later, for many apparently valid reasons, they were thought to belong to the Protozoa, but one by one these reasons for looking on them as animals rather than bacteria are falling away and many biologists at the present time relegate them to their old place among the Bacteria. They still serve as a bone of contention, however, between bacteriologists and protozoölogists, and at present we can only look upon them as occupying an intermediate position between the Bacteria on one hand and the Protozoa on the other.

Like Bacteria the spirochætes lack any distinct nucleus; their multiplication is commonly by transverse division, although the more typically protozoan longitudinal division has also been claimed for them by some investigators; and no unquestionable conjugation or other sexual process has been observed. Like Protozoa, on the other hand, some of the spirochætes have a membrane, the "crista," which reminds one somewhat of the undulating membranes of trypanosomes; they react to certain stains and chemicals in a protozoan manner; and they multiply in a specific intermediate host which serves as a means of transmission to a new host. Until recently it was believed that some spirochætes passed through a distinct phase of development in such intermediate hosts as ticks or bedbugs, but some doubt has been cast on this, and it is now the commonly accepted belief that the organisms live and multiply in the body of a tick or insect just as bacteria do in artificial cultures, without going through any phase of their life history which does not at least occasionally occur in the vertebrate host.

Spirochætes are excessively slender threadlike animals, spirally 
twisted like corkscrews. They are very active in movement, and dart back and forth across the field of a microscope so swiftly that they can hardly be followed by the eye. The movement is apparently by wave motions passing through the body, often accompanied by a rotation of the body in corkscrew fashion. Swiftly moving spirochætes show many small waves in their bodies, while the more slowly moving ones have larger and more graceful curls. They also have the power of bending their bodies to and fro, and of oscillating while attached to some object by one end. Spirochætes ordinarily divide by a transverse division of a single thread into two; a spirochæte in the act of such division can be seen in Fig. 6. The result of growth in length and transverse division is that the spirochrtes of any given species are very variable in size. Often individuals can be found which have incompletely divided and which hang together in long chains. Another interesting method of reproduction in spirochætes, the details of which have been worked out largely by Fantham and his students, is by "granule-shedding," i.e., the production of tiny granules by a breaking up of the body substance inside the delicate enclosing membrane into a chain of round "coccoid bodies," resembling coccus forms of bacteria (Fig. 4). These minute bodies are set free either by a disintegration of the enclosing membrane or by a rupture of the latter at one end. The elongation of the granules, the taking on of the sinuous form and the ultimate development of diminutive spirochætes are said by several investigators to have been observed by them in living cultures of these organisms. It is probable that the granule-shedding occurs at regular periods in the life of spirochrtes, and that it is comparable to the process of sporulation in malarial parasites. It appears to be particularly associated with the existence in the intermediate host if there is one, but it also occurs in the blood of the vertebrate host, sometimes apparently in preparation for the transfer to the intermediate host, sometimes as a protection against adverse conditions. It is quite likely that some spirochætes may be able to resist atmospheric drying up while in 
the granule stage and may thus be transmitted in dust or on the bodies of flies. Spirochoeta bronchialis, causing a form of bronchitis, is probably transmitted in this way.

There is a wonderful variation in the size and form of spirochætes and also in their mode of life. A few species are freeliving and of very large size, in fact almost visible to the naked eye $\left(\frac{1}{5} \mathrm{~mm}\right.$. in length), and there are many large species which live as harmless commensals with various mollusks. The disease-causing species (some examples of which are shown in

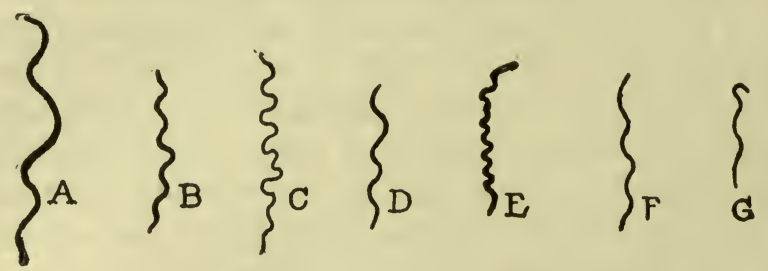

Fig. 5. Types of parasitic spirochætes. A, Sp. duttoni; $B, S p$. novyi; $C, S p$. pallida; $D, S p$. refringens; $E, S p$. balanitidis; $F, S p$. vincenti; $G, S p$. icterohemorrhagice. $\times$ about 1500 . (After various authors.)

Fig. 5) are very much smaller, often being so delicate and slender as to be hardly visible under the highest powers of the microscope. Not all the small spirochætes of vertebrates are pathogenic however; two species occur almost invariably in the human mouth, living on the tartar of the teeth and about the roots of the teeth, and yet, normally at least, cause no ill effects. One of these inhabitants of our mouths, $S p$. buccalis, is a relatively short blunt species, but the other, $S p$. dentium, is excessively slender, and practically indistinguishable when living from the spirochæte of syphilis. Other harmless spirochætes occur in various stagnating secretions or excretions of the body, about the tonsils, and in the intestinal mucus.

Spirochætes and Disease. - There is some question about how many distinct human diseases are caused by spirochætes. The mere presence of spirochætes in sores or diseased tissue is not sufficient reason for believing that they are the direct cause of the diseased condition, for, like many bacteria, they are often found in exposed sores which are known to be due to other causes. Spirochætes are often found associated in sores or ulcers with certain kinds of bacteria, and both bacteria and spirochætes 
have been thought by some workers to be different stages in the life history of a single organism.

Spirochætes living in animal bodies have a strong tendency to localize in definite parts of the body or in special tissues. The spirochætes which choose the mouth, the teeth or the digestive tract as a habitat have already been mentioned. Spirochceta bronchialis confines itself to the respiratory tract, causing a certain type of bronchitis. Sp. schaudinni localizes in skin tissue, causing ulcers, in certain tropical countries; Sp. icterohemorrhagia, although probably invading many parts of the body, especially affects the liver and kidneys; the spirochrtes of the various types of relapsing fever confine themselves to the blood; $S p$. pertenuis, the cause of yaws, produces a local sore followed by a general invasion of the body, but it returns to the skin tissues and settles there; $S p$. pallida, of syphilis, is able to produce lesions almost anywhere in the body, but in any given case usually attacks some special organ or tissue, such as the central nervous system, skin, bones, reproductive system, arteries, etc. Other spirochætes have been found in connection with many different maladies, for instance, $S p$. orientalis in "ulcerating granuloma of the pudenda," an ulceration which spreads over the skin and mucous membranes of the external genital organs; $S p$. vincenti in Vincent's angina, a diphtheria-like affection of the tonsils and throat; Sp. bronchialis in certain types of bronchitis; and $S p$. balanitidis in balanitis, an erosion or ulceration of the glans of the penis. There seems to be more or less evidence that the spirochætes found in connection with these diseases, often associated with bacteria of various kinds, may be at least partially responsible for them, but to prove this is a very difficult matter.

In general the diseases caused by spirochætes may be divided into three groups. The first of these is the type in which the organisms live in the blood and cause general symptoms, such as fever, spleen enlargement, and anemia, and have a tendency to cause relapses. Of such a nature is rat-bite fever and the various forms of relapsing fever. Second, there is the type in which there are general constitutional symptoms often preceded by a local lesion of some kind, followed later by a localization of the organisms in special organs or tissues. This type, characterized by continued or remittent attacks rather than by short relapses, 
includes such diseases as syphilis, yaws, and infectious jaundice. The third type is that in which occur only local ulcerating sores of skin or mucous membrane; of such a nature are the other diseases named above.

\section{Relapsing Fever}

In every continent in the world, with the possible exception of Australia, there occurs a form of relapsing fever caused by spirochætes in the blood. In Africa it ranks next to malaria and sleeping sickness as a scourge of that disease-cursed country. In India it is hardly less severe, while in Eastern Europe and America it is a mild disease. The clinical effects of these various strains of the disease vary, especially in the number and duration of the relapses. The mode of transmission also varies and the parasites are apparently distinguishable and are therefore given different scientific names. The African spirochæte, Spirochœeta

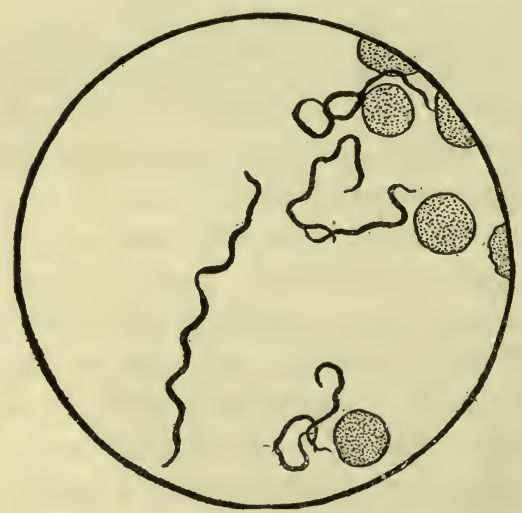

FIG. 6. Spirochata duttoni in blood of experimentally infected rat. Upper individual shows transverse fission. $\times 1000$. (After Novy and Knapp.) in the closeness and regularity of the coils. Each type, however, is quite variable within itself, and one is likely to be misled as to size by the hanging together of several individual or partially divided spirochætes in a chain. The varying symptoms of the different types of the disease and the fact that immunity to one does not give immunity to another are reasons for considering the relapsing fever spirochætes as constituting several species. 
Although relapsing fever was known to physicians over a century ago, it was not until 1873 that Obermeier discovered the hitherto unseen agitator which causes it; he made his discovery during one of the epidemics which spread from Russia over Poland and Prussia.

Many great epidemics have swept Russian, Austrian and Balkan cities. Early in the present European war Serbia was held in the grip of an epidemic of relapsing fever of unusual severity and of high fatality. In Bombay and other Indian cities the oriental type of the disease is nearly always present, and it sporadically appears in various parts of North Africa, China and Japan. In tropical Africa it occurs over a large part of the continent occupied by the tick which transmits it. It is also probably widely distributed throughout Mexico and Central and South America. In the United States it occurs chiefly as irregular epidemics among immigrants. Just recently a small epidemic occurred in Colorado.

Transmission. - In Africa, where the disease is commonly known as "tick fever," it was thought for a long time to be the result of the poisonous nature of the bite of a common houseinfesting tick, Ornithodorus moubata (see p. 360, and Fig. 155). This tick, which inhabits the huts of natives throughout Central Africa, is the chief if not the only transmitter of the Central African relapsing fever spirochæte, Spirochoeta duttoni. It can infect both man and monkeys by its bite.

It has been shown that the spirochætes can live for a long time in the ticks though they apparently disappear from the digestive tract after nine or ten days, many of them penetrating to the blood-filled body cavity while still in the spirochæte form. Leishman found that the spirochætes break up into a series of tiny granules which penetrate many of the organs of the tick, including the ovaries and eggs. When the ticks are exposed to a temperature of $95^{\circ} \mathrm{F}$. for a few days the spirochrtes reappear. The ticks may remain infective a year and a half after feeding on an infected person though frequently fed on clean blood in the meantime, and a single tick may, therefore, infect a number of people. By means of the granules the spirochætes may be passed on to a second, or even to a third, generation of ticks through the eggs. Young ticks reared in the laboratory from infected parents have been found capable of transmitting the 
disease. Indeed, the tiny unfed nymphs are very infective, and on account of their small size are particularly dangerous since they are not easily detected. The ticks do not usually transmit the parasites by means of the beak but deposit a bit of infected excrement beside the wound they make; from here the spirochætes make their way into the blood, aided by the scratching which follows the tick bite. However, when the tick is kept for a few days at a temperature of $95^{\circ} \mathrm{F}$. the salivary glands as well as nearly all other organs become infective, and the disease may then be transmitted in the usual insect manner, by injection with saliva. The relapsing fever of Abyssinia and Somaliland is transmitted by a closely allied tick, Ornithodorus savignyi. African tick fever is said to have been imported into Persia, where it is transmitted by $O$. tholosani. The complete life cycle of Spirochceta duttoni is shown diagrammatically in Fig. 7.

The other types of relapsing fever spirochætes do not appear to have such definite and invariable transmitters. Nicolle and his fellow workers have shown that in Algeria the head and body lice are undoubtedly the means of spreading the disease. In experimental work they have shown that there is a rapid temporary disappearance of the spirochætes from the body of the louse after they have been sucked with blood from an infected person; during this time they are presumably in the granular stage. After about eight days the spirochætes reappear and are abundant in the body cavity of the louse for some 12 days before they finally disappear for good. The lice are infective while spirochætes are present in their usual form, and also just before they reappear at the end of eight days. It is by crushing the louse and allowing the juices from its body cavity to contaminate the wound that infection is obtained.

In experimental work in Algeria a man experimented upon was bitten several thousand times by infected lice without contracting the disease, but one louse crushed, and the body fluids placed on the conjunctiva, caused the disease to develop. The same result would undoubtedly have occurred if the crushed louse had come in contact with a wound of the skin.

In some cases the spirochætes are transmitted through the eggs to the next generation of lice, just as in the case of ticks and the African disease. The louse has been shown to be the transmitter of relapsing fever in India also. 
In Europe several different pests are probably implicated in the transmission of relapsing fever. In Persia and neighboring countries the miana tick, Argas persicus (see p. 364, and Fig. 159), is probably the chief offender, while in Russia, Serbia and the

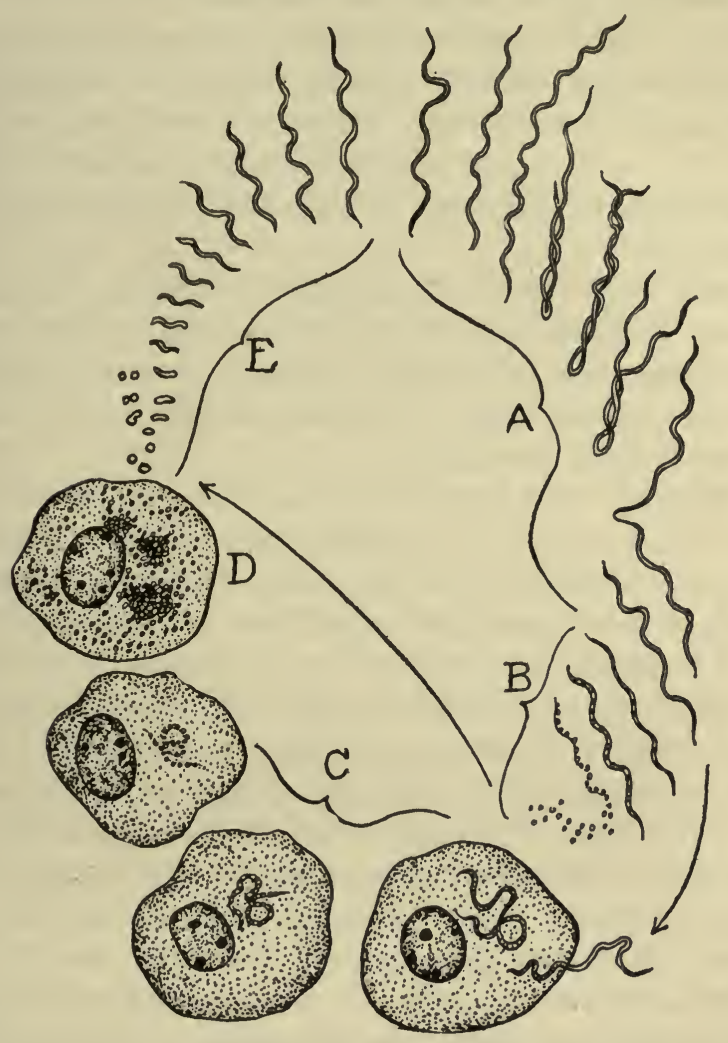

Fig. 7. Life cycle of Spirochata gallinarum, applicable also to Sp. duttoni of relapsing fever. $A$, multiplication by transverse division in vertebrate blood; $B$, formation of coccoid bodies in vertebrate blood: $C$, infection of cells of tick and formation of coccoid bodies: $D$, multiplication of coccoid bodies in tick; $E$, development of spirochæte forms from coccoid bodies after reëntering vertebrate blood. $\times 1500$. (After Hindle.)

Balkan States lice and probably also bedbugs are the transmitters (see p. 378). It is noteworthy that relapsing fever always thrives best in those countries where body cleanliness is neglected, and where vermin are in consequence abundant. In fact, the prevalence of relapsing fever in any country, as of typhus, 
is in inverse proportion to the prevalence of the use of soap and water. Relapsing fever, in countries where it is transmitted by lice, always spreads most rapidly in cold weather when people are huddled together in stuffy, filthy houses, thus giving the lice ideal opportunities for doing their evil work.

In Mexico and Central America it is believed that certain ticks, Ornithodorus talaje and $O$. turicata, which in form and habits closely resemble the African relapsing fever tick, transmit the disease, but this has not been proved. O. turicata is said to be the transmitter in Colombia, but the bedbug and other ticks are also suspected.

Relapsing fever is not a contagious disease as was formerly supposed. A typical case in the Bellevue Hospital in New York failed to spread the infection to anyone else during the 89 days' stay of the patient, although no special precautions were taken to prevent it from spreading.

The Disease. - In the human body the spirochætes appear to live exclusively in the blood, where they become fairly common, though never abundant, at regular intervals. In the meantime they apparently disappear though they are undoubtedly present either in the granular form, or else in such limited numbers as to be practically impossible to find. The repeated increase and decrease of the spirochætes in the blood goes hand in hand with a recurring fever broken by periods of apparently almost normal health. The time of incubation of the disease varies from two days to two weeks, but in most cases the initial attack comes on the third or fourth day. It usually begins with severe chilly sensations, headache and shooting pains in the limbs. The ensuing fever lasts intermittently for several days, being accompanied by such symptoms as rapid pulse, enlarged spleen, constipation, nausea and mental disturbances. After several days the temperature suddenly drops below normal and remains so for a period of seven or eight days, during which time the patient recovers rapidly, feels perfectly well and thinks it unnecessary to remain at home or in the hospital any longer. Then comes the first relapse, repeating all the symptoms of the first attack, sometimes in somewhat milder form. Following this there is a second period of apparently normal health, usually followed by a second relapse, this time much milder. The number of relapses varies: in the European and allied types the second relapse is mild, and 
is the last one felt; in the African type, on the other hand, there are usually four or five relapses, of shorter duration and more irregular in occurrence. In a Gibraltar case Manson observed eight distinct relapses, but this is very unusual. Hemorrhages under the skin and in various organs of the body often occur, and cases have occurred recently in Hungary in which the meninges (tissues covering the brain and spinal cord) were severely affected, causing various nervous disorders. Spleen, liver and other organs are frequently affected.

Even the African type of the disease does not ordinarily have a high mortality, though some epidemics are more serious than others. In an epidemic in Tonkin in 1912, 48 per cent of 703 cases were fatal. In India the fatality is often high on account of the well-meant but pernicious habit of depriving fever-stricken people of food, thus often increasing the exhaustion caused by the disease. Abortion is a common result in pregnant women. A single attack gives permanent immunity to any one particular type of the disease but not to others.

Treatment and Prevention. - Ehrlich's famous spirochæte poison, "No. 606," or salvarsan, destroys the spirochætes of relapsing fever more readily, if anything, than it does other species of spirochrtes, since the parasites live in the blood stream into which the drug is directly injected. A single injection nearly always causes the disappearance of the parasites from the blood and prompt recovery from all symptoms of the disease. Preventive and curative inoculations of the serum of highly immune animals has been found to be effective in rats and monkeys. The power of the immune serum can be so increased by repeatedly inoculating an animal that very small injections of it are sufficient not only to cut short the course of the disease in these animals but also to give an immunity of considerable duration. It is probable that the same serum would immunize human beings as well.

Eradication of vermin from person and home and avoidance of places where infected parasites might be acquired are the most important protective measures in places where an epidemic is raging. Methods for the control of ticks are discussed on page 369 , of lice on page 400 and of bugs on page 383 . Since the parasites are not ordinarily introduced directly into the blood by the beak of the transmitter, but are simply voided with the 
excrement in the vicinity of the wound, careful disinfection, with alcohol or carbolic acid, of the wound before the removal of the parasite is a good means of prevention if the suspected transmitter be caught in the act of biting.

\section{Syphilis}

History. - There are few diseases which mean more to the human race as a whole than syphilis, due in part to its almost universal distribution, and in part to its insidious and deceiving course, thereby leading to untold misery and disaster. Rosenau says "civilization and syphilization have been close companions"; the one has followed in the wake of the other like the guerillas behind an army. Unlike most diseases, syphilis is one of whose origin among civilized nations we have strong evidence. There are many reasons for believing that syphilis was acquired by the members of Columbus' crew when they discovered the island of Haiti, and that it was carried back to Spain by them on their return. These adventurers promptly joined the army of Charles VIII of France in its invasion of Italy in 1494. Soon after the army had triumphantly set up a court in Naples it became weakened through the ravages of a terrible venereal disease of unusual intensity, hitherto apparently unknown in Europe. The following year the army retreated almost in a rout and was broken up, the miscellaneous troops scattering all over Europe to their respective home countries, and carrying the new disease with them. In the next four years the disease had spread to practically every country in Europe, and was soon carried by the Portuguese to Africa and the Orient. The venereal nature of the disease was fully recognized, and its foreign origin was well known, each nation trying to shift the responsibility to another by name, many peoples calling it the "French disease," others the "Spanish disease," etc., while the Spanish alone seemed aware of its real origin in America and called it "española" which then meant Haiti. The absence of any reference to a disease resembling syphilis in the historical records before the discovery of America; the absence of any bones showing evidence of syphilitic attack in the abundant pre-Columbian remains in Europe, and abundance of such bones in American remains, many of which must certainly be pre-Columbian; the positive 
evidence of Spanish physicians and historians at the time of the return of Columbus; and the severity of the great epidemic in the latter part of the 15th century, - it being almost invariable for an infectious disease, when first introduced among a new people, to rage with unwonted severity; all these facts point strongly to the American origin of syphilis.

Interesting as is the early history of the disease, the recent history is infinitely more so. By the beginning of the twentieth century medical men had come to the end of their rope in knowledge and treatment of the disease, and found themselves at a standstill. But in 1902 the disease was successfully transmitted to animals where it could be conveniently studied; in 1905 Schaudinn discovered the spirochæte, Spirochæeta (or Treponema) pallida (Fig. 5C), which is believed to cause the disease. In 1906 Wassermann demonstrated the possibility of detecting latent syphilis by the reaction which bears his name; in 1910 Ehrlich made the epoch-making discovery of his famous drug, "No. 606," or salvarsan, a deadly poison for spirochætes of all kinds, and a cure for syphilis in nearly all stages; in 1913 the direct relation of syphilis to insanity, paralysis and other diseased conditions of the central nervous system was demonstrated by the discovery of the organisms in the cerebrospinal fluid, and in the same year a method of destroying the parasites in the central nervous system was discovered. There is no other instance in the history of medical science where such wonderful strides have been made in such a short time in the knowledge and control of a disease. At the beginning of the twentieth century syphilis was one of the most horrible, hopeless and tragic diseases known to ravage the human body; it is now a disease which can be readily recognized even in latent stages; it can be cured in its early stages; and the terrible tragedies resulting from apparent but imperfect cure can be avoided. Its eradication, however, will not soon, if ever, be accomplished, since in this are involved some of the most intricate moral and social questions with which we have to deal.

Prevalence. - The prevalence of syphilis is difficult to determine for at present the recording of syphilitic cases is practiced to a very slight extent, and accurate data can be obtained only in military organizations and certain public and private institutions. Sir William Osler places syphilis as third or fourth 
of the killing diseases. The use of the Wassermann reaction for the detection of syphilis has greatly extended the possibility of arriving at an estimate of the prevalence of the disease, and has shown that it is far more common than was formerly believed. Yet even the Wassermann test fails in about 10 per cent of cases. It is now known that the disease may be present in latent but nevertheless infective form for many years after all active symptoms have disappeared. The recently published report of the British Royal Commission on Venereal Diseases concluded that the numier of people infected with syphilis cannot fall below ten per cent in large cities, and that at least one-half the registered still-births are due to this disease. They found that in Britain this as well as other venereal diseases is most prevalent in the unskilled labor class, and least among miners and agricultural laborers. Fournier estimated that in Paris 15 per cent were infected. In China syphilis is, next to tuberculosis, the most common disease. In the United States conditions are no better than elsewhere; some cities, notably San Francisco, are much more heavily infected than others. Of 111 cases admitted to the Children's Hospital in Boston 31 per cent were infected with syphilis. Of 102 children admitted to a Chicago hospital, none of them for syphilis, 30 were syphilitic. In the "red light" districts of cities, which undoubtedly serve as the centers of distribution for the disease, the per cent of syphilitic prostitutes is very high. Dr. Browning found every one of 104 prostitutes in Glasgow infected, and a like condition among 109 men, women and children classed as "vagrants".

According to Capt. E. B. Vedder of the U. S. Army, the statistics compiled from over 1000 new recruits in two widely separated camps (in New York and Ohio respectively) showed that over 19 per cent of all applicants for enlistment, approximately one in five, are probably syphilitic, although only a trifle over 2 per cent showed any symptoms of the disease which would exclude them from the army as the result of a rigid physical examination. From this Vedder concludes that there is a good reason for believing the percentage of syphilis among the young men in civil life, between the ages of 20 and 30 , to be fully 20 per cent. "It means that when a man's daughter marries, the chances are just one to five that she will become the victim of 'damaged goods'." Vedder shows further that in the relatively select class 
of young men at West Point from two to five per cent are probably syphilitic. In the U. S. Army, as a whole, Vedder believes an estimate of 16 per cent of syphilis among the whites is conservative, and his statistics show that the per cent increases steadily with the ages of the enlisted men, and as the years of service increase. Among enlisted negroes, who are notoriously more syphilitic in civil life than are whites, syphilis is two or three times as prevalent as among white enlisted men. "This study confirms observations that have already been published indicating that syphilis is so prevalent among negroes that it is possibly the greatest single factor in the production of disability and high mortality rates among the race." The figures obtained from an examination of 531 Porto Rican enlisted men are most startling of all - over 50 per cent show evidence of being probably syphilitic.

Transmission. - Syphilis is fundamentally a venereal disease, transmitted by sexual intercourse, and over 90 per cent of cases are undoubtedly of such origin. It is a common belief that this is the only way in which the disease can be acquired, and sometimes an unjust stigma of shame and disgrace is attached to a perfectly innocent case of syphilis. As already remarked, in the vast majority of cases the parasites are directly acquired from their usual habitat in the underworld, but over 20,000 cases of innocent syphilis have been reported, and five per cent of infections occurring in the army are of innocent origin. A horrible case is on record where seven young women at a church social in Philadelphia acquired syphilis from kissing a young man who had a syphilitic sore on his lip. A case recently occurred in one of our western cities which was ultimately traced to the eating of apples sold by an Italian who was in the habit of spitting on his fruits and rubbing them on his sleeve to shine them. Public drinking cups, public towels and soiled bed-linen serve admirably as temporary abodes for the spirochætes of syphilis, but fortunately these curses of civilization are in most places abolished by law. Unsanitary barbers and dentists can easily spread infection, and dentists and physicians often themselves contract the disease from handling syphilitic patients, the spirochætes readily entering the smallest cut or abrasion of the skin. Midwives and wet nurses are likewise exposed to infection from diseased babies, as are the babies from diseased nurses. 
Indeed, when we think of the many ways in which syphilis spirochætes may be transmitted from person to person it is surprising that the number of innocent cases is not much greater.

The Spirochætes. - The spirochætes of syphilis, Spirochøeta

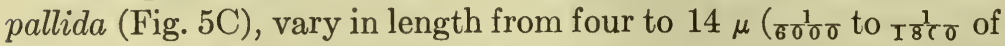
an inch) and are immeasurably slender. They are more closely curled than the spirochætes of relapsing fever, having usually from six to 14 very regular, short, sharp curls, quite different from the long graceful curves of a relapsing fever parasite. The living organisms are very active and dart with great speed across a slide, threading their way between blood corpuscles or cells. The spiral turning of the body reminds one of the undulating movements of a swimming snake. Another spirochæte, $S p$. refringens (Fig. 5D), is often found associated with $S p$. pallida.

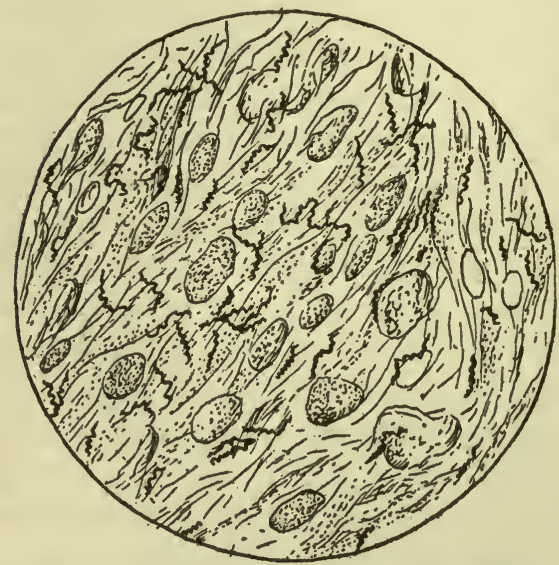

FIg. 8. Spirochata pallida in liver tissue of a congenital syphilitic.

During the early stages of their sojourn in the body the spirochætes can always be found in the primary and secondary lesions, and in the neighboring lymph glands. During the second phase of the disease and also toward the end of the first phase the spirochætes occur in variable numbers in the blood, and very early make their way into the cerebrospinal fluid in the brain and spinal cord. After it was found that the spirochætes actually invade the central nervous system, and cause diseases of it, it was supposed that this occurred only occasionally in late stages of the disease. During the last year or two it has been shown, however, that the great majority ( 80 per cent) of syphilitics show distinct pathological changes in the spinal fluid, due to spirochætes in it, from the date of the primary sore, and are therefore possible candidates for syphilis of the nervous system. During the second phase the spirochætes make a general invasion of the entire body, later showing some special predilection for 
certain tissues or organs. The gummy sores or "gummas" which often break out during the third stage of the disease have usually been considered non-infective, and spirochætes could not be found in them. Recently, however, the parasites have been found in some of these lesions, also. In congenital syphilis the parasites often multiply in enormous numbers in the unborn child, penetrating practically every organ and tissue of the body. The liver especially is often found literally teeming with spirochætes (Fig. 8).

The Disease. - Syphilis is a disease which has no equal in its deceptive nature. It is largely due to this fact that so many tragedies result from its ravages. Its effects on the individual are often horrible enough, leading to disease of almost any tissue or organ in the body, but it is only when judged in the light of the additional damage that is done to the innocent wife or husband, as the case may be, and to the next generation, that the true meaning of syphilis can be measured. Syphilis may remain latent and unsuspected for twenty years or more, and the carrier still be infective. Meanwhile, perhaps in ignorance of his condition, he may infect a hitherto sound person whom he has taken for a life companion, and cause her, or him, to be ravaged and slowly destroyed by this horrible disease. Worse than this his chances of having healthy children are small. It has been shown that about 45 per cent of those who later become victims of general paralysis from syphilis never can have any children, either on account of sterility or of repeated abortions. The author of the statement in the Bible that "the sins of the fathers shall be visited upon the heads of the children unto the third and fourth generations " may well have had in mind the hereditary effects of venereal diseases, but he might have stated further that often there is no third or fourth generation. The only pity of it is that this is not always the case, for those who are brought into the world are in the majority of cases hopelessly handicapped either mentally or physically. Feeblemindedness is five times as common in syphilitic families as in normal ones. There is some reason for believing that the hideous mentally deficient children known as mongols are the result of syphilis in parents. And finally, as if all this were not enough, the carrier of latent syphilis may later develop general paralysis, or some other disease of the nervous system or other organs, which 
will render him an ineffectual social unit, and make him and his family a burden to the community.

In the majority of cases the disease begins with a hard sore on the skin or mucous membrane known as the "primary chancre." This usually appears at the point of infection in from ten days to three weeks after the infection occurs. In some cases such a chancre never develops. The chancre gradually heals up and the second stage begins, in which general constitutional symptoms appear, as fever, anemia and a general run-down condition during which the patient is very susceptible to other diseases, such as tuberculosis. Often there is an extensive breaking out on the body, production of scaly patches of skin, and inflammation of the mucous membranes of the mouth and throat.

From this point on the course of the disease depends on what particular tissues or organs the spirochætes especially attack, for although the parasites, as said before, may produce disease almost anywhere in the body, in any given case there is usually a localization. It seems that certain strains of the parasites have special preference for certain tissues. The differences in this respect have been shown by Nichols to hold good through many transfers from animal to animal, and visible differences in the parasites can be observed. In about 40 per cent of cases syphilis settles in the nervous system, causing a great variety of evil effects, such as feeble-mindedness, tabes, or locomotor ataxia, general paralysis, epilepsy, insanity and moral defectiveness. Often it settles in the skin and mucous membranes, producing the gummy sores or "gummas" which were formerly supposed to be the usual tertiary stage of syphilis. It may select the bones, muscles, arteries, heart, reproductive system, or any other part of the body, in each case producing a different set of symptoms, but in every case weakening the vitality and leading ultimately to an early grave.

An active attack on one tissue or organ of the body seems to have an inhibiting effect on other attacks. It is well known that an infected person presumably with an active attack of the spirochætes on some organ in his body will not develop new lesions when re-infected. Possibly this explains why there is often a relapse of the nervous system after incomplete treatment of skin syphilis. The spirochætes in the nervous system which 
are not reached by the drugs may flare up and produce a serious attack after the spirochætes in other parts of the body have been killed and the skin lesions healed. On the other hand paralytics with an active attack on the central nervous system seldom show any other symptoms. Unborn babies seem not to be subject to such specialized attacks, but, as already pointed out, are often found with every organ and tissue in the body full of spirochætes. There is a form of the disease occurring in adults known as "malignant syphilis" in which ulcerating sores appear early and gradually eat away large portions of the skin. It is marked by extreme anemia and great weakness, and usually causes an early death.

Diagnosis. - The modern methods of diagnosing syphilitic infection have revolutionized our knowledge of the disease, and have done much toward placing its treatment and control on a scientific basis. In at least 50 per cent of syphilitic cases there are no symptoms which can be attributed positively to syphilis, but we now have several tests for the disease, two of which are of wide application, and, together with the characteristic lesions in certain stages of the disease, make it possible to detect syphilis in practically any phase.

The simplest of these indicators for syphilis is the "luetin test." This consists of the injection into the skin of a sterile emulsion of the dead bodies of the spirochætes from a culture. If the test is positive, i.e., if syphilis is present, the inoculation results in a solid or a pus-filled pimple, usually appearing in a few hours but sometimes not for several days. This test is applicable to latent, tertiary and congenital syphilis and does not give positive results during the active primary and secondary stages of the disease. Its value lies in the fact that it is sometimes sensitive to latent infections which the Wassermann reaction, now to be described, does not demonstrate.

The Wassermann reaction, although it fails to reveal syphilis in rare cases, is one of the most valuable and dependable means of diagnosis known in medicine. It is now almost universally used in well-equipped laboratories. The reaction is also positive to some other diseases, such as yaws (also a spirochæte disease), leprosy, malaria, scarlet fever and other diseases, but all of these can be diagnosed beyond doubt by other means and thus prevent a false diagnosis of syphilis. The reaction in brief is as 
follows: A little serum from the suspected person is mixed with an extract of liver or heart and some guinea pig serum and incubated a short time. A suspension of blood corpuscles and a hemolytic serum is then added and the mixture is incubated again. If the suspected serum is not syphilitic the blood corpuscles are dissolved by this mixture and the serum becomes red, whereas if the serum is syphilitic no change in the blood corpuscles takes place, and they sink to the bottom. The more highly syphiFra. 9. Wassermann litic the individual the more complete is the
eaction. Neg., nega- sedimentation of the corpuscles. As stated Reaction. Neg., nega- sedimentation of the corpuscles. As stated
tive; Pos., positive. before there are possible sources of error in this test, but if properly made with standard reagents, and with sufficient control tests, it can be confidently relied upon.

Treatment. - There are many quack doctors who are still practicing the same inefficient methods of curing syphilis that were in vogue several centuries ago. Syphilitic sores are powdered and cauterized and cured, and the patient is given to believe that his disease is cured. Unfortunately, as we have seen, the course of the disease is of such a nature that the doctor's claim of having cured may be borne out for months or years before the insidious disease appears again, this time in a much more destructive and perhaps incurable state. Superficial treatment of syphilis sores, accompanied perhaps by a few "tonic" pills, in no way destroys the virulence of the parasites or alters the future course of the disease. It merely makes the chance of correctly diagnosing the disease more difficult, and it frequently results in an unsuspecting victim carrying the disease untreated to a stage where it has wrought irreparable damage to himself, his life-mate and his children.

Treatment of the disease formerly consisted in the administration of mercuric chloride. While this sometimes effected an apparently complete cure, over 80 per cent of syphilitics suffered relapses in spite of the most persistent treatment. In 1910 Ehrlich, after years of experimentation, offered humanity his famous preparation, "No. 606," known as salvarsan, an arsenic compound which is deadly to spirochætes. When this drug is injected into the veins of a syphilitic, it almost immediately kills all the spirochætes except a few which have stowed away in 
inaccessible parts of the body, and these must be caught by continued administration of the drug, or by special methods. The most successful method of treatment is an alternate use of mercury and salvarsan, this apparently being more effective than salvarsan alone. There is now on the market a modified form of salvarsan, known as neosalvarsan, which is milder in its effects on the body but usually considered less powerful in destroying the spirochætes. Several other more or less valuable substitutes for salvarsan are now prepared. On account of the war, salvarsan itself, a German product, is at present difficult to obtain.

Salvarsan injected into the veins does not reach the spirochætes in the central nervous system, and since it is too injurious to be injected directly into the spinal fluid, the usual treatment of syphilis is inapplicable to syphilitic infections of the nervous system. An injection of salvarsan into the lymph spaces under the fibrous coverings of the brain is sometimes used, but is not always successful. Swift and Ellis, of the Rockefeller Institute, discovered in 1913 that the blood serum of a syphilitic who had recently been given salvarsan was destructive to spirochætes and could be injected into the spinal fluid without injurious results. Out of this grew the so-called Swift-Ellis treatment of syphilis of the nervous system by the use of "auto-salvarsanized serum," i.e., the serum of the patient himself after having been given salvarsan an hour before. This serum is heated for half an hour to make the salvarsan in it more active, then diluted and injected into the spinal canal. While complete cures in late cases of paralysis and other nervous diseases could hardly be expected from this or any other method, the results which have been obtained are very encouraging. It has been suggested that in all cases of syphilis the Swift-Ellis treatment be made routine as a protective measure since, in the majority of cases, the spirochætes invade the nervous system in the early stages of the disease and the consequences of their establishment there are so terrible as to warrant every possible preventive measure.

The modern methods of diagnosing syphilitic infection have given a definite standard of cure, and the success or failure of treatment can be positively demonstrated. A uniform negative Wassermann reaction given several times during a year, and absence of any symptoms, can be looked upon as an indication of 
cure, though some doctors consider a negative Wassermann reaction for two years necessary to indicate a certain cure on account of rare cases of relapse, even after a year of apparent absence of the spirochætes. In contrast to the 85 per cent of relapses which occurred when mercury alone was used to treat syphilis, less than four per cent of relapses occur after treatment with both mercury and salvarsan. Certainly salvarsan may justifiably be considered "one of the mightiest weapons in medicine."

Prevention. - The control and ultimate eradication of syphilis is, in spite of our present methods of diagnosis and treatment, a dream of the distant future. In its prevention are involved so many social and moral problems upon which people will not agree that the task is beset with great difficulties.

According to Dr. Snow of the American Social Hygiene Association, the means of controlling and preventing syphilis fall into three groups: (1) care and treatment of existing cases with a view to preventing their spreading the infection, (2) protection of the uninfected by education and administrative measures, (3) the development of social defenses against the disease.

As regards the first type of preventive measures, practically all medical men and public health workers are agreed. Adequate means for the diagnosis and treatment of syphilis should be provided in all cases. At present not only are there no laboratories for diagnosis or free hospitals or clinics for treatment provided at public expense, but most of our private physicians and hospitals shun syphilitics, and refuse to care for them. Many physicians at the present time have little knowledge of venereal diseases. The suggestion of the British Royal Commission on Venereal Diseases urging that this subject be given a prominent place in all medical schools is certainly worthy of being put into practice immediately. In many of our large cities and in most of the small ones there is not a single hospital which will admit a patient for a venereal disease. Of 30 general hospitals in New York City, only ten receive patients with recognized syphilis in the infective stages. Theoretically a syphilitic in the infective stages should be as carefully watched and cared for as a leper or smallpox patient, yet the syphilitic, the victim of immorality usually, but sometimes only of the carelessness of some other culprit, is turned loose without treatment, but with full power to infect all with whom he comes in contact directly or indirectly. We 
seem to have made little advance since 1496, when the Parliament of Paris decreed that all persons found infected with syphilis should leave the city within 24 hours.

The British Royal Commission urged the provision of ample facilities for free diagnosis of these diseases and for free treatment when necessary. Such measures have already been attempted in a few instances in our own country and their ultimate success on a large scale is insured. The New York City Health Department in a single year examined 59,614 specimens of serum for the presence of syphilis and three-fourths of these were received from private physicians. A few public health institutions are doing splendid work in the operation of a department for the diagnosis of venereal diseases and the giving of personal advice. The Oregon State Board of Health is undertaking an extensive correspondence with persons in all parts of the state who write for information in response to venereal disease placards posted in appropriate places. The provision of ample facilities for the free treatment of syphilis in the way of hospital service when necessary, of proper medication, and of the extension of Social Service hospital work is something which we have only begun to touch upon, but which will undoubtedly come in time. The fact that no facilities have hitherto been provided for the care of syphilitics either at public expense, or in the private practice of physicians and hospitals, is a disgrace to our civilization and a menace to our health. The medical prevention of syphilitic infection after exposure to it is possible and succeeds in the great majority of instances if attended to within a few hours after exposure. The use of self-applied medical treatments has been fairly successful in military life, but as shown by Dr. Snow it is of doubtful value in civil life, since the intelligence required to apply medical preparations properly is lacking in those who need it most - immature boys, drink-befuddled men, defective girls, and the average prostitutes. These classes constitute the bulk of the citizens who become exposed to infection and since the personal supervision of a physician is necessary in most cases, it might best be required in all. Private physicians, dispensary officers and the health department staff are the persons qualified to employ medical treatment designed to prevent infection after exposure to it. Avoidance of exposure constitutes the best and only safe preventive measure before exposure. 
As to the second type of prevention, the protection of the uninfected by education and administrative measures, great advances are being made. One of the most important measures, and one to which we are slowly coming, is the compulsory notification of the Public Health Department of all cases of venereal diseases so that whatever action seems best may be taken to safeguard the public health. There can be no question but that such a recording of venereal diseases would work for the best good of all concerned, both the patient and the public. Laws compelling the notification of health departments of venereal diseases now exist in eleven states and a number of cities in the United States, but only in rare instances are they enforced. Such a law in modified form has been passed and is being enforced in Western Australia.

With the notification of venereal diseases, many other practical measures could be inaugurated, such as the exclusion of infectious syphilitics from occupations connected with the preparation and serving of food; the careful instruction of syphilitics concerning various phases of their disease, and possible means of transmission, thus in many cases securing their active coöperation; and the effective prevention of the marriage of syphilitics. The last is one of the most important measures that could be adopted. Many states at present prohibit the marriage of persons with venereal diseases but without enforcement of notification these laws are worse than useless, since they may give a false sense of security. Knowing the awful consequences of inherited syphilis it is the duty of society to prevent the marriage of syphilitics even with the full knowledge and consent of both parties. The Royal Commission urged only the full information of the undiseased party in marriage, allowing the union to be made if then consented to. In this they seem not to have given due consideration to the rights of the next generation. With compulsory notification of venereal diseases, and a law refusing a marriage license to any person who has or has had syphilis and cannot pass the accepted laboratory tests for the disease, the pitiful results of hereditary syphilis could be largely prevented. Even the remote possibility of the spectacle of a diseased wife and of stillborn, insane, or physically imperfect children should be enough to induce any man worthy of the name to take every precaution to avoid such a tragedy, but if 
he is unwilling to do this for himself and his posterity, social laws should do it for him.

Sanitary laws are in effect in many places which help to prevent infection from such sources as public drinking cups, towels, bed-linen, and other articles, but such laws, excellent as far as they go, are inadequate, since no law can cover all the articles which may be rendered infective by contact with a syphilis sore. One common source of infection, though more for gonorrhea than for syphilis, is the improperly constructed toilets in public schools. These are usually built so high, and of such a type that school children, little girls especially, are exposed to infection every time they use them. Many cases of venereal diseases in school children, particularly in larger cities, have been traced to this source.

No preventive measure which does not strike directly at the primary source of infection can be adequate in coping with any disease. Just as we fight malaria through the mosquito, sleeping sickness through tsetse flies and typhoid through contaminated water and houseflies, so we must fight syphilis and other venereal diseases through prostitution. The abolishment of this vice would unquestionably mean the abolishment of venereal diseases. At present, at least in many places, this is certainly not possible. The abolition of "red light" districts is invariably followed by a parallel increase in clandestine prostitution, luring many who would abstain from unmasked brothels, to say nothing of the increase in seduction and rape of innocent girls. The most feasible plan at present, as successfully tried in many European cities, especially Germany, is the municipal supervision of restricted "red light" districts. By continuous medical attendance, and the enforcement of strict sanitary measures, the normal spread of disease from this source has been reduced to a great extent. It may be argued that municipal control of prostitution implies public sanction of it, and is therefore morally wrong. This perhaps is true but there can be no question about the futility of attempting, at the present state of our civilization, to abolish prostitution or even to lessen it materially by passing laws against it. In view of this it is merely a question of a greater or lesser evil, and there can be no moral crime in lessening the dangers from an evil which we are powerless to destroy. It may be said that the lessening of danger from disease in houses of 
prostitution will increase their popularity. The same argument might be used, and has been used by the ultramoralists, to show that it is morally wrong to attempt to cure venereal diseases, since this lessens the terror of them. Such arguments might have more force if syphilis were a disease which affects only the individual, and was not a source of danger and burden to the community. Moreover it seems doubtful whether the person whose character is such a combination of moral weakness and cowardice that he shuns houses of prostitution only from dread of disease, will not spend his time in seducing innocent girls, or in other hardly less despicable crimes. It may further be pointed out that disease and immorality go hand in hand. A healthy body is conducive to a healthy mind, so by eliminating disease we would be doing at least as much toward giving a death stab to immorality as toward extending it.

The medical supervision of prostitution, adopted as a temporary measure, should be accompanied by efforts toward its ultimate reduction. The abolition of alcoholic drinks, the improvement of conditions in slums, the furnishing of decent surroundings and wholesome sports and exercises, and the enforcement of minimum wage laws for women are all measures which tend toward the reduction of prostitution, but foremost of all such measures should be education; in this lies our most powerful weapon against immorality and venereal disease. Hospitals, public schools, churches, libraries and the lecture platform all have the power of spreading the gospel of sex hygiene, each in its own way, each in a way especially suited to its listeners. Even the theatre can enter the field of education and it has done so. The play, and the motion picture patterned after it, entitled "Damaged Goods," in the estimation of the author, has done a great deal of practical good. Yet many ministers, teachers and newspapers, often in total ignorance of the real nature of the play, and in absolute neglect of their own opportunities for educating, have severely criticized the play as "immoral." Such men and women, who should know better, are nothing short of a disgrace to the institutions they represent and are largely responsible for the present popular ignorance concerning one of the matters of most vital interest to humanity - sex hygiene. 


\section{Yaws}

A common feature of nearly all tropical countries is the disease known as yaws or frambesia. In the Fiji Islands all healthy children are expected to pass through an attack of yaws and are sometimes inoculated with it by their parents. It is common in many parts of equatorial Africa, particularly on the West Coast. In the West Indies it is also a very common disease, especially in the islands which are largely inhabited by negroes. There is some evidence that yaws was imported to America from Africa with the slaves as were some others of the most troublesome American diseases. In Brazil the disease is called "buba brasiliensis" and is often confused with Leishmanian diseases.

The parasite which is the cause of this loathsome disease is a spirochæte, Sp. pertenuis, which is hardly distinguishable from the spirochæte of syphilis, and was for a long time thought to be identical with it. Recent investigations, however, have shown that there are some slight differences in the two parasites, though not enough to be recognizable by anyone but an expert. Like the spirochæte of syphilis, $S p$. pertenuis inhabits many different organs and tissues of the body, being found especially in the spleen and lymph glands and in the tumor-like "yaws." It is not yet conclusively proved that yaws and syphilis are not slightly different types of the same disease, though most workers believe in their distinctness, and for practical purposes, at least, it is best to consider them as distinct. One of the arguments in favor of the unity of the two diseases is that typical syphilis seldom occurs where yaws is prevalent, and vice versa, but this may be due to a reciprocal immunity, i.e., yaws giving immunity to syphilis, and syphilis to yaws.

The Disease. - In from 12 to 20 days and occasionally longer after infection constitutional symptoms appear, such as fever, rheumatic pains, and general illness. These symptoms are sometimes very severe, but usually they are slight and often hardly noticeable. After several days of such symptoms there appears a peculiar powdery scaling-off of the skin, sometimes almost invisible but at other times making white marks, especially conspicuous on the dark skin of negroes. After several days little pimples appear over the hair follicles in the patches of powdery skin. As these grow the raw flesh from beneath pushes the horny 
epidermis up, causing it to crack over the surface in such a way as to give the little tumor the appearance of a raspberry. Little yellow summits soon develop on the tumors, composed not of pus but of a cheesy material. Some of the pimples grow no

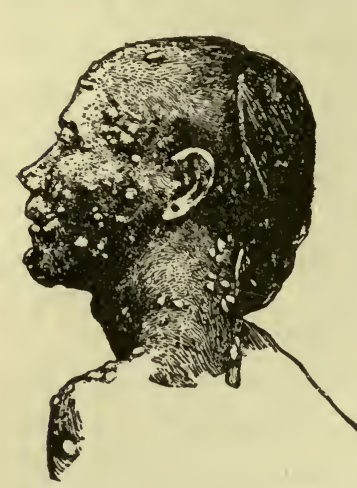

Fig. 10. A case of yaws. (After Manson.) further, but most of them become capped over with the yellow cheesy substance which catches and holds particles of dust, and thus become very dirty. These are the "yaws" from which the disease takes its name. During their formation they cause some itching, but are not painful. They reach the height of their development in 12 or 14 days and then usually begin to shrink, the dirty yellow cap, now dark colored, falling off and leaving a sound patch of pale skin. Sometimes, however, though in less than ten per cent of cases, ulceration of the yaws takes place, but this is probably due to complicating infections. The time that the disease lasts varies greatly according to the general health and constitution of the patient. In normal mild cases it may be all over in less than two months, while in weak or sickly individuals crop after crop of yaws may appear for months or years, recurring at irregular intervals. There is some evidence also that there may be a rare tertiary stage of yaws corresponding to a similar stage in syphilis, characterized by a diseased condition of the bones of the arms and legs, ulcers, etc., though this may often be due to mixed infections with syphilis or other diseases. The disease known as gangosa, prevalent in Guam and other East Indian Islands, is thought by some to be a consequence of yaws. Yaws is very seldom a fatal disease except in young children. Like syphilis it is very contagious, but the parasites are not transmitted from mother to baby before birth or by nursing.

Treatment and Prevention. - Care of the general health of yaws patients and conditions leading to the free eruption of the yaws aid much in shortening and alleviating the course of the disease. Salvarsan is poisonous for the parasites of yaws as it is for other spirochætes, and is an almost sure cure at any stage of the disease when injected either into the veins or muscles. In experimental animals the parasites disappear within 24 hours 
after the injection of salvarsan. Galyl and other arsenical substitutes for salvarsan are also effective against the disease.

The suppression of yaws in communities where it is common consists largely in affording isolated hospitals or houses for yaws patients and in preventing the patients by proper care and treatment from spreading the disease by contagion. Personal care on the part of the patient is often more than could be expected, considering that yaws is most common among halfcivilized and ignorant tropical races. However, the lure of a comfortable and congenial ward where he could get good treatment would undoubtedly induce many a native to submit to the practice of being sanitary, however it might grate upon his nerves at first. His accounts of the good treatment received would help in luring others, and what few ideas of sanitation he might have retained would help in spreading the gospel of sanitation. In this way the prevalence of the disease, at least in local areas, could be greatly reduced, and public money used for such purposes could be considered well spent.

\section{Infectious Jaundice or Weil's Disease}

In parts of Europe and in Japan, and also reported from various parts of North America, there occurs a disease characterized especially by fever and jaundice (i.e., affection of the liver causing a marked sallow color due to bile pigments in the blood), the cause of which has long been a puzzle to medical men. It has often been confused with yellow fever and with bilious typhoid, and it is not certain even now that the latter is not a very severe type of Weil's disease. Early in 1915 the connection of a new species of spirochæte, Leptospira icterohoemorrhagice, with the disease was discovered by two Japanese investigators, Inada and Ido. Later in the same year, and independent of the Japanese work, the same organism was discovered in Germany in cơnnection with Weil's Disease, the German investigators suggesting the name $S p$. nodosa. One could almost wish that the German name had been given first!

The Disease. - A week or more after infection the first symptoms appear rather suddenly in the form of headache, high fever, and a feeling of leaden fatigue in the legs which soon changes to intense pains. The muscles become so tender that 
even a slight touch is unbearable. Usually the spirochætes are abundant in the liver, suprarenals, blood and other organs and tissues during this initial "febrile" stage of the disease, but they are destroyed in the liver and suprarenals by antibodies usually by the seventh day. During the second week of the disease, termed the "icteric" stage, the fever subsides and marked jaundice, accompanied by swelling and pain in the liver, usually appears, though this symptom is sometimes evident as early as the third day. In some cases, in Europe at least, jaundice may not appear at all. The fever usually reappears in milder form about the end of the second week, but it is of short duration. Such symptoms as vomiting, nose bleed, upsetting of the digestive system, swollen spleen, weak but rapid pulse, and meningitis are usually associated with the disease, and kidney trouble is nearly always present, and is sometimes more severe than the jaundice. A

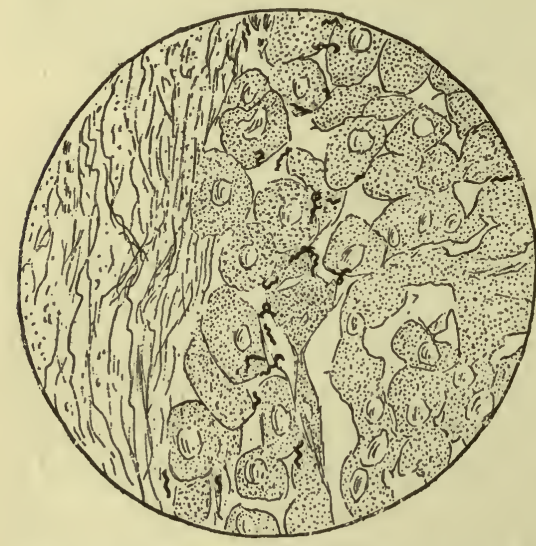

FIG. 11. Liver of patient who died from Weil's disease on sixth day, showing Spirochota icterohemorrhagice in tissue. $\times 200$. (Sketched from figure by Inada et al.) tendency for the mucous membranes and various organs to bleed is a common and dangerous symptom. During the icteric stage of the disease the spirochrtes disappear from the blood, and are gradually destroyed in other parts of the body; they persist longest in the kidneys, since the antibodies which destroy them elsewhere are apparently ineffectual against those situated in the kidney tubules. They continue to be excreted with the urine for six or seven weeks, though nearly all symptoms usually disappear much earlier. If death occurs, it nearly always comes between the eighth and sixteenth days of illness. The disease is said to be not as severe in Europe as in Japan, the mortality among infected soldiers in Flanders being less than six per cent.

The spirochætes are found in the blood, the cerebrospinal fluid, and in many of the tissues of the body, especially the liver and the kidneys. They vary in length from only four or five $\mu$ to $20 \mu$. 
( ${ }_{5000}^{\frac{1}{0}}$ to $\mathrm{I}_{2}^{\frac{1}{2}} 30$ of an inch) and are characterized by pointed and usually hooked ends (Fig. 5G). According to the workers in Japan the undulations are irregular and more like those of the relapsing fever spirochætes than like those of the spirochæte of syphilis. Noguchi, however, states that the number of coils in a given length is greater than that in any spirochæte hitherto known, there being ten or twelve coils in five $\mu$ ( $5 \frac{1}{50 \sigma}$ of an inch). The figure (Fig. 5G) shows only the gross undulations of the organism and not the individual coils. From their descriptions it would seem that the Japanese workers have mistaken these gross undulations for the true coils. Noguchi believes this spirochæte to have characteristics sufficiently distinctive to warrant its being placed in a new genus, Leptospira. The spirochætes become most numerous from the 13th to the 15th day of illness, and begin to diminish and degenerate by the 24th or 25th days, though they may continue to be excreted with the urine for six or seven weeks.

It is probable that the spirochætes gain access to the body either by way of the alimentary canal or directly through the skin. The disease can be experimentally transmitted to guineapigs by applying an emulsion of diseased liver to the shaved but uninjured skin, infection taking place in as short a time as five minutes. Infection is more certain if any abrasion of the skin exists.

It has been shown that both the urine and the fæces of infected people contain living spirochætes and that these excretions are infective. Since infection can occur directly through the skin contact with contaminated ground is dangerous and probably accounts for the prevalence of the disease in certain mines in Japan. Rats have been shown by Japanese investigators to serve as a reservoir for infectious jaundice. The spirochætes are very common in rats, especially in the kidneys, being constantly excreted with the urine. Examination of 86 rats in cities and coal mines in Japan where infective jaundice occurs showed that nearly 40 per cent carried virulent spirochætes in their kidneys, in most cases demonstrable by microscopic examination of kidney tissue or urine as well as by experimental inoculations. In America the parasites have been demonstrated in wild rats caught in the vicinity of New York City and in Nashville, Tenn. The ease with which rats may contaminate food with their ex- 
cretions makes it appear probable that these animals are an important means of spreading the disease, and this most readily explains the common occurrence of epidemics in families. Two cases have been reported as having resulted from the bites of rats. That rats serve to spread Weil's disease in Europe also appears evident from its common occurrence where rats are abundant. In Europe butchers are especially prone to it, and severe epidemics of it have broken out in the rat-infested war trenches.

Treatment and Prevention. - The Japanese investigators find evidence that salvarsan is destructive to $S p$. icterohemorrhagice, but their results are far from convincing and the German investigators say that salvarsan does not destroy the parasites. More investigation and experimentation needs to be done before this question can be settled.

Investigators of both countries have had greater success in treating the disease by injection of the serum of a convalescent or of an animal which has become immune. The Germans found the convalescent serum effectual, either as a preventive or for cure, when diluted 100 times. Japanese workers, on the other hand, put far more faith in active immunization. They inject spirochætes which have been weakened by subjection to very dilute carbolic acid and left on ice for a week. Guinea-pigs can be immunized by such injections, or even by injections of dead parasites or the products of their disintegration.

Prevention of this disease, as of plague, evidently resolves itself largely into rat destruction by poisoning, trapping and rat-proofing. Some reduction should be obtained by keeping food where rats cannot get access to it, and, of course, where it cannot become infected, directly or indirectly, by the excretions of human patients. However, since the parasites are able to penetrate directly through thin skin, especially if there are any abrasions, care should be taken to prevent contamination with urine of objects or surfaces which are likely to come in contact with the hands or other parts of the body of other people. As remarked before, epidemics in mines are largely due to insanitary habits and contamination of the ground. In mines or other places where sanitary conditions are difficult to enforce, wholesale immunization would probably be effective, but good results can also be obtained by disinfecting the ground. According to 
Japanese authors, two epidemics in coal mines have already been prevented by the latter method, combined with removal of inundated water.

\section{Yellow Fever}

Distribution. - Yellow fever is a disease which is especially characteristic of the seaport towns of tropical America, although it is also endemic on the west coast of Africa, whence many think it was imported to America with the slaves. Its approximate distribution is shown in Fig. 12. In the past, before the days of the strict quarantine laws now enforced, serious epidemics of this dread disease appeared during the summer in numerous seaports of subtropical and temperate countries. At one time

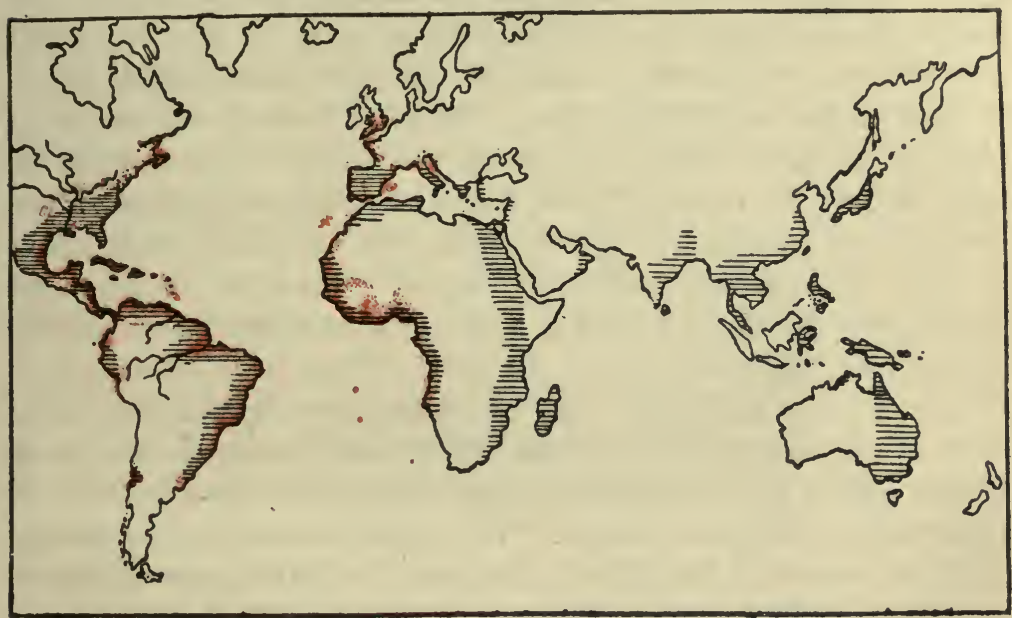

FIG. 12. Map showing geographic distribution of the yellow fever mosquito, Aëdes calopus (black lines), and former distribution of yellow fever (red stipple).

there was no city on the whole Atlantic and Gulf coasts of the United States which was exempt from yellow fever epidemics, and the disease exerted a serious influence on the economic conditions, especially of our Southern States. In New Orleans there have been epidemics which have cost thousands of lives, the last one occurring in 1905. In temperate cities the epidemics always ended with the coming of frost and destruction of the transmitting mosquitoes. Now the situation is quite different and there is no reason to believe that the world will ever again see such a sight 
as was formerly only too common - a frantic, terrorized city helpless in the grip of a deadly yellow fever epidemic. No epidemic has occurred in the United States since 1905 and many of the tropical cities, such as Havana, Manaos and Rio de Janiero, which were formerly famous as endemic centers of the disease, and from which it was carried to seaports in all parts of the world, are now practically free from it. It is only in such notoriously unsanitary cities as Merida in Yucatan and Buenaventura in Colombia that yellow fever still rages, with little or no attempt on the part of the inhabitants to stamp it out.

Nature of the Disease. - Our present knowledge of the nature of yellow fever and of its dissemination, which has made possible the scientific checking of the disease and will undoubtedly result ultimately in its complete extermination, is largely the result of the noble and self-sacrificing work of the American Yellow Fever Commission appointed in 1900, consisting of Reed, Carroll, Lazear and Agramonte. Three of these illustrious men, Doctors Lazear, Reed and Carroll, lost their lives directly or indirectly as the result of their work, but their achievements are of inestimable value to the human race and their names will not soon be forgotten. The discovery by Noguchi of the organism causing the disease in 1918 has opened up new possibilities in the way of immunization.

Yellow fever was shown by the American Commission to be not a contagious disease, but one which can be transmitted only by the yellow fever mosquito, Aëdes calopus, or by injections of blood from an infected person. The organism causing the disease was discovered by Noguchi at Guayaquil in 1918, and was found to be a spirochæte-like organism closely related to, and very closely resembling, the spirochæte of infectious jaundice. It was named by Noguchi Leptospira icteroides. The organism can be found in the blood serum and also in the tissues of the liver, kidneys and other organs. It has been obtained in pure culture both from blood of patients and from inoculated animals. It is an extremely delicate filament varying in length from 4 to $9 \mu$, and tapering gradually toward the extremities which end in immeasurably thin sharp points. The filament is minutely wound at short and regular intervals, the length of each spiral measuring about one-fourth of a micron. The windings are so placed as to 
form a zigzag line by the alternate right angle change of direction of each consecutive spiral. The organism is smaller than that of infectious jaundice and is actively motile.

Since the mosquitoes cannot transmit the disease by biting until 12 or 14 days after sucking infected blood the parasites evidently undergo a cycle of development in the mosquito. The appearance and habits of the yellow fever mosquito are described on page 443.

The Disease. - Yellow fever has an incubation period of from three to six days. The first symptoms are severe headache and aches in the bones, followed by a sudden fever during which the face is flushed and swollen and the skin dry. This fever slowly subsides, and after three or four days there is a period of "calm" during which the temperature is near normal but the pulse very slow. By the third day the skin usually becomes a characteristic yellow color, which, as the disease progresses, changes to a deep coffee brown. A striking but not invariable symptom, and one of ill omen, is the "black vomit," a gushing up through the œesophagus of a coffee-colored or even black fluid, consisting largely of fragments of red blood corpuscles and freed hæmoglobin, and sometimes even pure blood. The period of "calm" may lead to recovery in a few days or there may be a second fever which lasts irregularly for a longer time than the first.

Yellow fever is a very fatal disease. During the French operations at Panama relay after relay of laborers were stricken with the yellow plague and were turned loose to die without mercy or help, to be replaced by a new set. Not only the laborers but the engineers, nurses and others were stricken down. One vessel is reported to have brought over 18 young French engineers, all but one of whom died of yellow fever within a month after their arrival.

Fortunately yellow fever gives a permanent immunity after one attack has been successfully withstood. In children the disease is often very mild so that it is frequently not even recognized, yet the immunity it gives is permanent. Natural immunity is unknown in any race, sex or age, though the negroes suffer less from the disease and have a much lower per cent of mortality than the whites.

Treatment and Prevention. - Until the discovery of the organism causing yellow fever by Noguchi and its isolation in pure 
culture, the treatment of yellow fever consisted only of careful nursing and in the maintenance of the best hygienic conditions: there are no drugs known which have any value as specifics against the disease. Recently, however, Noguchi and his fellow workers at the Rockefeller Institute have demonstrated the development of antibodies in animals and man inoculated with killed cultures of the leptospira, and from the results of vaccination in guinea pigs, concluded that with the inoculation of a sufficient quantity of dead organisms they were rendered immune to subsequent infection. Distinctly encouraging results have been obtained from the vaccination of human beings. Of 8000 nonimmune persons vaccinated in tropical America, excluding those exposed to the disease just before or immediately after vaccination, no cases of yellow fever have developed, while among unvaccinated persons during the same period and in the same locality there have been about 700 cases of the disease. A therapeutic serum has also been prepared for treatment of yellow fever. According to Noguchi persons treated with a sufficient quantity of the serum before the third day of illness have invariably recovered. By the fourth day of illness the injuries to organs are so great as to be irreparable in severe cases. The usual 50 to 60 per cent mortality from yellow fever was reduced to 9 per cent by the use of the serum, while still in an experimental state.

The eradication of yellow fever consists largely in the application of sanitary measures to exterminate the yellow fever mosquito Aëdes calopus, but in the face of an epidemic this can now be supplemented by vaccination to cut off the supply of nonimmunes from infected mosquitoes. According to Noguchi a threatening epidemic in Central America in 1920 is reported to have been checked by these methods within a month, i.e., before the development of the second set of cases. The value of immunization as an emergency measure does not, however, minimize the importance of anti-mosquito campaigns, since the elimination of both factors - non-immune human beings and infected mosquitoes - is necessary for the eradication of yellow fever. The habits of the mosquito, as described on p. 444, are such that it is not difficult to combat and successful campaigns against it, with a resultant obliteration of yellow fever, have been made in many places. Panama, Havana, Rio de Janiero, and recently Manaos and 
Yquitos, are conspicuous examples of tropical cities which have been cleared of the disease which once made them highly dangerous to visitors and a menace to the rest of the world. In connection with an incessant war on the transmitting mosquito in all stages of its life history, all known or suspected cases of yellow fever should be carefully screened so that mosquitoes can have no access to them.

\section{Rat-bite Fever}

In many parts of the world, especially in Japan, there occurs a disease which follows a rat bite, and is therefore known as " ratbite fever." It has been reported from various localities in the United States. Some inflammation occurs at the place of the bite and the neighboring lymph glands swell up. After several weeks a high fever ensues, preceded by chills and headache. The apparently healed rat bites become inflamed and there is usually a red rash which spreads all over the body. In from three to seven days the fever subsides but it recurs, usually within a week, with similar symptoms, and the rash is more constantly present than in the first attack. In some cases there are still more relapses.

The similarity of the disease to such spirochæte diseases as the relapsing fevers is obvious, and its spirochæte nature was long suspected by Japanese physicians, especially when they found salvarsan to be effective in its treatment. Within the past few years some Japanese physicians (Futaki, Takaki, Taniguchi and Osumi) discovered in seven out of eight patients numerous actively moving spirochætes in the broken-out skin and in swollen lymph glands. Animals were successfully inoculated with the disease by means of bits of skin tissue and blood containing spirochætes. The organism, which has been named Spirochoeta morsus muris, is described as being an actively moving animal, larger than Sp. pallida of syphilis, but smaller than the relapsing fever spirochætes. It is rather short and thick with an attenuated portion or flagellum at each end. Long spirochætes, at first thought to be specifically distinct from the short thick forms, also are found in human infections. According to Kaneko and Okuda these are probably degenerate forms resulting from the action of antibodies.

The Japanese investigators have been unable to find the spiro- 
chæte in the saliva of infected rats or of other rodents, but only in their blood. From this the conclusion has been drawn that the source of infection in a rat bite is blood from hemorrhages of the gums or tongue which contaminates the teeth.

Both mercury and salvarsan are effective in the treatment of this disease as of most other spirochæte diseases.

Attention should be called to the fact that rat bites may often give rise to diseases which may be of quite different nature from the "rat-bite fever" described above. It is well known that many different infective organisms live in the mouth and around the teeth of such animals as rats, and it is not surprising that infections of divers kinds may result from rat bites, and that these infections should have been confused with typical rat-bite fever. Several investigators have described a vegetable organism, Streptothrix, as the cause of rat-bite fever. Recently Ruth Tunnicliff has shown that a form of pneumonia in rats is produced by a Streptothrix very similar to, if not identical with, that described in some cases of rat-bite fever. It is very probable that these cases were really infections with the pneumonia-causing organism, and quite distinct from the Japanese disease.

\section{Other Spirochæte Diseases}

Spirochætes, often in association with bacteria of various kinds, have been found in a number of other human diseases, and are in all probability at least partially the cause of them.

The common spirochæte, Sp. buccalis, which lives about the gums and roots of the teeth in almost all human mouths is thought by some investigators to be entirely harmless, living only on waste matter. By others it is thought to become pathogenic under some circumstances, and, in partnership with certain cigar-shaped bacteria, to be the cause of Vincent's angina, a diphtheria-like ulceration of the tonsils and throat; of noma, an ulceration of the mouth cavity and cheeks; of ulcerations of the nose, teeth and lungs; and of balanitis, an ulceration of the genital organs which may occur after unnatural sexual relations. In central America there is a common disease "mal de boca" (disease of the mouth) which is marked by swollen, spongy and tender gums over which a whitish pellicle forms. It is infectious and is probably caused by a delicate spirochæte found on the 
lesions. Some workers believe that some or all of these afflictions are due to different species of spirochætes and bacteria, but the fact that both organisms are found together in all these diseases, and that they show only such slight differences from the organisms in the mouth as would be expected under altered conditions, makes it seem quite possible that Spirochoeta buccalis and its constant companion, a cigar-shaped bacterium, are the causes of all of them. The conditions which seem to favor the growth and disease-producing propensities of these organisms are heat, moisture, filth and absence of air. Wherever these conditions prevail, and these ordinarily harmless organisms can get a foothold, sores and ulceration are likely to result, accompanied by more or less fever and digestive disturbance due to absorption of poisonous substances from the decaying tissues.

The treatment of these affections must vary with their location. For the sores on the tonsils or mouth cavity in Vincent's angina or noma either salvarsan or silver nitrate is effective. It should be daubed on the injured tissue with a piece of cotton. The silver nitrate is less dangerous than salvarsan and equally effective for these superficial ulcers. Treatment of the infected parts with a two per cent solution of silver nitrate for a few days results in a rapid healing. In case of balanitis, ordinary cleanliness and exposure to air is sufficient to cause a spontaneous healing in four or five days. Washing with hydrogen peroxide, which liberates oxygen in the presence of organic matter, is very destructive to such organisms as these, which thrive best in the absence of air.

In the Sudan region of Africa, and also in Colombia, South America, there is found a certain type of bronchitis, marked by fever and often by hemorrhages along the respiratory tubes, which is accompanied by the spirochæ:: Sp. bronchialis. This parasite has very slender pointed ends, and averages eight to nine $\mu$ ( $\frac{1}{300}$ of an inch) in length, but its most marked characteristic is its variability. These spirochætes reproduce by the peculiar method of "granule shedding," breaking up into tiny round bodies which later develop into new spirochætes. It is probable that these little particles of living matter can resist drying up in air, especially in humid atmospheres, and may therefore be transmitted with dust or with little droplets of moisture propelled by coughing. 
Tropical Ulcer. - Still another human disease that has been attributed to spirochætes is tropical ulcer, also known by the more impressive name, "tropical sloughing phagedæna." This is a type of sore on the skin, most commonly of the leg, which originates either in some slight abrasion of the skin or in some preëxisting wound or sore, especially in persons debilitated by some other disease or by alcohol. It begins in a tiny blister which soon bursts, and the sore thus exposed spreads very rapidly, constantly sloughing a yellowish, moist and exceedingly fetid matter. After a few days,

Fig. 13. Tropical ulcer. (Drawn from photo by Halberstadter in Kolle and Wassermann.)

while the sore is still spreading, the center of the slough begins to liquefy and is gradually sloughed off and heals. Usually the ulceration confines itself to the skin but sometimes it goes deep into the muscles, nerves and bloodvessels, even injuring the bones and joints. Sometimes permanent deformity or even death results from these extensive excavations, death resulting especially from the opening of some large bloodvessel.

Tropical ulcer occurs in nearly all hot damp tropical countries. Although not definitely proved, it is usually accepted that the spirochætes, Spirochœeta schaudinni, which can almost always be found in the ulcers, together with cigar-shaped bacteria found in association with them, are the ringleaders in producing it. The treatment usually recommended is a thorough cauterization of the sore, followed by antiseptic washes and applications. Salvarsan and other arsenic compounds have been found beneficial in many cases. Finocchiaro and Migliano in Brazil claim to have found a specific cure for this loathsome disease in an application of powdered permanganate of potash or in a compress of a one to ten solution of this substance. They achieved a complete cure in from ten to thirty days in every one of seven cases.

Ulcerating Granuloma. - Of a somewhat similar nature to tropical ulcer, and of wide distribution in the tropics, is " ulcerating granuloma of the pudenda," a sore which spreads, very slowly however, over the external genitals and along the moist folds of skin in neighboring regions. Both spirochætes and bacteria have been found deeply situated in the tissues at the bases of these sores, but to what extent either or both are responsible for 
the condition is not known. The disease is peculiar in being very refractory to treatment by any of the usual methods of cauterization or application of drugs. Recently, however, it has been found to succumb to X-ray treatment, and this method is now extensively employed. Aragao and Vianna in Brazil and Breinl and Priestley in Australia have obtained excellent results from intravenous injections of tartar emetic.

Japanese Seven-Day Fever. - A number of Japanese workers have recently described this disease as resembling infectious jaundice but usually without the occurrence of jaundice. It has a fairly wide distribution in Japan among field workers. A spirochæte morphologically indistinguishable from Leptospira icterohomorrhagice, but distinct in its immune reactions, has been found in blood and urine of human cases, and in inoculated animals. The organism was named Spirochceta hebdomadis, but if related to the organism of infectious jaundice should be called Leptospira hebdomadis. A spirochæte believed to be identical with this was found in the field mouse, Microtus montebelli, and the Japanese believe that this mouse acts as a reservoir for the disease as does the rat for infectious jaundice.

Other Spirochætes. - Spirochætes have been found in connection with still other human afflictions, and it is possible that they may be the cause of them. In most cases, however, it is more probable that spirochætes which are normally harmless and live only on dead matter find congenial surroundings in tissues diseased by some other cause, and that this accounts for their presence. Often, however, such ordinarily harmless spirochætes may change their habits under suitable conditions and become pathogenic, thus aggravating the diseased condition. The pathogenic propensities of spirochætes have been demonstrated in so many cases, however, that they may rightly be looked upon as one of the most destructive groups of human parasites. 


\section{CHAPTER V \\ LEISHMAN BODIES AND LEISHMANIASIS}

Leishman Bodies in General. - While investigating the cause of a deadly disease of tropical India known to the natives as kala-azar or dumdum fever, Leishman, in 1903, and at about the same time, Donovan, discovered in the spleen of victims numerous little round parasites. These looked to Leishman exactly like the non-flagellated stage of a trypanosome (see Chapter VI), and he naturally took them to be developmental stages of trypanosomes, and added another terrible disease to the credit of those murderous animals. Later, however, it was found that while these little round organisms resemble a certain stage in the life history of a trypanosome, yet they never reach this fully developed form. Nevertheless it was discovered that when transferred to the intestine of certain insects, or when grown on artificial cultures, they undergo a wonderful transformation. They become elongate in form and develop a waving flagellum, assuming what is known as a "Herpetomonas" form (see Fig. 14L), and they move about so actively that it is difficult to believe that they are really transformed from the un-animal-like round bodies found in diseased human bodies. Such flagellates, under the name "Leptomonas" or "Herpetomonas," had been known before, and were recognized as common parasites of insects, belonging to a primitive group of the class Flagellata. They were also known to present, during their development, this unflagellated round condition, but always in the bodies of insects. Here was a vicious form of the parasite which was not content with life in an insect, but must adapt itself to live in the bodies of warm-blooded animals. There is reason to believe that some of the flagellates which normally live exclusively in the intestines of blood-sucking insects have the power, if injected into warm-blooded animals, to adapt themselves to the conditions they find there, causing more or less local inflammation and sores. In Panama, for instance, sporadic cases of sores occur in which are found Leish- 
man bodies in small numbers, resulting from the bite of horseflies (Tabanidæ) of various species. There is every reason to believe that the parasites in these sores are normally parasitic in the insects only, but are able to adapt themselves to their new environment in human flesh and to multiply there for a time. They are permanently sidetracked, however, and have no further chance of completing their life history or of reaching new hosts, unless a suitable fly should, by some infinitesimally small chance, suck blood from the sore in which they were developing, and thus rescue them.

Several investigators have recently shown that a number of typical insect flagellates, if injected into mice and rats or other mammals, or even birds, may become pathogenic and even cause the death of the animal. That the well-established Leishmania diseases of man and other animals originated from insect flagellates can hardly be doubted, but it is possible that in some cases the parasites may have adapted themselves to their new type of host to such an extent as to have become quite independent of the insects from which they originated. Fantham suggests that all forms of Leishmania and Herpetomonas may be mere physiological races of a single species which is variously adapted to live in a variety of different hosts, and perhaps able to adapt itself anew to unaccustomed hosts under certain conditions.

Leishmania and Herpetomonas belong to a group of the class Flagellata known as the Hæmoflagellata. This group presents a series of forms from the simple Leishmania, which at times is a non-motile, unflagellated organism, through the increasingly highly developed Herpetomonas and Crithidia to the trypanosomes (see Fig. 18). Some reach only the Herpetomonas stage as adults, others only the Crithidia stage, while others pass through the entire series of developmental stages and reach the final trypanosome stage. All of them are probably primarily parasites of the guts of insects or other invertebrates, and only comparatively few of them have adapted themselves to spending part of their existence in the blood or tissues of vertebrates. Apparently only the Leishmania and trypanosome forms are adapted for existence in vertebrates, since the other forms are not found in them, except in rare instances when Herpetomonas forms are found in the blood of Leishmania-infected individuals. A number of species have become thoroughly adapted to life in 
vertebrates and are now normal parasites of them, and others, as already shown, if accidentally introduced may be able to multiply sufficiently to cause local or temporary sores, or even a fatal infection.

All the species of hæmoflagellates which normally live in warmblooded animals in the form of Leishman bodies are grouped together in the genus Leishmania. A number of human diseases are known to be caused by them. Kala-azar of southern Asia, already mentioned, is the most severe one. A similar disease, infantile kala-azar, occurs around the Mediterranean, especially in children. There are also a number of Leishmanian diseases which, instead of causing constitutional disturbances, cause sores or ulcers on the skin or mucous membrane. One type, oriental sore, also called by various local names, is widespread throughout many tropical countries, especially southern Asia and around the eastern end of the Mediterranean, and possibly in tropical South America. It causes temporary sores on the skin, chiefly of the exposed parts of the body; the sores may or may not ulcerate. In South America there occurs a much more vicious type of the disease in which the skin sores are followed by ulcers spreading over extensive areas of the mucous membranes of nose and mouth, often resulting fatally. A parasite, Aphthomonas infestans, believed to be allied to Leishmania, has recently been described by Stauffacher as the cause of foot-and-mouth disease.

The clinical manifestations of these ulcers and sores on the skin or mucous membranes are extremely variable and indicate the possibility of there being a number of different species or at least varieties of Leishmania causing them. There are some parasitologists who believe that all the different kinds of Leishmaniasis - internal, cutaneous or mucosal - are caused by different strains of the same species, while others believe in the existence of several species. Usually four species are recognized, as follows: Leishmania donovani, causing kala-azar; L. infantum, causing infantile leishmaniasis; $L$. tropica, causing cutaneous sores; and L. americana (brasiliensis), causing sores or ulcers of long duration on the skin and mucous membranes. However, until we are familiar with the adult forms of all the various types of Leishmania, and know more about their life histories, we can only guess at their classification. 


\section{Kala-azar}

About 1870 there began a great epidemic of a strange and deadly disease in Assam, India, which spread up through the Brahmaputra Valley. It was believed to have been imported by the British from Rangpur, where a similar epidemic had been raging for some time before. Whole villages and settlements were depopulated and the country was terrorized by the "black sickness." It is said that victims of the disease were driven out of the villages, sometimes being made unconscious with drink, taken into the jungle, and.burnt to death. Some villages completely isolated themselves from the outside world, and still others were entirely deserted for new and uninfected districts. The natives were most severely affected, no doubt due both to their filthiness and unsanitary habits and to their weak condition as the result of almost universal malaria a hookworm. Before the true nature of the disease was discovered it was usually diagnosed as "severe malaria"; one physician concluded that it was excessive hookworm infection, since he found hookworms almost universally present in kala-azar sufferers.

This Assam epidemic, which lasted for many years, is the only recent case of a great epidemic of kala-azar, although the disease now occurs endemically in many parts of India and Southern China, and is spreading in the Sudan region of North Africa. It has been pointed out that the endemic parts of China, chiefly along the north bank of the Yangtse River and its tributaries, correspond closely in latitude and climate to a considerable part of southern United States, and since kala-azar is believed by some to be spread by bedbugs and perhaps other vermin, there is danger that once introduced it might become endemic in America. A single case has been found in Brazil, contracted in a region where another form of Leishmaniasis is prevalent. How this case should be explained is difficult to know.

Transmission. - In spite of numerous experimental investigations to discover the mode of transmission of the kala-azar parasite, Leishmania donovani, the question is still obscure. Captain Patton, of the British Medical Service in India, adduced some evidence that the common Indian bedbug, Cimex hemipterus (rotundatus), is the normal intermediate host and transmitter of kala-azar. Using laboratory-bred bugs, Patton succeeded in 
getting abundant growths of the parasites in the intestines of the bugs after they had been fed on infected blood. When sucked up by the bugs, the Leishmania-laden cells in the blood are digested and the parasites set at liberty in the stomach. Here after several days they begin to go through their remarkable transformations and active flagellated Herpetomonas forms develop similar to those which occur in artificial cultures (Fig. 14, $\mathrm{F}$ to $\mathrm{O}$ ). After several days of free active life the parasites round up again, lose their flagella, and are then presumably ready for inoculation into a new host. All these changes occur during a period of 12 days. Patton has not, however, shown that the bedbug is capable of transmitting the parasites to other victims by means of its bites, though it is possible that scratching of the bites and crushing of the bugs might cause infection. Developmental stages have also been traced in the mosquito, Anopheles punctipennis, but here again there is no proof of the insect's method or power of transmitting the parasites to new hosts. The facts connected with the spread of the disease in India seem to favor the theory of transmission by a household insect. Rarity of the parasites in the circulating blood has been claimed as an argument against the insect transmission theory, but Patton has shown that almost every smear of blood from an infected person contains white blood corpuscles with the Leishman bodies in them. On the other hand the manner of spreading of the disease in Sudan is rather opposed to a theory of insect transmission, and it has been suggested that infection may take place through the medium of contaminated water or food, since experimental animals occasionally become infected when fed on infected material. The suggestion is also made that an intestinal wound of some kind may be necessary to allow the entrance of parasites into the blood and organs of the body. Bodies resembling Leishman bodies have been found in the fæces of infected persons, so that fæces may in some way have to do with the transmission of the parasites. The difficulty experienced in inoculating the disease into experimental animals makes the investigation of its transmission very difficult. The parasites develop readily in artificial cultures at relatively low temperatures, presenting the series of changes shown in Fig. 14. These forms are practically identical with those found by Patton in the intestine of the bedbug and undoubtedly represent part, at least, 
of a possible cycle in an insect host. That this phase of the life history of the parasite may normally be omitted is nevertheless quite possible.

Human Cycle. - After entering the human body, the parasites probably utilize the blood and lymph streams to obtain transportation to all parts of the body, but do not live free in

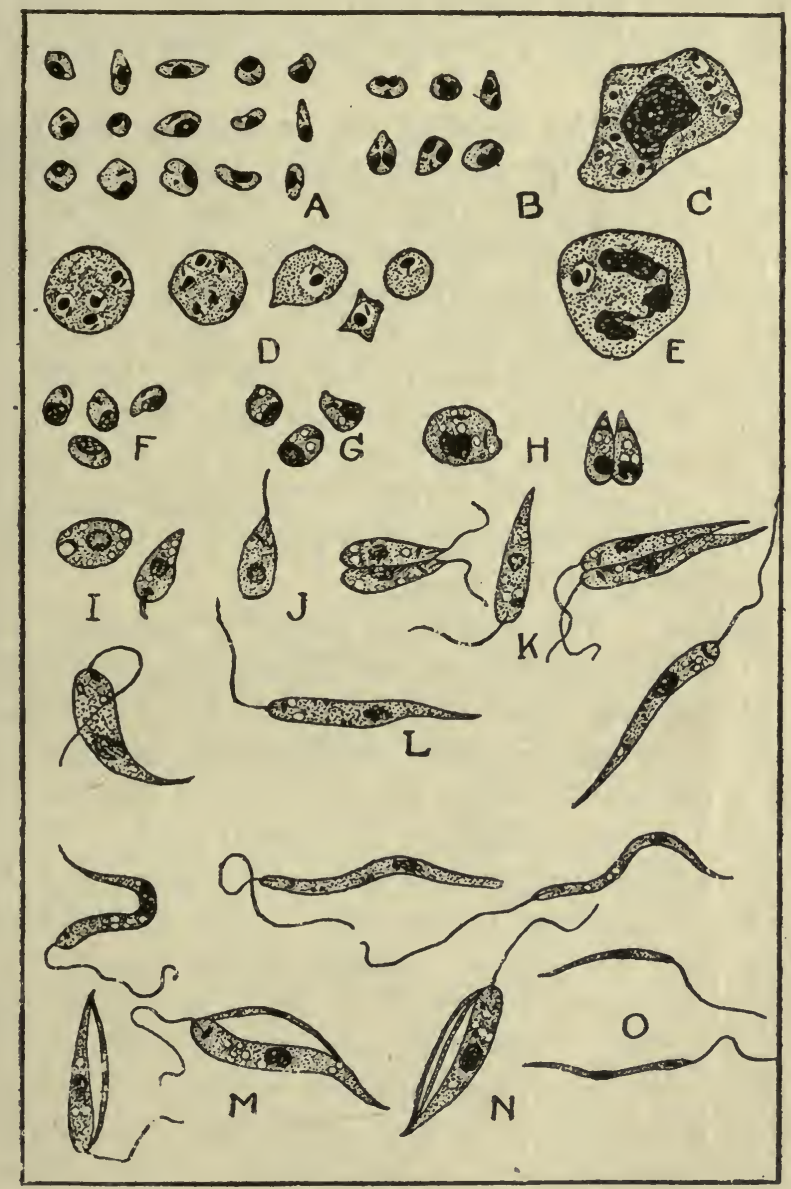

Frg. 14. Parasites of kala-azar, Leishmania donovani; $A$, isolated parasites irom spleen; $B$, dividing forms from liver and bone marrow; $C$, spleen cell with parasites; $D$, group of cells with parasites; $E$, parasite ingested by leucocyte; $F-O$, from cultures; $F$ and $G$, early stages after ingestion; $H$, large dividing forms; $I$, development of flagellum; $J$, small flagellated form; $K$ and $L$, flagellated Herpetomonas forms; $M$ and $N$, unequal division; $O$, parasites resulting from unequal division shown in $\boldsymbol{M}$ and $\boldsymbol{N}$. $\times$ about 1500. (After Leishman.) 
the body fluids. They enter the delicate endothelial cells which line the blood and lymph vessels, and also the cells of the spleen, liver and lymph glands. Within the cell they have entered they grow and multiply rapidly (Fig. 14C and D). The individual parasites (Fig. 14A) are exceedingly small, about two $\mu$ (less than $\frac{1}{10.000}$ of an inch) to four $\mu$ in diameter. They are round or oval in form with a large nucleus and a smaller parabasal body shaped like a little rod and set more or less at a tangent to the nucleus.

In a short time, by dividing and re-dividing, the Leishman bodies completely fill the cell they inhabit, causing it to enlarge to many times its normal size (Fig. 14C). There may be as many as several hundred parasites in a single enlarged cell. The parasitized endothelial cells often seem to " run amuck," breaking loose from their normal position on the lining of bloodvessels and becoming free-living carnivorous cells like the white blood corpuscles. When these parasite-filled cells finally burst, the liberated parasites enter new cells, or are gobbled up by the white blood corpuscles (Fig. 14E). It is probable that the parasites are ingested by bedbugs while inside free endothelial cells or white corpuscles in the blood.

The Disease. - The incubation period of kala-azar after infection is not definitely known, but Manson cites one case where an Englishman was attacked by a fever, which terminated in kala-azar, within ten days after arriving in an endemic locality.

A high fever usually marks the onset of the disease, and this persists more or less irregularly for several weeks. Meanwhile the spleen and liver enlarge enormously, increasing and decreasing with the fluctuations of the fever. After several weeks the fever drops and the patient becomes almost normal for some time, only to be attacked by the fever again, with an enlargement of spleen and liver. These remittent attacks gradually dwindle to the steady low fever, accompanied by sweating spells, rheumatism-like aches, high pulse rate, anemia and a general wasting away, with the skin often a dark earthen color. Dysenteric symptoms, with discharges of blood and mucus, are common, especially in the late stages of the disease, and frequently after death the intestine is found to be extensively ulcerated, with numerous parasites in the walls of the ulcers. Parasites are usually found most abundantly in the spleen, liver capillaries, 
bone marrow and lymph glands. When the chronic condition is reached the patient presents an appearance not unlike that resulting from chronic malaria, and it is little wonder that the diseases were long confused. Usually complication by some other disease, especially dysentery, which gets a severe hold on account of the low vitality of the victim, causes death (according to Rogers in 96 per cent of cases), but in a relatively small per cent of cases there is recovery. A steady gain in weight, however slight, is said by Mackie to be a fairly accurate sign of recovery.

Treatment. - Within the past few years (1914-1921) the remarkable destructive effect of antimony, especially in the form of tartar emetic, on Leishman bodies has been thoroughly established. Tartar emetic as a cure for Leishmanian diseases was first tried out in 1912 with astonishing success by Vianna, a Brazilian investigator, on the Leishmanian ulcers of the face and nasal mucosa. Similar treatment has been applied with equal success to oriental sores and to infantile kala-azar. Its application to the more severe Indian kala-azar has been attended with great success, and even advanced stages of the disease can sometimes be cured by its use. Rogers and Hume have used it extensively in India. Injections of metallic antimony have also been found of great benefit in treatment of this disease.

The usual method of giving tartar emetic is by injections into the veins, as in trypanosome diseases. From one to ten cc. of a one per cent solution is given, the dose being gradually increased in accordance with the age and tolerance of the patient. The drug is a powerful one, and if given in over-doses may cause severe disturbances of the digestive tract and of the kidneys, but if it is given in small quantities to begin with, and its effects carefully watched as the doses are increased, it can be used without danger and constitutes a treatment as specific in its effects as is quinine on malarial parasites, or salvarsan on spirochætes.

Prevention. - On account of the uncertainty which exists concerning the mode of transmission of kala-azar, victims of the disease and those who have closely associated with them should be quarantined and their houses thoroughly disinfected to kill any bedbugs or other vermin, as well as any Leishman bodies which might exist in any body excretions. The safest method in the case of the native huts which are hopelessly filthy and 
unsanitary is to burn them with all their junk. This method of stamping out the disease before it has had time to spread has been successfully used on some of the tea estates in Assam. For the successful prevention of the spread of the disease, an isolation of 300 to 400 yards has been found sufficient, a fact which exonerates most flying insects as transmitters. Houses in endemic regions should be kept scrupulously free from bedbugs, and any place where bugs might be acquired should be carefully avoided.

Since the parasites have been shown to exist in the fæces of infected persons, careful and thorough disposal of the fæces should be attended to. The possibility exists that non-bloodsucking flies which frequent human fæces may be instrumental in spreading infection. Until proved otherwise, precautions against this should be taken in endemic places.

\section{Infantile Kala-azar}

In many of the countries bordering the Mediterranean-Algeria, Tunis, Malta, Crete, Greece, southern Italy, Sicily, Spain and other regions - there occurs a disease which in many respects closely resembles true kala-azar, but differs from it very strikingly in other ways. While true kala-azar attacks young and old alike, the Mediterranean disease attacks infants and children almost if not quite exclusively. Children between one and two years old are most frequently subject to it, while children over six years old are practically exempt. While true kala-azar is not readily communicable to other animals than man, the Mediterranean disease occurs naturally in the dogs of endemic regions and in some places where the disease is not known to occur in children. It can be experimentally transmitted not only to dogs but also to rats, mice and, with more difficulty, to monkeys. Cats cannot be successfully inoculated. It is believed by some that the disease in dogs is different from that in children, but the similarity in symptoms, and geographic distribution, as well as the fact that dogs can be infected by parasites from human beings, and other dogs from these dogs, all point to the identity of the diseases.

Transmission. - A remarkable fact connected with artificial inoculation of the disease is the great quantity of infective ma- 
terial which is necessary to produce infection. Injection of infected material under the skin does not transmit the disease, although in nature this is probably the mode of transmission. Evidently, then, the natural means of transmission must be by more powerful or virulent parasites than can be obtained from already infected animals. The common opinion is that the dog flea, Ctenocephalus canis (see p. 416), is the usual transmitting agent, and that this insect serves as an intermediate host for the parasites. This opinion is based on the fact that parasites apparently identical with those in infected children have been found in the tissues and fæces of fleas. Brumpt, however, believes that there has been confusion with an apparently harmless flagellate which is frequent in fleas even where infantile kala-azar does not occur. Patton suggests that the kala-azar of dogs may really be an infection quite distinct from the infantile disease and caused by infection with the common intestinal flagellate of fleas, Herpetomonas ctenocephali. The possibility that the human disease may also be caused by this flagellate seems to have been overlooked; the fact that the fleas do not readily become infected from sucking an infected child does not necessarily argue against the origin of the human parasites from fleas. Recent work by da Silva and Spagnolio in attempting to infect fleas by allowing them to feed on a naturally infected child has been unsuccessful. They fed 25 fleas on an advanced case of the disease and secured no infection of the fleas in 484 feeds. These authors do not believe in the relation of fleas to infantile kala-azar, and point out that the disease is at its height in the spring before fleas have become very abundant.

Infection of bedbugs with Leishmania infantum is not successful. If fleas do serve as the usual transmitting agents, it is probable that after development in the flea the Leishman bodies, as suggested above, become more resistant and are able to establish themselves in situations where they would otherwise be destroyed before they had a chance to multiply and adapt themselves.

Since so many dogs around the Mediterranean are infected, although only a small number give any indication of it, they probably serve as a reservoir for the disease. Not infrequently children attacked by kala-azar have been known to have played with diseased dogs, and it is easy to see how the fleas which almost always infest dogs in these regions could infect children. 
The Disease. - The disease is much like true kala-azar in most of its clinical manifestations, though differing in details. It is characterized by fever, aches and anemia, and by excessive enlargement of the spleen, the liver also enlarging somewhat. Though in some places comparatively mild, in others it is extremely fatal. Recovery is rarer the younger the patient; in $\mathbf{1 3 0}$ cases reported from Palermo and Naples, 93 per cent of the children under two years old succumbed to it, while 87 per cent of the older children died. Similar high mortality has been reported in other parts of Italy.

Usually after recovery from a single attack immunity is given, almost always lasting until the susceptible age is passed.

Treatment and Prevention. - Wonderfully successful results have been obtained in the treatment of infantile kala-azar with tartar emetic as described on page 81. Of eight children treated with tartar emetic in Italy, all of whom were between five and 27 months old, except one boy of six years, seven recovered completely. In the last stages of the disease the vitality is so weakened that recovery is impossible even with the destruction of all the parasites.

Prevention obviously lies in keeping infected dogs away from children. In endemic regions dogs should be kept scrupulously free from fleas, and all dogs showing the slightest symptoms of feverishness, enlarged spleen or emaciation should be killed, and their bodies burned to destroy fleas. Even if this were done it would not be sufficient to stamp out the disease completely, since so many dogs carry the infection in latent condition, serving as a reservoir for it without showing any appreciable symptoms. Basile showed the value of attacking the disease in dogs by destroying all obviously infected dogs in a certain township in Italy. In the year the dogs were destroyed there were seven new cases of the disease in children in a population of 2000 , but in the following year not a single new case appeared, and in the year after only one.

\section{Oriental Sore}

One of the commonest sights in many tropical cities, particularly in the cities of the eastern Mediterranean region and southwestern Asia, is the great number of children, usually under three years of age, who have on the exposed parts of their bodies un- 
sightly ulcerating sores, upon which swarms of flies are constantly feeding. The exudations from such sores are teeming with Leishman bodies, Leishmania tropica (Fig. 15A and B), which very closely resemble those of kala-azar. In some cities infection by these parasites is so common and so inevitable that normal children are expected to have the disease and visitors to the cities seldom escape a sore as a souvenir, even if present for only a short time. In Bagdad, Wenyon has shown that almost as soon as the children are relieved of the wrappings in which they are covered as babies, and allowed to run free and play in the streets, they are almost certain of infection. Since one attack gives immunity, oriental sores appearing on an adult person in Bagdad brands him as a new arrival, and the same is undoubtedly true

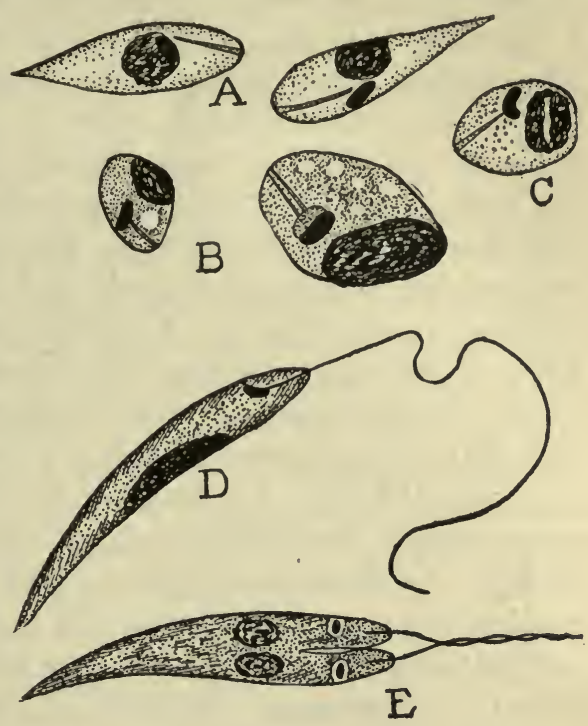

FIg. 15. Parasites of oriental sore (Leishmania tropica); $A, B$ and $C$, parasites from sore, the torpedo-shaped forms $(A)$ being found outside the cells, the others $(B$ and $C)$ within the cells; $D$, Herpetomonas form taken from bedbug 48 hours after feeding on sore; $E$, the same, dividing form. $\times 4000$. (After Wenyon.) in many other tropical cities. The disease is prevalent from India through Persia, Syria and Arabia and along the south shore of the Mediterranean as far as Morocco. True oriental sore probably occurs commonly in many cities of tropical South America, though here it is obviously difficult to distinguish it from the skin sores of espundia.

Transmission. - Though oriental sores may appear at any time of the year, they are particularly abundant in the autumn months in most cities of the Old World. Since the usual time of the appearance of the sore, as nearly as can be judged, is about two months after infection, though sometimes much less and often much longer than this, infection must usually occur during the hot mid-summer months. This fact suggests the probability of 
the parasites being transmitted by some biting insect which appears during this season. There can be no doubt that the myriads of flies which collect on the sores must mechanically carry the parasites in many cases from infected individuals and deposit them on wounds or cuts of others where they gain access to the body. It may be that one or more kinds of insects act as intermediate hosts; in fact, it has been claimed that in India the bedbug is an intermediate host for this as well as for the kala-azar parasite. In Teheran, where a large proportion of the dogs (in one case 15 out of 21 street dogs) show Leishmanian sores, the parasites have been found in the gut of a fly, Hippobosca canina, common on the dogs. Camels and horses are also subject to infection in some places. A number of French workers in North Africa have suggested that the sandfly, Phlebotomus minutus, which is very abundant there, is the transmitter of the disease and that the common Algerian gecko, Tarentola mauritanica, may play the rôle of a reservoir for the disease. The sandflies swarm about the lizards in large numbers, and also bite man readily. Leishman bodies have been found in the blood of a number of geckos near Tunis. On the western face of the Andes in Peru there occurs a similar disease known as uta, which has been shown by Townsend, of the U. S. Bureau of Entomology, to develop in the intestine of two little gnats, Forcipomyia utce and Forcipomyia townsendi, very closely allied to the American "punkies." Inoculation of the gut contents of these insects into guinea-pigs produces sores believed to be identical with uta, and Townsend believes the insects transmit the disease in nature by voiding the Leishman bodies from the anus while sucking blood, the puncture being contaminated in this way. Whether this disease is a very mild form of espundia, described below, or is more closely allied to true oriental sore, is difficult to say. According to the description given by Dr. Strong and his colleagues of the Harvard expedition to Peru, uta is not so mild, and may attack the mucous membranes as does espundia. Possibly both diseases occur there. Dr. Strong has pointed out that the flagellated stage of the uta parasite differs from that of other Leishmania in possessing a basal granule in addition to the nucleus and parabasal body.

The Disease. - Although oriental sore often has a long incubation period, and produces such profound constitutional changes 
as to build up an immunity which is usually effective for life, the general symptoms are so mild as to be usually unnoticed. Slight fevers and general malaise are frequent at all times in tropical countries, and it would be extremely difficult to connect such non-characteristic symptoms with a sore appearing perhaps months afterward. There is some evidence, however, that at least in some cases fevers do occur which are attributable to the parasites of oriental sore.

The nature and course of the sores vary to some extent in different localities. The sore usually begins as a small dark pimple which causes very slight itching and little if any inflammation of the surrounding skin. The pimple grows slowly and develops in one of two ways, forming either an ulcerating or a non-ulcerating sore, more popularly known as female and male sores respectively. In the former type, under a flaky scab which soon falls away or is scratched off, there develops a shallow ulcer exuding a foul-smelling yellow fluid. Usually the sore covers an area about the size of a dollar, the older portion often healing while the ulcer is still extending in another direction. The surface of the ulcer is covered by red granulations which bleed readily. In most localities these ulcers are not painful, but those occurring along the eastern slope of the Andes in Peru and Bolivia are said to be very painful.

The non-ulcerating or male sores grow slowly and develop a covering of white flaky scales over a thin red skin, below which is a mass of red granulations where the parasites may be found in large numbers. The non-ulcerating sores sometimes break down and ulcerate, but usually grow to about the same size as do the "female" sores, and then gradually shrink, finally healing as do the others.

The sores, of either kind, nearly always occur on exposed parts of the body, as the face, neck, arms or legs. Occasionally they occur on the lips or edges of the nose and spread to the mucous membranes, but this must not be confused with the mucous membrane ulcerations of American leishmaniasis. A single sore is the most common condition, but secondary sores sometimes develop in its vicinity, and sometimes a great many sores may occur on an individual. In the Peruvian uta several sores seem to be the rule, these occurring at the sites of the bites of gnats and possibly other insects. 
The sores usually last for a year, more or less, gradually healing over, but leaving permanent scars. The uta sores of the Peruvian Andes, which have a much shorter incubation period, may run their course and heal in much less time, according to Townsend in as short a time as 15 days. Except in rare cases, after an oriental sore has once run its course and healed a person is permanently immune to any further attacks.

Treatment and Prevention. - The use of tartar emetic as a cure for oriental sores is as productive of good results as it is in the case of other Leishmanian infections. With the usual one per cent or two per cent solutions of this drug injected into the veins the sores yield promptly and, if treated at an early stage, can be prevented from leaving scars.

In badly infected places there might be some advantage in allowing the sore to run its course, inoculating it on an inexposed part of the body where it could cause no visible disfigurement, since in this way a permanent immunity to further infection could be prevented. It is better to keep the sores open than to allow a scab to form over them, since the scab shuts in the pus and results in more extensive ulceration and inflammation. Applications of various kinds which will soothe the inflammation and keep the sores as dry and clean as possible are beneficial. The use of hypodermic injections of dead cultures of the parasite, as in anti-typhoid vaccinations, has been found to hasten the healing. The inoculation of the active disease germs on inexposed parts of the body, especially in young children in whom the sores are never very extensive, is easily accomplished, and has been practiced in Bagdad and other cities where the disease is so prevalent as to make avoidance of it almost impossible. Such a procedure tends to lessen the number of exposed sores, to which flies or other insects might get access. Unless the disease should be found to be transmitted by insects which suck the parasites from the circulating blood, which seems very unlikely, the protection of the sores will greatly reduce the prevalence of this unpleasant feature of tropical cities.

It is possible that an immunity may be established by the inoculation of dead parasites as in the case of typhoid fever, but this has not yet been demonstrated. 


\section{Espundia or American Leishmaniasis}

In many parts of Brazil, Paraguay, Bolivia, Venezuela, French Guiana and other countries of tropical South America there occurs a horrible form of Leishmanian ulcers which attack both the skin and the mucous membranes of the nose and mouth cavity. These ulcers do not grow to a limited size and then heal, but slowly and constantly spread further and further, lasting for a period of five, ten, fifteen or more years. The disease goes by a great variety of local names of which espundia is the most common. The best name of all is probably "American Leishmaniasis." The name "buba braziliensis" has been given it by some writers, but erroneously, since this name properly belongs to another tropical disease, yaws. A few cases of Leishmania ulcers have been observed in dogs in South America. Monkeys can be experimentally inoculated. The organism causing these intractable ulcers has been named Leishmania americana (braziliensis). It is a very minute animal, and is found usually in rather scanty numbers in the sores; it can be distinguished from the parasite of oriental sore, L. tropica, of which many authors believe it is a mere variety, rather by its pathogenic effects than by any peculiarity of form. Flagellated forms of the parasite are occasionally found in the sores.

Skin Sores. - The sores on the skin, which do not always ulcerate, usually begin as one or two itching spots that seem to be produced by the bites of insects. If the sores are of the nonulcerating type there is produced a great deal of red granular tissue, raised slightly above the surrounding skin, and bleeding easily. The surface, which is rosy in color, is rough, resembling, according to one author, a cauliflower. An intolerably foulsmelling fluid is constantly emitted which sometimes dries over the sore to form a crust of varying thickness. The fluid given off is infectious and starts new sores if it comes in contact with any broken skin on the same or another individual.

In the ulcerating type of the disease in the skin the same fetid fluid is emitted, but instead of the sore being elevated, it is extensively excavated and has raised borders. Often an enclosing crust forms over it and it is improperly called a "dry sore." In this case the fluid is shut in between the crust and the sore and causes even more intensive destruction of the tissues. Some- 
times nearby lymph glands also become infected. Such general symptoms as evening fever, pains in the joints, headache, etc., sometimes accompany the ulceration, probably due to the absorption of toxins.

As remarked before, the exudations from the sores are extremely infectious for either the same individual or another one. Consequently it is not infrequent to find on a single individual a great many sores, up to 50 or more, in all stages of development, though more often there are only a few. In one case recorded from Brazil there were 35 active sores and 29 extinct ones, and these were arranged in a more or less symmetrical manner, suggesting the influence of the nervous system on their location. The sores become secondarily infected with bacteria and spirochrtes and are sometimes attacked by screw-worms and other fly maggots. The rarity of Leishman bodies in the late stages of the sores suggests that the secondary infections may then play an important rôle, though the prompt cure which follows treatment destructive to the protozoans shows that the latter still play a leading part.

Mucous Membrane Ulceration. - A far more vicious manifestation of the disease and one which follows the cutaneous sores is the ulceration of the mucous membranes of the nose and mouth (Fig. 16). It may be several months or over a year after the skin sores develop and often after they have healed that the mucous ulcerations appear. In rare cases ulcers have been known to occur in the vagina also. Ordinarily the infection commences as a tiny itching hardness or swelling of the mucous membrane, usually in the nose, the infected membrane becoming inflamed, and marked either with small granular sores or with blister-like swellings. The lymph glands in the infected regions become swollen and turgid. A granular ulceration begins in a short time, invading all the mucous membranes of the nose and spreading, by means of infective fluid which flows down over the upper lip, into the mouth cavity, attacking the membranes of the hard and soft palate. Its advance is obstinate and slow, and gives rise to serious complications. The nostrils become too clogged to admit the passage of sufficient air and the patient has to keep his mouth constantly open to breathe. His repulsive appearance and fetid breath help to make his life miserable. Affections of the organs of smell and hearing, and even sight, 
often supervene, and the voice is weakened or even temporarily lost. The digestive tract becomes upset from the constant escape down the throat of the exudations from the ulceration, mixed with saliva or food. A spreading of the nose due to the eating away of the septum is a characteristic feature. Although in late stages of the disease the entire surface of the palate and nasal cavities is attacked, and the septum between the nostrils destroyed, the bones are left intact, a feature which readily distinguishes a Leishmanian ulcer from a syphilitic one. Usually the victim of espundia, after long suffering, sometimes for 20 or 30 years, succumbs to the disease from pure exhaustion and from poisoning by exuded liquids which are swallowed.
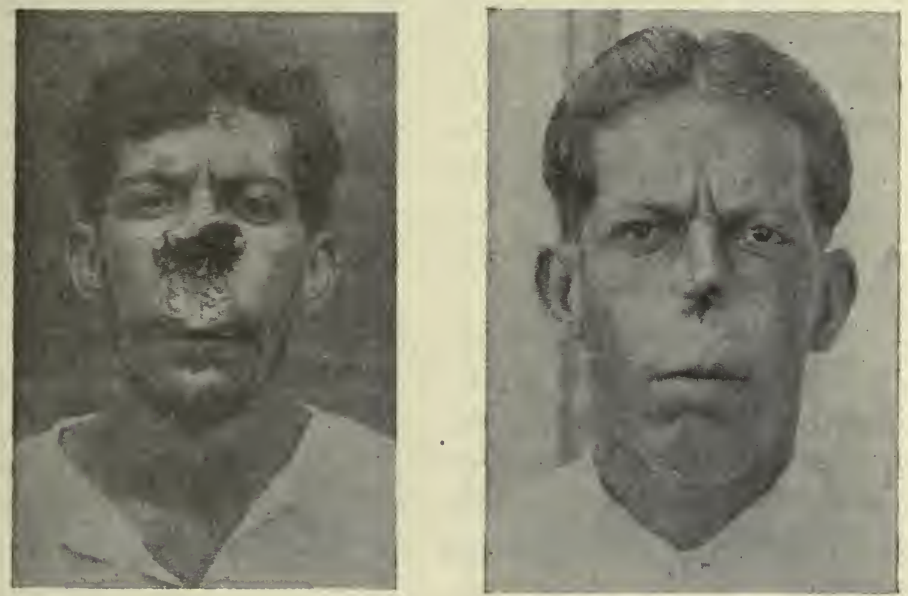

Fig. 16. A case of espundia before and after treatment with tartar emetic. (After d'Utra e Silva.)

Treatment and Prevention. - It was in connection with ulcers caused by Leishmania americana that the curative action of tartar emetic was first worked out by Vianna in the Instituto Oswaldo Cruz at Rio de Janeiro. The treatment of espundia with this drug, injected into the veins, has been thoroughly tried out in the past two years with great success. Although the mucous membrane ulcers do not yield to the treatment as readily as do skin sores, yet they can be cured with persistent treatment, even in cases in which the nose and throat had been infected for several years. The tartar emetic is injected as a one to two per cent solution, as for other Leishmanian diseases, five to ten cc. 
being injected daily for from five to 40 days. As remarked elsewhere, it must be administered very carefully and slowly since it is likely to produce much irritation.

Practically nothing can be said about the prevention of the disease, since its method of transmission is unknown. The natives of South America believe that it results from the bite of some jungle insect, probably a horsefly (tabanid), but nothing definite is known about it. Blackflies, mosquitoes and ticks have been suggested as transmitters also. Since the disease is contracted in forests in the daytime, and the sores usually develop on exposed parts of the body, tabanids seem to be incriminated by circumstantial evidence. However, it is possible that houseflies or other non-biting insects may carry the infection, the punctures of biting insects serving merely to open a door of entrance for the parasites. Natives of Paraguay believe that rattlesnakes harbor the parasites and that the latter are transmitted to man either by blackflies or ticks, both of which attack the snakes. Although only a popular belief, this is interesting in view of the incrimination of geckos as reservoirs of oriental sore parasites in Algeria.

It would seem obvious that in case a skin sore of the espundia type develops, great care should be taken not to allow the mucous membranes to become infected by contact. Yet a case is cited by da Matta where an ignorant wood-cutter who had been tormented by espundia of the skin for five years and who persistently cleaned his nose with infected fingers, never developed the slightest affection of the mucous membranes. In other cases, simultaneous affection of the mucous membranes and skin is common. 


\section{CHAPTER VI}

\section{TRYPANOSOMES AND SLEEPING SICKNESS}

Importance of Trypanosome Diseases. - One of the blackest clouds overhanging the civilization of tropical Africa is the terrible scourge of sleeping sickness, a disease caused by protozoan parasites known as trypanosomes. The destiny of the equatorial parts of Africa depends largely on the issue of the struggle of medical science against this haunting malady. The ravages of the disease were well known to the old slave traders, and the presence of "lazy niggers" lying prostrate on wharves and decks with saliva drooling from their mouths, insensible to emotions or pain, was a familiar sight. It did not take these astute merchants long to find that death was the inevitable outcome of the disease, and they very soon recognized swollen glands in the neck as an early symptom and refused to accept as slaves negroes with swollen glands (see Fig. 24). Nevertheless sleeping sickness must often have been introduced with its parasites into various parts of North and South America, as it frequently is even at the present time, and only the absence of a suitable means of transmission has saved the Western Hemisphere from being swept by it.

Up to about thirty years ago sleeping sickness was confined to a limited part of tropical West Africa, but with the opening up of Central Africa by whites and the consequent movements of disease-carrying inhabitants to new portions of the continent, the afflicted country was greatly extended. The great explorer Stanley, in his expedition to reach Emin Pasha, was almost unquestionably responsible for the introduction of the scourge into Uganda and the lake regions of Central Africa in 1888, where it had hitherto been unknown. In one district of Central Africa the population was reduced from 300,000 to 100,000 in the course of seven years, from 1901 to 1908 , and there are records of whole villages and islands being depopulated.

In 1909 there occurred a case of sleeping sickness contracted in 
Rhodesia in southeastern Africa occasioned by a distinct and apparently newly originated type of trypanosome, as indicated by its sudden appearance and startlingly rapid spread. This type of sleeping sickness is more deadly than the older type and there is reason to fear that unless efficient methods of controlling it and stamping it out are discovered it will spread over a large part of tropical Africa. The disease has already spread over a great part of Rhodesia, Nyasaland and Portugese East Africa, and has been reported from German East Africa. There is apparently a rather high natural immunity to the disease, which alone is responsible for the small number of the victims.

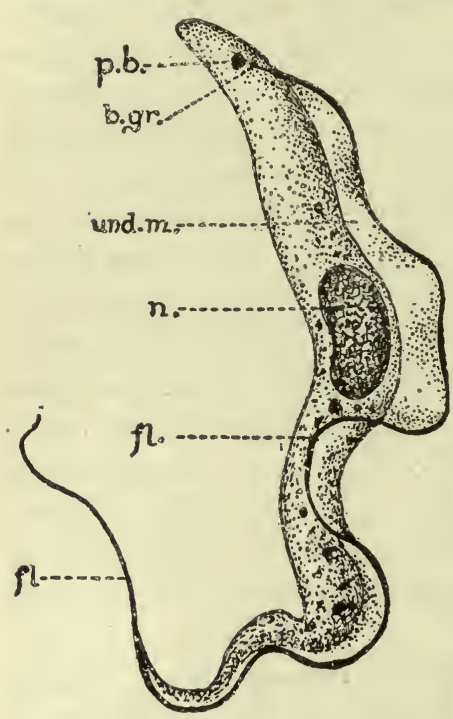

FIG. 17. Trypanosoma gambiense, slender form. p. b., parabasal body; b. gr., basal granule; und. m., undulating membrane; n., nucleus; fl., flagellum. $\times 4000$. ing diminutive "artistic dolphins" (Fig. 17). They are about $25 \mu$ (about $\frac{1}{1000}$ of an inch) or even less in length, spindle-shaped, and somewhat flattened from side to side like an eel. Along the "back" runs a flagellum connected with the body by an undulating membrane, like a long fin or crest. This terminates at what is really the anterior end in a free tail-like flagellum. It is by means of the wave motions of the membrane and the lashing of the flagel- 
lum that the animal moves through the blood or other fluids of the body, either forward or backwards, so rapidly that it is difficult to observe under the high power of a microscope as it wends its way between the blood corpuscles on a slide. The body of the animal contains, in addition to the large round nucleus near the middle, another deeply-staining structure, the parabasal body (see p. 31) at the posterior end near where the flagellum originates. The body also contains other granules of various sizes.

There are a great many kinds of trypanosomes inhabiting many different animals. Those living in cold-blooded animals have no apparent effect on their hosts but the species infesting mammals almost always cause disease. In man their effect is particularly deadly and the African species usually cause death if allowed to run to the sleeping sickness stage. Unlike many kinds of parasites most trypanosomes can live in a great many different hosts. The common sleeping sickness trypanosome, for instance, can live not only in man but also in monkeys, dogs, rodents, domestic animals and a large number of the wild game animals of Africa.

Most kinds of trypanosomes, like the malarial parasites, live only part of their life histories in the blood or other fluids of their vertebrate hosts, undergoing another phase of it in the digestive tracts of insects or other invertebrates. In their intermediate hosts they undergo remarkable transformations; the whole series of forms through which trypanosomes may pass in their development, and which may represent a phylogenetic as well as an ontogenetic series, is shown in Fig. 18. The first or Leishmania form, which stands at the foot of the series, is a rounded body with a large central nucleus and small rod-shaped para-
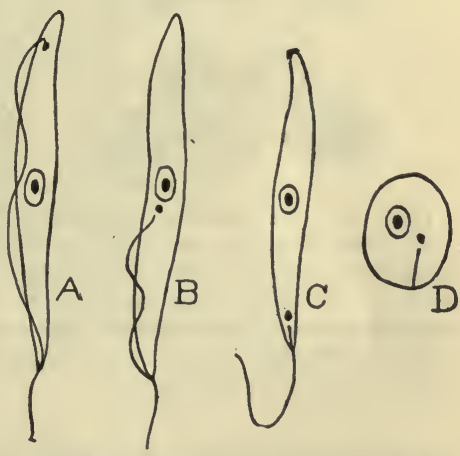

Fig. 18. Diagram of developmental types of trypanosomes; $A$, trypanosome form; $B$, Crithidial form; $C$, Herpetomonas form; $D$, Leishmania form. (After Wenyon.)

basal body usually set at a tangent to the nucleus (Fig. 18D). Next in development comes the Herpetomonas form which differs in having a long slender body and in having a flagellum produced from the parabasal body (Fig. 18C). Next comes the Crithidia 
form, differing from the preceding in that the parabasal body has moved back to near the middle of the body, and the flagellum is connected with the body for half its length by an undulating membrane (Fig. 18B). This type is a very common developmental phase in nearly all trypanosomes, but it is also the adult condition of many insect parasites. Finally there occurs the fully-developed trypanosome form (Fig. 18A), apparently especially adapted in form and structure for life in vertebrate
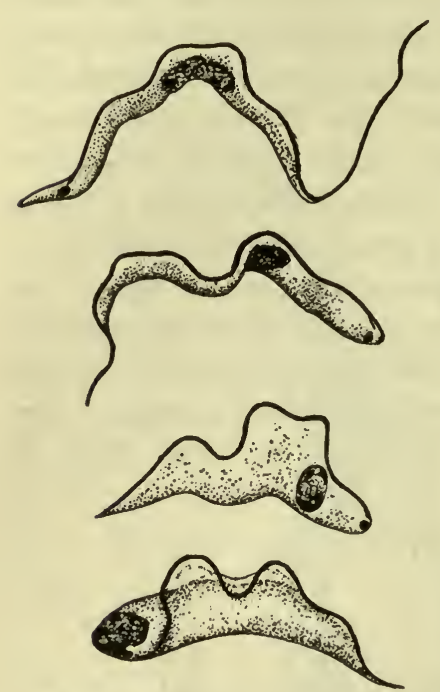

Fig. 19. Trypanosoma rhodesiense, from blood of monkey inoculated from case of human sleeping sickness. Note posterior position of nucleus in short blunt forms, especially in lower figure. $\times 2000$. (After Kinghorn and Yorke.) bodies. The method of development of this form from a crithidial type can easily be seen from Fig. 18 . Only the first or Leishmania form and the last or trypanosome form normally occur in vertebrate bodies, though all of the four types are found in the digestive tracts of invertebrates. The fact that some flagellates never develop further than the Herpetomonas form, and others never further than the $C$ rithidia form, makes a study of this group of Flagellates very confusing, since when a Herpetomonas or crithidial type is found in an insect gut it is very difficult if not impossible to say whether it is an adult animal which never undergoes any further development or is only a developmental phase of a trypanosome of a vertebrate animal.

It is often very difficult to distinguish different species of trypanosomes; of over 70 known species only a few can be distinguished on morphological grounds. Average size, position of nucleus and parabasal body, length of snout, and presence or absence of a free flagellum are sometimes useful in identifying them. More reliable, however, are their socalled "biological characteristics," such as pathologic effects on different animals, susceptibility of different hosts, the effect of serum immune to certain species, and the "cross-immunity reactions." The last is the most certain method. Thus, if an 
animal has recovered from an attack by one strain of trypanosome it is rendered immune and will not succumb to second attacks of the same strain, though it is still susceptible to others.

As remarked before there are at least three species of trypanosomes which are known to cause disease in man, two in Africa and one in South America. The African species, causing sleeping sickness, are the most deadly but the South American species is frequently fatal, especially to children, and often renders a life worse than useless. The Gambian trypanosome, Trypanosoma

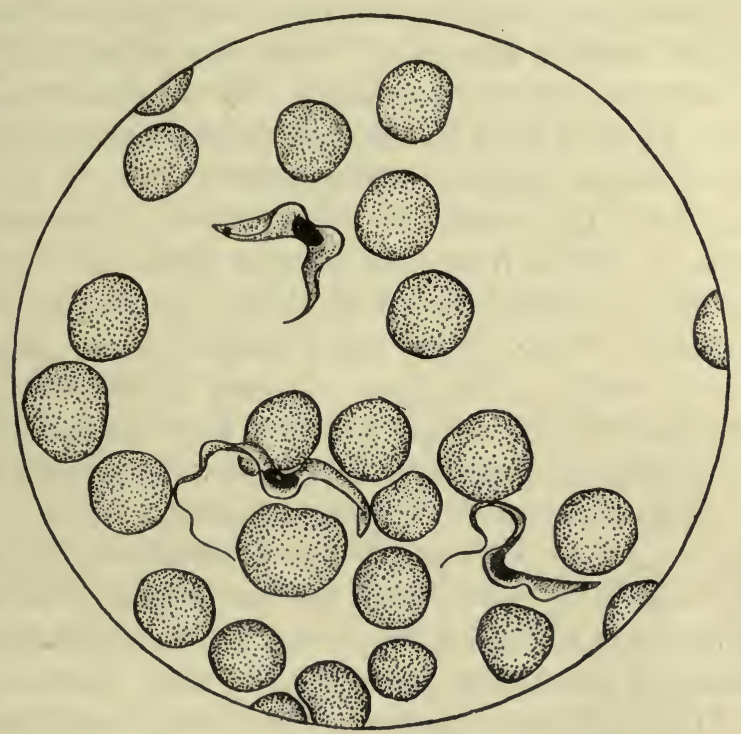

FIG. 20. Trypanosoma gambiense in rat blood, showing long, intermediate and short forms all in one microscopic field. $\quad \times$ about 1200 . Drawn from microphotograph by Minchin.

gambiense, is the cause of the commoner and more widespread form of sleeping sickness, while the Rhodesian species, T. rhodesiense, is the cause of the recently established East African form of the disease. The most salient distinguishing characteristic between these two species of trypanosomes is the posterior situation of the nucleus of the Rhodesian parasite in a certain per cent of individuals when they are developed in rats and some other animals (Fig. 19). This is a feature never observed in the Gambian trypanosome. Both species vary a great deal in form, 
and three distinct types may be observed at once in the blood of an infected animal, a long slender form with a long free flagellum, a short stumpy form with a short flagellum and a form intermediate between these (Fig. 20). Some investigators regard the trypanosome causing the mild sleeping sickness of Nigeria as a distinct species, named $T$. nigeriense.

\section{Sleeping Sickness}

Transmission. - Sleeping sickness of either type, and also many trypanosome diseases of lower animals, is transmitted primarily by certain species of tsetse flies which act as intermediate hosts for the trypanosomes. The Gambian parasite, as first shown by Sir David Bruce, is normally transmitted by the tsetse fly, Glossina palpalis, and its distribution is now almost coincident with the range of this species. It occurs on the west coast of Africa from the Senegal River to the State of Mossamedes in Portuguese West Africa, including nearly all the tributaries of the Niger and Congo Rivers. Eastward it extends to the valley of the Upper Nile and Lake Victoria Nyanza in Uganda and along the east shore of Lake Tanganyika.

The Rhodesian trypanosome depends on the more widespread and less easily controlled Glossina morsitans. This species of tsetse fly occurs all the way from northeastern Transvaal to northern Nigeria in West Africa and to southern Sudan in the basin of the Nile in East Africa. So far, the disease caused by the Rhodesian parasite is limited to a small portion of East Central Africa but it is spreading both north and south.

Experimentally other species of tsetses are able to transmit the Gambian disease, but it is doubtful whether any except G. palpalis are important transmitting insects in nature. As intimated above it is only the absence of tsetse flies in other parts of the world that we have to thank for the fact that sleeping sickness when introduced is not propagated. Experiments show that it is also possible for the Gambian trypanosome to be transmitted mechanically by the stable-flies, Stomoxys, though this probably seldom happens in nature. Macfie has shown that in Nigeria the human trypanosomes undergo developmental stages in a stable-fly, $S$. nigra, but circumstances did not allow him to determine whether the salivary glands become infective as in 
tsetse flies. There is evidence that sleeping sickness, like surra, a trypanosome disease of horses, may also be transmitted sexually or through abrasions of the skin, but this is certainly not the usual method of transmission.

The tsetses are blood-sucking flies resembling stable-flies, which inhabit the brushy borders of lakes, streams or swamps, - the so-called "fly-belts." The distinguishing characteristics of the tsetse flies and of the various disease-carrying species are discussed in the chapter on biting flies, p. 490.

For a long time it was thought that the tsetse flies could transmit trypanosomes only in a simple mechanical way, the parasites adhering to the proboscis, and being subsequently injected into the blood of another person. It is now known that the trypanosomes of sleeping sickness can be transferred in this manner only for a few minutes after an infective feed, but that the fly again becomes infective after a period of three or four weeks. Meanwhile the parasites have undergone a series of changes in the gut of the insect and finally become stored in the salivary glands from which they are poured with the salivary juices into the blood of a new victim.

Life Cycle in Fly.-According to observations on Trypanosoma gambiense in Glossina palpalis by Miss Robertson the critical time for the trypanosomes after they are sucked up by the fly is when the fly feeds the next time, since in many cases they are swept out of the body with the new influx of blood, or digested. Having stood their ground until they have become established in the new influx of blood they multiply so rapidly that permanent infection of the fly is almost certain. The difficulty experienced by the parasites in establishing themselves in the gut of their insect hosts largely accounts for the relatively low percentage (usually less than five per cent) of infections which result from feeding of tsetse flies on infected blood. When conditions are favorable for development in the fly the parasites multiply first in the middle intestine, producing long-snouted forms such as shown in Fig. 21B. After the tenth to fifteenth day long slender forms (Fig. 21C) are developed, and these move forward in the digestive tract. These slender trypanosomes have long snouts and differ most strikingly from the earlier forms in the appearance of the nucleus (Fig. 21C). After several days more the trypanosomes make their way to the fly's salivary glands, to the walls of which 
they attach themselves by their flagella (Fig. 21D) and, rapidly multiplying, undergo a crithidial stage. As multiplication continues free-swimming trypanosome forms are again produced

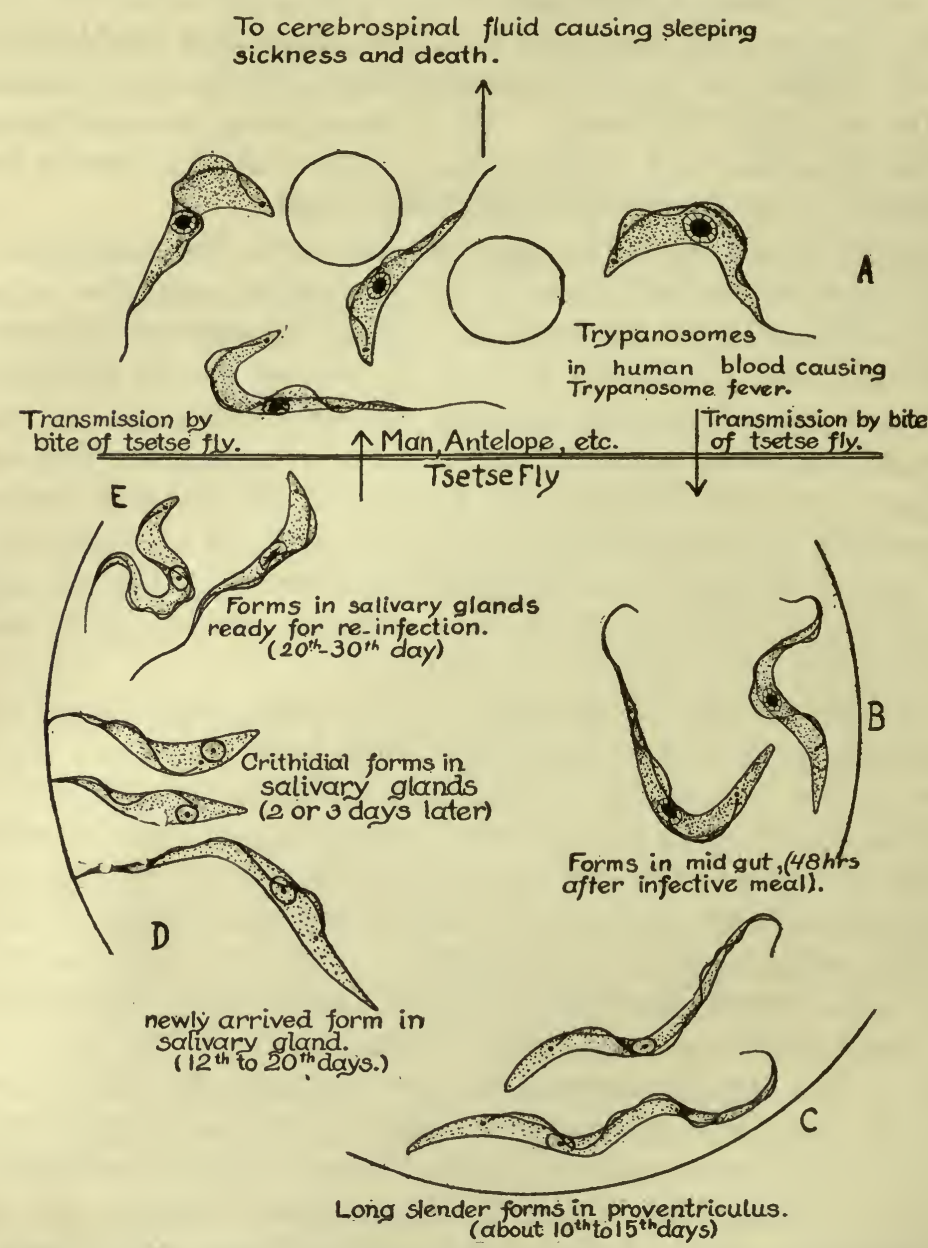

Fıg. 21. Life History of Trypanosoma gambiense. $\times 1500$. (Constructed from figures by Miss Robertson.)

which very closely resemble the parasites in vertebrate blood (Fig. 21E) and which are now capable of infecting a vertebrate host. The whole cycle in the fly usually occupies from 20 to 30 days. According to Kinghorn and Yorke the time required for 
Glossina morsitans to become infective varies from 11 to 25 days, but under unfavorable conditions the parasites may remain in the fly in an incomplete stage of development for at least two months. A temperature between $75^{\circ} \mathrm{F}$. and $85^{\circ} \mathrm{F}$. is necessary for the full development of the parasite $n$ the fly, ending in invasion of the salivary glands. For two days after the trypanosomes have been swallowed by the fly they remain infective if injected into a vertebrate, but after this time they must pass through the crithidial stage before they are again infective.

The reader will note that no sexual reproduction, such as is so conspicuous in the mosquito cycle of the malarial parasites, has been described in this fly cycle of the trypanosome, though the general features of the cycle are so parallel. It can hardly be doubted that sexual reproduction of some kind, or at least something which takes the place of it, does occur in the tsetse fly, but it has not yet been recognized by scientific observers.

Life Cycle in Man. - The parasites, when injected into man or other susceptible animals by a tsetse fly,

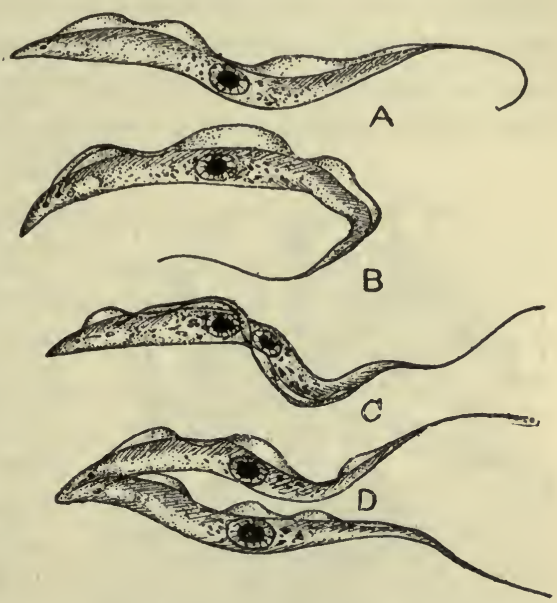

FIG. 22. Method of division in trypanosomes. $A$, elongated form ready for division; $B$, form with divided parabasal body and partially split undulating membrane; $C$, form with double parabasal body, double undulating membrane, and double nucleus; $D$, almost completely divided forms, adhering by posterior ends.

live and multiply in the blood, swimming free in the serum without entering the corpuscles (Fig. 21A). They obtain nourishment by simply absorbing food material through the delicate cuticle which covers them. The method of division is the usual protozoan type of simple fission. When about to divide the trypanosome elongates (Fig. 22A) and the parabasal body at the posterior end divides first (Fig. 22B). Then the flagellum and undulating membrane begin to split from the posterior end forward, the central nucleus divides (Fig. 22C), and the animal splits into two parts which hang together longest by the "snouts" or posterior ends (Fig. 22D). 
The fast-multiplying parasites do not remain in the blood of their victim but penetrate many of the tissues and organs of the body, especially the liver, spleen, lungs and lymph vessels and glands. The last mentioned are probably one of the main strongholds of the parasite in the body and the swelling of lymph glands, especially in the neck, has already been mentioned as one of the characteristic symptoms of sleeping sickness. The parasites are not present in constant numbers in the blood, but periodically appear in large numbers and then apparently disappear at fairly regular intervals. Often the trypanosomes are so few in the blood that their presence can be proved only by causing disease through the injection of some of the blood into a susceptible animal or by causing the parasites to multiply, as

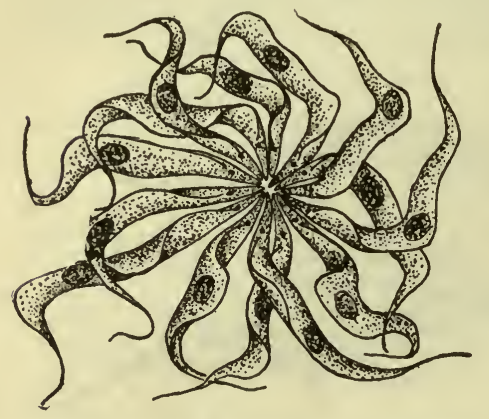

FIG. 23. Agglutination of trypanosomes, T. lewisi, in blood of immunized rat. (After Laveran and Mesnil.) they will quite readily do, in a suitable artificial culture medium. In Nigeria the parasites are hardly ever seen in the blood of infected persons, but they can be found by puncturing a lymph gland. According to recent investigations by Fantham the trypanosomes, probably as a reaction against antibodies which tend to destroy them; shrink into rounded sporelike bodies without locomotory organs but with a protective shell. In this condition they remain until conditions again become favorable for them when they once more elongate, develop a flagellum and undulating membrane, multiply and reappear in the circulating blood. After several months or years the parasites penetrate the cavity of the brain and spinal cord and live in the cerebrospinal fluid which fills it; this invasion of the central nervous system is the direct cause of the dread sleeping sickness stage of the disease.

While under normal and favorable conditions the trypanosomes merely live and multiply in the way described above, they are capable of reacting in a very peculiar manner when exposed to unfavorable conditions, such as the presence of drugs, low temperature or administration of serum from an immune animal. 
Under such circumstances they have a tendency to mass together in large numbers, up to a hundred or more, like sheep in a storm, all with their flagellated ends projecting from a common center (Fig. 23). Such " primary agglomerations " may adhere to form "secondary agglomerations" comprising altogether many hundreds of parasites. When the unfavorable conditions disappear, the trypanosomes disentangle themselves without any apparent ill effects, although a few of them remaining agglutinated may die and disintegrate. Another peculiar habit described by some investigators is the extrusion from their bodies of very minute granules, really tiny buds from the nucleus, which ultimately develop into new trypanosomes. This is said to occur just before the temporary disappearance of the trypanosomes from the blood.

The Disease. - The course of the disease caused by trypanosome infection is insidious and irregular in the extreme. The Gambian and Rhodesian diseases are essentially alike in their symptoms and in the course they run, except that the latter is usually more rapid in development and more virulent in effect, as a rule causing death within three or four months after infection. The variety of the Gambian disease found in Nigeria is comparatively mild and of long duration.

The bite of an infected tsetse fly is usually followed by itching and irritation near the wound. After a few days fever is felt and a peculiar tenderness of the muscles develops, so that striking against an object causes undue pain. Usually the fever comes and goes at irregular intervals of days or weeks or even months, an infected person sometimes carrying the para-

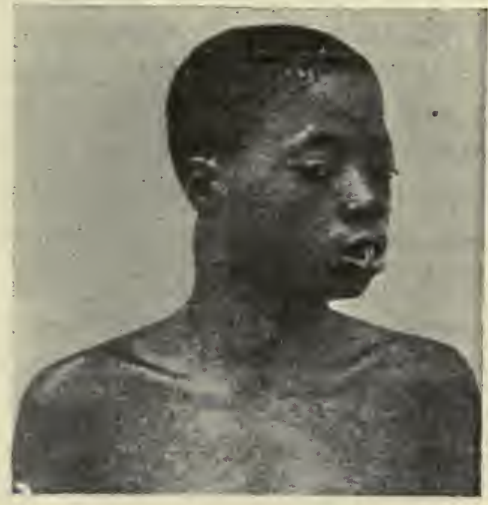

Fig. 24. Negro infected with trypanosomes, showing enlarged cervical glands. (After Kolle and Wassermann.) sites in his blood, as shown by its infectivity when injected into susceptible animals, for months at a time without any appreciable fever, and in insufficient numbers to be seen readily by microscopic examination. When the attacks of typical trypanosome fever do 
come they generally are worse in the evening, unlike malarial fevers. After a variable time the victim becomes weak and anemic, probably due to toxins secreted by the parasites, his pulse becomes rapid, and various lymph glands, especially those of the neck, tend to swell up and become tender. Often an irritating rash breaks out on the skin during the early stages of the disease. Loss of ambition and vitality usually figure prominently, and childbirth is seriously interfered with. It is possible that after weeks or months or years of irregular fever and debility the disease may spontaneously disappear, and never become more than trypanosome fever. Usually, however, the parasites ultimately succeed in penetrating to the cerebrospinal fluid in the cavity of the brain and spinal cord, and "sleeping sickness" results. In some cases the onset of this horrible disease has been known to be delayed for seven years after the beginning of the disease, but usually it comes in the course of a few months.

Sleeping sickness is ushered in by an increase in the general physical and mental depression, the symptoms being not unlike those of hookworm disease but more pronounced. The victim wants to sleep constantly and lies in a stupor; his mind works very slowly, and even the slightest physical exertion is obnoxious. Eventually the sleepiness gets such a hold on him that he is likely to lose consciousness at any time and even neglects to swallow his food. After weeks of this increasing drowsiness, his body becomes emaciated, a trembling of the hands and other parts of the body develops, with occasional muscular convulsions and sometimes maniacal attacks. He finally passes into a state of total loss of consciousness ending in death, or death may end the unhappy condition earlier during an unusually intense convulsion or fever, or through the agency of some complicating disease. If untreated, death is the inevitable outcome. A large per cent of infections occur among people of middle age. Old people are significantly few in number in sleeping sickness districts. The presence of these few may be due to a natural or acquired immunity. In Nigeria the disease predominates in young people, possibly because they are water-carriers and are therefore more exposed to the bites of tsetse flies.

Treatment. - Various drugs have been tried in the treatment of trypanosomiasis, especially arsenic and antimony compounds. Until recently the most effective drugs in use have been arsenic 
in the form of atoxyl or salvarsan, injected intramuscularly, or antimony in the form of tartar emetic, injected intravenously. Atoxyl is injected in weak solution, the doses being repeated every few days for a period of many months, even long after the symptoms of the disease have disappeared. A serious objection to atoxyl is the slight tolerance which many people have for it, and its serious effects on the optic nerve and digestive apparatus. Tartar emetic is also injected in weak solutions, care being taken not to allow it to escape into the muscles or connective tissues, to which it is very irritating. Usually a high fever follows the administration of either drug, probably due to toxic substances liberated from dead trypanosomes. Recently the Rockefeller Institute has produced a new arsenic compound, tryparsamide, which promises to be more valuable than any other drug yet tried. It is very soluble in water, can be given either intramuscularly or intravenously, produces practically no untoward effects, effects very rapid destruction of the parasites in the blood, brings about very marked improvement in the condition of the spinal fluid in advanced cases, and results in remarkable general beneficial effects.

The chief difficulty in the use of any of these drugs is that the trypanosomes tend to build up a tolerance for them, in much the same way that a man may build up a tolerance for opium or other drugs. This tolerance is hereditary and gives rise to "arsenic-fast" or "antimony-fast" strains of trypanosomes. In such cases the parasites cannot be destroyed. It is an interesting fact that in at least one species of trypanosome, $T$. lewisi of rats and mice, and probably others as well, when strains immune to atoxyl are passed through their intermediate host, a louse, where they presumably undergo sexual reproduction or some process which takes its place, the tolerance is entirely lost. Thus the sexual process at a stroke eliminates acquired characters which have been maintained through thousands of asexual generations in passages from mouse to mouse or from rat to rat. This fact, if invariably true, is of considerable importance in the outlook for the treatment of sleeping sickness, since it would prevent what would otherwise inevitably happen, the evolution of a permanent strain of trypanosomes immune to both arsenic and antimony. The fact that parasites resistant to arsenic may not be resistant to antimony, and vice versa, makes it advisable 
in treating trypanosome fever to give both drugs either together or alternately.

When the disease has reached the sleeping sickness stage and the parasites have penetrated the cerebrospinal fluid, a cure has so far never been accomplished. The arsenic and antimony compounds which are so destructive to trypanosomes will not permeate the nervous tissue and diffuse into the cerebrospinal fluid, and they are too poisonous to be injected into the spinal canal. The success that has been attained in the use of salvarsanized serum (see p. 57) against spirochætes in the cerebrospinal canal gives hope that a similar mode of treatment may be used in the case of sleeping sickness. All that can be done for sleeping sickness now is to alleviate the suffering and postpone the inevitable end.

Although the use of immune serum from animals which have recovered has been very successful in curing and immunizing various lower animals against certain trypanosome diseases, this has not yet been accomplished for man.

The Rhodesian trypanosome consistently resists both arsenic and antimony treatment, and no successful drug has been found for combating this parasite.

Prevention. - Since the tsetse flies, Glossina palpalis and $G$. morsitans, are by all odds the most important means of transmitting the Gambian and Rhodesian trypanosomes respectively, the prevention of the diseases resolves itself into the problem of avoiding or exterminating these insects. Methods for controlling and destroying tsetse flies are discussed in the chapter on Biting Flies, p. 501.

In places where tsetse fly extermination has not or cannot be accomplished the best safeguard is the avoidance of the "flybelts." In the case of G. palpalis these belts consist of narrow strips along the brushy edges of water, but with G. morsitans they are not so closely limited, the flies being sometimes found at considerable distances from water. Villages or camps should always be removed from fly-belts, and travel through the belts, when absolutely necessary, should be done on dark nights when the flies seldom bite. Occupations carried on in fly-infested areas should be discouraged or prohibited. In Uganda fishing along the fly-infested streams and lake shores is one of the chief occupations indulged in by the natives, who go naked and are 
constantly bitten. The deadly epidemic of sleeping sickness in Uganda was fostered by the fishing industry. It has been suggested that by importing dried sea fish to trade for agricultural products the natives might be induced to change their occupation. In Congo the rubber industry is the one which is the most deprecated. Personal protection against tsetse flies is discussed on page 501.

Another method of protection is suggested by the researches of Van den Branden who has found that a single injection into the veins of salvarsan or neosalvarsan or some of their compounds will sterilize the blood against trypanosomes for a period of several months - in the case of salvarsan copper for 19 to 24 months.

Infected individuals should not only be kept away scrupulously from places where flies can possibly get access to them, but should also be prevented from traveling to new places. Some strains of trypanosomes seem to be much more virulent than others, and the introduction of a virulent strain to a region where a mild strain previously existed has occasionally caused a considerable increase in the disease. The strict quarantine of infected persons, while unquestionably worth while, is not a measure sufficient to stamp out the disease, since many of the wild animals of Africa serve as reservoirs for the disease, harboring the parasites in their blood but not succumbing to them. Tsetse flies on the shores and islands of Lake Victoria, after the entire population had been stringently kept away for three years so that the flies could not have fed on human blood during this time, were found to be still infective. The situtunga antelope and other wild game undoubtedly served as a reservoir. It has been suggested that a war of extermination be made on the rich and interesting wild game of the countries infected with the Rhodesian trypanosome in the hope of checking the rapid spread of the disease (see p. 503). It has recently been shown by Taute, however, that a large proportion of the wild game of Nyasaland is infected with a trypanosome indistinguishable from Trypanosoma rhodesiense in all its general characters but nonpathogenic to man. Taute evidently had the courage of his convictions since he tried several times to infect himself with this trypanosome without success. It is possible, however, that a high natural immunity to the parasite may exist in many people, and thus explain Taute's negative results. 
Probably the deadly Trypanosoma rhodesiense is merely a strain of this wild game trypanosome which has undergone some physiologic change or mutation, making it possible for it to live in the human body. Bruce and some others consider it identical, in every respect except its ability to live in human bodies, with the well-known and widespread $T$. brucei which causes nagana in wild and domesticated animals.

A concrete example of sleeping sickness extermination is to be found in the fight against it on the Island of Principe by the Portuguese Sleeping Sickness Commission. Sleeping sickness had been a scourge on the island for years when the Commission began its work in 1911. Its efforts were directed against the tsetse fly, but this was accompanied by an active campaign against pigs, dogs and other trypanosome carriers, and the thorough care and treatment of human victims. The methods used are discussed in Chapter XXVI. The Commission cleared the island of sleeping sickness in a four years' campaign, but the tsetse flies are not yet totally exterminated, and the present condition on the island can only be maintained by constant work in the future, though at comparatively slight cost.

\section{Chagas' Disease}

A very different but hardly less destructive disease is caused by a trypanosome, Trypanosoma (or Schizotrypanum) cruzi, in

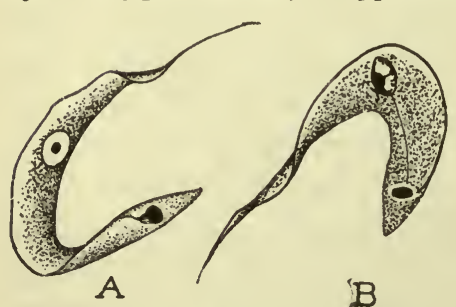

FIg. 25. Trypanosoma cruzi in blood of experimentally infected monkey. $A$, so-called male form; $B$, so-called female form. (After Chagas.) certain parts of South America. Chagas, of the Oswaldo Cruz Institute, first investigated the disease in the state of Minas Geraës in Brazil. He found that nearly all children in the endemic regions were stricken with the disease, usually before they were one year old. The mortality was found to be very high, and those who survived the initial acute attack usually passed over into a chronic diseased condition, very often being left to live a worse than useless life as paralytics, idiots or imbeciles. The disease has since been found in other parts of Brazil and in neighboring countries. Large bloodthirsty 
bugs of the genus Triatoma serve as intermediate hosts; bugs of a number of species infected with trypanosomes morphologically indistinguishable from.T. cruzi have been found all the way from Central America to Argentina, but the disease in man has been recognized only in a small part of this extensive area, though it is suspected of existing in northern Argentina and may occur in many more places than is now known.

Human Cycle.The trypanosome causing this disease very closely resembles the sleeping sickness trypanosomes in form but it is quite different in its life history. In the human body, Chagas recognized two distinct types which he believed to be male and female forms, but subsequent work indicates that these two types are merely young and adult forms of the

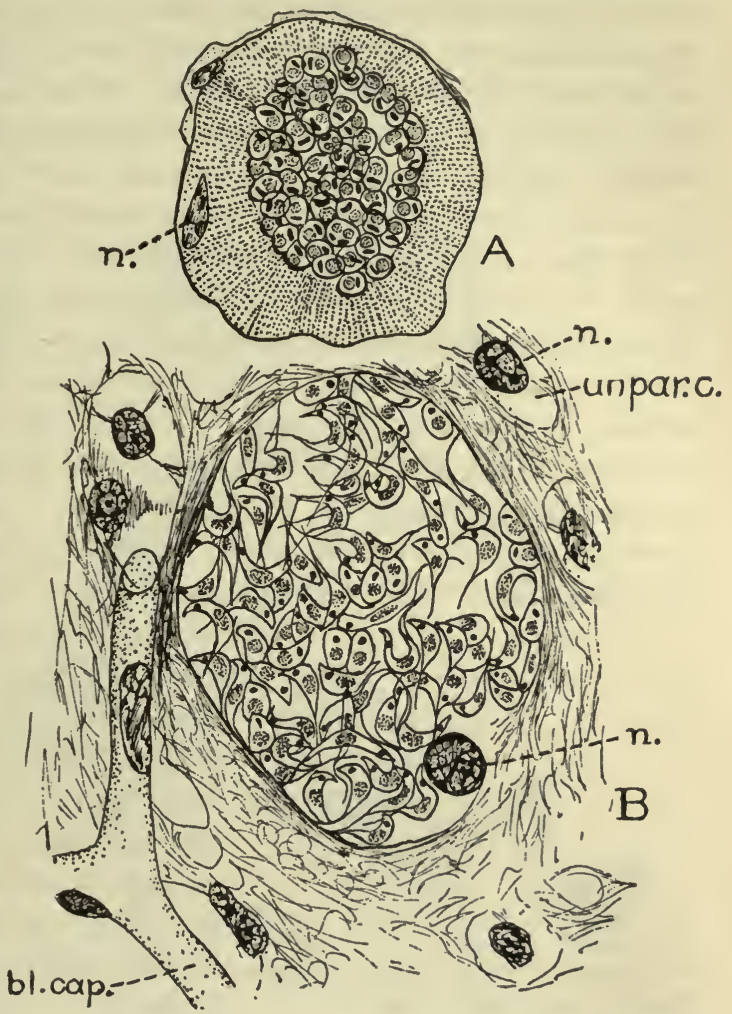

Frg. 26. Trypanosoma cruzi. A, cyst containing Leishmania forms in muscle fiber of guinea-pig, cross section; n., nucleus of muscle fiber. $B$, older cyst, containing trypanosome forms, in neuroglia cell in gray matter of cerebrum; n., nucleus of parasitized cell; bl. cap., blood capillary; unpar. c., unparasitized cells. $\times 1000$. (After Vianna.)

parasite. Unlike other trypanosomes this species as found in the blood never exhibits stages in division, and this fact led Chagas to search for some other form of multiplication. He found in the lungs of infected animals what he thought to be a process of division of the trypanosomes into eight parts, but this later was found to be a stage in the life history of an entirely 
different parasite. The real method of multiplication was first discovered by Vianna in the bodies of man and animals who had died of the disease. Vianna found in various tissues, especially in the walls of the heart, the striped muscles, the central nervous system and various glands, greatly swollen cells which served as cysts, enclosing a mass of rapidly dividing trypanosomes, varying in number from just a few to many hundreds. In younger cysts the parasites are round in form and exactly resemble Leishman bodies (Fig. 26A), while in older cysts the flagellum can be seen on many individuals and the trypanosome form becomes evident (Fig. 26B). When the enclosing cell has swollen to the bursting point, the swarming mass of trypanosomes is liberated. Each parasite, unless destroyed, then penetrates a new cell somewhere in the body, usually near where it originated, and begins the process of reproduction again. Only in the early acute stage of the disease can the parasites live in the blood, since the blood serum rapidly reacts by the formation of antibodies, and becomes deadly to trypanosomes. Chagas believed that the parasites could live within the corpuscles as well as in the serum, but later work does not confirm this.

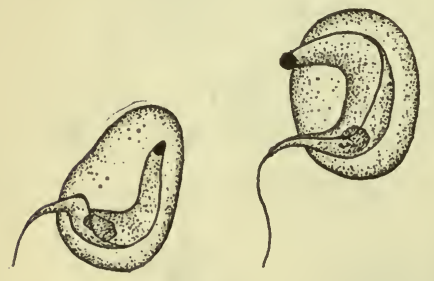

FIG. 27. Trypanosoma cruzi in blood of ape, said to be inside corpuscles. (After Chagas.) On account of the development of antibodies in the blood serum, the parasites are very seldom found in the blood of chronic cases of the disease, though their cysts may be abundant in various tissues and glands in the body.

Life Cycle in Bugs, and Transmission. - The intermediate host of Trypanosoma cruzi is a large black and red bug, Triatoma megista, known to the natives as "barbeiro." It is related to the cone-nose, Triatoma sanguisuga, of our southern states. The barbeiro is a fierce blood-sucking insect which infests the dirty thatched or mud houses of the natives, coming out at night and skillfully secreting itself in the daytime (see p. 379, and Fig. 168).

It was found that the bugs in the houses where Chagas' disease had been observed were invariably infected with trypanosomes in their gut, and from this fact and from the habits of the bug Chagas rightly deduced and later proved that the bug was the 
transmitting agent of the trypanosome. A few hours after a bug has fed on infected blood the trypanosomes begin to change form in the midgut, becoming round and Leishmania-like in form, losing the flagellum and undulating membrane (Fig. 28A, B and C.) Then comes a period of very rapid increase in number, the parasites gradually pushing backward toward the hindgut by sheer multiplication. After about two days Crithidia forms begin to develop and become numerous in the hindgut, being
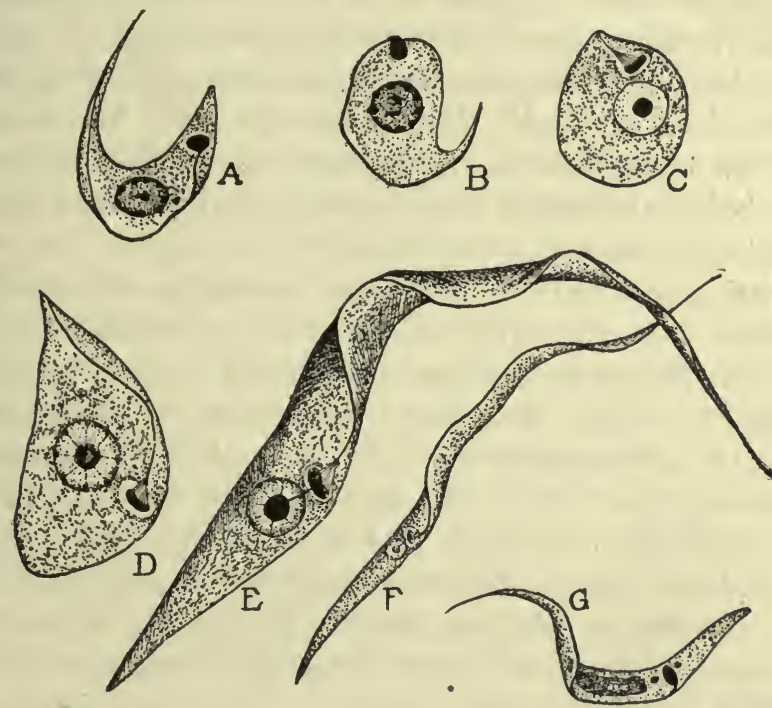

Fig. 28. Development of Trypanosoma cruzi in digestive tract of bug (Triatoma megista). A, freshly ingested form; $B$, rounding up and loss of flagellum, 6 to $10 \mathrm{hrs}$. after ingestion; $C$, Leishmania-like form in midgut, 10 to $20 \mathrm{hrs}$. after ingestion; $D$, redevelopment of flagellum and undulating membrane, $21 \mathrm{hrs}$. after ingestion; $E$ and $F$, crithidial forms in hindgut, $25 \mathrm{hrs}$. after ingestion; $G$, trypanosome form from salivary gland, 8 days or more after ingestion. (After Chagas.)

voided with the excrement from time to time (Fig. 28D, E and F). It has been suggested that these crithidial forms do not play any part in the transmission of the disease to man but that they represent a return to a primitive condition suited to existence in the bugs, and that they may be transmitted from bug to bug in this form, since the bugs are known to prey to some extent upon each other and a'so upon their excrement. Torres, however, considers transmission of the flagellates from bug to bug as very doubtful.

Chagas believes that there is a second cycle of development in 
the bugs which he interprets as sexual reproduction. After about ten days there occasionally occurs in the midgut of the bugs round organisms with thick capsules, and in a few cases Chagas has observed, after about six days, what seemed to be a division of this body into eight individuals each presumably giving rise to a trypanosome of a new generation. Whether or not the infective trypanosomes arise in this way they appear in the midgut from the eighth day onward. The occurrence of the infective parasite in the salivary glands (Fig. 28G) is very irregular. The fact that parasites have occasionally been found in the body cavity of bugs suggests that the trypanosomes may make their way through it to reach the salivary glands. As already remarked the trypanosomes in the bugs have never been found infective before the eighth day, but once the infective forms have developed they persist in the bugs for over a year.

The barbeiro is not the only insect capable of acting as an intermediate host for Trypanosoma cruzi. Several other South and Central American species of Triatoma have been found to be naturally infected with this trypanosome or with one morphologically indistinguishable from it, and experimentally the trypanosomes develop in the cosmopolitan species, T. rubrofasciata and other cone-noses and in bedbugs. Nor is man the only vertebrate host. Experimentally apes, dogs and guineapigs are subject to infection, and in nature the common Brazilian armadillo, Dasypus novemcinctus, and various rodents have been found infected, their infection undoubtedly being carried by the common bug, Triatoma geniculata, which infests their burrows. The fact that infected bugs occur in some places where Chagas' disease is not known to occur suggests that, as is the case with Trypanosoma rhodesiense and the trypanosomes undistinguishable from it except by their harmlessness to man, not all strains of the parasite cause human disease. It is interesting to note that a very similar trypanosome has recently been discovered by Kofoid and McCulloch in Triatoma protracta of southwestern United States. This bug is common in nests of wood-rats and frequently attacks man also. This discovery suggests one of two things: either the trypanosome described as T. triatome, and which is admitted by the discoverers to differ from $T$. cruzi only in slight characteristics of questionable importance, is really identical with or a mere variety of $T$. cruzi, 
or else others of the trypanosomes observed in species of Triatoma from Argentina to Central America may not be identical with the trypanosome which is the cause of Chagas' disease.

The Disease. - In endemic regions Chagas' disease is so prevalent that children are usually attacked within a few months after birth, and at this tender age are often unable to withstand its effects. If death does not result the disease passes over into one or other of its various chronic forms. As a result it is very rare to find acute cases in anyone but young children or new arrivals. The latter, however, usually come from other infected regions and show marks of the chronic disease, and so are not susceptible to a new acute infection.

The acute infection is marked by a constant high fever, lasting from ten to thirty days, often without remission, and by a characteristic swollen face, noticeable from a considerable distance. The skin has a peculiar feeling of "crepitation" due to the mucous infiltration of the tissue under the skin. The lymph glands especially in the neck and arm pits swell up, the liver and spleen become enlarged, and the thyroid gland becomes swollen as in goitre. In fact, most of the symptoms are connected with interference with the thyroid gland which, while becoming massive in size, becomes reduced in function, thereby causing a number of nervous and constitutional symptoms. This interference is due, apparently, not so much to invasion by the parasites as to a specific effect of the toxins produced by them and carried by the blood. These are the constant features of the disease; the other symptoms vary according to the localization of the parasites. Frequently they multiply in the heart muscles, and the functions of the heart may be seriously interfered with. Very often, and with the most dire results, the parasites invade the brain and spinal cord. When this happens the mortality is high, and it is only a pity that it is not higher, since it would be better if death always eliminated these unfortunate trypanosome victims who are spared only for an unproductive, piteously mutilated life, doomed to grow up with the intellect of an infant, or as paralytics, idiots or imbeciles.

The chronic forms of the disease follow the acute form by the development of a substance in the blood which is deadly to the trypanosomes, so that the latter are restricted to the protecting tissue cells in which they multiply. The commonest chronic 
form is that in which the predominating symptoms result from an enlarged but insufficient thyroid gland-goitre, cretinism, convulsions, swollen skin and various functional disturbances, including imperfect heart and intellectual defects. Another chronic form is that in which the heart is especially affected. The fact that the parasites have a special predilection for the heart muscles makes this form of the disease very common. The results of localization of the parasites in the nervous system have already been mentioned. The intensity of the motor disturbances, varying from paralysis or spasmodic convulsions of a single muscle to complete paralysis or convulsions of the whole body, has no relation to the degree of intellectual affection, which may vary from a simple cretinoid condition to complete idiocy or infantilism.

It is doubtful whether the disease is ever recovered from entirely if left to run its course. Sometimes the symptoms become gradually less intense, in other cases they become worse and new ones develop, or recurrences of acute symptoms may develop, due either to reinfection or to a loss of the trypanocidal power of the blood.

Treatment and Prevention. - The treatment of Chagas' dissease is still in the experimental stage but there is some evidence that tartar emetic may prove to be of great value in dealing with it, at least in early stages. In fact it was the success obtained by Vianna in combating the disease with tartar emetic that first suggested to him its use against Leishmanian diseases.

Prevention of the disease consists largely in avoiding and exterminating the barbeiros. It is practically impossible to keep the bugs out of mud or thatched houses. For this reason the rebuilding of houses with other material is being urged everywhere in Brazil and with good results. The town of Bello Herizonte, for example, which was formerly termed " a nest of cretins" is now nearly free from Chagas' disease, due to the remodeling of the houses. People traveling through infected districts can readily protect themselves by sleeping under mosquito nets and by avoiding the native houses. There is said to be no danger of being bitten by the bugs in daytime or in the presence of artificial light, since they come forth only in the dark.

The extermination in the vicinity of villages of armadillos and of the various rodents which harbor the trypanosomes would be a valuable aid in the reduction of the disease. 


\section{CHAPTER VII}

\section{INTESTINAL FLAGELLATES AND CILIATES}

The human intestine furnishes a habitat for a considerable number of animals belonging to all four classes of Protozoa, though it is not so subject to such infections as are the digestive tracts of many lower animals, especially the ruminants. By far the most important intestinal protozoan of man is an ameba, Endamœba histolytica, discussed in connection with other parasitic amebæ in a subsequent chapter. Probably next to the amebæ from a pathogenic point of view should stand the ciliate, Balantidium coli, which is, however, not common in most parts of the world. The various flagellates of the intestine, from the simple bi-flagellate forms, such as Bodo, Cercomonas and Embadomonas, some of which are probably only accidentally parasitic, to the highly organized multi-flagellate forms, such as Trichomonas and Giardia, which are very common human parasites, differ greatly as regards their pathogenic importance, and opinions do not agree concerning the importance of particular ones.

General Characteristics of Intestinal Protozoa. - In some respects nearly all the Protozoa which make their home in the digestive tracts of animals resemble one another. Nearly all of them secrete for themselves resistant transparent cysts which protect them from drying up or from the presence of an unfavorable medium. In the encysted state intestinal protozoans are able to exist under the unfavorable conditions found outside the body of the host, and are capable of remaining in this state in a sort of torpid condition for long periods of time until they gain access to a new host. The cysts of intestinal protozoans are analogous to the resistant eggs of intestinal worms, and like worm eggs their presence in the fæces of infected persons serves as a convenient means of diagnosis. The unencysted protozoans which may be carried out of the intestine die quickly and probably could not produce a new infection even if swallowed immediately, since in some species at least they are unable to 
withstand the action of the acid juices of the stomach. None of the human intestinal Protozoa require a second host to transmit them as do the blood-dwelling parasites. While outside the body they remain dormant in their cysts for weeks or months until they can gain access to a host again through food or water. There is still much doubt as to the extent to which intestinal protozoans are confined to particular hosts. Some workers believe that each animal has its own species peculiar to it, and that these species are not capable of infecting different hosts. Evidence is accumulating, however, to show that in some cases at least this is not so, and that many intestinal protozoans of man are able to live in such animals as rats, mice and hogs. Most intestinal protozoans are of very wide geographic distribution, their abundance in any given place being largely determined by the warmth of the climate and the sanitary, or rather unsanitary, conditions.

As remarked before there has been much discussion concerning the effect produced by various species of flagellates in the intestine. Naturally these parasites are seldom discovered except when there is some intestinal ailment, since in normal health fæces are seldom submitted for examination. Where routine examinations have been made regardless of physical condition, it has been found that a large per cent of people in unsanitary places are infected. Stiles, in a town in one of our southern states, found that from 50 to 100 per cent of the children were infected, and it would probably be easily within the bounds of truth to say that 75 per cent of all people in warm countries living in places where unsanitary conditions prevail are subject to infection with one or several species of intestinal Protozoa. As Stiles has pointed out, such infection usually means that the infected person has swallowed human excrement, since it would be impossible for any natural agency to separate the microscopic protozoan cysts from the fæces in which they are found. This fact, impressed upon the mothers of infected children, especially when accompanied by the remark that one could not tell whether the infection had come from the excrement of a white or a negro, was found by Stiles to be one of the most powerful means of improving sanitary conditions in the South.

Facts which support the view that intestinal flagellates are of more importance pathogenically than has commonly been sup- 
posed have been furnished recently by the findings in returned British soldiers, in whom uncomplicated infections with flagellates have been found in many dysenteric cases, and also by the investigations of Lynch, Barlow, Escomel and others in various parts of the world. Still further evidence is furnished by the fact that parasites very closely allied to species found in man have recently been shown to be unquestionably of pathogenic importance, at least under certain conditions, in lower animals.

Obviously, however, in view of the large number of infected persons, the intestinal protozoans must often have little or no pathogenic effect. There is, nevertheless, much individual difference in susceptibility, and different strains of the same parasite seem to vary in the effects they produce. Moreover it is highly probable that a great many slight and perhaps almost unnoticed symptoms, resulting in a certain amount of interference with the digestive tract and in a general lowering of the health, may find their ultimate cause in intestinal parasites, either protozoans or worms or both. The health of people living in warm and tropical countries, even aside from the effects of malaria and other warm-climate diseases, is proverbially less perfect than that of people in the usually more sanitary northern countries. It is quite probable that intestinal Protozoa may play a part in this lowering of the tone of health.

In the paragraphs below a brief account of the more important intestinal flagellates and ciliates is given, with what is known of their pathogenic effects, in the following order: (1) the bi-flagellate forms, Bodo, Cercomonas and Embadomonas, (2) the multiflagellate forms, Trichomonas, Enteromonas, Chilomastix and Giardia; and (3) the ciliate, Balantidium.

\section{Bi-flagellate Protozoa}

Most primitive of the intestinal flagellates are the bi-flagellated forms, several genera of which have been found in the human intestine. The relation of these animals to the still more primitive mono-flagellated trypanosomes and their allies is shown by the parasites of the genus Trypanoplasma. The animals of this genus resemble trypanosomes in the general form of the body and in the possession of a parabasal body and an undulating membrane, but have an additional free flagellum. In Cercomonas 
(Fig. 29C), according to Wenyon, the trailing flagellum is attached to the side of the body as far as the posterior end, usually being continued as a free flagellum. According to others Cercomonas has only a single flagellum, the free one at the anterior end. Bodo (Fig. 29B) has two free flagella, one waving anteriorly,

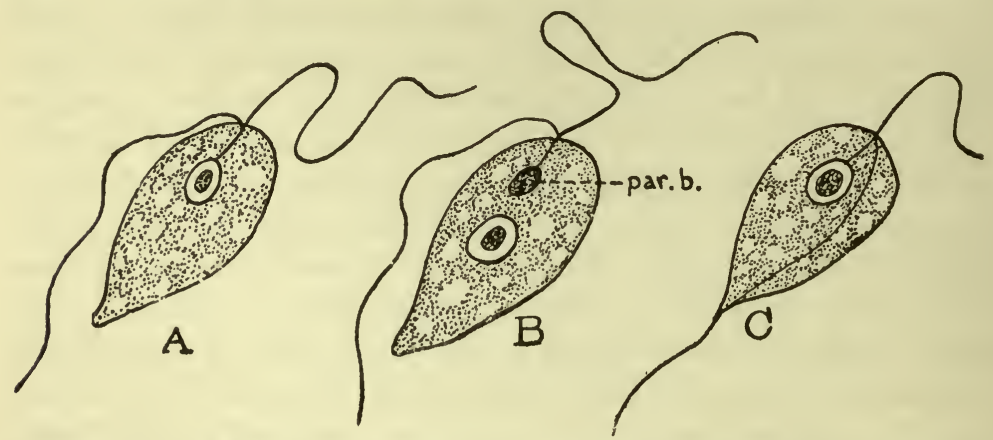

Fig. 29. Bi-flagellated parasites A, Prowazekella, separation from Bodo proposed on account of absence of parabasal body. B, Bodo, note parabasal body (par. b.). C, Cercomonas; note trailing flagellum attached to side of body. This is not recognized as a flagellum by some workers. $\times 2000$. (After Wenyon.)

the other trailing behind; some species not showing a parabasal body have been placed in a genus Prowazekella (Fig. 29A) of doubtful validity. Most of these flagellates are coprozoic, i.e., saprophytic fæcal protozoa, rather than true parasites, the cysts being accidentally ingested with water or food.

A slipper-shaped flagellate, Embadomonas (Waskia) intestinalis, first described by Wenyon and O'Connor in Egypt, and since recorded by Kofoid, Kornhauser and Plate in American troops returning from overseas, and by Hogue from a person who had never been out of the United States, is probably a true human intestinal parasite. It is only from 4 to $8 \mu$ in length, has a rather large cytostome, an anterior nucleus, and two flagella, one of which is short and thick and lies in the cytostome. The cysts are very small and pear-shaped. A larger species, Embadomonas sinensis has been found by Faust in China.

\section{Multi-flagellate Intestinal Protozoa}

Trichomonas hominis. - Of the several flagellates which have been found in the human digestive tract and fæces, Trichomonas is the commonest. It makes its home in the upper 
part of the large intestine and cœcum, often multiplying in prodigious numbers. Trichomonas also lives in the vagina and in the urinary tract, being quite often found in vaginal discharges, especially in cases of leucorrhea. It has been commonly believed that the vaginal parasite, which is larger than that of the intestine, is a distinct species, and it has been given the name $T$. vaginalis, but there is reason for believing that it is identical with the intestinal parasite. Other intestinal parasites are sometimes found in the urinary tract. This or a closely allied species is also occasionally found in the mouth, about the tartar of the teeth. According to Goodey the mouth form differs from the intestinal form to a sufficient extent to warrant its being given a distinct name, at least provisionally, and he proposes the name Trichomonas (Tetratrichomonas) buccalis.

Trichomonas hominis (Fig. 30) is a pear-shaped flagellate averaging about eight to $15 \mu\left(\frac{1}{3000}\right.$ to $\frac{1}{1500}$ of an inch) in length, the size being inversely proportional to the rapidity of multiplication. Typically it has three vigorously moving anterior flagella arising from the blunt anterior end, and a fourth wavy one which turns backward and is attached to the side of the body by an undulating membrane. Some

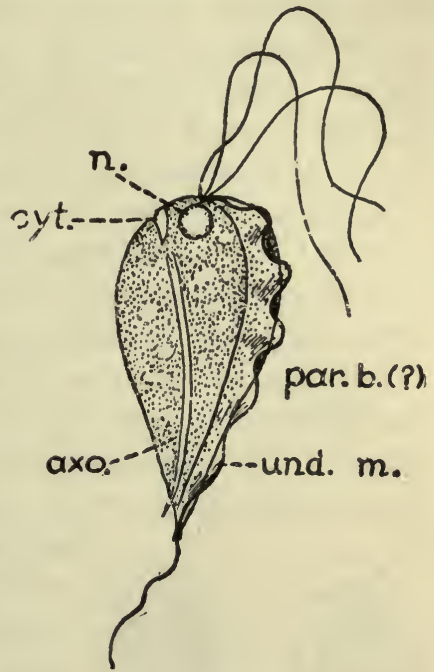

FIG. 30. Trichomonas hominis; n., nucleus; cyt., cytostome; axo., axostyle; par. b., parabasal body (?); und. m., undulating membrane. $\times 2400$. (After Wenyon.) investigators however, record four free flagella and others five. On this basis new species and genera have been created but, as pointed out by Faust, it is more probable that all of them may be included under a single species, with races among which the number of flagella may vary, but within any one of which it tends to remain constant, usually either three or four. Goodey describes the mouth form of the parasite as having four flagella. Along the line of attachment of the undulating membrane to the body is a structure which takes a deep stain, called the chromatic basal rod or "costa." It is believed by some workers to 
be a modified parabasal body; in some forms it is broken up into a row of chromatinic granules. At the point of insertion of the flagella, and serving as the center of the neuromotor apparatus is a basal granule or blepharoplast. Arising near the anterior end and running through the body is a sort of supporting rod called the " axostyle," which, according to Kofoid and Swezy, is also used as an organ of locomotion. At the anterior end at one side of the point where the flagella originate is a slight depression or "cytostome" which serves as a mouth. The small round nucleus lies in the body just behind the origin of the flagella.

Trichomonas swims by active lashing movements of the free flagella and by wave motions of the undulating membrane. The body revolves as the animal wends its way through the semi-liquid substances in which it lives. Multiplication is by longitudinal division of the body, the flagella and undulating membranes and internal structures all being duplicated before the animal splits into two. A process of multiple fission resulting in the formation of eight individuals has also been described.

Encystment, such as occurs in other intestinal protozoans, has definitely been observed only recently in Trichomonas. Some of the flagellates, after escaping from the body with the fæces, soon degenerate, gradually losing all their appendages

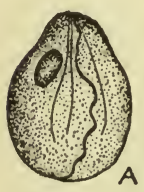

Fig. 31. Trichomonas intestinalis; $A$, pre-encystment stage; $B$, encysted form. $\times 2400$. (After fynch.) except the undulating membrane. Without their flagella, and with their ameboid movements, these animals closely resemble amebæ but can usually be identified by the undulating movement which persists at one side of the body. Others, without losing their appendages, become round and motionless as if in a cyst, but with no cyst wall around them. When warmed up they stretch themselves out and resume an active life. It is probable that these forms are preparing for encystment, since they correspond with pre-encystment forms (Fig. 31A) recently described by Lynch. Lynch, who found considerable numbers of cysts in a heavily infected case in South Carolina, describes the cysts (Fig. 31B) as thin-shelled, pearshaped bodies, about three-fourths the size of the active flagellates. The oval body of the animal with its appendages can be seen clearly through the cyst wall in properly prepared microscopic 
slides. It is the opinion of many protozoologists, however, that these so-called cysts are really degenerate forms, and that cyst formation has not yet been satisfactorily demonstrated. Lynch and others have succeeded in cultivating Trichomonas and in infecting rabbits with it, but specimens cannot be kept alive in water or fæces for more than a few days under the most favorable conditions.

Trichomonas is generally regarded as a harmless parasite, but there seems to be strong evidence that it often causes diarrhea, sometimes very severe and of long duration. Epidemics of diarrhea and mild dysenteric symptoms in man apparently caused by Trichomonas have been reported from Peru, Brazil, China, South Carolina and Indiana, and it is probable that the parasite is at least mildly pathogenic wherever it occurs, tending to aggravate other intestinal ailments if not causing them directly. Haughwout suggests the following possible ways in which this parasite may damage its host: (1) production of growth-inhibiting substances; (2) production of substances directly toxic; (3) liberation of injurious metabolic products; (4) mechanical irritation of mucous membranes; (5) interference with absorption by adherence to intestinal walls; (6) invasion and destruction of tissues. On the other hand Wenyon and O'Connor regard the presence of Trichomonas in cases of diarrhea as usually accidental, and fixed upon as the cause because the most conspicuous organism present. A case has recently been reported of an Oriental who was suffering from a foul-smelling decay of the jaw, accompanied by pains in the joints, in which numerous Trichomonas were found in the jaw lesion. After treatment with emetin there was rapid improvement, which suggests that Endamœba may also have been present.

No specific drug for use against Trichomonas has yet been found. Methylene blue in weak solutions is absorbed by the parasites and causes them to become round and quiet. Castellani recommends taking methylene blue both by mouth and by means of an enema, i.e., irrigation of the large intestine. With this treatment the flagellates are said to decrease rapidly and to disappear usually within a few days. Escomel, who has found Trichomonas an important factor in diarrhea in Peru, advises an enema consisting of one grain of iodine in a liter of water, taken in the evening on three successful days. Unless the parasites 
have established themselves in the membranes high up in the intestine they are said to disappear after this treatment. As with other intestinal Protozoa infection occurs through polluted food or water.

Enteromonas hominis. - This minute spherical or nearly spherical organism, 5 to $6 \mu$ in diameter, was first described by Fonseca in Brazil. It possesses two equal anterior flagella, and a larger trailing flagellum; the nucleus is situated anteriorly as in Chilomastix. It is probable that Tricercomonas and Diplocercomonas, described as distinct human parasites, are really identical with Enteromonas.

Chilomastix (or Macrostoma) mesnili. - A parasite which closely resembles Trichomonas in many respects is Chilomastix
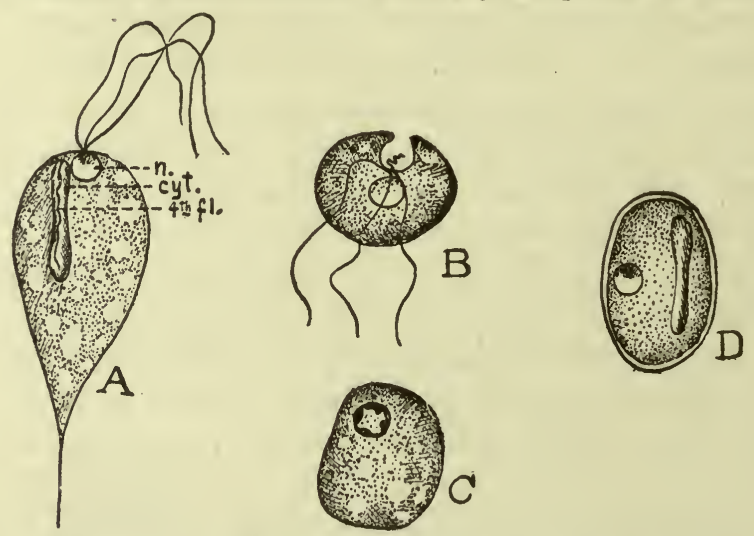

Fig. 32. Chilomastix (or Macrostoma) mesnili; $A$, adult parasite (n., nucleus; cyt., cytostome, 4 th ff., fourth flagellum); $B$, end view of adult parasite, showing cytostome with flagellum in it; $C$, degenerating form, resembling an ameba; $D$, cyst, showing nucleus and cytostome. $\times 2000$. (After Wenyon.)

mesnili (Fig. 32). It is smaller than the former, averaging about eight or ten $\mu$ ( $\frac{1}{3000}$ of an inch) in length. It has three slender anterior flagella like Trichomonas but has no conspicuous undulating membrane. It has a large and conspicuous slit or cytostome along one side which corresponds to the very small mouth cavity of Trichomonas. Within the cytostome is a fourth inconspicuous flagellum which seems to be attached to a small undulating membrane. The posterior end of the body is drawn out into a long point. As in Trichomonas the nucleus lies just behind the origin of the flagella. The rest of the body contains numerous vacuoles filled with bacteria, the latter apparently serving as the staple article of diet. 
The ordinary multiplication of Chilomastix is no doubt similar to that of Trichomonas. When ready to leave the body oval cysts are formed from 7 to $8 \mu\left(\frac{1}{3500}\right.$ of an inch) in length, within which the animal with its nucleus and large cytostome can be seen (Fig. 32D). Wenyon has found Chilomastix cysts with four nuclei and thinks that some multiplication may occur within the cysts as it does in Endamœba. The methods of transmission and means of prevention differ in no way from those of Trichomonas.

Giardia (or Lamblia) intestinalis. - Next to Trichomonas, Giardia is the most common flagellate in the human digestive tract. Unlike most of the other intestinal protozoans it establishes itself in the upper part of the small intestine. It is one of the oddest-shaped little animals known. Wenyon aptly describes it as follows: "In shape it resembles a pear split into two parts along the longitudinal axis. There is a flat surface on which there is a sucking disk with raised edge, and a convex surface. The tapering extremity or tail can be turned over the convex back, and it terminates in two flagella. There are three other pairs of flagella, the arrangements of which are best seen by referring to the plate." (Fig. 33.)

Giardia is remarkable in being perfectly bilaterally symmetrical, every organelle, including the nucleus, being accurately reproduced on each side of the middle line. Between the two small nuclei are a pair of rodlike structures (Fig. 33, par. b.) thought by some workers to be parabasal bodies, from which the flagella arise. As seen in face view the parasite has a comical owl-like

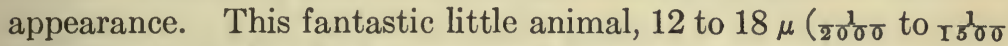
of an inch) in length fastens itself to the convex surface of an epithelial cell by means of its sucking disk, resting with its flagella streaming like the barbels of a catfish (Fig. 33F). Sometimes long rows of them can be found resting on the surface of the epithelial cells of digestive glands. Miss Porter, who has studied Giardia infections in British soldiers from Gallipoli, estimated recently that in one case the number of cysts, each having been an active flagellate in the intestine, exceeded 14,000,000,000 in a single stool. The number of cysts in an average stool in a case of moderate infection she estimated at $324,000,000$.

Evidently this flagellate multiplies very rapidly, but its method of multiplication is not fully understood. Division of unen- 
cysted forms has very rarely been observed, and some writers have even gone so far as to say that it does not occur. It is well known that division into two individuals takes place after encystment, and Wenyon has recently expressed the opinion that if the division is completed before the cyst is expelled from the body of the host, the cyst may burst and liberate the two animals,

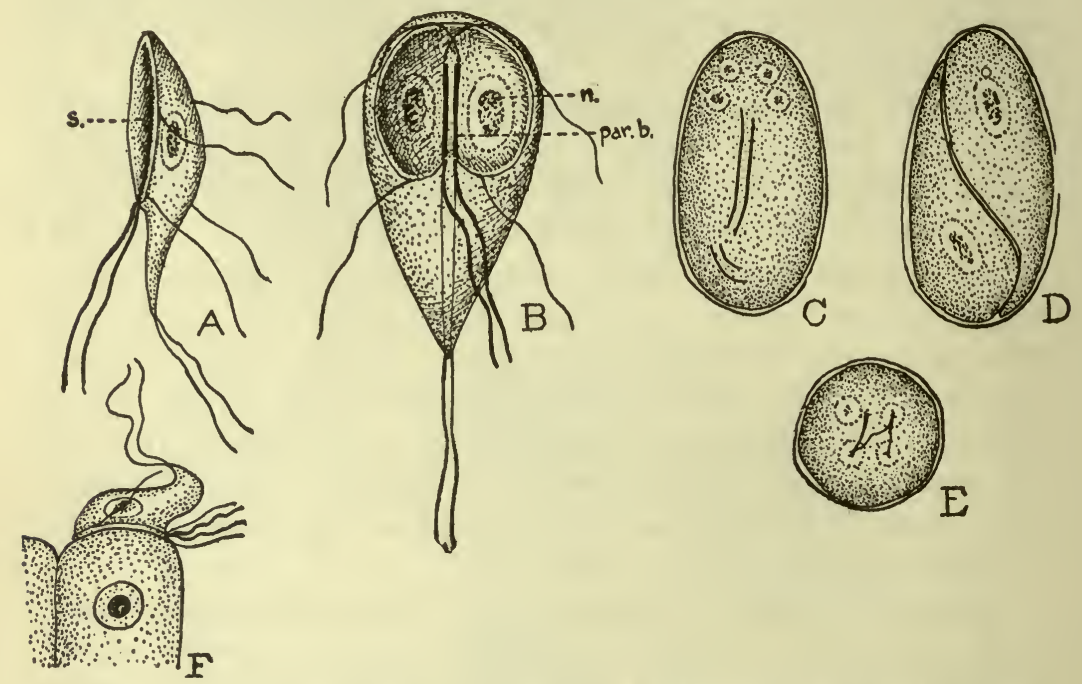

FIG. 33. Giardia (or Lamblia) intestinalis; $A$, side view (s. sucker-like depression); $B$, ventral view (par. b., parabasal bodies, n., nucleus); $C$, young cyst with four nuclei; $D$, mature cyst containing two parasites; $E$, end view of young cyst; $F$, parasite resting on epithelial cell. Figs. $A-E, \times 2000$, after Wenyon; Fig. $F$, $\times 1000$, after Grassi and Schewiakoff.

the cysts thus serving as a means of multiplication. Kofoid and Christiansen have recently succeeded in finding numerous individuals of an allied parasite of the mouse, Giardia muris, in process of division into two and also into four and eight individuals, both in the free and in the encysted state. That a similar process really occurs in the human parasite can hardly be doubted, both from its similarity to the mouse parasite and from the enormous numbers which may occur in an infected person at one time.

The free active parasites become motionless and die soon after leaving the body of the host with the fæces, but encysted forms (Fig. 33C, D and E) may retain their vitality for a very long time. 
The cysts usually form around single animals which then proceed to divide into two or more individuals. The commonest condition is that of two parasites lying with their anterior ends at opposite ends of the cyst (Fig. 33D).

According to Wenyon, Giardia is a very persistent flagellate, often keeping an individual infected for years. It is sometimes noticeably pathogenic, causing intermittent diarrhea in which blood and mucus is passed, swarming with parasites. Between such attacks the infected person passes apparently normal stools, with only the cysts of Giardia in them. An active increase of parasites accompanied by attacks of diarrhea is likely to occur after exposure to weather, irregular diet, or other weakening conditions. Many cases of dysentery and diarrhea in British soldiers invalided home from Gallipoli were found to be due to Giardia infection. The acute symptoms last from one to six months, after which the symptoms practically disappear for a variable length of time. Strangely enough there is always spontaneous improvement upon a change to a cooler climate.

Giardia infections are extremely difficult to get rid of, and some infections seem to survive every attempt at treatment. They do not respond to emetin, though they are sometimes destroyed by beta-naphthol. The latter drug in combination with bismuth salicylate has been found successful in some cases. Escomel in Peru uses a method of dieting followed by calomel and castor oil and claims to rid his patients of the parasite by the third day. The difficulty experienced in expelling these parasites is probably due to their habit of lodging themselves in the digestive glands outside the main passage of the intestine, where it is difficult for drugs of any kind to reach them.

Like other intestinal protozoans, Giardia is transmitted in the encysted state with polluted food and water. Stiles has shown that flies play an important rôle, carrying the cysts on their feet from open privies and depositing them on human food. By capturing flies known to have fed on Giardia-infected material and shaking them up in distilled water, Stiles was able to recover Giardia cysts from them.

Deschiens has recently adduced strong evidence by experimental infections that the Giardia of rats and mice is really only a variety of the human species, in which case the fæces of the rodents would afford a means of dispersal. Cats also may serve as a reservoir and means of dissemination for the infection. 


\section{Ciliates}

Balantidium coli. - Although several species of ciliates have been recorded as human parasites, there is only one species, Balantidium coli (Fig. 34A), normally parasitic in hogs, which is common enough to be of any importance. This large ciliate stands next to Endamoba histolytica among the Protozoa as a
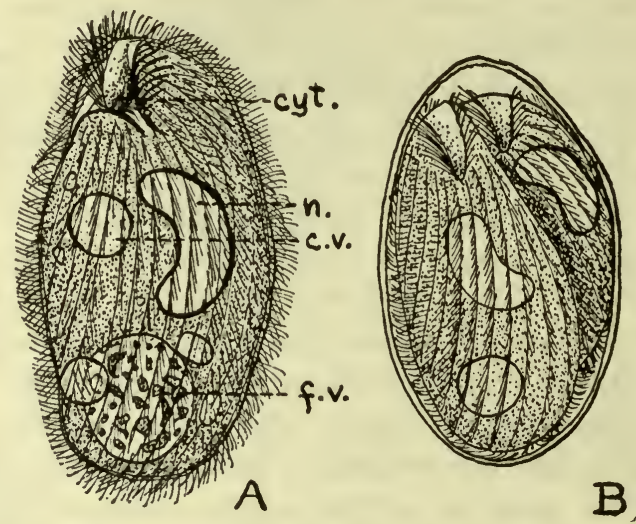

Frg. 34. Balantidium coli; $A$, free ciliate from intestine; n., nucleus; c. v., contractile vacuoles; f. v., food vacuole; cyt., cytostome. $B$, cyst, as passed in fæces, containing two parasites. $\times$ about 500. (After Wenyon.)

cause of human dysentery. It is a large animal for a protozoan, averaging from 50 to $100 \mu$ ( $\frac{1}{50}$ to $\frac{5}{2} \frac{1}{0} 0$ of an inch) in length, and thus being visible to the naked eye. Its body is oval and entirely covered with cilia, and at the anterior end there is a gashlike slit leading to the mouth or "cytostome" (Fig. 34, cyt.). The large bean-shaped nucleus (Fig. 34, n.) lies near the middle of the body and near each end is a pulsating cavity or contractile vacuole (Fig. 34, c.v.) which excretes waste matter. These parasites multiply by transverse division, often so rapidly that the animals do not have time to grow to full size and so become very small. When ready to leave the body they form an oval cyst about themselves. Sometimes two occupy a single cyst (Fig. 34B), and later fuse together. Since the ciliated bodies of the protozoans can be seen, under a microscope, inside the large transparent cysts, their identification is not difficult. The cysts can exist outside the body for a long time, awaiting an opportunity for reinfection. 
Balantidium swims about in the contents of the large intestine devouring particles of fæcal material. As long as the animal confines its activities to this, no ill effects result, but it also has the power, like Endamceba histolytica, of invading the tissues and causing ulceration, perhaps after an injury from some other cause has given an opening for invasion. Although many infected persons do not show any dysenteric symptoms, these are likely to appear at any time. When they do appear, they are of a very serious nature, and cause a high mortality. On post mortem examination the large intestine is often found in a horrible condition, ulcerated from end to end, with shreds of mutilated or dead tissue hanging from the walls.

Unfortunately there is no specific treatment for balantidial dysentery as there is for the amebic disease. In some cases emetin and alcresta ipecac (see p. 135) have caused a disappearance of the parasites, but these are not reliable remedies. Salvarsan and methylene blue have also been recorded as successful in some cases. Organic compounds of silver seem to have some value in destroying Balantidium, and there are other drugs and herbs of much local fame which are undoubtedly sometimes effective. Rest and care of the general health are always required.

Prevention of balantidial dysentery consists not only in the sanitary disposal of human fæces, as in the case of other human intestinal protozoans, but also in the proper care of hogs, since Balantidium is a common parasite of these animals, and is probably normally a hog parasite. A large proportion of hogs are infected in almost all warm and temperate countries, and it is nearly always in hog-raising countries, and in places where there is too close association between hogs and man, that balantidial dysentery occurs. Around Manila, where the disease is fairly common, the majority of the hogs are infected and pass encysted parasites in their fæces almost constantly. In Colombia the disease is found only in those altitudes where hogs are raised and among those who raise them. 


\section{CHAPTER VIII}

\section{AMEB $\mathbb{E}$}

Thоse of us who have had an opportunity, in studying microscopic life in water, to observe the restless movements of the tiny bits of naked protoplasm which we call amebæ, having watched them slowly creep along the surface of a slide, extending a portion of the body as a finger-like projection or "pseudopodium "and then allowing the rest of the body to flow up to the new position; having seen them creep up on tiny protozoans or other single-celled organisms and devour them by merely wrapping themselves around them, thus engulfing them in an improvised stomach; and having seen them propagate their kind by simply constricting in the middle and dividing in two; - those of us who have observed these acts on the part of such tiny and simple animals have come to be fascinated by them and to like them, and find it hard to realize that certain species are nstrumental in causing some important human diseases. Amebæ are found almost everywhere in water, soil and carrion. They have even been found recently to exist in large numbers in the sunbaked sands of the Egyptian deserts, lying dormant in their cysts which protect them from evaporation, ready to emerge and resume an active life when they become moistened. In view of the wide adaptability of these animals it is not surprising to discover some living as parasites, finding congenial surroundings in the bodies of higher animals.

Classification. - Amebæ are protozoans belonging to the subclass Sarcodina, a group characterized by a body without a cuticle, though sometimes protected by a shell or cyst wall, and by their peculiar method of locomotion. In the adult form they have neither flagella nor cilia, but simply outgrowths of protoplasm, called pseudopodia. In the amebæ and their close relatives the pseudopodia can be projected anywhere on the surface of the body, now here, now there, though the number, form and activity of the pseudopodia are quite different in different species. 
The life history also varies in the different species, many freeliving forms possessing a flagellated stage. On the basis of structure, life history, and habits the old genus Amœba has been broken into a number of genera distinguishable from each other principally by the structure of the nucleus and the nature of the cysts. The life history is simple in all of them, so far as it is known. The cases of spore formation, multiple fission, conjugation or sexual processes described by various investigators have not been substantiated by subsequent work. All of the parasitic amebæ are characterized by the absence of contractile vacuoles. One species, Endamoba histolytica, habitually feeds on blood corpuscles and living tissues, and another, Councilmania lafleuri, does so to some extent. The other species apparently feed principally on bacteria and other organic material with which they are associated in their normal habitat.

The principal amebæ occurring in human beings may be tentatively grouped in the five genera Endamoba, Councilmania, Endolimax, Iodamcba, and Dientamcba, but the classification of the parasitic amebæ cannot yet be considered as satisfactorily settled. The characteristics of these genera are briefly as follows:

Endamoeba; nucleus vesicular with chromatin arranged in a peripheral layer of beadlike granules of fairly uniform size, and a small compact karyosome; a capsule-like structure can usually be seen surrounding the karyosome. Cysts, if produced, with normally 4 or 8 nuclei of similar structure to those of the free forms, and including also glycogen masses and refractile "chromatoid" bodies, though these masses and bodies commonly disappear before or soon after the cysts become mature. (See Figs. 36 and 37.)

Councilmania; nucıeus with chromatin distributed in a peripheral zone on nuclear membrane and in an irregular karyosome. Cysts normally with 8 nuclei which have relatively large and very variable karyosomes, and very little peripheral chromatin. Buds containing single nuclei escape from a pore in the cyst wall until the cyst is emptied of all its nuclei. (See Fig. 41.)

Endolimax; nucleus vesicular without a distinct peripheral layer of chromatin. A fairly large compact mass of chromatin (karyosome) in the interior, usually more or less eccentric and connected by threads or processes with one or more smaller masses. Mature cysts oval, with four nuclei in the single known species, similar in structure to those of the free forms. The cysts contain, 


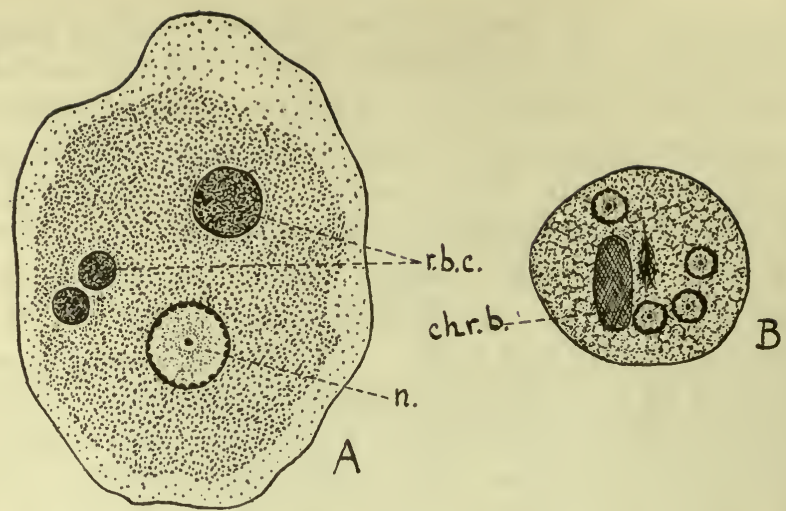

Fra. 36. Endamaba histolytica. $\times 1650 . A$, stained vegetative ameba; $B$, cyst with four nuclei; $n$., nucleus, showing peripheral chromatin granules and central karyosome; r. b. c., ingested red blood corpuscles; chr. b., chromatoid body. (After Dobell.)
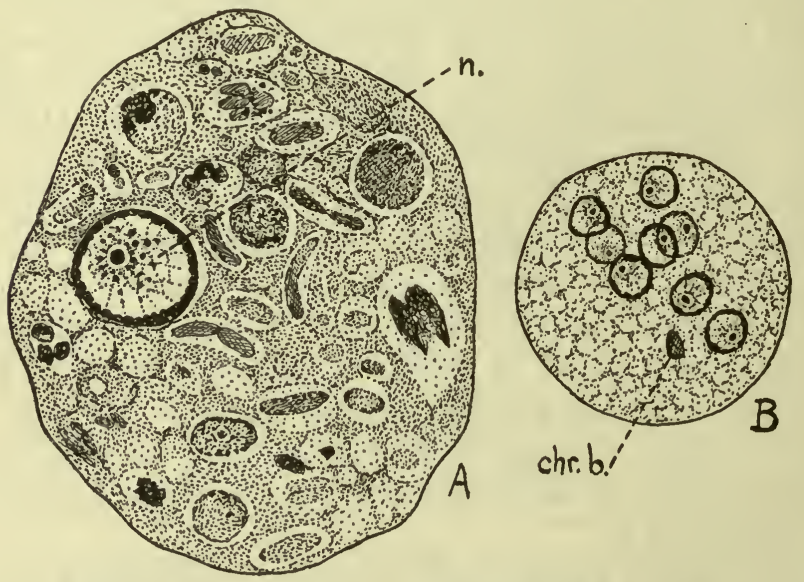

Fig. 37. Endamœba coli. $\times 1650 . A$, stained vegetative ameba; $B$, cyst, with eight nuclei; n., nucleus, showing coarse peripheral chromatin granules, chromatin granules in "clear zone" between periphery and karyosome, and eccentric karyosome; chr. b., remnant of chromatoid body. Note large number of food vacuoles in vegetative ameba. (After Dobell.)
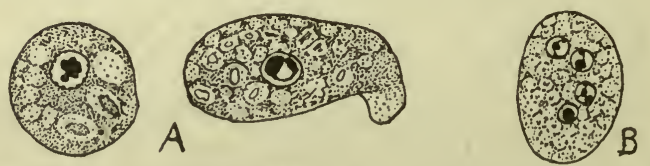

Fig. 38. Endolimax nana. $\times 1650$. A, two stained vegetative forms, showing nuclei with large irregular karyosome, and numerous food vacuoles. $B$, cyst with four nuclei. (After Dobell.) 

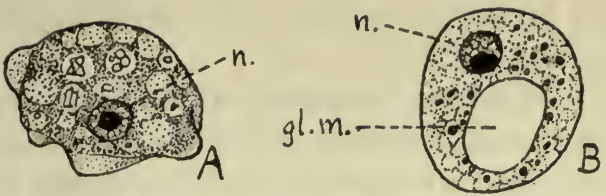

F1G. 39. Indamœba bütschlii. $\times 1650$. A, stained vegetative ameba, showing numerous food vacuoles and nucleus (n.), the latter with large central karyosome and a single layer of granules between karyosome and nuclear membrane. $B$, cyst, showing nucleus (n.) with peripheral karyosome, and glycogen mass (gl. m.) or "iodophilic body," from which these cysts received the name "Iodine or I. cysts." (After Dobell.)
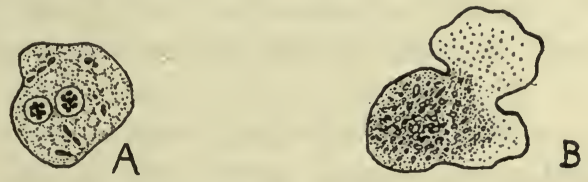

Frg. 40. Dientamøba fragilis. $\times 1650$. A, stained vegetative ameba, showing two nuclei with granular karyosomes, and tood vacuoles; $B$, living ameba, showing leaf-like pseudopodia. ( $A$, after Dobell; $B$, after Jepps and Dobell.)

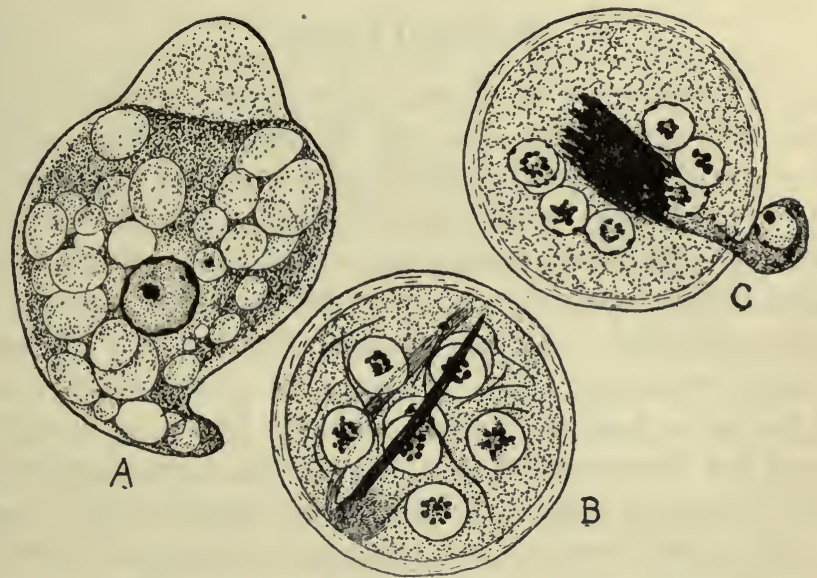

Fig. 41. Councilmania lafleuri. $\times 1650 . A$, stained vegetative ameba with a pseudopodium in early phase of protrusion and one nearly retracted and filled with endoplasm; nucleus showing peripheral chromatin and large irregular, eccentric karyosome; endoplasm filled with food vacuoles. $B$, eyst showing 8 nuclei, thick wall, and chromaphile ridge (dark) nearly encircling body, and chromatoid bodies in form of scattered threads. $C$, cyst showing bud escaping with one nucleus; large chromatoid body in center of cyst. (After Kofoid and Swezy.) 
in addition to the nuclei, a number of small refractile granules of a substance known as volutin. The young cysts also contain masses of glycogen. (See Fig. 38.)

Iodamœba. - Nucleus vesicular with moderate-sized central karyosome and well developed membrane without a distinct peripheral zone of chromatin, but with a single layer of rather large granules between the karyosome and the outer membrane; cysts very characteristic, formerly known as Iodine cysts or I. cysts, of very irregular shape, containing, besides a single nucleus, a number of brightly refractile granules and a relatively large clearly defined solid mass of glycogen which stains very deeply in iodine. The nucleus is peculiar in that the karyosome comes to lie peripherally in contact with the nuclear membrane. (See Fig. 39.)

Dientamœba. - Mature individuals with two similar nuclei; these are vesicular with a large central karyosome consisting of a number of granules; nuclear membrane very delicate without distinct peripheral chromatin; cysts not found. (See Fig. 40.)

\section{Intestinal Amebæ}

The intestinal amebæ of man, according to Dobell, consist of Endamoeba histolytica, E. coli, Endolimax nana, Iodamœba bütschlii ind Dientamœba fragilis, and to these must be added Councilmania lafleuri recently described by Kofoid and Swezy. A number of other species have been described by various writers, but these have probably been either deteriorated examples of the known species, body cells mistaken for amebæ, or a confusion of two other organisms. The discovery by Craig of an intestinal ameba having a flagellated stage, and now known as Craigia hominis, and the discovery of an allied species, Craigia migrans, by Barlow, have not been corroborated by other workers, and, as Dobell has pointed out, these organisms are probably in reality a mixture of known amebæ and flagellates. A number of species of free-living amebæ are occasionally found in fæces of human beings, apparently being able to pass through the digestive tract uninjured, but unable to thrive there. These amebæ are usually placed in the genus Vahlkampfia, in which there is not a flagellated stage in the development. Another species of Sarcodina occasionally found in human fæces is Chlamydophrys stercorea. This is a common 
organism in the fæces of various animals and in sewage, but there is no reason to believe that it should be considered a human parasite.

Endamoeba histolytica. Endamœeba histolytica, (Figs. 35 and 36) the dysentery ameba, is a large and active species, $18 \mu$ to $40 \mu$ ( $\frac{1}{1400}$ to $\frac{1}{600}$ of an inch) in diameter, the majority being between $20 \mu$ and $30 \mu$ in diameter. They are rather transparent in appearance, with blunt pseudopodia, and when perfectly fresh from the intestine creep along in a sluglike manner. After they have been outside the body for a short time they remain in one place and throw out large clear pseudopodia composed of ectoplasm sharply separated from the endoplasm (Fig. 35). The nucleus is very indistinct and usually eccentric in position. The most characteristic feature of the living ameba, however, is the presence of ingested blood corpuscles and tissue cells. Ordinarily there are from one to ten of these, but according to Dobell there may be forty. The amebæ after being fixed and stained can be distinguished from the closely allied $E$. coli by the nucleus. This has a peripheral layer of small uniform granules of chromatin, no chromatin in the clear zone between this layer and the karyosome, and a small centrally placed karyosome surrounded by an indefinite clear halo. (Fig. 36A.) E. coli has a thicker layer of peripheral chromatin, a number of chromatin granules in the clear zone, and a larger karyosome, eccentric in position, with a more distinct " halo" (Fig. 37A).

There are two stages in the life history of this ameba, the vegetative and the cystic. As long as conditions in the intestine are favorable for their growth and development, the amebæ continue in their active vegetative condition, multiplying by simple division of the body into two. When conditions have become unfavorable for them, however, as in later stages of the disease, they decrease in size, become rounded, and begin to develop a cyst wall. This is known as the precystic stage. From this stage they pass rapidly into the cystic stage by the completion of the cyst wall and the division of the nucleus into four daughter nuclei, thus forming the well-known "tetragena" cysts, long supposed to belong to a distinct species (Fig. 36B). The size of the cysts varies from $5 \mu$ to $20 \mu$ ( $\frac{1}{5000}$ to $\frac{1}{1200}$ of an inch) in diameter and there are a number of races or varieties of the ameba which differ from each other in the average size of the cysts. 
The nuclei have exactly the same structure as in the free amebæ but are smaller. The majority of freshly passed cysts contain blocks or masses of a highly refractile substance, called chromatoid bodies by Dobell (Fig. 36B, Chr. b.). These gradually disappear in the course of a few days. The normal cysts of the dysentery ameba are distinguishable from those of $E$. coli by the number of nuclei, and also by the central position of the karyosomes. The cysts pass out with the fæces of the infected individual, and live outside the body for a number of weeks if kept cool and moist. They are destroyed by desiccation and degenerate rapidly at high temperatures.

As cysts the amebæ are dispersed by " night soil " when used as fertilizer, by seepage into water, by flies and other insects, or by other means. If by any of these means they reach human food or water and thus secure entrance to the digestive tract, the cyst wall is dissolved by the pancreatic juice and four little amebæ, each containing one of the daughter nuclei which were formed when the cyst first developed, are set free in the intestine and begin to grow and multiply. The active vegetative amebæ from an acute case of dysentery are destroyed in the stomach if swallowed, and only the parasites in the encysted stage, with an enclosing capsule to protect them from being digested, can reach the intestine and cause disease.

The dysentery amebæ inhabit the tissues of the walls of the large intestine, where they cause more or less extensive ulceration. From this point they are carried by the lymph or blood stream to various other parts of the body, where they form local abscesses. Such abscesses are commonest in the liver but occasionally occur in other organs, such as the lungs, brain and spleen. The amebæ probably live entirely on the living tissues and blood corpuscles, the tissues being destroyed by means of a powerful cell-destroying substance produced by them.

Until recently the dysentery ameba was thought to be almost exclusively a parasite of tropical and subtropical countries, but in recent years it has been shown to be widespread throughout temperate climates as well, having been found endemic in nearly all parts of the United States and in the British Isles and Central Europe. The amount of endemic infection in temperate parts of both Europe and America has been greatly increased since the European war. Kofoid Kornhauser and Plate found 10.8 per 
cent infection among returned American overseas troops in contrast to 3 per cent in home service troops. In India, on the other hand, MacAdam estimates that one-third of the inmates of hospital wards are infected with $E$. histolytica.

Amebic Dysentery and Liver Abscesses. - One of the most serious menaces in the tropics is dysentery; people who have always lived in temperate countries have no conception of the severity of this ailment. In many tropical countries dysentery ranks next only to malaria as a cause of death, and very often it finishes the work of such diseases as malaria, kala azar and other fevers. There are many different types of dysentery, especially in the tropics, each showing somewhat different symptoms and having to be treated in different ways. Some cases of dysentery are due merely to improper diet, some to disturbances of the digestive tract due to other diseases, and the majority to intestinal parasites of some kind, either bacteria, protozoans, or worms. In a restricted sense "dysentery" is used for intestinal diseases caused by bacteria or protozoans. The diseases caused by protozoans other than amebæ are discussed in the chapter preceding this. "Bacillary dysentery" is a bacterial disease and need not be discussed here except in comparison with other types of dysentery. It occurs in temperate as well as in tropical countries and is very common in epidemic form in armies, prisons and asylums. Amebic dysentery, on the other hand, is uncommon outside of warm climates, but is of frequent occurrence in local areas in almost all tropical and subtropical countries, where frequently a large majority of dysentery cases are caused by amebæ. The proportion of cases of amebic dysentery to infections with the amebæ seems to be higher, and the disease more severe, in warm than in temperate climates. Many of the cases of "trench diarrhea" which occurred in France were a mild form of amebic dysentery.

The rôle played by amebæ in dysentery was in doubt for a long time. The presence of amebæ in perfectly healthy individuals and the fact that amebæ grown in artificial cultures would never give rise to dysentery experimentally confused the problem. There are several reasons for this confusion. In the first place there are common species of intestinal amebæ, especially Endamoba coli and Endolimax nana which do not attack the tissues or cause dysenteric symptoms, but which are easily confused with $E$. histolytica. In the second place, non-parasitic amebæ 
normally found in water or soil, may pass through the intestine uninjured, and these are the ones which have commonly been obtained in cultures from fæces. In the third place infection with Endamoba histolytica does not by any means cause dysentery in all cases; in fact, it may be said that in a normal infection there is sufficient resistance on the part of the host so that no appreciable harm results from the attacks of the parasites. The injury done to the tissues is repaired rapidly, and while a certain amount of ulceration probably exists in practically all cases, it may not be sufficient to cause dysentery. Individuals who thus harbor amebæ without being greatly inconvenienced by them are the so-called "carriers" of the disease. It is probable that only a small percent of infections, possibly only 10 per cent, actually give rise to dysenteric symptoms. The carriers constitute the principal means of dispersal of the parasites, since, to protect themselves against the reactions of the host, the amebæ are constantly encysting, and the cysts are constantly being liberated with the fæces. In acute cases only free amebæ, which cannot infect other individuals, are passed; convalescents, however, usually become carriers for variable lengths of time, and may frequently suffer relapses.

The Disease. - Experiments made by Walker and Sellards in feeding ameba-infected material to animals and human volunteers showed that dysenteric symptoms, if they appeared, occurred in from 20 to 94 days, averaging about two months. The most marked symptom is an acute diarrhea in which the stools consist largely of blood and mucus. In a typical case from Alabama a patient passed as many as fifteen or twenty stools in an hour. This condition had been going on for years, recurring about three or four times a year, lasting a month at a time. In the intervals between these attacks the symptoms were mild and the patient passed only two or three stools a day. Sometimes the attacks are more regularly chronic, or may recur at long intervals. Often the dysentery is accompanied by evening fever and anemia from loss of blood in the bowels.

Instead of producing ulcers on the mucous surface of the large intestine such as occur in bacillary dysentery, the amebæ commonly work deeper into the muscular linings of the intestines. Local swellings first appear, followed by an ulceration of the mucous membrane. This produces a portal for the entrance of 
the amebæ to the tissue underlying the mucous membrane, and here they make extensive excavations. The lesions are most common in the upper half of the large intestine but can be found from the lower part of the small intestine to the rectum. The exposed ulcerations vary from the size of a pinhead to that of a silver dollar, their ragged edges tending to roll into the craterlike areas. Often the tunnel-like excavations under the mucous membrane connect with one another.

Liver abscess is a common result of infection with Endamœba histolytica. Often these abscesses are of large size, filled with a slimy and somewhat bloody chocolate-colored pus. Over a quart of such pus has been removed from an amebic liver abscess. The parasites are found at the edges of the abscess, eroding more tissue and enlarging the pus cavity. How they reach the liver to do their damage is not certainly known, but it seems probable that they bore into blood vessels in the walls of the diseased large intestine and are carried by the portal vein to the liver, where they find a fertile feeding ground.

Infections with $E$. histolytica are very persistent, and, without treatment, may persist throughout the lifetime of the host. In many cases dysenteric symptoms never develop, while in other cases there may be intermittent attacks or an attack after the infection has persisted harmlessly for months or years.

Treatment and Prevention. - One of the greatest discoveries in the field of medical treatment since the production of salvarsan by Ehrlich is the discovery of emetin as a specific against amebic dysentery. Emetin is an alkaloid prepared from ipecac, the extract of the roots of a Brazilian herb. It was long known that ipecac sometimes had a very marked effect on dysentery, but since amebic dysentery has only recently been differentiated from other forms very variable results were obtained from its use. Ipecac has a decided disadvantage in that it causes violent vomiting, but its alkaloid, emetin, in the form of emetin hydrochloride, while possessing all the beneficial properties of ipecac, can be used in the form of injections into the veins, and therefore does not cause vomiting. Experiments with cultural species of amebæ showed that emetin (emetin hydrochloride) is destructive to amebæ when diluted 500,000 times, and the intestinal amebæ on a microscope slide become round and motionless and apparently dead when subjected to this very dilute solution. It is now 
thought however, that emetin acts rather by its effect on the tissues of the host than by its direct poisonous action on the amebæ.

Emetin is given in hypodermic injections. Almost without exception the effect of the drug on the disease is certain and rapid. Severe cases which have been running on for years can be cured in four or five days by this simple treatment. One of the chief disadvantages is that the treatment is often discontinued too soon. The dysenteric symptoms disappear as if by magic and the patient is often not willing to be subjected to continued drug injections until every trace of the amebæ has disappeared. Emetin is powerless against encysted amebæ and an apparently cured patient may continue to harbor and scatter these dangerous microscopic particles of living matter for some time, thus endangering other members of the community. Possibly selfinfection from the cysts lodged in the tissues of the intestine is the cause of the frequent recurrence of infection after inadequate treatment, but it is more probable that some of the free parasites escape the action of the drug and continue to multiply and develop new cysts. Under continued treatment the cysts gradually disappear from the intestine, but their exodus is hastened by purges.

Bismuth subnitrate has been used with good success in conjunction with emetin, the bismuth acting as a sedative on the intestine and aiding in healing of the lesions, and also as an amebicide. A daily enema of saline solution also seems to aid the efficiency of emetin.

Another preparation of emetin, alcresta ipecac, is effective against dysentery amebæ, though not so certain in its action as the hydrochloride. It has an advantage in that it can be taken in the form of tablets when a physician is not available and the apparatus for hypodermic injection is not at hand. Some doctors in .southern United States have advocated the use of extract of a common southern plant, Chaparro amargosa, to destroy intestinal amebæ. This extract is very cheap and entirely devoid of danger in ordinary doses, but its use in place of emetin has not yet been sufficiently justified.

Walker and Emrich have recently (1917) reported the successful use of oil of chenopodium for treatment of mild cases of amebic dysentery, and especially of "carriers." It is pointed out that emetin in its various forms is often inefficient in treatment of carriers on account of its powerlessness against encysted amebæ 
and its inability to eliminate them. These investigators emphasize the importance, before giving the oil, of a preliminary purgation with Epsom salts (magnesium sulphate) sufficient to produce fluid bowel movements, the purpose being both to remove excess fæcal matter from the intestine and to bring the amebæ out of their protective cysts and subject them in the unencysted condition to the action of the chenopodium. The treatment found most effective by Walker and Emrich is as follows: (1) magnesium sulphate, from one-half to one ounce, at 6 A. м.; (2) oil of chenopodium, 16 minims in gelatine capsules (to obviate disagreeable odor and taste), at 8 A.M., 10 A. M. and $12 \mathrm{M}$., and (3) castor oil, one ounce, containing 50 minims chloroform, at 2 P.M. This or any other treatment should be followed by examination of the fæces at intervals for some weeks after treatment, to make certain of the cure.

The keynote to the prevention of dysentery whether it be caused by amebæ or other protozoans or bacteria is sanitation. The efficacy of sanitary measures was well illustrated by the fact that during the first month of the occupancy of Vera Cruz by the Americans in 1914 there were four times as many cases of dysentery as during the second month when sanitary measures had been taken and were enforced. The fact that only the encysted parasites as found in the fresh or dried fæces of infected individuals can cause disease suggests a simple remedy in the proper disposal of infected fæces. In tropical countries, however, such a preventive measure is not so simple as it sounds. In many districts where amebic dysentery is endemic the first rudiments of sanitation are unknown and every possible method of transmission of amebic dysentery is given full opportunity. Polluted drinking water, uncleanliness, transmission by flies, and the almost universal use of "night-soil" (human fæces) for fertilizer, all help the cause of dysentery and account for its prevalence.

The segregation and cure of dysentery patients, and the careful disposal of their fæces, is not enough to eradicate the disease entirely since there are many immune carriers of the disease who, though apparently well, harbor the encysted amebæ in their fæces and thereby constitute a source of danger to the community. Thorough sanitation throughout the community is the only preventive measure which is adequate. 
Still anotner possible factor in the distribution of dysentery amebæ is the rat. Dr. Lynch of Charleston, S. C., states that in that city rats suffer from amebic dysentery as well as man. The fact that rats became infected by eating infected human fæces, the frequent occurrence of the disease in rats in houses where human amebic dysentery has occurred, and the ready transmission of the disease from rat to rat indicate that the rat infection is identical with that in man, and is not due to the ameba peculiar to rats, $E$. muris, and that rats may play an important rôle in the spread of the human infection. It may be that rat destruction will prove to be an important preventive measure against amebic dysentery. Chatton has recently transmitted the disease to guinea pigs also.

Other Intestinal Amebae. - As stated above there are a number of other species of amebæ which occur as human parasites, but as compared with $E$. histolytica most of them are of little importance. They are not tissue parasites, except possibly Councilmania lafleuri, but feed principally if not exclusively on bacteria or dead organic matter with which they are associated in the intestine. Their principal importance lies in the possibility of their confusion. with the dysentery amebæ.

One of the commonest species is Endamoba coli. In stained preparations this species can be distinguished from $E$. histolytica, which it resembles in size and habitat, by the characteristics given on p. 133, (cf. also Figs. 36 and 37). E. coli is usually more sluggish in movement than $E$. histolytica. It never contains blood corpuscles, but the body is often filled with numerous food vacuoles containing bacteria or other material ingested from the intestinal contents (Fig. 37A). The cysts of $E$. coli (Fig. 37B) are, on the average, larger than those of $E$. histolytica, but as with the latter species there are races distinguishable by the size of the cysts. The average sizes in different races vary from $15 \mu$ to about $22 \mu$. The mature cysts normally contain eight nuclei in contrast to the four usually found in $E$. histolytica. Even in the four-celled stage the cyst can readily be distinguished from those of $E$. histolytica by the eccentric position of the karyosomes of the nuclei. According to Dobell " $E$. coli has been found living as a harmless commensal in the colon of man wherever and whenever it has been sought: no race, 
nor any country, has yet been discovered in which infections with this species are not common."

There is no effective treatment for $E$. coli infections. Since the parasites do not live in the tissues they are, like the intestinal flagellates, unaffected by emetin.

Councilmania lafleuri (Fig. 41), formerly confused with Endamoba coli, has recently been described by Kofoid and Swezy. The free amebæ are very active, and creep about with remarkable rapidity. They commonly produce only a single pseudopodium at a time, and this is composed entirely of clear ectoplasm. The pseudopodia are peculiar in that they are shot out almost instantaneously for the greater part of their length. The endoplasm is usually loaded with food vacuoles containing bacteria, fæcal particles, and also frequently red blood corpuscles, the latter fact indicating that this parasite, like Endamoba histolytica, is in part a tissue parasite. The most striking and distinctive characteristic is the method of escape of amebulæ from the cysts by a repeated process of budding, a single nucleus at a time slipping out through a pore in the cyst wall, surrounded by a part of the cytoplasm (Fig. 41C). It is probably the only parasitic ameba in which the cysts act as a means of multiplication without transfer to a new host.

Another common intestinal ameba of man is Endolimax nana, (Fig. 38), the principal characteristics of which are given under the genus Endolimax on p. 129. It is a small ameba measuring from $6 \mu$ to $12 \mu$ ( $\frac{x}{4000}$ to $\frac{1}{2000}$ of an inch) in diameter. It creeps sluggishly like $E$. coli, and, like that species, often contains numerous food vacuoles filled with bacteria. The four-nucleated cysts (Fig. 38B) might be confused with those of $E$. histolytica, but are distinguishable by their small size (usually 8 to $10 \mu$ by 7 to $8 \mu$ ), their oval shape, and the peculiar structure of the nuclei, described on p. 129. This is a very common human parasite, having been found in as high as 33 per cent of some series of examinations made by competent workers. Although frequently found in dysenteric patients associated with Endamœba histolytica, there is no evidence that this species is at all pathogenic. Like $E$. coli, this ameba cannot be eliminated by emetin or any other drugs although it temporarily disappears during emetin treatment. Its exact habitat in the intestine is not known, but it is certainly not a tissue parasite. 
Iodamøbe bütschlii (Fig. 39) is a small ameba (usually $9 \mu$ to $13 \mu$ in length) the characteristics of which have been sufficiently described on p. 132. The eysts of this species (Fig. 39B) were until recently thought to be of vegetable nature and were known as Iodine or I. cysts. The cysts are not smaller than the free amebæ; they are of very irregular shape, as if formed under pressure. This ameba, also, has a wide geographic distribution and, while not so common as the intestinal amebæ hitherto discussed, the available data indicate its occurrence in from 3 per cent to 5 per cent of all human beings in temperate or tropical climates.

Another very small human ameba, Dientamœba fragilis (Fig. 40), has recently been described by Jepps and Dobell. This species averages only about 8 or $9 \mu\left(\frac{1}{300}\right.$ of an inch) in diameter, thus resembling Endolimax nana. The free amebæ are active, showing well marked ectoplasm and endoplasm. The pseudopodia are of ectoplasm, and are flat and leaflike (Fig. 40B). The peculiar features of the organism are described on p. 129. The most characteristic feature of this ameba is the division of the nucleus shortly after cell division has occurred, so that mature individuals have two similar nuclei. No cysts have yet been found, yet it is highly improbable that the infection is transmitted by the free amebæ, since the latter are very short-lived outside the body. The habits of the organisms are probably similar to those of the harmless amebæ already described. Only ten cases of infection have so far been discovered, but the evidence afforded by these cases indicates a wide geographic distribution.

\section{Mouth Amebæ}

The occurrence of amebæ in the human mouth has been known for many years, but particular interest has attached to them only recently, since a number of investigators have brought forth evidence to show that the common mouth ameba, Endamoba gingivalis, is pathogenic, and an important factor in pyorrhea.

Pyorrhea, or Rigg's Disease, in some stage afflicts the majority of all adult people, and over 50 per cent of all permanent teeth which are lost are lost as the result of pyorrhea. The apparent relation between this disease and the presence in the mouth of the above-mentioned ameba, E. gingivalis (or buccalis), was first demonstrated in 1914 by Smith and Barrett. Since 
then there has been much controversy as to the rôle played by the amebæ in connection with the disease, and most authorities are now inclined to look upon the amebæ as having little or no important part in the pathogenic process, but this question cannot be considered as settled. The prevalence of amebæ in the mouth, even in young children, is well shown by a recent investigation by Anna Williams of the mouths of over 1600 school children in New York City. Of the children between five and seven years of age 35 per cent were found infected, while of those between five and fifteen years 60 per cent were infected.

The mouth ameba, $E$. gingivalis, can be found by placing a bit of the pus from a tooth pocket, or the scrapings from the teeth, on a microscope slide. Here the parasites will be found intermingled with pus cells and bacteria. They are from one to three times the diameter of the pus cells, usually from $12 \mu$ to $20 \mu$ ( $\frac{1}{2000}$ to $\frac{1}{1200}$ of an inch) in diameter, and have a granular appearance; the nucleus is relatively very small. Often when stained they show dark bodies inside of them which are probably the nuclei of other organisms or of semi-digested pus cells. When living the amebæ prowl about sluggishly, pushing out a blunt pseudopodium now on one side of the body, now on the other, then drawing up the body, and pushing out more pseudopodia, thus slowly working their way about between the pus cells and fragments of tissue. The outer layer of the body, or ectoplasm, which serves as a sort of protecting envelope, like the rind on a melon, is clear and transparent but is not readily distinguishable except when the animal is moving. The pseudopodia are always formed first out of this clear ectoplasm, the more granular, grayish inner substance or endoplasm pouring out into it later. The reproduction of these little animals is by a simple division of the body into two when they have grown large enough to feel cumbersome as single individuals. Although cysts have been described by some workers, there is insufficient evidence for their existence, and it is more probable that infection is spread by simple direct or indirect transfer of the free amebæ from mouth to mouth.

Some investigators have suggested the possible identity of $E$. gingivalis and $E$. histolytica, but, as pointed out by Craig, the sluggish movements, small nucleus, inability to produce dysentery when swallowed and other characteristics all indicate that without doubt the mouth ameba is quite distinct from the intestinal amebæ. 
Endamoeba gingivalis and Disease. - As intimated above, although the presence of amebæ in the mouth has been known for many years, these parasites attracted little interest until 1914 when several investigators called attention to an apparent relationship between the amebæ and the presence of pus pockets between the teeth and gums, a disease known to dentists and physicians as "pyorrhea alveolaris." The amebæ do not thrive on exposed surfaces in the mouth, but find a congenial environment in any little secluded pockets between the teeth and gums, in crevices between close-fitting teeth, or where a bit of food forms

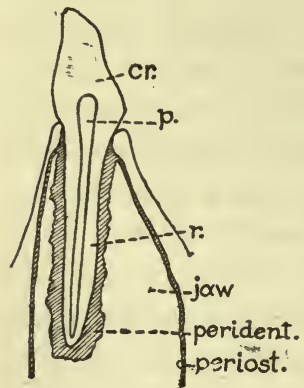

Fig. 42. Sketch of tooth showing peridental membrane, which is the tissue attacked by Endamoba gingivalis and the seat of pyorrhea, perident., peridental membrane; periost., periosteum; cr., crown; r., root; p. pulp. (After Bass and Johns.) a protected spot for them. Stowed away in such places, and invariably accompanied by bacteria and often spirochrtes, they multiply rapidly. That they feed largely on other organisms cannot be doubted, but that they prey also on the living tissue cells is very probable. Eventually the delicate peridental membrane surrounding the roots of the teeth (Fig. 42), corresponding in a general way to the periosteum of bones, is eaten away and becomes ulcerated. The eating away of the living membranes of the teeth and gums is accompanied by a constant formation of pus, and a marked proneness for the gums to bleed, often without provocation. The swallowing and absorption of the pus and of the poisonous waste products generated by the parasitic organisms are probably the cause of the more or less noticeable constitutional symptoms which accompany the disease. These may consist of feverishness, disordered digestion, nervous troubles, rheumatic pains in the joints, anemia, or various combinations of these ailments. We have long known that unhealthy mouths were the cause of general bad health, but we never until recently had any definite clue to the reason why.

As the ulceration of the membrane continues, the tooth is gradually loosened from the gum. Just as meadow mice girdle fruit trees, so these amebæ, or the bacteria or spirochætes which accompany them, eat away the living "bark" of the teeth and 
gums, eventually causing the teeth to fall out. As already stated, over 50 per cent of all permanent teeth which are lost fall out as the result of pyorrhea.

Whether the formation of the pus pockets is initiated by the amebæ or by other organisms is not known, but certain it is that Endamaba gingivalis is with few exceptions found in the lesions, and at the very bottom of them, often burrowing into the inflamed tissues to a depth of several times its own diameter, devouring cells and transporting bacteria. The belief in the rôle of the amebæ is based on these facts and on the fact that often, though not always, the disease is greatly improved by treatment with emetin, which has a specific action on amebic dysentery. Some investigators, notably Craig, consider it, to quote from Craig, "more than doubtful that Endamoba gingivalis is the cause of pyorrhea alveolaris, this conclusion being based upon the following facts: the occurrence of the parasite in a large per cent of healthy mouths and in the material that can be scraped from healthy teeth and gums; the occurrence and persistence of the parasite in patients treated with emetin, even when marked improvement in the clinical symptoms have occurred; the absence of the parasite in some typical cases of pyorrhea; the lack of improvement with emetin shown in numerous instances of the disease, although the endamebæ may disappear; and the fact that emetin acts upon other organisms as well as upon endamebæ and the possibility that the improvement that often follows its administration may be due to such action or to a favorable action on the tissue cells." That these facts argue against the causation of pyorrhea by amebæ alone is unquestionable. These facts, however, are not only not opposed to the possibility of amebæ being partly or indirectly responsible for the disease, but may be interpreted as being in support of such a view. It is entirely in accord with the known facts about the disease to suppose that the pus pockets may be initiated or enlarged by the action of amebæ, the damage being then continued by bacteria which have been given a portal of entry. This would account for the occasional absence of amebæ in typical cases of pyorrhea and for the occasional cases of the disease which are not improved by emetin. It is further quite conceivable that the amebæ may live for a long time in crevices in the mouth without doing any damage, and yet be capable of causing or aggravating pus pockets 
under suitable conditions. Perhaps some slight injury to the membranes or the combined action of the amebæ and certain bacteria is necessary to start the process. Parallel cases of parasites which may live for a long time as harmless messmates and then, under favorable conditions, become pathogenic are well known; one of the best examples is the intestinal ciliate, Balantidium coli. This would account for the presence of Endamœba gingivalis in healthy mouths. It is significant that in her investigation of school children in New York, Anna Williams found only 30 per cent of apparently healthy mouths, and 94 per cent of mouths with spongy and bleeding gums, infected. As to the statement that amebæ still exist in pus pockets after treatment with emetin, even when there is marked improvement in clinical symptoms, there is no doubt but that the number of amebæ is greatly reduced, and those on the frontier where the most damage is done are undoubtedly killed, since they are most exposed to emetin in the blood. The ineffectiveness of emetin against amebæ which are not directly in the tissues has been demonstrated in the case of the intestinal amebæ which are not tissue parasites (see p. 139). Again, were the improvement following treatment with emetine due to favorable action on the tissue cells, such improvement would invariably follow. That emetin affects other organisms besides amebæ is true, but it is more active against these protozoans than against any other organisms, as far as is known. The complete cure of pyorrhea which emetin sometimes effects, the usual improvement shown after its use, and the occasional failure of it, all point to the instrumentality of amebæ in causing or aggravating the disease, but indicate that they may be aided and abetted, or entirely replaced, by bacteria or other organisms.

There is some evidence that chronic tonsilitis also is often caused by $E$. gingivalis, since this parasite is found in the majority of diseased tonsils, irritating the tissues and opening the road for bacteria.

An indirect relation of this same mouth ameba to certain types of goitre also has been shown to be very probable. Evans, Middleton and Smith found that diseased tonsils and nasal passages and enlarged thyroid glands (goitre) are frequent companions in the goitre belt of Wisconsin. They believe that the amebæ injure the tissues sufficiently to give ample opportunity 
for bacteria to enter and multiply in enormous numbers, and that certain of these bacteria produce poisonous substances which exert a stimulative effect on the thyroid glands, thus causing goitre. The effect of the presence of amebæ, indirect as it is, can be fully demonstrated by destroying them with emetin. In 18 out of 23 cases of goitre treated with emetin the size of the thyroid mass was obviously reduced.

Prevention and Treatment. - Ordinary cleanliness of the mouth by frequent brushing of the teeth, rinsing of the mouth, and care of imperfect teeth is the most important factor in keeping the gums healthy and free from an injurious degree of amebic infection. In the investigation of school children in New York already mentioned the number of ameba-infected mouths was reduced one-half by ordinary cleanliness and care. Such methods, however, are of little value if the amebæ have established themselves in a pus pocket, since in such situations they cannot be reached by the usual methods of mouth cleansing. In the New York investigation it was found that mouths could almost always be freed of amebæ by using a mouth wash with a weak solution of emetin, the latter being a valuable preventive measure. In older people, however, where the amebæ have often already succeeded in stowing themselves away in little crevices and pockets where mouth washes cannot reach them, some other method must be employed. The ideal method is to open up and thoroughly clean out any pus pockets which can be found. This should be followed by a hypodermic injection of emetin, repeated on a few successive days to destroy the amebæ if possible. The effect of the emetin on Endamœba gingivalis is not as marked as was formerly thought, and Dobell goes so far as to say " it thus seems probable that emetin has no specific action upon $E$. gingivalis, and that the original claims were based upon insufficient evidence." Usually with the administration of emetin the soreness ceases, the pus formation stops, the gums stop bleeding and the general health rapidly improves. Of course it takes time for the injured tissue to heal and the part destroyed is never replaced. There is also constant danger of reinfection and the already eroded pocket forms an excellent place for fresh amebæ to take up a claim and begin their work. Furthermore there are cases of pyorrhea which do not respond to treatment with emetin, probably because the work is continued by bacteria. Emetin, diluted 
200 to 400 times in alcohol and applied with a tooth brush, is usually sufficient to kill recently implanted amebic infections. A thorough mouth rinse with a drop or two of emetin in half a glass of water is an excellent protective measure but even with the use of these means of prevention some apparently cured cases of pyorrhea get reinfections within a few months.

As intimated before, the prevention of infection with Endamoba gingivalis is largely a matter of ordinary mouth hygiene. Infection can be avoided to a large extent by care in eating and drinking. One should never eat or drink with the same articles that have been used by other people. The practice of promiscuous kissing is, of course, a ready means of transmission for these parasites as for many others.

Occasional infection with Endamceba gingivalis is, however, almost inevitable. If the mouth is kept scrupulously clean and in as near perfect condition as possible the amebæ may find no congenial place to settle down, but in the vast majority of mouths there is an abundance of fertile ground for them. 


\section{CHAPTER IX}

\section{MALARIA}

Importance. - Of all human diseases there is none which is of more importance in the world today than malaria, and this in spite of the fact that we have a very full knowledge of its cause, the manner of its spread, its cure, and means of prevention. It has been estimated to be the direct or indirect cause of over onehalf the entire mortality of the human race. Sir Ronald Ross says that in India alone it is officially estimated that malaria kills over one million persons a year, a greater number of deaths than was caused by the great European war in the first two years of its existence. When there is added to this the thousands from the rest of Asia, Africa, Southern Europe, South and Central America, and the southern part of our own country who are annually sacrificed on the altar of the malarial parasite; the millions of others who are broken in health, incapacitated for work and made easy victims of other diseases; the valleys, countries, and even continents which have been barred from full civilization and development by this more than by any other cause; then only can we get a glimpse of the real meaning of malaria to man. Ross argues convincingly that the downfall of the great Greek empire and the present poverty-stricken blighted condition of many parts of Greece is probably due primarily to the invasion of that country, not by burning and devastating armies of men, but by the malaria parasite, an infinitely more terrible though unseen foe which destroyed the newborn infants, undermined the health of the children or killed them outright, rendered the richest agricultural lands uninhabitable, and, in a word, sapped the vitality of the people until the boasted power and glory of Greece is but a mocking memory.

Though historians and economists have failed to recognize it, the rôle of malaria and other endemic diseases must have played an enormous part in the history of the world and in the progress of nations. Malaria and its powerful accomplice, the hook- 
worm, are largely responsible for the present deplorable condition of some parts of our own South. Dr. Howard estimated in 1907 that there were nearly 12,000 deaths a year in the United States from malaria. This, however, is probably almost inconsiderable when the amount of damaged health and weakened resistance to other diseases is taken into consideration. Dr. Von Ezdorf, of the U. S. Public Health Service, in a recent attempt to estimate the prevalence of malaria in the United States, obtained data, based on morbidity reports, which indicate that at least four per cent of the population of eight southeastern states $-1,000,000$ people - is affected by the disease annually, and found by 13,526 blood examinations that over 13 per cent harbored malarial parasites in their blood, the percentage being much higher in negroes than in whites. Dr. Howard thinks that an estimate of $3,000,000$ cases of malaria a year in the United States would not be too high. Millions of acres of fertile land in this country are rendered useless or only imperfectly cultivable. Taking everything into consideration, Dr. Howard makes the astounding but wellfounded statement that the annual financial loss to the United States from malarial diseases is not less than $\$ 100,000,000$. This is the condition in the United States, a large portion of which is relatively free from malaria, and in no part of which is the disease so prevalent or so destructive as in the tropical portions of Asia, Africa and South and Central America. In a broad way one-third of the population of highly malarial countries suffer from the disease annually. According to Ross the number of deaths from malaria in India must reach $1,300,000$ every year. Obviously the importance of this disease to mankind is not likely to be overestimated.

History. - "Malaria" means bad air, and was therefore applied to a number of fevers which were commonly associated with the bad air of swampy regions. The idea that malaria is caused by bad air, unwholesome odors, damp night winds, or impure drinking water is even yet adhered to not only by some of the populace but even by a few unenlightened medical men. Ross says that it takes ten years for the world to grasp a new idea, but his estimate is far too low; it is now (1917) 37 years since the organism causing malaria was discovered and 19 years since its transmission by mosquitoes was experimentally proved. It was in 1880 that Laveran, a French army surgeon in Algeria, 
discovered a parasitic "germ " which he proved to be the true cause of malarial fevers. Dr. King, of Washington, in 1883 suggested the probability of malaria parasites being spread by mosquitoes, adducing much circumstantial evidence in support of his views. It was not until 1898, however, that Sir Ronald Ross, an Englishman in the Indian Medical Service, experimentally proved that the malaria parasite is absolutely dependent upon certain species of mosquitoes for its transmission from man to man. Only six years ago (1911) the parasites of malaria were first successfully cultured outside the human body by Bass and Johns at New Orleans, a feat which will eventually lead to new and valuable discoveries. Other workers deserve no less credit, perhaps, for suggestive ideas, or for additional facts concerning the life and control of the malarial parasites. The ultimate results of their discoveries have only begun to be felt, but already such enterprises as the building of the Panama Canal have been rendered possible. The Canal could never have been built under the old régime of medical ignorance. Statues of the pioneers in the work of unraveling the truths about malaria and yellow fever might well have occupied conspicuous places at the Panama Pacific International Exposition at San Francisco.

Malarial Parasites. - Malarial fevers, of which there are several different kinds, we now know to be caused by protozoan parasites which live at the expense of the red blood corpuscles, and are injected into the human body and transmitted from person to person only by the bite of certain species of mosquitoes.

The malarial parasites belong to the protozoan class Sporozoa, or spore animals, so called from their habit of reproducing by breaking up into a number of small parts or spores, instead of simply dividing into two as do most of the Protozoa. All of the Class Sporozoa are parasitic and have no organs of locomotion when full grown. Although there are many different kinds which live as parasites in other animals, very few normally attack man and only the malarial parasites, belonging to the genus Plasmodium, are of primary importance. There is still considerable disagreement as regards the classification of the human malarial parasites. Nearly all workers on the subject agree that there are at least three well-defined species of Plasmodium causing human malaria, and there is some evidence that distinct subspecies or varieties of some of these occur. The commonest 
and most widely distributed species is Plasmodium vivax, which causes tertian malaria. Of somewhat more limited geographic range, being confined to tropical and subtropical countries, but of infinitely more importance on account of the deadly nature of its attacks, is Plasmodium falciparum, the cause of the æstivoautumnal type of malaria, also called malignant tertian or subtertian fever. During the hot part of the year in the tropics 96 per cent of malarial cases are of the æstivo-autumnal type. The third species, Plasmodium malarice, causing quartan malaria, is relatively uncommon, though more frequent in temperate than in tropical countries. These three species of malarial parasites differ from each other in a number of important details of structure and life history and in the diseases which they produce.

Life History of Plasmodium falciparum; Human Cycle. The life history of malarial parasites may well be exemplified by that of the malignant æstivo-autumnal parasite, Plasmodium falciparum, as diagrammatically shown on Fig. 43 . When first injected into the human blood by a mosquito the animal is exceedingly minute (Fig. 43A). It immediately enters or attaches itself to a red blood corpuscle, where it grows until it occupies one-half or two-thirds of the corpuscle, meanwhile undergoing a number of different forms. It first goes through a "signet ring" stage (Fig. 43B), the ringlike appearance being due to the presence of a transparent area occupying the middle of the parasite, while the tiny round nucleus occupies a position at one side of the parasite, simulating the setting in a ring. As the parasite grows larger it becomes irregular in shape (Fig. 43C) and quite active, constantly changing its form, thrusting out little clublike processes or pseudopodia, now here and now there. Although it has been taken for granted that malarial parasites penetrate the blood corpuscles and live inside of them recent investigations by Mary $\mathrm{R}$. Lawson (Mrs. Johnson) indicate that this may not be the case at all, but that the parasites may attach themselves to the surface of the corpuscles, squeezing up little mounds of the substance of the corpuscles and encircling these mounds with their bodies, just as a bit of skin might be squeezed up between the fingers. Sometimes several parasites attach themselves on top of each other around a single mound. A number of facts give support to Mrs. Johnson's theory: it affords a logical explanation for the ring forms of the parasite; it 
LIFE HISTORY

151

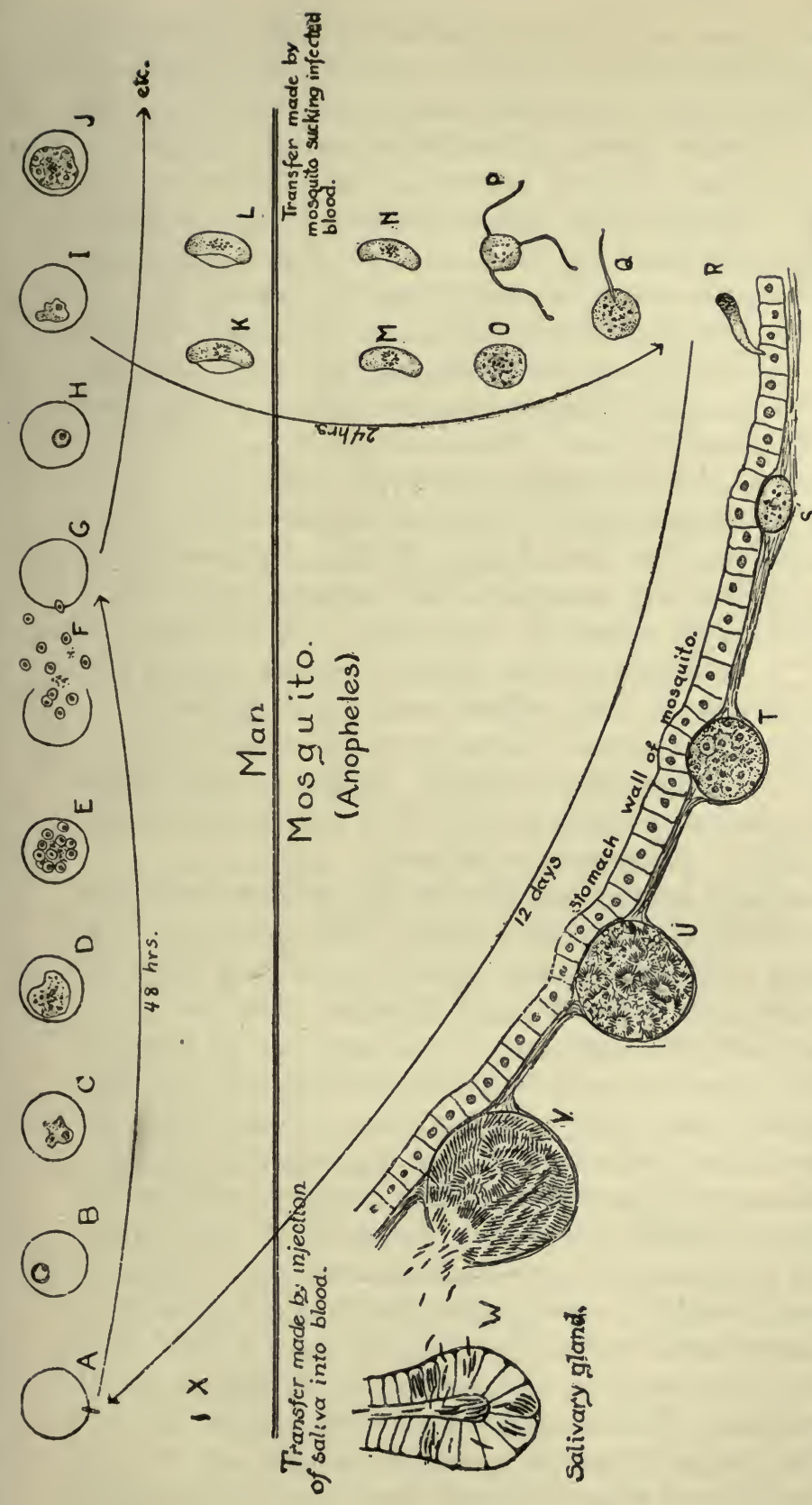

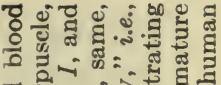

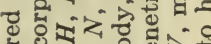

on 0.08.

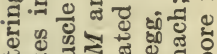

द्वे क्ष

०

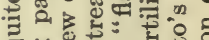

on

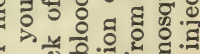

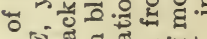

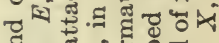

ज़

ᄀ 3 . R 2

का कू

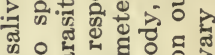
운

ह $\rightarrow 200$

ฐँ

o

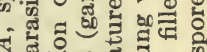

$\checkmark$ का

牙解

है

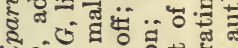

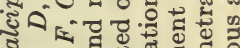

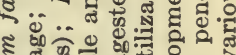

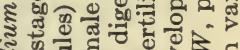
के है 융 \$에 을

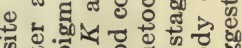

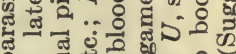

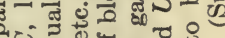

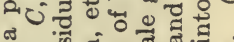

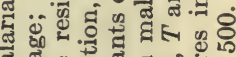

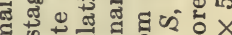

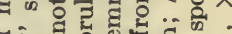
पै

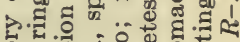
政 구의.

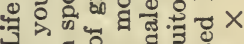
\%

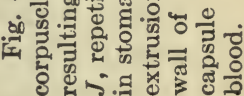


explains the occasional distinct projection of the parasites at the periphery or edge of the corpuscles (Fig. 44); and it accounts for the ease with which the parasites may be distorted in making
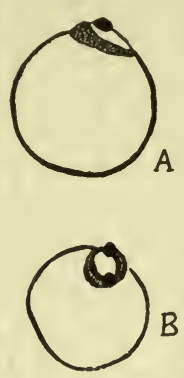

Fig. 44. Blood corpuscles showing malaria parasites at periphery. $B$ shows two parasites resting one above the other. (Sketches from microphotographs by Mary Lawson [Mrs. Johnson].) blood smears. Another argument in favor of this theory as opposed to the intracorpuscular theory is that the hæmoglobin in the corpuscles is believed to be in a more or less solid state, and would therefore make it difficult for the parasites, if situated inside, to indulge in such active movements as they do. The majority of protozoölogists, however, have not accepted Mrs. Johnson's conclusions.

As the parasite develops there is a distinct tendency for the affected corpuscles to clump together, thus clogging the tiny capillaries which are large enough to allow the passage of only a single corpuscle at a time. In this way the capillaries of such organs as brain, spleen, bone marrow and others may be obstructed to a fatal degree. Three-fourths of the life cycle of the parasites is usually passed in the plugged capillaries so that only during one-fourth of their cycle can they be found readily in the circulating blood.

After about forty hours the nucleus of the parasite divides into a variable number of fragments, usually from ten or 15 to as many as 32 , i.e., under favorable conditions it may split five times, into two, four, eight, 16, and 32 parts. The rest of the body divides itself into portions, one surrounding each fragment of the nucleus, thus forming a little heap of "spores " (Fig. 43E) ready to burst apart and leave the corpuscle on which the parent parasite had been feeding. In the center of the heap can be found a little mass of coal-black pigment granules, the waste products resulting from the digestion of the oxygencarrying red substance of the blood, hæmoglobin. When the parent parasite bursts the young parasites formed by this rapid process of multiplication are set free (Fig. 43F) in the blood where each enters a new corpuscle and repeats the process of growth and reproduction. The pigment and other waste products which are left behind when the parasite multiplies are released into the blood stream where they are carried to all parts of the 
body and deposited in the spleen or other organs or under the skin, causing the sallow color so characteristic of malarial patients. It is at the time of the bursting of the corpuscles and release of the waste matters which act as poisons that the characteristic chills and fever of malaria are felt. Since the cycle from one generation to the next is usually about 48 hours in the æstivoautumnal parasite the attacks of ague are felt at these intervals. In this malignant type of malaria the bursting of all the parasitized corpuscles and release of poisonous waste matter does not occur so nearly simultaneously as it does in the other species, the result being that the paroxysms of chill and fever are drawn out over many hours.

A "quotidian" type of malignant malarial fever in which agues occur every 24 hours is occasionally met with, the parasites of which are thought by some authors to constitute one, or even two, distinct species. The majority of cases of malaria with daily-recurring fevers are due to double or triple infections, the different broods maturing on different days.

This rapid process of multiplication in the human blood results in a short time in an enormous number of parasites, sometimes many billions. The actual quantity of parasites in a human body in a case of severe æstivo-autumnal malaria has been estimated at 600 cc., or over one pint. It may or may not mean more to the reader to know that such a quantity of malarial parasites would number $3,000,000,000,000$. A better conception of the real meaning of such a number may perhaps be gained when it is realized that to count off this number at the rate of 100 per minute day and night without cessation would require 30 times the period of time that has elapsed since the birth of Christ. Eventually, however, either the parasite kills its host, which very commonly happens with this particular species, or the host, by the development of a temporary immunity in his body, kills or, as it more often happens, suppresses the parasite. Such a course of events unaltered, would lead to a very early and complete extermination of the parasite. There is a second chapter in the life history of Plasmodium which saves it from such an early death.

After the parasites have been developing in the blood for about two weeks or more there are developed special sexual forms or gametocytes, male and female, in the form of sausage-shaped 
crescents (Fig. 43K and L). Just as in the case of other kinds of animals and plants, nature has adapted these animals to cope with their environment. As long as the blood of their host forms a suitable environment they continue to multiply in the normal manner, but when conditions due to the formation of antibodies become unfavorable they produce these sexual crescents in large numbers and patiently await rescue at the hands, or rather the beak, of a mosquito. The crescents may persist in the blood for several weeks, gradually disappearing after all other symptoms of infection have vanished. Only slight differences can be seen between the male and female gametocytes, the female being more granular in appearance, and with the pigment particles arranged in a more regular triangular manner (Fig. $43 \mathrm{~K}$ and L).

Mosquito Cycle. - When sucked into the digestive tract of the mosquito these gametocytes begin a complex developmental cycle, providing conditions of temperature are favorable. The most favorable temperatures are between $75^{\circ}$ and $85^{\circ} \mathrm{F}$. The digestive fluids dissolve the remnant of the blood corpuscles, but the crescents resist digestion (Fig. 43M and N) and become more obviously sexually differentiated. The male gametocyte develops into a " flagellated body" (Fig. 43P), a little sphere from which several long slender filaments project. These are very active, constantly lashing to and fro, and ultimately break loose and wriggle about in the stomach of the mosquito like little spermatozoa, which, in effect, they are. The female gametocyte develops into an inactive sphere or gamete (Fig. 430) and one of the filaments from the flagellated male enters to fertilize it (Fig. 43Q). How perfectly the process simulates the act of fertilization of an egg by a spermatozoan in the higher animals!

The result of the union of the filament from the flagellated body with the inactive female gamete is a body which corresponds in every way to a fertilized egg of a higher animal. This new individual, the beginning of a new generation, grows, elongates, and becomes quite like a little worm (Fig. 43R). It now wriggles and worms itself about in the stomach of the mosquito and penetrates the wall, lodging itself between the inner and outer linings of the stomach (Fig. 43S). Here more rapid growth takes place and a heavy capsule develops, protruding on the outer surface of the mosquito's stomach like a wart (Fig. 45). Meanwhile the contents of the capsule undergo important changes, 
dividing into daughter cells (Fig. 43T) from each of which slender spindle-shaped bodies project like the "stickers" on a chestnut burr (Fig. 43U). Ultimately the cells lose their identity and the entire capsule or cyst becomes crammed full to the bursting point with myriads of these spindle-shaped bodies which have now developed into spores (Fig. 43V). Such a capsule may contain over 10,000 spores, and there may be as many as 500 capsules on a single mosquito's stomach (Fig. 46). About 12 days or more, according to temperature, after the infected blood was swallowed by the mosquito, the capsule becomes mature and bursts, releasing the spores into the body cavity of the mosquito. From here the little parasites

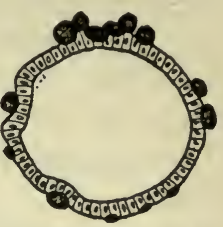

Fig. 45. Cross section of stomach of Anopheles showing capsules (oöcysts) of subtertian malaria. $X$ about 30. (After Grassi.) make their way to the three-lobed salivary gland (Fig. 46, sal. gl.) lying in the fore part of the thorax and connecting with the sucking beak. They assemble in the cells lining the salivary

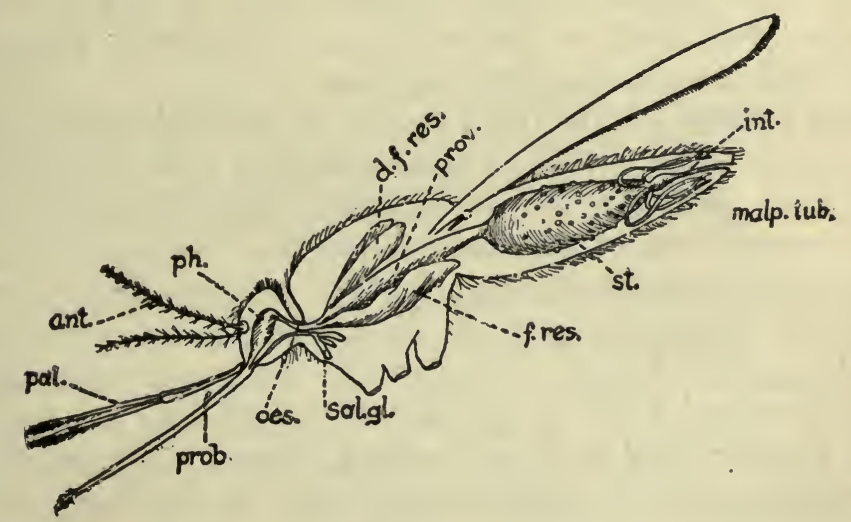

Fig. 46. View of digestive tract of Anopheles, showing spore-filled capsules of malaria parasites on wall of stomach. pal., palpi; prob., proboscis; ant., antennæ; ph., pharynx; œs., œsophagus; sal. gl., salivary glands; f. res., ventral food reservoir; d. f. res., dorsal food reservoirs; prov., proventriculus; st., stomach; malp. tub., malpighian tubules; int., intestine. $\times 10$.

glands (Fig. $43 \mathrm{~W}$ ) and remain there perhaps for weeks, until the mosquito bites. When this happens the parasites flow with the poisonous saliva into the puncture made by the mosquito and, should the prey of the mosquito be a human being, the whole 
process of asexual multiplication in the human blood corpuscles begins over again. Since it takes ten or 12 days for the sexual cycle to be completed in the case of æstivo-autumnal malaria, an infected mosquito is not dangerous for at least this length of time after biting a malarial patient. However, once the new generation of spores has been developed, the mosquito remains dangerous for several weeks and may infect many persons, as not all the parasites are poured out of the salivary glands at one biting.

It is commonly believed that malaria parasites not only do not develop but cannot live in the mosquito at winter temperatures, and conflicting results have been obtained from experimentation. King in America and Wenyon in Macedonia have succeeded in exposing infected mosquitoes to low temperatures without destroying the parasites, whereas Mayne (Mitzmain) found that in Anopheles infected prior to hibernation, healthy normal sporozoites did not last through the winter in the salivary glands. Roubaud obtained similar results in France. It is still doubtful whether the malaria parasites normally pass the winter in the mosquito hosts in places where the mean temperature falls much below $60^{\circ} \mathrm{F}$. for any considerable time.

Other Species. - The other species of malarial parasites differ only in minor details of their structure and development. The tertian parasite, Plasmodium vivax, during the early stages of its development in the blood corpuscles is extremely active. Its unceasing restless changing of shape is fascinating to watch under the microscope and one feels that it was very appropriately named "vivax." Unlike the malignant parasites of æstivo-autumnal malaria, the tertian parasites do not tend to clump together, and so do not become plugged in the capillaries but remain constantly in the circulation. To this fact, as will be shown later, is due the "benign" nature of this and also of the quartan parasite. The tertian parasites have the peculiarity of growing very large and of causing the corpuscles which they parasitize to enlarge and become unhealthy in appearance. The number of spores which result from the sporulation every 48 hours ranges from ten to 25. According to Ross the normal number of splits of the nucleus is four, which would result in 16 spores. One of the most striking points of difference from the "malignant" parasites is the fact that the gametocytes 
are not in the form of crescents, but instead resemble mature parasites ready to sporulate. A comparison of Fig. $47 \mathrm{~A}, \mathrm{~A}^{\prime}$ and $\mathrm{A}^{\prime \prime}$ with $\mathrm{B}, \mathrm{B}^{\prime}$ and $\mathrm{B}^{\prime \prime}$ and $\mathrm{C}, \mathrm{C}^{\prime}$ and $\mathrm{C}^{\prime \prime}$ brings out the principal differences among the three species of parasites as regards size at maturity $(\mathrm{A}, \mathrm{B}, \mathrm{C})$, number of spores $\left(\mathrm{A}^{\prime}, \mathrm{B}^{\prime}, \mathrm{C}^{\prime}\right)$ and form of gametocytes $\left(\mathrm{A}^{\prime \prime}, \mathrm{B}^{\prime \prime}, \mathrm{C}^{\prime \prime}\right)$.
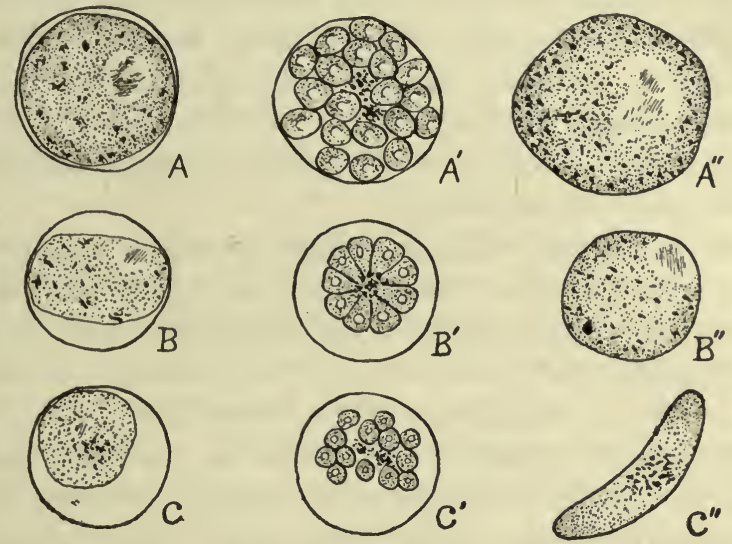

Fig. 47. Comparison of three species of malaria parasites $\times 2000$ (figures selected largely from Manson). $A, A^{\prime}$ and $A^{\prime \prime}$, Plasmodium vivax; $B, B^{\prime}$ and $B^{\prime \prime}$, Plasmodium malaria; $C, C^{\prime}$ and $C^{\prime \prime}$, Plasmodium falciparum.

$A, B$ and $C$, mature parasites in red corpuscles.

$A^{\prime}, B^{\prime}$ and $C^{\prime}$, segmented parasites ready to leave corpuscles.

$A^{\prime \prime}, B^{\prime \prime}$ and $C^{\prime}$, mature gametocytes.

The quartan parasite more closely resembles the tertian parasite in flexibility of body and form of gametocytes (Fig. 47B" ), but it differs in that it does not cause the corpuscle to enlarge (Fig. $47 \mathrm{~B}$ ) and is never active in movements. It produces only from five to ten spores, the nucleus normally undergoing three splits. The spores form a very regular rosette or "daisy-head," arranging themselves petal-like around the dark mass of pigment in the center (Fig. 47 $\mathrm{B}^{\prime}$ ). Unlike either of the other parasites this one causes ague by its sporulation once in 72 hours instead of in 48 hours. A comparison of certain phases of this parasite with the same phases of the others will be found in Fig. 47.

Propagation. - As remarked above infection with malaria is now known to take place exclusively through the bites of certain species of mosquitoes, all belonging to the genus Anopheles (including its subgenera). While over a hundred species of Anoph- 
eles have been described, less than one-third have been proved to be carriers of malaria. Some species will carry certain types of malaria and not others (see p. 439). A knowledge of the malaria-transmitting ability of various species of mosquitoes and their habits is of the utmost importance in any attempt to exterminate malaria by exterminating mosquitoes. The knowledge that $A$. malefactor of Panama, breeding in cavities of stumps and trees, was not a malaria carrier saved several hundred thousand dollars in the anti-malarial campaigns in the Canal Zone. The distinguishing characteristics of Anopheles and a brief account of a few of the more important malaria-carrying species will be found on pp. 439-441.

Reports of malarial outbreaks have occurred which were said to be due to some other cause than mosquito transmission, but when completely investigated there has always been found to be a "leak" somewhere. Sometimes the presence of mosquitoes was unsuspected, sometimes other fevers have been mistaken for malaria, and sometimes the malarial parasites have been harbored for weeks or months in "latent" form. This is a phase of malaria which is little understood, but it is a well-known fact that long after symptoms of the disease have disappeared, and the parasites can no longer be found in the blood, a fresh outbreak may occur, coincident with some loss of vitality, or some physiological shock on the part of the host from some other cause. Often a mere change of climate and environment is sufficient to precipitate "latent" malaria. It is highly probable that the ordinary blood parasites are carried in the meantime in such small numbers as to be practically impossible to find. Ross has pointed out that if 1000 parasites in the body were able to withstand the unfavorable conditions and existed there during the "latent" stages, a man working 12 hours a day searching blood smears would have a chance of finding one only once in five years. Some authors have advanced the theory that the gametocytes, suddenly stimulated by some unknown cause, develop by parthenogenesis, i.e., without the ordinary sexual mosquito cycle, and thus cause the relapse. This idea has been widely accepted but there seems to be little ground for it and some positive evidence against it. The parasites naturally thrive best when their host is weakened by some other influence which then acts as an accomplice for them. Such influences are ex- 
posure to sudden changes in climate, fatigue, dissipation and other sickness. Even educated people often come to believe that malaria is directly caused by these conditions.

Suffice it to say that many experiments, carried out with the utmost care and accuracy, and checked by numerous repetitions, have proved beyond doubt that the mosquito is the necessary transmitter and intermediate host of malarial parasites. A few investigators think it possible that other animals besides man may serve as hosts for the malarial parasites, so that malaria may occur even in uninhabited regions. Although many parasites are able to live in a number of different kinds of animals, this does not seem to be true with the malarial parasites, and all attempts to infect even monkeys have so far failed. Until some definite proof of the rôle of some other animal as a host for human malarial parasites has been brought forward we may look upon this as very improbable. Possibly the alleged presence of malaria in uninhabited regions may be explained by the malarial parasites in the mosquito passing into the eggs of the mosquito, and thus being carried on generation after generation. Though the germs of some diseases are known to do this in their insect hosts, experiments with hereditary transmission of malarial parasites in mosquitoes have so far been unsuccessful.

The Disease. - Malaria as a disease is extremely variable. A "typical" case of malaria, in the tropics at least, is a rather unusual thing. As we have seen, there are at least three different kinds of malarial parasites, each of which produces a somewhat different disease. While ordinarily all the parasites of a brood mature at regular intervals, a person in a malarial district may be infected with two or more broods maturing at different times, and the case may be farther complicated by a "mixed" infection, that is, by more than one species of malaria at a time. Varying degrees of immunity, the effects of insufficient quinine or other drugs, the presence of complicating diseases and the virulence of the particular strain of parasites all have a hand in modeling the effects produced by " malaria." It is little wonder that in some places practically every ailment or feeling of " malaise" is attributed to malaria. In the tropics such a diagnosis would be correct in a great many cases. However, the habit of attributing any indisposition which cannot be accounted for otherwise to malaria has been transplanted into non-malarial 
places, and it is not uncommon to hear of a person having a "touch of malaria" when in reality he has only indigestion, a cold or a light case of la Grippe. It is largely due to this fact that malaria is looked upon in non-malarial districts as of such small consequence.

The early stages of all types of malaria are similar except that the quartan type produces the intermittent fevers on every third, instead of every second, day. During the incubation period of the disease there is a feeling of ennui with headache and perhaps slight fever. After about a week, when the parasites have multiplied to $150,000,000$ or more, the regular intermittent fevers set in. Each attack begins with a shivering chill, sometimes accompanied by convulsions, so severe that the teeth chatter and goose-flesh stands out all over the body. Yet the temperature will be found to be several degrees above normal, and still going up. In the wake of the chill comes a burning and weakening fever, with violent headache and vomiting and a temperature from six to eight degrees above normal. The fever stage in turn is followed by a period of sweating, so profuse that the clothes or bedding may become wringing wet. The sweating gradually subsides, the temperature drops rapidly, often below normal, and the patient, after from six to ten hours in the case of benign infections and about 20 hours in malignant infections, rests fairly easy until the next attack. The fact that the attacks most commonly occur between midnight and noon, instead of in the evening, is often useful in distinguishing malaria from other intermittent fevers.

In the case of "benign" (tertian and quartan) infections after these agues have recurred for about ten days or two weeks, the symptoms gradually subside and the patient experiences a rally. From this point either he may recover completely (even if untreated) or he may suffer a relapse with all the old symptoms of regular agues. Then comes another rally and a second relapse, this continuing for months or years, aided, perhaps, by constant reinfections. During all this time general symptoms of emaciation, sallowness, anemia and enlarged spleen constantly increase at a diminishing rate with each elapse, and decrease at a similarly diminishing rate with each rally, so that eventually a fairly constant state of spleen-enlargement, emaciation, anemia, sallowness and general run-down condition is arrived at - the 
well-known condition of chronic malaria, or malarial cachexia, common especially in children. The spleen enlargement is the most readily recognizable symptom of chronic malaria and therefore the "spleen rate," i.e., the percentage of enlarged spleens in a community, gives a fairly accurate measure of the prevalence of malaria to which some degree of immunity has been developed. Usually, unless the weakened condition has given some other disease a chance to put an end to it all, a general improvement ultimately begins. This is especially true in children, so that by the time they reach adult life they are in fairly good health and immune to malaria.

In the case of æstivo-autumnal or malignant malaria the course of the disease is often not so light, and early death is not a rare occurrence. The fact that the bodies of the malignant parasites clump together and plug the capillaries, thus preventing the proper flow of blood in the vital organs, is probably the chief cause of their malignant nature. One of the most certain symptoms of a malignant attack of malaria is a total loss of consciousness or coma, due to a plugging of the capillaries in the brain. Indeed, 50 per cent of the deaths from malaria are said to be caused by a plugging of the brain capillaries. The type of brain disease which may be caused is very variable but some mental disturbance almost always occurs, and may take place at almost any time during the course of the disease, though it never occurs during the first fever fit, probably because the parasites are not yet numerous enough to do any great damage.

In connection with malarial fevers there must be mentioned a much dreaded and little understood condition known as "blackwater fever." This is a disease in which something destroys the red blood corpuscles in large numbers, causing the coloring matter of the blood, hæmoglobin, to be liberated, eventually to be voided with the urine, giving the latter a very dark color. At the same time there is a more or less irregular fever, bilious vomiting and severe aches. In a great many cases it results in death. This disease has usually been considered as an outcome of severe malaria, since it always occurs in malarial countries and usually follows or accompanies an attack of malaria. It is not uncommon in southeastern United States, some parts of tropical Africa, southern Europe and many parts of tropical Asia and the East Indies. In many other malarial districts it 
is entirely absent. It is suggested by Manson that the fever is caused by a distinct organism, and that malaria is merely a predisposing cause.

Immunity and Epidemics. - Absolute immunity to malaria is rarely if ever acquired but, as already remarked, oft-repeated infections especially in childhood tend to build up a high degree of tolerance to the effects of the parasites and a diminution in the number of parasites in the body. The protection afforded by a single infection is very slight, and is retained for only a short time in the absence of reinfections. Even the cumulative effect of numerous infections disappears rapidly in the course of a few years. Some authors divide malaria into two types. There is a "tropical" form, occurring in places where reinfections can occur practically throughout the year on account of the continued warm temperature. The other, a "subtropical" form, is found in regions where cold weather causes an annual seasonal interruption of infection by a cessation of breeding on the part of Anopheles, and by a discontinuance of growth on the part of the parasites in the mosquitoes. In tropical malaria a fairly constant degree of immunity is maintained, and epidemics are rare if they occur at all. In Java and other tropical places, according to Robert Koch, nearly every native child, under four years of age, has his blood teeming with malaria parasites from which he suffers little inconvenience. These parasites gradually become scarcer in older children and are often practically absent in adults who, however, have been shown to be passive "carriers" of small numbers of the parasites and therefore a source of danger to the community. The "carriers," though relatively immune to the more acute symptoms of the disease, are left in the run-down condition of malarial cachexia. As pointed out by Gill, there is a striking analogy between the confirmed opium-eater and the malarial cachectic. Both have purchased their immunity at a heavy price. In the former the emaciated frame, sallow complexion and other signs of debility proclaim the victim of a drug habit; in the latter the enlarged spleen, the lack of physical and mental energy, and the shrunken body bear witness to the havoc wrought by long-standing malaria. In the case of neither does death often take place as the direct effect of their respective poisons, but both readily fall victims to intercurrent affections. In subtropical malaria, on 
the other hand, the average tolerance of the community to the disease suffers an annual relapse, and may constantly decrease for a number of years. When the immunity of the community as a whole becomes quite low, and there is a sudden increase in the probability of infection by a great increase in number of mosquitoes, accompanied possibly by an influx of infected people, an epidemic of the disease may occur of such extraordinary severity as to involve almost the entire population, and to cause a mortality of several hundreds per thousand. Such devastating epidemics, probably of the subtertian type of malaria, have been termed "fulminant malaria" and are believed to occur quite extensively in malarial countries lying just outside the region of "tropical" malaria. Fulminant malaria in especially severe form occurs periodically in parts of India and in Italy.

It was formerly thought that considerable racial immunity protected the negro races, but it has been shown that in many cases, at least, the immunity has been acquired by constant exposure to the disease, and that it disappears upon removal from infected regions. The whites in southern United States are said to suffer markedly more from malaria than do the negroes though the latter are more frequently parasitized, but this may be due, in part at least, to the more permanent residence of the latter in the malarial districts. As said before, individual resistance to the effects of the disease is variable. Occasionally there is found a fortunate individual who is naturally absolutely immune, but this is a very rare occurrence.

Treatment. - It is one of the greatest blessings in the world that we have for malaria a definite and specific cure as near to being a "sure cure" as has been discovered for any disease. Quinine has been found absolutely destructive to malarial parasites. While a dose of quinine given during a fever attack will not act quickly enought to cut it short, it will, if given immediately after an attack, prevent the next one, or at least alleviate it. Meanwhile the organisms disappear from the circulation. It is usually supposed that they are directly killed by the quinine, which acts as a virulent poison for them, though this is doubted by some workers. The methods of administering quinine must, of course, vary with the age and condition of the patient, and the state of the disease. Sometimes very speedy action is needed, and it is not safe to wait for quinine to be slowly absorbed from 
the stomach. Many a patient has died from malaria with enough quinine in his stomach to have saved his life had it been properly given. In such cases injections into the muscles, or still better, directly into the veins, is necessary. In malignant malaria quinine does not reach the parasites plugged in the capillaries and therefore can destroy them only as they sporulate and get back into the circulation. Since the parasites of this type often sporulate at irregular intervals a constant supply of quinine at a killing concentration must be kept in the blood. However, overdosing with quinine is not an uncommon fault with physicians. Quinine poisoning in some respects resembles malarial symptoms and the physician, thinking the latter are not abating, gives still more quinine until the patient succumbs to it. Not a few malarial deaths are really due to excessive quinine. Malarial specialists, such as Professor Bass of New Orleans, say that it is never necessary to give more than ten or possibly fifteen grains of quinine at a time, if given as the case requires it. Twenty grains of quinine sulphate a day taken by mouth in several doses for a period of two weeks is said by Bass to disinfect anyone. Quinine must be avoided during or immediately following an attack of blackwater fever, since the symptoms of this malady are intensified by its use.

In case of severe malarial cachexia, the only safe course is for the patient to leave the malaria-infected country in which he has been living, and stay away for an extended period of time. He should take regularly small doses of quinine to kill any lurking parasites which may remain in his body, and do everything possible to build up his general health and to regain his lost vitality.

Prevention. - The prevention of malaria is a problem that should be solved not by individuals but by civic effort. Ross says: "It (malaria) is essentially a political disease - one which affects the welfare of whole countries; and the prevention of it should therefore be an important branch of public administration. For the state as for the individual health is the first postulate of prosperity. And prosperity should be the first object of scientific government."

Since the malarial parasites have two hosts, man and mosquito, the possibility of exterminating them in either host presents itself. Stephensport, in New Guinea, was practically cleared of malaria 
in a few months by destroying the parasites in man by wholesale "quininization." In most places, however, the difficulties connected with this method of extermination are even greater than those associated with its alternative, the destruction of malarial mosquitoes. The relation of partially or entirely immune "carriers" to the spread of malaria is of extreme importance and is usually greatly underestimated. The number of such apparently healthy carriers in malarial districts is astonishingly large. Eradication of malaria by attacking it in man would entail the persistent and thorough quinine treatment of all these carriers as well as of patients.

Undoubtedly in practically every case, if accompanied by as extensive a use of quinine as is possible, eradication of malarial mosquitoes is the most effective and most permanent preventive measure. A discussion of methods of reducing and controlling such mosquitoes will be found on pages 455-462.

Complete extermination of malarial mosquitoes is not necessary to reduce or even to eradicate malaria entirely. Ross has shown by mathematical computation that a relatively high number of malarial mosquitoes per person is necessary in a community to propagate malaria successfully. A small deviation above or below a certain number of malarial mosquitoes, probably between 40 and 60 per person during a month, a deviation too small to be detected readily, will mean the difference between an ultimate extermination of the disease and its permanent establishment. Ross also shows that the relation between the amount of malaria in a given region and the number of malarial mosquitoes is so definite that it can be mathematically computed. These facts are of importance in the fight against malaria since they demonstrate to us that we do not have to exterminate totally even the malaria-carrying species of Anopheles in order to exterminate malaria, and our task becomes much less difficult. By this partial extermination some of the most malarial districts in the world have been practically freed. Up to 1900 over 16,000 deaths a year from malaria occurred in Italy; now they may be counted in hundreds. One of the first demonstrations of what could be accomplished by mosquito extermination was made by Major Ross in 1902 at Ismailia on the Suez Canal where from 1100 to 2500 cases of malaria occurred annually in a population of less than 10,000. Four years later not a single new case 
occurred there. The same thing on a much larger scale was accomplished in the Canal Zone at Panama by Surgeon-General Gorgas and his staff. On this relatively large malaria-infested area the death rate for the total population of about 100,000 was reduced 64 per cent in four years. The deaths from malaria were reduced about 85 per cent in less than four years, and yellow fever was totally eradicated. Similar feats have been accomplished at Havana, Staten Island, and other places. One of the most recent examples of what can be done was furnished by the American occupation of Vera Cruz in 1914. The American troops were severely attacked by malaria of all three types, and an antimosquito campaign was immediately inaugurated. It cost the Sanitary Department $\$ 5000$ a month to oil the pools, drain the low parts of the city and its environs, and dispose of the standing water in street gutters, refuse heaps, etc., but in a few months Vera Cruz, one of the most deadly malarial districts in the world, was practically freed from Anopheles, and danger of malaria reduced to almost nothing.

Obviously the wholesale reduction or extermination of malarial mosquitoes can be accomplished only by communities or by government aid. San Antonio has freed itself of mosquitoes and mosquito-borne diseases by enlisting the services of the school children. In our southern states, where there are $65,000,000$ acres of swamp land, and where the chief malarial mosquitoes are swamp breeders, malaria can never be destroyed until state and federal governments are willing to invest money as readily to take water off the land in these parts of the country as they now invest it to put water on the land in the arid western parts.

Much can be done toward reduction of malaria in selecting dry brushless sites for houses and in constructing them in mosquito-proof fashion. The houses one sees in the American Government settlements on the Canal Zone, built well up off the ground and with open sleeping porches, wide verandas and airy windows, all carefully screened, are ideal for tropical districts where malaria and other insect-borne diseases are common. They present a happy combination of airiness, sanitation, and complete protection from insect pests.

In well-known malarial districts it is a good personal safeguard to use screens as much as possible and to take regular doses of 
quinine at all times as a preventive measure. In the pine swamps and along the coasts of Florida malaria is practically absent on account of the effectiveness of screening necessitated by the abundance of non-malarial mosquitoes. Three to five grains of quinine daily, or ten to fifteen grains once a week, is an almost certain malaria preventive. Quinine, however, is apt to cause abortion in pregnant women, though less so than is a severe attack of malaria. Some people are naturally very susceptible to quinine and cannot take it; such people should carefully avoid malarial districts. Tea, coffee and other mild stimulants are also said to be beneficial, but the safest course is always the same - quinine. 


\section{CHAPTER $\mathrm{X}$}

\section{OTHER SPOROZOA, AND OBSCURE OR INVISIBLE PARASITES}

Although the class Sporozoa includes a very large number of species, all of which are parasitic, and many of them the cause of fatal diseases in vertebrate as well as invertebrate animals, yet very few other than the malaria parasites, already discussed, are normally parasitic in man, and none of these can be looked upon as of prime importance in the causation of human disease. Of greatest importance, perhaps, are the Coccidiida or coccidians, which in lower animals are frequently the cause of fatal diseases and have been known to be fatal to man, though in some cases causing very little inconvenience. Another sporozoan parasite which is of importance where it occurs is Rhinosporidium, which produces tumors in the nose. A group of muscle-dwelling Sporozoa, the Sarcosporidia, occur accidentally or sporadically in man.

There is another group of Sporozoa, the Piroplasmata, related to the malaria parasites, which are the cause of some of the most fatal diseases of domestic animals, including Texas fever and East Coast fever of cattle, biliary fever of horses, etc. These diseases are invariably, as far as known, transmitted by ticks. There is one human parasite, Bartonella bacilliformis, the cause of Oroya fever of Peru, which is thought to belong to this group of organisms. Possibly the Rickettsia-like organisms of typhus, trench fever, Rocky Mountain spotted fever, and the related Japanese disease, kedani, are related to the Piroplasmata.

There are a number of other diseases, some of them of great importance, of which the "germ " either has never been seen or is of obscure nature. It is not always possible to guess at the nature of such undiscovered parasites but in some cases we can get a fairly accurate conception of them from a study of the course of the diseases they cause, the conditions under which they thrive and their means of dissemination. One by one the villains be- 
hind the screens are brought to light, experimented with, and brought under control but there are still some which have defied the most ardent researches of modern science and have never yet been discovered. The fact that many of them are able to pass through filters of certain kinds, as shown by the infectiveness of fluids containing them after having been passed through the filters, demonstrates that at least in some stages of their development they are actually too small to be visible under the highest power of the microscope.

However, in the case of some of these unseen parasites we have sufficient knowledge of their habits and life histories to wage a fairly intelligent war against them, at least as regards prevention. The parasite of yellow fever, for instance, was not discovered until 1918. Yet we knew much about its probable nature, we knew its full life history in a general way, and to a large extent we knew how to combat it, far better, in fact, than we know how to combat some of the well-known parasites. There are two other diseases, dengue and phlebotomus fever, which are probably caused by parasites related to that of yellow fever, but they have not yet been discovered.

The organisms of typhus fever, trench fever, spotted fever and kedani have only recently been discovered. They are all minute bodies showing various morphological types, which occur in both vertebrate and arthropod hosts. They are here grouped together as "Rickettsia-like organisms." Their affinities, whether with bacteria or protozoa, are at present entirely problematical, but for the present they may best be considered, like the spirochætes, to represent a more or less intermediate group.

Several other diseases, some of them of prime importance, of which the parasites are of obscure nature, are believed by some workers to be caused by Protozoa: such are hydrophobia or rabies, trachoma, smallpox, verruga peruviana (not Oroya fever), foot-and-mouth disease, measles, scarlet fever and a few others. The parasites or parasite-like bodies which are associated with these diseases are in some cases minute, in other cases, e.g., hydrophobia, of relatively large size. In most of these diseases the "germ" or virus is capable of passing through ordinary bacterial filters, as shown by the infectiveness of filtered material. It is also evident from this that the viruses live out- 
side the cells or blood corpuscles, at least during part of their life history. On the other hand, in these diseases there have been discovered bodies of various kinds within the cells, interpreted by some workers as true parasites, by others as reaction products of the cells. These bodies have received zoölogical names, e.g., the Negri bodies of hydrophobia were named Neuroryctes hydrophobice, the cell inclusions in smallpox Cytoryctes variolce, and so on. It is now a commoner belief that these bodies consist of material extruded from the nucleus of the cell into its cytoplasm where it surrounds one or many of the minute organisms during the intracellular portion of their life history.

For these problematical organisms, minute in size, of uncertain life history, and apparently enshrouded in a mantle of extruded nuclear material during their intracellular life, the name Chlamydozoa (meaning mantle animals) has been given. Whether these bodies have been correctly interpreted as described above and whether they should be considered Protozoa is open to question. Their anmal nature has not been sufficiently demonstrated to warrant more than brief mention of them and the diseases they cause in a treatise on animal parasites.

In the following paragraphs the sporozoan parasites and obscure or invisible parasites which have been briefly mentioned above will be discussed in a little more detail in the following order: (1) coccidians, (2) Rhinosporidium, (3) Sarcosporidia, (4) oroya fever, (5) dengue and phlebotomus fever, (6) Rickettsialike organisms, (7) Chlamydozoa.

\section{Coccidians}

There are a number of serious diseases of animals which are caused by parasites of the class Sporozoa known as coccidians. These are very small animals, without distinct organs of locomotion, which have both an asexual and a sexual phase in their life history (Fig. 48). The asexual phase is not unlike what takes place in the asexual phase of malaria parasites, except that the parasites live inside of cells lining the intestine instead of in the blood. Like the malaria parasites, a coccidian, within the epithelial cell in which it is living (Fig. 48A-C), divides into two, four, eight, sixteen, or perhaps twenty or more daughter cells, arranged somewhat like the segments of an orange (Fig. 48D). The young coccidians, escaping from the 
host cell which has been preyed upon and destroyed, invade fresh cells, multiply again, and thus eventually destroy large portions of the lining of the digestive tract. The daughter coccidians are not adapted for withstanding conditions outside the intestine

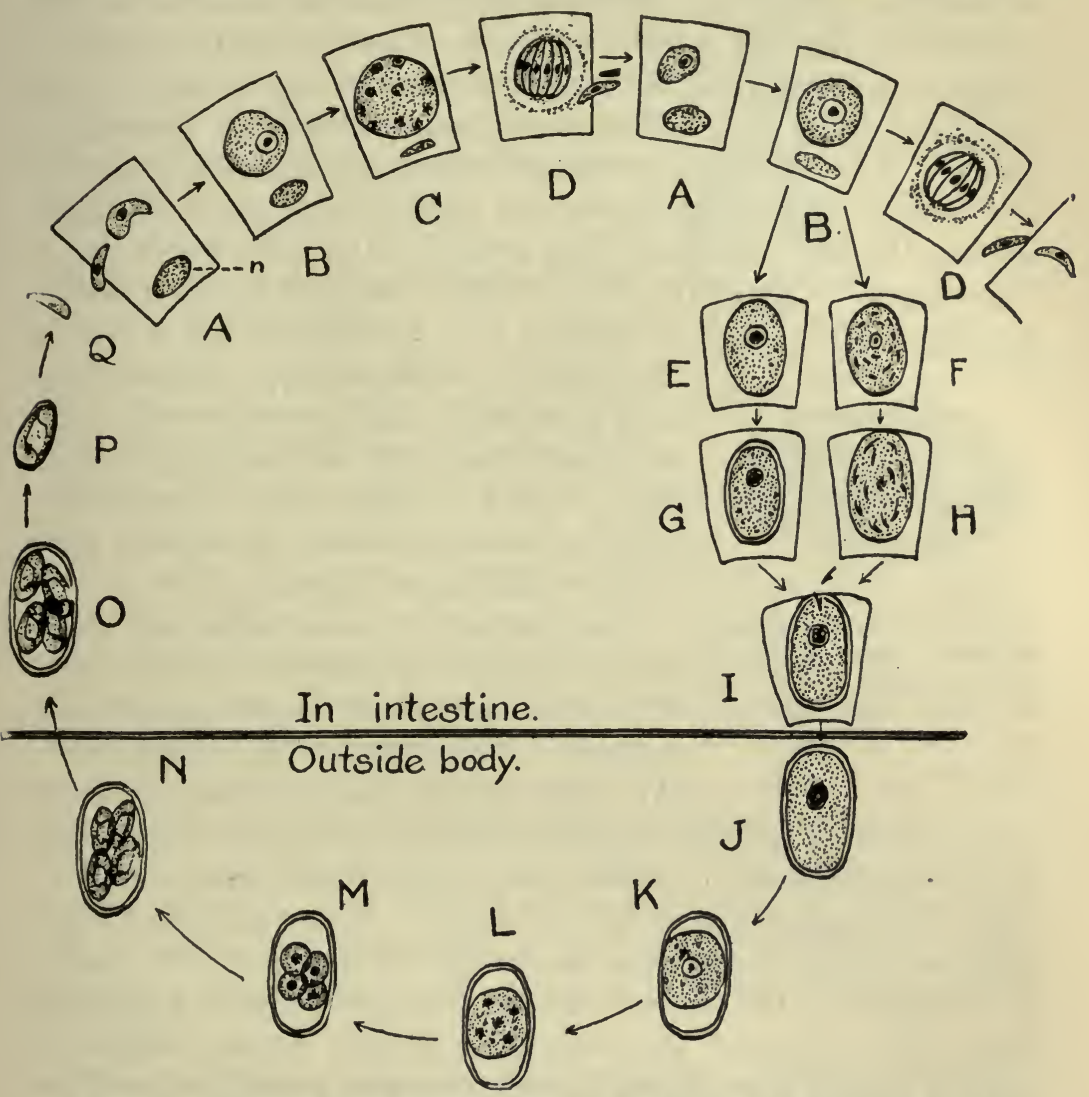

FIg. 48. Life history of Eimeria avium. A, infection of epithelial cells of intestine by sporozoites ingested with food or water; $B$, growth inside cell; $C$ and $D$, sporulation and formation of young spores; $E$ and $G$, formation of female gamete; $F^{\prime}$ and $H$, formation of male gametes; $I$, fertilization; $J$, fully developed oöcyst as passed out with fæces; $K, L$ and $M$, formation of four sporocysts; $N$, complete development of sporocysts, each containing two sporozoites; $O$, same, ingested by susceptible animal; $P$, sporocyst liberated from oöcyst in alimentary canal; $Q$, liberated sporozoite ready to infect epithelial cell, as shown in $A$.

of the host, and therefore the parasite would be exterminated with the death of its host were it not protected in some manner against this calamity. The sexual phase of its life history serves 
this important purpose. At intervals the coccidians, instead of multiplying in the usual manner, differentiate into sexual forms, corresponding to eggs and sperms (Fig. 48G and H). One of the spermlike individuals penetrates an egglike individual and fuses with it (Fig. 48I), in precisely the same manner as a spermatozoön fertilizes an egg in higher animals. The fertilized individual develops a thick resistant cyst wall and is then known as an "oöcyst" (Fig. 48J)! The parasite is now ready to hazard the dangers of an exit into the outside world, and is passed out with the fæces. Eventually, sometimes within a few days, the contents of the oöcyst divide into several parts, each known as a "sporocyst" (Fig. 48K, L and M). Each sporocyst in turn develops within itself a number of "sporozoites" (Fig. 48N), each capable of infecting a separate cell

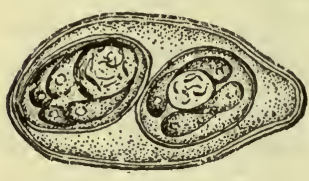

FIG. 49. Oöcyst of Isospora from British soldier returned from Gallipoli. Note presence of only two sporocysts, each with four sporozoites. $\times 1000$. (After Wenyon.) in a new host. The oöcysts with their contained sporocysts and sporozoites can exist in soil or dust for a long time, awaiting an opportunity to enter a new victim with food or water.

Infection with coccidians has not often been observed in man but it is possibly more prevalent than is commonly thought. A few cases of coccidian infection of the human liver have been recorded and it has been generally assumed that the parasite was identical with Eimeria stiedce of rabbits, but Dobell has pointed out that there is no sound reason for this belief. Recently Wenyon has reported the not uncommon occurrence of oöcysts of two species of coccidians in the fæces of British soldiers returning from Gallipoli. The cysts of the commoner species, Isospora hominis, contain a single mass of protoplasm when first passed, but in three or four days they become fully developed and contain two sporocysts, each with four sporozoites (Fig. 49). The cysts measure $12 \mu$ to $14 \mu$ by $7 \mu$ to $9 \mu$ (about $\frac{1}{2000}$ by $\frac{1}{3000}$ of an inch). The cysts of the other species, Eimeria wenyoni, are fully developed when passed, and possess four sporocysts with two sporozoites each (Fig. 50) They measure $20 \mu$ in diameter. Only four cases of infection are known. A single case each of infection with two other species have been recorded: $E$. oxyspora, with large spherical cysts (36 $\mu$ in diameter) and straight sharp-pointed spores, recorded by Dobell; and E. sniideri, with still larger 
cysts but smaller spores, by Snijder in Sumatra. Little is known of the symptoms produced by these parasites, but since they live inside epithelial cells of the intestine or liver they must be injurious. Wenyon has recently reported dysenteric symptoms in a case in which no intestinal parasites except Isospora were present. Coccidians are undoubtedly spread by means of water or food polluted by mud and dirt, by unsanitary habits, and by flies.

\section{Rhinosporidium, a Parasite of the Nose}

In natives of India there is occasionally observed a peculiar infection of the

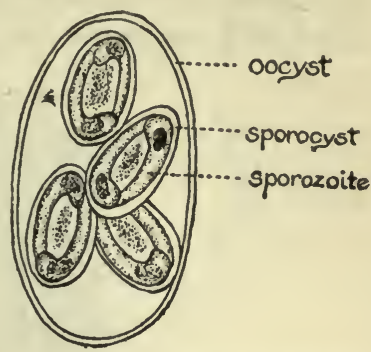

FIG. 50. Oöcyst of Eimeria containing four sporocysts, each with two sporozoites. nose in which a red tumor, flecked with whitish spots, and likened by some authors to a raspberry, grows out from the partition or septum of the nose, remaining attached by a narrow stalk. The tumors are not very painful, but they tend to block the nasal passages. It has been suggested that this disease, known as nasal polypus, may have the same influence on the intellect of children that o her impediments of the nose and throat are known to have.

When the tumor is cut the white spots visible on the surface are seen to be scattered throughout the tissue and to be of very variable size. Microscopic examination shows them to be the cysts of a protozoan parasite in various stages of development. The parasite has been named Rhinospo idium kinealyi, and is classified as a member of the group of Sporozoa known as Haplosporidia.

The cysts in the tumor are filled with great numbers of spherical or oval bodies, the pansporoblasts, each of these in turn containing from one to a dozen closely-packed spores (see small portion of a cyst in Fig. 51). The manner of development of the cysts and of the tumor can readily be discovered from the various stages of development of different cysts and parts of cysts which can be observed in a single tumor. The youngest cysts are small granular masses of protoplasm, more or less irregular in shape. As one of these minute animals grows there are developed within it small bodies with definite shape which are destined to become the pansporoblasts already mentioned. However, the proto- 
plasm at the periphery of the animal continues to grow, constantly becoming differentiated into new pansporoblasts. The young pansporoblasts (Fig. 51, yg. pansp.), at first simple masses of protoplasm, soon form within themselves one, two, four, and ultimately as many as 12 spores, tightly clumped together so

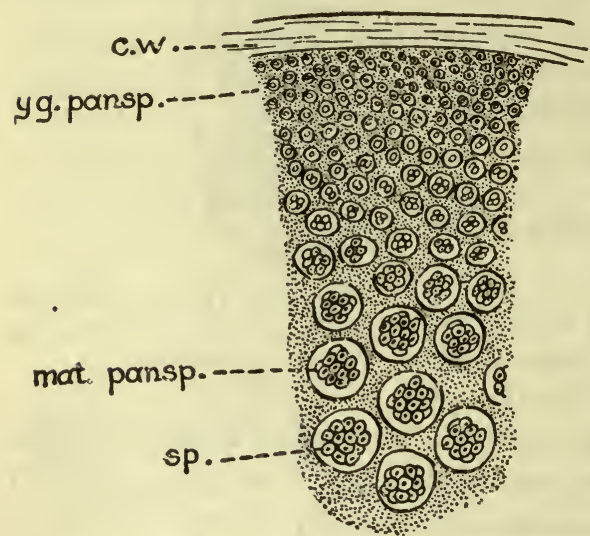

Fig. 51. Portion of fully developed cyst of Rhinosporidium; c. w., cyst wall; yg. pansp., young pansporoblasts; mat. pansp., fully developed pansporoblasts containing spores, sp. $X$ about 100. (After Fantham and Porter.) as to resemble little mulberries (Fig. 51, mat. pansp.). From the mode of development of the cysts it is clear that the older pansporoblasts are the ones near the center of the cyst, the younger ones those toward the periphery. When the cysts have reached a certain size the growth of the periphery ceases, all the pansporoblasts mature and the cyst ruptures, liberating the spores into the surrounding tissue, each to develop into a new cyst. How the parasites are transmitted to new hosts is not known.

A similar disease was found some years ago in South America and a parasite, then named Coccidium seeberi, has been described from the tumors. It is possible that this may be the same organism as that of Indian nasal polypus, but according to Fantham, who was one of the original describers of Rhinosporidium, there are a number of differences between them.

\section{Sarcosporidia, Parasites of the Muscles}

Brief mention should be made of a group of Sporozoa known as the Sarcosporidia which develop relatively enormous cysts in the muscles of vertebrate animals, especially in mammals. These parasites are usually found in the striped muscles but they also occur in other muscles. Infected muscles (Fig. 52B and D) appear to have white streaks or patches in them, sometimes 
several inches in length. Microscopic examination shows that these patches are cysts containing thousands of tiny spores, segregated into chambers (Fig. 52A) which correspond to the pansporoblasts of Rhinosporidium. The spores (Fig. 52C), escaping from the cyst, ultimately develop into new cysts in much
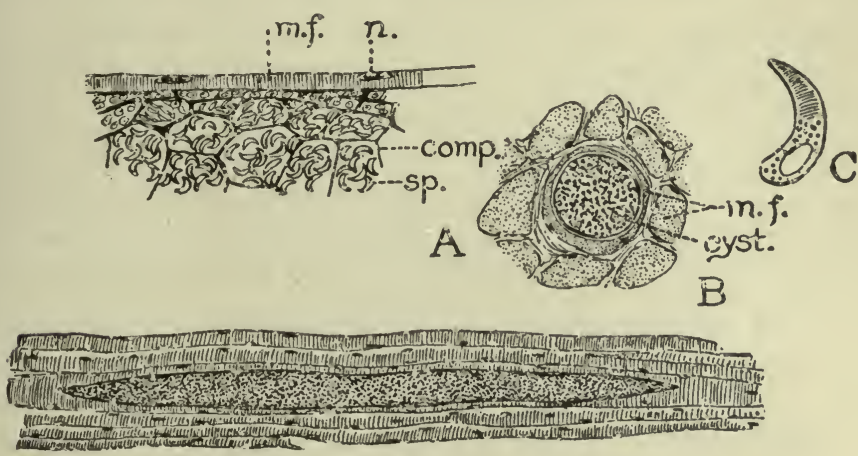

D

FIg. 52. Sarcosporidia. A, Sarcocystis blanchardi of ox, longitudinal section of infected muscle fiber (m. f.) showing spores (sp.) in chambers of compartments (comp.); n., nucleus of muscle fiber, $\times 265$. (After von Eecke from Wasilewsky.) $B$, cross section of sarcocyst from human larynx, probably S. tenella, $\times 200 . \quad D$, same, longitudinal section. (After Baraban and St. Remy.) C, spore of S. tenella of sheep. (After Laveran and Mesnil.)

the same way as is the case with the nose parasite. Although the muscle parasites have been known to parasitologists for many years there are portions of the life history which are not yet known. Darling and others have suggested that these peculiar protozoans may be "side-tracked varieties of parasites of invertebrate animals." We have no definite knowledge of the normal means of transmission although a number of possible methods are known. It has been found that infections can be spread by cannibalism, and that the fæces of infected mice can infect other mice; it has also been stated that spores occur in the circulating blood, which would mean that blood-sucking arthropods may be instrumental in the transfer. Fleshflies may also play a part in dispersing the spores. .

Erdmann has shown that when spores of Sarcosporidia develop in the intestine a very powerful toxin, called sarcocystin, is discharged and destroys the neighboring epithelial cells of the intestine and thus breaks a way for the young parasite into the 
lymphatics and ultimately into the muscles. Crawley has recently described in Sarcocystis muris of mice what he interprets as sexual differentiation of the spores and fertilization within 18 hours after the spores have been ingested by mice. Crawley believes the Sarcosporidia to be closely allied to the Coccidia, and suggests that there may be an unrecognized stage of development in a carnivorous animal. It is quite evident from the various hypotheses and speculations mentioned above that there is much yet to be learned about these enigmatic parasites.

Only a few scattered cases of Sarcosporidia in man have been recorded, and these may be looked upon as purely accidental. The parts affected have been the muscles of the heart and larynx. Many speculations as to how these infections occurred have been made, but nothing definite is known about it. It is probable that the human infections are due to Sarcocystis muris, a species which produces a very fatal disease in mice, and infections may have been due to contamination of food or water with the excrement of infected mice. The use of meat of Indian buffaloes infected with another species, Sarcocystis tenella bubali, seems to have no injurious effect on man, but ingested spores cause irregular fever.

\section{Oroya Fever}

The Disease. - Since at least the time of the Incas, Peru has suffered from a strange disease which has swept over the country from time to time in the form of frightful epidemics, some of which have cost thousands of lives. One of the severest recent outbreaks occurred among the workmen building the Peruvian Central Railway between Lima and Oroya and it is estimated that at least 7000 individuals died in it. In 1906 at least onetenth of 2000 workmen employed building tunnels and bridges on the Central Railway died of the fever, and one bridge in particular, which was the scene of a great many deaths from the disease, has come to be known as the Oroya Fever Bridge (Fig. 53).

The disease is at present endemic in the deep cleft canyons or quebradas (Fig. 53) characteristic of the west face of the Andes, at an elevation of between 2500 and $8000 \mathrm{ft}$., but it is probable that it has a wider distribution than is now supposed. It shows a marked seasonal prevalence, most of the cases occurring from January to April, especially toward the close of the warm, rainy season. 

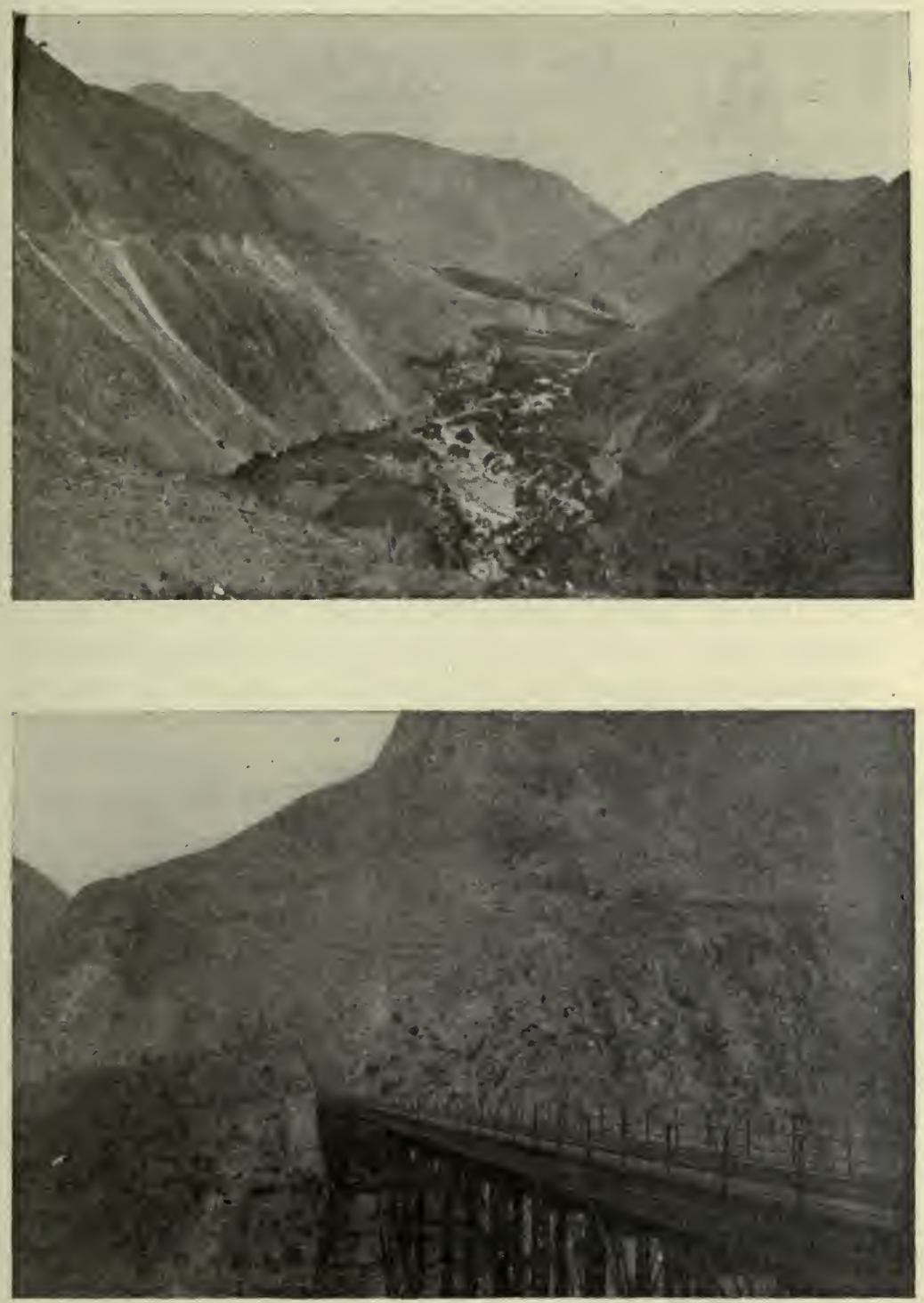

Frg. 53. Above, a typical "quebrada" or canyon on the west slope of the Andes where Oroya fever abounds. Below, the famous "Oroya Fever Bridge" on Peruvian Central Railway where hundreds of lives were lost from Oroya fever. (Photos kindly lent by Harvard School of Tropical Medicine, previously published by Strong et als) 
Oroya fever has been constantly confused with other diseases and it was not until the South American expedition of the Harvard School of Tropical Medicine, under the leadership of Dr. R. P. Strong, made an investigation of the disease that some order was brought out of the confusion. Malaria, paratyphoid, and particularly verruga peruviana are the diseases. which have been most frequently confused with Oroya fever. Mixed infection of these diseases and others such as yaws and tuberculosis with true Oroya fever has still further complicated matters. From the time of the Incas verruga peruviana and Oroya fever have been associated and regarded as different phases of the same disease, and this view is still held by some investigators. The fact that the characteristic nodules of verruga were usually associated with a very mild form of fever and sometimes with none at all, while oroya fever was of very severe type causing very high fatality, raised some question as to the distinctness of the diseases. To settle this point a Peruvian medical student, Daniel Carrion, vaccinated himself with blood from a verruga nodule. Five or six weeks later he died of a severe fever, and the question of the identity of the disease was apparently settled, and the fever was called "Carrion's Fever" in his honor. The notes regarding Carrion's illness have been lost and it is now believed that he may have died of some other disease or that the patient from whom he inoculated himself may have been suffering from some other disease in addition to verruga.

As a result of their own studies, Dr. Strong and his colleagues believe that the diseases are quite distinct. They have shown that Oroya fever is caused by a very minute parasite living in the red blood corpuscles and multiplying in the endothelial cells, and that it cannot be inoculated into animals; verruga peruviana, on the other hand, is caused by a virus which is ultra-microscopic, probably related to the smallpox virus, and can be successfully inoculated into laboratory animals. It is easy to understand how the two diseases were confused, since to a large extent their ranges overlap and a visitor to endemic regions would be likely to contract both. Verruga, being less quickly contracted and having a longer incubation period, would tend to appear later than Oroya fever, and would therefore be looked upon as a later stage of the same disease. The native belief that a general erup- 
tion was favorable to recovery, a belief undoubtedly based upon the benign nature of verruga, leads to the adoption of all sorts of methods to invoke a breaking out of the skin, such as applications of turpentine, rubbing with irritant leaves, etc., and undoubtedly a great many cases of eruptions following Oı oya fever are really only the eruptions caused by the artificial irritation of the skin.

Oroya fever, after an incubation period of about 20 days, begins with a general feeling of malaise and aches in the joints, followed by chills and fever, which last irregularly for many weeks. The fever is accompanied by a rapid pernicious anemia, the red blood corpuscles being reduced in some cases to one-fifth, or even less, of their normal number. This causes severe prostration and in a large per cent of eases death results within three or four weeks. The skin assumes a yellowish waxy color, and there are often slight hemo:rhages of the mucous membranes and various internal organs, as demonstrated by post mortem examinations. The liver and spleen become moderately enlarged, and the lymph glands are swollen.

The Parasite. - The true parasite of Oroya fever was first discovered by Barton, of Lima, Peru, in 1905 and confirmed by him in 1909, at which time he suspected that it might be a protozoan. The parasites were more thoroughly studied by the Harvard expedition in 1913 and 1914 and named Bartonella bacilliformis. Dr. Strong and his colleagues describe them as minute rods or, more rarely, rounded bodies occurring inside the red blood corpuscles (Figs. 54 and 55). These parasites, the rod form of which are only 1.5 to $2.5 \mu$
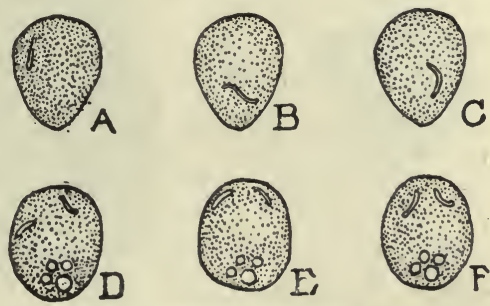

Fig. 54. Bartonella bacilliformis, living. $\quad 1, B$ and $C$, successive drawings of a single red corpuscle showing movements of parasite within it; $D, E$ and $F$, corpuscle containing two rod-shaped and four round parasites, showing migrations of the rodshaped individuals. $\times 2000$. (After Strong et al.)

(less than $\frac{1}{10.000}$ of an inch) in length and the round bodies 0.5 to $1 \mu$ in diameter, are definitely motile, moving about freely inside the corpuscles. In severe infections there may be found from one to ten parasites in a single corpuscle.

A multiplicative stage of the parasite occurs in large swollen endothelial cells in the lymph glands and spleen. In these swollen 


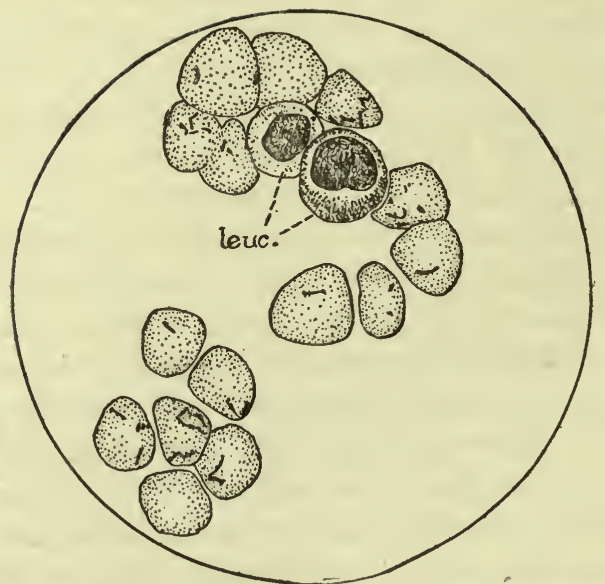

Fra. 55. Bartonella bacilliformis in stained blood from Oroya fever patient. Some cells show chains of parasites. Bodies with large dark nuclei are leucocytes (leuc.). $\times$ about 1000 . (After Strong et al.)

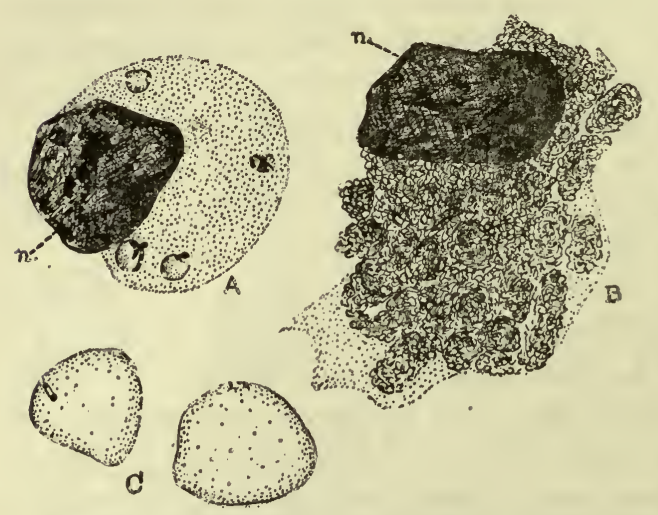

Frg. 56. Development of Bartonella bacilliformis in endothelial cells. $A$, endothelial cell, with large nucleus (n.) at left, containing five roundèd bodies in early stage of development; $B$, endothelial cell showing rounded bodies developing large numbers of small rod-shaped parasites; $C$, red corpuscles lying near with parasites identical with those escaping from such a cell as shown in $B . \times 2000$. (After Strong et al.)

\section{a parasites known as}

the Piroplasmata, including the Texas fever parasite of cattle and a number of other disease-causing parasites of wild and domestic animals. Its exact classification cannot yet be determined, and rounded bodies, sometimes a few, sometimes great masses of them (Fig. 56B). Some of these rounded bodies contain only one, two or four deep staining granules (Fig. 56A), while others contain large numbers of them. It appears that these granule-filled bodies break up into a large number of parts each containing one granule; these become elongated, and finally appear as distinct rods containing the granule at one end. In this condition they are identical with the parasites which occur in the red blood corpuscles (Fig. 56C) and indicate the manner in which the corpuscular parasites arise. Dr. Strong and his colleagues believe Bartonella bacilliformis to be a protozoan probably related to the group of

cells are minute 
it is simply looked upon as possibly belonging to a group of microorganisms intermediate, as perhaps are the spirochætes, between Bacteria and Protozoa, but with a decided leaning toward the latter. Possibly they are related to Rickettsia. (See p. 185.)

Transmission. - The method of transmission of Oroya fever is still in doubt, but it seems practically certain that some arthropod acts as a transmitting agent. All the other parasites of the group to which Bartonella belongs are transmitted by ticks, but there is apparently no tick having habits compatible with the occurrence of the disease. The Harvard expedition attempted to obtain the development of Bartonella in a mosquito, Phalangomyia debilis, which is common in the infected zones, but without success. That the transmitting agent is a nocturnal blood-sucker of very limited distribution but abundant within its range is strongly indicated by the limitations of the disease and by the fact that in many cases a single night in the infected zone is sufficient for contracting it, whereas there is apparently no danger a short distance from the infected zone, or within it in the daytime. According to Townsend, who spent two years investigating verruga (which he considers identical with Oroya fever) the only arthropod which fulfills all the conditions is a sandfly, Phlebotomus verrucarum, which is a very abundant nocturnal blood-sucker apparently limited in its distribution to the verruga zones. Townsend attempted experiments with the transmission of the disease through the agency of this insect but his results have not been generally accepted. Whether or not Phlebotomus is instrumental in transmitting Oroya fever is a matter which will have to be proved by further research but the circumstantial evidence against the insect is strong.

As pointed out by Townsend, the portions of Peru which are haunted by Oroya fever and verruga have one of the most perfect and healthful climates in the world and they would be ideal for sanatoriums and resorts were it not for these diseases. It is to be hoped that the diseases may soon be more thoroughly worked out and gotten under control. However, if Phlebotomus is instrumental in the transmission of either, the hope of eradicating them in the near future is slight, judging by the difficulties which have been experienced in Mediterranean countries in controlling these minute rock-breeding insects. 


\section{Dengue and Phlebotomus Fever}

These two diseases are wide-spread in tropical and subtropical countries, and are frequently confused. They have commonly been associated with yellow fever, none of the parasites causing them having been discovered until Noguchi discovered $a^{\cdot}$ Leptospira as the causative organism of yellow fever in 1918. It is possible that the other two diseases will also be shown to be caused by species of Leptospira. Dengue is a much milder disease than yellow fever and is seldom fatal, though its after-effects linger for many months. Phlebotomus or three-days' fever is a still milder disease and, as its name implies, of short duration. Like dengue it has lingering after-effects but they are not so severe or so persistent as in the former disease.

Both diseases are caused by blood parasites, so far undiscovered, which are transmitted by dipterous insects in which they apparently undergo part of their life cycle. The diseases each begin with a sudden high fever and headache, and pass through essentially similar stages, intense rheumatism-like aches being very characteristic of all. Each disease confers immunity, often of long duration in dengue and often transitory in phlebotomus fever. It seems not improbable that the parasites of these diseases and of yellow fever are closely allied.

\section{Dengue}

Dengue, seven-days' fever, or breakbone fever, is a disease of tropical and subtropical countries. It is very common in the West Indies and great epidemics have swept through Panama, the eastern Mediterranean and southern Asian countries, the Philippine Islands and various South Sea Islands. An epidemic has recently been reported from Argentina and Uruguay, the disease supposedly having been introduced from Spain. Dengue also occurs in southern United States where it is probably often overlooked, being diagnosed as something else. In some places, e.g., southeastern Europe and India, there is some confusion between dengue and phlebotomus fever. Both diseases vary somewhat and mild types of the former and severe types of the latter may easily be, and frequently are, confused. Dengue occurs in the form of sudden and rapidly spreading epidemics which sweep 
over limited areas, affecting a large per cent of the population.

Nearly every fluid and organ of the body has been examined in an effort to find the organism causing dengue, but although many supposed parasites have been found, the true cause of the disease is still unknown. In at least one stage of its life history the parasite, like that of yellow fever, is ultra-microscopic. The disease has been proven by experimentation to be transmitted commonly by the tropical house mosquito, Culex quinquefasciatus, and its distribution geographically coincides very closely with that of this mosquito. However, Aëdes calopus has also been incriminated, and in Formosa another closely related species, Desvoidea obturbans.

Unlike yellow fever, dengue has a very short incubation period in the mosquito - in one experiment it was only 48 hours. This fact, together with the short incubation period in the human body (from two to five days), explains the rapidity with which dengue epidemics spread.

The disease begins with startling suddenness. Within a few hours a normal healthy individual acquires a prostrating fever, a severe headache and terrible aches in the bones and joints which make it necessary to lie still. His face and sometimes his whole body becomes flushed and purple with congested bloodvessels, and the patient is to say the least very miserable. In a day or two the fever moderates, and usually is terminated by a sudden crisis of nose-bleed and diarrhea, relieving the congestion which has been felt in all parts of the body. Then follows an interval of apparently normal condition during which the patient feels perfectly well. After a few days there is a return of more or less severe fever and aches accompanied by a measleslike rash. The latter fades in from three to five days and is followed by a powdery scaling off of the skin. If lucky, the patient now quickly recovers but more often he has lingering and recurring aches in various joints, especially the knees and ankles, and he may be thus afflicted for several weeks before final recuperation, whence the name "breakbone fever." The disease is dangerous to life only if complicated in some way.

An attack of dengue usually confers immunity on an individual but this sometimes lasts only a year and is sometimes not established at all, since more than one attack during a single epidemic have been known to occur. 
There is no specific remedy known that will cure dengue. Care of the general health, including measures to lessen the fever, headache and bone aches, help in making life worth living during the eight or ten days of suffering.

Once an epidemic has broken out, it is almost as useless to attempt to stop it as to stop a tidal wave, as far as the mass of people is concerned. Houses screened against mosquitoes, if available, are havens of refuge, but the tropical villages or cities in which there are enough screened houses to care for even a small per cent of the population are hopelessly lacking, and the rapidity of the spread of the disease makes the isolation of early cases in mosquito-proof wards almost futile. Anti-mosquito campaigns, conducted not merely during an epidemic but at all times, are the only methods now known of preventing epidemics of dengue or of lessening their local prevalence.

\section{Phlebotomus Fever}

Of the same general nature as yellow fever and dengue, and concluding this series of gradually milder diseases, is phlebotomus or three-days' fever. This disease occurs especially on the shores of the Mediterranean and in India, and possibly also in other parts of the world. In endemic countries it occurs in the form of annual epidemics. It is estimated that in the earthquake regions of Italy where the disease is especially prevalent, 50,000 persons are attacked annually, incurring a financial loss of over $\$ 7,000,000$ by the prolonged incapacitation for work which follows the disease. In central India, every non-immune person is said to be attacked by the end of June each year.

Phlebotomus fever begins suddenly, like dengue, with a high fever, severe headache and aches in the bones and joints. The nervous symptoms are marked, and the pulse and respiration are accelerated. Usually the fever subsides on the third day, though in India it often lasts four or five days. The aches and general depression continue for ten or twelve days or even longer after the disappearance of the fever.

The disease, the parasite of which has never been discovered, is transmitted by the gnat or sandfly, Phlebotomus papatasii (see p. 470, and Fig. 212), which is extremely abundant in the regions where phlebotomus fever is endemic. The appearance and habits of this insect are described on p. 471. The prevalence 
of phlebotomus fever in the earthquake districts is due to the abundance of ideal breeding places for the gnats furnished by the ruined walls. It is possible that other species of Phlebotomus may also transmit the disease.

The gnats become infective about a week after feeding on an infected person. The incubation period of the disease in man is about four or five days. Natural immunity is extremely rare, but in most cases an immunity of variable duration results from an attack of the disease.

No specific cure has yet been discovered. Prevention lies in avoiding the bites of Phlebotomus papatasii and in reducing their numbers as far as possible by methods described on p. 473 . In case of prolonged residence in an endemic region, there is little hope of escaping infection, and willful exposure to it at a time when the disease will be least inconvenient is usually advisable, in view of the usually persistent immunity which results.

\section{Rickettsia-Like Organisms}

Within the past few years there have been described as the probable cause of a number of human diseases, organisms of very minute size, frequently less than half a micron in diameter, which may show a number of morphological types. Round or diplococcal forms are of most frequent occurrence, but there also occur minute rod-shaped bodies and occasionally threadlike forms. The relationships of these organisms is very obscure. They are looked upon by some investigators as bacteria, and by others as protozoa, while still others consider them merely by-products of the infection. In their staining reactions they resemble spirochætes rather than bacteria, and the possibility of their representing modified granule stages of spirochætes has been suggested. They are non-motile, and very difficult to cultivate. They occur in a number of kinds of arthropods, usually multiplying on or in the epithelial cells of the alimentary canal, but some species, at least, occur in other organs also, including the reproductive glands, and are hereditarily transmitted. The resemblance to spirochætes is further borne out by the way in which reproduction takes place by granuleshedding from elongated threadlike forms, and by their hereditary transmission among arthropods. Species of Rickettsia or closely allied forms have been found in human lice (Pediculus), Mallophaga, fleas, bugs (Cimex), sheep ticks (Melophagus), and true 
ticks (Dermacentor), and undoubtedly will be found in other arthropods. Their occurrence in non-bloodsucking insects, aside from the fact that no vertebrate disease is known to be associated with many of these infections in parasitic insects, is evidence for the fact that these organisms, like the intestinal flagellates of insects (see p. 75), are primarily parasites of arthropods, a comparatively few species of which have become adapted to live in the blood of vertebrate animals, where they may cause disease.

The human diseases with which Rickettsia-like bodies have been definitely associated are typhus, trench fever, and Rocky Mountain spotted fever. In the Japanese disease, kedani or tsutsugamushi, which shows some affinities with spotted fever, parasites have been described which may be referable to this group.

Typhus Fever. - This deadly disease, which so frequently breaks out when human beings are crowded together where personal care and cleanliness are either neglected or impossible, has for ages been associated with wars and prison camps. In some parts of Europe and North America typhus persists in an endemic or mild epidemic state, ready to burst into flame when opportunity comes, giving rise to terrible epidemics. The disease is endemic on the Mexican plateau, where it is known as tabardillo.

Typhus has a sudden onset, and is characterized by high fever, headache, aches in the bones and muscles, bronchial troubles, congestion of peripheral blood-vessels and a red rash later giving rise to dark blotches. In an endemic state the mortality is very low, but when an epidemic breaks out there may be 50 to 79 per cent mortality.

Typhus has been conclusively shown to be transmitted principally, and perhaps solely, by body lice. Details of the rôle of the lice, and an account of some recent epidemics, will be found on pp. 397 to 399 .

Attention was first directed to minute granules associated with typhus fever by Ricketts in 1909, but these bodies were first described in detail from typhus-infected lice by Rocha-Lima in 1916 and named Rickettsia prowazeki. The organism occurs in abundance in the gut of infected lice as single granules, diploid bodies, or in clumps evidently resulting from continued multiplication without separation of the individuals; occasionally bacillus-like or thread forms occur. Multiplication seems to 
take place inside the epithelial cells of the insect. This organism has been found in the blood and tissues of typhus patients also.

A great deal of dispute concerning the relationship of Rickettsia prowazeki to typhus has arisen from the fact that Rickettsia also occurs in lice which could not have been infected from typhus patients. It is now generally believed that there is a distinct species or variety of Rickettsia, named $R$. pediculi, which is an inhabitant of the gut of the body louse, but which is non-pathogenic to man, in fact probably limited to the insect host.

Trench Fever. - This disease, which was unknown before the great war, but which was one of the most important diseases afflicting troops participating in it, was shown in 1918 by a commission of the Medical Research Committee of the American Red Cross and also by a British committee to be normally transmitted by body lice (see p. 399), although the possibility of transmission by body excretions also exists. A form of the disease occurring in Central Europe was known as Volhynian fever.

After an incubation period which is probably usually between 14 and 30 days, there is a sudden onset of fever accompanied by headache, dizziness, muscular pains and other symptoms. The fever may last continuously for several weeks, or it may be more or less definitely relapsing. In most cases the spleen is enlarged, and there is a rash on the back, chest and abdomen. Usually the patient recovers in from five to six weeks, but the illness may be greatly prolonged.

In 1916 Toepfer first described a species of Rickettsia, R. quintana, as occurring in lice fed on trench fever patients. This organism closely resembles that of typhus fever but is more constantly rounded or diploid in form, and most writers agree that it does not occur inside the epithelial cells of the gut of the louse. This parasite may be merely a pathogenic variety of $R$. pediculi, mentioned in the preceding section, which has acquired the ability to thrive in human blood. In infected lice the organisms are found attached to the surface of the cells of the midgut. They gradually fill the lumen, and are eventually voided with the fæces.

According to Hindle, who is inclined to regard the organisms as bacteria rather than protozoa, no distinct portion of the life cycle occurs in the louse, this insect merely acting as a culture tube as does the flea for the bacilli of plague. The negative period of four or five days after a louse becomes infected until it is ca- 
pable of transmitting the disease, Hindle thinks is probably due to insufficient numbers of Rickettsia in the lice. In the human host the parasites, as shown by transmission experiments, occur in the blood plasma and also in various body excretions. They are ordinarily not filterable, but in some stages of their development will pass through bacterial filters.

Rocky Mountain Spotted Fever. - For many years certain limited districts in the Rocky Mountain region of northwestern

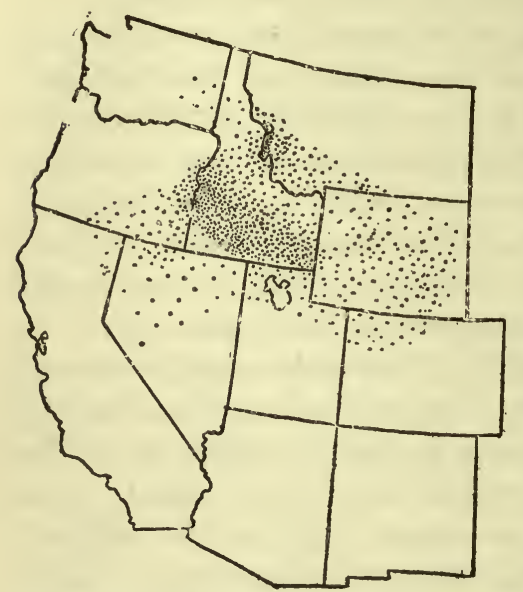

United States (Fig. 57), particularly Idaho and Montana, have been known to be affected by this very serious disease. Its yearly occurrence in welldefined areas has given rise to panic and hysterical fear of entering the " haunted" places. Houses were deserted, land depreciated in value, and some of the richest valleys in the Northwest left unpopulated. In 1906 it was shown by Ricketts that the disease was invariably preceded by the bite of a common

FrG. 57. Map showing distribution of local wood-tick, Dermacentor Rocky Mountain spotted fever. Compiled from U. S. Public Health Reports. venustus (see p. 361, and Fig. 156), which was experimentally shown to be the intermediate host of the parasite. Wolbach in 1919 demonstrated the regular appearance of minute organisms, which he named Dermacentroxenus rickettsi, in the cells of bloodvessels of infected mammals, and also in nearly all the tissues of infected ticks. The organism shows several morphological types, but most frequently appears as paired lanceolate bodies, the length of a pair being about $1 \mu$. It is transmitted by ticks to their young with the eggs. It requires from two to ten hours feeding for a tick to become infective, but after an incubation period of a few days it may remain infective for many months. Although $D$. venustus appears to be the only transmitter in nature, experimentally other ticks have been shown to act as suitable hosts.

In man the incubation period is usually from four to seven 
days. The disease begins with a general feeling of illness followed by chills and aches. A constant fever gradually increases until the tenth or twelfth day, when death is likely to occur. In mild cases the fever gradually subsides during the five or six days following. Usually on the third day a rose-colored rash breaks out on the head and upper part of the body, followed a day or two later by a characteristic spotting of the arms and legs, and later of much of the body, caused by the bursting of blood capillaries in the skin. The spots often become brownish or grayish in color, giving the spotted appearance from which the disease takes its name. In Montana, especially in the Bitter Root Valley, the disease has a high fatality, 75 per cent or more of the cases ending in death. The fatality is also high in eastern Oregon, but in other endemic regions is very much lower. The disease appears only in spring and early summer when ticks are abundant. So far no specific remedy has been discovered.

There is evidence that spotted fever may be harbored by some of the wild mammals on which the wood tick normally occurs, but this has not yet been proved. The transmitting tick, $D$. venustus, is a species which requires two years to reach maturity. In its immature stages it infests many of the local rodents, nearly all of which are susceptible to the disease, and capable of transmitting it to uninfected ticks. As adults the ticks live on many of the larger wild animals and on domestic animals, especially cattle and horses. Whether some of these animals may be carriers of spotted fever has not been determined.

Prevention of spotted fever consists primarily in fighting ticks by various methods (see p. 368), and in destroying rodents, both to reduce the number of host animals for the young ticks, and to prevent the possibility of their acting as carriers of the disease.

There is grave danger that spotted fever may be introduced into other parts of the country where suitable ticks for transmitting it can be found. The exportation by railroad of wild deer, elk, goats or other tick-infested animals to zoölogical parks or government preserves is a dangerous proceeding unless great care is taken to destroy all ticks and to exclude any individuals which might be harboring the disease germ. The occasional occurrence of the disease in various parts of the United States should be carefully watched for, and every precaution taken to prevent local ticks from getting access to the infection. 
Kedani or Japanese Flood Fever. - In certain parts of Japan there occurs a disease usually called kedani, or tsutsugamushi, which is reminiscent of American spotted fever. It begins after an incubation of five or six days or longer with a fever and breaking out of the skin, the fever reaching its height between the third and seventh days. It lasts from one to three weeks and is accompanied by a swelling of the lymph glands, especially in the vicinity of the point of infection. This is usually the armpit, neck or groin region, where a small ulcerous wound can be found resulting from the bite of a mite.

The disease is transmitted by the bite of a very small reddish mite, probably an immature mite of the genus Trombidium, or harvest bug. These mites live in great numbers on a very abundant local field-mouse, Microtus montebelli. The mice are not only the hosts of the mites but are also subject to the disease and undoubtedly are an important factor in its distribution and control. Kedani is apparently most common in laborers working in hemp fields in July and August, on the plains which are annually flooded by the overflow of certain rivers.

In Sumatra a similar disease, which is either identical or closely related to kedani, occurs commonly among Chinese and Japanese laborers in the tobacco fields. The disease as it occurs in Sumatra, where it is called pseudo-typhus,

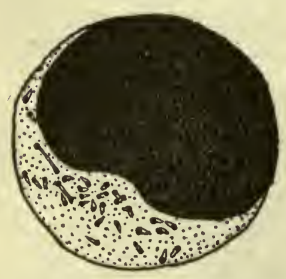

Fig. 58. "Granular bodies" in mononuclear leucocyte, supposed to be parasites of Kedani (Theileria tsutsugam differs in some slight respects from the typical Japanese disease and has a very much lower fatality. In its incubation period, eruption, and general course it resembles spotted fever more closely than does the Japanese disease, and Schueffner, who has worked most with it, thinks it may be transmitted by ticks as well as mites. The disease has also been reported from the Philippines, and about 150 cases have been reported in the Malay States. It is not improbable that it will be found to be widely distributed in southeastern Asia, having been incorrectly diagnosed as other diseases.

A number of organisms thought to be the cause of Kedani have been described. Hayashi has recently found granular bodies of several morphological types in both the plasma and cells of the 
blood of infected individuals, and in the primary sore. These he believed to be piroplasma-like organisms, and named them Theileria tsutsugamushi. From the description and figures of these organisms, however, it seems more probable that they are related to Rickettsia or Dermacentroxenus. The blood of infected individuals injected subcutaneously causes the disease. The organisms do not pass through bacterial filters.

Prevention in the endemic regions obviously consists in avoiding mites by skin applications or other means. The extinction of the field-mice and with them most of the mites would undoubtedly lessen the danger of the disease.

\section{Chlamydozoa}

The protozoan affinities claimed for the parasites or parasitelike bodies included in the so-called Chlamydozoa is, as said before, doubtful, and new investigations do not, in most cases, tend to substantiate the claim of these structures to consideration as animal parasites. A brief account of the parasites or cell inclusions in some of the principal diseases attributed to this group is all that can be given here.

Smallpox and Vaccine. - The youngest forms of the parasite are minute granules or "elementary bodies" measuring about $0.5 \mu\left(\frac{1}{50,000}\right.$ of an inch $)$ in diameter. As growth takes place the granules increase in number and become surrounded by material which is usually interpreted as a reaction product of the cell, forming the "Guarnieri bodies" (Fig. 59A). These eventually rupture, liberating the granules to infect new cells. The smallpox parasites differ from those of cowpox in that they attack the nuclei as well as the cytoplasm of the cells.

Scarlet Fever. - In the skin cells of scarlet-fever patients are found characteristic inclusions which have been referred to the Chlamydozoa and named Cyclasterion scarlatince (Fig. 59B). These bodies in one stage of their development are of irregular shape with numerous enclosed granules, while in another stage the granules become radiately arranged around a larger central body.

Hydrophobia or Rabies. - There usually occur in certain brain cells of animals suffering from hydrophobia specific bodies which are popularly known as "Negri bodies" in honor of their discoverer, and which have been given the scientific name Neuroryctes 


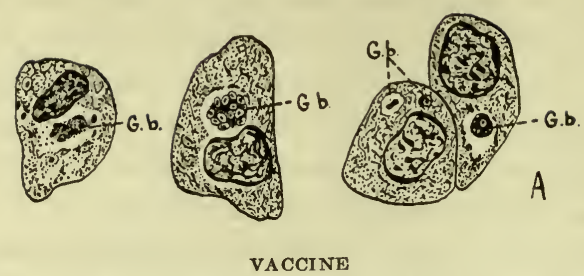

Cornea cells showing developmental stages of Guarnieri bodies, Cytoryctes vaccinice. (After Tyzzer.)

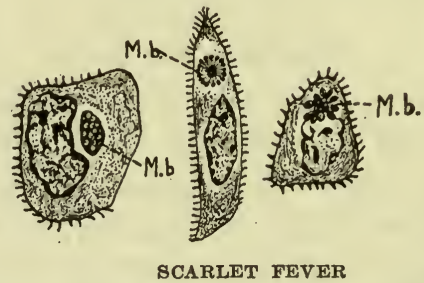

Epithelial cells of skin showing various stages in development of Mallory bodies, Cyclasterion scarlatince. Radiate form shown in middle figure. (After Mallory.)

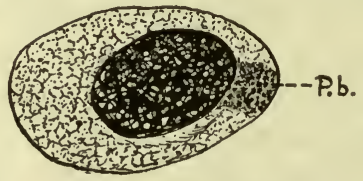

TRACHOMA

C

Epithelial cell of conjunctiva showing Prowazek bodies. (After Halberstaedter.)

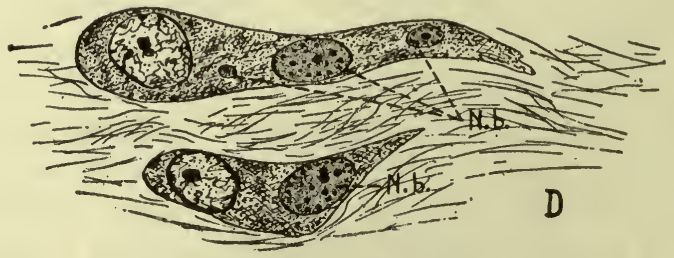

RABIES OR HYDROPHOBIA

Nerve cells of Ammon's horn of cerebrum showing Negri bodies, Neuroryctes hydrophobice. (After Maresch.)

Fig. 59. - Various types of Chlamydozoa. Note that in each case the parasitelike bodies are enclosed in a ground substance supposed to be extruded by the nucleus. 
hydrophobia (Fig. 59D). At first thought to be simple parasites, these bodies are now generally regarded, as are other Chlamydozoa, as reaction products of the host cell surrounding one or many minute granules which are the true parasites. The minute size of the granules and the difficulty of identifying them when they are separated from their "mantles" probably accounts for the negative findings in infective parts of the nervous system in which Negri bodies are not found, and also in the saliva, which is highly infective. The weight of evidence seems to favor the protozoan affinities of the microörganism of hydrophobia, but the nature of the parasite is still shrouded in uncertainty.

Trachoma. - The belief in the protozoan nature of the parasite of trachoma, a disease of the eyes causing inflammation of the conjunctiva, rests on similar ground. In the affected portions of the eye are found numerous tiny granules known as "Prowazek's bodies " (Fig. 59C), sometimes within the cells and even within the nuclei and at other times free in the serum, which have been thought to be the cause of the disease. The fact that these bodies are sometimes found in other affections has thrown some doubt on their relation to trachoma. Recent investigations by Anna Williams of 4000 school children in New York with eye infections or inflammations, none of which were typical cases of "trachoma," showed "trachoma inclusions " to be common, and gave evidence that these inclusions were in reality " nests " of growing bacteria, of various kinds, in the epithelial cells of the conjunctiva. Miss Williams' investigations throw doubt on the existence of a specific disease to which the name trachoma can be applied.

\section{Other Diseases Caused by Obscure Parasites}

The diseases mentioned above are those which are most commonly thought to be caused by organisms of this problematic group, Chlamydozoa. There are a number of others, however, which may belong here, but on which much further investigation is necessary. Among. these are foot-and-mouth disease, in which Stauffacher has recently found an organism, Aphthomonas infestans, which, however, is probably more closely related to Leishmania (see p. 76); verruga peruviana, which in some respects resembles smallpox; the ubiquitous measles; and a number of diseases which are of rare or of more or less limited distribution. That all of these diseases, or even all of those separately discussed 
above, are caused by protozoan parasites is very doubtful, and only further investigation can determine the true status of their causative microörganisms. The recent development of knowledge concerning such groups of pathogenic organisms as the Spirochætes and Rickettsia-like organisms, on the border line of visibility, leads to speculation as to whether some of these other diseases may not be found to be caused by parasites of similar nature. 


\section{PART II - WORMS}

\section{CHAPTER XI \\ INTRODUCTION TO THE "WORMS"}

Classification. - The name worm is an indefinite though suggestive term which is popularly applied to any elongated creeping thing which is not obviously something else. There is hardly a branch or phylum of the Animal Kingdom which does not contain members to which the term "worm" has been applied, not excepting the great group Chordata, to which the backboned animals, including man himself, belong. In fact some animals, such as many insects, are "worms" during one phase of their life history, and something quite different during another.

In a more restricted sense the name "worm" is applied to three great groups of animals, with a few outlying forms, which superficially all resemble one another in being unquestionably " wormlike," though in life and structure they are widely different. To these animals, together with a few other heterogeneous forms, the collective name "Vermes," meaning worms, was applied by the early workers on zoölogical classification. Upon more detailed study it became obvious that different types of the "Vermes" differed from one another to such an extent that they would have to be divided into several great branches or phyla of the Animal Kingdom. At the present time the majority of these animals are classified in three phyla, as follows: the Platyhelminthes or flatworms, the Nemathelminthes or roundworms and the Annelida or segmented worms. There are a number of "worms" which will not readily fit into any of these groups but are incerto sedis, showing affinity to one group in some respects and to another in others. Some species are so profoundly modified by their peculiar modes of life that it is practically impossible even to guess at their true relationships. All three of the phyla of "worms" contain parasitic species, though none of them contain parasites exclusively.

Flatworms. - The group of lowest organization is the Platyhelminthes. The worms included in this phylum are flattened 
from the dorsal to the ventral side, whence the common name "flatworms." Unlike nearly all other many-celled animals they have no body cavity, the organs being embedded in a sort of spongy "packing" tissue. The digestive tract has only a single opening which serves both for mouth and vent (Fig. 60A), and in the tapeworms the entire alimentary canal is absent. The nervous system is very simple. Performing the function of kidneys is a system of tubes, the terminal branches of which are closed by "flame cells," so called from the flamelike flickering of a brush of cilia which keeps up a flow of fluid toward the

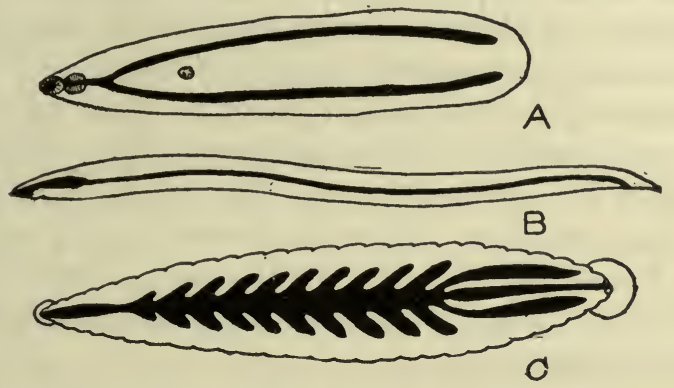

Fig. 60. Types of digestive tracts in worms; $A$, fluke, - note branching and absence of anus; $B$, roundworm, - note simple form, with only pharynx differentiated, and presence of anus; $C$, leech, - note extensive pouches or cœea which serve as reservoirs for surplus food.

larger branches of the system and ultimately to the excretory pore, thus conducting the waste products out of the body. The absence of any kind of blood system or other apparatus for transporting food or waste products in the body necessitates a branched condition of the digestive and excretory systems. The most highly developed system of organs and one which occupies a large portion of the body is that concerned with reproduction. Usually there is a complete male and female system in each worm and in some tapeworms there is a double system of each kind.

The flatworms are usually divided into three classes, the Turbellaria, the Trematoda and the Cestoda. The Turbellaria are for the most part free-living animals and include the "planarians " which can be found creeping on the under side of stones in ponds. The Trematoda include the flukes, all of which are parasitic, some externally on aquatic animals, others internally 
on aquatic or land animals. They are flattened animals, usually oval or leaf-shaped, furnished with suckers for adhering to their hosts. The flukes which live as external parasites of aquatic animals have a comparatively simple life history, while those which are internal parasites of land animals have a complex life history, in the course of which they pass through two or three different hosts. The third class, Cestoda, is comprised by the tapeworms. As adults they are all parasites of the digestive tracts of various animals and are profoundly modified for this kind of an existence. Their peculiar method of multiplication by budding results in the formation of a chain of segments, sometimes of great length, which collectively constitute a tapeworm; each segment, however, is practically complete in itself and capable of separate existence if it had some method of retaining its position in the host's intestine. Some tapeworms have a life history comparable in its complexity with that of the flukes but as a rule it is much simpler. With the flatworms are usually associated the Nemertinea, marine worms some of which are more or less parasitic. None of them is of any interest in connection with human parasitology.

Roundworms. - Of somewhat higher organization than the flatworms is the phylum Nemathelminthes or roundworms. These worms are cylindrical instead of flattened, they possess a body cavity, and they have an opening at each end of the digestive tract (Fig. 60B). The excretory system usually consists of simple tubes running the length of the body. The presence of a fluidfilled body cavity through which food and other substances can diffuse obviates the necessity for having branched organs. The sexes are separate, and the reproductive systems are much simpler than in the flatworms.

Usually there is only a single class recognized as belonging to this phylum, namely, the Nematoda or nematodes. Some of the nematodes are not parasitic but many of them parasitize either plants or animals. There are many important human parasites among the Nematoda, for instance, the hookworms, pinworms, Ascaris and other intestinal worms, Trichinella, Filaria and the guinea-worm. In some of these the life history is fairly simple while in others it is more complex and involves two different hosts.

Some zoölogists associate with this phylum two other classes 
of worms, the Acanthocephala and the Nematomorpha. The former class, as indicated by the name, include the spiny-headed worms. These are cylindrical worms of peculiar anatomy, notable for the complete absence of a digestive tract as in the tapeworms. The head is furnished with a proboscis which is armed with rows of thornlike hooklets. The adults live in the digestive tracts of their hosts, burying the thorny proboscis deep into the mucous membranes. They have a complex life history, the larval stage being passed in insects of various kinds. Several species are occasionally but rarely found in man.

The class Nematomorpha is comprised by the "horse-hair snakes," so called from the popular belief that they develop from horse hairs which fall into water. They are exceedingly long slender worms, usually parasitic in insects. Occasionally they are accidentally swallowed by man with drinking water and are usually vomited, much to the surprise and horror of the temporarily infected person.

Annelids. - The most highly organized phylum of worms is Annelida, including the segmented worms or annelids. In three important respects these worms are the first animals in the scale of evolution to develop the type of structure characteristic of the vertebrate animals, consisting, namely, in a division of the body into segments, in the presence of a blood system, and in the presence of "nephridia" or primitive excretory organs of the same fundamental type as are the kidneys of higher animals. In addition the digestive system is highly developed and there is a well-developed nervous system distinctly concentrated in the head. In some annelids the sexes are separate, while in others both reproductive systems occur in the same individual.

At least three classes of Annelida are usually recognized, namely the Archi-annelida, including a few primitive marine forms; the Chrtopoda, including the worms which are furnished with bristles or setæ, such as the earthworms and marine sandworms; and the Hirudinea or leeches, in which there are two suckers but no setæ. There are a number of other groups of worms which many zoölogists include with the annelids, but as their systematic position is doubtful and as they include no parasitic forms they need not be mentioned here. The only class of annelids which includes parasites of man are the Hirudinea or leeches. These animals superficially resemble flatworms but they can readily 
be recognized externally by the segmentation of the body; the internal anatomy is totally different. Both sexes are represented in each individual.

The number of different species of worms in these three phyla which have been found in man is well up in the hundreds. In the following pages each group of these worms which contains important human parasites will be dealt with, but only those species which are important, or which are particularly interesting from some other point of view, will be individually considered.

Parasitic Habitats. - As to the parts of the body which may be attacked by worms of one kind or another, there is hardly any organ or tissue which is exempt. There are flukes which habitually infest the intestine, liver, lungs and bloodvessels, and one species occasionally wanders to the muscles, spleen, brain and many other organs. The adult tapeworms are all resident in the small intestine, but larval tapeworms are found in various locations in the body. The majority of the parasitic nematodes of man are found in the intestinal canal but there are exceptions to this. The adult Trichinella, for instance, inhabit the intestine, but the larvæ are found in the muscles; the adult Filarice usually live in the lymph vessels, whereas the larvæ swarm in the blood; the guinea-worm and some other nematodes creep under the skin in the connective tissue; the lungworm of the hog, Metastrongylus apri, which occasionally occurs in man, infests the lungs and bronchial tubes; and Dioctophyme renale (or Eustrongylus gigas) is an occasional human parasite which occurs in the kidneys and rarely in the body cavity. The leeches, on the other hand, are parasitic on the surface of the body or in the cavities of the nose and mouth.

Life History and Modes of Infection. - The life history and mode of infection of worms varies with the habitat in the body. Every parasitic worm must have some method of gaining access to the body of its host, and must have some means for the escape of its offspring, either eggs or larvæ, from the host's body in order to continue the existence of its race. Many species utilize intermediate hosts as a means of transfer from one host to another; others have a direct life history, i.e., they either develop inside the escaped egg and depend on such agencies as food and water to be transferred to a new host, e.g., pinworm, or they 
develop into free-living larvæ which are swallowed by or burrow into a new host when opportunity offers, e.g., the hookworms.

Most of the intestinal parasites apparently enter their host by way of the mouth, and the eggs escape with the fæces. Many species enter as larvæ in the tissues of an intermediate host which is eaten by the final host. Of such a nature are most of the tapeworms and flukes and some nematodes, e.g., Trichinella. Some nematodes of the intestine, as the pinworm and whipworm, enter contaminated food or water as fully developed embryos in the eggs. Still other species, as the hookworms and Strongyloides, usually reach their destination in an indirect way by burrowing through the skin. All the intestinal worms except Trichinella produce eggs or larvæ which escape from the body with the fæces. In Trichinella the larvæ encyst in the muscles and in order for them to be released the host must be eaten by another animal. Many of the worm parasites of other organs of the body also enter by way of the mouth and digestive tract, though they have various means of exit for the eggs or larvæ. The liver flukes enter and escape from the body as do ordinary intestinal parasites; the lung flukes enter by the mouth, but the eggs are expelled with sputum; the blood flukes enter by burrowing through the skin, and the eggs escape either with fæces or urine; the Filario, like blood-dwelling protozoans, enter and leave the body by the aid of blood-sucking insects; the guineaworm enters by the mouth, and the larvæ leave through the skin. The larval tapeworms which infest man enter either by the mouth or by accidental invasion of the stomach from an adult in the intestine. Like Trichinella they are usually permanently sidetracked in man, since they can escape only by being eaten with the tissues in which they are imbedded.

Effects of Parasitism. - The effects produced by parasitic worms depend in part on the organs or tissues occupied, in part on the habits of the worms and in part on the poisonous qualities of their secretions or excretions, to which the susceptibility of different individuals is very variable. The effects of some kinds of worms is a much disputed point. Some investigators tend to minimize the damage done by worm parasites, especially intestinal ones, while others undoubtedly overestimate it. Improved facilities for discovering infection have demonstrated the presence of intestinal parasites in so many unsuspected cases that we are 
likely to incriminate them in nearly every morbid condition for which we cannot, with equal readiness, discover another cause. It cannot be doubted, however, that many of the morbid conditions really are, in part at least, produced by intestinal worms. Much of the difference of opinion regarding the effects of these parasites is no doubt due to the variable susceptibility of different individuals.

The amount of nutriment which is absorbed by worms such as Ascaris and the pinworm, which live on semi-digested food in the lumen of the intestine, is probably in most cases relatively slight: Leuckart states that Tcenia saginata, for instance, gives off about 11 proglottids a day, which would amount to one and two-thirds pounds in a year. This would not, of course, represent more than a fraction of the food materials used. Such a loss would, however, be inappreciable in adults, though it would be felt in growing children unless compensated for by increased appetite. Many intestinal parasites, as the hookworms, devour cells of the mucous membrane and suck blood, sometimes causing extensive bleeding.

The most serious injury from intestinal worms is undoubtedly the toxic effects of their secretions and excretions. We know that the diseases caused by most Bacteria and Protozoa are the result, not of the actual damage done by the parasites in devouring tissues, but of the poisonous waste products and secretions given off by these organisms. Until recently little was known about the toxic effects of worms, but that toxins were produced by them was evident from symptoms disproportionate to the mechanical injury the parasites could do, and from effects which could in no way be the direct result of mechanical injury. In 1901 a French worker, Vaullegeard, actually obtained from certain tapeworms and from Ascaris toxic substances which acted upon the nervous system and upon the muscles. Recent investigations by Flury have shown that Ascaris, a nematode, contains certain substances which are very irritating to mucous membranes, other substances which have blood-destroying and tissue-destroying properties, and still others which have an intoxicating effect on the nervous system, causing hallucinations, delirium and other disturbances. These toxins, derived from the body and excretions of Ascaris, when introduced into a vertebrate animal, cause the same symptoms which often accom- 
pany Ascaris infection, but which have usually been attributed to other causes. With such an array of formidable chemical compounds in the body substance of intestinal worms, it is not necessary to search for mechanical factors to explain intestinal disturbances, abdominal pains, nervous and mental symptoms, and the various other apparently unrelated conditions which accompany infection with such worms. When, as in the case of hookworms, such effects are combined with blood-sucking and bleeding from wounds, facilitated by secretions. which prevent coagulation of blood, it is not difficult to understand how such profound anemia and loss of vitality are produced by comparatively few small worms. The presence in blood of toxins absorbed from worms in the intestine is further indicated by changes in the blood itself. The anemia of hookworm disease, due both to reduction of blood corpuscles and to diminution in percentage of hæmoglobin, is so well known that anemia is sometimes used as a synonym for hookworm disease. Similar though usually less marked anemia occurs in cases of infection with other worms, e.g., the fish tapeworm, Dibothriocephalus latus, the blood flukes, etc. Another symptom of the presence of worms in the body is a change in number and kinds of leucocytes or white blood corpuscles. An almost universal symptom, though one which is occasionally absent even in the infections in which it is most characteristic, is an increase in the number of so-called "eosinophiles," white blood corpuscles containing granules which stain red with eosin. These cells are supposed to be for the purpose of destroying toxins in the blood just as some of the leucocytes are apparently for the purpose of capturing and destroying bacteria or other foreign cells. The mere presence of an increased number of them is, therefore, sufficient reason for assuming the presence of toxins for them to destroy. The normal number of eosinophiles varies from one per cent to four per cent of the total number of leucocytes, whereas in infections with such parasites as trichina, blood flukes, echinococcus cysts, etc., the number nearly always rises to five per cent or higher, and in some cases reaches over 75 per cent.

Another factor which is undoubtedly of prime importance is the portal of entry which intestinal worms give to Bacteria and Protozoa. We have awakened to the importance of a "whole skin" and the danger which accompanies the piercing of it by the 
unclean probosces of biting flies, bugs or other insects. We have not yet fully awakened to the importance of an uninjured mucous membrane. As has been pointed out by Shipley, the intestinal worms play a part within our bodies similar to that played by blood-sucking arthropods on our skins, except that they are more dangerous since, after all, only a relatively small per cent of biting insects have their probosces soiled by organisms pathogenic to man, whereas the intestinal worms are constantly accompanied by bacteria, such as Bacillus coli, which are capable of becoming pathogenic if they gain access to the deeper tissues as they are able to do through the injuries made by hookworms, whipworms, tapeworms, etc. Weinberg, for instance, found that whereas he was unable to infect unparasitized apes with typhoid bacilli, apes infested with tapeworms or whipworms readily contracted typhoid fever, the bacteria presumably gaining entrance through wounds in the mucous membrane made by the worms. The relation of intestinal worms to appendicitis is more than hypothetical, and it is probable that far more cases of appendicitis are the outcome of injury done by worms than is usually supposed. Although it has been objected that very few of the thousands of appendices removed yearly are reported to contain parasites, it must be pointed out that parasites are very seldom sought, could easily be overlooked, and might not be recognized as such if found. It is furthermore possible that parasites which initiated the inflammation and ulceration might no longer be present in the appendix upon its removal, since they are able to move about freely in the digestive tract. Shipley remarks that appendicitis is a commoner disease now than it was when vermifuges were more frequently given.

Diagnosis. - The diagnosis of infection with various species of worms depends principally on the identification of their eggs or larvæ as found in the fæces or other excretions by microscopic examination. Nearly every species of parasite has recognizably distinct characterics of the eggs, the chief variations being in size, shape, color, thickness of shell, stage in development, appearance of the embryo if present, and presence or absence of an operculum or lid. Some of the commoner worm eggs are shown in a comparative way in Fig. 61. 

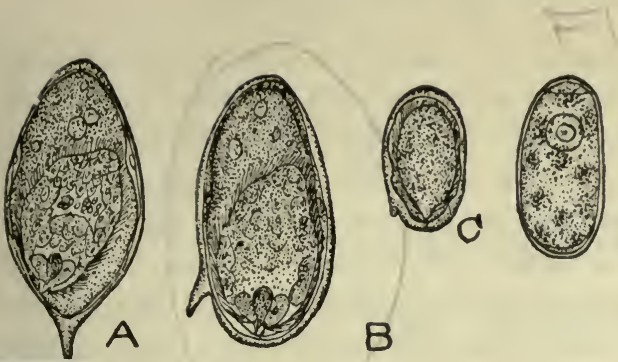

D
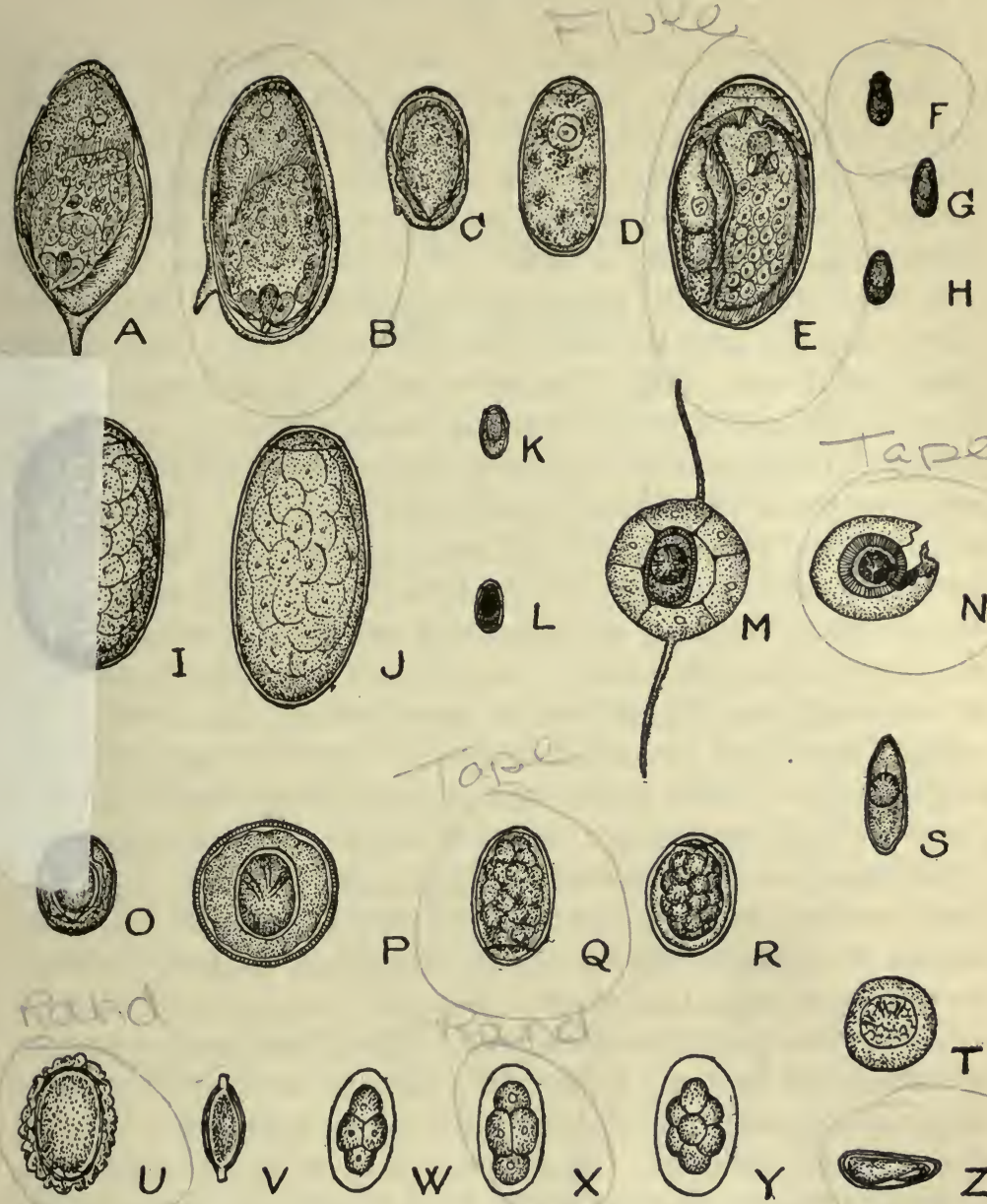

B

I
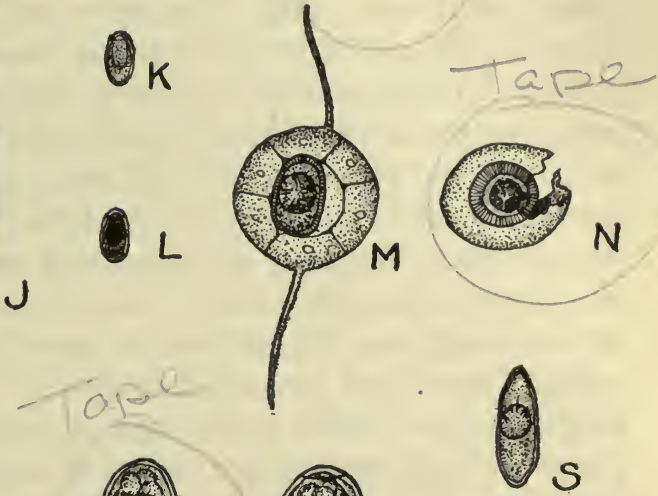

FIG. 61. Eggs of parasitic worms, drawn to scale. $\times 200$. (After various authors.)

A, Schistosoma hæmatobium, voided with urine.

$B$, Schistosoma mansoni, voided with fæces.

C, Schistosoma japonicum, voided with fæces.

D, Paragonimus ringeri, voided with sputum.

$E$, Fasciola hepatica, voided with fæces.

$F$, Clonorchis sinensis, voided with fæces.

$G$, Opisthorchis felineus, voided with fæces.

$H$, Opisthorchis noverca, voided with fæces.

I, Fasciolopsis buski, voided with fæces.

$\vec{J}$, Gastrodiscoides hominis, voided with fæes.

$K$, Heterophyes heterophyes, voided with fæces.

$L$, Yokagawa yokagawa, voided with fæces.

$M$, Tonia saginata, voided with fæces, usually in proglottids.

$N$, Tania solium, voided with fæces, usually in proglottids.
O, Hymenolepis nana, voided with fæces, usually in proglottids.

$P$, Hymenolepis diminuta, voided with fæces, usually in proglottids.

Q, Dibothriocephalus latus, voided with fæces.

$R$, Diplogonoporus grandis, voided with fæces.

$S$, Davainea madagascariensis, voided with fæces, usually in proglottids.

$T$, Dipylidium caninum, voided with fæces, usually in proglottids.

$U$, Ascaris lumbricoides, voided with fæces.

V, Trichuris trichiura, voided with fæces.

W, Ancylostoma duodenale, voided with fæces.

$X$, Necator americanus, voided with fæces.

$Y$, Trichostrongylus orientalis, voided with

$Z$, Oxyuris vermicularis, voided with fæces. 
In cases of heavy infection it is usually possible to find the eggs in the fæces or other excretions by simple microscopic examination of a smear made directly from the fæcal sample. Many light infections, however, escape detection by this method, and various means of concentrating the eggs so that they can be more readily found have been devised. One method of concentration is to mix with water, strain, and centrifuge, thus eliminating coarse material and throwing the relatively heavy eggs to the bottom of a tube. The principal objection to both methods is the small size of the sample which is examined.

Several methods for the quick and efficient examination of large samples of fæces have recently been devised. One method, known as the glycerine-salt method, developed by Barber, consists in thoroughly mixing a fairly large fæcal sample with a mixture of equal parts of glycerine and saturated magnesiun sulphate solution. The eggs present float to the surface and can be poured off on large slides for examination. By centrifuging negative specimens and again floating the eggs in a glycerine-salt solution a still higher percentage of positives can be found. In a test trial in Siam 35.5 per cent of 45 fæcal samples were found to be hookworm-infected by examination of two slides by the plain smear method, whereas 84.4 per cent were positive by the examination of a single slide by the glycerine-salt method, and 86.6 per cent by examination of two slides.

A still more accurate method is the brine flotation-loop method of Kofoid and Barber. A large fæcal sample is thoroughly mixed with concentrated brine in a paraffin paper container of two or three ounces capacity. The coarse float is forced below the surface by means of a disk of No. 9 steel wool, and the container is allowed to stand an hour for the eggs to float to the surface. The surface film is then wiped off with wire loops one-half inch in diameter, the material transferred to a large slide, and examination made. The number of positives escaping detection by this method is practically negligible. Another very simple and effective method is to mix the fæcal sample thoroughly with a concentrated salt solution in a container so filled that there is a slight meniscus. After standing a few minutes the eggs rise and adhere to a glass slide touched to the surface of the meniscus. This method is very effective especially for hookworm eggs, but is said not to be useful for operculated eggs or for larvæ. 


\section{CHAPTER XII}

\section{THE FLUKES}

General Account. - The flukes are animals of a very low order of development in some respects and of very high specialization in others. In shape they are flat and often leaflike, with the mouth at the bottom of a sucker at the anterior end and with a second little sucker, for adhesion, on the ventral side of the body. They are all parasitic when adult and attach themselves to their hosts, either externally or internally, by means of their suckers, sometimes aided also by hooks. The development of the nervous system is of a very low grade, and the only tendency towards a brain is the presence of a small ganglion at the forward end of the body which gives off a few longitudinal nerves. Sense organs are almost lacking - there is usually no sense of sight and none of sound; in fact no sensations whatever except a meager sense of touch falls to the lot of these lowly animals. There is no blood or blood system, the result being that the digestive tract and excretory system are branched, often to a surprising extent, in order to carry food to all parts of the body and to carry waste products out from all parts. In these respects the flukes are very primitive animals, but in other respects they equal or surpass any other animals in their complexity. We would have to look long to find more intricate and highly specialized reproductive systems than they possess, and their life histories are so marvelously complex as to tax our credulity. We are accustomed to think of a butterfly as having a wonderful life history in that it passes through two phases of life, the first as a caterpillar, the second as a mature butterfly, the two being separated by a third inactive phase of existence. But by comparison with the flukes this life history appears simple. Many flukes, especially those which live as internal parasites in the land animals, pass through four and sometimes even five distinct phases of existence, during some of which they are free-living, and during others may parasitize successively two or even three different hosts. 
In all flukes except those of the family Schistosomidæ both male and female reproductive systems occur in the same individual, and occupy a large portion of the body of the animal. We are familiar with animals which appear to live almost wholly to eat; the flukes are animals which seem to live merely for reproduction. They are reproductive machines, all the other organs of their bodies being developed only to a sufficient extent to ensure the proper development and maturity of the eggs. The eggs proper and the shell materials are produced by separate glands, and sometimes the canal for conducting the sperms from another individual into the body to fertilize the egg is distinct from that which conducts the eggs out of the body. The male system consists of two or more glands or testes for the production of the sperms, two sperm

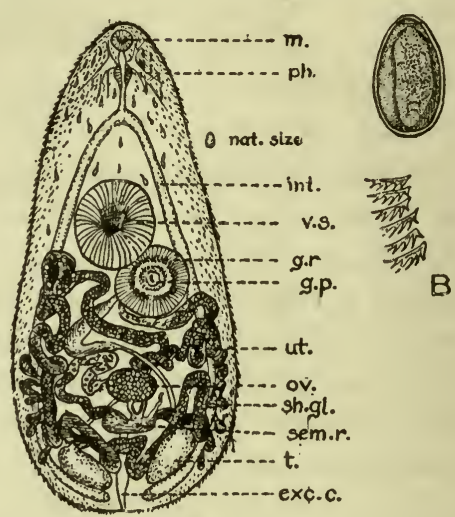

4

Fig. 62. Heterophyes heterophyes, a very small intestinal fluke of man; $A$, adult; $B(\times 350)$, spines from genital ring; g. r., genital ring; g. p., genital pores; other abbrev. as in Fig. 74. $\times 33$. Egg shown above, $\times 500$. (After Looss.)

ducts which meet and enlarge into a "cirrus pouch" for storing the sperms until ready to be used, and a rectractile copulatory organ. All these complex sexual organs in a single animal which may be no larger than the head of a pin (Fig. 62)!

Almost as soon as the fluke reaches its final host and assumes its mature form, development of the reproductive systems begins. Although both sexes are usually in the same individual, mutual cross-fertilization generally takes place, the sperms of two individuals simultaneously fertilizing each other. The number of eggs maturing in a single fluke is enormous, and while it undoubtedly varies in different species and in different individuals, the eggs are probably always to be reckoned in the thousands, and sometimes in the hundreds of thousands.

Life History. - The life histories of all the flukes which are internal parasites have much in common, and all of them undergo a series of marvelous transformations from egg to adult.

The fluke which is most thoroughly known in every respect 
is the almost cosmopolitan liver fluke, Fasciola (or Distomum) hepatica, of sheep, goats and other ruminants. This species occasionally establishes itself in man also, but it can be looked upon only as an accidental parasite as far as man is concerned. Its life history (shown diagrammatically in Fig. 63) will be described in some detail since it is more thoroughly known than is that of most of the flukes, and because it is typical of the group.

The adult of the liver fluke (Fig. 63A) lives normally in the bile passages and liver tissue of its host. About three weeks after the flukes have reached their destination in or near the liver, reproduction commences. Eggs (Fig. 63B) begin to pass out through the uterus of the fluke, and are carried by the bile of the host to the intestine and thence out of the body by the fæces, a single fluke producing as many as 50,000 eggs. Since there may be over 200 flukes in a single host, the number of eggs voided may amount to many millions. These eggs, if they chance to fall into water of moderate temperature, hatch out little ciliated embryos known as miracidia (Fig. 63D), which resemble ciliated protozoans. They are about $100 \mu\left(\frac{1}{5} \frac{1}{0}\right.$ of an inch) in length. Each of the embryos swims about for a day or two, by means of its cilia, in an effort to find a suitable intermediate host, in this case certain species of snails of the genus Limncea (Fig. 63E), and if successful it bores into the snail by means of a little pimple-like projection at the anterior end of the body. It is obvious that only a small per cent of the embryos are likely to survive the double risk of not reaching water, and if safely in water of not reaching a suitable snail to bore into. However, once safely within the tissues of the snail, the embryo begins the second phase of its existence, during which it reproduces to make up for the enormous mortality encountered in the transfer from sheep to snail.

In the course of some days the ciliated embryo transforms into a saclike body or "sporocyst" (Fig. 63F), the inner " germinative" cells of which act as parthenogenetic eggs (i.e., eggs which do not need fertilization), each developing into a larva of a new type, known as a redia (Fig. 63G). The latter, when nearly mature, burst the wall of the mother sporocyst and migrate into other tissues of the snail. The rediæ are very simple organisms with a sucker and an unbranched blind pouch for a digestive tract. Like the sporocyst they contain germinative cells within 


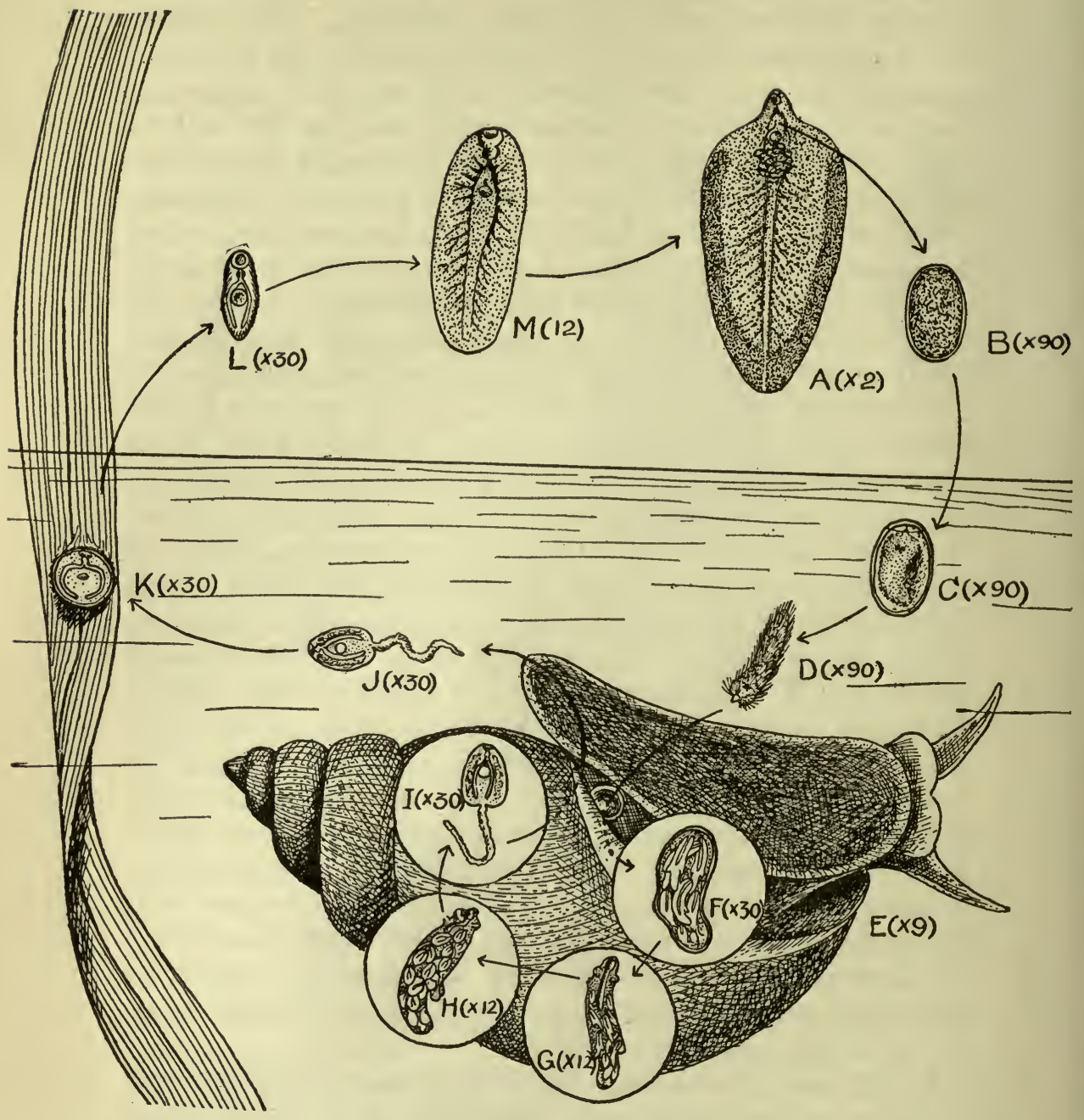

Ftg. 63. Life history of liver fluke, Fasciola hepatica; $A$, adult in liver of sheep; $B$, freshly passed egg; $C$, egg with developed embryo, ready to hatch in water; $D$, ciliated embryo in water, about to enter pulmonary chamber of snail $(E) ; F$, sporocyst containing rediæ; $G$, redia containing daughter rediæ; $H$, redia of 2 nd generation containing cercariæ; $I$, cercaria; $J$, same, having emerged from snail into water; $K$, ccrcariæ encysted on blade of grass; $L$, cercaria liberated from cyst after ingestion by sheep; $M$, young fluke developing in liver of sheep. 
their bodies and these develop into a second generation of rediæ (Fig. 63H) ultimately escaping from a little "hatching pore" in the body wall of the parent. In this way even a third generation of rediæ may be developed, but usually the second generation of rediæ produce from their germinative cells a new type of larva, the cercaria (Fig. 63I), quite different from either the embryo or the redia. The cercariæ are furnished with a sucker and a forked digestive tract, and have an actively moving tail. They worm their way out of the body of the snail in which they were developed and swim about in the water by means of lashing movements of their tails (Fig. 63J). Eventually they attach themselves to a submerged blade of grass or aquatic plant, lose their tails, secrete a cyst about themselves (Fig. 63K), and wait to be eaten or drunk by a sheep or a goat. When so swallowed the cyst is dissolved off in the stomach, and the little parasite (Fig. 63L) wends its way up the bile duct to the liver, there to begin again the reproduction of eggs and start a repetition of the entire cycle.

Such is the life history of the liver fluke. In some flukes this strange life is further complicated by the invasion of a third host by the cercariæ. In some fluke parasites of frogs, for instance, the rediæ inhabit certain snails, while the cercariæ inhabit insect larvæ, and infect their ultimate host by being eaten with the insects. Several human flukes, including the lung fluke, Paragonimus ringeri (westermani) have a life history of this type. The encysted cercariæ of the lung fluke are found in the tissues of several species of fresh-water crabs and in the earlier stages are believed to be parasites of a snail on which the crabs feed. The Chinese liver fluke, Clonorchis sinensis, parasitizes successively a snail, a fish and a mammal.

In some species of flukes daughter sporocysts are formed instead of rediæ, and in some the sporocysts give rise to cercariæ directly. The known types of life histories of flukes are graphically shown by the following diagram, copied from Leiper:

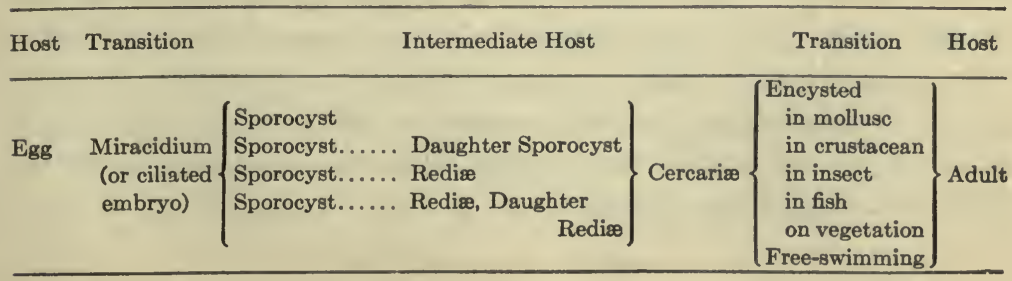


tube of an aquatic animal, another in the conjunctival sac in the eyes of birds.

The flukes which infest man may be divided for convenience into four groups, the blood flukes, the lung flukes, the liver flukes and the intestinal flukes. Altogether over 20 different species have been found in man, but only those which are common or important will be considered in the following pages.

Control. In all known cases freshwater snails act as intermediate hosts for the flukes which infest man and domestic animals. A practical and efficient method of destroying these snails would make the ultimate eradication of fluke diseases, in spite of the difficulty in treating them, a matter of brigher prospect than the eradication of hookworm and other intestinal parasites, in which sanitary disposal of fæces must be relied upon.

Recent experiments by Chandler show that copper salts exert a powerful effect upon snails, even in very high dilution, apparently acting by inhibition of necessary enzymes. In experiments on a number of snails of six different families, including members of all the families of which species are known to act as intermediate hosts for pathogenic flukes, it was demonstrated that copper sulphate in proportions of one part in from 500,000 to $2,000,000$ parts of water destroys the snails in all cases within 48 hours. From the point of view of expense, harmlessness and convenience in use, copper sulphate is preferable to any other substance which has been tried or suggested for destroying snails. In the dilute solutions which are necessary the water is not injured either for human or animal consumption, for bathing, or for agricultural purposes. It is destructive to very few other organisms in the water, except algæ. It is, however, injurious to some species of fish, especially the young, and its use may be objected to for this reason in some places where fluke diseases are prevalent, e.g., in oriental countries, where fish are extensively kept in the snail-infested waters and form an important part of the native diet. The eggs of the snails are not destroyed by the copper salts. With government aid and supervision, the work being carried out under the direction of scientifically trained men or commissions, it seems entirely possible that whole states or countries, at least in the vicinity of towns or villages, could be freed of human fluke diseases. 


\section{Blood Flukes}

The most important flukes parasitic in man are three species of Schistosoma (or Bilharzia) which live in the large bloodvessels of the abdominal cavity.

Schistosoma is one of the few genera of flukes in which the sexes are separate. The relation of the sexes is one of the most remarkable in nature. The mature male worm (Fig. 64) has a cylindrical appearance due to the fact that the sides of the flat body are folded over to form a ventral groove. In this groove, projecting free at each end but enclosed in the middle, is the longer and slenderer female, safe in the arms of her lord. While young the sexes live apart, but as soon as sexual maturity is attained they couple together and spend the rest of their lives in this manner.

Unlike the liver flukes, the blood flukes do not develop great numbers of eggs all at once, but instead develop them one by one and have only a few in the oviduct at any one time. Such a method of reproduction is facilitated, of

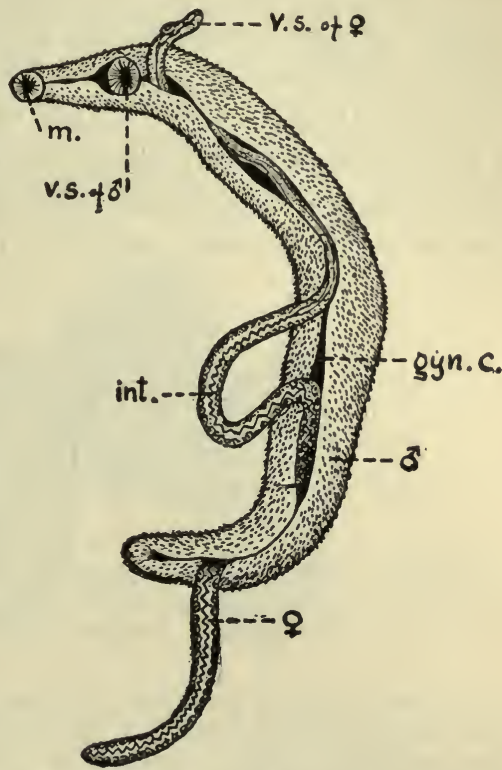

Fig. 64. Blood fluke, Schistosoma homa. tobium; male ( $\delta$ ) carrying female $(\xi)$ in ventral groove; int., intestine; gyn. c., gynecophoric canal or ventral groove; m., mouth; v. s., ventral sucker. $\times 8$. (After Looss.) course, by the constant juxtaposition of the male and female worms. The blood flukes live correspondingly much longer than the liver flukes, often persisting for many years.

Schistosoma haematobium. - The most important species from the pathogenic point of view is Schistosoma homatobium (Fig. 64). This parasite is common in the countries surrounding the eastern end of the Mediterranean, southern Asia and many parts of Africa, especially the east coast. In Egypt over half 
the population is said to be infected, and in an examination of 54 boys in the village of El Marg, near Cairo, 49 were found infected.

These flukes, about one-half inch in length, abound sometimes in hundreds in the abdominal veins of their host, living especially in the portal vein and its various branches. The eggs of the worms, which are oval with a stout spine at one end (Fig. 65A), and about $0.16 \mathrm{~mm}$. ( $\frac{1}{160}$ of an inch) long, are carried to the small vessels on the surface of the urinary bladder. By means of the sharp spine they penetrate to the wall of the bladder and are voided with the urine. As the eggs enter the bladder they cause a certain amount of bleeding, resulting in a bloody urine. From this symptom the disease caused by infection with Schistosoma hæmatobium is often called "parasitic hæmaturia." Except
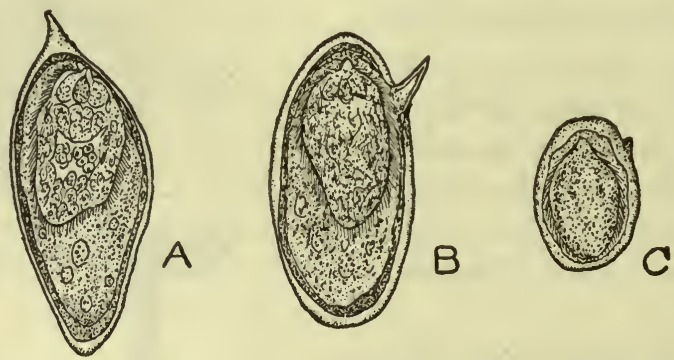

FIG. 65. Eggs of Schistoma; $A$, terminal spined egg or $S$. næmatobium from urine; $B$, lateral spined egg of $S$. mansoni from fæces; $C$, egg of $S$. japonicum, with only rudiment of spine; note developed embryos in all. $X$ about 200. ( $A$ and $B$ after Looss, $C$ after Leiper.)

in severe infections no serious symptoms appear, but when numerous the worms cause much pain and give rise to a great variety of abnormal conditions of the bladder. The damage they do is partly the result of blocking of the veins, and partly the result of inflammation and bleeding of the bladder caused by its penetration by the spined eggs. Sometimes the kidneys, ureters and other urino-genital organs are attacked and seriously affected. In addition there can be little doubt but that the worms excrete poisonous matter into the blood, as practically all parasitic worms do to some extent, and this probably accounts for part at least of the anemic and debilitated condition so common in infected 
people. Recent experiments by Fairley seem to corroborate this view. It is reported that of 625 British soldiers who became infected with blood flukes in South Africa during the Boer war, 359 were still on the sick list in 1914 exclusive of those permanently pensioned. The cost to the British government for permanent and "conditional" pensions for these soldiers amounted to nearly $\$ 54,000$ a year.

The life history of Schistosoma hoematobium has only recently been worked out by Leiper, of the British Army Medical Corps, in Egypt. It was long known that a ciliated embryo or miracidium developed inside the egg shells, even before they left the body of the host, and that these embryos hatched out and swam
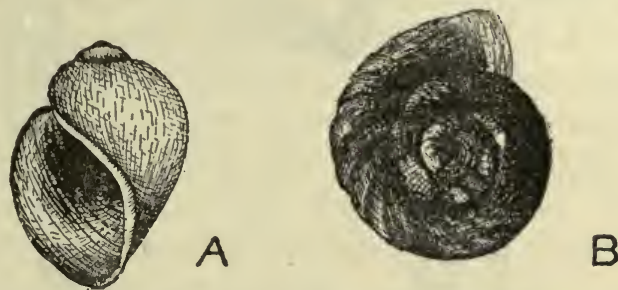

FIG. 66. Egyptian snails which serve as intermediate hosts for blood flukes; $A$, Bullinus contortus, an intermediate host for Schistosoma homatobium; B, Planorbis boissyi, an intermediate host for Schistosoma mansoni. (After Leiper.)

about when the eggs were immersed in water, but beyoud this point the life history could only be conjectured from analogy with the liver fluke. Leiper, who had already made some investigations in regard to the life history of S. japonicum in China, worked on the life history of this species, chiefly at El Marg, near Cairo, Egypt. He found that Schistosoma embryos are attracted by several species of fresh-water snails and that they penetrate the bodies of three species, Bullinus contortus (Fig. 66A), B. dybowskii and Planorbis boissyi (Fig. 66B). Here they undergo transformation into sporocysts, from which daughter sporocysts bud off (Fig. 67). After leaving the mother cyst the daughter sporocysts migrate into the tissue of the liver and grow rapidly. They become greatly elongated and eventually ramify throughout the organ, so increasing its bulk and color that an infected snail can be detected at a glance. The sporo- 
cysts move by wriggling movements, and absorb nourishment directly through the body wall. When they become over-distended with the cercariæ developing within them the wall ruptures and the cercariæ are set free in the snail. The cercariæ are discharged from the mollusc in "puffs," a number being periodically shot into the water.

Examination of molluses which were collected in the El Marg canal resulted in finding 17 species of cercariæ, among them the cercariæ with forked tails and no bulb in the œesophagus, the

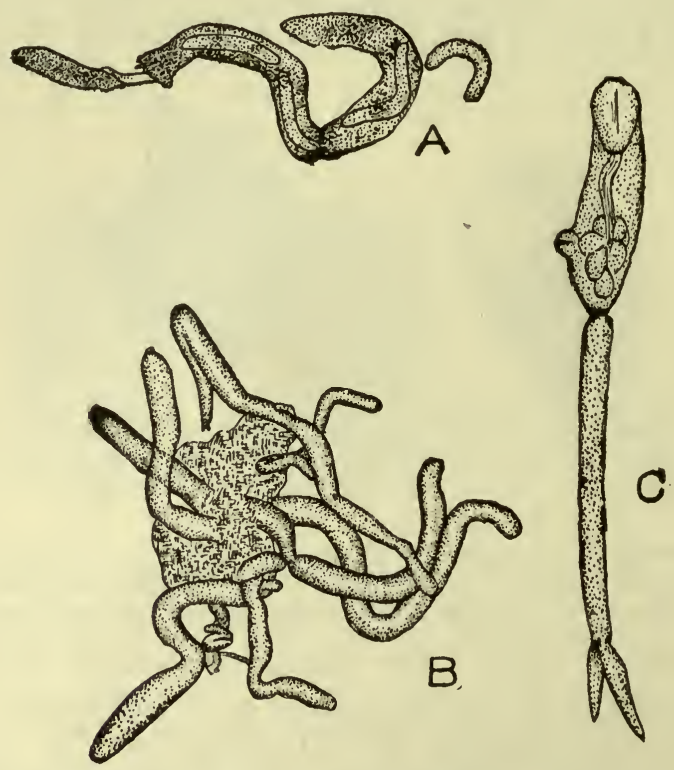

Fig. 67. Larval forms of blood flukes teased from liver of Planorbis; A, sporocyst containing daughter sporocysts; $B$, daughter sporocysts in liver tissue; $C$, cercaria. Note forked tail, characteristic of Schistosoma cercariæ. (After Leiper.)

typical form of Schistosoma cercariæ (Fig. 67C). Infected molluses may continue to liberate cercariæ for several weeks. Leiper later found that $S$. homatobium developed only in the species of Bullinus, the cercariæ from Planorbis belonging to another species, S. mansoni. In Natal and the Transvaal a small 
dark-colored snail, Physopsis africana, acts as an intermediate host.

When fully developed the cercariæ escape from the snails and swim about in water in search of a final host. They do not live at best as long as 48 hours, so a vast majority of the larvæ must perish from failure to find a suitable host. It has been shown that not only man but also various species of monkeys and rodents may be infected by the cercariæ.

Infection may occur in two different ways: by drinking water containing cercariæ, or by bathing in it, since the cercariæ are able to penetrate either the mucous membranes or the sound skin, migrating through the body until they reach their destination in the abdominal veins. The natives of some parts of Africa realize that infection may result from bathing, but from the nature of the disease they believe that infection takes place by way of the urinary passages and therefore employ various mechanical devices to prevent infection in this manner. There is little doubt, when infection occurs from drinking water, that the cercariæ adhere to the walls of the mouth and throat and bore through them, since passage through the acid juices of the stomach is apparently fatal for them.

The disease usually develops in from two to four months after infection.

Treatment and Prevention. - Recent work by Christopherson has shown that tartar emetic has a specific action on both the worms and the eggs, and leads to a rapid improvement in the condition of the infected individuals. The drug is given in the form of intravenous injections on alternate days beginning with $\frac{1}{2}$ grain in 40 to 50 minims of saline solution, and increasing the dose by $\frac{1}{2}$ grain with each injection until a dose of 2 to $2 \frac{1}{2}$ grains is reached. The injections are continued until all of the voided eggs are sterile and show evidence of deterioration. Usually the total amount of tartar emetic required is between 20 and 30 grains. Christopherson's work has been confirmed by a number of other investigators. The dead eggs imbedded in the tissues may continue to cause inflammation for a time, but the symptoms gradually disappear. Even without reinfection some of the worms, if the disease is left untreated, may continue to live and produce eggs for years, but in most longstanding cases reinfection probably occurs frequently. 
Now that the life history and modes of infection are known, definite preventive measures can be taken. Prevention of contamination of drinking water by infected urine is, of course, the ideal preventive measure, but in countries where the disease is most prevalent, as in Egypt, coöperation of the natives in such a matter is more than can be expected. Leiper has pointed out, however, that the disease can be eradicated without such coöperation by other means, depending upon local conditions. In large towns and cities it is practical to destroy the free-swimming infective stage of the worm by filtering or impounding water, while in rural districts the worm must be deprived of its intermediate host. Cairo, for example, obtains its water supply from the Nile, part of it being unfiltered. Water used for irrigation purposes above Cairo, and frequently contaminated, is turned back into the river and is probably the chief source of infection at Cairo, where 10,000 children are said to become infected annually. In towns where filtering is impractical the water could be rendered uninfective by impounding it in protected raservoirs for 48 hours, since the cercariæ die in this time. The objection to this is that the water loses valuable sediment, but it is doubtful whether the agricultural loss from lowered vitality resulting from Schistosoma infection is not greater than is the loss in fertility from impounding water.

It seems very probable that the use of copper sulphate for destroying snails in ponds or irrigation ditches in agricultural districts, as outlined on p. 211, will prove to be an effective means of controlling the disease. At present a preventive measure used in Egypt is the intermittent flow of water in irrigation canals, under government control. The snails which serve as intermediate hosts for Schistosoma are said to die if the water in which they live is dried up. It is customary for water to be turned out of most irrigating canals for periods of $\mathbf{1 5}$ days at a time, which Leiper says would be sufficient to destroy molluses in them except in puddles left by an uneven floor, which must be treated by chemicals, or the floors leveled. However, in view of the remarkable resistance which most snails have to drouth and to other adverse conditions, this conclusion ought to be proven by extensive experimentation. Infected water to be used for washing can be 
rendered non-infective by the addition of one part of cresol in 10,000 parts of water.

Leiper's work shows that transient collections of water are not sources of infection after recent contamination, whereas all permanent collections of water, as in rivers, canals and marshes, are dangerous if inhabited by a suitable intermediate host. The removal of infected persons from a given body of water would have no immediate effect in reducing its infectiveness, since the snails discharge cercariæ into the water for a prolonged period. The preventive measures briefly outlined above were worked out by Leiper for the special conditions existing in the infected parts of Egypt but in a broad way they are applicable wherever Schistosoma hømatobium occurs.

Other Species. - There are two other species of Schistosoma which are pathogenic to man. One, S. mansoni, was long confused with $S$. hoematobium, the only apparent differences having been observed in the eggs. The eggs of S. mansoni (Fig. 65B) are provided with a lateral instead of a terminal spine, and are voided in the digestive tract and its appendages, whence they are liberated with the fæces, instead of making an exit from the body by way of the urinary organs. By experimental infections of mice Leiper showed that cercariæ from the snail Planorbis boissyi (Fig. 66B) developed into worms somewhat smaller than those from the species of Bullinus, with certain distinct differences in anatomy. The cercariæ from Planorbis produced only lateralspined eggs which were voided with the fæces, thus showing that $S$. mansoni was really a distinct species and not merely an abnormal type of S. hoematobium. This parasite occurs in common with the foregoing species in many parts of Africa and is also common in the West Indies, Venezuela and perhaps other parts of tropical America. Like the hookworm, it was probably introduced from Africa in the slave days. The intermediate host in Venezuela has recently been shown by Iturbe and Gonzales to be the snail Planorbis guadelupensis, whereas in Brazil Lutz has shown $P$. olivaceus to be the intermediate host.

The anemia and debility caused by $S$. mansoni is similar to that caused by $S$. homatobium. The irritation and inflammation of the urinary organs is replaced, however, by similar symptoms of the intestine, and a kind of dysentery often results.

The manner of transmission of the parasite is similar to that 
of Schistosoma homatobium except that infected fæces instead of urine contaminates water inhabited by a suitable intermediate host, as Planorbis boissyi.

A third species of Schistosoma, S. japonicum, is endemic in parts of Japan, China and the Philippine Islands, and perhaps in many other oriental countries. It is slightly smaller than the other species (about two-fifths of an inch in length) and produces eggs (Fig. 65C) which do not have the spine that is so characteristic of the other species of Schistosoma, but only a rudiment in the form of a little lateral knob. The eggs of $S$. japonicum, like those of $S$. mansoni, are voided from the intestine with the fæces. They also frequently become lodged in the liver gall bladder, walls of mesenteric bloodvessels, spleen, pancreas, and sometimes other organs, not even the brain being exempt. The female worm must in some way deposit her eggs outside the bloodvessel in which she lives since they are apparently carried to their destination by the lymph streams. Severe infections with this parasite usually prove fatal sooner or later, and post-mortem examinations show many of the organs of the body to be badly affected. Infection with $S$. japonicum is associated with a skin disease known to the natives as "kabure," and probably caused by the burrowing of the cercariæ in the skin. According to Laning of the U. S. Navy, it is not an uncommon thing for large per cents of the crews of patrol gunboats in the Yangtze River to be completely disabled by infection with this parasite. Laning divides the disease caused by $S$. japonicum into three stages. The first stage, lasting from three to six weeks, is marked by high afternoon temperatures, slow pulse, respiratory troubles, transient œdema and rash on the skin and mucous membranes, abdominal pains, digestive irregularities and sometimes mental disturbances. The second stage is marked by enlarged liver and spleen, dysenteric symptoms, anemia and irregular fever. The third stage, which does not always occur, but may appear in from three to five years where there are constant reinfections, is marked by diseased liver, œdema of legs and arms, emaciation, anemia and dysentery, and death from exhaustion is not uncommon.

The life history and mode of infection in the case of S. japonicum is undoubtedly very similar to that in other species of Schistosoma. Miyairi, a Japanese investigator, found reproductive stages of 
S. japonicum in a species of Limnoea in Japan. Miyairi and Suzuki, in further work on the life history of this fluke, found that after sporocysts have developed in the tissues of infected snails rediæ are produced, 50 or more from each sporocyst. The long, coiled rediæ crowd the liver and produce cercariæ, the latter reaching maturity in about seven weeks. If in autumn the cercariæ have become fully developed but have not left the snails they remain in their hosts over winter.

Leiper obtained development of the characteristic fork-tailed cercariæ in another small snail, Blanfordia (or Katajama) nosophora, common in the rice fields of Japan. There is an interesting tale connected with Leiper's experiments on infection with these parasites. With great care this investigator experimented with the infection of snails which he had imported from Japan to work on in his laboratory at Shanghai. After having succeeded in obtaining infection of the snails, he teased out the livers in water to liberate the cercariæ. Four laboratory-bred mice, which are difficult to obtain in the Orient, were immersed in the water

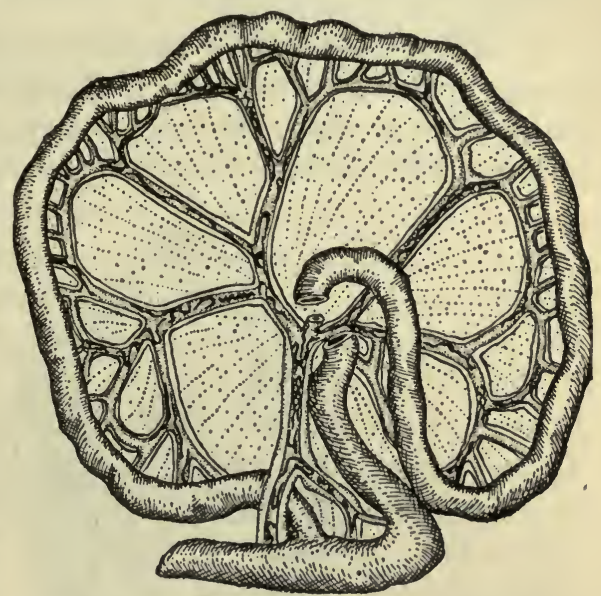

FIG. 68. Mesentery of mouse with bloodvessels infected with Schistosoma. (After Leiper.) in which the cercariæ had been liberated, and a start was made for England. But alas! a woman in a neighboring stateroom objected to the presence of the mice so near and demanded their relegation to the butcher's cabin, where three of them died. At Aden the few remaining infected molluses were sacrificed and the last mouse was subjected to infection. A month later when the animal was examined in the laboratory of the London School of Tropical Medicine many blood flukes, males and females in couples, were found in the portal bloodvessels (Fig. 68).

It should be remarked in concluding this discussion of the blood flukes that many snails, including members of the genera 
Planorbis and Limnoea, which could very probably act as intermediate hosts as well as the species in which the development has actually been observed, are abundant in the United States, and there is great danger that if once introduced, at least in the warmer parts of the country, these blood flukes might become endemic. Careful examination of immigrants from endemic countries and exclusion of Schistosoma-infected persons is important if the infection is to be kept from becoming established. An ounce of prevention in this country is worth a pound of cure.

\section{Lung Flukes}

In Japan, China, the Philippines, and other oriental countries, a region which seems to be particularly afflicted with fluke diseases, there occurs a very serious lung disease caused by a species of fluke, Paragonimus ringeri (westermani) (Fig. 69). It is also

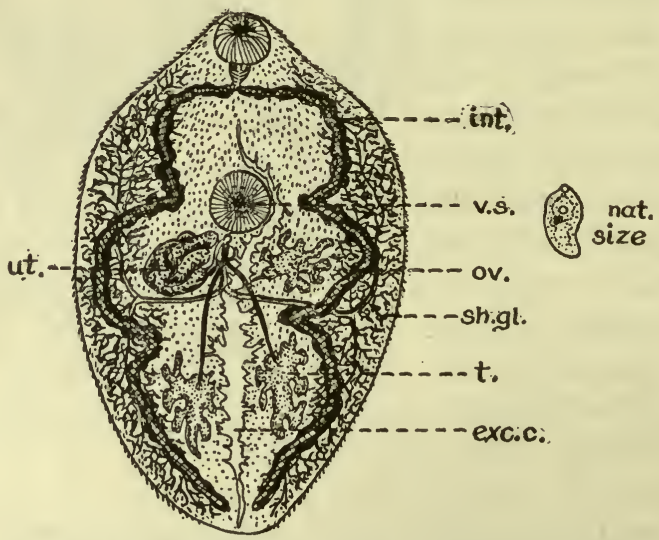

FIG. 69. Lung fluke, Paragonimus ringeri. Abbreviations as in Fig. 74. $\times$ about 7. (Partly after Looss, partly after Leuckart.)

found in dogs. In some parts of Formosa fully 50 per cent of the population is infected. A closely allied species, P. kellicotti, occurs in hogs in the United States, and probably in other parts of the world.

The lung fluke is about half an inch in length, reddish brown in color, and relatively very broad. The adult lives most frequently in the lungs of its host, where it produces cavities an inch or two in diameter. The cavities become filled with various 
tissues through which the parasite tunnels out its burrows and in which it deposits its eggs (Fig. 70). These excavations in the lung connect with the bronchial tubes, through which the blood, parasite eggs and other products are voided, thus causing the characteristic blood-spitting. The expectorations, resembling those of pneumonia, are of a peculiar brownish red color, due in part to the blood corpuscles present and in part to the dark brown fluke eggs, which are often very abundant.

Occasionally the lung fluke burrows in other organs and glands of the body, such as the liver, spleen, muscles, intestine and

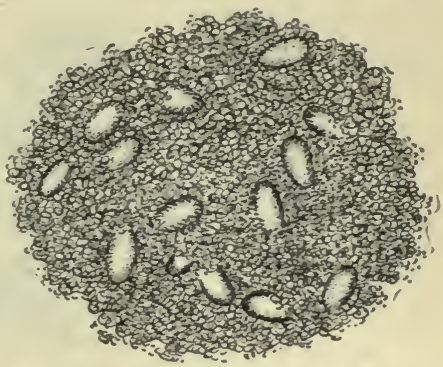

FIG. 70. Eggs of lung fluke in contents of cyst in lung of hog. $\times$ about 50. (After Stiles and Hassall.) brain. Musgrave found in the Philippines that sometimes many parts of the body are infested at once, and in one case he found over a hundred mature parasites in a muscular abscess. When they burrow in the brain they cause epileptic fits and usually in time cause death.

The eggs of the lung fluke (Fig. 71A) when immersed in fresh
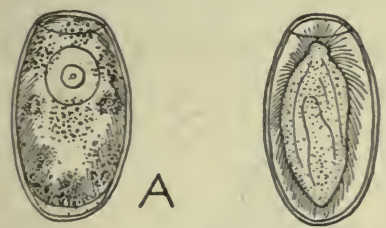

Fig. 71. $A$, freshly passed egg of lung fluke; $B$, egg of lung fluke with fully developed embryo. $\times$ 250. (After Katsurada.) water for several weeks develop within themselves typical ciliated embryos or miracidia (Fig. 71B). The latter burst away the little cap at the end of the egg and emerge as free-living animals.

Nakagawa has recently shown that if these miracidia are placed in water with certain species of snails, particularly Melania libertina, the miracidia swarm about the snails and burrow into them, shedding their cilia as they go. The entire cycle of development in the snail has not been worked out but it is probably very similar to that of Schistosoma. Sporocysts of various sizes occur in the liver and other tissues of the snail, and it is probable that these produce the cercariæ directly.

Nakagawa discovered the encysted cercariæ of this species, proved to be such by experimental infection of animals, in three 
species of crabs in Formosa, and Yoshida, another Japanese investigator, acting on the discovery of his countryman, found the larvæ in a fourth species of crab in Japan. The crabs most commonly infected are Potamon obtusipes, a coarse-shelled, chestnut-

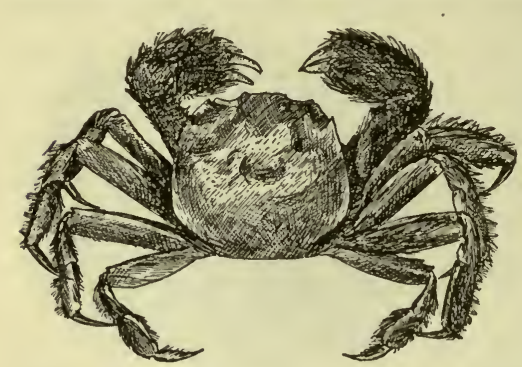

FIG. 72. A common fresh-water crab of Japan, Eriocheir japonicus, which serves as a host for lung fluke. (After Yoshida.) colored crab about one and a half inches in diameter, and $P$. dehaanii, a slightly smaller species, grayish black or reddish in color. Both these crabs bound in the shallow waters of mountain streams, and the former species is sometimes used as food. Another implicated species, Eriocheir japonicus (Fig. 72), is abundant in all plains rivers in Japan and is a common article of diet throughout the country. It is a larger crab, reaching a diameter of three inches, and has large hairy claws. The fourth species, Sesarma dehaani, is of medium size, dark in color with light reddish claws, and inedible. Miyairi has shown that in Korea another crab, Astacus japonicus, is the intermediate host.

The lung fluke cercariæ encysted in these crabs (Fig. 73A) were found chiefly in the liver while young, but when older they

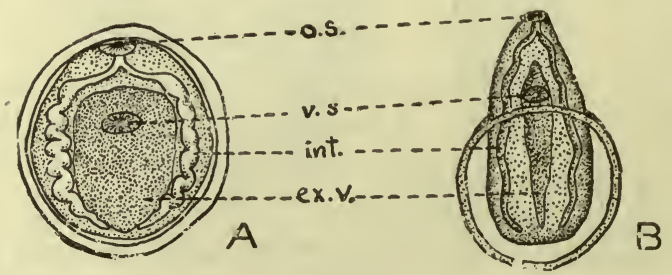

Fic. 73. A, encysted cercaria of human lung fluke, Paragonimus ringeri, from gill of crab; $B$, larva emerging from cyst. o. s., oral sucker; int., intestine; ex. v., excretory vesicle; v. s., ventral sucker. $\times 50$. (After Yoshida.)

occur in the gills. They vary in number from a few to several hundred. In some localities a very high per cent of crabs are infected, Nakagawa reporting that practically 100 per cent are infected in one district in Formosa where the lung fluke is very common. The cysts containing the cercariæ are nearly round, $0.5 \mathrm{~mm}$. ( $\mathrm{B}^{\frac{1}{6}}$ of an inch) or less in diameter, and have relatively 
thick walls. The enclosed cercaria lies straight, unlike most encysted cercariæ, and the body is entirely covered by short spines. In fully-developed specimens the suckers, digestive tract and other parts of the anatomy of the enclosed cercariæ can be seen (Fig. 73A). While still in the cysts the cercariæ are fairly resistant to unfavorable environmental influences, but are easily destroyed after hatching.

When an encysted cercaria is swallowed by a susceptible animal the cyst wall is dissolved off in the intestine, the active liberated larva (Fig. 73B) bores through the intestinal wall, wanders about in the abdominal cavity for some time, then bores through the diaphragm into the pleural cavity, whence it eventually penetrates the lungs from the outer surface. It becomes mature in about 90 days. Occasionally the worms apparently get lost and bore through the abdominal wall and muscular connective tissues. It is probably in this way that other organs than the lungs are penetrated by the flukes.

There are two ways in which man may become infected, namely, by eating infected crabs which are not thoroughly cooked, or by drinking water containing cysts discharged from infected crabs. As already remarked, the mature cysts make their way to the gills, whence they can easily be removed, and whence they probably escape readily under natural conditions, thus becoming free in the water. Here they may remain alive for some time, probably 30 days or more. Yoshida states that the cysts sink to the bottom, in which case human infection could occur only rarely if ever from infected water. Nakagawa, however, observed that 20 per cent of the larvæ when freed float on the surface of the water.

Prevention of infection consists either in the destruction of the snails which act as the first intermediate host by the use of copper sulphate, as outlined on p. 211, or by abstinence from the use of raw crabs as food and in avoidance of water for drinking which may possibly be infected. Whether or not other animals may serve as hosts for the cercariæ is unknown, but if the allied Paragonimus kellicotti is truly endemic in the United States, where no fresh-water crabs are found, some other animal must serve as an intermediate host, possibly certain species of crayfish. The fact that the lung fluke is not known as an endemic human parasite in this country suggests that the intermediate host may be an anima! 
which is not used as food and the habits of which give little opportunity for the parasites to gain access to the human body. The disease is said to have increased in Peru, having been introduced there by Japanese and Chinese laborers. If this is true some Peruvian animal, probably a fresh-water crab, must serve as an

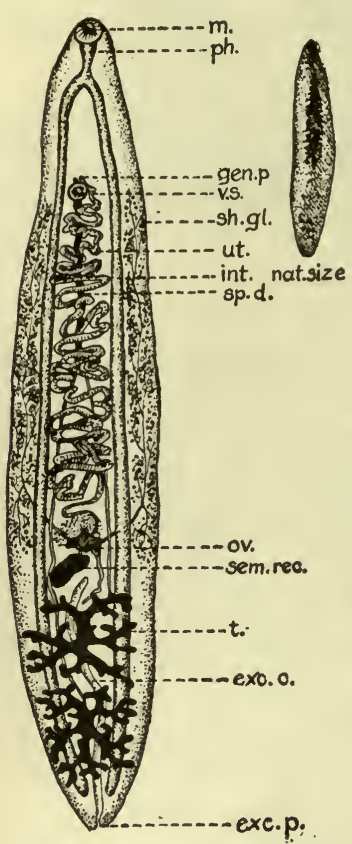

FIg. 74. The Chinese fluke, Clonorchis sinensis. $\times 3 \frac{1}{2}$. m., mouth in oral sucker; ph., pharynx; gen. p., genital pores; v. s., ventral sucker; sh. gl., socalled vittelline or yolk glands, really shell glands; ut., coiled egg-filled uterus; int., intestine; sp. d., sperm duct; ov., ovary; sem. rec., seminal receptacle, where sperms for fertilizing eggs are temporarily stored; t., testis; exc. c., excretory canal; exc. p. excretory pore. (After Stiles.) intermediate host. This suggests that the disease if once introduced might flourish in other countries, especially where fresh-water crustaceans are eaten. Lung fluke infection is evidently another disease for which a quarantine should be established.

\section{Liver Flukes}

Although the liver fluke of the sheep, Fasciola hepatica, and other flukes of herbivorous animals are occasionally found in man, they cannot be looked upon as usual human parasites. Adult liver flukes are sometimes accidentally eaten with raw liver, in which case they attach themselves to the membranes of the throat, causing irritation, congestion, a buzzing in the ears, difficult breathing, and other quite alarming symptoms. Vomiting to expel the worms usually gives immediate relief.

There are several species of flukes, however, which are apparently especially adapted for parasitizing carnivorous animals, and which are common human parasites in some countries. Japan, China, the Philippines and other oriental countries are especially afflicted by these flukes. The commonest species in man is the Chinese fluke, Clonorchis sinensis (Fig. 74) which is found in all of southern Asia from India to Korea. In some parts of Japan about 60 per cent of the population are said to harbor it in their livers, sometimes in hundreds or even thousands. 
In some districts in Korea 80 per cent of the inhabitants are said to be infected. Leiper found it common in both dog and man in the vicinity of Shanghai. It is also found in the liver ducts of cats, hogs, and probably other flesh-eating animals. It is from one-half to three-quarters of an inch in length, and nearly four times as long as wide. The ventral sucker is very small, and is situated on the anterior third of the body. Some authors believe that a small variety of this fluke found in Japan constitutes another species, $C$. endemicus, but this view is assailed by recent investigations. In Europe there occurs a species, Opisthorchis felineus (Fig. $75 \mathrm{~A}$ ), which is very common in domestic cats and dogs and is by no means uncommon in man; there is one record of its having been found in eight or nine out of 124 post mortem examinations in Siberia. A very closely related species, O. pseudofelineus (Fig. $75 \mathrm{~B})$, has been found in cats and coyotes in the central parts of the United States. From its similarity to the Old World species it would not be surprising to find it occasionally parasitic in man.

The European species, $B$ Fir. 75. A, Cat fluke, Opisthorchis felineus; Opisthorchis felineus, is viations as in Fig. $74 . \times$ about 5 . (A, after usually a little less than

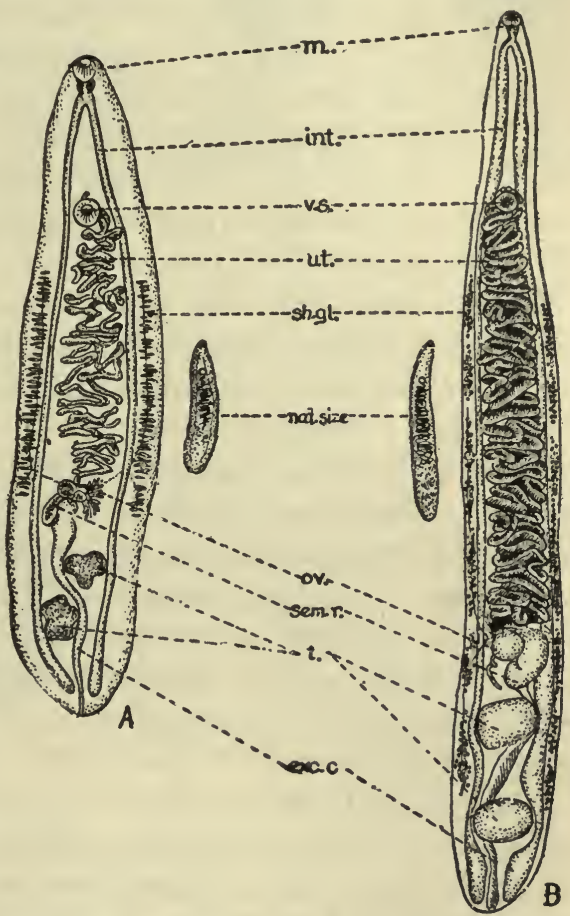
Stiles and Hassall; $B$, after Stiles.)

half an inch in length, and shaped very much like Clonorchis sinensis. The Amcrican O. pseudofelineus is somewhat longer and slenderer than the European species. Another species of the same genus, $O$. noverca, occurs commonly in pariah dogs in India, and occasionally in man. It differs from the European species chiefly in having the skin thickly beset with spines. 
Little is known. of the life history of any species except the Chinese fluke, C. sinensis. The eggs (Fig. 76A) are of characteristic shape, and hatch in water into miracidia (Fig. 76B). The encysted cercariæ of this fluke (Fig. 77A) have been found
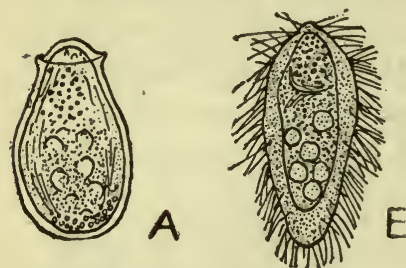

Fra. 76. Egg and ciliated embryo of Chinese fluke, Clonorchis sinensis. $\times$ 700. (After Katsurada.)

in the subcutaneous tissues and muscles of 12 different species of fresh-water fish. The cysts, which are very small, measuring only about 0.14 by $0.10 \mathrm{~mm}$. $\left(\frac{1}{180}\right.$ by $\frac{1}{250}$ of an inch), are usually more abundant in the superficial than in the deeper tissues. Although cysts can be found in fish throughout the year, the younger ones are more frequently met with in late summer and early autumn.

When infecte fish are eaten, according to experiments recently made with animals by Kobayashi, the larval flukes escape from the cysts (Fig. 77B) within three hours, and in fifteen hours they may already have reached the bile duct and gall bladder. The parasites reach maturity and eggs are found in the fæces of the host within 26 days. The young flukes have a spiny cuticle until nearly mature, but the spines finally disappear.

Muto recently found cercariæ in the snail Bythinia cercaria encysted in fish; $B$, larva freed striatula var. japonica which from cyst; m., mouth in oral sucker; v. s., encyst in fish of various ph., pharynx; int., intestine. species. When these fish are fed to dogs and mice, infection with Clonorchis sinensis results. On the other hand, controls fed with fish parasitized by cercariæ from the snail Melania libertina, which Kobayashi believed to be the first intermediate host, developed infections with Metagonimus only. Sporocysts develop in Bythinia about three weeks after infection with the miracidia.

It is probable that the European liver fluke, O.felineus, and its 
Indian and American allies all have histories very similar to that of the oriental species. Their occurrence in man in countries where fresh-water fish is a common article of diet, and their frequency in animals which eat raw fish, strongly suggest fishes as intermediate hosts.

These liver flukes, like the sheep fluke, live chiefly in the gall bladder and bile ducts where they often cause much mechanical obstruction on account of their large numbers. Severe infections such as occur in countries like Japan where raw fish is commonly eaten cause symptoms of a very serious nature. One of the most prominent of these is enlargement of the liver accompanied by more or less bloody diarrhea; the latter becomes more and more constant as time goes on. The liver sometimes becomes painful, and jaundice is a frequent symptom. The patient becomes anemic, emaciated and weak, and is ready prey for other diseases. There are often periods of partial recovery followed by relapses, probably due to reinfections, and the patient ultimately becomes exhausted and succumbs to a cold, an attack of malaria, or other ailment from which one would ordinarily recover readily.

There is no specific treatment for the disease. The only measures that can be taken are to remove the patient from any possible source of reinfection and to keep him in the best possible general health, with wholesome diet, good air and proper exercise. How long the flukes persist in the liver is not known.

Means of prevention of the disease are suggested by what is known of the life history of the parasites. The most important measure is unquestionably the suppression of the habit of eating uncooked fish in places where the disease is endemic. Kobayashi has shown that while the larvæ of $C$. sinensis are killed at once on exposure to a boiling temperature and in a short time when exposed to considerably lower temperatures, they are not destroyed by exposure to vinegar for five hours, nor by refrigeration.

Much could also be done by destroying the snails which act as the first intermediate hosts by means of copper sulphate, as suggested on page 211. Another measure, which is far less reliable, is the prevention of contamination of water in which fish live. It is impossible to prevent some contamination of water by the lower animals which carry the infection, and it is nearly as difficult to prevent contamination by human fæces. The almost universal use of night soil (human fæces) for fertilizer in oriental countries 
is a serious hindrance to the sanitary disposal of such infected material. Leiper suggests that this problem may be solved by a chemical treatment of night soil which would destroy all parasite eggs or cysts and yet not injure its value as a fertilizer.

\section{Intestinal Flukes}

There are several species of flukes which appear to be common parasites of the human intestine in certain parts of the world, especially in the oriental countries where the other human flukes abound the most. Many of these flukes are very small, but they may occur in great numbers, producing practically the same effects as do tapeworms, - anemia, emaciation and general debility. Many species are probably only accidental human parasites, normally living in some other host but occasionally finding their way into the human intestine with food or water and establishing themselves there.

The smallest fluke parasitic in man is Yokagawa (or Metagonimus) yokagawa, named after a Japanese parasitologist. It is widely distributed in Japan, Korea, Formosa, parts of China, and probably other oriental countries. It infects mice and dogs as well as man. Muto infected fish with the encysted cerceriæ by feeding to them cercaria-infected snails, Melania libertina. Encysted cercariæ occur in a number of Japanese fresh-water fishes which are commonly eaten raw, particularly the "ayu". The cysts are most numerous in the connective tissue under the skin and about the fins, especially early in the season, indicating that the fish become infected by free-swimming cercariæ which bore through the skin, and not by cercariæ eaten with another host. The encysted cercariæ closely resemble those of Clonorchis sinensis, The development in the final host is said to take only from seven to ten days. $\quad Y$. yokagawa inhabits the upper portion of the small intestine, sometimes in considerable numbers, but it never seems to do enough damage to cause more than a slight intestinal catarrh. It is remarkable for the lack of a ventral sucker and is only about $1 \mathrm{~mm}$. (about $\frac{1}{25}$ of an inch) in length, and about half as broad. Its body is covered with a great many microscopic spines.

A very similar fluke, Heterophyes heterophyes (Fig. 62), only slightly larger, occurs from Egypt to Japan in a variety of animals and occasionally parasitizes man. Two species of Echi- 
nostoma normally parasitic in other animals occur occasionally in man in the Malay countries. They are distinguished from other flukes by the crown of spines around the mouth sucker. One species, $E$. ilocanum, about one-fifth of an inch long, was found endemic among some Filipinos in a prison in Manila. The other, E. malayanum, about two-fifths of an inch long, occasionally parasitizes man in the Malay countries.

Gastrodiscoides hominis (Fig. 78) is a species which is characterized by the expansion of the posterior end of the body into a great
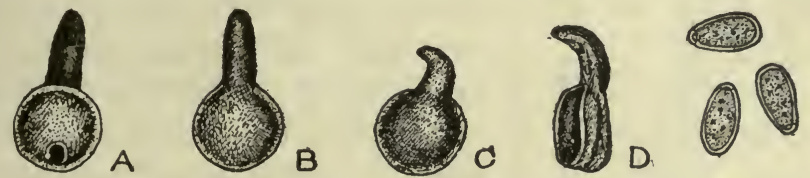

Frg. 78. Gastrodicoides hominis. $A$, ventral view, showing disc-like expansion and posterior position of ventral sucker; $B$ and $C$, dorsal views; $D$, lateral view; $E$, eggs. $A-D, \times 3 ; E, \times 65$. (After Lewis and McConnell.)

concave disc. It is a small reddish brown parasite a little over one-fourth of an inch in length, which inhabits the cecum and large intestine of hogs, and occasionally of man, in India. A closely allied species occurs in horses and asses in many parts of Africa. Watsonius watsoni (Fig. 79) is a related species, also reddish brown in color, found in the small intestine of West African negroes. A closely related species, Paramphistomum cervi, is found in the stomach of sheep and cattle in Egypt and has a life history almost identical with that of the sheep liver fluke. This or a very similar species occurs in the stomach of cattle in the United States.

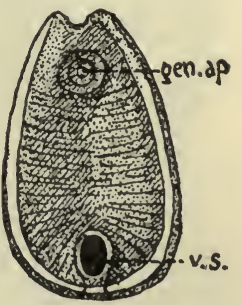

Frg. 79. Watsonius watsoni. Note prominent genital aperture (gen. ap.) and large

Several large flukes of the genus Fasciolopsis $\begin{aligned} & \text { posteri o r ventral } \\ & \text { sucker (v. s.). } \times \text { about }\end{aligned}$ occur occasionally in man, especially $F$. buski 35 . (After Shipley, (Fig 80) found in many East Asian countries, from Stiles and Gold(Fig. 80), found in many East Asian countries. berger.)

This species reaches a length of over an inch

with a width of about half an inch, and has the ventral sucker very close to the mouth. It normally inhabits the small intestine of the hog but occasionally parasitizes man. Goddard found it in 5.5 per cent of all dispensary patients at Shaohing, China, during 17 months, and in 28 per cent of 304 cases admitted to the 
hospital. According to Goddard there are three stages in the disease caused by this parasite. There is first a period of latency during which there is some asthenia and mild anemia. This is followed by a period of diarrhea, in which there is more or less

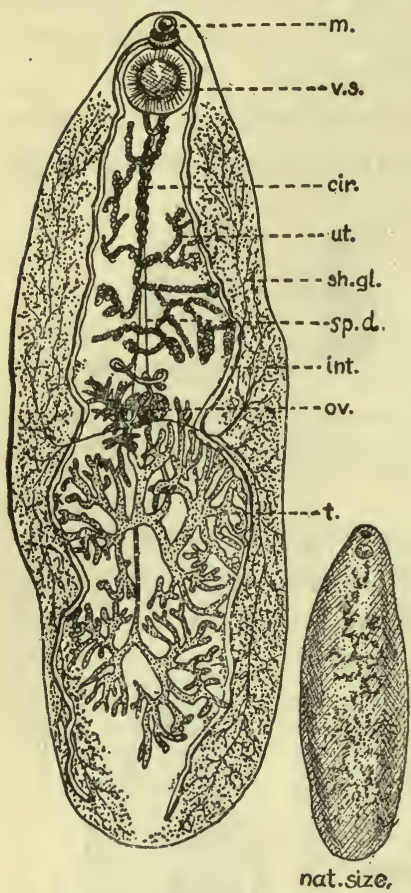

FIg. 80. Fasciolopsisbuski, large intestinal fluke of man. $\times 2 \frac{1}{2}$. Abbreviations as in Fig. 74. (After Odhner.) intestinal disorder; there is however, no blood in the diarrheal stools. There is always noticeable anemia, and this may be extreme. A combination of chronic diarrhea and anemia is said to be characteristically the result of Fasciolopsis infection in Shaohing. Often the abdomen is protuberant in children. The third stage is characterized by increased anemia and a distressing amount of œdema, which affects the abdominal cavity first, then the legs, and finally the upper portions of the body. It gives the affected parts of the body a very characteristic puffy and swollen appearance. Goddard believes that the infection is derived from eating imperfectly cooked snails, some species of which may act as the intermediate host. This would imply that a second intermediate host was not necessary in the life history. On the other hand it has been claimed that the cercariæ encyst in shrimps, but Leiper had no success in infecting hogs with the cysts which he found in shrimps.

The full life history of none of these intestinal parasites is known, and we can only guess at them by analogy with more or less closely related parasites about which we have more knowledge. None of them do enough damage to cause more than slight intestinal irritation or catarrh, and sometimes light dysenteric symptoms. They are susceptible to most of the drugs used for expelling tapeworms and roundworms. Some species are said not to be affected readily by santonin, though they are expelled by thymol and naphthalene, and presumably by oil of chenopodium. 


\section{CHAPTER XIII}

\section{THE TAPEWORMS}

General Structure. - Even more peculiar and remarkable in their structure and life than the flukes are the tapeworms. A mature tapeworm is not an individual, but a whole family, consisting sometimes of many hundreds of individuals one behind the other like the links of a chain (Fig. 81). In some respects the tapeworms are more degenerate than flukes, due to their invariably parasitic life in the digestive tract of their hosts. Being continually bathed in semi-digested fluids in the intestine they can readily absorb food all over the surface of their bodies, and have no need for a digestive system of their own. The digestive tract, therefore, is entirely lacking, not even a vestige of it remaining as an heirloom from less dependent ancestors.

In general form the majority of tapeworms are very long tapelike organisms which attach themselves to their host's intestinal walls by a "head" or scolex at what is really the posterior end of the chain of segments. This scolex is furnished with suckers and often hooks as well (Fig. 82). Next to the head there is a narrow region or "neck" which continually grows and forms

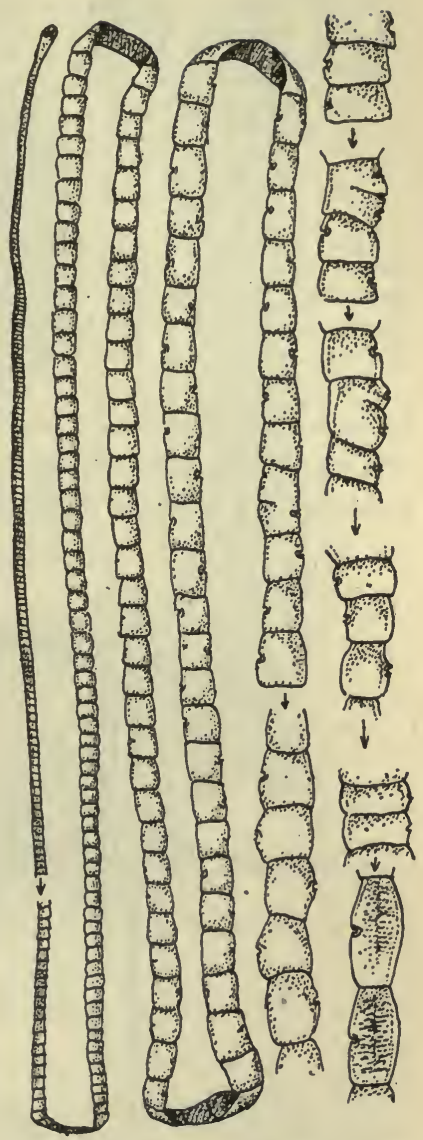

Frg. 81. Beef tapeworm, Trenia saginata, $\times \frac{2}{3}$. Note small head, gradual change in size of proglottids, and irregular alternation of sides of genital apertures. (After Stiles.) segments as it grows, each new segment thus produced pushing forward the segments previous'y formed. This process eventu- 
ally produces the characteristic chain of segments, each of which is known as a proglottid. Obviously the oldest proglottid is the one at the end of the chain, those just back of the neck being
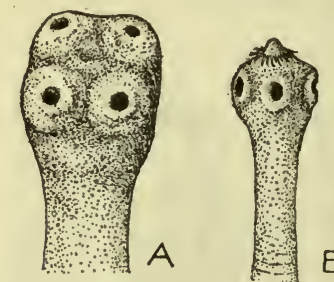

Fig. 82. Armed and unarmed tapeworm "heads" or scoleces; $A$, unarmed head of Tœnia saginata; $B$, armed head of Tania solium. $\times 10$. flukes, if not more so (Fig. 83). The female system consists of an ovary, a pair of shell glands (usually spoken of as yolk glands), a seminal receptacle for receiving and holding the sperms until used for fertilization, a vagina for the

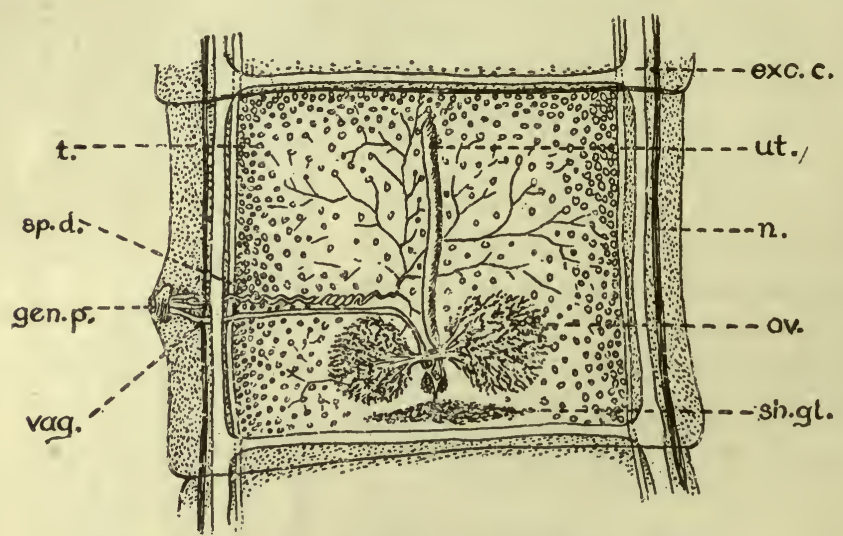

FIg. 83. Sexually mature proglottid of beef tapeworm, Tania saginata; exc. c., excretory canal; n., nerve cord; ut., uterus; ov., ovary; sh. gl., shell gland, usually called yolk gland; vag., vagina; gen. p., genital pore; sp. d., sperm duct; t., testis. $\times 7$. (Partly after Leuckart.)

entrance of the sperms, and a uterus for the storage of the mature fertilized eggs. The male system consists of a number of scattered testes for production of sperms, all connecting by minute tubes with the sperm duct. The latter, near where it opens at the surface of the body, enlarges into a "cirrus pouch" where 
the mature sperms are temporarily stored. The sperm duct ends in an extensible copulatory organ for conducting the sperms into the vagina of the same or another proglottid. Though hermaphroditic, i.e., with both sexes in a single individual, a
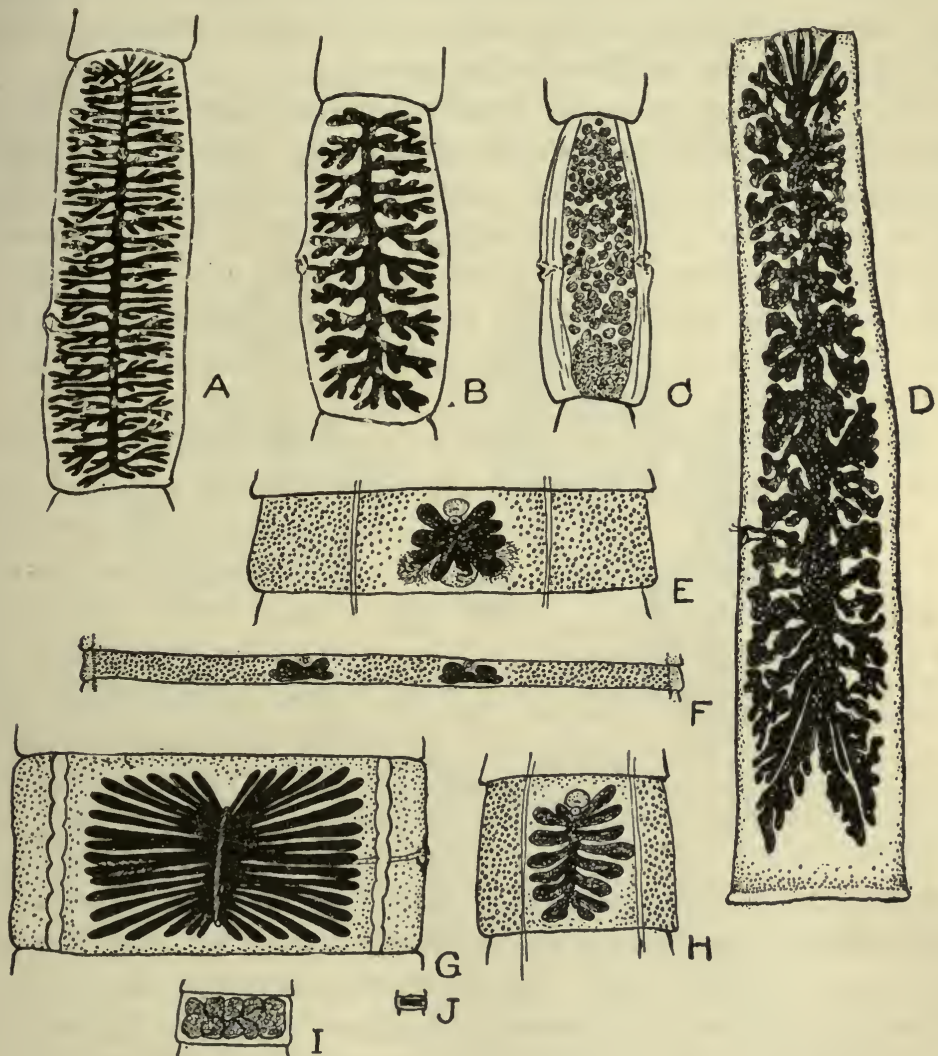

$\Rightarrow \mathrm{J}$

Fig. 84. Ripe proglottids of various tapeworms of man, drawn to scale according to average measurements: $A$, Tænia saginata (after Leuckart). $B, T$ Tenia solium (after Stiles). C, Dipylidium caninum (after Diamare). D, Tæenia confusa (after Guyer). E, Dibothriocephalus latus (after Leuckart). F, Diplogonoporus grandis (after Ijima and Kurimoto). G, Dibothriocephalus cordatus (after Leuckart). $H$, Tania africana (after von Linstow). I, Hymenolepis diminuta (after Grassi). $J$, Hymenolepis nana (after Leuckart).

proglottid does not necessarily always fertilize its own eggs, but cross-fertilization may often occur. This is generally insured by the fact that the male reproductive system usually becomes mature before the female. In the pork tapeworm, 
for instance, the male reproductive system reaches maturity when the proglottid has been pushed back to about the 200th position, whereas the female system does not mature until it has been pushed 200 or 300 proglottids farther back. Copulation then takes place by the doubling back of the chain of proglottids on itself, bringing the young mature male segments into contact with the older mature female segments.

After copulation, when the mature fertilized eggs begin to form, great changes take place in the proglottid. The uterus begins to enlarge and branch until it nearly fills the segment, crowding aside and absorbing the other organs. Segments thus distended with eggs are spoken of as "ripe" proglottids and are ready to break loose from the chain to be voided with the fæces of the host. Ripe proglottids of a number of species of tapeworms found in man are shown in Fig. 84.

Life History. - The life histories of all tapeworms are much alike. Usually before the ripe proglottids become detached and

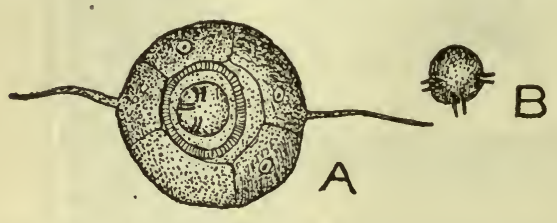

Fig. 85. $\quad A$, egg of beef tapeworm, T. saginata; - note contained embryo and external filaments; $B$, freed six-hooked embryo. $\times 300$. (After Leuckart.)

which acts as an intermediate host. Most often the adult form of the worm occurs in carnivorous animals, while the intermediate host in which the larva develops is a herbivorous animal, but there are numerous exceptions to this. There is no active search for a new host on the part of the tapeworm embryo as there is by the embryos of flukes, but instead merely a passive transfer.

When eaten by a suitable intermediate host, the shell enclosing the six-hooked embryo is dissolved off, and the embryo is released (Fig. 85B). It migrates into the organs and tissues of the body, aided by the blood and lymph circulation of the host, some species having preference for certain organs, others establishing themselves with equal readiness in any parts which they happen to reach. 
Having reached the organ or tissue in which it is destined to develop, the embryo loses its hooks and grows into some form of bladderworm, that is, the body undergoes a series of transformations which usually result in the formation of a bladder-like body filled with a watery fluid. Into the bladder there grows an invagination and at the bottom of this, pushed inside out into it, there develops a head or scolex. There are different types of bladderworms which go under different names, as follows: (1) the cysticercus (Fig. 86A), the simple type described above; (2) the cysticercoid (Fig. 86B), in which the bladder part of the
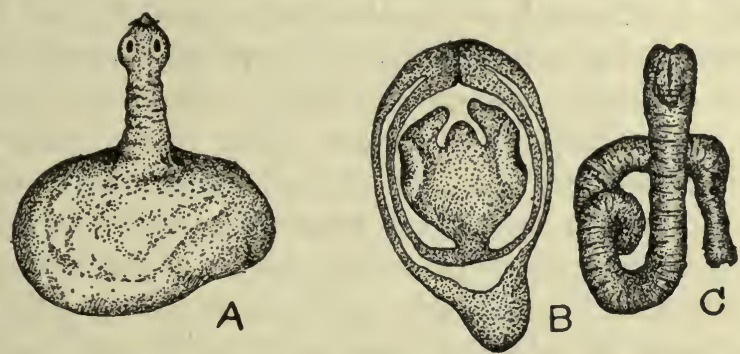

FIG. 86. Types of tapeworm larvæ: $A$, cysticercus of Tania solium with head and neck evaginated, $\times 3 ; B$, cysticercoid of Hymenolepis nana, $\times 12 ; C$, plerocercoid of Dibothriocephalus latus, with head invaginated. ( $A$, partly after Stiles, $B$, from several figs. by Grassi and Rovelli; $C$, partly after Braun.)

worm is poorly developed; (3) the cœnurus, in which multiple heads form in the single bladder; and (4) the hydatid, in which the bladder buds into multiple daughter cysts, each with multiple heads (Fig. 95). The larvæ of the tapeworms of the family Dibothriocephalidoe are quite unlike the bladderworms of other tapeworms. They grow as long wrinkled wormlike bodies with the head invaginated in a little projection at the anterior end. Such a larva is called a plerocercoid (Fig. 86C).

When the organs or tissues in which the larval stages are developed are eaten by an animal of the kind from which the eggs originally came, all but the scolex of the bladderworm is digested off, the latter turns right side out, attaches itself to the wall of the small intestine with the aid of its suckers and hooks, and begins to bud off proglottids of another generation. Tapeworms are usually looked upon as inert animals, but in reality they are quite active, and their movements can often be felt. 
Damage to Host. - The amount of damage which adult tapeworms do to their hosts is a much disputed question. There are those who believe that the presence of an adult tapeworm is more or less of a joke and as such is to be gotten out of the system but not to be taken seriously. The experience of physicians who have had wide dealings with tapeworms does not ordinarily bear out this idea. The mere mechanical obstruction of the intestine which a large tapeworm may cause must be considerable. The amount of food taken from the host for nourishment of such a worm might well be compared with the food absorbed by a growing embryo, and it usually produces a ravenous appetite. The injury to the wall of the intestine caused by the adhesion of the worm by its suckers and hooks is often the cause of serious conditions, allowing the entrance of bacteria and sometimes resulting in destructive ulceration. The waste products and other toxic substances given off by tapeworms must be very considerable and their poisonous properties cannot be doubted. Only recently there came before the notice of the author a case of tapeworm infection illustrating the toxic effect of the worms. A patient came to a local physician for treatment, thinking he had tuberculosis and having been so diagnosed by another doctor. He was in an extremely anemic condition and was very weak and easily exhausted. His cheeks were sunken, his eye staring and he was subject to occasional mental disturbances. Within a fortnight after the worm had been expelled he was practically a new man although he had been suffering for over a year.

Abdominal pains, anal itching, disordered appetite and digestion, emaciation, anemia and many types of nervous derangements, as giddiness, partial paralysis, false sensations and epileptic fits, are common symptoms of tapeworm infection. The degree to which each of these symptoms is felt varies remarkably in different individuals. The nervous symptoms are all due to intoxicating substances liberated by the worms. Sometimes a partial immunity to the toxic effects of worms is acquired by infected people, and in such cases the worms may be present unnoticed for years.

The damage done by bladderworm stages of tapeworms is often more serious, especially in the case of hydatids, the large multiple bladderworms of Echinococcus granulosus. The bladderworms which occur in man most commonly develop in the lung 
or liver, but may attack other parts of the body such as the muscles, eye and even the brain. They do injury both by mechanical interference with the organs and tissues, and by the accumulation of poisonous waste products which may be accidentally liberated. Only by surgery can such bladderworms be removed, and surgery is often impossible on account of the numbers and positions of the bladders.

Treatment. - Preparatory to treatment for adult tapeworm infections the patient is put on a light diet and his bowels cleared out so that the parasite may meet with no obstruction in its passage through the intestine. The drugs which have been found most useful in expelling tapeworms are male fern, pelletririne and thymol. These drugs are dangerous if not taken properly, and none should be taken without the supervision of a physician. Thymol, for instance, while ordinarily quite harmless since it is not absorbed by the intestine, is soluble in alcohol and certain other substances and may cause death if taken along with these things. Oil of chenopodium, which has recently come into great favor for expelling hookworms and is even more efficient for certain other nematodes, has been found valuable for expelling dwarf tapeworms, Hymenolepis nana, and would probably be equally effective for other species.

After the drug is administered a purgative is given which tends to drive the parasite out. The latter should be passed into a vessel of warm water, since sudden contact with cold stimulates the nervous system of the worm and causes it to contract suddenly, thus often breaking it before it has been completely expelled. A careful search for the head should be made, and if not found the treatment should be repeated in the course of a week or ten days.

Prevention. - Prevention varies, of course, with the species of tapeworm and its intermediate host, but since infection with all the common human species, with the exception of the species of Hymenolepis, occurs from eating raw or imperfectly cooked meat of some kind in which the bladderworms have developed, the exclusive use of thoroughly cooked meat is the best preventive measure. Experiments show that pork bladderworms are killed when heated to $127^{\circ} \mathrm{F}$. and beef bladderworms to $120^{\circ}$ or even less, but the difficulty of heating the center of a large piece of meat even to this point is shown by the fact that 
in an experiment to test the penetration of heat, a ham cooked by boiling for two hours had reached a temperature of only $115^{\circ}$ in the center. When roasted, pork should always be cut into pieces weighing no more than three or four pounds to insure thorough penetration of heat. Beef which has lost its red or " rare" color is quite safe.

Since bladderworms are unable to survive the death of their host for more than a limited time, they are eventually destroyed by ordinary cold storage - within three weeks in the case of the beef bladderworm, Cysticercus bovis, but not always so soon in the case of the pork bladderworm, C. cellulosce. According to Dr. Ransom temperatures of about $15^{\circ} \mathrm{F}$. kill beef bladderworms within five days. Thorough curing or salting of meat is also destructive to the parasites.

Infected persons should be careful not to contaminate the food or water of domestic animals with their fæces, bearing in

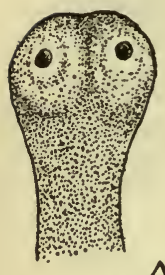

A
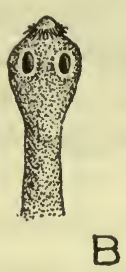

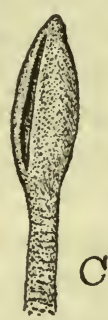

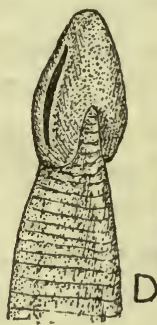
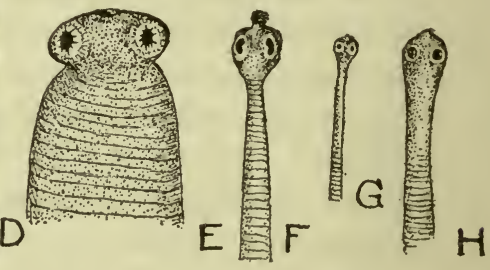

FrG. 87. Heads of some adult tapeworms found in man, drawn to scale; $A$, beef tapeworm, Tania saginata; $B$, pork tapeworm, $T$. solium; $C$, fish tapeworm, Dibothriocephalus latus; $D$, heart-headed tapeworm, Dibothriocephalus cordatus; $E$, African tapeworm, $T$. africana; $F$, double-pored dog tapeworm, Dipylidium caninum; $G$, dwarf tapeworm, Hymenolepis nana; $H$, rat tapeworm, Hymenolepis diminuta. $\times 10$.

mind the various ways in which the eggs may be disseminated - by streams, rain, flies, etc.

The eggs of the dwarf tapeworm, Hymenolepis nana, are thought to be able to develop through the bladderworm stage to the adult in a single host, and should therefore be guarded against by different measures (see p. 243). The larvæ of other species of Hymenolepis develop in insect larvæ such as mealworms, and are therefore subject to still different means of prevention.

The tapeworms of man belong to two quite distinct families, the Tæniidæ, in which the scolex is rounded and furnished with four cup-shaped suckers (Fig. 87, A, B, E, F, G and H), and the 
Dibothriocephalidæ, in which the head is flat and possesses two slitlike suckers (Fig. 87C and D). The latter family also differs from the Tæniidæ in having eggs with lids like those of the flukes (Fig. 88A), and without developed embryos when passed in the fæces.

\section{Family Tæniidæ}

Beef Tapeworm. - The commonest human tapeworm in most parts of the world is the beef tapeworm, Trenia saginata. The adult of this species as it occurs in the human small intestine consists of over 1000 proglottids, and grows to a length of 15 or 20 feet; cases have been reported of specimens of this tapeworm which measured 35 to 40 feet, though in some of these cases there were probably several tapeworms infesting a single person. When two or more worms are expelled in pieces it would naturally be easy to measure them as parts of a single one.

The scolex of the beef tapeworm (Fig. $82 \mathrm{~A}$ ) is hardly larger than the head of a pin. It possesses four small suckers for adhering to the wall of the intestine, but there is no crown of hooks. The suckers are apparently quite sufficient for maintaining a hold, if one should judge from the difficulty experienced in dislodging the worm from the intestine.

The proglottids gradually increase in

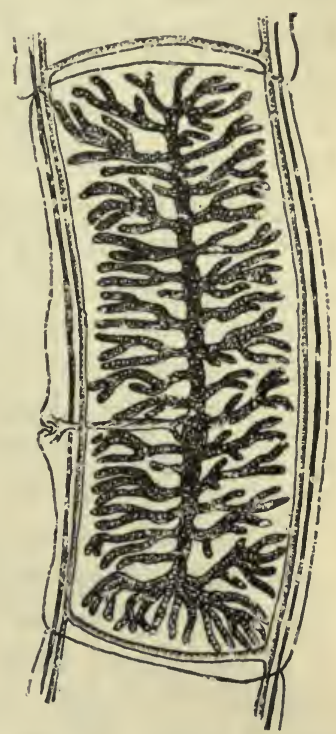

Fig. 88. Gravid segment of Tania saginata. $\times 4$. (After Stıles.) size as they get farther from the scolex (Fig. 81), and the organs contained in them develop slowly. The general form of a sexually mature proglottid and the appearance and arrangements of the organs are shown in Fig. 83. Shortly after sexual maturity has been reached and the sperms for fertilizing the eggs have been received, the uterus begins to grow and develop lateral branches to accommodate the rapidly forming eggs. When fully developed the gravid proglottid enlarges, becoming three or four times as long as when sexually mature, and resembles a pumpkin seed in shape. The greatly developed uterus, distended with eggs, 
occupies practically the whole segment, while nearly all the other organs degenerate (Fig. 88).

A man infested by a beef tapeworm expels several hundred proglottids a month, each one gorged with many thousands of eggs. Fortunately the majority of these never get an opportunity to develop further but it is easy to see how some of the eggs may reach their intermediate hosts, cattle, if the people who harbor the worms are at all careless. Disseminated by rain water, washed by streams into drinking troughs, carried about on the feet of flies, adhering to the heel of a boot, and in many other ways the eggs passed with the fæces may be transferred to the grass or water eaten by cattle. In India, where this tapeworm is common, cattle are said to devour human excrement if they have access to it.

When eaten by cattle or other ungulates, as the pronghorn antelope, giraffe and llamas, the six-hooked embryos (Fig. 85) escape from the eggs and migrate into the muscles of the new host, attacking especially the muscles of mastication. Here in the course of from three to six weeks they grow into bladderworms, Cysticercus bovis, about one-third of an inch in length. They are grayish white in color with a little yellow spot where the head is invaginated. The fact that the cysts lack any marked contrast to the muscle tissue, and if not very numerous may be obscured by it, causes them to be overlooked frequently. If present they can usually be found most readily in the muscles of mastication or in the heart; these are the portions of the carcass regularly examined in meat inspection. Beef which contains bladderworms is said to be "measly."

Infection of man results, of course, from eating measly beef which is raw or only partially cooked. In Abyssinia the Mohammedans, who are forbidden by religious law to eat raw meat, are practically free from tapeworm infection, whereas practically all the non-Mohammedans are infected. The ripe proglottids begin to appear in the fæces, several at a time, in the course of two or three months after infection, and may continue to be developed for years.

Pork Tapeworm. - Common in some parts of the world, but very rare in the United States, is the species Trenia solium, which passes its bladderworm stage in hogs. Wherever raw or imperfectly cooked pork is eaten, infection with this tapeworm is 
likely to occur. The infrequence of these tapeworms in the Philippines where the bladderworms are very common in hogs is worthy of note.

The adult Trenia solium differs from the beef tapeworm chiefly in the form of the scolex, which in addition to four suckers is armed with a double row of hooks, arranged on a conical projection or "rostellum" at its apex (Fig. 82B). The worms are usually of less length than beef tapeworms, seldom exceeding from six to ten feet; they consist of about 800 or 900 segments. The ripe proglottids (Fig. 84B) can in most cases be distinguished from those of the beef tapeworm by their usually smaller size and fewer branches of the uterus (compare Figs. 84A and B).

The eggs, passed in the ripe proglottids with the fæces, develop into bladderworms when eaten by hogs or certain other animals. The usual filthy way in which hogs are housed and fed gives ample opportunity for infection if the infested people are at all careless in their personal habits, or if privies are built so that they leak and the hogs have access to the surrounding ground or outflowing streams. Young pigs are especially likely to become "measly" from eating tapeworm eggs.

As soon as the eggs reach the intestine the six-hooked embryos are liberated from the enclosing capsule and make their way

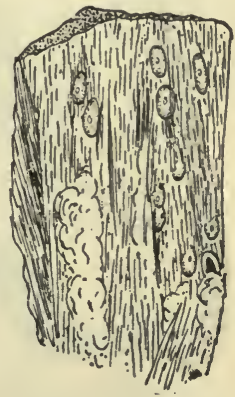

FIG. 89. Fragment of measly pork. (After Raillet.) through the wall of the intestine, to be carried by bloodvessels to the place where they are to develop. They may develop in almost any or all of the muscles or organs of the hog's body, but they especially favor the tongue, neck and shoulder muscles, and, next in order, certain muscles of the hams. Sometimes the bladderworms, technically known as Cysticercus cellulose, become so numerous as to occupy over one-half of the total volume of a piece of flesh examined, i.e., several thousand to a pound. They appear as small elliptical bladders from 6 to $18 \mathrm{~mm}$. (onefourth to three-fourths of an inch) in length (Fig. 89). They have a whitish spot at about the middle of the length, in the center of which is the opening where the head is invaginated.

Unlike the beef tapeworm, Tonia solium can pass its bladderworm stage in a number of animals, namely hogs, man and dogs. 
They have been reported in a considerable number of other animals also but the cases are very doubtful. The fact that the larval stage can develop in man makes the species particularly dangerous on account of possibility of self-infection, either by contaminated hands or by a reversal of the peristaltic movements of the intestine which throws the ripe proglottids of the worm back into the stomach where the embryos in the eggs are liberated by the gastric juices. This is discussed further on p. 251.

/ The Dwarf Tapeworms. - The dwarf tapeworm, Hymenolepis nana (Fig. 90A), is the smallest tapeworm found in man, but

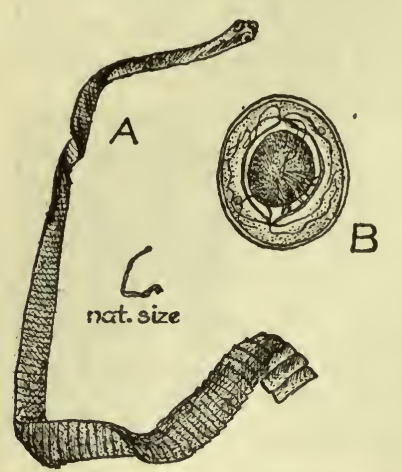

Fig. 90. $A$, dwarf tapeworm, Hymenolepis nana, $\times 7$ (after Stein); $B$, egg of $H$. nana, $\times 700$ (after Ransom). it often occurs in such numbers as to cause much irritation in the intestine. It is a common parasite in Italy, and occurs throughout the warm parts of Europe, Asia, Africa and America. It is probably much more common in the United States than is generally supposed, since it can easily be overlooked unless the fæces are microscopically examined for eggs. It is probably a common parasite of rats and mice as well as of man, though the rodent parasite is believed by some parasitologists to be a distinctspecies, H. murina. Stiles considers the rodent parasite a sub-species, $H$. nana fraterna.

The adult worm, which consists of from 100 to 200 proglottids, is usually little over an inch in length and less than one $\mathrm{mm}$. $\left(\frac{1}{25}\right.$ of an inch) in width. The scolex (Fig. 87G) has four tiny suckers and a crown of little hooks. The ripe proglottids (Fig. 84J) differ from those of the large tapeworms in being much wider than long, with the enlarged uterus in the form of a solid mass, partially divided into compartments instead of being branched.

As regards life history, the dwarf tapeworm is commonly believed to pass both its larval and adult stages in a single host, contrary to what occurs, so far as is known, in any other tapeworm. The eggs (Fig. 90B), eaten by a rat or man, liberate sixhooked embryos in the small intestine, where they enter the villi and transform into cysticercoid bladderworms (Fig. 86B), which in turn fall into the cavity of the intestine, attach themselves 
by the armed head, and become adult. It is said that eggs of this parasite can be found in the fæces within a month after an egg of the preceding generation has been swallowed. Selfinfection with these eggs ${ }^{\circ}$ rarely occurs, since the eggs will not develop unless acted upon by the gastric juices. There is still room for doubt as to whether an insect is not commonly involved as an intermediate host as in other species of Hymenolepis; in fact, several investigators have found cysticercoids in rat fleas which they ascribed to this species. Ransom thinks there is room for doubt as to whether the larvæ of Hymenolepis found in the intestinal villi of rats and mice break out and become mature in the lumen.

The common presence of this parasite or a variety of it in rats and mice indicates that infection in man may occur from accidentally swallowing the "pills" of these animals infected with the eggs or ripe proglottids of the worm. Since a single mouse "pill" might contain hundreds of eggs, each of which could develop into an adult in another rat or mouse, or in man, it is

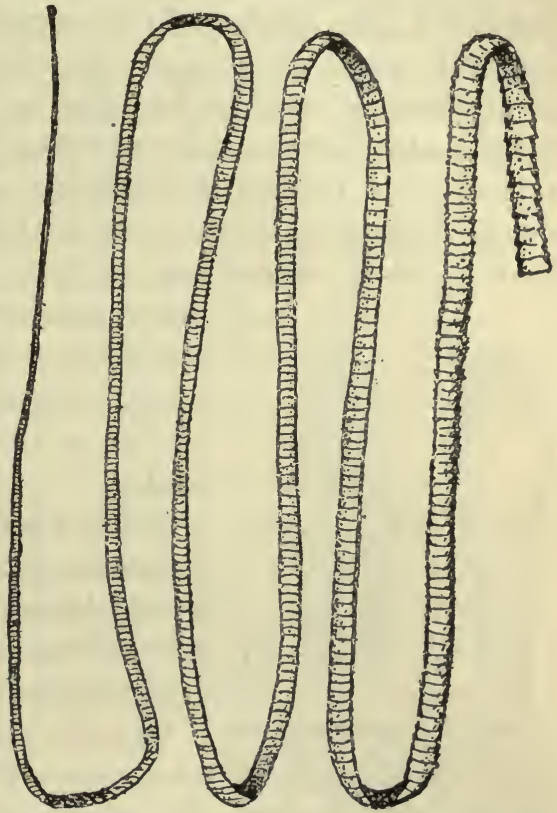

FIG. 91. Rat tapeworm, Hymenolepis diminuta, from house mouse in Oregon. Natural size.

not difficult to understand the great numbers of this worm which are often found in a single intestine.

The unique life history of this species, if true, makes it subject to entirely different preventive measures from those used against most other tapeworms. Since infection results not from eating bladderworm-infected meat, but probably from swallowing egginfected fæces, especially the "pills" of mice and rats, and possibly also from swallowing infected insects which are acting as intermediate hosts, prevention consists in the elimination of 
rats and mice from the household and in keeping food out of their reach, and in guarding against the accidental ingestion of such possible intermediate hosts as fleas.

A closely allied species, $H$. diminuta (Fig. 91), occurs rarely in man. It closely resembles the dwarf tapeworm but is of larger size (four to 24 inches in length) and has no hooks on the scolex (Fig. 87H). . The eggs develop in the larvæ or adults of the mealworm, Asopia farinalis, and in other insects, forming cysticercoids. When these are eaten by rats, mice or man they transform into adults. In an experiment on man the eggs of the adult worm were found in the fæces 15 days after the eating of an infected mealworm. The larvæ of a number of species of fleas also become infected when they ingest the eggs. It is evident that prevention consists in guarding carefully against the accidental swallowing of mealworms with cereals or other foods, and in cautioning children against putting beetles or

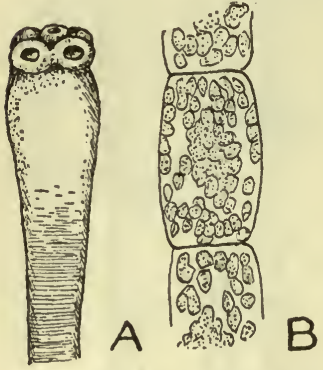

Fig. 92. Davainea madagascariensis; $A$, head and neck, $B$, gravid proglottids. $\times 8$. ( $A$, after Blanchard, $B$, after Daniels.) other insects into their mouths. Although the worm is rare in man it is common in rats and mice in many parts of the world, and occurs in nearly all parts of the United States.

Other Tapeworms (Tæniidæ). - A considerable number of other tapeworms of this family have been found in man, accidentally occurring in him, or having a very limited distribution.

Of those with limited distribution should be mentioned two species of Davainea. One, D. madagascariensis (Fig. 92), is a small tapeworm reaching a length of ten or twelve inches. It is found, chiefly in children, in many tropical countries, especially.in islands and seaports and on ships. The suggestion has been offered that the intermediate host, so far unknown, may be the ubiquitous sea-going cockroach. This tapeworm is interesting in that there is not only a crown of hooks on the head, but there are hooks on the suckers also. The other species of Davainea is $D$. formosana, recently described by Akashi from children in Formosa and Tokyo. It differs from the preceding species in its larger size, lack of hooks on the suckers, larger size of egg masses in the ripe proglottids and in other minor details. 
The African tapeworm, Tcenia africana, is a species found in German East Africa. It is about four feet in length with no hooks on the scolex (Fig. 87E) and with an unusual fanlike arrangement of the uterus in the ripe proglottids (Fig. $84 \mathrm{G}$ ). Von Linstow, who described the worm, suggests that the zebu may be the intermediate host since its flesh is eaten raw by the natives.

A medium-sized tapeworm, Tcenia philippina, reaching a length of about three feet, has been found among prisoners at Manila. It very much resembles the African tapeworm but has smaller proglottids. Other species have been described from various parts of the world, especially southern Asiatic Russia, but they are of such rare occurrence, some having been found only once, that they need no description here.

Two specimens of the species Taenia confusa (Fig. 84D,) were found by Ward in Nebraska many years ago, and recently another specimen was reported by Chandler from Texas. It probably has been endemic in the central states in the meantime, but confused with other species. It is possibly identical with $T$. bremneri of Africa.

Of the accidental tapeworms of man there should be mentioned especially the dog tapeworm, Dipylidium caninum. This species is abundant in dogs, and sometimes cats, in all parts of the world. It is a species about a foot in length, with three or four rows of hooks on the rostellum (Fig. $87 \mathrm{~F}$ ), and a double set of reproductive organs in each proglottid (Fig. 84C). The larva, a cysticercoid, occurs in lice and fleas. It is stated that the eggs of this tapeworm cannot be sucked up by the dog-infesting fleas, but that they are readily swallowed by flea larvæ. The eggs hatch in the intestine of the fiea larvæ, the embryos pass to the body cavity and the cysticercoids remain through the metamorphosis of the larvæ to the adult fleas. Children who play with dogs are occasionally infested by this worm, probably by accidentally swallowing lice or fleas or by crushing them and then putting infected fingers into the mouth.

\section{Family Dibothriocephalidæ}

The tapeworms of this family, as remarked before, are characterized by a flattened head with two slitlike suckers (Fig. $87 \mathrm{C}$ and D). The larvæ, which usually develop in fishes, are of the 
plerocercoid type, i.e., they have long wormlike bodies with an invaginated head at one end (Fig. 86C).

Fish Tapeworm. The common fish tapeworm of man, Diphyllobothrium latus, is an important species in the districts in which it occurs. It is found in all countries where fresh-water fish is extensively eaten, and especially in countries where it is commonly eaten raw. In the Baltic countries, Switzerland, Russia, Japan, and about the Central African lakes this parasite is particularly common. Relatively few cases have been reported in the United States, though the larvæ are said to be found frequently in fish from the Great Lakes.

The fish tapeworm is a large species and commonly reaches a

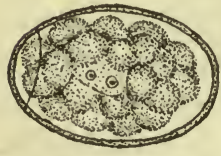

length of from six to 30 feet, or even more, with from 2000 to 4200 short, broad proglottids, only the terminal ones of which are as long as broad. The scolex (Fig. 87C) is almond-shaped. Unlike the tapeworms of the family Tæniidæ, the genital openings

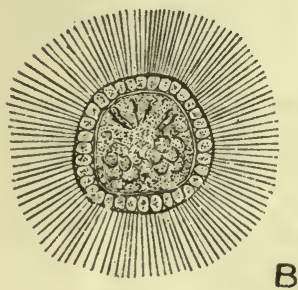
are near the middle of the under surface of the proglottids, instead of at one side. In the ripe proglottids (Fig. $84 \mathrm{E}$ ) the uterus is in the form of a rosette near the center of the segment. The proglottids do not usually retain the eggs until they break off

B from the chain, but void them, as do flukes,

Fig. 93. An egg of fish tapeworm, D. latus, - note segmented condition and operculum; $B$, ciliated embryo of same. $X$ about 300. (After Looss.)

through the genital pore. The empty proglottids, shrunken and twisted, are broken off in short chains from time to time.

The eggs (Fig. 93A), which are large and brown with a lid at one end as in fluke eggs, contain six-hooked embryos which are furnished with a covering of cilia (Fig. 93B). The eggs hatch in water in from eight to fifteen days, and the embryos swim about by means of their cilia, though they often slip out of their ciliated envelope and creep on the bottom. The embryos have recently been shown by Rosen and by Janicki to develop in certain species of Cyclops and Diaptomus, copepod crustaceans, into larvæ which are called procercoids. In 12 to 15 days these larvæ are about 0.4 $\mathrm{mm}$. ( $\frac{1}{60}$ of an inch) in length, sausage shaped, with a spherical caudal appendage carrying the embryonic hooks. This append- 
age shrinks and a depression appears at the opposite end; a large portion of the body is occupied by a pyriform central mass. The full development is reached in two or three weeks, the larvæ being then about $0.5 \mathrm{~mm}$. long. Small trout fed on the infected copepods were successfully infected. The passage through the intestine and body cavity of the fish is slow; it requires about six days for the larvæ to reach the liver in young fish, and in older fish it may take from two to four weeks. In the fish the worms develop into plerocercoid larvæ in the muscles. Pike, perch and other carnivorous fish are most frequently infected.

When eaten by a susceptible host in raw or imperfectly cooked fish, the larva, except the head, is digested, and the head, attaching itself to the wall of the small intestine, begins to grow into an adult worm at the rate of about 31 to 32 proglottids a day. The mature eggs begin to appear in the fæces within a month.

The fish tapeworm is especially active in the production of toxins which cause intense anemia. Its head has been found to produce oleic acid, a substance which has blood-destroying properties. Often the nervous symptoms produced by this species are also very marked.

Two other species of Dibothriocephalidæ have been found in man. One of these, Diphyllobothrium cordatus (Figs. 84G and 87D), occurs in dogs, seals and other fish-eating animals in Greenland. It only accidentally establishes itself in man. Diplogonoporus grandis is a very large species, found in Japan, in which there is a double set of reproductive organs. The genital openings are arranged in two longitudinal grooves on the ventral side of the worm (Fig. 84F). This species is rare in man.

\section{Larval Tapeworms in Man}

There are several species of tapeworms which inhabit the human body in the larval or bladderworm stage. Three types are found in man. Most important are the huge multiple cysts or " hydatids" of Echinococcus granulosus, a small tapeworm of dogs. Second, there are the bladderworms of the common pork tapeworm, Tcenia solium, which often occur in large numbers, and may be of very serious nature if located in important organs. And, finally, there are two species of Sparganum. This is not 
a true genus but is a collective group of larval tapeworms of the plerocercoid type which cannot be definitely classified because the adult is unknown.

Echinococcus hydatids. - In some parts of the world infection with the hydatids or larvæ of Echinococcus is very common, especially in children. In Iceland, where there is very close association between the human and the canine population, two or three per cent of the inhabitants are afflicted, and in certain districts as high as ten per cent. In Australia, also, this tapeworm is common in dogs and its larvæ occur in a considerable proportion of human beings as well as in stock. In the United States, especially in the southeastern states, it is fairly common.

The adult of Echinococcus (Fig. 94) is a minute tapeworm found in dogs and sometimes in other carnivorous animals. It measures only from one-tenth to one-fifth of an inch in length. The mature worm consists of a tiny scolex with four suckers and a double crown of hooks, followed by an unsegmented neck and

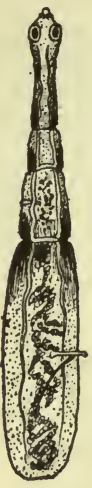

Frg. 94. Echinococcus granulosus from dog. $\times 10$. (After Leuckart.) three gradually larger proglottids, the ultimate one of which is larger than all the rest of the worm and contains about 500 eggs in the uterus.

Echinococcus may occur in hundreds or even thousands in the intestine of dogs, though it often escapes notice on account of its small size.

The eggs of the worm, dropped in pastures with the fæces of infected dogs, ordinarily develop in sheep, cattle or other herbivorous animals. Human infection usually results from too intimate association with dogs, and children especially are liable to infection by allowing dogs to " kiss" them or lick their faces with a tongue which, in view of the unclean habits of dogs, may be an efficient means of transmission for the tapeworm eggs.

The hydatids develop in many different organs of the body. The liver is the favorite site, after which, in order of frequency, come the lungs, kidneys, spleen, intestinal walls, membranes lining the body cavity, heart, brain and various muscles. Sometimes a single host is invaded by the hydatids in several different organs.

The development of the embryos is very slow indeed. In a 
month after reaching their destination in the liver or other organs they are in the form of little globular bodies, enclosed by a capsule produced at the expense of the host. A cyst measures about one $\mathrm{mm}$. ( $\frac{1}{25}$ of an inch) in diameter. By the end of the fifth

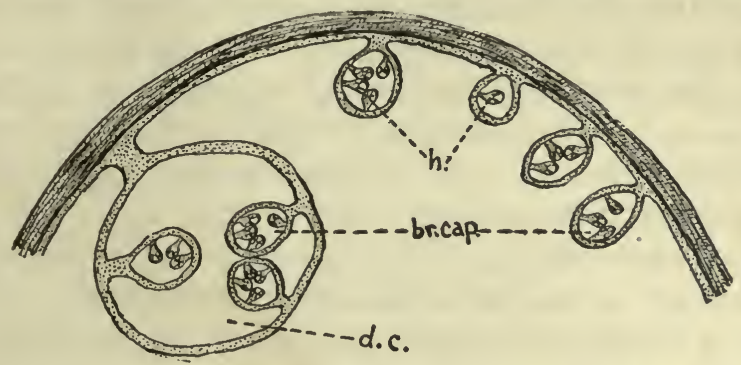

FIG. 95. Diagram of portion of small Echinococcus cyst showing daughter cyst (d.c.), brood capsules (br. cap.) and invaginated heads (h.). $x$ about 5 .

month it has grown to the size of a walnut. The membrane of the bladderworm itself is very delicate, but the capsule formed by the host is thick and tough. The enclosed fluid is transparent and nearly colorless, and is composed of various materials which have permeated in from the blood and tissues of the host, and of the waste products produced by the growth of the parasite.

When the hydatid has reached this stage in its development (Fig. 95) there grow into its cavity from the inner surface little vesicles or brood capsules, on the inner surface of which in turn there grow a number of little heads or scoleces. Each of the heads has the power ultimately to grow into an adult worm. As there may be a dozen or more of the scolex-bearing brood capsules in

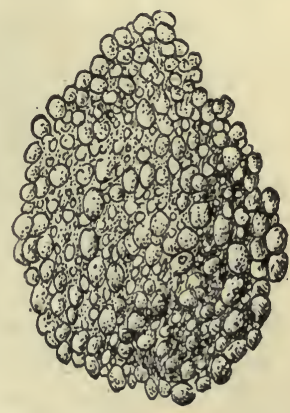

FIG. 96. Multilocular cyst from liver of steer, $\frac{2}{3}$ nat. size. (After Ostertag from Stiles.) a single hydatid, and from six to 30 heads scoleces produces may be enormous. Sometimes there may be still further multiplication by the development of secondary cysts either inside or outside of the original hydatid which may develop a whole series of scolex-bearing vesicles of their own.

Sometimes instead of forming the usual large vesicles and secondary vesicles, the growth results in the formation of a great mass of small separate vesicles (Fig. 96), varying in size from a 
pinhead to a pea, with few and scattered heads. These masses of vesicles, known as "multilocular" cysts, may be six inches. or more in diameter; they are most frequently found in the liver. Unless surgically removed they usually prove fatal sooner or later.

The fact that the "multilocular" cysts are not found in Iceland or Australia where the ordinary cysts are so common, and that they occur to the almost total exclusion of the ordinary kinds in some countries, especially in parts of Germany, suggests that they may belong to a different species indistinguishable from $E$. granulosus in the adult state.

The great size to which hydatids may grow makes them dangerous on account of the mere mechanical damage they may do, especially if they occur in such organs as the heart, brain, kidneys or liver. The liver of an ox containing hydatids has been

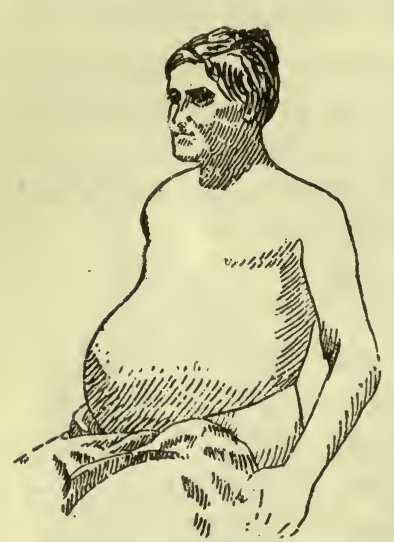

FIG. 97. Echinococcus cyst in liver of man. (After Huber from Stiles.) found to be a hydatid. Ordinarily growth does not increase beyond the size of a baseball. The only treatment is a surgical operation.

The conditions which exist in places where hydatid disease in man is common gives us an idea of what to avoid in order to prevent infection. In Iceland from 30 to 100 per cent of the dogs in different regions are said to be parasitized by Echinococcus. A large proportion of the sheep and many cattle are infested with the hydatids. The dogs are fed on the uncooked entrails and waste meat of slaughtered animals, and the dogs in turn are 
allowed to run at will over the pastures, dropping the egg-laden proglottids with the fæces in places where the water or food of the stock may be infected. Dogs are allowed the free run of the houses, are given unbounded liberty in playing with children, and not infrequently eat from the same dish as their human companions. The resulting prevalence of Echinococcus in both dogs, stock and man is hardly to be wondered at.

The precautions which should be taken to prevent the spread and to bring about the control of this disease may be summarized as follows: (1) avoidance of too great familiarity with dogs, (2) exclusion of dogs from shores of lakes or reservoirs from which drinking water is taken, (3) extreme cleanliness in handling of food, (4) prevention of dogs from eating the entrails or meat scraps of animals which may be infected with hydatids.

Cysticercus of Taenia solium. - The fact that the bladderworms of the pork tapeworm, Trenia solium, sometimes occur in man has already been mentioned. Since self-infection with the eggs of the worm is a dangerous possibility, the presence of a pork tapeworm in the intestine is to be looked upon much more seriously than infection with other tapeworms.

The bladderworms, technically named Cysticercus cellulosce (Fig. 86A), develop from the six-hooked embryos which are freed from the enclosing egg-shell by the gastric juices. The embryos bore through the intestinal wall and migrate to various organs and tissues to develop.

The effect of Cysticercus infection depends entirely upon the number present and upon their location in the body. A few of them in the muscles or in the connective tissue under the skin are quite harmless. In the eye, heart, spinal cord, brain or other delicate organs their presence may be very serious, the symptoms being due chiefly to mechanical injury. Infection of the brain is usually accompanied by epileptic fits, convulsions and other nervous disorders. There is no treatment except a surgical operation, and this is often obviously impossible, both on account of the number and position of the parasites. Moreover, in a great many cases a correct diagnosis of this infection is made only in a post-mortem examination.

Sparganum. - The group name Sparganum has been given to plerocercoid larvæ of tapeworms of the family Dibothriocephalidæ, of which the adult form is unknown and the true genus therefore indeterminable. 
The most common type of such tapeworm larvæ is Sparganum mansoni (Fig. 98), an elastic worm three to 14 inches in length.

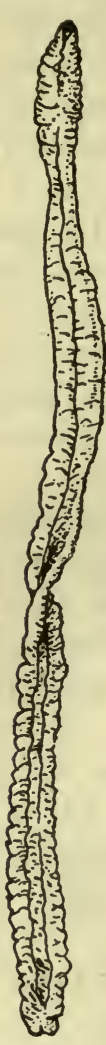

It is not segmented, but appears so since it is transversely wrinkled; there is an invaginated scolex at the broader end. These parasites are found coiled in the connective tissue of man and other animals, often causing tumors. The majority of human cases have occurred in Japan, but cases are recorded from many other parts of the world also, including Texas.

Recently it has been shown that this worm is the larva of Diphyllobothrium mansoni of dogs and cats, which has a life history similar to that of D. latus. The first larva, or procercoid, develops in Cyclops leuckarti, but not in related copepods. The plerocercoid or sparganum stage develops in a large number of different animals, including man, but probably the normal second intermediate hosts are cold-blooded vertebrates, especially frogs and snakes, in which they are very common. When these animals are eaten by dogs or cats the parasite completes its development to the adult form.

Another type of Sparganum, which has been termed $S$. proliferum, was discovered by a Japanese investigator, Ijima, in a Japanese woman in 1904. The skin on a large part of Fig. 98. Spar- her body was much swollen and presented ganum mansoni; numerous hard pimples. Examination showed
nat. size. (After Ijima and Mur- thousands of worms which were identified as ata.) larval tapeworms of the Sparganum type, imbedded in little oval capsules varying in size from less than one $\mathrm{mm}$. ( $\frac{1}{25}$ of an inch) in length to six or eight $\mathrm{mm}$. ( $\frac{1}{4}$ of an inch). Young slender worms not yet encysted were also found. In 1907 a similar case occurred in a fisherman in Florida, and the parasites were believed by Dr. Stiles to be either identical with or closely related to the Japanese worm. Two 
other Japanese cases, discovered in 1907 and 1911 respectively, have also been reported. In one of these the worms, most but not all of them in capsules, were found in countless numbers not only in the subcutaneous tissue but also in the muscles and throughout most of the internal organs, including even the brain.

The worms of this species (Fig. 99) are in all cases white, flattened organisms of very variable shape and size. They usually vary from three $\mathrm{mm}$. to $12 \mathrm{~mm}$. ( $\frac{1}{8}$ to $\frac{1}{2}$ an inch) in length, and from $0.3 \mathrm{~mm}$. to $2.5 \mathrm{~mm}$. ( $\frac{1}{80}$ to $\frac{1}{10}$ of an inch) in width, but in one Japanese case they were uniformly larger, reaching a length of three inches. Their peculiarly irregular shape is due to the unique method of proliferation by the growth of buds or supernumerary heads. These apparently become detached, leave the cyst, and become encapsuled themselves after migrating in the subcutaneous tissue. This explains the increasing numbers of acne-like spots or nodules containing worms, which were reported by the patients.

Attempts made by Ijima to produce adult worms by feeding the larvæ to various domestic animals failed, and nothing is known of the life history or mode of infection beyond a suspicion that the eating of raw fish is responsible for it. Dr. Gates, who discovered the Florida

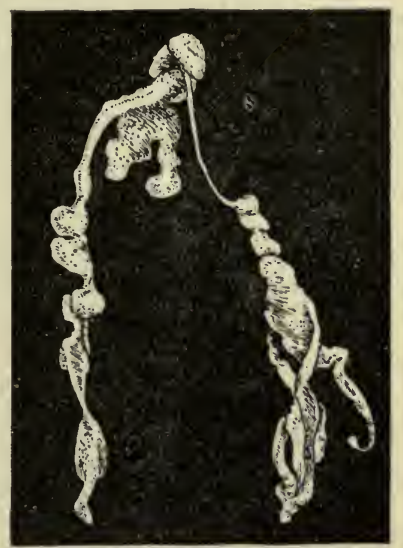

FIG. 99. Sparganum proliferum, from man in Florida. Much encase, reported that there was probably larged. (After Stiles.)

a similar case in Florida a few years before, the patient having moved to California where he died "eaten up with worms."

The rare occurrence of this peculiar and serious parasitic disease is evidence that the mode of infection is unusual. The suspicion that it results from eating raw fish is sufficient reason for discrimination against this kind of food even in places where this or other human para sites which come from raw fish are not positively known to occur. 


\section{CHAPTER XIV}

\section{HOOKWORMS}

History. - For many years it was customary in the United States to look upon the shiftless people to be found in our South as the product of wanton laziness and an inborn lack of ambition. For decades the more fortunate Northerners considered the "poor whites" of the South a good-for-nothing, irresponsible people, worthy only of scorn and of the sordid poverty and ignorance which they brought upon themselves as the fruits of their own shiftlessness. When it became known, largely as the result of investigations by Dr. C. W. Stiles, of the U. S. Public Health Service, that these hopelessly incapable and pitifully emaciated and stunted people were the victims, not of their own unwillingness to work or learn, but of the attacks of intestinal worms which sapped their vitality, poisoned their systems, and stunted both their mental and physical growth, and that over two million people in our own southern states were the victims of these parasites, the "poor whites" and "lazy niggers" of the South became objects of pity and help rather than of scorn.

The hookworm, which is the cause of this deplorable condition, is by no means a newly discovered parasite. A close cousin of the American hookworm was discovered in Italy over 75 years ago, and has subsequently been found to be prevalent in parts of every warm country in the world, in some places infesting nearly or quite 100 per cent of the inhabitants. It would probably be well within the truth to say that over half a billion people in the world are infected with hookworms. The disease caused by hookworm, which has recently come to be used as a symbolism for laziness, was known for ages before the cause of it was discovered, in fact it was probably one of the ailments most familiar to the ancient Egyptians, and descriptions of symptoms probably representing hookworm disease appear in the medical papyrus of 3500 years ago. The disease has gone by many names: malcoeur or mal d'estomac in the West Indies, tuntun in Colombia, 
opilagço in Brazil, tunnel disease and miner's itch in Europe, and chlorosis in Egypt.

The American hookworm, Necator americanus, was probably introduced into America from Africa by slaves. In many parts of the latter continent as well as in parts of Asia, especially Ceylon, this hookworm is very common. It occurs in the gorilla as well as in man. In the United States it is occasionally found in all but the most northern states, but is a great menace only in the southern ones - North and South Carolina, Georgia, Florida, Alabama, Mississippi, Louisiana and Texas. It also presents a serious problem in Cuba, Porto Rico and Brazil. In most other warm parts of the world a closely allied species, the Old World hookworm, Ancylostoma duodenale, is more prevalent. It is impossible now to know what was the origin or natural distribution of either species, since both worms have been introduced by infected travelers into every quarter of the globe. In Europe Ancylostoma duodenale is far the more common. It first attracted attention there as the cause of "tunnel disease" at the time of the building of the St. Gothard tunnel. The infected laborers, dispersing after the completion of the tunnel, spread the infection to all parts of Europe, and serious epidemics broke out in the coal-mining districts of Hungary, Germany and Belgium.

The Parasites. - The two species of human hookworms are similar in structure; they agree in all important details of life history; and both produce the same symptoms, require the same treatment, and can be prevented in the same ways. They are round worms, belonging to the great group of nematodes, which as adults live in the small intestine of their hosts and suck blood. An allied species, A. ceylanicum, found in civet cats and dogs in southern Asia occasionally occurs in man. The American hookworm, Necator americanus (Fig. 100), is smaller than the Old World species, Ancylostoma duodenale, the measurements being about eight $\mathrm{mm}$. (one-third of an inch) and ten $\mathrm{mm}$. (twofifths of an inch) respectively in the males, and ten $\mathrm{mm}$. and 15 $\mathrm{mm}$. (three-fifths of an inch) respectively in the females. They are normally whitish in color but when gorged with blood they are reddish brown. The females, which are much more numerous than the males, have simple cylindrical bodies, largely occupied by the threadlike ovaries and egg-filled oviducts. In the Old 
World species the mouth (Fig. 101A) is armed with a number of chitinous hooklike teeth, which in the American species are replaced by hard ridges or lips (Fig. 101B). The male worms are
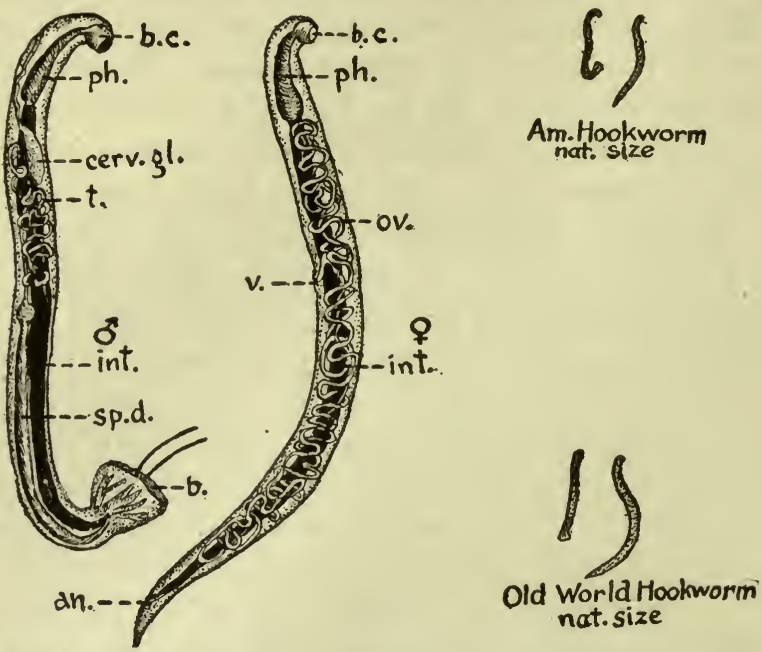

Fig. 100. American hookworm, Necator americanus, male ( $\hat{\delta}$ ) and female ( $\$$ ); b.c., buccal cavity; ph., pharynx; int., intestine; cerv. gl., cervical gland; t., testis; sp. d., sperm duct; b., bursa; ov., ovaries and oviducts; v., vulva or genital opening; a, anus. $\times 8 . \quad$ (Partly after Manson.)

also cylindrical but instead of tapering at the tail end they possess an umbrella-like expansion known as a bursa, which is sup-
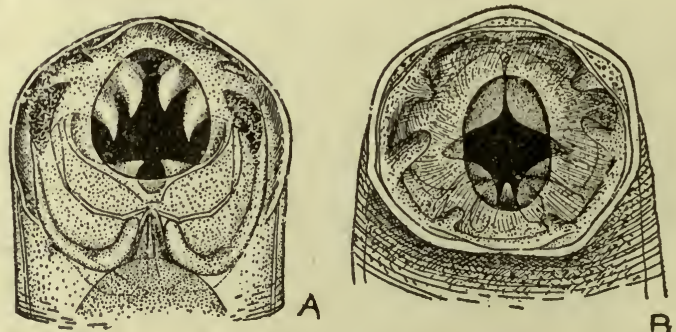

Fig. 101. Buccal cavity and mouth of Old World hookworm $(A)$, and American hookworm $(B)$, showing teeth in former and cutting ridges in latter. $A, \times 100$; $B, \times 230$. (After Looss.)

ported by clawlike rays somewhat suggestive of the ribs of an umbrella (Fig. 102). The bursa is used for holding the female during copulation. It was the clawlike ribs of this "umbrella" 
which first suggested the name "hookworm" for the parasites, though the hooklike teeth in the mouth of the Old World species might just as readily have suggested the name.

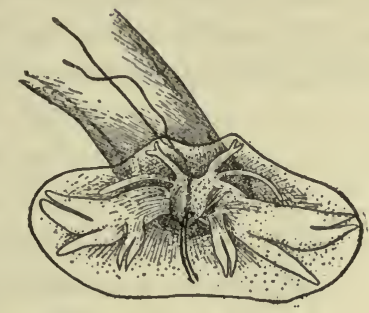

Fig. 102. Bursa of American hookworm. (After Stiles.)

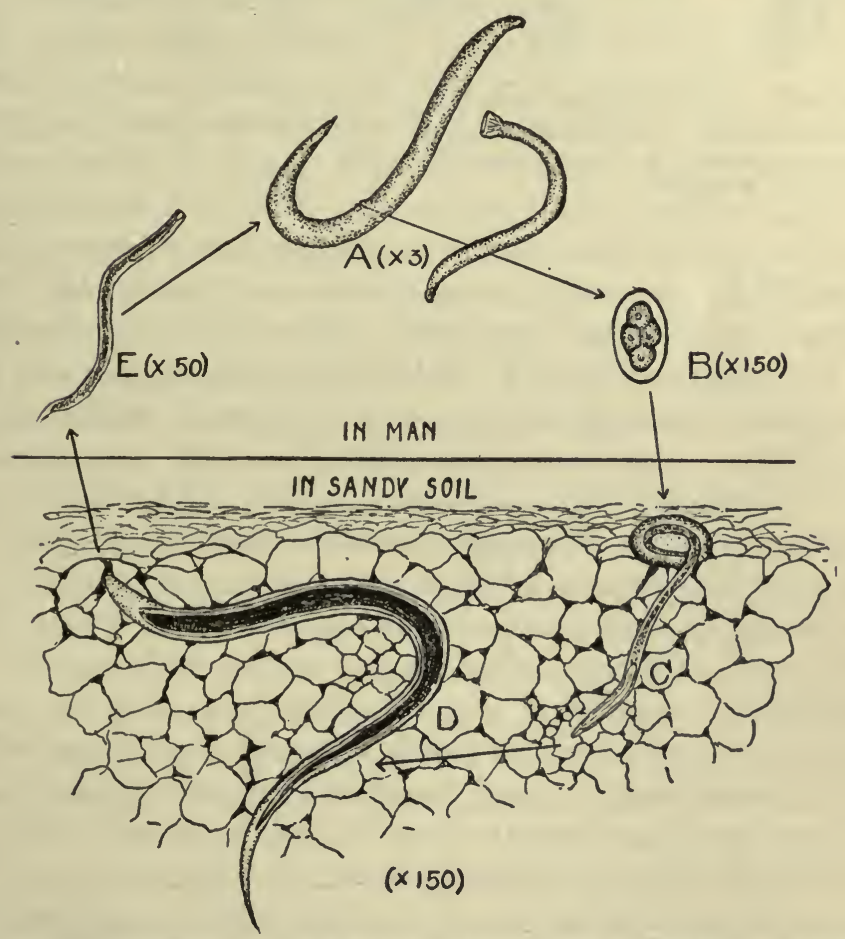

Fig. 103. Life history of hookworm; $A$, adults, female and male, in intestine; $B$, egg as passed in fæces; $C$, embryo hatching in ground, 24-48 hours later; $D$, fully developed larva, enclosed in sheath, ready to infect human being; $E$, larvæ released from sheath, migrating in body of new host.

Life History. - (Fig. 103.) The female worms produce an enormous number of eggs which are poured into the intestine of 
the host, usually in a continuous stream, but occasionally with intermissions, to be passed with the fæces. The thin-shelled eggs, which are about $60 \mu$ by $35 \mu$ ( $\frac{1}{40} \overline{0}$ by $\frac{1}{70} \sigma$ of an inch) in size, and slightly larger in the American species, undergo the first stages of development while still in the intestinal canal, and by the time they are voided with the fæces they are segmented into
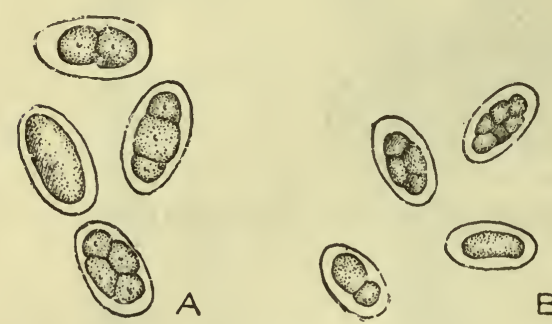

from two to eight cells (Fig. 104). The segmented condition, together with the fact that they are clear and not yellow or brown from bile stain, distinguishes the eggs from those $B$ of many other worms found in the intestine.

FIG. 104. Eggs of hookworms in early stages of segmentation, - four-segmented type most common in fæces; $A$, Necator americanus; $B$, Ancylostoma duodenale.

Further development does not take place until the fæces are exposed to air, when, if moisture is present and the temperature is moderately high $\left(65^{\circ}\right.$ to $85^{\circ} \mathrm{F}$.), the development continues and the embryo hatches in from 24 to 48 hours (Fig. 103C). Below $65^{\circ} \mathrm{F}$. development is very slow, and above $85^{\circ} \mathrm{F}$., although development is very rapid, the eggs and larvæ are likely to die. The newly hatched worm is about $0.2 \mathrm{~mm}$. (less than a hundredth of an inch) in length with a bottle-shaped œsophagus, a simple intestine, and practically no reproductive organs. The most favorable conditions for the development of the larvæ, in addition to the temperatures mentioned, are a moderate degree of moisture, presence of air, plenty of food in the form of decomposing organic matter, and not too rapid putrefaction. According to Looss, the larvæ will not develop well in fæces derived from a purely vegetable diet, a small proportion of animal matter being essential for food. Enough animal food for some development would always be provided by blood from intestinal hemorrhages. On the other hand a purely meat diet is unfavorable on account of the rapid putrefaction. If suitable conditions are present, the larva grows rapidly for four or five days, shedding its skin at the end of the second day. In about five days, under ideal conditions, the skin begins to become detached again but is not shed. It is retained as a flexible protecting sheath for the larva, but does not hinder free motion 
(Fig. 103D). The larva has by this time grown to several times its original size, being over $5 \mathrm{~mm}$. ( ${ }^{\frac{1}{2}} \frac{\mathrm{o}}{\mathrm{b}}$ an inch) in length, and is now in the infective stage and ready to begin its parasitic life. No further food is taken but the parasite begins an active migration in the neighboring soil or water. If even a trace of moisture is present in the soil the larvæ are capable of traversing considerable distances and may thus give rise to infection far from the place where the fæces were originally deposited. They are said to be able to travel through moist soil at a rate of probably not less than five feet per hour, which, if kept up constantly in a straight line would mean a wandering of forty yards in twentyfour hours. While such continued travel in a straight line probably would never occur, it is evident that a single infective stool would easily be able to infect the ground for several square yards. Complete drying up is fatal to both eggs and larvæ in all stages.

The larvæ may remain just under the surface of moist soil or mud or in water for a long time, awaiting an opportunity to enter a human host. They have been kept alive in the laboratory in plain water at a temperature of about $60^{\circ} \mathrm{F}$. for 18 months and unless attacked by predaceous insects or other animals would undoubtedly live fully as long under outdoor conditions. They are much more resistant to unfavorable conditions than are the eggs or newly hatched larvæ. They can exist under deprivation of air for a long time and may survive burial in snow for at least six days.

It was formerly thought that infection occurred by way of the mouth only, the larvæ entering with impure food or water. It is now believed, however, that this means is not only not the usual one, but that direct infection by swallowing may never occur, since there is evidence to show that the parasites are unable to resist the acid juices of the stomach before they have first passed through the blood and tissues of the body. It was discovered purely by accident that the hookworm larvæ can readily penetrate the skin and bore through the tissues until they reach a vein. The feet of plantation laborers are often in a bad state of soreness and ulceration due to the boring of the larvæ and to subsequent infection by bacteria. Walking on infected ground with bare feet is undoubtedly the mode of infection in the majority of cases. By the blood or lymph vessels the worms are carried eventually to the heart and thence to the lungs; 
from the lungs they pass by way of the trachea to the œsophagus, and thence to the stomach and intestine. Experiments show, however, that the larvæ may reach the intestine by other routes, leaving the trachea and œsophagus out of the circle of migration, but in any case they follow a rather roundabout path in the bloodvessels. Probably in cases of infection by food or drink the worms bore through the mucous membranes of the mouth or œsophagus during the swallowing of the food and thus, even when eaten, reach their ultimate destination by an indirect route. The larvæ shed their skins twice more after entering the human body, each time attaining more and more of the adult characteristics and growing in size at the expense of the blood and mucous membranes on which they feed. After the last moult the sexes are differentiated but the larvæ are still less than a fourth their full size and require five or six weeks from the time of infection to become fully mature. The length of life of individual hookworms in the intestine is variously estimated in months or years. The readiness with which reinfection usually occurs makes this a difficult point to determine.

The Disease. - The disease to which hookworms give rise varies to a very great extent in different individuals, and is not always dependent upon the number of worms present. It was formerly supposed that the anemia and loss of vitality produced by hookworms was due solely to the loss of blood devoured by the parasites. In cases of severe infection, where perhaps several thousands of worms may be harbored by a single patient, the amount of blood devoured must be sufficient to account for a considerable degree of anemia. However, in cases of infection with relatively few worms the symptoms are sometimes fully as marked and cannot be explained on this basis. The injuries from hookworm infection result apparently from a number of causes which may be summed up as follows: (1) ulceration or infection of the skin from wounds made by the boring of the parasites, often giving rise to an extensive affection of the feet in the form of pimples or sores called "ground itch," "water sores," etc., caused partly by entrance of bacteria into the wounds, and partly by the irritation produced by the boring of the worms; (2) loss of blood devoured by the parasites; (3) loss of blood from the bleeding of wounds into the intestines, sometimes very considerable, due to a secretion from the mouth of the worm which 
prevents the coagulation of blood; (4) the entrance of harmful bacteria and other microscopic organisms into the wounds made by the worms, resulting in the absorption of bacterial toxins and in the formation of dangerous lesions; (5) a thickening and degeneration of the mucous walls of the intestine; and (6) the secretion of poisonous substances or toxins from glands in the heads of the worms. These poisonous secretions, which have blood-destroying properties, probably account for more of the symptoms of hookworm disease than does anything else, though apparently they have widely different effects on different individuals. Sometimes the presence of eggs in the frees is the only indication of infection. Negroes as a class show far less susceptibility

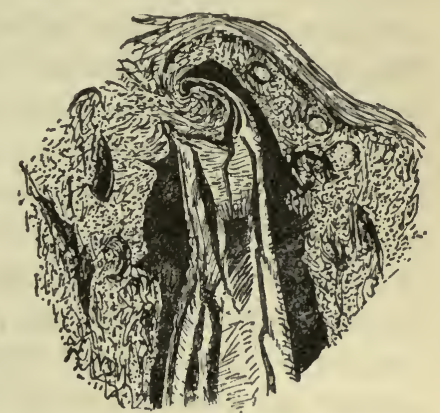

FIG. 105. American hookworm; section showing manner of attachment to intestinal wall. (After Ashford and Igaravidez, from photo by Dr. W. M. Gray.) to the poisons produced by hookworms than do the whites; this is especially well demonstrated in our southern states. The symptoms are more severe in summer than in winter, very probably due to the greater abundance of worms in the summer.

Hookworm disease is almost always preceded by a case of ground itch, due, as remarked above, to irritation from the boring of the worms and to secondary infection with bacteria. The commonest symptom of the disease is anemia, usually accompanied by some fever or dyspeptic trouble, though often in mild cases there is no evident emaciation. The significant name " el palido" (the pale one) is applied to the hookworm victim on the coffee plantations of Porto Rico. In severe cases of long standing the anemia and loss of vitality become extreme and so weaken the patient that he succumbs to the least unfavorable circumstance; his unhappy career is usually ended by some slight illness which in normal health he could easily have resisted. In Porto Rico about 30 per cent of all deaths are attributed to hookworm.

Both the mental and physical development become abnormal. A child of 12 or 14 years may have the degree of development which should belong to an average child of six or eight and a 
young man or woman of 20 may present the general development of a child of 12 or 14, though the face may appear either very childish or prematurely old. Girls who are affected from childhood lack development of the breasts, but in general there is no marked loss of flesh. The face has a stupid bloated appearance, and the eyes have a hollow stare which is very characteristic. The bloating carried to the abdomen results in "pot-belly." The appetite, at first ravenous, diminishes with the progress of the disease, and frequently becomes perverted so that patients become dirt-eaters, i.e., have a mania for swallowing earth or mud, possibly a reaction involuntarily prompted by the irritation of the intestinal tract by the parasites. Over 25 per cent of the hookworm patients of one physician in our southern states confessed to " dirt-eating." The diseased appetite, of course, only adds to the infection. The nervous symptoms, which are rather late in appearance, consist of dizziness, headache and profound stupidity.

The loss of efficiency from hookworm infection is startling, and the slow development of many countries may be largely attributed to the handicap placed upon the citizens by the hookworm. The effect of the disease can be appreciated from the following examples: the managers of large coffee "haciendas" in Porto Rico state that hookworm reduces the average efficiency of the laborers from 35 to 50 per cent. On a cocoa plantation in Ecuador not over 33 per cent of the work which should have been obtained from 300 laborers was available, due to anemias of hookworm and chronic malaria. On a sugar plantation in British Guiana, after the laborers had been treated for hookworm on a large scale, the working power of the gangs increased 100 per cent. Dr. McDonald of Queensland, Australia, reports that hookworm "is sucking the hearts' blood of the whole community." The loss of efficiency of the miners in a single California mine, due to hookworm, has been estimated at 20 per cent. Estimating only 50 per cent of the miners to be infected, the annual economic loss in this one mine would be $\$ 20,000$ per year. The economic loss due to the infection of 2,000,000 or more people in the southeastern United States or to the infection of from 60 to 80 per cent of the $300,000,000$ people of India must be almost incalculable.

The retarding effect of the disease in education and civilization 
is not less terrible. There are many families in our South where for at least four generations illiteracy and ignorance have resulted from disablement by hookworm disease. In many communities large proportions of the children are kept out of school on account of physical or mental disablement from this cause. Unlike many diseases, this one has no tendency to weed out the weak and unfit; it works subtly, progressively, undermining the physical and intellectual life of the community, each generation handing down an increased handicap to the next.

Treatment. - Treatment of hookworm disease consists, primarily, of the administration of a drug which will kill and expel the worms from the intestine. In severe cases this is followed by treatment with a tonic to bring back some of the lost health and vitality. Recently it has been shown possible to hasten recovery after expulsion of the worms by vaccinations prepared from bacteria which are found in abundance in the fæces. This indicates that some of the evil effects of hookworm disease are due to absorption of bacterial toxins through the injured intestine.

Until recently the classical remedy for use against hookworm has been thymol. This is a drug which is poisonous to the human system but under ordinary circumstances is not absorbed by the digestive tract. It is, however, very soluble in alcohol, ether and various oils, so that certain precautions have to be taken in its use, and it should not be taken except under medical supervision. Thymol is not highly efficient except in repeated doses, taken some days apart, and this is a severe handicap in its use. During the five-year period from 1909 to 1914, however, the American Hookworm Commission, largely by the coöperation of local physicians, treated nearly 700,000 hookworm patients in southern United States with thymol.

A few years ago oil of chenopodium came into favor in some parts of the United States as a remedy for hookworm, and is now rapidly supplanting all other remedies in all parts of the world. It is made from a common weed, usually called Jerusalem oak or goose-foot, and is therefore very cheap and the supply inexhaustible. It is more effective than thymol and is if anything less dangerous to the patient. According to Hall and Foster, oil of chenopodium is not entirely harmless, and among other effects is distinctly constipating. To hasten the elimination of the chenopodium as well as to counteract the constipating effect 
and the slow absorption through the intestinal walls, Hall and Foster strongly advise giving castor oil with the chenopodium and also afterward; this gives a maximum of both efficacy and safety. The usual method of giving oil of chenopodium is five to 15 drops at two hour intervals; each dose should be accompanied by castor oil.

A number of investigators have pointed out the superior effect of oil of chenopodium when given with chloroform. Hall and Foster, by means of extensive experiments on dogs, have demonstrated that chloroform itself is more efficient against hookworms than any other drug with which they have experimented, and they could find no evidence of superior efficiency of a combined use of both drugs, except in case of accompanying infection with Ascaris, against which oil of chenopodium is particularly effective. Chloroform dissolved in castor oil can be given internally in from three to four gram doses with as great a degree of safety as can other drugs in common use for worms, its safety lying in its rapid elimination from the system. A dose of chloroform should not be repeated, however, in less than three weeks, since it does some temporary damage to the liver which may not be completely repaired in less than that time.

Beta-naphthol is considered by some physicians better than thymol, especially when distributed to laborers for use without medical supervision, since there is less chance of bad results, and it can be taken safely by an ignorant person with a few simple directions. This drug is used for treatment of coolie laborers in Ceylon, and new consignments of coolies are treated with it whether infected or not, since a great majority of them are parasitized.

Male fern is sometimes used for expelling hookworms but is more dangerous than either thymol or beta-naphthol, is more expensive and is if anything less efficacious. Oil of eucalyptus has also been used with some success. It has the advantage of being less unpleasant and less dangerous than some of the other drugs in common use.

Prevention. - Methods of prevention of hookworm disease are suggested by the mode of infection, namely, contact with soil or water contaminated by infected fæces. The ways in which such contact may be made are numerous, and vary with the habits, occupation and wealth of the inhabitants. Plan- 
tation workers on our sugar and cotton plantations and on the coffee plantations of Central and South America, and the coolies working on the estates of China, India and other tropical countries, practically never wear shoes. The necessity for shoes is unknown, the discomfort of using them when the habit of going without them has been long established makes their use difficult to encourage, and there are very few who could afford such luxuries even if their value were appreciated. As shown above, the hookworm larvæ in soil or water commonly gain access to their hosts through bare feet. The readiness with which infection may occur by contact with contaminated ground or water is shown by the case of a prominent American in Porto Rico who became infected by removing his boots and wading in a small pool. Kneeling bare-kneed or resting the bare hands on the moist ground beside a stream or pool to drink; drinking water which has been directly or indirectly polluted; dirt-eating, which is a common perversion of the appetite in intestinally diseased people; eating with soiled or dirty hands; the chewing of dirty fingernails; in all these and a hundred other ways the agricultural laborer may become infected.

Miners, working underground where they are continually in contact with earth, are exposed equally as much as agricultural laborers, and more so in relatively cold countries such as those of central Europe, since the warmth resulting from subterranean location allows the parasites to thrive where on the surface they would perish. Dirty hands, unsanitary habits and polluted water are the cause of the high percentage of hookworm infection in mines where no special preventive measures are practiced.

Sanitation. - Prevention of hookworm disease, were it not for the inevitable ignorance and stupidity of many of the people to be dealt with, would be a relatively easy matter. The comparative ease with which infections can be discovered, the readiness with which the parasites, once discovered, can be expelled, and the ease with which heavy infection, even in badly infested countries, can be prevented by cleanliness, sanitation and care of exposed parts of the body are factors which should make the hookworm relatively easy prey for the hygienic reformer. But the hookworm has a valiant ally in the stunted brain and will of its victim and in the unsanitary habits, established by countless generations, which characterize the natives of almost every hook- 
worm-infested country, and for these reasons alone the eradication of the disease has in many cases been a greater stumbling block to medical science than that of even malaria or yellow fever. Mosquitoes are easier to control than are the hopelessly ignorant and stupid victims of hookworm disease!

The keynote in the prevention and eradication of hookworm disease is the prevention of pollution of the soil, in other words, proper sanitation. Not only the hookworm, but almost all of the true nematode parasites of the human intestine, are the direct outcome of unsanitary conditions. The early stages of development, so far as is known, are invariably passed in water or moist soil, and for this reason the sanitary disposal of fæces would forever put an end to such of these parasites as are peculiar to man. The difficulties involved in this simple hygienic principle are infinitely greater than the average civilized and cultured person would suspect. In southern United States, 68 per cent of the rural homes are estimated to be without privies of any kind. In many rural districts where privies $d o$ exist, their use is restricted to the women and children or to the family of the manager. In most tropical countries where coolie laborers are employed, practically all of whom carry infection, no attempt is made to provide any kind of place for defecation, not even a simple hole in the ground. The condition in this regard among the "jibaros" or plantation laborers of Porto Rico, for instance, is fairly represented by this case - of 61 hookworm patients at Utuado, 55 never had used privies of any kind, and of the six who did occasionally use them only two lived in rural districts! The extent to which the unhygienic conditions may go, and the readiness with which infection with various intestinal worms may take place, is demonstrated by the occurrence in Brazil of three species of intestinal worms in a baby three months old.

The time when the value accruing from proper sanitation will be realized to an extent sufficient to make man as careful concerning his personal habits as are some of his domestic animals is still in the future; but it is reasonable to hope that it will soon be at hand. It is a significant fact that the domestic cat, which sanitarily covers up its excreta, has, on the average, fewer intestinal parasites than the less careful dog. Dr. Stiles has recently tested the effect of sanitation and consequent reduction of intestinal parasites, both protozoans and worms, by examination of 
school children from sewered houses and from houses with privies. The statistics compiled from the data obtained showed that the children from sewered houses possessed fewer parasites and averaged a higher grade in school than those from houses with privies, even though the difference was undoubtedly reduced by the fact that sewered homes suffered from proximity to the privies of unsewered homes and from consequent infection by flies and other agents of transmission.

The most important and effective preventive measure against hookworm and other intestinal nematodes which can be inaugurated is the enforcement of the building of privies or latrines of some sort, if it be only a ditch which is occasionally covered with earth or disinfected, for the use of laborers on plantations and estates, and the placing of a penalty or fine for unnecessary pollution of the ground or water where there is any danger of spreading hookworm infection, especially along roads or on plantations. Naturally such practices as the use of night-soil (human fæces) for manure, which is extensively practiced in China, should be stringently forbidden, unless the material can be disinfected by chemical treatment, as suggested by Leiper. The fæces of all infected persons, as well as those of any suspected persons, should be carefully disinfected. The use of common salt as a disinfectant against hookworm has been found efficacious, but it must be used in rather large quantities. Nicoll, in Australia, in experiments recently conducted with hookworms, obtained rather unsatisfactory results with salt treatment of infected fæces, unless the salt was used in very large quantities and was very thoroughly mixed with the infective material. The spraying of the earth walls and floors of mines with a strong salt solution or other disinfectant, and a similar treatment of factories, yards, etc., which are known to be infected, is a preventive measure which is said to bring good results, but in the light of Nicoll's experiments this should be reinvestigated. Wearing of boots or shoes by mine workers, agricultural laborers and all who work with brick, pottery, earth roofing, etc., is recommended as a protective measure by the boards of health in some countries. This certainly is a good recommendation when it can be followed, but it should be remembered that many who need protection the most are unable to invest in such luxuries as shoes, and that at best little advance towards the final eradication of the disease is 
made by such measures. . If money sufficient to buy shoes and other protective garments were invested in improving sanitary conditions much more permanent good would result. This does not mean, however, that such protection as is gained by the use of shoes and spraying of ground is not well worth while for such individuals as can afford it and who are forced by occupation or other circumstances to come in contact with polluted soil.

The isolation and treatment of infected persons is to be highly recommended, especially in case of immigrants or new arrivals from infected regions. In 1910 the Board of Health of San Francisco made an examination of a shipload of Hindus which had just arrived and found 90 per cent to be infected, whereupon a quarantine was established, and has since been maintained, for hookworm patients. Every colony of Hindu coolies in California is a center from which hookworm disease is spreading. Had a hookworm quarantine been established years before; California would have been to a great extent free from this parasite. Q Quarantine measures have been taken in Natal, where all infected immigrants are treated before being assigned to plantations. When the infection does appear in a mine or plantation the infected persons should be treated, and not allowed to return to work until their fæces are free from eggs.

The treatment of hookworm disease is of such vital importance to the public of any endemic region that it should be supervised and aided by the government. Such aid should consist in the establishment of free dispensaries for hookworm patients, the supply of necessary drugs at cost for the treatment of hookworm disease, the appointment of inspectors to enforce sanitary regulations, and the distribution of information regarding the disease by free pamphlets, public lectures and school instruction. In the United States this work has been done largely by the American Hookworm Commission, financed by a gift of $\$ 1,000,000$ from John D. Rockefeller. In 1914 the Rockefeller Foundation extended the work of hookworm eradication "to those countries and peoples where conditions invite." Such work has been begun in a number of West Indian islands, Central America and Egypt.

It has been pointed out that demonstration as well as instruction is necessary to impress the natives of hookworm districts with the advantages of sanitation and hygienic conditions. It is absurd to rely upon the ability of the average native, dwelling 
in a filthy environment in which he was born and brought up, to form a conception of community cleanliness, which he has never seen, resulting in public benefits which he has never known. The erection of schools, hospitals, residence sections, etc., which are models of simple but efficient sanitation, would go much further toward securing the coöperation of natives in duplicating such conditions than would any amount of instruction without such practical demonstrations. 


\section{CHAPTER XV}

\section{OTHER INTESTINAL ROUNDWORMS}

General Account. - As compared with the hookworms all the other intestinal roundworms, except trichina, which will be discussed in the following chapter, sink into relative insignificance, but there are several species which are very common in some parts of the world and some which are of very wide distribution. The pathological effects of some of these worms appear to be slight or almost entirely negligible, - while others, at least in individual cases, cause severe symptoms and may even be a direct cause of death. Recently, as has been remarked in a preceding chapter, more and more suspicion is being aroused against various intestinal worms, especially those which habitually inhabit the cœcum and appendix, as playing a leading part in producing appendicitis. The relation of intestinal worms to bacterial infections is discussed on pp. 203-204.

As regards the selection of a drug for treatment of any of these rarer intestinal parasites, certain general principles should be of value. As has been pointed out by Hall and Foster, " almost all anthelmintics (i.e., drugs used against worms) are poisons, intended to kill or stupefy or otherwise disable and remove worms, while at the same time inflicting a minimum amount of damage on the host animal by virtue of the comparative insolubility of the drugs or their rapid elimination." For worms situated in the upper portions of the digestive tract, drugs such as chloroform, which are rapidly absorbed and eliminated, can be used, whereas for worms situated in the lower portions of the digestive tract, insoluble drugs would in general be better. That certain drugs have more or less specific action against certain species of worms is true, as evidenced by the case of oil of chenopodium against ascarids, and chloroform against hookworm. It is quite probable, however, that this apparently specific action may be due rather to a mode of life of the worm affected which makes it particularly easily reached by the drug. Hall and Foster, for instance, suggest that the striking efficiency 


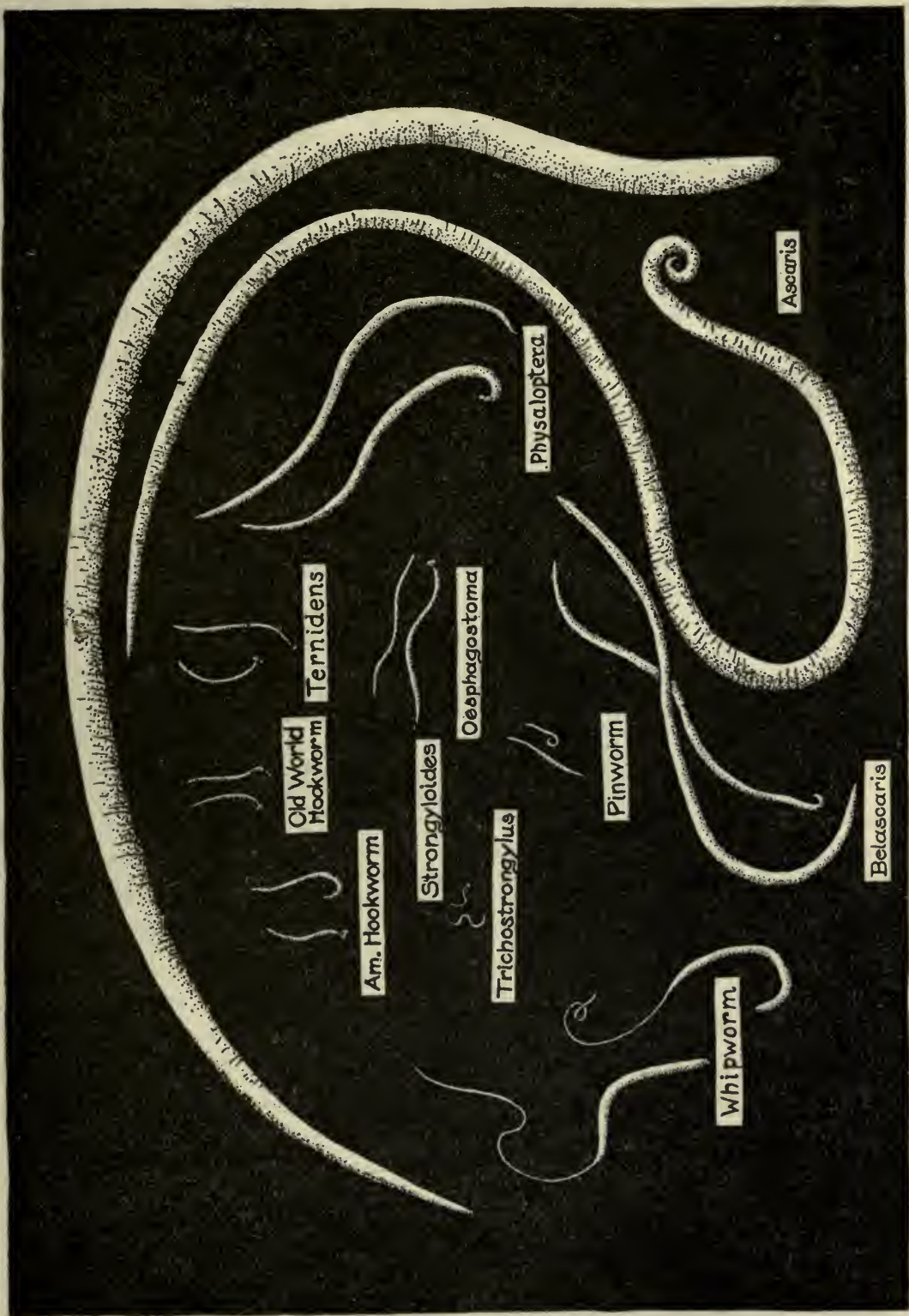

Fig. 106. Intestinal nematodes of man, natural size. Male and female of each species shown, except Strongyloides, in which only the females is known. The female of Esophagostoma is immature, the mature form being unknown. 
of chloroform against hookworms may be due to the fact that hookworms are blood-suckers and that the chloroform rapidly absorbed by the blood is ingested by the hookworms in amounts sufficient to cause stupefaction or death.

The presence of intestinal worms of most species can be determined by the finding of the eggs in the fæces, and in most cases the eggs are characteristic enough to make a determination of the species fairly easy. It often facilitates the search for parasite eggs to concentrate them in the following manner: Mix a portion of the fæces the size of a walnut with $60 \mathrm{cc}$. of distilled water, strain through several thicknesses of wide-mesh surgical gauze and centrifuge at high speed for about ten seconds. Pour off most of the liquid, add more water, shake thoroughly and centrifuge again. The material thrown to the bottom of the tube contains the eggs, which can readily be found under a microscoper A bit of the centrifuged material is placed on a slide with a little distilled water. In two or three minutes the eggs will settle on the slide, and the excess liquid can be poured off. The eggs of parasitic worms vary in size, shape, color, surface markings and state of development. Most eggs are colored yellow or brown from bile in the fæces but the eggs of the hookworms, Strongyloides, and a few others remain clear and colorless. The characteristics of the eggs of the commoner parasitic worms are shown in a comparative way in Fig. 61, p. 205. In the case of a few intestinal nematodes eggs do not appear in the fæces. In the pinworms, for instance, the adult female containing the eggs usually passes out entire, whereas in Strongyloides the eggs hatch before leaving the host.

Preventive measures against practically all of the true nematode parasites of the intestine consist mainly in proper sanitation, a discussion of which will be found on p. 265. It is possible that some of the intestinal nematodes may occasionally, at least, utilize an intermediate host of some kind, but even if this were true sanitary disposal of human fæces would, as said before, be sufficient to exterminate such parasites as are peculiar to man. The nematodes which occur in other animals as well as man have to be guarded against by other means also. The spiny-headed worms, which are transmitted in the bodies of insects which serve as intermediate hosts, are, of course, subject to quite different prophylactic measures. 
Ascaris or Eelworm. - Of greatest importance of these lesser intestinal parasites is the eelworm, Ascaris lumbricoides (Fig. 106). Ascaris is one of the largest nematode parasites known, the female averaging about ten inches in length, and occasionally measuring a foot and a half, while in diameter the body is about as large as an ordinary lead pencil. The males are usually several inches shorter. These worms are among the most frequent human parasites. They occur in all parts of the world and are found, especially in children, in the majority of temperate countries, even in countries as far north as Greenland and Finland. In the tropics they are abundant and are almost universally present in children, each individual harboring anywhere from two or three to several hundred worms.

Ascaris can be recognized immediately by its large size and robust form. The males (Fig. 107) can be distinguished by the

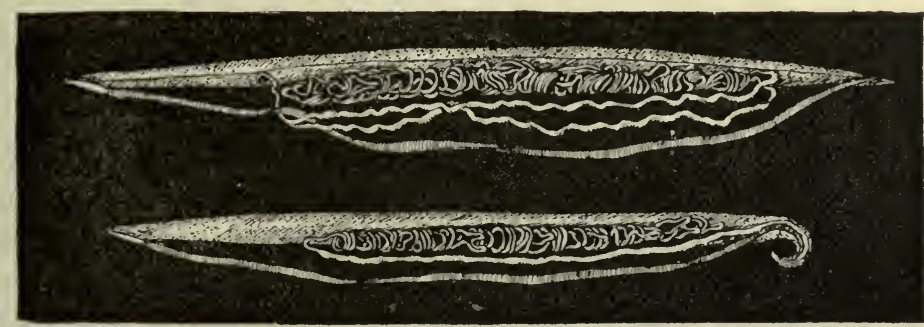

Fig. 107. Ascaris, dissected to show anatomy; female above, male below. Note ribbon-like intestine (cross-barred) with pharynx at its anterior end; the coiled threadlike ovaries in female and testıs in male; the large kinky oviducts in the female, uniting to form a vagina near the external opening on the anterior third of the body; and in the male the large sperm duct opening at the ventrally-curved posterior end of the body in common with the intestine.

sharp downward curve of the posterior end of the body, the female (Fig. 107) having a straight and rather stumpy tail. Both sexes are more slender at the head than at the tail end. The sexual organs occupy the greater part of the body. In the female they consist of two coiled threadlike ovaries (Fig. 107) and a pair of large oviducts in the form of kinky tubes which open about one-third of the way back from the anterior end. In the male there is a single coiled threadlike testis and a single sperm duct (Fig. 107), the latter opening at a cloaca at the posterior end of the body. The size and simplicity of the organs makes Ascaris a favorite subject for class-room dissection. The human species, 
Ascaris lumbricoides, is now usually looked upon as a variety of the species which occurs in hogs in almost every country in the world, and which is sometimes known as $A$. suilla.

The life history of Ascaris is usually thought to be very simple. The eggs, of which thousands are deposited by a single female, develop within the eggshell outside of the human body, in water, soil or manure piles, wherever the proper conditions of temperature can be found. The eggs (Fig. 108) are about $0.06 \mathrm{~mm}$. long by $0.04 \mathrm{~mm}$. wide ( $\frac{1}{40} 0$ by $\frac{1}{60}$ of an inch), elliptical in form

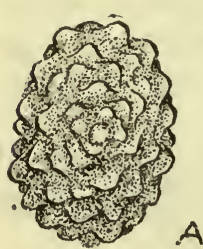

Fr 108. Egg of Ascaris; A, surface view showing warty albuminous coat; $B$, same in "optical section," i.e., with microscope focused on center of egg instead of on surface. with a thick transparent shell, usually bile-stained, covered over outside by irregular albuminous coats which give them a rough warty appearance. When passed from the digestive tract no sign of segmentation can be secn. Under favorable conditions of optimum temperature $\left(33^{\circ}\right.$ C. $)$, moisture, and oxygen supply the eggs develop in from 10 days to a month, but may be retarded for a long time by low temperatures, excessive dryness or insufficient oxygen. Although continued dryness at high temperature may be fatal to the eggs, yet under natural conditions in soil there is probably almost always sufficient moisture tc allow development to proceed, and the developed eggs may live for several years. The eggs are extremely resistant to most chemical reagents, even when used in very strong dilutions, so that ordinary methods of disinfection would be of little value in destroying them. The use of human fæces (night soil) as a fertilizer undoubtedly results in wholesale contamination of vegetables and other garden products.

The eggs only rarely hatch outside of the body; ordinarily the larvæ escape only after the eggs have been ingested and have reached the small intestine. Since eggs injected under $\ell_{1}$ e skin will hatch it is evident that some other factor besides the digestive juices is influential in liberating the larvæ.

Stewart, in recent experiments in China, was the first to demonstrate that Ascaris might not develop directly in the intestine of its host. Subsequent experiments by Stewart and also by Ransom and Foster have fully corroborated Stewart's work. 
Ripe eggs ingested by rats and other rodents hatch in the small intestine. Some of the newly-hatched larvæ may be eliminated in the fæces, but others penetrate the wall oi the intestine and go to the liver and lungs and occasionally other organs, including the abdominal cavity. The newly-hatched larvæ measure between 0.2 and $0.3 \mathrm{~mm}$. in length, but during their migrations and during their sojourn in the lungs they grow rapidly, reaching a length of from 1 to $1.5 \mathrm{~mm}$. by the tenth day, when ready to leave the lungs. They may reach the liver as early as two days after

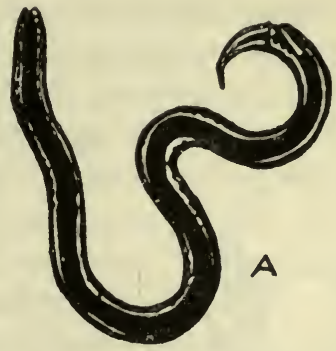

FIG. 109. Developmental stages of Ascaris; $a$, freshly hatched larva; $b$, larva from lung of rat on tenth day after infection. $\times 130$. (Adapted from Stewart.)

infection, and the lungs on the

third day. From the sixth to the tenth days the larvæ pass from the blood-vessels into the air sacs and bronchial tubes of the lungs, and thence through the trachea to the mouth. Some larvæ escape from the mouth, but the majority are swallowed and pass out with the fæces apparently dead, the host becoming free from parasites in about two weeks. It is not probable that, as at first suggested by Stewart, the rat normally acts as an intermediate host, but rather the development of Ascaris to the lung stage and failure to complete the development in the intestine may be considered as a case of imperfect adaptability of the worm to this host. It was subsequently shown by Stewart himself and also by Ransom and Foster that a similar development in the lungs takes place in pigs and other animals as well as in rats, and it is reasonable to suppose that a direct development in a single host normally takes place. However, this has been shown to be the case only in a few instances, and in cases where a massive infection would be expected from feeding enormous numbers of eggs to susceptible animals only a small number of Ascaris developed, and in some instances none at all.

Both in the hog and in man, susceptibility to Ascaris infection decreases with age. The higher rate of infection in children is probably, however, due to careless habits as well as to greater susceptibility. 
The symptoms produced by Ascaris infection vary greatly with different individuals. In some cases a great number of Ascaris may be harbored with practically no ill effects. Often, however, even when small numbers are present, peculiar mental and constitutional ailments occur, such as feverishness, anemia, restlessness, epilepsy, insomnia and deliriousness. In combination with these nervous troubles there is usually some dyspeptic trouble, such as irregular appetite, nausea and stomach aches. The nervous and other constitutional symptoms are the result

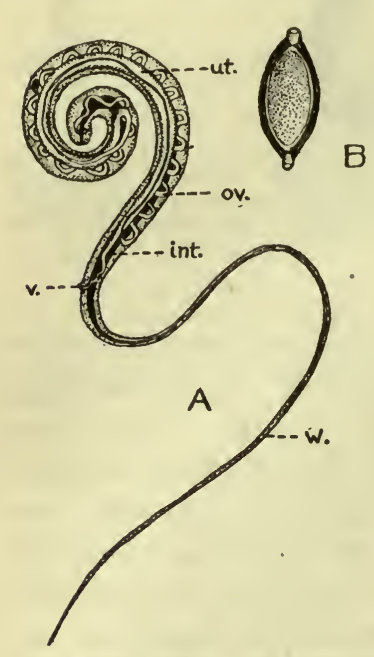

Fig. 110. Human whipworm, Trichuris trichiura: $A$, female; ov., ovary; ut., uterus; v., vulva; int., intestine; w., whiplike anterior end containing ossophagus. $\times 3$. $B$, egg; note barrel shape and pluglike bodies at ends. $\times 560$.

drug for expelling Ascaris, but oil of chenopodium has recently been demonstrated to be considerably more effective. According to Hall and Foster oil of chenopodium, properly administered (see Chap. XIV, p. 264), is almost 100 per cent effective for ascarids, and is more dependable than any other drug commonly used for worms.

Whipworm. With the possible exception of the hookworms and Ascaris, the whipworm, Trichuris trichiura (Figs. 106 and 110 ), is the most common worm parasitic in man. It is a nema- 
testine occupy the thicker posterior part of the body. The female whipworms, which are always far more numerous than the males, are about two inches long, while the males are a little smaller.

The human whipworm is found in almost every part of the world, but is especially prevalent in warm countries; it parasitizes both man and monkeys. It usually makes its home in the cœcum but occasionally establishes itself in the appendix or large intestine. It is usually said to transfix the wall of the eœcum with its threadlike anterior portion, but there is some evidence to show that it merely buries its long head and "neck" between the folds of the intestinal wall.

Usually the only evidence of the presence of whipworms is the appearance of the characteristic dark-colored, barrel-shaped eggs (Fig. 110B) in the fæces. These eggs, like those of Ascaris, develop in water or moist soil. The embryo-containing eggs are very resistant to adverse conditions and may live for years without losing their vitality. Infection, as far as known, occurs as in the ease of Ascaris. The worms may attain maturity and produce eggs in less than a month after the eggs have been swallowed. Although the whipworm feeds on blood to some extent, and undoubtedly produces toxins, as evidenced by the increase in eosinophiles (see p. 203) in the blood which nearly always occurs in case of whipworm infection and by the occasional mental disturbances and other nervous symptoms, this worm usually produces very slight, in fact often unnoticeable, effects. It is, however, thought by some workers to be one of the intestinal parasites most frequently involved in causing appendicitis. It is very difficult to dislodge the whipworm by the usual methods used for expelling intestinal parasites, probably due to its very firm attachment by the long slender " neck." Oil of chenopodium administered as for hookworm (see p. 264) is probably the most effective remedy.

Pinworm. - One of the most frequent and widely distributed intestinal parasites of man is the pinworm, Oxyuris vermicularis (Figs. 106 and 111). This parasite occurs almost universally in children at one time or another in temperate as well as tropical countries; it inhabits the lower part of the small intestine and the cœcum.

The adult females (Fig. 111 \&) are whitish worms about two- 
fifths of an inch in length, and have about the diameter of an ordinary pin. The males (Fig. $111 \delta$ ) are only about half as large and have the posterior end of the body rolled ventrally.

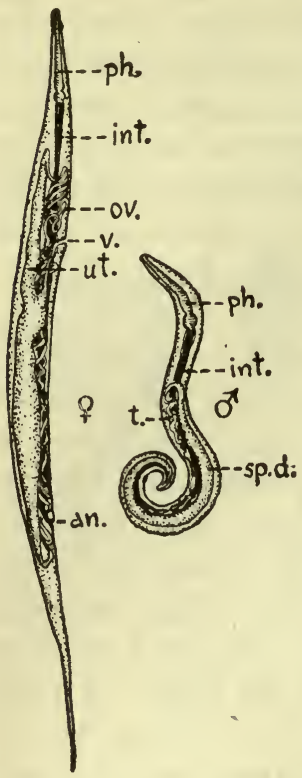

FIG. 111. Pinworm, Oxy uris vermicularis; , female; $\hat{\delta}$, male; ph., pharynx; int., intestine; ov., ovary; ut., uterus; an., anus; v., vulva; t., testis; sp. d., sperm duct. $\times 8$. (After Claus, from Braun.)

The adult females filled with eggs leave the small intestine and cœeum and wander back to the rectum whence they are passed out with the fæces or creep out of the anus, especially in the evening or at night, causing intense itching. These egg-filled females, or the free eggs which already contain coiled embryos, live in the moist groove between the buttocks, in girls sometimes creeping forward to the vagina. From the scratching and rubbing which results from the itching in the vicinity of the anus the fingers and fingernails become infected with the eggs. The eggs may then be transferred to the mouth directly or indirectly, thus causing reinfection, or they may be transmitted from person to person by unclean hands. Infection may also occur by swallowing the mature egg-filled female worms, or by the eating of raw vegetables or other foods which have been polluted by the eggs. As in the case of other parasite eggs, those of the pinworm may also be scattered by flies which have visited infected fæces.

When first deposited, the eggs, often hanging together like short strings of beads, contain larvæ which resemble tadpoles (Fig. 112A). In the fæces or in the moist groove between the buttocks the larvæ, still in the eggs, transform within a few hours into worms of typical nematode form (Fig. 112B). Later stages are shown in Figs. $112 \mathrm{C}$ and D.

After infection, which probably nearly always occurs by way of the mouth, about two or three weeks elapse before sexual maturity is again attained and the eggs and females reappear in the fæces.

While usually no inconvenience is felt from the presence of 
even large numbers of pinworms, since they do not suck blood and seldom cause intestinal lesions, yet they sometimes produce reflex nervous symptoms, probably by secretion of toxins, and they may interfere with the normal action of the bowels. As
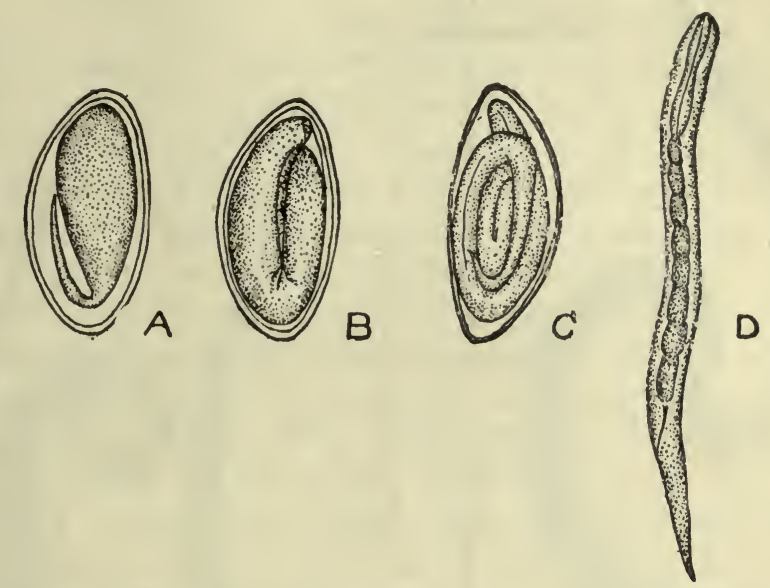

Fig. 112. Early development of pinworm, Oxyuris vermicularis. A, newly laid egg containing tadpole-like larva; $B$, egg $12 \mathrm{hrs}$. later with nematode-like larva; $C$, egg with fully developed embryo; $D$, newly hatched embryo. $\times 500$. ( $A$ and $B$ after Braun; $C$ and $D$ after Leuckart.)

remarked elsewhere pinworms are believed to be sometimes, and perhaps frequently, the original cause of lesions in the appendix which culminate in appendicitis. The intense itching which they produce by creeping in the vicinity of the anus is usually the most disagreeable effect of their presence.

On account of their situation in the lower part of the intestine, treatment for pinworms should be by drugs which are not rapidly absorbed from the intestine but are relatively insoluble. Thymol, male fern and, best of all, oil of chenopodium are effective remedies.

Strongyloides. - Another parasite of the intestine which is of wide distribution and locally very common is Strongyloides stercoralis, a very small worm about one-tenth of an inch in length which bores deep into the mucous membrane of the intestine. The female strongyloid (Figs. 106 and 113A), which is the only sex known, can be recognized by its small size, and microscopically by the chain of six or eight eggs, lying near the middle of 


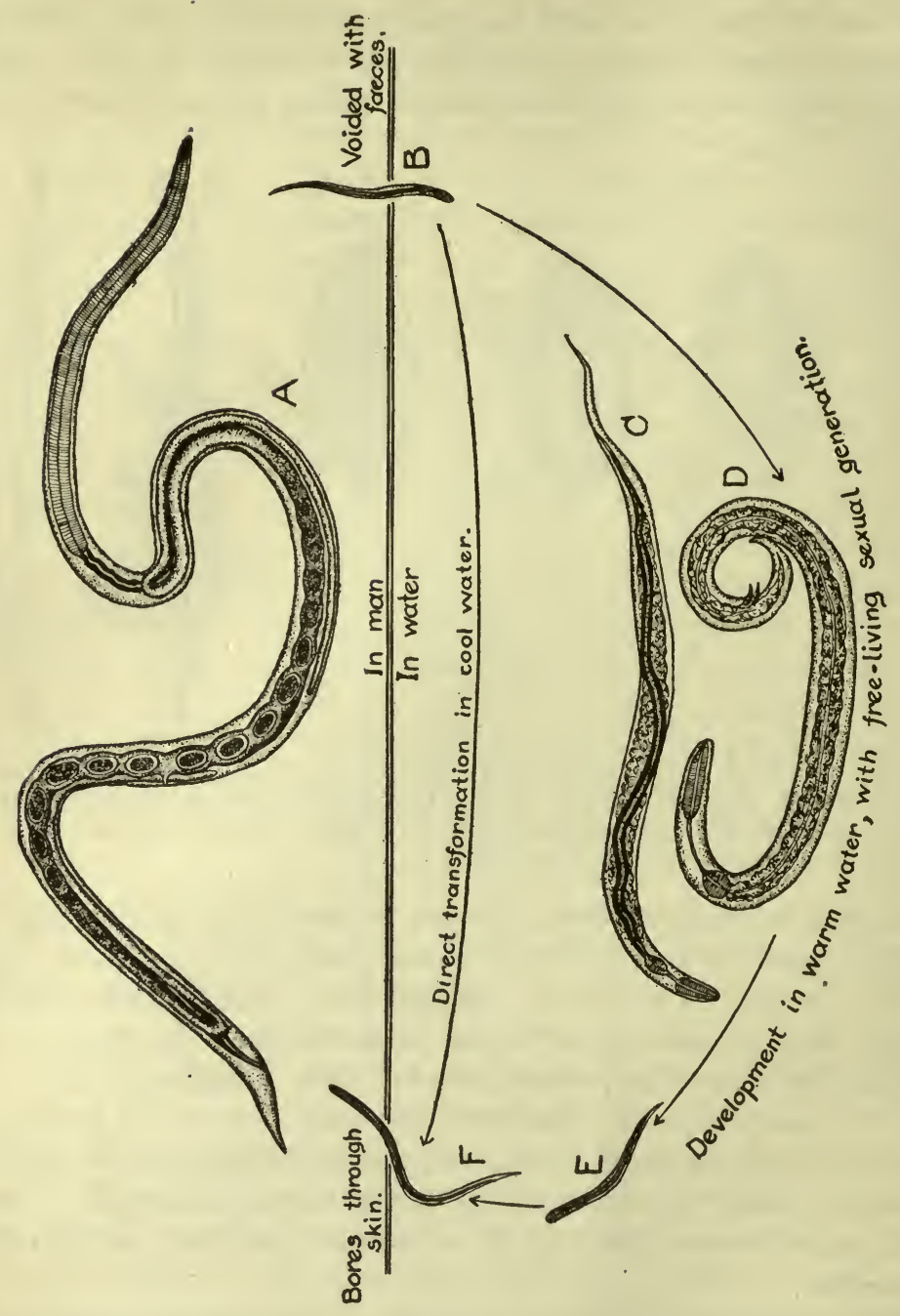

Fig. 113. Life history of Strongyloides stercoralis. A, adult female in intestine (note long pharynx, egg-containing uterus and vaginal opening on posterior third of body; $B$, newly born embryo as passed with fæces; $C$ and $D$, adult female and male, respectively, of free-living generation; $E$, "rhabditiform" larva, from female of free-living generation; $F$, filariform larva, resembling grandparent, and formed by metamorphosis of $E$, ready to infect by boring through skin. $\times 75$. (Partly after Looss.) 
the body, visible through the delicate body wall. The eggs, which are deposited deep in the intestinal coat, normally hatch before leaving the digestive tract of the host and grow considerably, so that when the fæces of an infected person are examined microscopically the active writhing larvæ (Fig. 113B), $250 \mu$ ( $1 \frac{1}{0} \delta$ of an inch) in length, can be seen darting about in snakelike fashion. Further development of the larvæ takes place in water of fairly high temperature, such as would be found under the burning rays of a tropical sun. Under such conditions the larvæ attain a sexually mature form, male and female (Fig. $113 \mathrm{C}$ and $\mathrm{D}$ ), in which they are quite different from their parents. They now copulate, and the females lay 30 or. 40 eggs, all within two days. This second generation of eggs hatch into tiny freeliving larvæ (Fig. 113E) resembling the parents, but after their first moult they lose the parental characteristics and become like their grandparents (Fig. 113F). After having reached this stage, they soon die unless they gain entrance to the digestive tract of a human being again. An unusual phenomenon is shown by these worms in that the life cycle, under less favorable conditions, can be abridged, and the alternation of generations eliminated. If, for instance, the larvæ in the fæces be exposed to the cooler water of a temperate country, they do not become sexually mature and reproduce, but transform directly into the parasitic type and reinfect without further reproduction.

The method of infection is similar to that of the hookworms. While the larvæ may occasionally gain entrance to their host with polluted water or food, they are able to bore through the skin as do the hookworm larvæ, and it is probable that this is the more usual method.

As a rule Strongyloides does not cause very serious ill effects from its pursuit of life and happiness in the intestine. Nearly all cases of diarrhea and dysentery, in which the strongyloids were formerly supposed to be the chief agent, can now be ascribed to some other cause, the strongyloids being more or less innocent bystanders. Barlow, however, reports that in 23 cases in Honduras, five of them uncomplicated, such symptoms as intermittent diarrhea without blood or mucus in the stools, colic and certain nervous symptoms were in evidence. In many cases where a diseased condition of the intestine is brought about by 
some other causes, the strongyloids increase in number and undoubtedly intensify the bad condition.

The worms are not so readily expelled by drugs as are most of the intestinal parasites, being able, on account of their small size, to stow themselves away in the folds and villi of the intestine where drugs do not reach them.

Since the strongyloid occurs in the same countries as do the hookworms, though more limited in distribution, and has a similar mode of transmission and infection, the same preventive measures which are used against hookworm are of service against this comparatively harmless companion of it.

Other Species. - There are a great many other worms which occasionally make their home in the human digestive tract, some being locally common, others merely sporadic in their occurrence; some, in fact, are not truly parasites at all, but have merely established themselves temporarily after having been swallowed with infected food. Stephens lists 59 species of nematodes as having been observed in man. None of those not already mentioned can be considered of great importance, since they seldom cause serious ailments and are most of them rare. Only those which are true parasites and have been recorded from man more than once need be mentioned here.

Belonging to the same family as Ascaris (Ascaridæ) or to closely allied families are: Belascaris cati (or Ascaris mystax) (Fig. 106) and Toxascaris limbata (or Ascaris marginata), small Ascarids two or three inches in length, normally parasitic in cats and dogs respectively, found practically all over the world but only occasionally in man; and Physaloptera mordens (Fig. 106), a worm one and a half to two inches long, which appears to be not uncommon in negroes in central East Africa.

Allied to the hookworms and having an expanded umbrellalike "bursa" at the posterior end of the male are several species of Trichostrongylus (or Strongylus). T. instabilis (subtilis) (Figs. 106 and 114) is a small worm from four to six mm. (one-fifth of an inch) in length, somewhat resembling a hookworm but much more slender. It is normally parasitic in the small intestine (duodenum) of sheep, camels, baboons and other animals and occasionally occurs in Egyptian "fellahs." A closely allied species, $T$. orientalis, is found in the duodenum of Japanese. Other species of this genus normally found in herbivorous ani- 
mals in Egypt occasionally parasitize man. The eggs of Trichostrongylus (Fig. 61Y) resemble those of hookworms, but they are a little larger and frequently contain more than four cells. The life history is similar to that of the hookworms. Ternidens (or Triodontophorus) deminutus (Fig. 106) is a worm about half an inch in length, normally found in the large intestine of monkeys in central East Africa, and not uncommon in natives; Esophagostomum apiostomum (brumpti) is a parasite which forms tumors in the large intestine of monkeys and occasionally man, in central Africa and in the Philippines. It produces symptoms of

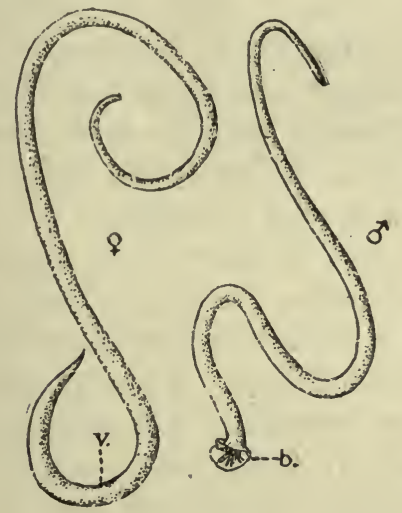

FIg. 114. Trichostrongylus instabilis; $A$, female, showing pointed tail and vulva $\left(v_{0}\right) ; B$, male, showing smaller size and bursa (b.). $\times 25$. (After drawings and measurements by Looss.)

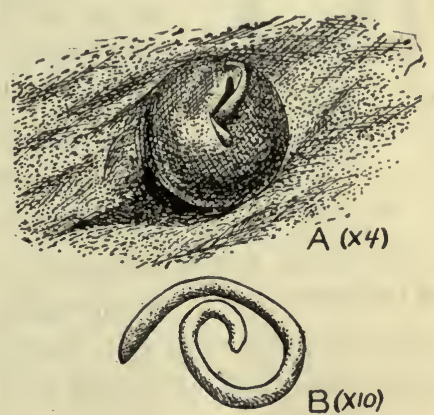

Fig. 115. Essophagostoma stephanostomum var. thomasi. $A$, immature female in eyst in large intestine of man in Brazil; $B$, same, removed from cyst. (After Thomas.)

dysentery. An allied worm, O. stephanostomum var. thomasi, a variety of a species normally found parasitic in gorillas in Africa, has been found once in man in Brazil. In this case there were 187 tumors (Fig. 115A) in the small and large intestines each containing one worm (Fig. 115B). This species will probably be found to be normally parasitic in some species of South American monkey.

These and a number of still rarer human parasites are of little interest as far as man is concerned, except as medical curiosities.

In connection with the intestinal nematodes there should be mentioned three species of spiny-headed worms (class Acantho- 
cephala) which occasionally have been found in man. These worms are not true nematodes but are distantly related to them. They are characterized by the presence, at the anterior end of

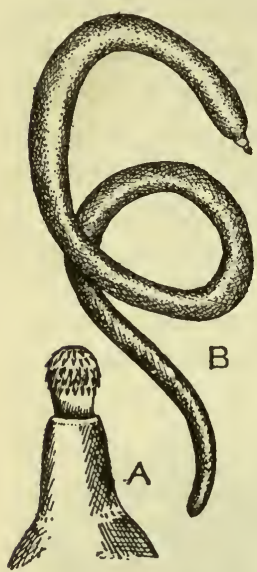

Fig. 116. Gigantoryhnchus hirudinaceus (or Echinorhynchus gigas). $A, \frac{1}{2}$ natural size, $B, \times 5$. (After Raillet from Neumann.)

is probably more frequently a human parasite is Moniliformis (or Echinorhynchus) moniliformis, normally parasitic in field mice, rats and marmots. The female worm is a
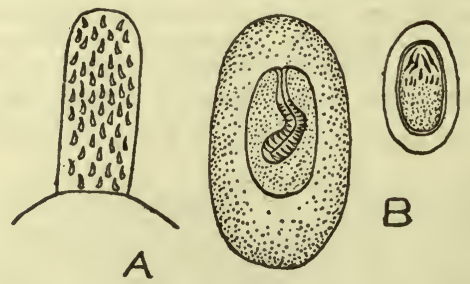

\section{C}

Fig. 117. Development of spiny-headed worm of rats and mice, Moniliformis (or Echinorhynchus) moniliformis. $A$, proboscis, $\times 50$; $B$, larva from cockroach, $\times 23 ; C$, egg, $\times 150$. (After Grassi and Calandruccio.)

little over three inches in length, the male about half this size. A species of cockroach serves as an intermediate host. Grassi and Calandruccio found by experimentation that the larvæ in cockroaches (Fig. 117B) would develop apparently equally well in white rats and in man. An allied or possibly identical species, 
M. clarki, has been described by Ward from a squirrel in Illinois; it is a worm about four or five inches in length with a very minute proboscis. Ward believes that this species would also probably develop in man if the larvæ were accidentally swallowed with some insect which presumably serves as an intermediate host. A Moniliformis which may be identical with $M$. moniliformis was found by the writer in about ten per cent of rats in Houston, Texas, and it has been reported in other parts of the United States also. 


\section{CHAPTER XVI}

\section{TRICHINA WORMS}

OF quite a different nature from other intestinal parasites is the trichina worm, Trichinella spiralis. As far as the injuriousness of its presence in the intestine is concerned it is much less serious than many of the other intestinal worms, since its length of life as an adult is relatively short. The serious and often fatal results of trichina infection are due to the peculiar life history of the worm and are concerned with the offspring of the infecting worms and not with these worms themselves.

There can be little doubt but that this worm, with the pork tapeworm as an accomplice, was responsible for the old Jewish law against the eating of pork. It was, however, many thousands of years later, in A.D. 1828, that the worms were first discovered. A little over 50 years later, 1880-1891, the trichina worm was the cause of international complications between the United States and Germany, and during this time American pork was excluded from German markets on account of the alleged frequency with which it was found to be infected. The outcome of this trouble was the beginning of the present American system of government meat inspection.

Prevalence. - Since the danger of infection from eating imperfectly cooked pork has been given wide publicity, and has come about as near to being a matter of common knowledge as any fact of parasitology, the prevalence of the infection has been greatly reduced, but even now trichina embryos are found in from 0.5 per cent to 2 per cent of the inhabitants of most civilized countries, as shown by post mortem examinations. According to Dr. Ransom, of the U. S. Bureau of Animal Industry, statistics based on microscopic inspection of $8,000,000$ hogs in the United States show only 1.41 per cent infection with live trichina worms, and a total of 2.57 per cent infection with live trichinæ and trichina-like bodies. 
In some European countries the infection is somewhat less. Some of the great epidemics of trichiniasis (or trichinosis) in Europe have been attributed to American pork, but according to Ransom there have been no authentic cases of the disease in Europe from American pork up to recently, and, so far as known, none recently. Our slaughterhouses have been referred to as the great breeding centers of trichina, but this is true only as to slaughterhouses not under federal inspection.

The rôle of the rat in the spread of trichiniasis can readily be appreciated when the statistics concerning the infection of these animals in slaughterhouses, stables, etc., are examined. Of 51 rats captured in the Boston abattoir some years ago 39 (77 per cent) were infected, and every one of 40 captured in a large exportation slaughterhouse in the same city was infected. Rats captured in stables where no hogs are kept, however, are usually less trichinized. Rats in localities where an epidemic of trichiniasis has recently swept through are usually extensively infected.

The prevalence of the disease in man is by no means parallel with its prevalence in other animals. The great controlling factor is the method of eating pork. Among such people as Americans, English and French, where pork is almost always eaten cooked, trichiniasis is rare and comes only from eating pork not thoroughly cooked, thus allowing a few worms to escape, though ordinarily not enough to cause serious disease. On the other hand very fatal epidemics have occurred among the Germans, Austrians and Italians, who are very fond of raw pork, especially in the form of sausage or "wurst." "Nearly all the epidemics in America have been among the Germans or Italians who still eling to their native habits.

According to statistics compiled by Dr. Ransom in the fiveyear period from 1909 to 1914,320 cases occurred in the United States, with 6 per cent fatality. The majority of all cases are reported as being caused by raw sausage or raw ham, and usually home-made or prepared in meat shops on a small scale. As stated by Ransom, " no cases of trichinosis have been reported which trace back to sausage prepared in establishments conducted on a large scale. While it is not impossible that such cases might occur, the chances seem very remote, for the reason that in such establishments any one lot of sausage is invariably made up of 
small portions from a large number of hogs, and the infection, if any be present among the hogs involved, is necessarily greatly diluted, with the result that no individual consuming the sausage is at all likely to ingest a sufficient number of trichinæ to produce an appreciable effect, even though the parasites should happen to survive the curing processes to which the commercially prepared sausage is usually subjected."

Life History. - The trichina worm, Trichinella spiralis, occurs in quite a large number of animals, but the readiness with which

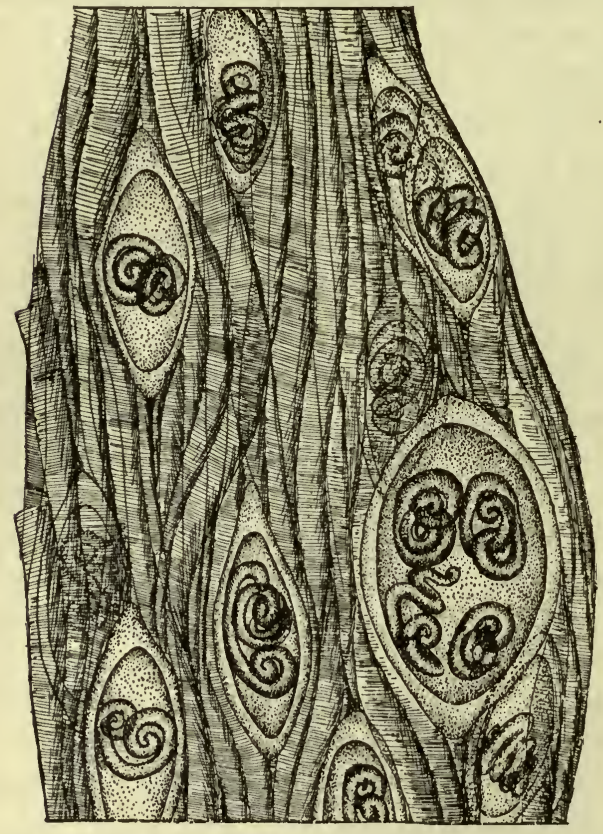

Frg. 118. Larvæ of trichina worms, Trichinella spiralis, encysted in striped muscle fibers in pork. Camera lucida drawing of eysts in infected sausage. $\times 75$. infection occurs in different species of animals varies greatly. In America hogs are most commonly infected, and infection is common in rats which have access to waste pork; in Europe dogs and cats commonly show a higher percentage of infection than hogs in a given locality. Man is highly susceptible, in fact so susceptible that he cannot be considered a normal host of the parasite. Rats and mice are sometimes thought to be the primary hosts of the worm, but the fact that these rodents succumb

easily to infection while the parasites are still in the intestinal stage tends to show that rats are not normal hosts. Rabbits and guinea-pigs are easily infected when fed meat containing the worms, and a number of other mammals can occasionally be infected artificially.

The worms gain entrance to the digestive tract as larvæ encysted in meat (Fig. 118). In the intestine of the host they are 
freed from their cysts and take refuge among the villi and folds of the mucous membrane of the small intestine. Here they mature and copulate as early as the third day after being swallowed. The female worms (Fig. 119) are from three to four mm. $\left(\frac{1}{6}\right.$ to $\frac{1}{8}$ of an inch) long, whitish in color, slender and tapering from the middle of the body toward the anterior end. The digestive tract of the worm consists of a long muscular pharynx, followed by a simple intestine. The forepart of the intestine has a very characteristic cross-barred appearance. The reproductive system in both sexes is single, i.e., with only one ovary or testis, and occupies a large portion of the body. The arrangement is different in the two sexes, the male reproductive system opening at the posterior end of the body with the anus while the female system opens on the anterior third of the body. The male worms (Fig. 119) are only about half the size of the females. The adult intestinal worms are essentially shortlived, the males usually passing out of the intestine soon after mating, and the females as soon as they have given birth to all of their offspring. The adults usually disappear within two or three months after infection.

Trichina worms are peculiar in that they bring forth living young, free of the eggshell. They do not nourish their young within the body as do truly viviparous animals, but merely retain

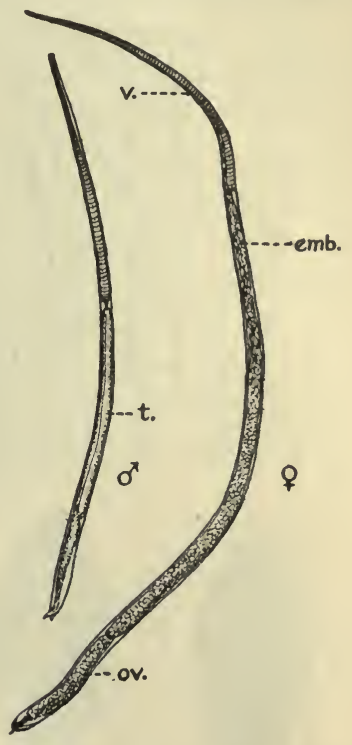

FIG. 119. Adult trichina worms, Trichinella spiralis, male $(\hat{\delta})$ and female ( $q$ ); v., vulva; emb., embryos in oviduct; ov., ovary; t., testis. $\times 5$. (After Claus, from Braun.) the eggs in the uterus until they hatch. Sometimes the young worms begin to be born within a week after the parents have been swallowed by the host. They are most numerous in the circulating blood between the eighth and 25th day after infection, though the greatest invasion occurs on the ninth and tenth days. When born they are scarcely $0.1 \mathrm{~mm}$. ( $\left(\frac{1}{5} \delta\right.$ of an inch) in length. The mother worms usually burrow into the walls of the intestine far enough so that the young can be deposited directly into a 
lymph or bloodvessel rather than into the lumen of the intestine. The larvæ are carried in the blood or lymph stream, and are distributed to nearly all parts of the body. They leave the capillaries in the striped muscles and penetrate into the fibers. Although young migrating larvæ may accidentally be carried to other tissues, and have even been found in the cerebrospinal fluid and in the mammary glands and milk of a nursing woman, they are apparently incapable of developing in any tissue except

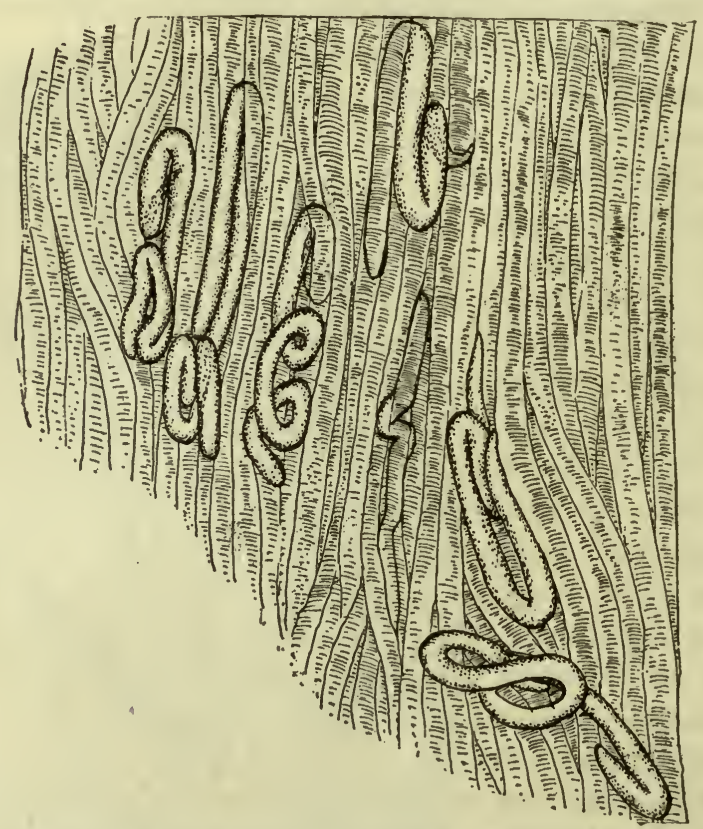

FIG. 120. Larvæ of trichina worms burrowing in human flesh before encystment. From preparation from diaphragm of victim of trichiniasis. $\times 75$.

voluntary muscle. They may settle in the heart muscle, but degenerate there without continuing their development. The muscles particularly favored by the worms are those of the diaphragm, ribs, larynx, tongue and eye, which, as noted by Stäubli, are among the most active muscles and the muscles with the richest blood supply and largest amount of oxygen. According to Flury trichinæ have a high glycogen content, and probably subsist on the glycogen stored in the striped muscles; in fact the abundance of glycogen may account for their location in these muscles. 
When the larvæ have arrived at their destination in the muscles they thread their way between the fibers towards the ends of the muscles (Fig. 120), ultimately penetrating the individual fibers where they coil up into loose spirals, constantly coiling and uncoiling as much as their close quarters will permit. When worms which are still boring are teased out of the flesh and warmed to blood heat, they can be seen constantly tightening and loosening their coiled form, reminding one of a fist being alternately clenched and unclenched. After entering muscle fibers the worms grow rapidly in size to a length of one $\mathrm{mm}$. ( $\frac{1}{25}$ of an inch), ten times their original size, and become sexually differentiated. The inflammation caused by the movements and waste products of the animals results in the degeneration of the enclosing muscle fibers and in the formation, beginning about a month after infection, of connective tissue cysts around the young worms. The cysts (Fig. 118), which are completely developed in from seven to nine weeks, are lemon-shaped, from 0.25 to $0.5 \mathrm{~mm}$. ( $\frac{1}{0} \sigma 0$ to $\frac{1}{50}$ of an inch) long, lying parallel with the muscle fibers. As a rule only one or two worms are enclosed in a cyst but as many as seven in a cyst have been observed. When first formed the cysts are very delicate and can only be seen by careful focusing with the microscope, but they gradually grow thicker and more conspicuous, and after seven or eight months there begins a deposit of chalky calcareous matter (Fig. 121A). This process ultimately results in the entire cyst becoming hardened into a calcareous
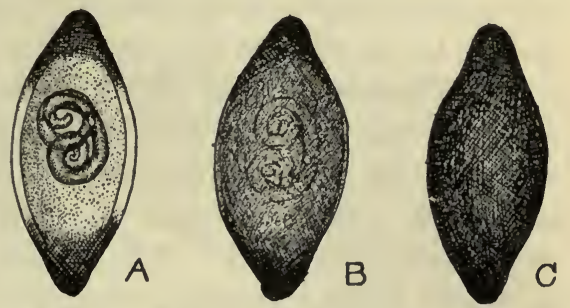

Fig. 121. Stages in calcification of trichina; $A$, ends calcified; $B$, thin layer of calcareous material over whole eyst, worm beginning to degenerate; $C$, complete calcification. (After Ostertag.) nodule (Figs. 121B and C), and even the enclosed worm, which usually degenerates and dies after some months, becomes calcified after a number of years. There are cases, however, where the trichina worms do not die and disintegrate so soon, and the calcification process is much slower. There are records of these worms found living in cysts in hogs 11 years after infection and in man 25 to 31 years after, though it is doubtful whether in some of these cases a fresh infection did not 
occur unknown to the patient or to the observers who made the records.

The larval worms, which, as pointed out by Ransom, on account of their advanced stage of development are comparable with the nymphs rather than the larvæ of arthropods, when encysted in the flesh of some susceptible animal never develop further until eaten by another susceptible animal. If they are eaten the cyst is dissolved off in the intestine of the new host, the larvæ are set free in the digestive tract, and within three days become sexually mature and copulate, to begin the performance all over.

Obviously man usually if not always becomes infected from eating infected pork, whereas hogs may be infected not only by eating scraps of raw pork but also by eating the bodies of infected rats and mice. The latter animals are infected in a similar manner. The number of trichina worms which may be harbored by a single host is almost incredible. According to the writer's investigations, the sausage which was the cause of a recent epidemic in Portland, Oregon, contained over 2,000,000 larvæ to the pound at a very conservative estimate, and in a bit of human muscle from the diaphragm of an Italian who fell victim to the disease the number of worms was even greater.

The Disease. - The disease caused by trichina worms is more fatal to man than to any other animal, the fatality sometimes rising to 30 per cent or more of the cases. Even in man the worms, if eaten only in small numbers, produce no serious or even noticeable effect. When eaten in great numbers, however, as would always happen in eating heavily-infected raw or underdone pork, the worms produce symptoms so much like typhoid fever that the disease is undoubtedly often diagnosed as such. The course of the disease, as described by Ransom, is somewhat as follows: the first symptoms of the disease - diarrhea, abdominal pains and intestinal catarrh - are the result of irritation of the intestine by the adult worms, especially the females, which burrow deep to deposit their young. Except in very light cases, a sort of general torpor is noticeable, accompanied by weakness, muscular twitching, etc. A very striking symptom, which appears in about a week and lasts for a few days, is a marked puffiness or edema of the face and especially of the eyelids. As pointed out by Ransom, the gravity of the case cannot be judged 
from the severity of the first symptoms. In some of the worst cases the first symptoms are very mild.

In nine or ten days or longer the second stage of the disease appears, accompanying the period of migration of the larvæ. This is the period which is frequently fatal. The most pronounced symptoms are intense muscular pains and rheumatic aches, with disturbances in the particular muscles invaded, interfering with the movements of the eyes, mastication, respiration, etc., the respiratory troubles becoming particularly severe in the fourth and fifth weeks of the disease, in fact sometimes so severe as to cause death from dyspnea or asthma. Profuse sweating and more or less constant fever, though sometimes occurring in the first stage also, are particularly characteristic of the second stage. The fever is commonly absent in children. The third stage, accompanying the encystment of the parasites, begins about six weeks after infection. The symptoms of the second stage become exaggerated, and in addition the face again becomes puffy, and the arms, legs and abdominal walls are also swollen. The patient becomes very anemic, skin eruptions occur, the muscular pains gradually subside and the swollen portions of the skin often scale off. Pneumonia is a common complication in the third stage.

Trichinella is unique among worms in causing constant fever. It is probable that the fever as well as certain changes in the blood corpuscles and chemical changes in the invaded muscles is due both to poisonous substances given off by the worms and to poisonous substances resulting from destroyed muscle tissue. Such substances have been found by Flury and Groll and others in cases of Trichinella infection. They are substances which act on the muscles themselves, on the nervous system, and on the bloodvessels. It is quite evident, as pointed out by Herrick, that with the invasion of the blood and tissues by millions of larvæ and with the breaking down of large amounts of muscle tissue a constant inoculation of the infected person with poisonous protein material is taking place, a condition which always gives rise to fever. Certain volatile acids are produced by the muscle degeneration, and these are considered by Flury to account for the muscular pains. Other toxic substances account for most of the other symptoms of the disease, e.g., the marked increase in certain kinds of white blood corpuscles, the eosinophiles. 
The duration and final outcome of the disease is variable, according to the heaviness of the infection. Death, as remarked before, may frequently result, and according to Ransom most commonly occurs from the fourth to the sixth week. It rarely occurs before the end of the second week or after the seventh. Recovery usually does not occur in less than from five to six weeks after infection, and often not for several months. Recurrent muscular pains and weakness may continue for years and a stiffness may persist indefinitely in the invaded muscles. Commonly cases in which a copious diarrhea appears early in the disease are of short duration and mild in type. Young children, due either to smaller quantities of pork eaten or to greater tendency to diarrhea, are likely to recover quickly.

Treatment and Prevention. - The search for a specific remedy for trichiniasis has so far been futile. Even the adult worms in the intestine are much more difficult to dislodge or destroy than are other intestinal worms, since they bore so deeply into the intestinal walls that the ordinary drugs do not affect them. Even were it possible to drive out the adults readily, this often could not be done in time to prevent disease or death, since the infection is seldom recognized before the larvæ are already produced and are migrating throughout the body. This is the critical stage of the disease; if the system can endure the irritation and inflammation produced by the burrowing of millions of worms and can withstand the effects of the toxins produced both from the worms themselves and from the destroyed tissues during the first and heaviest onslaught of the newly produced larvæ, the danger is past. The fever, the muscular pains, amounting to agony for a time, and the intestinal disorders continue for weeks but gradually subside. The treatment employed during all this time can only be systematic and of general nature - efforts to reduce the fever, to permit sleep, to keep the digestive system in as good order as possible and to do all that can be done to keep up the vitality and general health.

It is possible that if the trichina worms could be isolated and ground up, and injected into the blood, an active immunity could be built up as in the case of typhoid vaccinations. Passive immunity by injection of serum from a convalescent has been stated by Salzman to have some curative as well as preventive value, but this work needs confirmation. The disease, however, 
is not so prevalent or so difficult to prevent by other means as to make promiscuous immunization justifiable, even if possible. A more hopeful though so far unproductive line of research regarding the treatment of the infection lies in experiments with drugs or serum to kill either the adult worms in the intestine or the larvæ before they begin destroying the tissues.

Personal preventive measures against trichiniasis are easy and consist simply in abstinence from all pork which is not thoroughly cooked. Experiments by the U. S. Bureau of Animal Industry show that trichinæ are quickly destroyed by a temperature of $55^{\circ} \mathrm{C} .\left(131^{\circ} \mathrm{F}\right.$.), since the body protoplasm is coagulated at this temperature, but pork must be cooked for a length of time proportionate to its weight in order to insure the permeation of heat to the center. Experiments show that at least 30 to 36 minutes boiling should be allowed to each kilogram of meat (21 lbs.). Hurried roasting does not destroy the parasites as long as red or raw portions are left in the center. Cold storage for 20 days or more at temperatures below $10^{\circ} \mathrm{F}$. has been shown by Ransom to be destructive to trichinæ. The regulations of the U. S. Bureau of Animal Industry, relative to pork products customarily to be eaten without cooking, require freezing for 20 days at a temperature of not higher than $5^{\circ} \mathrm{F}$., or curing in accordance with certain specified processes. Temperatures above $10^{\circ} \mathrm{F}$. are more or less uncertain in their effects. Salting and smoking are not efficacious unless carried out under certain conditions. Thorough salting is effective, provided the meat is left for some time for the salt to permeate it. Large pieces of pork placed in brine have been known to contain living trichinæ for over a month. The parasites in sausages are destroyed in 24 hours by hot smoking whereas they resist cold smoking for three days.

Prevention of trichiniasis by meat inspection methods is at best only partial, and, while meat inspection might help to lessen the chances of the disease, it should not be implicitly relied upon. Probably in an ordinary meat inspection all heavy infections would be found, provided the inspector has been doing his work properly. The inspection usually consists in the microscopic examination of a bit of muscle from tongue and diaphragm; if the examination is negative, the hog is passed. Obviously light infections must frequently escape notice, and the false sense of security which is the result of knowledge that meat has been 
"inspected" may do much damage. There is no inspection for trichinæ in force in the United States at the present time.

Much could be done to prevent the prevalence of trichina infection in pork by preventing hogs from eating food which might be infected. Hogs should never be allowed access to the carcasses of other hogs or to the dead bodies of rats and mice, or to waste scraps of pork. Dead hogs or waste pork, if there is any possibility of their being infected, should not be thrown where rats and mice could prey upon them. If these principles were carefully followed out, there is no doubt but that trichiniasis could be reduced to a much greater extent than it has been.

The symptoms of trichina disease in hogs are much less evident than in man, and there is no certain diagnosis of it in living animals except by microscopic examination of the muscles for the detection of the larvæ. When heavily infected, hogs show severe intestinal disorders, abdominal pains and stiff muscles, but there is nothing diagnostic in these symptoms. A farmer who drives sick hogs to market, however, in order to get rid of them, without giving proper warning of their condition which might make possible the discovery of trichina infection if present, should be considered guilty of criminal negligence, and punished in accordance with the damage done by this negligence. This is particularly true if he feeds his hogs waste containing raw meat, or allows them to feed upon dead animals - a very common practice.

As has recently been pointed out by Stiles, there is no practical or proper method of inspecting meat by which the absence of Trichinella can be guaranteed, and it is therefore unjust to hold a butcher responsible for cases of trichiniasis which may result from the eating of pork sold by him. There are laws which provide that " diseased meat" shall not be sold and that an implied warranty of fitness for food goes with any sale of food. Neither of these laws, however, can be unreasonably enforced. Technically all meat is diseased, since there are no market animals which are not parasitized in some way. As to the "implied warranty," this can go only with an implied guarantee on the part of the buyer that the food will be properly prepared before being eaten. Clams in the shell, unhusked corn and uncooked beans are guaranteed as being fit for food only when properly prepared. In like manner pork is sold with the understanding 
that it will be properly prepared, i.e., thoroughly cooked. Raw pork, since it is likely to contain Trichinelloe which may cause disease, and since the absence of these worms cannot be guaranteed by any practical inspection now known, is unfit for food and therefore cannot be guaranteed if eaten raw. As Stiles has pointed out, great and unjustifiable loss may result from too stringent enforcement of the laws mentioned above. 


\section{CHAPTER XVII}

\section{FILARIE AND THEIR ALLIES}

General Account. - One of the most interesting and puzzling groups of human parasites are the members of the nematode genus Filaria. They are extremely common parasites in all tropical countries, have a unique and extraordinary life history, are associated with many serious pathological conditions and have figured prominently in the history of medical science.

Dr. Timothy R. Lewis first discovered these worms swarming in human blood, while working on tropical diseases in India. They had previously been observed in various bodily excretions but only in rare cases and in small numbers. They were found in enormous numbers in the blood, but only at night. The worms were evidently larvæ and since they only rarely and apparently accidentally escaped from the body with excretions, the thought occurred to Manson that they must be liberated from the blood by some nocturnal blood-sucking insect. Manson and others later proved this theory to be correct, and thus took the first step toward our present knowledge of the biological transmission of disease by insects, a step which marked the beginning of a new era in modern medicine.

Many species of Filaria from human blood have been described, some of which undoubtedly are not valid species. Some species apparently produce no pathological conditions whatever, while others are associated with, and are usually considered to be the direct cause of, a large number of diseased conditions. Some of the species are of limited geographic distribution while others are of world-wide range, probably due to differences in the extent of the distribution of the intermediate host. In some tropical localities 50 per cent or more of the population are parasitized by these animals. In South China ten per cent of the entire population is said to be infected and in some South Sea Islands over half of the inhabitants are infected. Recently in an examination of 949 natives from the Congo-Cameroon country of 
Africa, about 74 per cent of the men, 79 per cent of the women and 33 per cent of the children were found to be filariated.

The blood-dwelling filariæ which are readily observed are, as remarked above, only larvæ, the adults being much larger, long, slender worms which live in the lymphatic vessels, connective tissue or other tissues of the body. It is to these adult worms and not to the larvæ that the so-called "filarial diseases" are supposed to be due; the blood-living worms apparently cause no serious symptoms. The larvæ have been termed "microfilariæ " to distinguish them from the adult worms.

\section{Filaria bancrofti}

The most widespread species and most important from a medical point of view is Filaria bancrofti. This nematode occurs more or less abundantly in all warm climates of the world, north to southern United States and southern Europe and Asia, and south to southern Australia and Patagonia.

Life History. - The adult Filarice were not discovered for many years after the larvæ had been found in the blood, since they occur in the deepseated lymphatic vessels where they could

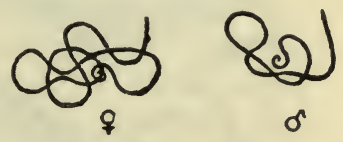

Frg. 122. Adults of Filaria bancrofti, female ( $\$$ ) and male (रे). Natural size. (After Manson.) be observed only on post mortem examinations. They are very long, slender nematodes (Fig. 122), the females three or four inches in length and hardly greater in diameter than a horsehair, and the males about half this size. In their

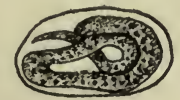
normal habitat in the lymph vessels the males and females live coiled up together, sometimes several pairs of them

Fig. 123. Microfilaria of in a knot. The male worms, in addition Filaria bancrofti as it emerges from uterus of parent, surrounded by delicate membrane. (After Bahr.) to their smaller size, may be distinguished from the females by the coiled tail which reminds one of a vine tendril. The greater part of the body of the female is occupied by a pair of uteri, which in the adult are always filled with eggs.

The eggs (Fig. 123) usually hatch before they are laid so that living young swarm forth from the parent worm, but in exceptional cases the eggs are deposited before hatching. The young 
worms reach the blood by way of the lymph stream and these grow to about $300 \mu$ (a little over ${ }_{1}^{1} \frac{1}{0}$ of an inch) in length. They are delicate colorless worms (Fig. 124A), blunt at the anterior end and tapering to a slender point at the tail end, and are entirely enclosed in a remarkably delicate transparent sheath, which, although it fits as tightly as a glove over a finger, is too long for the animal and can be seen projecting at either end. The sheath may be looked upon as a wonderful adaptation to prevent

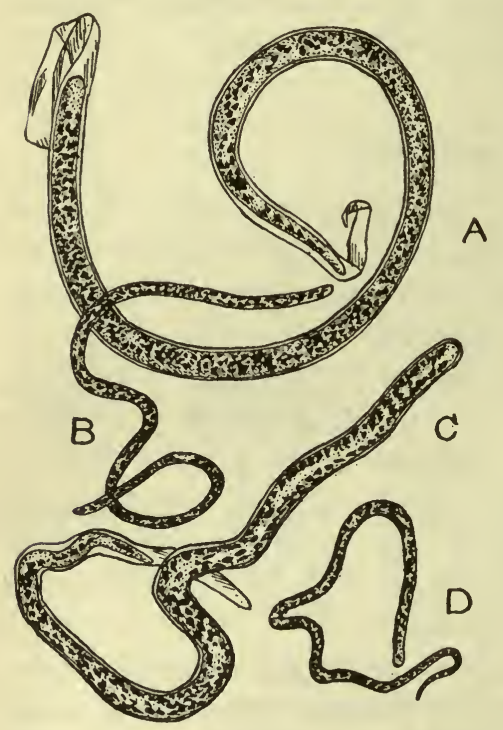

FIG. 124. Comparison of microfilariæ; $A, m f$. bancrofti (large with sheath); $B$, $m f$. perstans (small, blunt tail, no sheath); $C$, mf. loa (large, with sheath); $D, m f$. juncea (demarquaii) (small, sharp tail, no sheath). $\times 75 . \quad$ (After Manson.) the worms from being able to bore through the bloodvessels and escape from the blood, in which case they would miss their chance for "salvation." The internal organs are in a very rudimentary condition.

The most remarkable circumstance connected with the life of these microfilariæ is the periodical appearance and disappearance of them in the blood of the peripheral vessels. If the blood of an infected person is examined during the day few if any worms can be found, but as evening approaches they begin to appear and continue to increase until about midnight, after which they decrease again until morning. During the night when they are most abundant there may be as many as 500 worms in a single drop of blood. If the parasites are assumed to be evenly distributed throughout the peripheral circulation, this would imply the presence of several million worms in the body. The periodic appearance and disappearance of microfilariæ in the blood is not invariable. When an infected person is made to sleep in the daytime instead of at night, the appearance and disappearance of the parasites in the peripheral bloodvessels can be reversed, implying that the distribution of the parasites may be dependent 
on some physiologic condition of the host. Still stranger is the fact that in many of the South Sea Islands, Samoa, the Fiji Islands and the Philippine Islands, the microfilariæ show no periodic disappearance, although if a person infected in a place where the parasites do show periodicity be transferred to one of the above-named islands, the periodic phenomena still persist. As stated before, Manson, the great English parasitologist, with characteristic ingenuity, suspected that this parasite, so abundant in the blood, must make use of some blood-sucking insect as a means of transmission, and further concluded that the night swarming of the parasites in the peripheral circulation might be an adaptation to the nocturnal habits of an intermediate host. Working on this hypothesis, he discovered that certain mosquitoes acted as the liberating agents for the parasites. The fact that in those islands where no periodicity is shown the usual intermediate host is a diurnal mosquito Aëdes (or Stegomyia) pseudocutellaris, certainly bears out the adaptation hypothesis. On the grounds of the apparently distinct habits and different adaptation, the non-periodic microfilariæ have been separated into a distinct species, or at least subspecies, to which the name Filaria philippinensis was applied by Ashburn and Craig in 1906. Zoölogists are coming more and more to realize the importance of physiolologic as well as morphologic characteristics as a basis for separating species and subspecies. The case of these filariæ is by no means unique in the organic world. Physiologic and biochemical reactions are the main basis for the classification of the Bacteria, and some Protozoa can be distinguished better by their pathogenic effects and biochemical reactions than by their morphology.

To continue their development the larval worms must be sucked up by the females of certain species of mosquitoes. A considerable number of species of mosquitoes of several different genera, including Anopheles, Aëdes and Culex, may serve as intermediate hosts for $F$. bancrofti (see p. 449). The commonest and most widespread transmitting agent is the house mosquito of the tropics, Culex quinquefasciatus (fatigans), a species which also transmits dengue. A few hours after being swallowed by a susceptible mosquito the microfilariæ (Fig. 125A) become restless and endeavor to escape from their sheaths. This they eventually accomplish by butting against the anterior end, having gained as much impetus as their close quarters will allow. 
Once free, the little larvæ (Fig. 125B) move actively about in quite a different manner from the ineffective wriggling in which they indulged while enclosed in the sheath, and by means of which they were unable to "get anywhere." The active liberated

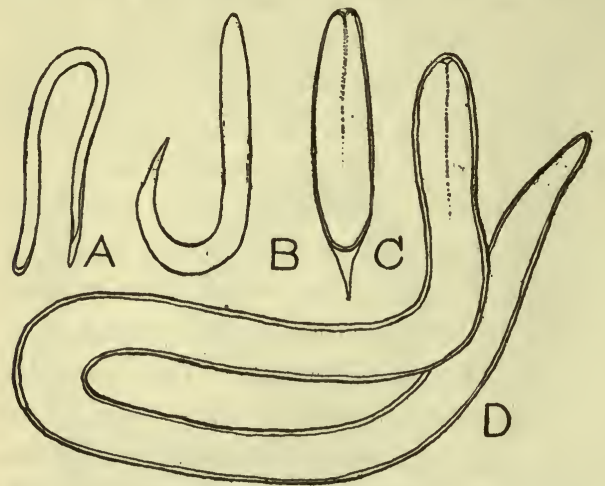

Fig. 125. Development of Filaria bancrofti in mosquito; $A$, as withdrawn with blood (first 24 hours) in stomach; $B$, form found in tissues just outside stomach (48 to 72 hours after ingestion); $C$, form found in muscles on fourth day; $D$, mature larval form, ready for transmission, in proboscis (two or more weeks after ingestion). $\times 150 . \quad$ (After Lewis from Nuttall.) internal organization of the animal undergoes a great change. The central core of cells gradually becomes differentiated into a digestive tract, separated from the body wall by a true body

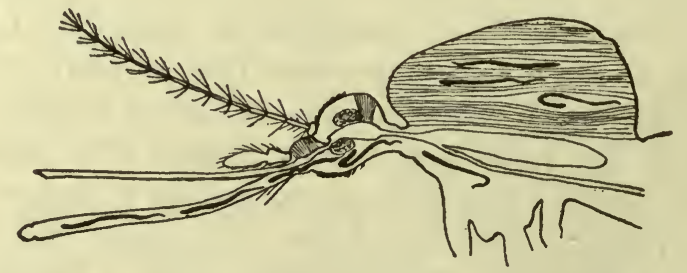

FIg. 126. Mature larvæ of Filaria bancrofti in thoracic muscles and proboscis of mosquito. (After Castellani and Chalmers.)

cavity. By the time the larva has reached its full size - about $1.5 \mathrm{~mm}$. ( $\frac{1}{16}$ of an inch) in length - the digestive tract is a complete tube with both mouth and anal openings. While these changes are taking place, the larval worm, though capable of activity, remains at rest between the muscle fibers (Fig. 126), 
but it now becomes active again and migrates into the connective tissue of the anterior parts of the body of its host, and ultimately into the proboscis (Fig. 126). Here the worms lie in pairs, or several pairs together, awaiting an opportunity to re-enter a human host.

The length of time required for the metamorphosis and development in the mosquito varies from about two weeks under ideal conditions to several weeks under less favorable circumstances. When the infected mosquito bites a human being, the worms emerge from the proboscis and bore through the skin in the immediate vicinity of the wound, though not directly through the puncture. Experiments have shown that the larvæ can not be deceived into entering vegetable tissue, such as a banana, even though for many days they have been at the tip of the proboscis, ready to emerge when the mosquito bites into warm-blooded flesh.

It is possible that these parasites may occasionally find entrance to the human body by other paths than the mosquito's bite but this has not yet been proved. The popular belief that bad water is the cause of filarial infection is probably due to the effect of stagnant water on the abundance of mosquitoes, and not to the emergence of the larvæ from the bodies of mosquitoes into water. Bahr has shown that the larvæ will live in water only seven hours.

Once back in a human body from this period of " purgatory" in the body of a mosquito the larvæ migrate to the lymphatic vessels, there to attain sexual maturity, copulate and reproduce. The larvæ of the next generation escape again to the blood as microfilariæ, and the cycle is complete. The adult worms may live for many years and even the microfilariæ are able to live for a considerable time, as shown by their continued presence after the death of the parents.

Filarial Diseases. - The disease symptoms which are associated with Filaria bancrofti can all be traced to interference with the lymphatic system. In many cases there are no ill effects of the infection felt for many years, or perhaps never, though sooner or later there is usually produced anemia, enlargement of the spleen and fever. More serious are the effects produced by obstruction of the lymphatics. This causes great enlargement of the lymph vessels and the diversion of the lymph 
from its normal channel, and results in varicose lymph glands (Fig. 127C) and vessels and in distended lymph sacs which may burst into the kidneys, bladder or body cavity. Often the microfilariæ disappear from the blood, probably on account of the death of the parents, but the obstruction of the lymphatics continues to exist, as do the evil effects resulting therefrom.
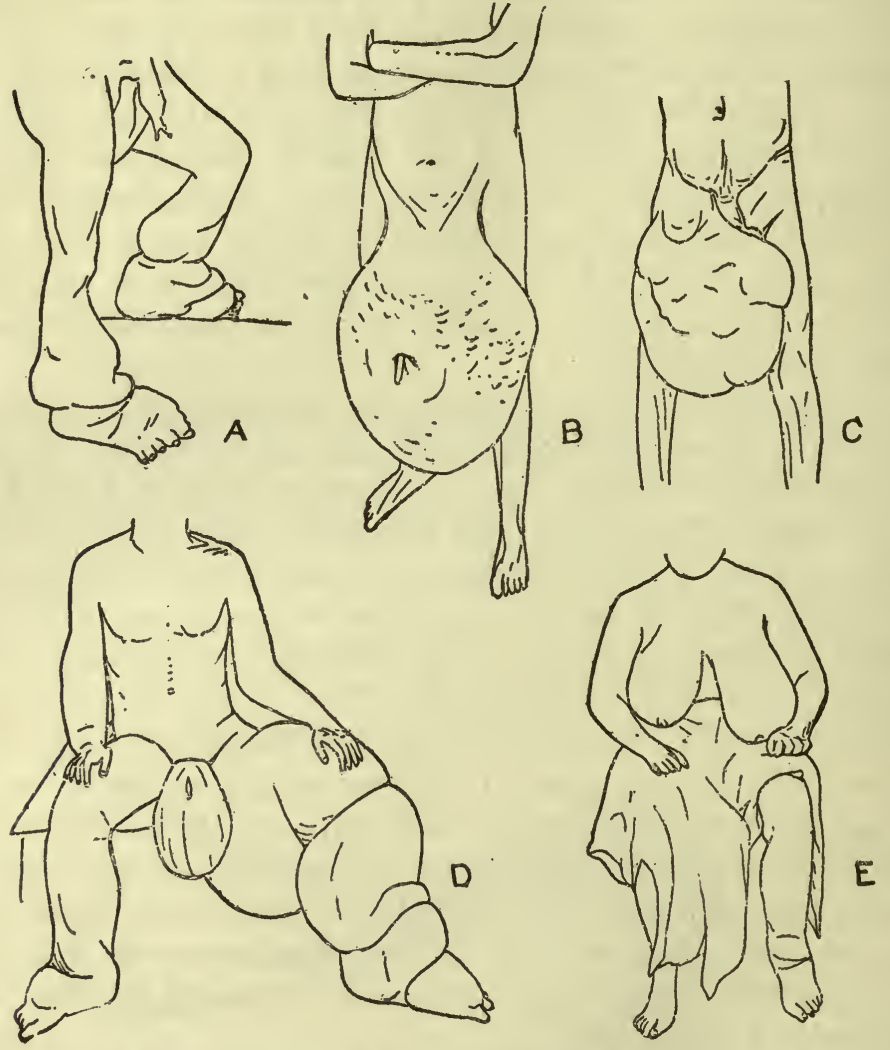

Frg. 127. A few extreme cases of elephantiasis; $A$, of legs and feet; $B$, of scrotum; $C$, varicose groin gland; $D$, of scrotum and legs; $E$, of mammary glands. ( $A$ and $B$ sketched from photos from Castellani and Chalmers; $C, D$ and $E$ from Manson.)

One of the most frequent results of a blocking of the lymph vessels is an enormous enlargement of the part of the body in which the blocking occurs, known by the suggestive name, "elephantiasis" (Fig. 127). In most cases the lower limbs and scrotum are the parts affected, though almost any portion of 
the body may occasionally become enlarged. In some South Sea Islands 50 per cent or more of the population are thus affected. The disease begins by repeated attacks, at intervals of from a month to a year, of "elephantoid" or filarial fever in which chills and high fever accompany a painful swelling of the parts affected. These attacks, also known as lymphangitis, end in an emission of lymph and a partial subsidence of the swelling. But each attack leaves a little more permanent tissue, so that in time the growth, which is hard and unyielding, develops to enormous proportions. Sometimes an affected leg may reach a diameter of several feet. In one case recorded by Manson, a scrotum affected by elephantiasis reached a weight of 224 pounds, though it must be admitted that this is unusual.

Another condition resulting from filarial infection is the escape of the contents of lymph vessels into the kidneys or bladder, a condition technically known as " chyluria." The urine is milky and coagulates after standing a short time. This condition lasts for a few days or weeks, then ceases and returns at irregular intervals. It produces severe anemia and a general feeling of ennui, and saps the vitality.

Occasionally the presence of dead filariæ in the body leads to the formation of abscesses which sooner or later discharge. If on any of the appendages, no further trouble results, but such abscesses in the internal regions of the body may have serious or fatal effects.

Though very probably some of these so-called "filarial diseases " are caused directly by the filariæ, the exact relation of $F$. bancrofti to all of the pathological conditions associated with its presence in the body is far from settled. Dutcher and Whitmarsh, of the United States Army, in investigations of filarial diseases in Porto Rico recently obtained pure cultures of a certain type of bacterium from the blood or serum of 15 patients, all but one of whom was affected by some form of filarial disease, whereas in unaffected individuals, with one exception which was looked upon as a "carrier," the cultures from the blood remained uniformly sterile. In a few cases in which filarial diseases were present the bacterium was not found but it was believed that either the infection was so light that the cultures did not happen to become contaminated, or that the infection had died out. A number of other observers have obtained cultures of bacteria 
from blood and tissues of elephantiasis cases. Others, however, have found the blood quite sterile. It is worth noting in this connection that the number of cases of elephantiasis or other filarial diseases in which microfilariæ are not present in the blood is considerably greater than those in which the larval parasites are present. This is usually explained by assuming that the parent filariæ have died or that the larvæ cannot reach the blood on account of a blocking of the lymph channels by fibrous growths. Cruickshank and Wright, for instance, in 130 cases of elephantiasis in Cochin, found only 12 with microfilariæ in the blood. The observations recorded above are certainly significant and may revolutionize our ideas in regard to filarial diseases. However, even if some of the "filarial diseases" were found to be due to bacteria, the filariæ might still be incriminated as carriers of the bacteria, and therefore as an indirect cause of the diseases.

Treatment and Prevention. - So far there is no widely-accepted treatment by which the parent filarix, and with them the microfilariæ, can be destroyed. The number of the larvæ is reduced, however, by injections of thymol, ichthyol and other drugs, and such injections might prove to be a useful preventive measure. McNaughton has recently reported five cases of filarial infection successfully treated by injections of salvarsan; one case was of ten years' standing. Usually the only course of the physician is to relieve as far as possible the abnormal conditions associated with the presence of the worms. Such relief, of course, varies greatly with the diverse pathological conditions which may arise. Varicose glands and vessels, unless causing great discomfort, are usually left alone, since they are lymph channels substituted for the normal ones in the body which have been blocked, and it is therefore dangerous to interfere with them. In cases of elephantoid fever the only treatment is such as would tend to relieve the pain in the swellings and the fever, and perhaps in severe cases the pricking of the swollen part to allow the exudation of the collecting lymph. In chyluria the treatment consists in rest and in making the pelvic regions as comfortable as possible to prevent pressure which would tend to burst the lymphatics and force the lymph into the kidneys or bladder. Elephantiasis, the commonest expression of filarial disease, is seldom completely recovered from. Formerly the only treatment was temporary reduction 
of the swellings and prevention of further growth by care of the general health, avoidance of violent exercise, massage and tight bandaging. In severe cases of elephantiasis of the leg physicians sometimes cut off great masses of the elephantoid tissue, grafting on new pieces of skin to cover the parts operated on. Removal of enlarged growths of the scrotum can usually be accomplished successfully. Another method which has been used with some success is an operation for the draining of the lymph from the tissue all the way into the bone or even from the bone itself.

Castellani has recently found a method of reducing elephantoid tissue which will probably supplant all of the above methods. This consists in the injection into the diseased tissues of a drug, fibrolysin, which, as its name implies, has the property of destroying fibrous connective tissue. Elephantoid swellings are reported to have been cured by this method in a few months.

Prevention of filarial diseases can best be accomplished by anti-mosquito campaigns. As far as is known at present mosquitoes are the only means of transmission which the parasites have. The same preventive measures, therefore, which serve as preventives against malaria, serve also against Filaria bancrofti, and since the former disease is found practically everywhere that the filariæ are found, it is possible to prevent the two diseases with one effort. People who carry filariæ in their blood should be prevented, as far as possible, from exposing themselves to mosquitoes. In the places where the microfilariæ are periodic and the transmitting mosquitoes are nocturnal this should be perfectly possible, although in such localities as the Philippines and Samoa, where the intermediate host is largely diurnal, it would present almost insuperable difficulties. In places where Filaria is abundant and mosquitoes are not exterminated the carrying at night of a bottle of disinfectant, as alcohol or dilute lysol, for immediate application to mosquito bites would be well worth while.

\section{Other Species of Filaria}

There are, as previously stated, a number of other species of Filaria which inhabit the human body. Filaria (or Acanthocheilonema) perstans is extremely common in the natives throughout Central Africa and also in parts of northern South America; 
it is confined to regions of heavily forested tropical swamps. In some districts in Uganda it has been found in 90 per cent of the inhabitants. The microfilariæ of this species (Fig. 124B) are smaller than those of $F$. bancrofti, have a blunt tail and lack the sheath which is so characteristic of $F$. bancrofti. Furthermore they show no tendency to disappear periodically from the peripheral vessels. The adult worm, which has rarely been found, is smaller than $F$. bancrofti (about three inches in length) and occurs in the connective tissue of the abdominal and pericardial cavities. The normal transmitting agent, probably some species of mosquito, is not certainly known. No disease symptoms which can be correlated with the presence of the parasite have yet been demonstrated.

Another species, $F$. juncea (demarquaii), of which the larva (Fig. 124D) is small and without a sheath, as in $F$. perstans, but with a sharp tail, occurs in the West Indies and northern South America. It is not known to cause any diseased conditions. The adults live in the mesenteric tissues. In many Indians in British Guiana $F$. perstans and $F$. juncea occur together in the blood, and in some cases the presence of $F$. bancrofti complicates the matter still more.

F. magalhãesi is another species about which very little is known. A pair of adult worms were found only once, in the heart of a child in Rio de Janeiro. They were of unusually large size, the female measuring over six inches in

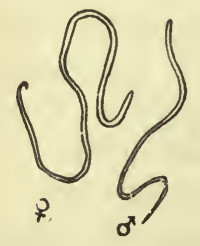

Fig. 128. Adult loa worms, female (q) and male ( $\hat{\jmath})$. Natural size. (After Looss.) length and the male about three and a half inches. Nothing is known of the life history or pathological effects.

The Loa Worm. - Of somewhat different nature from the above species of Filaria is $F$. loa or Loa loa (Fig. 128), a parasite found on the west coast of Africa, especially in Congo, which, as an adult, creeps in the connective tissue of its host just under the skin. The female varies, probably with age, from two to two and one-half inches in length, and is semi-transparent and very slender. The male resembles the female, but is only from one to one and one-half inches in length. Both sexes are characterized by numerous irregularly distributed pimple-like elevations of the skin. The loa worm shows a special preference for the connective 
tissue in and about the eyes, but may also be found creeping under the skin of fingers, breast, back, etc. A loa is said to travel at the rate of about an inch in two minutes, and to become especially active in the presence of direct warmth on the skin, as before a fire. The migration of the worms causes itching and a " creeping " sensation, and in some unexplained way gives rise to temporary swellings, from half an inch to four inches in diameter, known locally as "Calabar swellings." These swellings may shift their position an inch or more a day, and may disappear to reappear somewhere else. This relation of Loa to Calabar swellings has not been definitely proved but there is strong evidence for it. In one case Manson succeeded in finding great numbers of microfilariæ of Loa in lymph taken from one of these swellings, a fact which gives color to Manson's hypothesis that the swellings might be due to the emission of larvæ from the parent worm into the connective tissue. The larvæ of the parasite (Fig. 124C), very closely resembling the microfilariæ of $F$. bancrofti, occur in the blood in great numbers, but they have a periodicity directly opposite to that of the latter species in that they swarm in the peripheral blood in the daytime and withdraw to the larger vessels at night. The living larvæ of the two species cannot readily be distinguished from each other in fresh blood, but in dried and stained preparations the dead organisms can easily be identified. The micro-

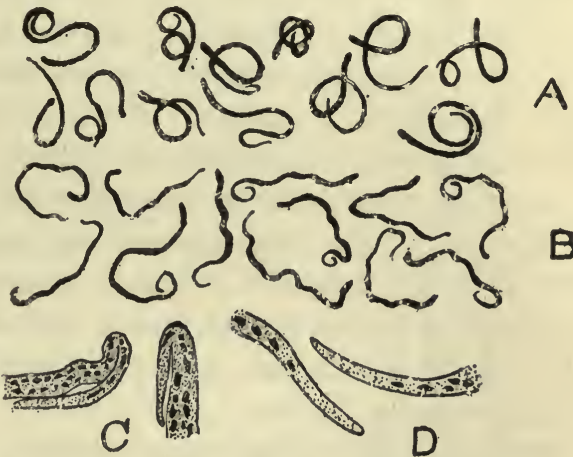

FIg. 129. Comparison of killed and stained specimens of Microfilaria bancrofti and $m f$. loa. A, $m f$. bancrofti, - note graceful curves; $B, m f$. loa, - note irregular scrawl-like curves; $C$, tails of $m f$. loa; $D$, tails of $m f$. bancrofti. (After Manson.)

filarice bancrofti are found lying in smooth graceful curves (Fig. 129A), while the microfilarice loa die in ungraceful and irregular scrawl-like positions (Fig. 129B), with the tail nearly always sharply turned back (Fig. 129C).

There is much evidence that the intermediate hosts of $L$. loa are mangrove flies of the genus Chrysops, which belong to the 
horsefly family, Tabanidæ, and resemble our deerflies (see p. 489 and Fig. 227). Leiper succeeded in obtaining a development of microfilaria loa in two different species of Chrysops. In recent investigations in a heavily infested district of Africa, Kleine found over five per cent of 600 Chrysops infected with larval filariæ, which he took to be Loa loa. The worms were found developing in the fatty connective tissue surrounding the tracheæ in the abdomen of the insects and later making their way forward toward the proboscis. In two cases larvæ were induced to emerge from the fly's proboscis into a few drops of salt solution. That these worms were really the larvæ of $L$. loa is entirely probable, but there is no definite proof of it.

The development of the parasites after they have been returned to a human body is extremely slow, in fact the evidence indicates that full sexual maturity is not reached for a number of years. The length of life of the worms is unusual; there are cases recorded in which these parasites were abstracted from patients who had been away from endemic regions for ten or 15 years. Microfilariæ are not invariably found in the blood of infected persons. Children, especially, are prone to infection with the creeping worms, usually sexually immature, without having any larvæ in their blood. Even sexually mature parasites apparently do not liberate larvæ constantly.

Surgical removal of the parasites when they present themselves in the eye or subcutaneous tissue is the only remedy so far known. Many of the parasites probably do not expose themselves at all, but remain in the deeper tissues and organs of the body. When they die in the tissues they probably become calcified as do the adults of other filariæ.

Onchocerca volvulus. - Closely related to the filariæ is another parasite of the subcutaneous connective tissue, Onchocerca volvulus. It occurs over a large portion of the west coast and central portion of Africa. Three cases of infection with the same or a closely allied species has recently been reported by Thézé from French Guiana. The adult female is several inches in length, and slender as a hair; the male is stouter, and little over an inch in length. The adults lie in couples in fibrous tumors which can be seen readily under the skin. The tumors vary in size from about one $\mathrm{cm}$. ( 2 of an inch) in diameter to the size of a pigeon's egg, and are found most commonly on the hip, 
sides of the chest and upper part of the back, and sometimes in the arm and knee pits and on other parts of the body. Each swelling consists of dense fibrous tissue in which several pairs of parasites are imbedded, and contains small cystlike spaces into which project the posterior end of the male with its copulatory organs, and the anterior end of the female with its vaginal opening. These cystlike spaces are usually swarming with sheathless microfilariæ. The latter are believed by some authors to leave the tumors and to find their way ultimately to the bloodvessels, whence they can be liberated by some blood-sucking insect. The intermediate host is unknown, but the stable-flies, Stomoxys, and tsetse flies, Glossina, have been suspected. The tumors are of long duration in man, and in some adults are said to have been present since childhood. It is significant that practically all cases of elephantiasis in the Welle district of Congo, where Filaria bancrofti is said not to occur, are accompanied by infection with Onchocerca volvulus. Recently Robles has described Onchocerca coecutiens causing subcutaneous nodules on the heads of natives in parts of Guatemala, and suspects certain species of Simulium as carriers.

The Guinea-worm. Another connective tissue parasite, more distantly related to the filariæ, is the guinea-worm, Dracunculus medinensis (Fig. 130). This is a frequent parasite in many parts of tropical Asia and Africa and has been known for a very long time. The "fiery serpents" which molested the Israelites by the Red Sea and were mentioned by Moses were probably guinea-worms. These parasites

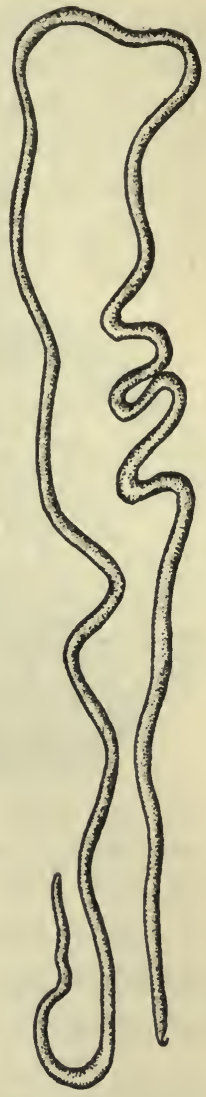

FIG. 130. Guineaw o r m, Dracunculus medinensis, female. Natural size. (After Leuckart.) creep in the deeper layers of the subcutaneous tissue where they can be more readily felt than seen, but the females always come to the surface of the skin to give birth to the myriads of wriggling young.

The adult female worm, which is the only sex certainly known, 
may attain a length of four feet or more, though the average length is about three feet, while the diameter is less than $\frac{1}{16}$ of an inch. The body is smooth, cylindrical and milky-white in color, with the tip of the tail sharply hooked. The male worms are believed to be much smaller than the females. When ready to bring forth her young, the guinea-worm is instinctively attracted to the skin, especially to such parts as are likely to, or frequently do, come in contact with cold water, such as the arms of women who wash clothes at a river's brink, or the legs and backs of water-carriers. The worm pierces the lower layers of the skin with the front end of her body and the outer layers of the skin form a blister over the injured spot. The blister

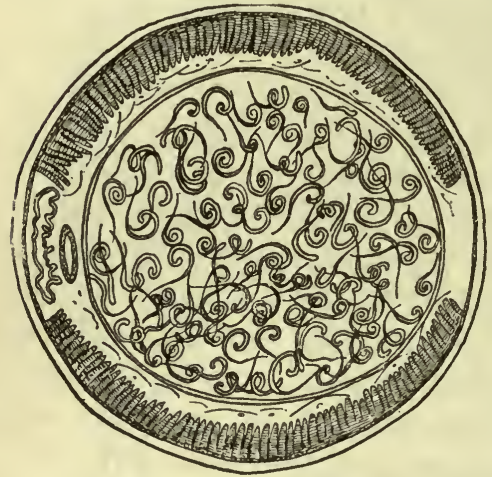

FIG. 131. Cross section of guineaworm showing uterus filled with embryos. $\times$ about 30 . (After Leuckart.) eventually breaks, revealing a shallow ulcer, about as large as a dime, with a tiny hole in the center. When the ulcer is douched with water a milky fluid is exuded directly from the hole or from a very delicate, transparent projected structure which is a portion of the worm's uterus. This fluid is found to contain hordes of tiny coiled larvæ with characteristic straight projecting tails. The larvæ (Fig. 131) are from 0.60 to $0.75 \mathrm{~mm}$. (about $\frac{1}{35}$ of an inch) in length. An hour or so later a new washing with cold water will bring forth a fresh ejection of larvæ and so on until the supply is exhausted, a little more of the uterus being extruded each time. After each ejection of the larvæ the protruded portion of the uterus dries up, thus sealing in the unborn larvæ. This process can be looked upon only as a wonderful adaptation for the preservation of the race. As we shall presently see, the tiny larvæ utilize various species of Cyclops (Fig. 132), small fresh-water crustaceans, as intermediate hosts. If the larvæ were not deposited in water, or if they were all poured at once into any bit of water with which the skin of the host came in contact, the chance of their reaching a suitable Cyclops would be very small. The result would usually be family suicide and eventually race 
suicide. The repeated birth of a limited number of progeny each time the skin of the host comes in contact with water is therefore a successful solution to a problem which to a blind burrowing unmeditative worm must otherwise present insuperable difficulties. When all her young have been deposited, under the stimulus of contact with water, the parent worm shrivels and dies and is soon absorbed by the tissues on which she formerly preyed and through which she roamed.

The embryo worms, safely deposited in water, unroll themselves and begin to swim about in a fashion peculiar to them-

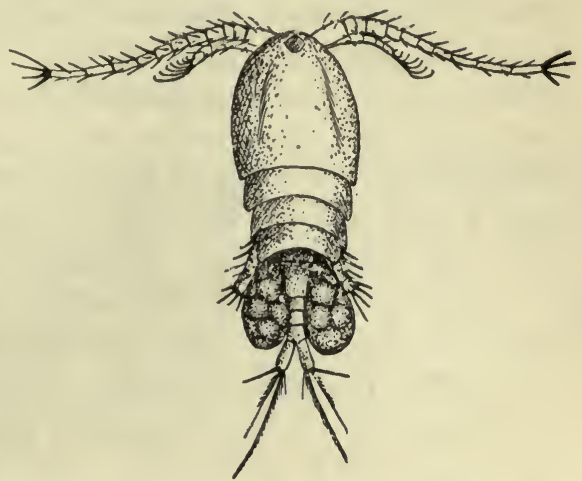

FIG. 132. Cyclops sp. (?), some species of which serve as intermediate hosts of guineaworms. $\times$ about 25 .

selves. Their bodies are somewhat flattened and they have a slender tail. They swim by a few quick sculling motions of the tail, followed by a pause, then a few more strokes, etc., in the manner of a tadpole. In turbid water they remain alive for two or three weeks but eventually perish unless they come in contact with a Cyclops, into the body of which they make their way.

They usually enter by way of the mouth, sometimes as many as six or ten entering a single Cyclops. In a day or two they leave the stomach of Cyclops and enter the body cavity. In spite of the relatively large size of the worms the crustaceans seem to feel very little inconvenience, and seldom succumb even to very heavy infection.

The young guinea-worms become fully developed in Cyclops in from four to six weeks, according to the temperature, meanwhile having undergone one and perhaps two moults. They are then about one $\mathrm{mm}$. ( $\frac{1}{25}$ of an inch) in length, and ready to infect a new host. Entrance to the new host is probably accomplished by the accidental drinking of a Cyclops with unfiltered water. The female worms become adult in their new host in about a year so the larvæ can again be deposited at about the time that Cyclops becomes abundant. 
The guinea-worm, though annoying and to one of fine sensibilities extremely disgusting, is not in any way dangerous if not interfered with. Should she come to an untimely end, however, or fail to pierce the skin, she may give rise to troublesome abscesses, though more often the body becomes calcified and may be felt for years as a hard twisted cord beneath the skin. The crude method of abstraction of the worm which is frequently practiced is the chief source of danger from infection with it. This extraction consists in winding out the extruded part of the worm around a stick, drawing it forth a little further each day. Sometimes this method is successful but frequently it results in the snapping in two of the worm beneath the skin, and the consequent liberation into the tissues of thousands of young worms with the fluid contents of the uterus. This gives rise to inflammation, fever, abscesses and even death from bloodpoisoning.

A much more effective and rational method of treatment is to bathe the part of the body occupied by a mature worm at frequent intervals until she has emptied her uterus, a process which takes two or three weeks. When the birth of embryos ceases, gentle pulling is likely to bring the worm forth, but if not her body is quickly absorbed by the tissues. A more recent and quicker method of dealing with a guinea-worm is to inject her body, or the tissue in which she is coiled, with a very weak solution of bichloride of mercury. This kills her and usually makes her extraction easy after a few hours. Promising results have recently been obtained by arsenobenzol injections into the host.

Prevention of guinea-worm infection consists obviously in keeping drinking water clear of Cyclops, or in thoroughly filtering it, or, if these measures are impracticable, in preventing infected persons from bathing in or otherwise contaminating rivers or other bodies of water from which drinking water may be taken. It has been suggested that portable steam generators be used to heat the water in wells, water holes, etc., in which infected Cyclops live, since these crustaceans succumb at a slightly elevated temperature. Addition of small quantities of potash to water is also effective in destroying Cyclops. The difficulty connected with an attempt to exterminate Cyclops locally is that the eggs resist desiccation and are blown about freely by the wind, so that a new colony is likely to spring up at any time. 


\section{CHAPTER XVIII}

\section{LEECHES}

THE annelids as a group are not of such primary importance as parasites as are the two other great groups of "worms." In fact only one class, the Hirudinea or leeches, contain species which are parasitic on the higher animals.

No boy who has ever experienced the unbounded delights of hanging his clothes on a bush and immersing his naked body for a swim in a muddy-bottomed river or pond is unfamiliar with leeches or "bloodsuckers." Still more familiar with them is any tourist who has journeyed on foot through the jungles of Ceylon or Sumatra, or any explorer who has walked through the warm moist valleys of the Himalayas or Andes, and who has been attacked by hordes of bloodthirsty land-leeches which infest these places. Nor is it likely that the thirsty traveler in North Africa or Palestine who stops to gulp a few mouthfuls of water from a pool or stream and who accidentally inbibes one of the leeches which infest such waters will not always remember the bleeding and unpleasant sensations, and perhaps dangerous symptoms, which follow the settlement of the leech in the mouth or nasal passages.

General Anatomy. - The leeches are segmented worms belonging to the phylum Annelida, in company with earthworms, kelpworms, etc. They are distinguished from other annelids by the absence of any bristle-like outgrowths from the body (setæ) and by the presence of two suckers, one at the mouth for sucking food, and a large one at the posterior end for adhering to surfaces. The rings of the body as seen on the surface do not correspond to true segments of the body as they do in other annelids; there are several rings to most of the segments. The bodies of leeches are extremely elastic, and can be stretched at will to several times the contracted length. In fact the usual method of locomotion, other than an undulating mode of swimming, is by alternately expanding and contracting the body, 
adhering first by the large posterior sucker, then by the smaller oral sucker and so forth.

Nearly all leeches feed exclusively on blood. The digestive tract (Fig. 60C, p. 197) is peculiar in that the œsophagus is supplied with a series of "crops" or side pockets in which blood can be stored up as a reserve supply to be gradually drawn back into the stomach and intestine and digested as needed. Since some leeches can fill up with three times their own weight in blood, and can live on this supply for a year or more, meals are few and far between. The saliva of the leech has the power of preventing the coagulation of blood, and therefore blood continues to flow for some time after the leech has "got his fill " and let go. Like other annelids, leeches have a true blood system and a series of nephridia, little coiled tubes, a pair in each segment, which function as primitive kidneys. There are no special gills or other respiratory organs; oxygen is absorbed directly through the skin which is constantly kept moist.

Leeches are hermaphroditic, i.e., both sexes are represented in the same individual, but the egg of one leech is always fertilized by a sperm from another. In most leeches the eggs are deposited in a stiff mucous cocoon which is secreted by a portion of the body. When the eggs are laid the cocoon is slipped over the head like a jersey, the ends closing together to form a capsule. After a little manipulation with the oral sucker the mother leech imbeds the cocoon in moist soil, near the edge of water in the case of aquatic species.

Importance as Parasites. - The ordinary pond and river leeches which adhere to bathers are of little or no economic importance as human parasites. Of these the well-known medicinal leeches, Hirudo, used for sucking out infections or bad blood, are the best known examples. They are furnished with powerful suckers and sharp-pointed pincer-like jaws, and can therefore easily penetrate the skin and suck blood from any part of the surface of the body. They can usually be persuaded to release their hold when removed from water.

With the weak-jawed members of the genera Limnatis and Homopis, commonly known as horse leeches, it is quite different. These animals seek to penetrate the natural openings of the body and fasten themselves to the mucous membranes, especially in the mouth and nasal cavities, where they may cause 
such extensive bleeding as to bring about the death of the host. Of perhaps even greater importance, because more difficult to avoid, are the bloodthirsty land-leeches which have already been mentioned as infesting many tropical countries. Leeches serve as intermediate hosts for many species of trypanosomes of fishes and other aquatic animals, and it is not impossible that they may be found to transmit some species to man.

Leeches in the Mouth or Nose. - The leeches which habitually settle themselves in the mouth or nasal cavities of men or animals are inhabitants of muddy-bottomed ponds, ditches, reservoirs, troughs, etc., and enter the mouth or nose of their host while he is drinking. According to Masterman, leeches of the species Limnatis nilotica become so abundant in northern Palestine in late summer and autumn that almost every horse and mule passing through these parts has a bleeding mouth. The Nile leech, Limnatis nilotica, is the most plentiful species around the shores of the Mediterranean, but leeches of the genus Hæmopis, with similar habits, also occur over a large part of Europe. Troublesome aquatic leeches have been reported by travelers in the lake regions of central Africa also, and in some other warm countries, especially Formosa.

The young leeches, which are usually the ones which enter the mouth or nose during drinking, are only a fraction of an inch in length, but the adults reach a length of several inches. The average length of Limnatis nilotica is about one inch or less.

A person while drinking from infected pools, especially in dusk or at night, is very likely to suck in one or more of these leeches. During the process of swallowing the parasites attach themselves to the walls of the mouth or pharynx and may migrate into the nose or larynx. Seldom, if ever, are the leeches completely swallowed, and even if they should reach the stomach they would probably be killed at once and digested. It is a peculiar and indeed unfortunate fact that, while the leeches which attack the surface of the body fill with blood and then let go, those which settle on the mucous membranes keep their hold for days or weeks, though they shift their positions, leaving the old bites to continue bleeding. As already stated, the loss of blood from the wounds made by the leeches is often sufficient to cause an extreme or even fatal anemia, though the hemorrhages of clear blood are never great in quantity at any one time. 
The blood flows out of the nose or into the throat or trachea, in the latter cases being constantly "hawked" up. Masterman describes the case of a man in Palestine, attacked by leeches, who for nearly a week had been "spitting blood" and had a spittoon full of practically pure blood by his side, every few minutes adding more. His lips were blue, and he was unable to speak above a whisper. Every few minutes he had a short cough. Often when the leech is attached in the larynx beside the vocal cords, the body flops back and forth during breathing, and has been known to cause asphyxiation by blocking the trachea. Cases are on record where leeches, having fallen into one of the bronchi, have died and disintegrated, and thus caused destructive bacterial infections to set in. The presence of leeches in the mucous membranes is often accompanied by severe headaches. Sometimes leeches which have settled in the nose have the revolting habit of protruding themselves from the nostrils and allowing a portion of the body to wander over the upper lip. They are, however, so elusive that they can be captured only with great difficulty.

The treatment employed for leech infestations of the nose or mouth varies greatly in different countries. According to Masterman the natives of Palestine transfix the leech, if within reach, with a thorn from a native tree, and muleteers extract leeches from mules' mouths with packing needles. When the parasite is beyond reach of this transfixing process these people smear some of the thick deposit which collects in their tobacco pipes on a splinter of wood and endeavor to touch the leech with it; this is said to cause the leech to lose its hold. Masterman found the most successful means of removing a leech to be either to seize it with a suitable forceps, or to paralyze it with cocaine. Much difficulty is often experienced in seizing the writhing, slippery creature with a pair of forceps even when it can be seen clearly with a mouth mirror, partly on account of the spasmodic contractions of the larynx and the frequent coughing. The paralyzing of the worms with cocaine is a very successful method: it is done by touching the worm with a 30 per cent cocaine solution on a bit of cotton. The worm becomes paralyzed in a few minutes after being touched, and releases its hold. To avoid the possibility of the leech falling into the trachea the patient is made to lie on a couch with his head hanging over the edge. 
Land-leeches. - Of perhaps greater importance, because far less easy to avoid, are the attacks of the land-leeches of many tropical countries. These leeches are found in Ceylon, Japan, Sumatra, Philippine and East Indian Islands, Australia, and the humid mountain meadows of the Himalayas in India and of the Andes in South America. Sir J. Emerson Tennent in his book on "The Natural History of Ceylon" writes as follows: "Of all the plagues which beset the traveler in the higher grounds of Ceylon the most detested are the land-leeches, Homadipsa ceylonica. They are not frequent in the plains, which are too hot and dry for them, but among the rank vegetation of the lower hill country, which is kept damp by frequent showers, they are found in tormenting profusion. They are terrestrial, never visiting ponds or streams. In size they are about an inch in length and as fine as a common knitting needle, but they are capable of distension till they equal a quill in thickness and attain a length of nearly two inches. Their structure is so flexible that they can insinuate themselves through the meshes of the finest stocking, not only seizing on the feet or ankles, but ascending to the back or throat, and fastening on the tenderest parts of the body. In order to exclude them the coffee planters who live among these pests are obliged to envelope their legs in "leech garters" made of closely woven cloth. The natives smear their bodies with oil, tobacco ashes or lemon juice, the last serving not only to stop the flow of blood, but also to expedite the healing of the wounds. In moving, the land-leeches have the power of planting one extremity on the earth and raising the other perpendicularly to watch for their victim. Such is their vigilance and instinct that, on the approach of a passerby to a spot which they infest, they may be seen amongst the grass and fallen leaves on the edge of a native path, poised erect, and prepared for their attack on man and horse. Their size is so insignificant and the wound they make is so skillfully punctured that both are generally imperceptible, and the first intimation of their onslaught is the trickling of the blood or a chill feeling of the leech when it begins to hang heavily on the skin from being distended with its repast. Horses are driven wild by them and stamp the ground in fury to shake them from their fetlocks, to which they hang in bloody tassels. The bare legs of the palankin bearers and coolies are a favorite resort, and as their hands are 
too much engaged to pull them off the leeches hang like bunches of grapes round the ankles. Both Marshall and Davy mention that during the march of troops in the mountains when the Kandyans were in rebellion in 1818, the soldiers, and especially

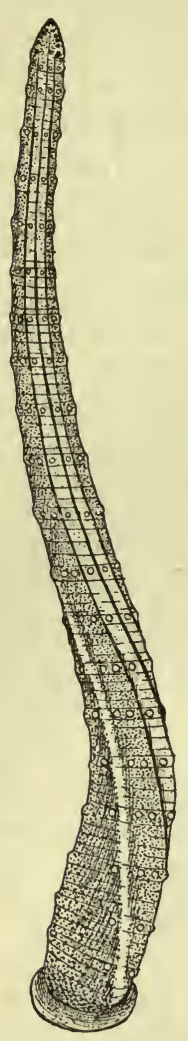

Fig. 133. Japanese land-leech, Hamadipsa japoni$c a$, extended. $\times 2$. (After Whitman.) the. Madras Sepoys, with the pioneers and coolies, suffered so severely from this cause that numbers perished.

One circumstance regarding these landleeches is remarkable and unexplained: they are helpless without moisture, and in the hills where they abound at all other times they entirely disappear during long droughts; yet reappear instantly at the very first fall of rain, and in spots previously parched, where not one was visible an hour before, a single shower is sufficient to reproduce them in thousands. Whence do they reappear! May they, like rotifers, be dried up and preserved for an indefinite period, resuming their vital activity on the mere recurrence of moisture?"

Similar reports come from travelers in other tropical countries. Alfred Wallace encountered land-leeches in Sumatra where he found them infesting the leaves and herbage by the side of the paths through the forests. At the approach of a traveler as indicated by footsteps or a rustling of leaves, the leeches stretched. themselves out at full length and attached themselves to any part of the passerby which they happened to touch. Their presence and the loss of blood was seldom felt during the excitement of walking, but a dozen or so had to be picked off every evenning. Dean C. Worcester in his book on the Philippines says "the moist earth swarmed with leeches which crawled through my stockings and bit my ankles until my shoes were soaked with blood." One species, H. japonica (Fig. 133), is common in parts of Japan. The land-leech of Australia belongs to a different genus, ${ }^{\circ}$ Philomon.

In any of the localities infested by land-leeches it is advisable 
to bind the feet and legs in leech-proof cloth, this being preferable to various ointments which are supposed to discourage the leeches from their meal. In a tropical climate where so many diseases and unfavorable conditions beset one on every side, it is important to take every precaution to keep in perfect health. The loss of blood from the attacks of leeches, and the portal given for entrance of bacteria and other organisms in the wounds made by them, might make all the difference between life and death in the struggle for existence in these disease-plagued climes. 


\section{PART III - ARTHROPODS}

\section{CHAPTER XIX \\ INTRODUCTION TO ARTHROPODS}

To the average person it is astonishing to learn that the insects and their allies, constituting the phylum Arthropoda, include probably more than four times as many species as all other animals combined. In this vast horde of animal forms are included some species which are distinctly valuable to the human race, such as bees, the silkworm, the thousands of insects (Diptera and Hymenoptera) parasitically destructive to injurious species and the predaceous beetles; a great number which are indifferent as regards their economic importance serving, perhaps, only to arouse admiration for their beauties or disgust for their loathsomeness; and many which are of great importance as crop pests or as annoyers of domestic stock or of man himself. Only relatively very few, a mere handful, are injurious to man as parasites or as disease carriers, but these few are of almost incalculable importance. As mere parasites the parasitic arthropods are of minor importance, but it is in their capacity as intermediate hosts of other parasites or as mechanical carriers of disease germs that these animals have to be reckoned with as among the foremost of human foes. Every arthropod, parasitic or otherwise, which habitually comes in direct or indirect contact with man must be looked upon as a possible disease carrier. The rôle of arthropods in the dissemination of disease is a matter about which practically nothing was known 35 or 40 years ago. A French physician, Dr. Beauperthuy, in 1853 was one of the first to express a belief in the dissemination of various diseases by mosquitoes and in the rôle of the housefly in the spread of pathogenic organisms. In 1879 Manson first proved insects to be intermediate hosts of human parasites, in the case of Filaria and the mosquito. Since that time many of the most important human diseases have been shown not only to be trans- 
mitted by arthropods but to be exclusively transmitted by certain species or genera. In the latter category, as far as we know at present, are malaria, by some physicians rated as the most important human disease; typhus fever, the unseen dragon of death which hovers over every war camp in the world; yellow fever, which formerly haunted South and Central America; sleeping sickness, the scourge of Central Africa; Chagas' disease of South America; relapsing fever; Rocky Mountain spotted fever; dengue; phlebotomus fever; Japanese flood fever; filarial diseases; guinea-worm infection; lung fluke infection; some tapeworm infections; and others of less importance. Some other important diseases, such as kala-azar and oroya fever, are believed to be transmitted by arthropods but the transmitting agents have not yet been discovered.

There are many other diseases which, although they may be transmitted in other ways also, are commonly disseminated by insects, often in a more or less mechanical way. Such are plague, tuberculosis, leprosy and others. In the case of some of these diseases, e.g., plague, the intestines of the transmitting arthropods serve as culture tubes for the disease germs, whereas in other cases, e.g., amebic dysentery, the arthropods are merely passive carriers of disease germs which adhere to their feet or bodies. It is evident that any insect may serve as a disseminator of disease in this mechanical way in direct proportion to the extent that it associates with man and that its habits bring it in contact with disease germs.

Relationships. - The insects and their allies, constituting the phylum Arthropoda, are the most highly organized of invertebrate animals, and stand at the head of their particular line of evolution. They find their nearest allies in the segmented worms or annelids, i.e., earthworms, leeches, etc., but most of them show a great advance over their lowly cousins. Like the annelids they have a segmented type of body, though in some types, such as the mites, all the segments become secondarily confluent. Like the annelids, also, the arthropods are protected by an external skeleton which usually consists of a series of horny rings encircling the body. The most obvious distinguishing characteristic of the arthropods is the presence of jointed appendages in the form of legs, mouthparts and antennæ. Internally they are distinguished from other invertebrates in that the body cavity, 
so conspicuous in the annelids, has been entirely usurped by a great expansion and running together of bloodvessels so that in the place of the usual body cavity or cœlom there is a large bloodfilled space. Within this space are bloodvessels and a so-called heart, which retained their individuality while the other vessels fused.

Classification. - The phylum Arthropoda is divided into five classes. One of these, the Onychophora, includes only a single genus of animals, Peripatus, which is very primitive, and helps to bridge the gap between the more typical arthropods and the annelids. Peripatus is a free-living wormlike animal and of no interest here. The remaining four classes are the Crustacea, Arachnida, Myriapoda and Insecta.

The Crustacea, including crayfish, water fleas, etc., are primarily arthropods of the water. They are geologically of great antiquity and among them are the most primitive of the typical arthropods. Their appendages are usually numerous and, taking the group as a whole, show a wonderful range of modifications for nearly every possible function. Crustaceans breathe by gills. Although many are parasites of aquatic animals, none can be considered as parasites of man or other land animals. In two cases Crustacea are known to serve as the intermediate hosts of human parasites, namely Cyclops as a host for the guinea-worm (see p. 312), and the Japanese land crabs as the second intermediate hosts of the lung fluke (see p. 222).

The Arachnida, including spiders, scorpions, mites, etc., are for the most part highly developed arthropods, representing the terminus of a separate line of evolution. They probably had a common origin with the Crustacea, but have become adapted to terrestrial life. The members of this class have four pairs of legs as adults, two pairs of mouthparts and no antennæ. They breathe by means of invaginations of the body which contain gills arranged like the leaves of a book, whence the name "book lungs." Some of the higher arachnids also have a system of branched air tubes or tracheæ in the body similar to those found in the insects and myriapods. Two orders of Arachnida contain parasitic species, namely the Acarina, or mites and ticks, and Linguatulina, or tongue-worms. Many ticks are disease carriers.

The Myriapoda, including centipedes and millipedes, are terrestrial arthropods which breathe by tracheæ. The body is 
furnished with a distinct head, followed by a considerable number of similar segments, each bearing one or two pairs of legs. There is a single pair of antennæ. Although some of the centipedes are poisonous, none of the myriapods are parasitic, nor are any of them known to be disease carriers.

The Insecta, or insects, represent the zenith of invertebrate life. They are terrestrial arthropods which, like the myriapods, breathe by tracheæ. Their appendages, however, are reduced to one pair of antennæ, two pairs of mouthparts and three pairs of legs with usually the addition, if not secondarily lost, of two pairs of wings. The wings are really mere outgrowths or folds of the integument or "skin" of the insect, between the two layers of which are branches of the tracheæ, represented by the "veins" in the wings of adult insects. There is a fundamental plan of arrangement of the veins which is variously modified in different insects, but absolutely fixed in any given species. The venation of the wings is often of great value in the identification of genera or species of insects. An insect is always readily divisible into three parts, the head, thorax and abdomen. The head, in addition to the antennæ already mentioned, bears two compound eyes sometimes of relatively enormous size, usually several simple eyes, and the mouthparts.

Mouthparts of Insects. - Incredible as it may seem at first thought, the mouthparts of all kinds of insects, from the simple chewing organs of a grasshopper to the highy modified piercing and sucking organs of biting flies and mosquitoes and the great coiled sucking tube of butterflies and moths, are modifications of a single fundamental type. This type is represented in its simplest form in the chewing or biting type, as found in grasshoppers and beetles (Fig. 134). The mouthparts in these insects consist (1) of an upper lip or labrum (Fig. 134, Lbr.); (2) a lower lip or labium (Fig. 134, Lbm.), really formed of a pair of organs fused together, each bearing a segmented appendage, the labial palpus (Fig. 134, Lab. p.); (3) a pair of hard, horny, toothed mandibles or jaws (Fig. 134, Mand.) lying just under the lower lip, which chew up food by a horizontal instead of vertical movement; (4) a pair of maxillæ (Fig. 134, Max.), lying between the mandibles and lower lip, each bearing a segmented appendage more or less like those on the lower lip, and called the maxillary palpus (Fig. 134, Max. p.) and (5) the 
hypopharynx (Fig. 134, Hyp.), a short fleshy organ lying in the midst of the other organs, and comparable in both form and function with the tongue of vertebrate animals. In addition to these parts there is a horny lining of the upper lip and roof of

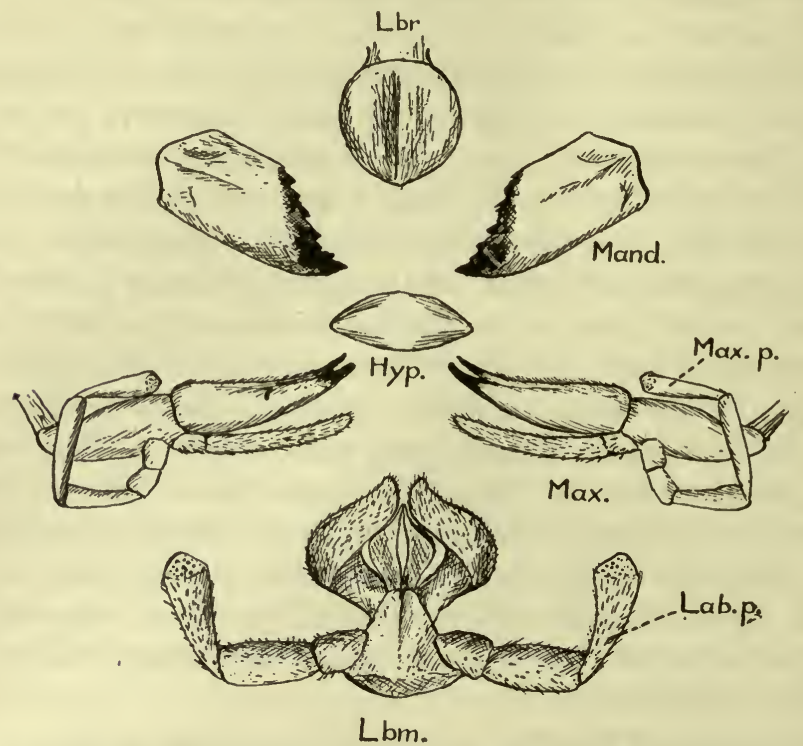

FIG. 134. Simple mouthparts of a chewing insect (Stenopalmatus); lbr., labrum, or upper lip; mand., mandible; hyp., hypopharynx or tongue; max., maxilla; max. p., maxillary palpus; lbm., labium or lower lip (really a second pair of maxillæ fused together); lab. p., labial palpus.

the mouth cavity known as the epipharynx. This structure is usually closely associated with the upper lip, so that the combined organ is spoken of as the "labrum-epipharynx."

The extent of the modifications which these mouthparts may undergo is wonderful, especially in insects where they are modified for sucking or piercing. In the true bugs the mandibles and maxillæ are prolonged into needle-like organs, the maxillæ often armed with sawlike teeth at their tips, and the lower lip is developed into a thick, fleshy, jointed proboscis, grooved on its upper side to form a sheath for the piercing organs (Fig. 164). The labrum is a short movable flap, and the hypopharynx is very slightly developed. In the Diptera, which include the mosquitoes, gnats, blackflies, tsetse flies and other biting flies as well as houseflies and blowflies, there are several different types 
of modifications. In mosquitoes the mouthparts (Fig. 191) are much as in bugs, but the labrum-epipharynx and hypopharynx are also modified into long piercing organs, and the latter is fashioned into a true hypodermic needle for injecting salivary secretions. In blackflies and tabanids (Figs. 220 and 225) the parts are similar but the piercing organs are shorter and more bladelike, resembling daggers rather than needles. In the tsetse flies and stable-flies (Figs. 220 and 225) the lower lip itself is the chief piercing organ, the labrum-epipharynx and hypopharynx contained in it being needle-like and capable of forming a sucking tube by apposition with each other. The mandibles and maxillæ are much reduced or rudimentary, but the maxillary palpi are conspicuous, and in tsetse flies form a perfect sheath for the proboscis. In the houseflies and their non-blood-sucking allies the mouthparts are most modified, being all molded together to form a fleshy proboscis especially fitted for lapping up liquid foods. In fleas the mouthparts (Fig. 178) are somewhat as they are in the biting flies, but the mandibles are not modified as piercing organs but as protective flaps, and the sheath for the piercing organs is formed from the labial palpi instead of from the labium or lower lip itself. The mouthparts of sucking lice (Fig. 171) are still not thoroughly understood but the piercing and sucking organs, whatever parts they really represent, can be retracted into a blind pouch under the pharynx. The mouth parts of such insects as moths, bees, wasps, etc., are also remarkable examples of structural adaptations, but they do not concern us here.

General Anatomy. - The digestive tract of insects (Fig. 135) is often highly developed and differentiated. The blood-sucking insects have a muscular pharynx in the head which acts like a suction pump. In the bedbug, for instance, the powerful muscles which are used to expand the pharynx and thereby produce suction occupy a considerable portion of the inside of the head, and the area on top of the head to which they are attached is distinctly visible on the outside. The pair of salivary glands open into the floor of the pharynx, but they themselves are usually situated in the thorax. Often they are very highly developed. In the true bugs they have connected with them accessory salivary glands, which in some species may serve at least in part as storage vats for holding the secretion temporarily. 
In mosquitoes (Fig. 189) the salivary glands consist of three lobes, one lobe being noticeably different in appearance and secretion from the others. The pharynx connects with the stomach by a slender œesophagus. Various means are used by blood-sucking insects to increase

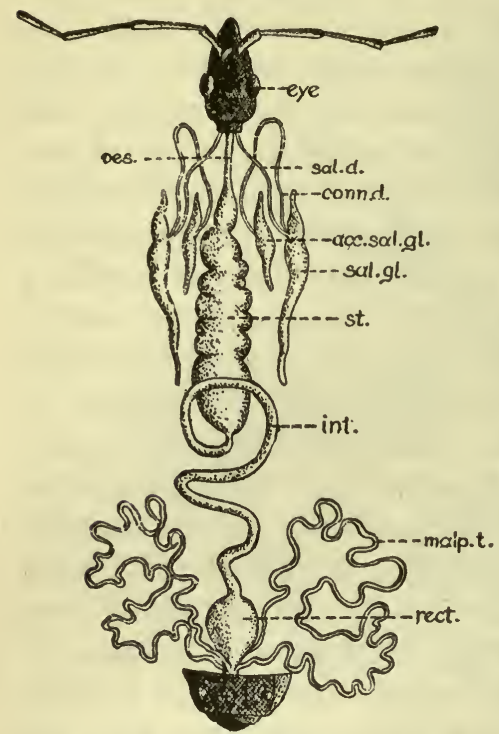

FIG. 135. Digestive tract of a Reduviid bug; acc. sal. gl., accessory salivary gland; conn. d., connecting duct between salivary glands; int., intestine; malp. t., malpighian tubules; œs., œesophagus; rect., rectum; sal. gl., salivary gland. (Partly after Dufour.) their capacity. In the bugs (Fig. 135) the stomach is extremely distensible and serves as a storage reservoir. In fleas and many biting flies there is an expansion of the œsophagus anterior to the true stomach, called the proventriculus; in mosquitoes there are capacious pouchlike food reservoirs or outgrowths from the œsophagus in addition to the proventriculus (Fig. 189). Just behind the true stomach at the beginning of the intestine there open a number of long slender tubes, the "Malpighian tubules" (Fig. 135, malp. t.). These are the excretory organs, corresponding to the kidneys of vertebrate animals. Their function is to collect the waste matter of metabolism from the blood and pour it into the intestine, whence it can ultimately be voided through the anus. The length of the intestine varies, being usually longer in vegetable-feeding insects than in carnivorous ones. It often has a marked expansion, the anal pouch, at its posterior end.

The tracheæ of insects, as already intimated, are really a ventilation system consisting of air tubes ramifying all through the body even to the tips of the antennæ and legs. They open by a series of pores along the sides of the insect known as spiracles, which function as do the nostrils of higher animals. The principle of oil sprays for insects is to form a film of oil over the spiracles, so that the insects will suffocate.

The nervous system of insects is very highly developed for 
invertebrate animals. In some species the instincts, especially those connected with providing for their offspring, simulate careful and accurate reasoning, and it is difficult not to look upon them as animals endowed with a high degree of intelligence.

Life History. - As regards life history, three different types can be recognized among insects. In the primitive order Thysanura alone there occurs " direct development" in which the newly hatched insect is nearly a miniature of its parent, and merely increases in size. The two common types of development are by incomplete and complete metamorphosis. Insects which have an incomplete metamorphosis are those which differ more or less from their parents when hatched, but which gradually assume the parental form with successive moults or sheddings of the skin. The young or "nymphs" of such insects invariably lack wings, and often have other characteristics different from their parents. In such insects as lice, in which the wings are absent in the adult, there is very little difference except in size between the young and adult forms. Insects which have a complete metamorphosis are those in which, as in butterflies, the newly hatched larva is totally different from the parent, and does not gradually assume the parental form. Instead, it retains its wormlike larval characteristics until full grown and then transforms, during a resting and more or less quiescent period of relatively short duration, into the adult form. This transformation, which may amount to nothing short of a complete remodeling of the entire body and all its organs, is sometimes accomplished in an amazingly short time. Many maggots transform into adult flies in less than a week, and some mosquito larvæ transform into perfect mosquitoes in less than 24 hours.

The length of life of insects in the larval and adult stages varies with almost every species and with environmental conditions. The larval stage may occupy a small portion of the life, as in the case of many mosquitoes and flies, or it may constitute the greater part of it. There are some mayflies, for instance, which live the greater part of two years as larvæ but exist as adults not more than a few hours. As a rule male insects are shorter lived than the females; the length of life of the latter is determined by the laying of the eggs - when all the eggs have been laid the female insect has performed her duty in life and is eliminated by nature as a useless being. The result 
is the paradoxical fact that ideal environmental conditions shorten the life of these insects, since they facilitate the early deposition of the eggs.

Classification. - The classification of insects is based mainly on three characteristics: the type of development, the modification of the mouthparts, and the number, texture and venation of the wings. All blood-sucking insects have mouthparts adapted in some way for piercing and sucking, but the types vary greatly in different groups. Many of the more thoroughly parasitic insects, e.g., lice, bedbugs and "sheep ticks," have secondarily lost their wings entirely, or have them in a rudimentary condition. In the whole order of Diptera the second pair of wings is reduced to inconspicuous club-shaped appendages known as halteres.

The only orders of insects which contain species of interest as human parasites are the Hemiptera (Rhynchota), or true bugs; the Siphunculata, or sucking lice; the Siphonaptera, or fleas; and the Diptera, or two-winged flies. These four orders may be briefly summarized as follows:

Hemiptera (suborder Heteroptera): metamorphosis incomplete; mouthparts fitted for piercing and sucking, the piercing organs being ensheathed in the jointed lower lip; first pair of wings, unless reduced, leathery at base and membranous at tip; second pair of wings, when present, membranous with relatively few veins. Human parasites: bedbugs, cone-noses, kissing bugs.

Siphunculata: metamorphosis incomplete; mouthparts fitted for piercing and sucking, and retractile into a pouch under pharynx; wings secondarily lost. Human parasites: sucking lice.

Siphonaptera: metamorphosis complete; mouthparts fitted for piercing and sucking, the piercing organs being ensheathed in the labial palpi, and the mandibles modified as protective flaps; wings secondarily lost. Human parasites: fleas, chiggers.

Diptera: metamorphosis complete; mouthparts fitted for piercing and sucking, for sucking alone, or rudimentary; first pair of wings (absent in a few species) membranous with few veins; second pair of wings represented only by a pair of clubshaped organs, the halteres. Human parasites: Sandflies, mosquitoes, midges, blackflies, gadflies, tsetse flies, stable-flies, maggots of various species. 


\section{CHAPTER XX}

\section{THE MITES}

General Account. - The mites and ticks, which constitute the Order Acarina of the Class Arachnida, are only slightly known by the majority of people. Popular knowledge of them is usually limited to a few species of ticks, chicken mites, and perhaps two or three other species of mites. Yet the group includes a large number of species, varying in size from some ticks which are half an inch or more in length to mites barely visible to the naked eye. The variety of body form is great and some species when magnified appear ridiculously grotesque. The majority of the species are more or less round or oval, with head, thorax and abdomen all in one piece, but many have the cephalothorax (head and thorax fused together) distinctly marked off from the abdomen, while a few are quite wormlike in form. Many mites are free-living and prey upon decaying matter, vegetation, stored foods and the like; some are predaceous and feed upon smaller animals; some are aquatic, even marine; and many are parasitic on other animals during all or part of their life cycle, and some of these serve as intermediate hosts for, and for dissemination of, dangerous disease germs.

Like other Arachnida (spiders, scorpions, etc.) the mites and ticks usually have two pairs of mouthparts and four pairs of legs, though the last pair of legs is not acquired until after the first moult. The first pair of mouthparts or cheliceræ are sometimes needle-like, sometimes shaped like a grapnel hook, and very often pincer-like, the pincers often being at the tip of a long exsertile needle-like structure. The second pair of mouthparts, or pedipalps, are simple segmented palpi. In many kinds of Acarina the anterior end of the ventral side of the body is produced as a sort of chin or lower lip, the hypostome, which may be needle-like or barbed and rasplike (Fig. 152).

The digestive tract is in most cases well developed. Waves of muscular contraction make a very efficient sucking organ of 
the pharynx. The stomach has pouches opening from it which act as food reservoirs (Fig. 149), so that one meal may last for a long time. The intestine is usually short and the excretory organs, malpighian tubules, open into it not far from the anus. The reproductive organs, as in other Arachnida, open on the ventral surface of the abdomen but at different places in different species. The nervous system is largely concentrated into a great mass, the "brain," lying near the anterior end of the body and pierced by the œsophagus. Many mites possess tracheæ, similar to those of spiders and insects, for breathing, while others, softskinned forms, simply absorb oxygen through the surface of the body.

Life History. - The life histories of mites and ticks are somewhat variable, but usually there are four stages in their development: the egg, the larva, the nymph and the adult (see Fig. 157). The eggs are usually laid under the surface of the soil or in crevices, or, in some parasites, under the skin of the host. After a varying period of incubation, which depends on climatic conditions, the larva hatches in the form of a six-legged creature, often quite unlike the parent. After a single good feed of blood or plant juices the larva rests, sheds its skin and appears with an additional pair of legs and a body form more closely resembling that of the parent but without developed sexual organs. The nymph thus produced feeds again, sheds its skin from one to three times and finally, after another period of rest during which its body is remodeled for the second time, moults again and comes forth as a fully adult male or female, ready for the reproduction of another generation. There are all sorts of modifications of this order of development, due to the slurring over of one phase or another. One of the most aberrant species is the louse-mite, Pediculoides. In this form the eggs develop within the parent's body and the adult males and females issue forth from the brood chamber improvised for them out of the abdomen of the mother (Fig. 139):

The popular opinion that all mites are parasitic is, as remarked before, far from being true. Over half of the known species are not parasitic at any stage in their life history, while many others are parasites only during part of their life cycle.

Parasitism. - The mites are an interesting group for the study of the origin of parasitic habits since, as Ewing has shown, para- 
sitism has apparently arisen independently in different families and genera at least eleven times. Nathan Banks in his treatise on the Acarina, after giving a number of interesting examples of peculiar parasitic habits, writes as follows: "We can only explain these remarkable habitats by the fact that mites, especially in their immature stages, have an incontrollable desire to go somewhere, and get into every cavity and crack they discover in their wanderings. When hungry they test their locality for food, and if not too different from their previous diet this new habitat may result in new species and genera."

A few species of mites have become adapted to live as internal parasites, but all the species normally infesting man are either external or subcutaneous in their operations. A few of the species which are not averse to human beings as food are troublesome and irritating enough to bring their whole tribe into disrepute. The families of mites which contain species annoying to man are the Ixodidoe and Argasidoe, the ticks; Trombidiidoe, the harvest mites and "red-bugs"; Parasitidae (Gamasidø), including the chicken mites; Tarsonemido, including the louse-mite; Tyroglyphida, including the cheese and grain mites; Sarcoptida, the itch mites; and Demodecidoe, the hair-follicle mites. For convenience we may include with the mites the very aberrant arachnid forms known as tongue-worms, now usually placed in a distinct order, Linguatulina. Since the ticks are popularly looked upon as quite distinct from other Acarina, and form a very important group of the order on account of their rôle as disease carriers, they will be considered in a separate chapter.

\section{Harvest Mites}

The six-legged larvæ of the harvest mites, family Trombidiidæ, known as red-bugs or chiggers, are very annoying pests, and one species, the Japanese "akamushi" or kedani mite, has been proved to be the carrier of a dangerous disease, kedani or flood fever. Harvest mites are little scarlet-red animals, and their larvæ are tiny pale-colored creatures bare.y visible to the naked eye (Fig. 136). According to one writer who had evidently experienced them a red-bug is a "small thing, but mighty; a torturer - a murderer of sleep; the tormentor of entomologists, botanists and others who encroach on its domains; not that it 
bites or stings - it does neither; worse than either, it just tickles."

The adult harvest mites (Fig. 137) are law abiding members of the community, and attack only such animals as plant-lice, caterpillars and other insects. They hibernate in soil or sheltered crevices and in the spring lay their eggs, several hundred apiece,

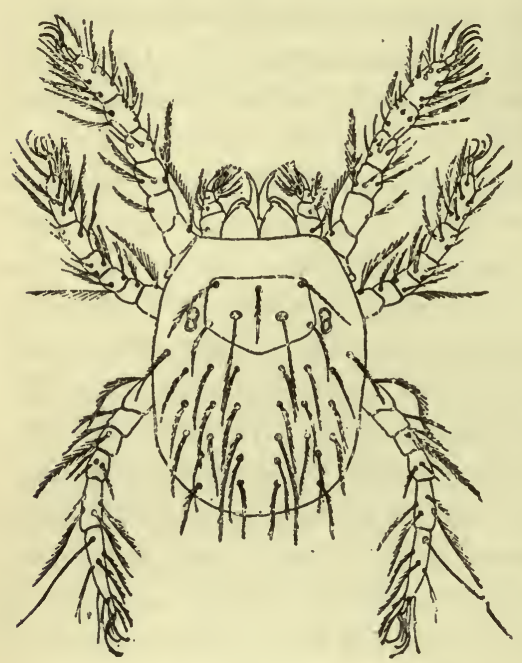

FIG. 136. European red-bug, Leptus autumnalis, larva of a Trombidium usually thought to be $T$. holosericeum. $\times 150$. (After Hirst.) in the ground or among dead leaves. The eggs are very small, round and brownish in color, and were once classified as fungous growths! The

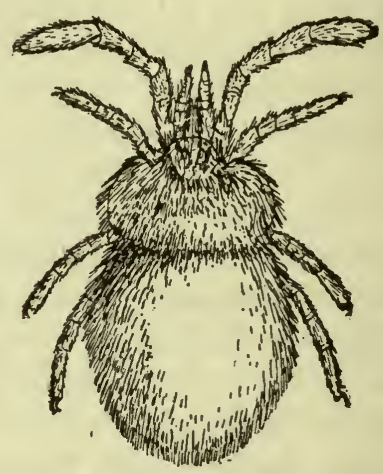

Fig. 137. An adult of the kedani mite, a Trombidiid. $\times 40$. (After Nagayo et al.)

newly hatched six-legged larvæ creep up on blades of grass or plant stems and await an opportunity to attach themselves to an insect. If successful in finding a host, or rather in being found by a host, the mites gorge themselves with the juices of the insect, then drop to the ground, crawl to some snug hiding place and undergo a transformation. The whole inside of the body is remodeled, a fourth pair of legs is acquired, and after a few weeks the skin is shed and an adult trombidiid mite crawls forth.

It is while the larval mites are hungrily awaiting the arrival of an insect upon which to feed that they attack human beings or animals which may pass their way. They are so small that they can easily pass through the meshes of ordinary clothing and reach the skin, where they set up a severe irritation and 
intense itching. Some authors claim that the mites burrow in the skin and produce inflamed spots, but ordinarily they do not go beneath the skin except sometimes to explore their way into the long tubes of the sweat glands. The habit of attacking warm-blooded animals is evidently abnormal, (and the love of blood proves ruinous to those individuals which get an opportunity to indulge it, since they soon die victims of their own perverted appetites. How like some human beings!

The irritation caused by the mites is probably due to a specific poison secreted by the mites rather than to any wounds that they make. The inflammation of the skin may not be felt for 12 or even 24 hours after infection by the mites. When the inflammation does commence there appear large red blotches on the affected parts of the body which itch intensely and are made worse by scratching. After a day or so the red blotches blister and finally scab over. Red-bug rash is most frequent on tenderskinned people and on those parts of the body which are most exposed, though it may spread over the whole body and torment the victim unbearably. Laborers who are continually exposed to these mites seem to develop an immunity to the mite poison, and suffer little or none from them. Herrick states that one of the severest infestations he ever knew was contracted by a delicate-skinned person who sat down on the ground for a few minutes on some golf links which had recently been laid out on an old pasture where there was still much long grass. This person's body became covered with large inflamed spots even to the neck. The torture was intense for a week, and the infection persisted for a still longer period. A Mexican species, known by the Ażtec name " tlalsahuate," meaning " grain of earth," shows a decided preference for the eyelids, armpits, groins and other thin-skinned portions of the body, where it induces itching and inflammation, and even ulceration when scratched. The "bête rouge " or "colorado" of the West Indies and Central America is a similar if not identical species.

Sprinkling sulphur on the legs and inside the stockings is a necessary preventive measure for those who are seriously affected by red-bugs, and who have to walk through tall grass or brush where these pests abound. A hot bath shortly after infection, with soap or with soda in it, gives much relief. To allay the itching weak ammonia or baking soda applied to the affected parts is 
good, and alcohol, camphor and other cooling applications are also useful.

Since in many instances the adults are unknown, the larval harvest mites are, for the sake of convenience, placed in a collective group, Leptus, and the name is used in the manner of a generic name. The common red-bug of Europe, for instance, which is supposed to be the larva of Trombidium holosericeum is known as Leptus autumnalis. The most abundant species of red-bug in the United States is Leptus irritans. It occurs throughout the southern United States and as far north as New Jersey and the upper Mississippi Valley. An allied species, Leptus americanus, also occurs in many parts of southern United States. On the northern border of its range this mite does not appear until the latter part of June and becomes especially annoying during August, but its season becomes earlier and earlier the farther south it occurs.

The European harvest mites, the commonest of which is Leptus autumnalis (Fig. 136), are well known pests throughout Europe, especially in Central and Western France, where they are known as the "bêtes rouges " or "rougets." They are said to attack small mammals, such as rodents, by preference. Unlike the American species, the European harvest mites become especially abundant in the fall of the year. Japanese investigators have recently cast doubt on the commonly accepted belief that Trombidium holosericeum is the parent of Leptus autumnalis since in Japan the parent of the kedani mite (Fig. 137), which very closely resembles $L$. autumnalis, is quite different from $T$. holosericeum, whereas an adult mite which very closely resembles the latter, produces larvæ quite different from $L$. autumnalis.

The Japanese harvest mite, larva of Trombidium akamushi, known locally as the akamushi (red-mite), tsutsugamushi (sickness mite) and kedanimushi (hairy mite), has been proven to be the carrier of a typhus-like disease known as kedani or flood fever. These larval mites occur in countless numbers on the local field mice, Micromys montebelloi, living especially on the inside of the ear. They frequently attack the farm laborers who engage themselves in harvesting and handling the hemp which is raised on the flood plains of certain Japanese rivers. It is among these people that the kedani or flood fever occurs, always following the bite of a mite. The bite, usually in the 
armpits or on the genitals, is at first painless and unnoticed, but the mite remains attached at the wound from one to three days before dropping to the ground to transform to the nymphal stage. The bite of the mite is said to develop into a tiny sore or inflamed spot in the region of which the lymph glands become swollen and painful and flood fever follows. The nymphs and adults of this mite have recently been found by Nagayo and his fellow-workers in Japan.

The transmission of kedani by this mite is the only positive instance of human disease carried by Acarina other than ticks.

\section{Other Occasionally Parasitic Species}

There are many species of mites, of several different families, which under abnormal circumstances or by sheer accident may become troublesome parasites of man. Nearly all mites secrete salivary juices which have a toxic effect when injected into the blood; therefore any mite which will bite man under any circumstances may become a pest. In nearly all cases the symptoms of attacks by mites are similar - hivelike or rashlike eruptions of the skin, intense itching and in severe attacks fever.

Louse-Mite. - One of the most important of the occasionally parasitic mites is the louse-mite, Pediculoides ventricosus (Fig. 138), belonging to the family Tarsonemidæ. This is a very minute species, barely visible to the naked eye, which is normally parasitic on grain-moth caterpillars and other noxious insects, and therefore beneficial. These mites live in stubble, stored grain and beans, cotton seeds, straw, etc., attacking the various insects which infest these products and becoming numerous in proportion to the abundance of their prey. The female has the remarkable habit of retaining the eggs and young in her abdomen 
until they have become fully developed males and females. Her abdomen in consequence becomes enormously distended so that the rest of the body appears as only a tiny appendage at one side of it. A gravid female (Fig. 139) fully distended may reach a diameter of $1.5 \mathrm{~mm}$. ( ${ }_{17}^{2}$ of an inch) whereas normally she measures only $0.2 \mathrm{~mm}$. ( $1 \frac{1}{2} 5$ of an inch) in length. Under the most favorable conditions only six days may elapse from the time the young females emerge from the mother before they reproduce a brood of

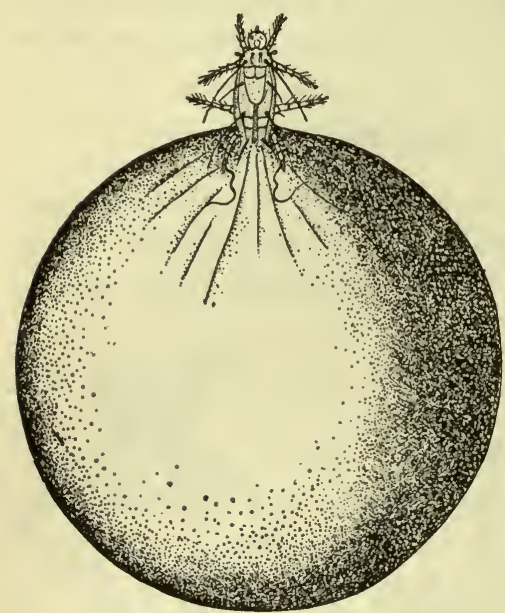

FIG. 139. Louse-mite, gravid female. $\times$ about 75. (After Brucker from Webster.) their own. The brood varies in number from a few dozen to over 200 .

Like many other beneficial things, these predaceous little mites may become a distinct nuisance, and many serious outbreaks of infestation of human beings by them are on record, especially among the grain threshers of the central portion of the United States and among laborers who handle stored grains and other dry foods. In our Middle West their attacks have often been attributed to harvest mites. In Italy the rash produced by louse-mites is called "miller's itch." Several outbreaks have occurred in the United States due to the use of new straw mattresses. The transformation of all the grain-moth caterpillars into moths leaves the mites with their normal food supply cut off, and they are then ready to feed upon any flesh to which they may have access in an effort to prevent starving to death.

The itching rash produced begins about 12 to 16 hours after exposure to the mites. At first they produce pale hivelike spots, which later become red and inflamed, and itch unbearably. Little blisters, the size of a pinhead or larger, appear at the sites of the bites and these later develop into little pustules. Scratching results in the formation of scabs, and when these fall off dark spots which are slow to fade are left on the skin. The rash and itching normally disappear within a week unless fresh 
detachments of mites are constantly acquired. In severe infestations the irritation and poisoning is sufficient to cause constitutional symptoms such as fever, high pulse, headache, nausea, etc.

Since the mites cannot thrive on human blood, and remain attached to the skin for only a short time, no treatment for destroying them is necessary. Remedies to relieve the itching, such as the application of soda or soothing ointments, or warm baths with a little soda, are called for. To prevent infection when handling infected produce, Dr. Goldberger, of the United States Public Health Service, suggested a greasing of the body, followed by a change of clothes and a bath after working with the infected material. Riley and Johannsen suggest the use of powdered sulphur as a preventive in view of its efficiency against harvest mites. Control of the mite consists largely in keeping grain and other dry produce as free as possible from the insects on which the mites feed. Burning stubble in winter and threshing wheat directly from the shock would tend to lessen the worms in stored wheat and with them the mites.

Grain Mites. - The family Tyroglyphidæ, including many species of mites which normally feed on grain, flour, sugar, dried fruits, cheese and other foods, contains several species which become annoying to man and produce an itching rash on people who handle infested goods.

According to Banks all the members of this family are pale-colored, soft-bodied mites, with prominent pincer-like cheliceræ and no eyes. Their bodies are about twice as long as wide and are furnished with a few scattered long hairs (Fig. 140).

The life history of some members of the family is quite remarkable in that there is added a phase of existence which does not

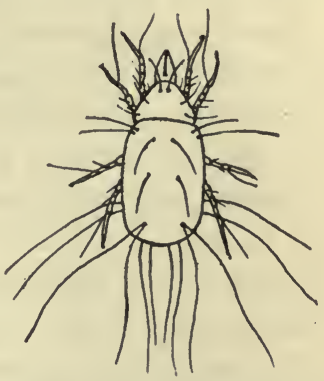

Fig. 140. Grain mite, Tyroglyphus longior. $\times 30$. (After Fumouze and Robin.) occur in other mites. All the species scatter their eggs haphazard over the infected material. Upon hatching the larvæ have six legs and acquire a fourth pair after moulting, in orthodox mite style. Some now develop directly into adults, while others go through what is called a "hypopus" stage. The hypopus (Fig. 141 ) is very different from the nymph from which it develops: 
the body is hard and chitinous, there is no mouth or mouthparts, the legs are short and stumpy, and there is usually a raised area on the ventral surface with a number of tiny sucking discs. By means of these suckers the hypopus attaches itself to insects or

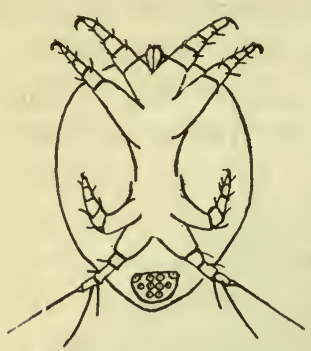

Frg. 141. Hypopus or traveling stage of Tyroglyphus, ventral view. Much enlarged. (After Banks.) other creatures and is thus transported to new localities, the entire object of the hypopus stage apparently being to secure passage to new breeding grounds. After dropping from its unwilling transporter the hypopus moults into an eight-legged nymph again, which, after feeding, develops into an adult.

The Tyroglyphidæ are all quite similar in appearance, and the characters which separate the species, and even the genera, are few and minute. A considerable number of species may attack persons who handle infested materials, and they are the cause of "grocers' itch." This affliction is caused especially by various species of Glyciphagus and Tyroglyphus. Of historical interest is a case of dysentery apparently due to a Tyroglyphus, $T$. longior, (Fig. 140) which occurred in one of Linnaeus' students. The mites were abundant in his fæces, and were found to live and multiply in a juniper-wood cup which he used. As shown by Castellani, an itching rash known as "copra itch," occurring among the laborers in the copra mills of Ceylon where cocoanut is ground up for export, is caused by a variety of this mite, called T. longior castellanii. Copra itch occurs also among stevedores who handle copra in London. Another species, Glyciphagus buski, was taken from beneath the skin on the sole of the foot of a negro in England; it had caused large sores. The negro attributed the affliction to the wearing of a pair of shoes loaned him by a similarly affected negro from Sierra Leone, Africa. Another species, Rhizoglyphus parasiticus, which lives on roots, bulbs, etc., in India, produces a skin disease among coolies working on tea plantations. It begins with blisters between the toes and spreads to the ankles, causing very sore feet.

Other Species. - A few species of the family Tetranychidæ, including the "red spiders" or spinning mites, occasionally become troublesome to man, although they are normally vege- 
table feeders and may do much damage to cultivated plants. One species especially, Tetranychus molestissimus, which lives on the undersides of leaves of a species of cockle bur, Xanthium macrocarpum, in Argentina and Uruguay, attacks man during the winter months from December to February. It produces symptoms similar to those of the louse-mite, with intense itching and some fever. The common "red spider," $T$. telarius, an almost cosmopolitan species, also is reported to attack man occasionally.

The common chicken mite, Dermanyssus gallinae, belonging to the family Parasitidæ (Gamasidæ), frequently causes much irritation and annoyance to those who come in contact with it. Although it can thrive and multiply only on certain kinds of birds, it sometimes remains on mammals for some time, causing an eczema or rashlike breaking-out on the skin, attended, as in other mite infections, by intense itching. Except in cases of constant reinfection chicken mites are usually troublesome to man for only a few days at most. Since these mites can live for several weeks without feeding on their normal hosts, places formerly frequented by fowls may be infective after the removal of the birds. The mites normally remain on their hosts only long: enough to fill up on blood, usually at night, spending the rest of the time in cracks and crevices in and about the coops. Various sprays of sulphur, carbolic solutions and oils are used to destroy them. An allied species, Holothyrus coccinella, living on geese and other birds on Mauritius Island, attacks man, causing burning and swelling of the skin, and frequently proves quite dangerous to children by entering the mouth.

A very small mite, Tydeus molestus, belonging to the family Eupodidæ, attacks man in much the same manner as do the harvest mites. It is common on some estates in Belgium, apparently having been imported many years ago with some Peruvian guano. It appears regularly each summer on grass plots, bushes, etc., in great numbers, disappearing again with the first frost. It causes great annoyance in red-bug fashion, not only to man but to other mammals and birds as well. 


\section{Itch Mites}

The itch mites, belonging to the family Sarcoptidæ, are the cause of scabies or mange in various kinds of domestic and wild animals, and of "itch" in man. This disease is one which has been known for a very long time but was formerly supposed to be caused by "bad blood" or other constitutional disorders such as cause the growth of pimples. Even at the present time the true cause of the disease is not understood by the majority of people.

The Parasites. - The itch mites (Fig. 142) are minute whitish creatures, scarcely visible to the naked eye, of which the females
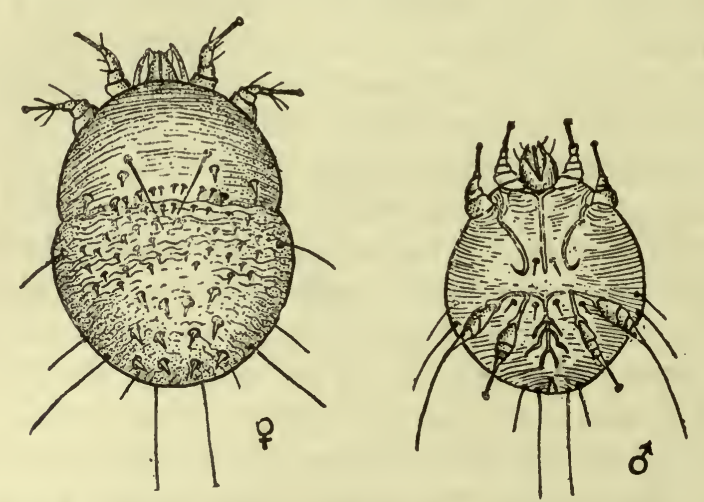

Fig. 142. Human itch mite, Sarcoptes scabiei; $\$$, female; $\delta$, male. $\times$ about 100. (Partly after Banks.)

burrow beneath the skin and lay eggs in the galleries which they make. They are nearly round and the cuticle is delicately sculptured with numerous wavy parallel lines, pierced here and there by stiff projecting bristles or hairs. There are no eyes or tracheæ. The cone-shaped mouthparts are covered over by the shieldlike upper lip. The legs are short and stumpy and are provided with sucker-like organs, called ambulacra, at their tips. In the female the two posterior pairs of legs terminate in a simple long bristle, whereas in the male only the third pair of legs terminates in bristles. The human itch mite, Sarcoptes scabiei, is only slightly distinguishable from the itch mites which cause scabies and mange in many of our domesticated animals. Each infected species of mammal has its own variety of itch 
mite, but many of them can be transferred readily from one host to another. In the common human species the male is only about $0.25 \mathrm{~mm}$. ( $\mathrm{I}^{\frac{1}{0} \sigma}$ of an inch) in length, while the female is about $0.4 \mathrm{~mm}$. ( $\frac{1}{60}$ of an inch) in length. A variety of this mite, S. scabiei crustose , causing the so-called "Norwegian itch," is found in northern Europe and occasionally in the United States, but is always rare. The disease caused by it differs in some respects from ordinary itch. Still another species, Notoedres cati, which causes a very persistent and often fatal disease in cats, temporarily infests man, but is apparently unable to breed in human skin, since the infection dies out in a week or two.

The impregnated females of itch mites excavate tortuous tunnels in the epidermis (Fig. 143) especially on such portions as

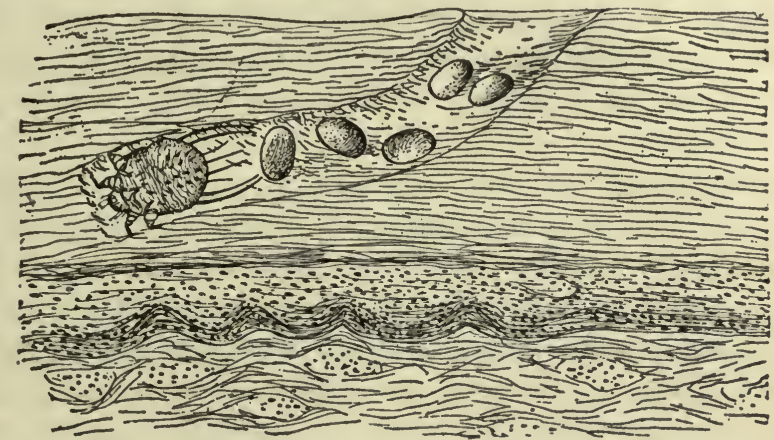

Fig. 143. Diagrammatic tunnel of itch mite in human skin, showing female depositing eggs. $\times$ about 30 . (Adapted from Riley and Johannsen.)

between the fingers and toes, on the groins and external genitals, and in the armpits, where the skin is delicate and thin. The tunnels are anywhere from a few millirneters to over an inch in length, and are usually gray in color from the eggs and excrement deposited by the female as she burrows. The daily excavations of a mite amount to two or three millimeters.

The eggs (Fig. 143) vary in number from 15 to 50 . They are laid in groups of from two to four, the mite resting after each oviposition. After they are all laid the female dies, usually at the end of a single tortuous burrow. The eggs hatch in from three to six days into six-legged larvæ. The latter transform in two or three days into nymphs. The nymphs commonly build burrows for themselves and moult twice, the second time becoming adult 
male and female mites. : The duration of the two nymphal periods is from three and one-half to six days, the entire development of the mites therefore requiring from nine to sixteen days. The mites are not necessarily nocturnal as was formerly supposed, but wander about on the surface of the skin when the latter is warm, which is most frequently the case when the host is in bed. Copulation takes place on the surface of the skin, and apparently the males do not burrow or enter the burrows made by the females, but merely hide under superficial dead cells of the epidermis. Since they die very soon after copulation, they are seldom found. The young impregnated females soon begin fresh excavations, and produce more eggs. Fifteen or twenty eggs each generation, of which approximately two-thirds are females, and a new generation about every four weeks, results in an enormous rate of increase. In less than six months the progeny of one pair of itch mites theoretically would number several millions!

The Disease. The "itch" is a disease which in the past has swept over armies and populations in great epidemics, but it has decreased with civilization and cleanliness, and is fortunately less common now, at least in civilized communities.

As its name implies, the disease is characterized by itching of the most intense kind where the mites burrow in the skin. The itching is probably due only to a very slight extent to the mechanical irritation in the skin, but is induced rather by poisonous substances secreted or excreted by the mites. Injection of fluid containing crushed mites produces an eruption and irritation similar to that caused by the burrowing of the living mites.

The excretions of the mites as they feed in their burrows form little hard pimples, about the size of a pinhead or a little larger, containing yellow fluid. When these are scratched, as they are almost certain to be on account of the unbearable itching, they frequently become secondarily infected and may give rise to larger sores. Ultimately scabs form over them.

Since the entire life history of the parasites is passed on a single host, generation after generation may develop from a single infection, and although the infection apparently may disappear temporarily, it persists recurrently for many years. Since the mites are sensitive to cold the infected areas of skin not only do not spread but may become restricted during the winter, to 
spread with renewed vigor with the coming of warm weather. So persistent is the infection that it is doubtful whether it ever spontaneously dies out." "Norwegian itch," caused by Sarcoptes scabiei crustosœ, is even more persistent, and, unlike ordinary itch, may occur on the face ard scalp as well as on other parts of the body. Gigantic crusts form oyer the infected parts.

Infection can result only from the passage of male and female mites, or of an impregnated female, from an infected to a healthy individual. Normally this takes place by actual contact, rarely in the daytime on account of the secretive habits of the mites, but commonly at night, especially from one bedfellow to another. Gerlach experimented to determine how long the mites could live away from their hosts and found that in the dry warm air of a room they lost vitality so rapidly that they could not be revived after three or four days. In moist places, on the other hand, such as in the folds of soiled underwear or bedcloths, they survived as long as ten days. From this it is evident that infection may take place by means of bedding, towels, underwear or other cloth which may come in contact with infected skin. The author once witnessed an epidemic of itch arising from the use of an infected wrestling mat in a college gymnasium. ( It is also possible for infection to be derived from mangy animals, though the mites, once adapted for several generations to a given host, do not survive a transfer to a different species of host more than a few days.)

Treatment and Prevention. - The treatment. of itch before the nature of the malady was understood was considered very slow and difficult, and even at the present time it is looked upon by many people as a disease which can be recovered from only after prolonged treatment. The fact that the mites burrow beneath the skin to lay their eggs makes careless superficial treatment almost as inefficient as the internal medicine which was once taken to "purify the blood." The most effective treatment for the itch is as follows: the patient rubs himself vigorously with green soap and warm water for about 20 minutes, and follows this with a warm bath for half an hour or more, during which the soapy massage continues. In this manner the skin is softened, the pores opened and the burrows of the mites soaked so that the application of mite poison which is to follow will penetrate more readily. When the skin is thus prepared 
some substance for destroying the mites is applied. Sulphur ointment made by mixing one-half an ounce of sulphur to ten ounces of lard, is excellent; its virtue lies in the formation of hydrogen sulphide in contact with the skin, sulphur itself being inert. A still more efficient though more expensive remedy is a beta-naphthol ointment, prepared as follows: beta-naphthol, 75 grains; olive oil, $2 \frac{1}{2}$ fluid grams; sulphur, $1 \mathrm{oz}$; lanolin, $1 \mathrm{oz}$.; green soap, $1 \mathrm{oz}$. One of these applications, or some other, is unsparingly rubbed into the skin of the infected portions of the body, and of a considerable area around them. When rubbed in for 20 or 30 minutes the patient goes to bed, leaving the ointment on his body until morning when it is washed off with another bath. Meanwhile the soiled underwear, bedclothes or other possibly infected articles are sterilized by boiling or baking. Since this course of treatment does not destroy the eggs it is repeated in about ten days in order to destroy any mites which may have hatched in the meantime.

For delicate-skinned individuals the treatment described above is too severe and may, of itself, give rise to inflammation of the skin not unlike that caused by the mites. In such case balsäm of Peru may be used satisfactorily instead of sulphur ointment, but should be rubbed in several times at intervals of a few hours. It does not cause any irritation.

Prevention of this annoying infection consists merely in avoiding contact with infected individuals, and of shunning public towels or soiled bed linen. A single infected individual in a logging or railroad camp may be a means of infecting the entire camp. Means should, therefore, be taken to guard against such individuals whenever possible, and to prevent the spread of infection from unsuspected individuals by care as regards the use of towels and bed clothes.

\section{Hair Follicle Mites}

The hair follicle or face mite, Demodex folliculorum (Fig. 144), of the family Demodecidæ, is a species which is most strikingly adapted for its parasitic life. It is a wormlike creature, very unmite-like in general appearance, which lives in the hair follicles and sebaceous glands of various mammals. In man it occurs especially on the face. 
The wormlike appearance of the adult mites is due to the great elongation of the abdomen which is marked by numerous fine lines running around it. The beak is short and broad, and the four pairs of legs, all similar, are short, stumpy, three-jointed appendages. The female mites are .35 to $.40 \mathrm{~mm}$. long (about $\frac{7}{64}$ of an inch), while the males are a little smaller.

The multiplication of these mites is slow. The eggs hatch into tiny six-legged larvæ in which the legs are mere tubercles. It requires four moults to bring the larvæ to sexual maturity.

In most cases these parasites cause no inconvenience whatever and their presence is not even suspected. In Europe a large proportion of people are said to be infected, but in America, according to Riley and Johannsen, there is reason for believing that the infection is far less common than is usually supposed. Since generation after generation may be produced on a single host the infection is potentially indefinite in its duration. When the mites become numerous in the hair follicles or sebaceous glands they sometimes cause "black-

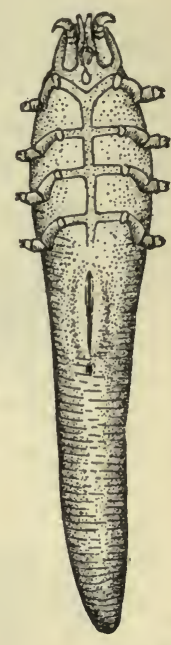

FIG. 144. Hairfollicle mite, Demodex folliculorum. $\times$ 200. (After Megnin.) heads" by causing a fatty accumulation to be produced, but they are not the only or even the usual cause of "black-heads." The skin disease known as "acne" has also been attributed to these mites, but probably erroneously. Follicle mites have been suspected also of spreading leprosy.

The method of transmission of the mites to another host is not definitely known but it is probable that the adults wander on the surface of the skin at times, and may then be transmitted by direct contact or by towels, as are itch mites. In dogs, where the follicle mite, possibly a different species, causes a very severe and often fatal form of mange, transmission from dog to dog takes place in a very irregular manner, and there are frequent instances cited of infected dogs associating for a long time with uninfected ones without spreading the disease. Experiments with transmission of the canine follicle mite to man have invariably failed. Little is known about treatment of 
Demodex infection, but it is probable that sulphur applications in some form would reach and destroy them.

\section{Tongue-worms}

Related to the mites, but now placed in a distinct order, Linguatulina, are the tongue-worms. These animals have become so modified by parasitic life that the adults have lost nearly all resemblance to the other members of their group, and have become

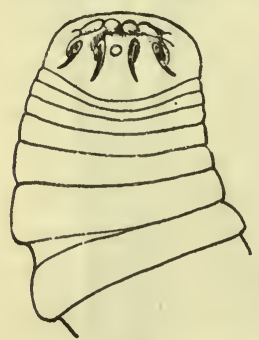

Fig. 145. Head of Porocephalus armillatus. $\times 3$. (After Sambon.) so wormlike, both in form and life history, as to have been classified by older writers with the tapeworms (Fig. 146A). Only the larval stage gives a clue to their real relationships. Their long bodies are either flattened or cylindrical, and distinctly divided into rings or segments as in leeches. There is no distinct demarcation between head, thorax and abdomen. On either side of the mouth are two hooks which can be retracted into grooves like the claws of a cat (Fig. 145). These are usually looked upon as the vestiges of some of the appendages. At the bases of the retractile hooks there open a number of large glands, the secretion of which is believed to have blood-destroying power. The internal organization of the body is degenerate in the extreme; there is no blood, no respiratory system, no special sense organs, no organs of locomotion; little more than the barest necessities of racial existence - a simple nervous system, a digestive tract and a reproductive system. The sexes are separate.

The adult worms live in the nostrils, trachæ or lungs of carnivorous reptiles and mammals, where they produce their myriads of eggs. The latter are voided with the catarrhal products of the respiratory system caused by the presence of the parasites. The egg-laden mucous excretions from the nose of an infected animal are dropped on vegetation and eaten by herbivorous animals, whereupon the eggs (Fig. 146B) develop into larvæ in the new host. These larvæ (Fig. 146C), hatched out in the stomach, are far more mitelike than the adults, inasmuch as they possess two pairs of rudimentary legs and primitive arthropod mouthparts. The larvæ migrate to the liver, spleen or other organs 
and there encyst (Fig. 146D). After a series of moults a second larval stage is entered upon, this time with a wormlike appearance much more like that of the adult (Fig. 146E).

At this stage a "wanderlust" seizes the tongue-worm and it begins an active migration in an endeavor to reach a more satisfactory site for adult life. The mites may settle in the res-

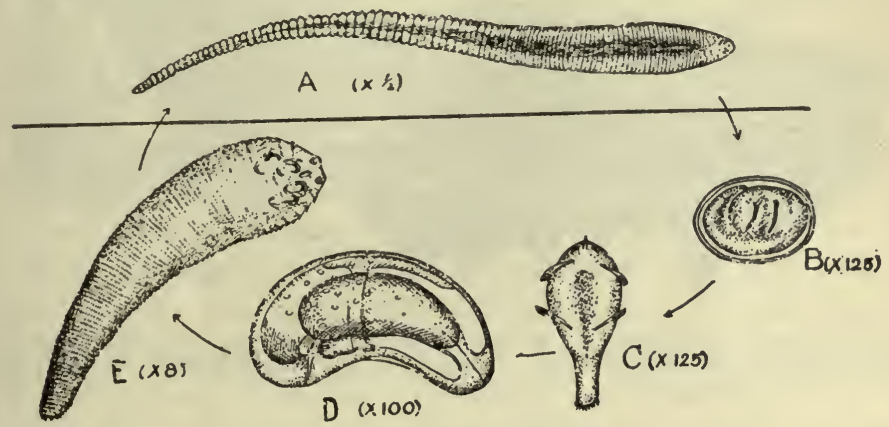

FIG. 146. Life history of tongue-worm, Linguatula rhinaria; $A$, adult female from nasal passage of dog; $B$, egg containing embryo; $C$, larva from sheep, man or other animals; $D$, encysted larva; $E$, 2nd larval stage, from liver of sheep or man.

piratory tract of their original host, or may abandon their host by way of throat or anus to take chances on being snuffed up or taken into the mouth cavity of another animal. Having gained access to their final habitat in the nostrils or lungs, they attach themselves by their hooks, moult, copulate and reproduce.

While both larval and adult stages of tongue-worms are occasionally found in man, the larvæ, as liver parasites, are more common.

The tongue-worm most frequently observed in man is Linguatula rhinaria. The male of this species is a small worm, whitish in color, about three-fourths of an inch in length, whereas the female (Fig. 146A), which is yellowish or brownish due to the eggs in her body, reaches a length of from three to five inches. The adults occur most commonly in the nasal passages of dogs (Fig. 147). The eggs (Fig. 146B) are dispersed with mucus during the violent fits of sneezing to which the presence of the parasite gives rise. The swallowing of food or drink, especially grass or vegetables, soiled by this infective mucus, results in the access of the larva-containing eggs to the intermediate host, which is most frequently sheep, goats, rabbits, etc., but occasionally 
man. In the course of five or six months the larvæ (Fig. 146C), having migrated to the liver or lymph glands, transform to the second larval stage (Fig. 146E), reach a length of about one-

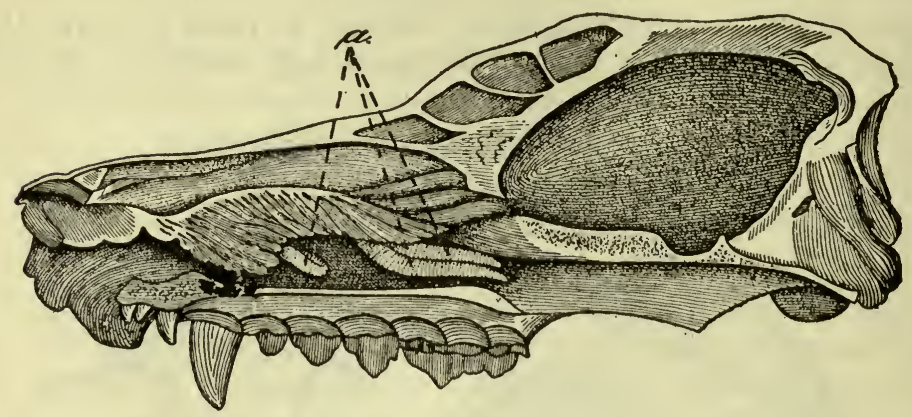

FIG. 147. Head of a dog split in half to show three tongue-worms, Linguatula rhinaria, (a) in the nasal cavity. Reduced in size. (After Colin, from Hall.)

fourth of an inch, and consist of from 80 to 90 rings or segments, each one with very fine denticulations on the hind margin. For

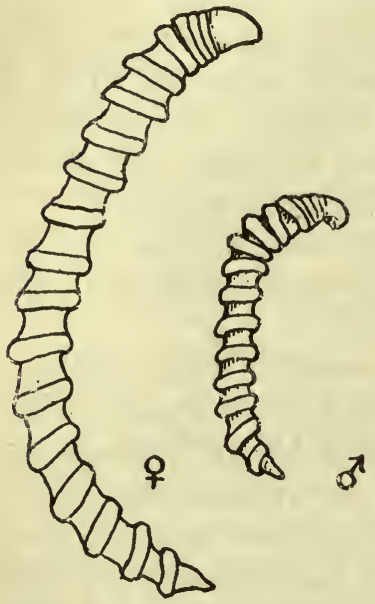

Fig. 148. Porocephalus armillatus; $\$$, female; $\hat{\sigma}$, male. Natural size. (After Sambon.) a long time this larva was looked upon as a distinct species. L. rhinaria is nowhere abundant, even in its normal hosts, though in some parts of Europe about ten per cent of dogs are said to be infected. The majority of human cases reported have been in Germany.

Another species which is occasionally found as a parasite in man during its larval stage is Porocephalus armillatus (Fig. 148). Unlike Linguatula, this worm has a cylindrical body, only about 18 to 22 rings of segments and a total length of about one-half an inch. The segments have no fine denticulations as they have in Linguatula. This species is said to spend its adult life in the lungs of the African python, the larvæ occurring occasionally in man, but more frequently in giraffes, monkeys and other African animals. Sambon thinks the eggs escape from the 
nostrils of pythons into water, and that infection occurs through drinking. The return of the larva from the intermediate host to the python probably takes place by the intermediate host being eaten. The larvæ as they occur in man or other animals may either be encysted or freely migrating in the tissues or body cavities. Such symptoms as emaciation, bronchitis, pleurisy and offensive discharges from the lungs may be present. From 75 to 100 larvæ have been known to be expectorated by a single patient. Recently Mouchet reports finding Porocephalus in over 22 per cent of post mortem examinations made at Leopoldville, in Belgian Congo.

A more slender species, P. moniliformis, bright yellow in color, occurs as an adult in pythons in southern Asia and the East Indies, and in two cases human infection has been reported. One case of human infection with a Porocephalus in Montana in 1876 is of interest, since, as pointed out by Sambon, it may have been the larva of $P$. crotali of rattlesnakes. 


\section{CHAPTER XXI}

\section{TICKS}

WhILE mites as a group are extremely annoying pests, with one exception they are not dangerous as disease carriers. The ticks, on the other hand, are not only annoying but dangerous. Several important diseases of domestic animals are transmitted solely by ticks, and several human diseases are likewise dependent on ticks for dissemination, especially African relapsing fever or " tick fever" and Rocky Mountain spotted fever. In addition to this, tick bites, at least those of some species, give rise to a serious form of paralysis, especially in children, which may end in death. Tick bites also frequently give rise to dangerous ulcerating sores which may result in fatal blood poisoning. The economic importance of ticks as parasites of domestic animals is not for consideration here, but it would not be amiss to state that the annual loss in the United States from cattle ticks alone is estimated at from $\$ 40,000,000$ to $\$ 50,000,000$. It is evident that ticks should be looked upon as worthy candidates for extermination wherever this is possible.

Although the ticks constitute only one of several divisions of the order Acarina, they are so readily distinguishable and so well known that in the popular mind the ticks are looked upon as a group quite distinct from all other mites, and equivalent with them. They are of relatively large size and usually exceed all other Acarina in this respect even in their larval stages. Some species when full grown and engorged are fully half an inch in length.

General Anatomy. - The body of a tick is covered by a leathery cuticle which is capable of great expansion in the females as they engorge themselves on their host's blood, filling the numerous complex pouches of the digestive tract (Fig. 149). When not engorged ticks are flat and oval or triangular in shape (Fig. 154), usually tapering to the anterior end, but after engorgement they resemble beans or nuts of some kind (Fig. 158). 


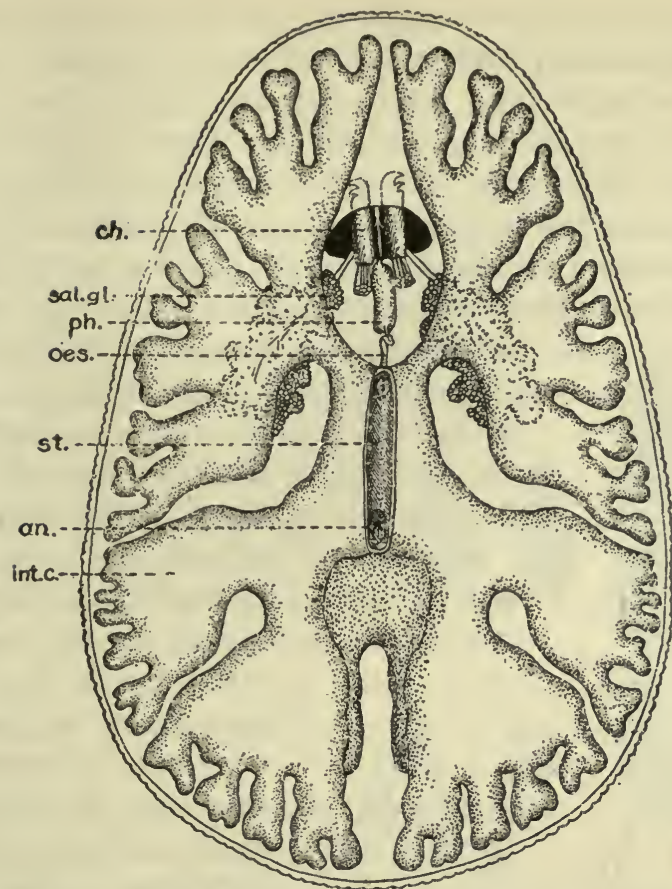

Frg. 149. Digestive tract of Argas persicus; an., anus; ch., chelicera; int. ce intestinal cœea; œs., œsophagus; ph., pharynx; sal. gl., salivary glands; st., stomach. $\quad \times$ about 20. (Adapted from Robinson and Davidson.)

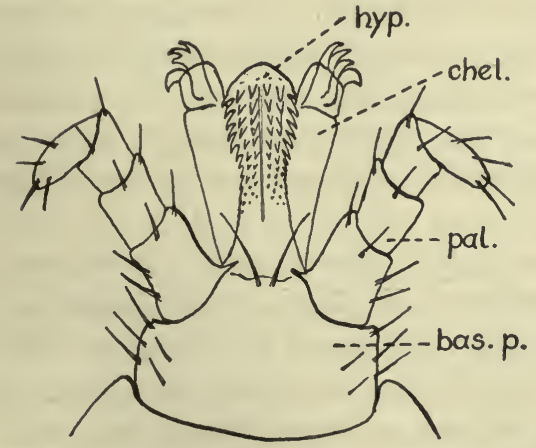

Fig. 150. Head or capitulum of tick; hyp., hypostome; chel., chelicera; pal., palpus; bas. p., basal piece. (Partly after Banks.)

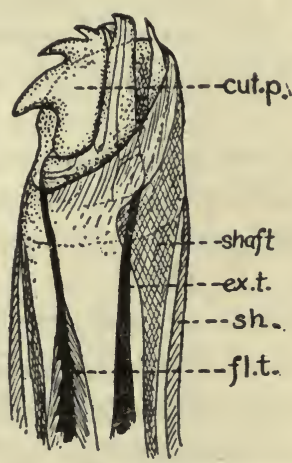

FIG. 151. Tip of chelicera of a tick, much enlarged; cut. p., articulated cutting part; shaft, shaft; sh., sheath; fl. t., tendon of flexor muscle; ex. t., tendon of extensor muscle. (After Nuttall, Cooper and Robinson.) 
Most ticks have a little shield or "scutum" on the dorsal surface, quite small in the females, but nearly or quite covering the back in the males (Fig. 156). Attached to it in front is a little triangular piece, the capitulum or "head" which bears the mouthparts (Fig. 150). The latter consist of a quite formidable piercing organ, the hypostome, a pair of cheliceræ or mandibles which are armed with hooks (Fig. 151), and a pair of blunt palpi which are probably tactile in function. The hypostome is a rasplike structure, beset with row after row of recurved teeth (Fig. 152). So firmly do these hold in the flesh into which the
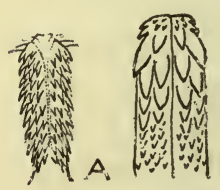

$A$
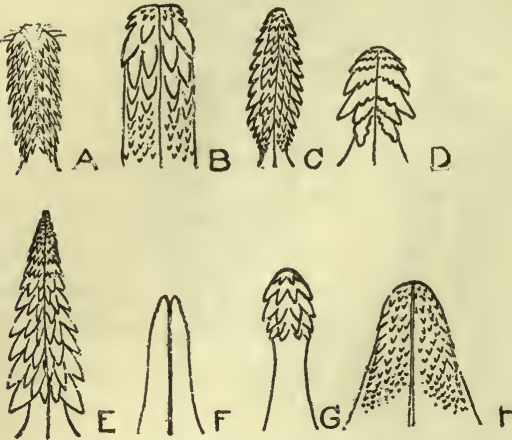

Fig. 152. Hypostomes of ticks; $A$, ear tick, Otiobius (or Ornithodorus) mégnini, nymph; $B$, Argas persicus, adult; $C$, Ixodes ricinus, adult female; $D$, same, male; $E$, Ixodes vespertitionis, adult female; $F$, same, male; $G$, Ornithodorus moubata, nymph; $H$, Ornithodorus savignyi, adult. ( $A$, after Salmon and Stiles; others after Nuttall.) proboscis is inserted that forcible removal of a tick often results in the tearing off of the body from the capitulum which remains attached to the host. Like other Arachnida, ticks have four pairs of legs. These are quite conspicuous when the body is empty but are hardly noticeable after engorgement. The breathing apparatus consists of a system of tracheæ which open by a pair of spiracles in the vicinity of the fourth pair of legs. The shape of the plates which cover the spiracles are sometimes used in distinguishing species. The ventral surface has two openings, the genital pore just back of the proboscis, and the anus some distance from the posterior end of the body (Fig. 154).

Habits and Life History. - All ticks are parasitic during some part of their lives. The majority of them infest mammals, though many species attack birds and some are found on coldblooded animals. A very decided host preference is shown by some species, whereas others appear to be equally content with any warm-blooded animal which comes their way. In many species the hosts or parts of hosts selected by the adults are not the same as those selected by the immature forms. 
The life histories of all ticks are more or less similar. After several days of mating the female ticks engorge and soon after drop to the ground and begin to lay their eggs (Fig. 153). These are deposited on or just under the surface of the ground. Some of the family Argasidæ engorge several times, laying a batch of from 20 to 50 eggs after each gluttonous repast. All of the Ixodidæ, on the other hand, lay their eggs after a single engorgement. The eggs number from a few hundred in some species to upwards of 10,000 in others and are laid in rather elongate masses in front of the female. Each egg as it is passed out by the ovipositor is coated with a viscid substance by glands between the head and dorsal shield of the tick and is then added to the mass in front. The process of egg-laying occupies several days, as not more than several hundred eggs can be passed out and treated with the viscid coating in the course of a day.

The eggs develop after an incubation period which varies with the temperature from two or three weeks to several months. Eggs deposited in the fall do not hatch until the following spring.

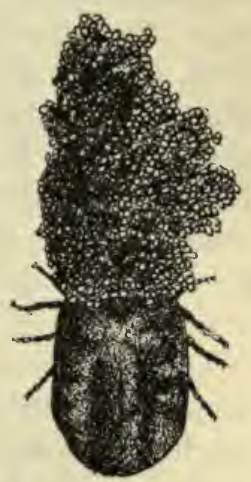

FIg. 153. Texas fever tick, Margaropus annulatus, laying eggs. (After Graybill.)

The larval ticks which hatch from the eggs are much smaller than the adult ticks and have only six legs (Fig. 157B). They are popularly known as "seed ticks." The seed ticks soon after hatching climb up on a blade of grass or bit of herbage and assume a policy of watchful waiting until some suitable host passes within reach. Seed ticks must be imbued with almost unlimited patience, since in many if not in the majority of cases long delays must fall to their lot before a suitable host comes their way like a rescue ship to a stranded mariner. The jarring of a footstep or rustle of bushes causes the ticks instantly to stretch out to full length, feeling with their clawed front legs, eager with the excitement of a life or death chance to be saved from starvation. If success rewards their patience, even though it may be after many days or weeks, they feed for only a few days, becoming distended with blood, and then drop to the ground again. Retiring to a concealed place they rest for a week or more while 
they undergo internal reorganization. Finally they shed their skins and emerge as eight-legged but sexually immature ticks known as nymphs (Fig. 157C). The nymphs climb up on bushes or weeds and again there is a period of patient waiting, resulting either in starvation or a second period of feasting. Once more the ticks drop to the ground to rest, transform and moult, this time becoming fully adult and sexually mature. In this condition a host is awaited for a third and last time, copulation takes place, sometimes even before a final host is reached, and the females begin their final gluttonous feeding which results in distending them out of all proportions. In some species, especially those which live on hosts which return to fixed lairs, copulation takes place off the host. When this occurs, as in many species of Ixodes, the male is often not parasitic at all, and may differ markedly from the female in the reduced structure of its hypostome (Fig. 152C, E and F). In all species the males die shortly after copulation.

This, in general, is the life history of ticks, but it is, of course, subject to considerable variation in different species. In many species there are two nymphal periods instead of one. In some species, as in the Texas fever tick, Margaropus annulatus, the moulting takes place directly on the host, thus doing away with the great risk of being unable to find a new host after each successive moult. In a few species the first moult is passed through on the host, but the second is passed on the ground. The most important asset of ticks to counterbalance the disadvantage of having to find new hosts is their extraordinary longevity. Larvæ of ticks have been known to live more than six months without food, and adults have been kept alive in corked vials for five years.

There are two families of ticks, the Argasidæ and the Ixodidæ. The Argasidæ include the bird ticks and their allies, which are distinguished from the Ixodidæ by the absence of a dorsal shield and in having the head partially or entirely concealed under the overlapping anterior margin of the body (Fig. 154). The females of this family do not become distended as do those of the Ixodidæ, but take more moderate though more frequent meals. They are chiefly inhabitants of warm countries. Both nymphs and adults feed at night, usually dropping off their hosts immediately after a meal, and thus seldom being carried from the 
lairs or abodes of their hosts. The Ixodidæ, on the other hand, inhabit the hosts rather than their lairs, and frequently remain attached for several days, or even longer. In the less capacious Argasidæ the females lay their eggs in a number of installments

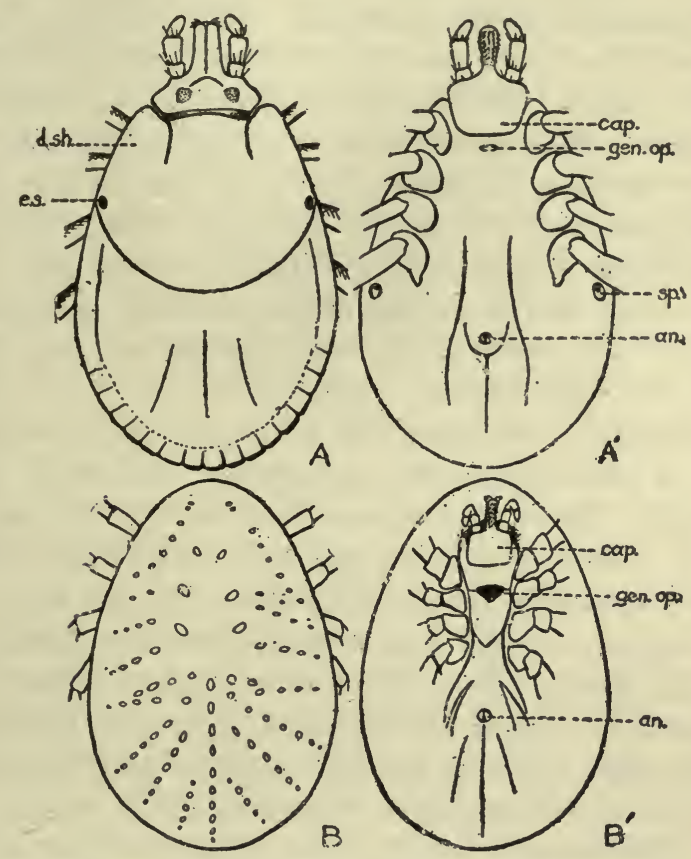

FIg. 154. Comparison of dorsal and ventral view of Ixodid and Argasid females; $A$, dorsal view of Ixodid $\$ ; A^{\prime}$, ventral view of same; $B$, dorsal view of Argasid \&; $B^{\prime}$, ventral view of same. An., anus; cap., capitulum; d. sh., dorsal shield; e.s., eye spot; gen. op., genital opening; sp., spiracle.

after successive feeds, and the total number of eggs may be counted in hundreds instead of thousands. The reason for this difference is readily accounted for by the difference in habits in the two families, since the progeny of the Argasidæ, reared in the lairs of the hosts, have far better chances of finding a host and of surviving than do the progeny of the Ixodidæ which live on their hosts and may drop off to lay their eggs almost anywhere in the wanderings of the host.

Tick Bites. - The status of ticks as human parasites, as stated before, is one not to be passed over lightly. Aside from the 
transmission of diseases tick bites are dangerous to man in a number of ways.

The wounds made by ticks, especially if the head is torn off in a forcible removal of the parasite, are very likely to become infected and result in inflamed sores or extensive ulcers, not infrequently ending in blood poisoning. The author, as the result of the bite of a tick in northern California (probably Dermacentor occidentalis), suffered from an ulcerating sore on his arm, over half an inch in depth and three-fourths of an inch in diameter. Blood poisoning set in early causing a very high temperature and great pain in the arm, and it was only a timely return to civilization and hospital care that saved his arm if not his life. Sanitary removal of ticks and cleansing of the wounds, as described on p. 367 , would be well worth the consideration of every inhabitant or traveller in a tick-infested country.

Tick Paralysis. - More serious than the painful wound made by ticks is a peculiar paralyzing effect of tick bites, known as tick paralysis. This occurs especially from tick bites on the back of the neck or on the head; it affects the legs first, but spreads forward in a few days to the arms and neck and may result in death. Paralysis in man and animals from tick bites has been reported from South Africa and Australia and in North America from the parts of Oregon and British Columbia inhabited by the spotted fever tick. Sheep are especially subject to tick paralysis, to such an extent in British Columbia as to present a serious problem. This peculiar effect of tick bites has been reproduced experimentally in sheep in places where it has not been known to occur normally, by allowing a spotted fever tick, Dermacentor venustus (Fig. 156), to bite along the spinal column. The bites of this tick are particularly likely to cause paralysis, though it is not yet known whether this is because of an especially toxic secretion produced by this species or because of its preference for biting along the spinal cord or on the head. There has been much controversy as to what really causes the paralysis, some authors believing that it is due to a microörganism injected by the tick, since it is usually six or seven days after the attachment of the tick before the effect is felt. The fact, however, that no such organism can be discovered, that inoculations of blood and other parts of diseased animals into healthy ones does not result in transmission of the disease, and that the paralysis 
is usually accompanied by little or no fever, makes this seem unlikely. A single attack of tick paralysis seems to confer immunity and it is probable that many children are naturally immune. The most reasonable explanation is that the ticks secrete a toxic substance, especially when rapidly engorging, which has a specific action on the motor nervous system. Possibly the bite must pierce or come in contact with a nerve or nerve ending in order to produce the effect.

Numerous cases of tick paralysis in children have occurred in British Columbia and in the Blue Mountains of Eastern Oregon. One doctor in the vicinity of Pendleton reported no less than 13 cases. The disease begins with paralysis of the legs and usually results in complete loss of their use; the paralysis ascends in the course of two or three days, affecting the arms and finally the thorax and throat. Unless the heart and respiration are affected, recovery follows in from one to six or eight days after removal of the ticks. The latter, often in pairs, are usually found on the back of the neck or along the middle line of the head, especially just at the base of the skull. If the ticks are not removed, the disease may result in death or in spontaneous recovery after a few days or a week.

Unfortunately in most of the cases of tick paralysis in children the ticks have not been identified, but it is well known that the spotted fever tick is the most frequent cause of paralysis in sheep and the only species by which such a disease has been reproduced experimentally. In South Africa, however, a similar paralysis in sheep results from the bites of Ixodes pilosus, and paralysis in children in Australia from the bites of other but undetermined species. The scrub-tick, Ixodes holocyclus, is said to be troublesome as a cause of paralysis in young stock in New South Wales. In the regions of Oregon and British Columbia where tick paralysis is especially prevalent there occur a number of different ticks, and there is no evidence that any tick which attacks man along the spinal cord or on the head may not cause paralysis.

\section{Ticks and Disease}

The rôle of ticks as disease carriers has been well established since Dutton and Todd in 1905 proved that African relapsing fever was transmitted by a species of tick known as the tampan, 
Ornithodorus moubata (Fig. 155). A year later Dr. Ricketts showed that spotted fever in the United Stated was dependent upon a tick, Dermacentor venustus, for its transmission. It is now known that ticks serve as intermediate hosts for a considerable number of disease germs of two different groups, the spirochætes and the Piroplasmata. The various forms of relapsing fever of man are caused by spirochætes, and it is possible that all the different types of this disease may be transmitted by ticks, though in some of the types other arthropods act as the usual transmitters. Many diseases of domestic animals are caused by organisms of the group Piroplasmata (see p. 182), including Texas fever of cattle in North America, East Coast fever of cattle in Africa, biliary fever of horses in Asia and Africa, and similar diseases of sheep, dogs, rats and monkeys. The only human disease positively known to be caused by an organism of this group is Oroya fever of Peru, caused by Bartonella bacilliformis (see p. 178). Whether or not a tick is instrumental in transmitting this disease is not yet known. Rocky Mountain spotted fever was at one time thought to be caused by a member of the Piroplasmata, but the parasite of this disease is still unknown. The fact that it is transmitted by a tick suggests that it may be found to belong either to the spirochætes or to the Piroplasmata. Ticks have also been suspected of carrying the East Indian form of kedani fever which in Japan is transmitted by a larval mite, but this has not been proved.

Ticks and Relapsing Fever.- The fact that tick bites frequently give rise to serious fever and illness, now known as relapsing fever, which not infrequently result in death, has been well known in Africa for many years, in fact Livingston in his "Darkest Africa " speaks of this disease as resulting from tick bites. The implicated ticks, Ornithodorus moubata, known as "tampans" or "carapatos," are very common pests in shaded places in the dirty thatched houses of the natives, and are difficult to avoid. They occur chiefly along the routes of travel, being readily carried and dispersed by caravans. They live also in the burrows of warthogs. A detailed account of the rôle played by the tick in harboring and transmitting relapsing fever spirochætes and a description of the disease can be found in Chap. IV, p. 42.

The tampan is a broad oval tick (Fig. 155), mud-colored, 
about five-eighths of an inch in length, belonging to the family Argasidæ. Like the other members of the family it has no dorsal shield and has the margin of the body produced in such a way as to conceal most of the head and legs. Unlike most ticks the larvæ are weak and do not feed but transform to nymphs very soon after the eggshell splits. The nymphs are said to produce more painful wounds than the adults and they can just as readily transmit relapsing fever.

An allied species, $O$. savignyi, occurs from Abyssinia through Arabia to India and Ceylon and attacks man, camels and horses. It is said to transmit the Indian form of relapsing fever in these countries.

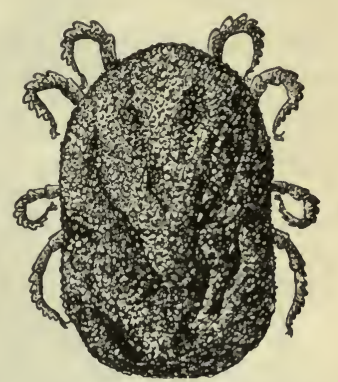

Fig. 155. The tampan, Ornithodorus moubata. $\times 3$. Like 0 . moubata it attacks its host in its resting place, hiding in the daytime in dust or sand in or around the squalid huts of the natives. Except in coastal towns, where it is abundant everywhere, it is found chiefly in camps of long standing inhabited by men and animals. Burrowing to a depth of an inch in dusty soil it can live without food for months. In Persia $O$. tholosani is said to transmit African relapsing fever which has been introduced there. O. talaje of Mexico and Central America has habits very similar to those of the tampan in Africa; it frequently occurs in the adobe houses and attacks the occupants at night. 0 . turicata, the "carapato" of Central America, is another very annoying species. Its bites are so severe that hogs are said to have been killed in a single night by its attacks. Though not proved it is very probable that one or both of these species may be instrumental in transmitting the milder American form of relapsing fever. It is almost certain also that another tick, the "miana bug" of Persia, is capable of transmitting European relapsing fever (see p. 364).

Ticks and Spotted Fever. - The tick which is responsible for the transmission of Rocky Mountain spotted fever (see p. 191) is a wood tick, Dermacentor venustus (andersoni) (Fig. 156). This is a handsome reddish brown species, the male of which has the whole back marked with black and silvery-white lines, while the female has only the small dorsal shield marked with silver, the abdomen being deep reddish brown. This species is one 
which requires two different hosts to complete the life cycle. The six-legged larvæ (Fig. 157B), of which there are about 5000 in a brood, attach themselves to any of the rodents which abound

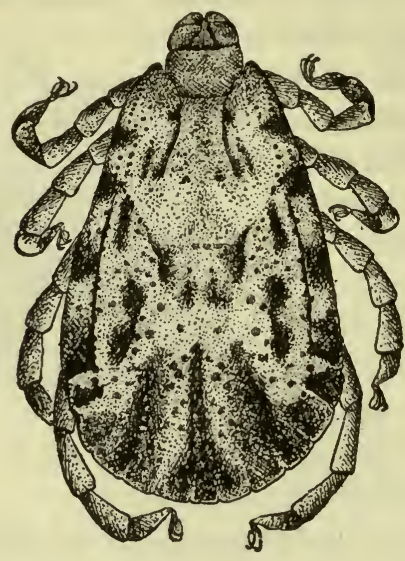

$\sigma^{\pi}$

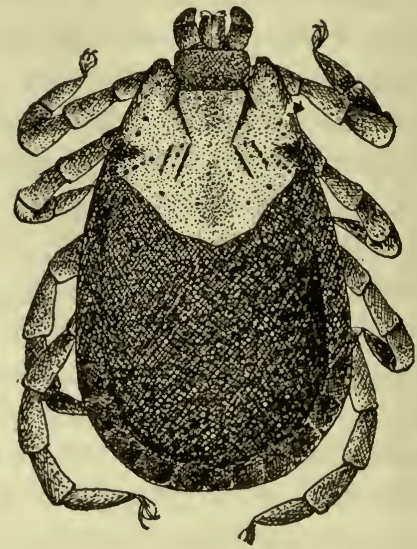

q

Fig. 156. Spotted fever tick, Dermacentor venustus, male $(\hat{\delta})$ and female $(\$)$. $\times 12$.
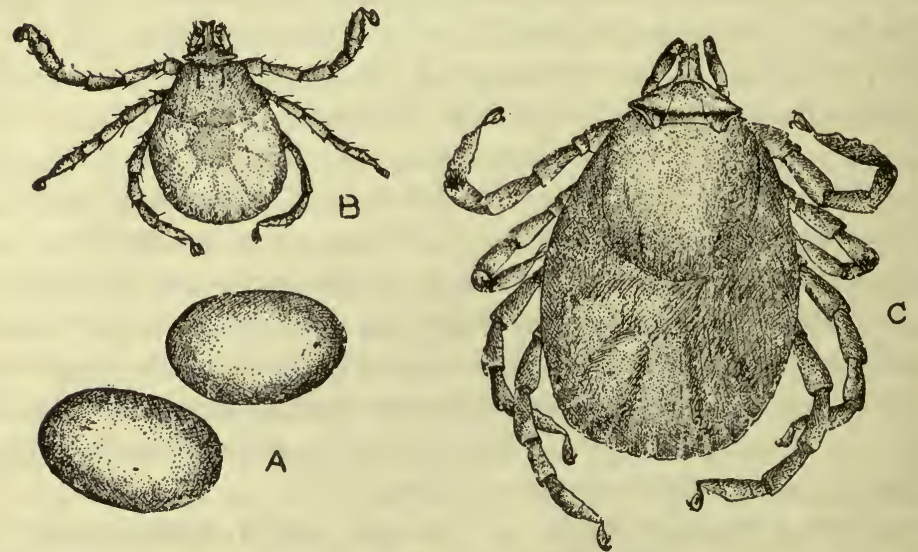

FIG. 157. Development of spotted fever tick, Dermacentor venustus; $A$, eggs; $B$, larva; $C$, nymph. $\times 30$.

in the country where the ticks occur, especially squirrels of various kinds. Usually the larvæ, and the nymphs also, attach themselves about the head and ears of their host. After a few days the larvæ drop, transform into nymphs (Fig. 157C) and 
again attack their rodent hosts. After dropping off these and transforming into adults they no longer pay any attention to the rodents but seek larger animals, especially preferring horses and cattle, though they readily attack other large wild and domestic animals and man. Their original wild hosts were probably the mountain goats, elk and other wild game of the region, but with the supplanting of these by domestic animals the latter have become the main host animals of the ticks. Unlike most ticks, this species may take two or even two and a half years to complete its life cycle under unfavorable conditions. The winter is passed in either the nymphal or adult stages.

Dermacentor venustus is found in a limited area in northwestern United States and British Columbia, east to eastern Montana and eastern Wyoming, west to the Cascade Mountains and south into Nevada and Colorado. This distribution somewhat exceeds the present distribution of spotted fever (Fig. 58, p. 191).

Several different species of ticks have been found capable of transmitting spotted fever from rodent

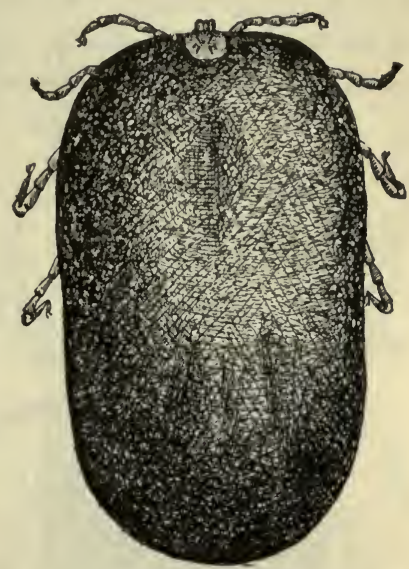

FIG. 158. Spotted fever tick, Dermacentor venustus; engorged female. $\times 4$. to rodent under experimental conditions. Several species of ticks other than $D$. venustus are found in the spotted fever districts, but none of these can have any hand in the transmission of the disease to man since they do not attack him. A tick closely related to $D$. venustus, the Pacific wood tick, $D$. occidentalis, occurs west of the Cascades and Sierras in Oregon and California and frequently attacks man. There is little doubt but that if spotted fever once got a foothold in the territory occupied by this tick, the latter would act as an efficient disseminator. In southern and eastern states other ticks which attack man would probably disseminate the disease were it once introduced. For this reason it is of the utmost importance that the infection should not be carried to parts of the country which are not now infected. Measures for the prevention of this are discussed in Chapter X, under "Spotted Fever." 


\section{Other Troublesome Ticks}

Although there are a large number of species of ticks which will attack man, there are a few in addition to the disease-causing species named above which deserve special mention on account of the particularly bad effects of their bites. The family Argasidæ includes a number of species which produce very venomous bites when they attack man. The various species of Ornithodorus, some of which have already been mentioned as carriers of relapsing fever, produce very painful bites. Another species worthy of mention is the famous " miana bug," Argas persicus (Fig. 159), which is especially renowned in Persia, but which also occurs in

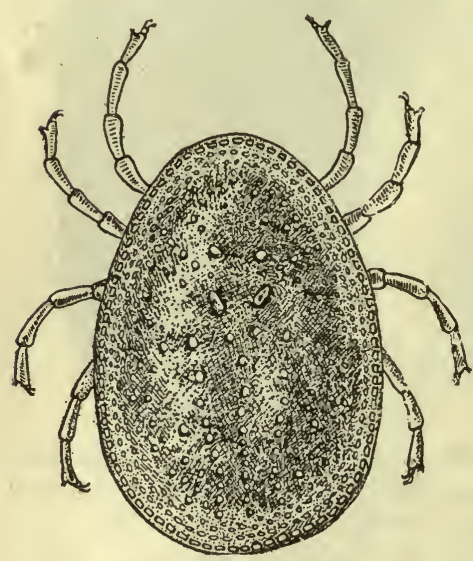

Fig. 159. Persian tick or fowl tick, Argas persicus. $\times 5$. (After Braun.) many other parts of the Old World. This species is often a great tormentor of human beings, especially in dirty huts where it can breed readily. It is primarily, however, a parasite of fowls, and is believed to be identical with the American fowl tick, Argas miniatus. The bites of the miana bug are dreaded not only on account of their painfulness, but also because they are believed to be a means of transmission of European relapsing fever, in common with lice and perhaps other insects. A closely allied species, $A$. reflexus, is a common parasite of pigeons in Europe and North Africa, and frequently attacks people who come in contact with infested birds or cotes.

Another argasid tick which deserves special mention is the "pajaroello," $O$. coriaceus of California. Herms states that " natives, principally Mexicans, in the vicinity of Mt. Hamilton fear this parasite more than they do the rattlesnake, and tell weird tales of this or that man having lost an arm or leg, and in one instance even death having ensued, as a result of a bite by the Pajaroello. There seems to be a suspicion in that region that three bites will result in certain death. The stories all 
agree in the essential detail that the bite results in an irritating lesion which is slow to heal and often leaves an ugly deep scar." The tick is about two-fifths of an inch in length, irregularly oval, with thick turned-up margins, roughly shagreened, and of a yellowish earthy color spotted rusty red. It occurs in the Coast Range mountains of California and in Mexico and according to Herms is most commonly found in the dry leaves under live oak trees where cattle or other animals are accustomed to lie in the shade. It passes through from four to seven moults to reach the adult state, occupying from one to two years to complete its life history, according to its success in finding suitable hosts. The bites of this tick produce sharp pain, accompanied by a considerable discoloration around the wound, and if on an arm or leg the whole limb may become greatly swollen as in the case of a snake bite. After scabbing over, the wound may continue to exude lymph and to be irritable for several weeks, and it is possible that infection and consequent blood-poisoning $\mathrm{might}$ readily occur, thus giving a basis for the tales mentioned above.

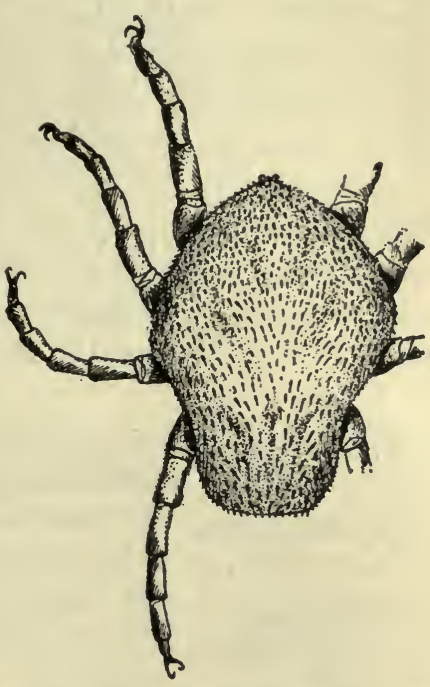

FIG. 160. Spiny nymph of ear tick, Otiobius (or Ornithodorus) mégnini. $\times 10$. (After Marx from Banks.) the Argasidæ is the spinose ear tick, Otiobius (or Ornithodorus) mégnini (Fig. 160), of southwestern United States and Mexico, and now becoming common in parts of South Africa. It is very troublesome to man as well as to horses and other domestic animals. The nymphs, which develop from the larvæ in the ears of their hosts, are peculiar in having very spiny bodies, quite different from the smooth larvæ and adults. The nymphs remain attached to their hosts for months but finally drop off to transform into adults. The adults are not parasitic but lay their eggs without further feeding. The pain and annoyance caused by the spiny nymphs in the ears of domestic animals is sufficient to cause them to be- 
come ill-tempered and emaciated. Children sometimes suffer a great deal from their attacks, and have difficulty in dislodging the invaders from their ears. This can readily be done, however, by pouring olive oil or some other harmless oil into the ears.

Although there are a large number of species of the family Ixodidæ which may attack man, they do not as a rule prove as great pests or produce as severe bites as some of the Argasidæ. The characteristics of some of the principal genera are given in
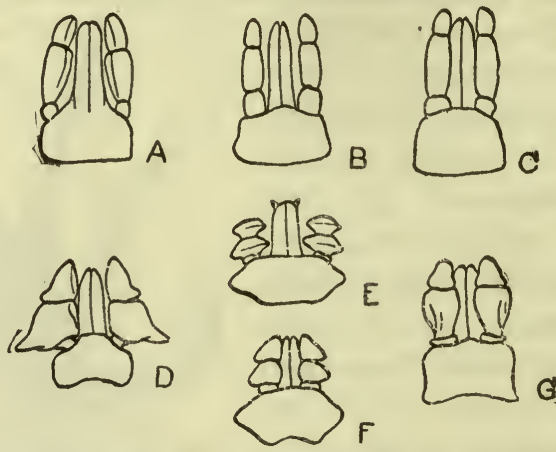

Fig. 161. Diagrams of rostra or capitula of important genera of Ixodid ticks, useful in identification. (After Nuttall.)

With long rostrum

Other characteristics

$A$, Ixodes.................Anal. groove in front of anus, no eyes, no festoons.

$B$, Hyalomma . . . . . . . . . . . . eyes present, festoons present.

C, Amblyomma..............eyes present, festoons present, ornate. or

A ponomma..............eyes absent, festoons present, ornate.

With short rostrum

$D$, Homaphysalis. . . . . . . . . . eyes absent.

$E$, Margaropus ................ circular spiracles.

$F$, Rhipicephalus . .............. comma-shaped spiracles.

G, Dermacentor. . . . . . . . . eyes present, ornate.

Fig. 161 and accompanying table. Only a few species need special mention here. Dermacentor venustus is, of course, of preëminent importance on account of its rôle as a transmitter of spotted fever and in producing tick paralysis. D. occidentalis, the "wood tick" of the Pacific slope of the United States, is another member of the genus which very commonly attacks man; its bites are particularly likely to cause ugly ulcerating sores. Experimentally, as said before, it has been shown to be capable of transmitting spotted fever, and it would probably act as an effi- 
cient disseminator if the disease were introduced into its territory. The same might be said of $D$. variabilis, the dog tick of eastern North America, though this species less commonly attacks man.

Of particular interest is the effect produced by the larvæ of certain ticks in southeastern Africa, especially the bont tick, Amblyomma hebrceum. Its larvæ produce itching and painful wounds which may be followed in a week or so by fever, headache, skin eruptions and other general symptoms. The name "tick-bite fever" has been applied to this malady. Whether it is caused by a microörganism is unknown. Immunity rapidly develops, so that usually only new arrivals are affected. In Europe one of the most troublesome species of Ixodidæ, as far as man is concerned, is the common dog tick, Ixodes ricinis, which attacks a great variety of animals, and is evidently quite fond of human blood. A particularly obnoxious species in tropical America is Amblyomma cajennense. Not only the nymphs and adults but also the larvæ of this species are pests of man.

Treatment and Prevention. - As shown above tick bites may be attended by a number of serious results, such as fever, ulcerating sores, paralysis or disease transmission. The treatment of the bites, therefore, may be of considerable importance. It has been shown that ticks, at least in the case of the relapsing fevers, do not ordinarily infect directly by biting, but by contaminating the wound with infected excrement. It is obvious, therefore, that disinfection of the wound after removal of the tick would be a precaution of great value in places where ticks carry diseases to which human beings are susceptible. Such treatment would also prevent bacterial infections of various kinds from entering the wounds and causing ulceration or bloodpoisoning.

Ticks should never be removed forcibly since if so handled the head is likely to tear off from the body and remain in the wound, held there by the ugly barbed proboscis. A drop of kerosene, creoline or some other oil on the head of the tick will cause it to withdraw its beak and drop off in the course of a minute or two. Disinfection of the wound with alcohol, weak carbolic, lysol or other disinfecting agent should follow immediately. 
Precautions against tick bites where serious diseases are likely to result are of the greatest importance but very difficult. King, while investigating spotted fever, spent a whole season in the heart of the Bitter Root Valley in Montana where spotted fever infection was most dangerous. He wore high-topped shoes and cotton outer garments soaked in kerosene and had pieces of khaki cloth soaked in kerosene sewed to the tops of his boots or fastened by drawstrings higher up on his leg. A leg covering of oil-proof material with crude oil applied on the outside would be of benefit, according to King. In Abyssinia the attacks of Ornithodorus savignyi are prevented by rubbing the feet with turpentine.

Means of control of tick pests vary considerably with the different species, depending on the hosts, their seasonal history, their varying life histories and other factors.

Most of the species of ticks which attack man are normally parasitic on domestic animals, and therefore means of exterminating ticks on the latter would tend to reduce the human pests.

Ticks on domestic animals may be destroyed either by hand treatment or by dipping, or by the elimination of ticks from pastures by starvation. The cattle tick, Margaropus annulatus, has been eliminated from many ranches by a skillful manœuvering of the cattle, driving them from field to field in such a way that in the course of a number of months the ticks would all have dropped and perished from starvation. Such a plan is not feasible for many species since a variety of hosts may be utilized, and long periods of starvation can be endured without injury.

Dipping of infested animals is a good control method. An arsenical dip has been found best adapted for destruction of ticks on their hosts, a description of which, with methods of preparing and using, is given in Farmers' Bulletin No. 378 of the U. S. Department of Agriculture.

Hand treatment with arsenical dip by means of rags, mops or sprays is sometimes found more practical.

The systematic dipping of domestic animals in the spotted fever districts of Montana for a period of three years has been recommended by the U. S. Department of Agriculture for the elimination of the spotted fever tick from these regions. In this particular case supplemental means of control consist in the 
destruction of indigenous rodents in a wholesale manner, and the clearing away of brush land in tick-infested areas.

Another means of destruction of spotted fever ticks has been found in grazing sheep on tick-infested lands. Range sheep have been found to destroy ticks in large numbers by the ticks becoming entangled in the wool and starved. Five hundred sheep were found to destroy 25,000 ticks in a season.

Ticks which infest the lairs of their hosts, attacking only at night and for brief periods, can be more easily handled. In this case thorough disinfection by fumigation or by spraying with a disinfectant, and thorough cleanliness in stalls, coops, kennels, huts or other host homes will effectually destroy them. The disease-carrying tampan, Ornithodorus moubata, of Africa is an example of a tick which can be controlled by such methods. Dirty, poorly kept native huts are the ideal habitats for tampans, which secrete themselves during the day in crevices, thatched roofs or débris, after the manner of bedbugs. Plastering houses with mud, building of smudges, fumigation and cleanliness are methods which usually succeed in keeping out ticks. Crevices, bed sheets and other places which might harbor ticks should be dusted with pyrethrum insect powder.

The nearly allied $O$. savignyi of Abyssinia, which conceals itself in dusty soil to a depth of one inch, can best be destroyed in infested camp sites, environs of wells, etc., by harrowing the surface of the ground, strewing dry grass and brush over it, and burning it from around the edge of the infested area toward the center. Spraying with antiseptics has been found practically useless, since even the total immersion of ticks in strong antiseptics for an hour or more fails to kill them.

The fowl tick or " miana bug," Argas persicus, and the American hut-infesting species of Ornithodorus, O. talaje and O. turicata, can be controlled by methods similar to those used for the tampan. 


\section{CHAPTER XXII}

\section{BEDBUGS AND THEIR ALLIES}

The Order Hemiptera. - The order of insects, Hemiptera (or Rhynchota), which includes the true bugs, contains a number of species which habitually or occasionally attack man. The most important of these are the bedbugs, which are found all over the world in temperate and tropical climates. There are few objects which are more disgusting than bedbugs to good housekeepers, yet there are few who, at one time or another, have not had to contend with them or at least guard against them. Belonging to an allied family are the cone-noses, larger than bedbugs and not devoid of wings, fiercer in disposition and capable of producing much more painful bites. A considerable

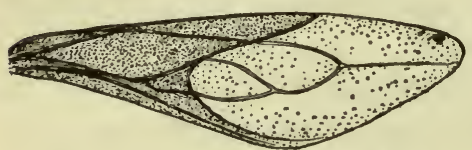

Frg. 162. A hemipteran wing (Reduviid). number of species of these bugs are known and are found in all warm countries. The relation of bugs to disease is still very imperfectly known, but these parasites are positively known to transmit at least one important disease, and are suspected of transmitting several others.

The true bugs, order Hemiptera, are characterized by having piercing and sucking mouthparts contained in a jointed beak and by an incomplete metamorphosis, i.e., not undergoing a complete transformation from a larval to an adult form during a period of rest, as do such insects as butterflies, beetles, etc. The newly hatched young may differ quite considerably from the adult, but the mature characteristics are gradually attained with each successive moult. The order is divided into two suborders, only one of which, the Heteroptera, concerns us here. In the members of this group the first pair of wings, if present, have a thickened, leathery basal portion and a membranous terminal portion (Fig. 162). The second pair of wings are always membranous when present. 


\section{Bedbugs}

General Account. - The bedbugs belong to the family Cimicidæ. They have broad flat bodies, and are devoid of wings, except for a pair of spiny pads which represent the first pair of wings (Fig. 163). The first segment of the thorax has winglike expansions at the sides which grow forward and partially surround the small head. In the male the abdomen is quite pointed at the tip, whereas in the female it is evenly rounded, the contour of the abdomen being almost a perfect circle in unfed bugs. The eyes project prominently at the sides of the head, the flexible four-jointed antennæ are constantly moved about in front of the head, and the jointed beak is folded under the head so that it is entirely invisible from above. The legs have the usual segments, the tarsi being threejointed. The greater part of the body is covered with bristles set in little cup-shaped depres-

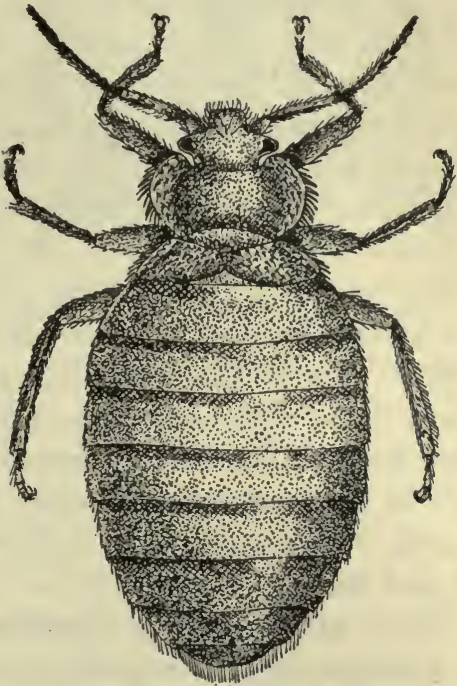

FIa. 163. Bedbug, Cimex lectularius, female. $\times 10$.

sions. These depressions are perforated at the bottom to allow for the passage of muscles which move the bristles. Murray describes having seen bugs raise the bristles upon meeting each other as cats raise their hairs or birds their feathers. The bristles are of two kinds, one a simple slender spine, the other with a stouter flattened end, with sawlike teeth along the thinner edge. In addition to both kinds of bristles, the legs also have a dense brush of hairs at the end of each tibia. When a bug is distended with blood a smooth shining band can be seen at the base of each abdominal segment where no bristles occur (Fig. 163). These bands are the portions of the segments which are not ordinarily exposed, being overlapped by the preceding segment.

One of the most striking characteristics of bedbugs is the peculiar pungent odor so well known to all who have had to con- 
tend with these pests. Many other bugs are characterized by similar odors, as, for example, the common "stink-bugs." The odor is produced by a clear volatile fluid secreted by a pair of

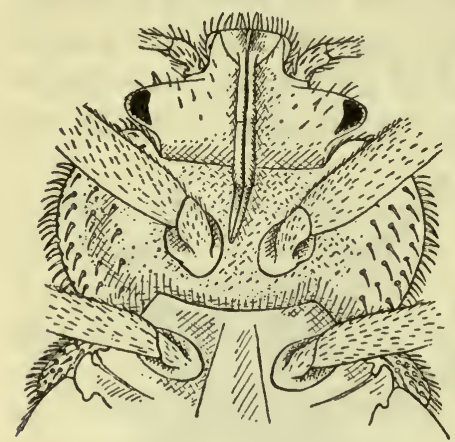

Fig. 164. Head and part of thorax of bedbug, ventral view. $\times 20$. Note jointed beak, eyes and stout spines. glands of very variable size which open between the bases of the hind pair of legs. Although in most "wild" bugs the stink glands are supposed to be distinctly beneficial in that they make the owners obnoxious to enemies which would otherwise prey upon them, they are a decided handicap to the domestic bedbugs in the struggle for existence, since the odor draws attention to the presence of bugs which might otherwise escape notice. Nor does the scent appear to be any protection to them against such enemies as cockroaches and red ants. Murray suggests that it may be of some use to them in their social intercourse in the dark recesses in which they spend their lives.

The nasty odor of bedbugs has evidently inspired some faith in their medicinal value. Seven bugs ground up in water was said by Pliny to arouse one from a fainting spell, and one a day would render hens immune to snake bites. Even at the present time there are places in civilized countries where bedbugs are given as an antidote for fever and ague.

There are a number of species of bugs in the genus Cimex, but some of the species confine their attentions to poultry and other birds, bats, etc.

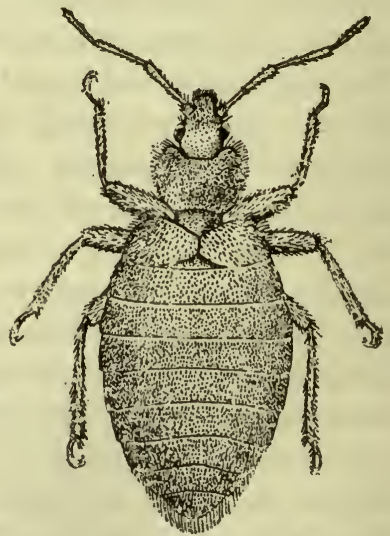

Fig. 165. Indian bedbug, Cimex heminterus (rotundatus), female. $\times$ 8. (After Castellani and Chalmers.)

There are two widely distributed species which attack man: one is the common bedbug, Cimex lectularius, found in all temperate climates; the other is the tropical or Indian bedbug, Cimex hemipterus (rotundatus), prevalent in many tropical countries, includ- 
ing southern Asia, Africa, the West Indies and South America. The tropical bug (Fig. 165) differs from the common one only in minor details, such as greater length of body hairs, darker color and more elongate abdomen. It is less dependent on human blood than its relative of temperate climates, and readily attacks not only rats and mice but also bats and birds. Both species are reddish brown in color, becoming deep red when gorged with blood. Another species, C. boueti, attacks man in Guiana.

Habits. - Bedbugs are normally night prowlers, and exhibit a considerable degree of cleverness in hiding away in cracks and crevices during the daytime. When hungry they will frequently come forth in a lighted room at night, and have even been known to feed in broad daylight. Favorite hiding places are in oldfashioned wooden bedsteads, in the crevices between boards, under wall paper, and other similar places, for which their flat bodies are eminently adapted. Like other animals which have long associated with man, bedbugs have developed much cunning in their ability to adapt themselves to his habits. Marlatt says " the inherited experience of many centuries of companionship with man, during which the bedbug has always found its host an active enemy, has resulted in a knowledge of the habits of the human animal and a facility of concealment, particularly as evidenced by its abandoning beds and often going to distant quarters for protection and hiding during daylight, which indicate considerable apparent intelligence." Their ability to gain access to sleepers at night is hardly less remarkable. Cases are reported of bedbugs creeping along ceilings and dropping down on beds in order to reach their hosts, but these may have been accidental.

The bedbug makes himself a great pest wherever he occurs by the unsparing use of his piercing and sucking mouthparts. The latter consist of four needle-like organs lying in the long, jointed lower lip or beak, a pair of flattened sharp-pointed mandibles and a pair of slightly shorter maxillæ with serrated edges. The beak is grooved in such a way that the sides of the groove almost close together, thus forming a protective sheath for the stilettos inside. When about to indulge in a meal the beak is bent back, and the piercing organs, gliding up and down past each other, are sunk into the flesh of the victim (Fig. 166). A strong sucking motion of the pharynx, into which a bit of sali- 
vary juice has already been poured, draws blood up through a tube made by the piercing organs, through a thickened "bottle neck" ring to the œsophagus and then into the relatively enormous stomach. The muscles for dilating the pharynx in order to make a suction pump out of it occupy the greater part of the

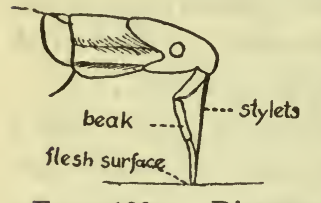

Frg. 166. Diagram showing bug in act of biting. Note bowing back of proboscis. (After Murray.) head. According to Cragg, who has worked on the alimentary tract and digestive process of bedbugs, there are about 70 pulsations of the pharynx per minute in young bugs, in which this can be observed through the body wall. Bugs seldom cling to the skin while sucking, preferring to remain on the clothing. Since a fresh meal apparently acts as a stimulus for emptying the contents of the rectum, the adherence to the clothing is a fortunate circumstance, inasmuch as it precludes to some extent the danger of bedbugs infecting their wounds with excrement, as do ticks.

In the course of ten or 15 minutes a full meal is obtained and the bug, no longer flat but round and distended with blood, retreats to his hiding place, having first deposited a bit of excrement. According to Cragg, in the case of C. hemipterus (rotundatus), a single meal, much of which is temporarily stored in the stomach which acts as a food reservoir as well as a digestive organ, is not fully assimilated for at least a week, although the bug is ready to feed again in a day or two, thus having parts of several meals in the stomach at once. This is quite a different condition from that found in most blood-sucking insects, where a meal is completely digested before another is sought. Observations made by several authors on $C$. lectularius do not indicate that this species has similar habits. As in other bugs, the digestive juices change the absorbed blood into a dense black mass, described by Murray as almost like lamp-black.

The bite of the bedbug seldom produces pain or swelling unless rubbed or scratched, a fact which indicates either that the saliva is not irritating or that it does not ordinarily reach the wound before sucking begins. In some people, however, a stinging, hard, white swelling is produced.

Under normal conditions the common bedbug, C. lectularius, has only rarely been found feeding on anything but human blood. The bugs which infest the nests of swallows and other birds are 
of different species from the human pests, and are not known to annoy man voluntarily, although they occasionally enter rooms from the nests of chimney swifts. Bats are often accused of carrying bedbugs into houses, but they, too, are attended by their own particular species which does not attack man. The assertion that bedbugs can be found under bark and moss out of doors also arises from a misapprehension. These bugs are really the immature stages of certain other species of bugs which resemble bedbugs closely enough to be mistaken for them by a casual observer.

Although human blood is their normal food, bedbugs are able to subsist on the blood of such animals as rats, mice, rabbits, cats, dogs and even chickens. It has also been shown that bugs will suck blood from freshly killed mice. By utilizing mice and rats as a food supply they are able to exist in deserted buildings for a long time. Furthermore they are able to endure long fasts; they have been kept alive without any food whatever for a year. Murray has found that bugs which have been starved even for a long time pass unaltered blood corpuscles in their fæces, and suggests that a small quantity of food may be retained undigested in the rectum to be drawn upon very slowly in time of need, though when a fresh supply of blood is obtained the old store is cleared out. Bugs also store up a great deal of fat for use in time of famine. Sometimes, however, after a house has been deserted for some time, and their normal supply of food is cut off, the bugs migrate in search of an inhabited house. In cold weather bugs hibernate in a semi-torpid condition and do not feed, but in warm climates they are active the year around. The common bedbug, according to Marlatt, is sensitive to temperatures of $96^{\circ} \mathrm{F}$. to $100^{\circ} \mathrm{F}$. or more if accompanied by a high degree of humidity, and is killed in large numbers under such climatic conditions. According to Bacot, unfed newly hatched bugs are able to withstand cold between $28^{\circ} \mathrm{F}$. and $32^{\circ} \mathrm{F}$. for as much as 18 days, though they are destroyed by exposure to damp cold after a full meal.

Life History. - The eggs of bedbugs (Fig. 167A) are pearly white oval objects, furnished with a little cap at one end which is bent to one side. As in the case of lice, the eggs are relatively large, being about one $\mathrm{mm}$. ( $\frac{1}{2} \mathrm{~s}$ of an inch) in length, and are therefore laid singly or in small batches. The ovaries hold about 
40 eggs at a time, all near the same stage of development, so they must undergo rapid increase in size shortly before being deposited. Girault, who has carried out extensive breeding experiments, saw one female lay 111 eggs during the 61 days that he had her in captivity, and another laid a total of 190 eggs. Often a female returns to lay more eggs in the same place so that batches of 40 or more can be found in the crevices where the adult insects hide.

The eggs hatch in from six to ten days during warm weather, but are retarded in their development by cold. A week of

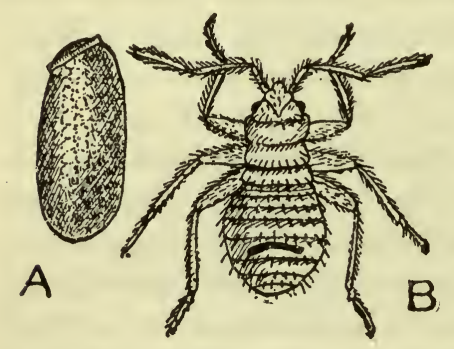

Fig. 167. Egg and newly hatched larva of bedbug. $\times 20$. (After Marlatt.) freezing temperature reduces the hatching to 25 per cent. The freshly hatched bugs (Fig. 167B) are very small, delicate and pale in color. After their first hearty meal they have a much more robust appearance, and grow rapidly. The skin is normally moulted five times before the final adult stage is reached, at least one gluttonous feed being necessary before each moult in order

to insure normal development and reproduction. Although apparently not necessary to its development, the bug may gorge itself several times between moults, at intervals of about one to six days. Marlatt found the average period of time between moults to be eight days. Allowing a similar length of time for the hatching of the eggs, the time occupied from laying of the eggs to maturity is about seven weeks. Girault has found the development from the hatching of the eggs to maturity to take place in as short a time as 29 days. On the other hand, starvation, cool temperatures and possibly other conditions may drag out the period of development to great length. Bacot found that the newly hatched larvæ could live unfed four and a half months and with one feed for nine months. The several larval stages of the insect resemble each other quite closely except in the constantly increasing size and deepening color. The wing pads appear only after the last moult.

Bedbugs and Disease. - The relation of bedbugs to human disease is a subject which, although a problem of the most vital 
interest in preventive medicine, is still very indefinitely known. Various authors have associated bedbugs with a number of human diseases but the evidence brought forth in support of these insects being the normal transmitters of the diseases in question rests on insecure foundations. Ordinarily bugs are handicapped in the extent to which they are able to spread disease by their non-migratory habits. Unlike many parasites they are not usually carried about by human beings, but remain permanently in places occupied by their hosts. It is obvious, therefore, that bugs are limited in the spreading of disease to the occupants of the infested place. Should this be a private home, spread of disease by bugs would be practically limited to a single family. In case of infested hotels, rooming houses, sleeping cars, boats, etc., conditions are ideal for the spread of disease by bugs, and it can hardly be doubted that it is in such places that most of the damage is done.

One of the first accusations against the bedbug as a disease carrier was made by Patton, of the British Medical Service in India, who in 1907 brought evidence against this insect as a carrier of Indian kala-azar (see p. 79). Patton followed what he believed to be developmental stages of the parasite of kalaazar, a species of Leishmania, in the gut of the Indian bedbug, Cimex hemipterus (rotundatus). Subsequent investigations, especially recent ones by Cornwall, have shown that infection of bedbugs by feeding on kala-azar patients is very rare, and that the bugs cannot, apparently, transmit the infection either by biting or by means of infected fæces. The rare infectivity of bugs which have fed on kala-azar patients, however, may be correlated with the fact that the kala-azar parasites are rare in the peripheral blood. As pointed out by Price and Rogers, even if only a small per cent of bugs become infective, where they are as numerous as they are in the coolie huts in India, they would be able to spread the disease successfully. Donovan believes the kala-azar parasites may utilize the Malay bug, Triatoma rubrofasciatus (see p. 381), as an intermediate host, but recent work is tending to throw doubt on the necessary instrumentality of any insect in transmitting the disease. Bedbugs have also been associated with another Leishmania disease, oriental sore, but it is doubtful whether the bugs act as more than mechanical disseminators of the parasite, if at all. Yaki- 
moff in Turkestan and Cornwall in India were unable to infect bedbugs with parasites of oriental sore even when the bugs were fed directly on the ulcers. On the other hand, the fact that one species of Cimex, C. pipistrelli, transmits a trypanosome disease of bats would lead one to suspect their ability to transmit a Leishmanian disease, since the two groups of parasites are certainly near relatives. Several workers have incriminated bedbugs as carriers of European relapsing fever, especially in Serbia and in the southeastern part of Europe, but there can be little doubt but that lice are the normal transmitters of the European as well as the North African form of relapsing fever. In Moscow, for instance, Bayon found that relapsing fever was practically unknown among the better class of people who were personally clean, even though living in bug-infested quarters, whereas the fever was very prevalent among the lower classes, most of whom were lousy, even though they were kept in hospitals where no bugs existed. On the other hand, Hagler, who worked with the American Red Cross expedition in Serbia in 1915 , points out that while typhus disappeared with the extermination of lice, relapsing fever continued to develop in the Belgrade hospital until the latter was fumigated for bedbugs. The Indian bedbug, C. hemipterus, is believed by some workers to be a common transmitter of Indian relapsing fever, though evidence points strongly to the instrumentality or lice and ticks in spreading the disease. Spirochoeta carteri, the organism of Indian relapsing fever, has been observed to remain alive for from four to seven days in the alimentary canal of bugs which have fed on infected monkeys, but bugs seldom become infected from human cases.

As remarked elsewhere, bedbugs have been found capable of acting as intermediate hosts for the trypanosome, T. cruzi, of Chagas' disease, but they usually remain infective for a much shorter time than do bugs of the genus Triatoma. Bedbugs have been found capable of transmitting the infection to guinea-pigs in from 21 hours to 77 days after an infective feed.

That bedbugs may act as mechanical spreaders of various diseases is unquestionable. Experiments show that the bacilli of bubonic plague can develop in the gut of bugs, though more slowly than in fleas, and with a much higher mortality for the bugs. That they may act as transmitters of the disease is quite 
certain, and bugs have been found to remain infective for 48 days if they did not early succumb to the disease. Leprosy also can probably be spread by bugs in a mechanical manner, and it is reasonable to believe that such diseases as tuberculosis and syphilis may likewise be carried by them.

\section{Other Parasitic Bugs}

Most of the other true bugs which may be looked upon as normally human parasites belong to the family Reduviidæ. This is a large family of rapacious bugs, many of them bright colored, which are especially numerous in the tropics. Most of them prey upon other insects, but nearly all of them produce painful wounds when they bite man. Nearly all are active runners and good fliers.

Triatoma. - By far the most important species are the members of the genus Triatoma (Conorhinus), popularly known as cone-noses, "big" bedbugs and by numerous local names. There are about 40 species, most of them in South and Central America. T. sanguisuga of southern United States is the wellknown "Mexican bedbug." It is a bug about one inch in length with a flat, dark brown body, the edges of which, not covered by the wings, are marked with pinkish bars. The long conical head is furnished with a strong beak. Its bite, like that of others of the family, is very painful and causes swelling, sometimes followed by effects which may last a year.

The salivary secretion is evidently very poisonous and not unlike snake venom in the extensive swelling and irritation which it causes. The adult bugs attack not only man but other mammals also, while the nymphs often annoy chickens. Unlike the bedbug this insect can fly, and will readily enter rooms at night to attack sleepers unless screened out. The eggs are white, oval objects when first laid, soon turning yellowish and then brownish; they are laid in small batches under logs or stones outdoors. They hatch in about. 20 days into young bugs which probably prey very largely on other insects. After four moults the insect reaches the adult winged condition in which it is most troublesome as an invader of houses. This species is replaced by $T$. protracta in southwestern United States.

The most important species of the genus are those which are 
naturally infected with Trypanosoma cruzi in South America. On account of its domestic habits, Triatoma megista (Fig. 168) is the most important species in the transmission of the disease to man. This bug is a large, handsome, black and red insect, locally known as the " barbeiro," which infests the dirty thatched houses of the natives in the state of Minas Geraes in Brazil. It is nocturnal in habit, coming forth from its hiding places in the thatch of the roof or in the débris of the floor to feed upon

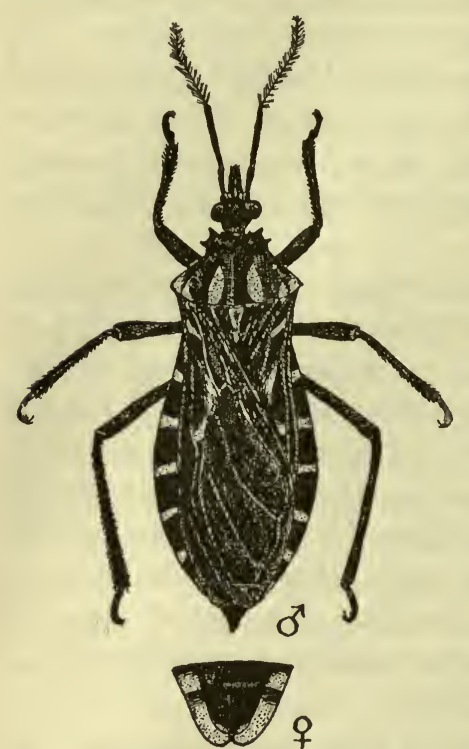

Fig. 168. The "barbeiro," Triatoma megista. $\times 1 \frac{1}{2}$. (After Chagas.) its human victims after the manner of bedbugs. The bugs are so active and hide so rapidly when a light is produced during their foraging in the dark that they can seldom be caught. The details of the development of the trypanosome of Chagas' disease in this insect and the relation of the insect to the disease are described in Chapter VI, p. 110. Torres believes the bugs almost invariably become infected by feeding on infected vertebrates, since Triatoma does not devour excrement of its own species, as does the allied Rhodnius prolixus, and cannibalism is rare among these bugs, except in young larvæ which sometimes feed on each other.

The life history of the barbeiro is quite like that of other members of the genus, except that the eggs are laid in or about human habitations. The eggs hatch in from 20 to 40 days and the young pass through five moults to reach maturity, the whole life cycle occupying about a year. The females begin depositing eggs about a month after the last moult. These insects suck blood at intervals of from four days to several months.

A number of other South and Central American species of Triatoma have been found to harbor Trypanosoma cruzi or a species indistinguishable from it. Triatoma geniculata, which inhabits the burrows of the armadillo and various rodents, is 
known to infect these animals in nature, and the armadillo is possibly an important reservoir of the disease. Triatoma chagasi which had fed on a rodent known as the "moco," Cerodon rupestris, in an uninhabited desert region was found to be infected. $T$. vitticeps, occurring near Rio de Janeiro, T. sordida of São Paulo and $T$. dimidiata of San Salvador in Central America have been found infected with trypanosomes thought to be identical with the species causing Chagas' disease, and these species have been shown to be capable of transmitting the infection to guinea-pigs. In Argentina, as well as throughout most of Brazil, T. infestans, the vinchuca or "great black bug of the Pampas," described by Darwin in his "Voyage of a Naturalist" as a vicious human pest, has been found to harbor a similar trypanosome, but whether or not Chagas' disease exists in Argentina is still in doubt. T. protracta of southwestern United States has been shown recently by Kofoid and McCulloch to harbor a trypanosome which exhibits only slight differences from Trypanosoma cruzi, and, as intimated by the discoverers, may possibly be merely a variety of the same species though named by them $T$. triatomee. The widely distributed $T$. rubrofasciata was shown by Neiva to become infected with trypanosomes after feeding on an infected guinea pig. From all this evidence, and from the fact that other species of bugs of different genera and families, including the bedbugs, are experimentally susceptible to the infection and capable of transmitting it to rodents, it is possible that all the species of Triatoma and allied genera in South and Central America may be potential transmitters of the infection. Cannibalism is common among many of these bugs, and may make possible a direct spreading of trypanosome infection from bug to bug.

The "Malay bug," T. rubrofasciata, of tropical Asia and some parts of Africa and Madagascar is a closely allied species. With its huge proboscis it produces a nasty sting which is followed in a few minutes by acute pain and swelling. Although it feeds on man by preference, it attacks a number of other mammals and even insects. Large nymphs or adults, which are an inch or more in length, are said to consume about one cc. of blood at a meal, and they feed at intervals of from three to six days. The breeding habits are similar to those of other cone-noses. In the islands of Mauritius and Réunion the stomach and intestines 
of this bug have been found to contain trypanosomes in all phases of development, and of very variable form, possibly representing several species. These trypanosomes can be inoculated into mice and rats and it is suggested that under certain conditions they or others living in the gut of the bug may cause disease in man. A number of cases are on record where irregular fevers have followed the bites of this insect. Since these fevers were shown to be non-malarial and showed symptoms of typical trypanosome infection, it is possible that such an infection may really be transmitted to man by this bug as well as by its close relatives in South America. It is also possible that the bug may serve as an intermediate host for the kala-azar parasite.

Other Species. - Several other species of bugs of this family occur in Africa. One, Acanthaspis sulcipes, has been thought to be the possible transmitter of a form of endemic goitre in tropical Africa. In North America the family is further represented by the "kissing bugs," of the genus Melanolestes. The common kissing bug or "black corsair," $M$. picipes, became very abundant in the United States a few years ago and gave opportunity for many startling newspaper stories. It is a large black bug with reddish marks on the back and legs. Its bite much resembles that of a wasp, though often much more serious, occasioning more than local symptoms and even vomiting. Allied bugs of the genera Reduvius, Rasahus

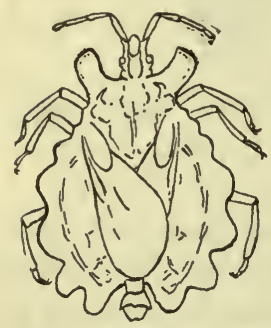

Fig. 169. Pito bug, Dysodius lunatus. (After Alcock.) and Melanolestes occur in the warm parts of North and Central America, and frequently attack man and other mammals, though their normal food in most cases is insects.

In Venezuela and other parts of northern South America a very common bug which infests houses is Rhodnius prolixus, a species which has been found capable of transmitting Trypanosoma cruzi. This species is not only cannibalistic in habits, but also devours excrement of other bugs, thus suggesting the possibility of direct dissemination of trypanosomes from bug to bug. Of other families, there are many bugs which occasionally attack man but few which commonly do so. One which is worthy of mention is the malodorous pito bug, Dysodius lunatus (Fig. 169), 
of South America, belonging to the family Aradidæ. It is a large broad bug which frequents houses and bites severely.

All the species of bugs which infest houses may be destroyed by the fumigation methods described below, but all but the bedbugs must be kept out by screening, since they are not handicapped in their migrations by degeneration of the wings.

\section{Remedies and Prevention}

Prevention of "bugginess," at least in the case of bedbugs, consists chiefly in good housekeeping, but occasional temporary infestations are likely to occur in almost any inhabited building. A number of remedies for bugs have been advocated, of which the best is undoubtedly fumigation with hydrocyanic acid gas, as described below. Sulphur is also valuable for fumigation but is not so harmless to household goods as is hydrocyanic acid gas. When the infested parts of houses or rooms can be easily located, good remedies are kerosene, gasolene, turpentine or other coal-tar products painted into all the infested cracks and crevices, especially in the woodwork of beds. An effective remedy of this nature is a mixture of one ounce corrosive sublimate, two cups alcohol, one-half cup turpentine. These substances should be applied several times at intervals of a week in order to destroy newly hatched bugs. Some housekeepers take infested beds apart and pour boiling water into the "buggy" parts, thus effectually killing both bugs and eggs in the bed but this does nothing against bugs which may hide elsewhere than in the bed. Bedbugs have a number of natural enemies, among which may be mentioned especially cockroaches, red ants and large predaceous bugs, but all of these are pests themselves, and are, therefore, hardly to be encouraged as bedbug hunters, efficient as they might be in this capacity.

\section{Fumigation}

Hydrocyanic Acid Gas. - Of the remedies for bugs mentioned above, fumigation with hydrocyanic acid gas is the most effective. This gas can be used with good success for fumigation of houses, mills, granaries, greenhouses or any other closed structure, against any kind of insect pest. But since the gas is extremely 
poisonous not only to insects but also to other animals and to man, its use must be accompanied by great care and precaution. A few deep breaths of the gas is sufficient to cause asphyxiation. On the other hand it has great advantages in that it is not inflammable or explosive, and, unlike sulphuric fumes, does no damage to dry foods or to household goods, except to tarnish nickel slightly. Wet foods may absorb some of the gas and should be removed before fumigation. Care should also be taken that there is no possible avenue of escape for the gas into adjoining rooms or houses which are occupied. The characteristic peach-kernel odor, however, makes its detection easy, thus removing danger of asphyxiation without warning.

The gas is generated by the action of sulphuric acid on potassium cyanide. The procedure as advised by Herrick is as follows: Estimate the number of cubic feet in the room or house to be fumigated, and allow one ounce of potassium cyanide to every 100 cubic feet. Make the room or house as near air tight as possible, stopping all the large openings such as fireplaces and chimney flues with old rags or blankets. Seal cracks about windows and doors with strips of wet newspaper. Such strips when thoroughly wet can be applied quickly and effectively over cracks and will stick tightly for several hours, and can be removed easily after the operation. While the room is being made tight someone should measure out the required ingredients for fumigation, allowing one fluid ounce of crude sulphuric acid and three fluid ounces of water to each ounce of potassium cyanide. The water first should be poured into a stone crock holding two gallons or more, i.e., large enough so that the reacting fluid will not spatter on floors or carpets. The crock had best be placed on several thicknesses of newspaper or on an old rug or burlap sack. The required amount of sulphuric acid should then be poured slowly into the water. Never pour the water into the acid. The cyanide should be weighed out and put into a paper bag beside the jar. All articles which might suffer from the gas or which will be needed before the operation is over should be removed from the room. When everything is ready the operator, holding his breath, should drop the paper bag of cyanide gently into the acid jar, and walk out shutting the door behind him. The time required for the acid to eat through the paper bag in order to reach the cyanide gives ample time to leave the room before the 
steamlike gas arises. If preferred, however, the paper bag may be suspended by a string passing through a screw eye in the ceiling and through the key hole of the door (Fig. 170). The operator may then lower the bag into the jar after leaving the room. When stringing a room in this manner, care should be taken not to place the acid jar under the bag until everything is ready. The fumigation should extend over a period of five or six hours at least, a good method being to start the operation toward evening and let it run all night. Better results will be obtained at a temperature of $70^{\circ} \mathrm{F}$. or above, than at a lower temperature.

Two or three hours after the doors and windows have been opened the gas will have disappeared sufficiently to allow safe entrance into the room, though it should not be occupied until the characteristic odor is gone. The contents of the generating

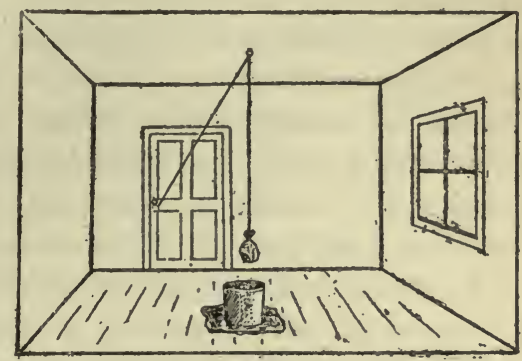

Fig. 170. A room "strung" for hydrocyanic acid gas fumigation from outside. The bag of cyanide can be lowered into the crock of sulphuric acid and water by means of the string. (After Herrick.) jar should be dumped in some safe place and the jar washed before being used again. When a whole house is to be fumigated each room should be made ready as described above and then set off in regular order beginning on the upper floor and working downward, since the gas is lighter than air and therefore rises. Herrick describes clearly and in detail the method which he has successfully used in the fumigation of large dormitories. For this account the reader is referred to Herrick's "Insects Injurious to the Household," pages 448 to 452 .

The effectiveness of this method of fumigation against bedbugs was proven by experiments conducted by Herrick. Bugs were placed in perforated pill boxes and wrapped in various manners, some with three inches of excelsior, some in two folds of a thick comforter, some in two inches of cotton batting and others in two folds of a woolen blanket. Others were placed in a cork stoppered vial, the cork of which was punched twice with a pair of curved forceps. In each box several newly laid eggs were enclosed to determine the effect of the gas on their hatching. In 
every case every bedbug was killed and none of the eggs showed signs of hatching in 12 days. According to experiments made by the U. S. Public Health Service five ounces of powdered potassium cyanide per 1000 cubic feet is sufficient for the destruction of bedbugs, four ounces for mosquitoes, two and one-half ounces for fleas and ten ounces for lice.

Sulphur. - The fumes of burning sulphur, sulphur dioxide, rank next to hydrocyanic acid gas as both a disinfectant and an insecticide, but they have a serious disadvantage in their tendency to bleach fabrics and to tarnish metals, especially in a humid atmosphere. Sulphur dioxide is considered the most effective remedy for mosquitoes in cellars, barns, etc., since it kills these insects even when very dilute, and it has remarkable penetrating power. The methods of sealing rooms or buildings are similar to those described for hydrocyanic acid fumigation. All dyed goods and metallic articles, however, must be removed or covered with vaseline. Two pounds of sulphur is used to $1000 \mathrm{cu}$. ft., more if the building cannot be tightly sealed. The sulphur is placed in some suitable dish with a little wood alcohol poured on it to make it burn more readily. In order to avoid danger of fire, the dish of sulphur should be placed on bricks or in a tub of shallow water before igniting. After two hours the place may be opened and ventilated.

Other Fumigants. - Another effective insecticide is the vapor of carbon bisulphide, a poisonous gas which is not nearly so virulent as hydrocyanic acid gas. As its vapor is heavy it settles rapidly. Its effect on many insects is less certain than in the case of the hydrocyanic acid gas and it has the additional disadvantage of being both inflammable and explosive. Recently cresyl or creolin, a very volatile substance, has come into favor as a fumigating medium, especially for destroying mosquitoes. It is not injurious to higher animals in the strength used (125 ce. to 1000 cubic feet), does not injure household goods and is destructive to all exposed insects. It is volatilized by means of an alcohol lamp. Cresyl does not, however, have the penetrating power of hydrocyanic acid gas or sulphur, and is therefore of less value for such secretive insects as bedbugs, though highly valuable for exposed insects, such as mosquitoes, since they may be destroyed without having the rooms vacated. Formaldehyde, though a valuable disinfectant, i.e., active in the destruction of microörganisms, is not an effective insecticide. 


\section{CHAPTER XXIII}

\section{LICE}

Although the disrepute of human lice has grown with civilization and with the knowledge that lousiness and cleanliness are incompatible, lice are even yet among the most important of external human parasites. In former times the louse apparently was not an object of disgust and loathing even among the better class of people. In Herrick's entertaining book, "Household Insects," the following quotation from Hooke, an English zoölogist of the 17th century, is given concerning the head louse. "This is a creature so officious that 'twill be known to everyone at one time or another, so busie, so impudent, that it will be intruding itself into everyone's company, and so proud and aspiring withall that it fears not to trample on the best, and affects nothing so much as a crown; feeds and lives very high, and that makes it so saucy as to pull anyone by the ears that comes its way, and will never be quiet till it has drawn blood."

Unfortunately, even at the present time, and in the face of present knowledge concerning the rôle of lice in the spread of disease, there are many individuals, many communities and even some races which make no effort to exterminate them. Still more unfortunate is it that there are many people who of necessity must associate with these unwelcome companions. In logging camps, jails, ships, railroad camps, etc., where close association with people who are dirty by nature is unavoidable, lice very often become prevalent. Most of all, however, are lice associated with war. The deadly typhus fever, which has ravaged the armies of almost every war in the history of the world, as far as is known, apparently is spread exclusively by lice. These parasites are the guerillas of war; they bring suffering and death not only to armies but also to the innocent non-combatant population of the war-stricken countries through which the armies have passed. This phase of the subject will be discussed in more detail under the section on "Lice and Disease." 


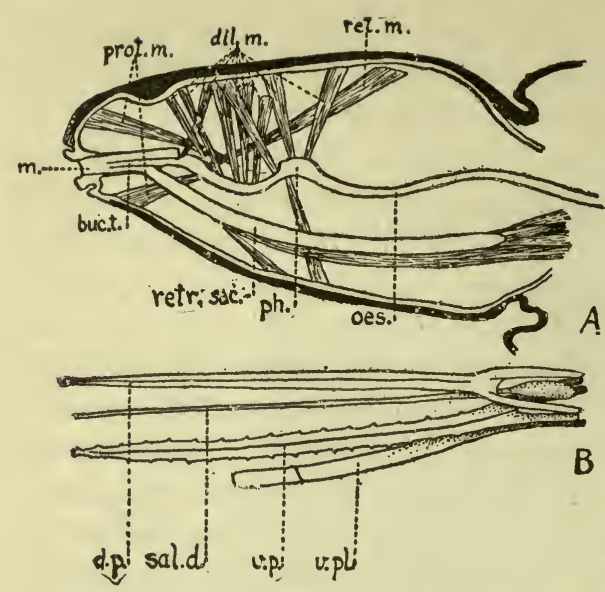

Frg. 171. Mouthparts of a body louse; $A$, longitudinal section through head; $B$, mouthparts from sac under pharynx and œsophagus; buc. t., buccal tube; m., mouth cavity; ph., pharynx; œs., œsophagus; retr. sac., retractile sack for mouthparts; prot. m., protractor muscles of pharynx; ret. m., retractor; dil. m., dilators; d. p., dorsal piercer; sal. d., salivary duct; v. p., ventral piercer; v. pl., ventral plate $=$ labium (?). (Adapted from Harrison.)

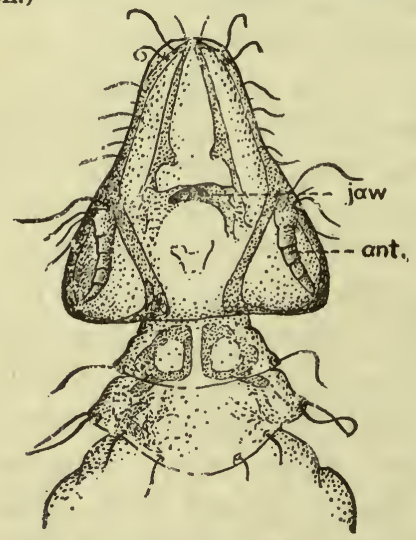

Fig. 172. Head of bird louse (from golden eagle); ant., antenna. Note breadth of head as compared with thorax, a feature which readily distinguishes bird lice from sucking lice. erally believed to be closely related. These two orders of lice are sometimes combined in an order Anoplura. The feet of the true lice are armed each with a very large curved claw, quite gro-

\section{General Structure. -}

Lice are small wingless insects constituting the order Siphunculata. They were formerly classified as a suborder of the Hemiptera or true bugs, but recent studies have shown the erroneousness of this grouping. The mouthparts are adapted for piercing and sucking. The piercing apparatus (Fig. 171B) consists of four needle-like organs, one of which is the delicate salivary duct, which can be withdrawn into a little pouch under the pharynx (Fig. 171 A). This type of mouthparts readily distinguishes the true lice from the bird lice, which constitute the order Mallophaga (Fig. 172). In the latter there are nipper-like mandibles fitted for biting instead of sucking, and these parasites feed only on hair, feathers, etc., and not at all on blood. In other respects the sucking lice and bird lice show a considerable resemblance to each other, and are now gen-
hese two orders of lice are 
tesque in appearance in some species, which closes back like a finger against a thumblike projection of the next segment of the leg (Fig. 173). There are not even rudiments of wings.

The body of a louse is clearly divided into head, thorax and abdomen (Fig. 174). The thorax is always broader than the head, a characteristic which distinguishes at a glance a true louse from the broad-headed bird louse (Fig. 172). The abdomen is divided into segments, normally eight of them in the human species; the terminal one is indented in the female, but is rounded in the male with the large spikelike copulatory organ often projecting at its tip (Fig. 174, p. 390). The digestive tract, as in

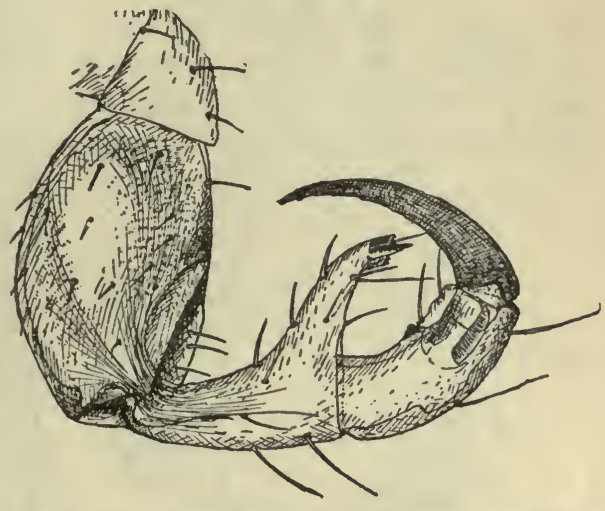

Fig. 173. Front leg of body louse, Pediculus humanus. Note huge claw and thumb-like opposing process of next segment. $\times 100$.

most other blood-sucking insects, is furnished with capacious pouches branching from the stomach, which serve as food reservoirs. The tracheal system is well developed and opens by prominent spiracles on the sides of the abdomen.

Most species of lice are quite closely limited to a single host, and sometimes even genera are thus limited. Kellogg has suggested that the evolutionary affinities of different birds and mammals may be demonstrated by the kinds of lice which infest them. The lice infesting man have generally been regarded as belonging to three species, each selecting a different portion of the body as a habitat; these are the head louse, Pediculus humanus (capitis), the body louse, $P$. corporis (vestimenti), and the crab louse, Phthirius pubis. The head louse and body louse are now thought to be mere races or varieties of a single species, $P$. humanus. Both Pediculus and Phthirius are probably normally confined to human beings.

Body Louse. The body louse (Fig. 174) is by far the most common, as it is the most important, louse infesting man. It closely resembles the head louse, but is often larger, more robust and less active. Fertile offspring result from hybridization 
of these two species. The females, which are somewhat larger than the males, reach a length of about one-eighth of an inch. Due to their dirty white or grayish color these lice are familiarly known as "gray-backs." This species is known to be instru-

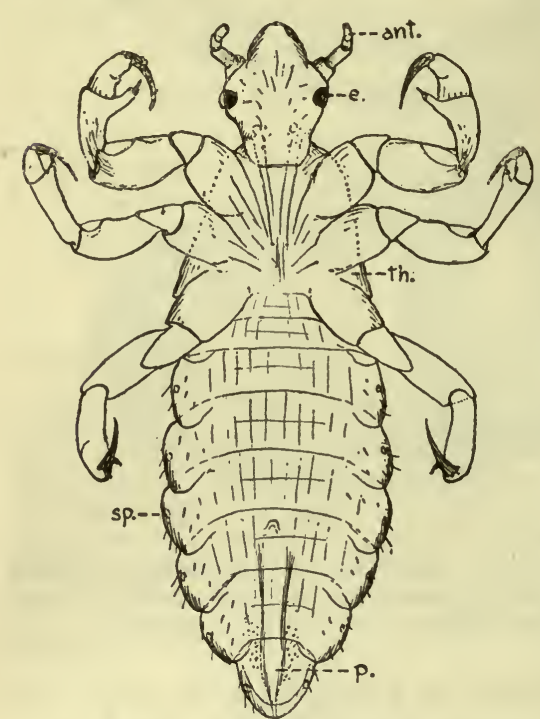

Fig. 174. Body louse, Pediculus humanus, male; ant., antenna; e., eye; p., penis; sp. spiracles; th., thorax. $\times 25$. mental in transmitting both typhus fever and European relapsing fever.

As the name "body louse" implies, this species inhabits the trunk rather than the head. The German name "Kleiderlaus", meaning "clothes louse", is still better, since this louse has so far adapted itself to its host as to have broken away from the custom, prevalent among all other species of lice, of living in the hair of the body, and to have established the habit of living on the clothing. Just when, in the process of our evolution from a hairy ancestor, this louse shifted its position from the waning hair to the more and more habitually worn clothes would be interesting to know. Not unlikely both this louse and the closely allied head louse have evolved from a species which once roamed the hairy bodies of our forefathers, each species coping with the unfavorable circumstance of the developing hairlessness of its host in a different way, the more conservative head louse withdrawing to the fine hair of the head, the body louse adapting itself to living on the clothing.

A person infested with thousands of body lice may remove his clothing and find not a single specimen on his body. An examination of the underwear will reveal them adhering by their long claws to the surfaces which were next to the body. Here they live and lay their eggs, leaving the clothing only long enough to suck a meal of blood, even then usually adhering to the clothes by their hind legs. 
Habits and Life History. - Although there has been very close association between lice and human beings probably since man's first appearance in the world, little definite knowledge concerning the life history of any of the three species was obtained until recently. The importance of lice in the great antiGerman war has stimulated much research on them.

One of the first experiments with the breeding of body lice was made by the great zoölogical pioneer, Leeuenhoek, in the 17th century. He placed two female lice in his stocking and tied them in; after six days he opened the brood chamber and found a cluster of 50 eggs beside one of the lice and another cluster of 40 eggs, probably laid by the other insect which had escaped. He found 50 more eggs in the remaining louse. He left the eggs in his stocking ten days more, when he discovered 25 young lice, whereupon he abandoned his experiment in disgust.

The eggs of lice, commonly called "nits," are oval, whitish objects fitted with a little lid at the larger end, through which

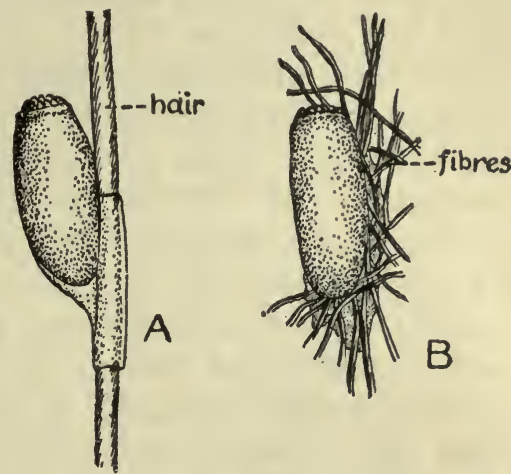

FIG. 175. $A$, egg of head louse, Pediculus capitis; $B$, egg of body louse, $P$. humanus. $\times 25$. (After Cholodkowsky.)

the hatching takes place. The eggs of the body louse are about one $\mathrm{mm}$. ( $\frac{1}{25}$ of an inch) in length. They are glued to the fibers of clothing (Fig. 175B) especially along seams or creases, although in all other lice the eggs are glued to hair. Under experimental conditions the body louse will sometimes lay eggs on hairs, but it nearly always selects the crossing point of two hairs and shows less skill in attaching the eggs. The body louse shows a marked " homing " instinct in laying her eggs and shows a strong desire to lay eggs where others have been laid, until clusters of from 50 to 75 or more have been formed.

According to recent experiments by Sikora in Germany and Bacot in England, the number of eggs laid by the single female body louse may frequently reach 200 or more. Bacot obtained 295 eggs from a single specimen in one case. During the first three or four days only two to four eggs are laid daily, the num- 
ber gradually rising, until after a week or so of egg-laying seven to ten or more eggs may be laid each day. A day or two before the end of egg-laying and the death of the louse the daily number drops again. Eggs are laid whether copulation has occurred or not, but in no case have unfertilized eggs been observed to develop. One copulation is not sufficient to fertilize all the eggs, but fertile eggs may be laid for at least 20 days after a single copulation. According to Sikora, copulation normally takes place at intervals of from one to three days. Egg-laying ceases at temperatures below $77^{\circ} \mathrm{F}$. and a daily exposure to a temperature of $60^{\circ} \mathrm{F}$. for only two or three hours causes a marked falling off in egg production.

According to Sikora the eggs hatch in about six days at the optimum temperature of $95^{\circ} \mathrm{F}$. At a temperature of $77^{\circ} \mathrm{F}$. the incubation period is prolonged to 16 days, whereas at $68^{\circ}$, lowered from $42^{\circ}$ to $60^{\circ} \mathrm{F}$. during the latter part of the night, or at a constant temperature of $60^{\circ} \mathrm{F}$., no hatching at all takes place. At temperatures above $95^{\circ}$, also, the eggs suffer a high mortality probably due to the difficulty in obtaining proper conditions of humidity rather than to the direct effect of the heat itself. Either excessive humidity or complete drying is fatal to the eggs. It is evident that in winter the laying off of the clothing at night in a cold room or the leaving of mattresses or bed clothes in the daytime is sufficient to prevent the laying of eggs or the hatching of eggs already laid, thus resulting in the extermination of the lice.

The newly hatched lice are almost perfect miniatures of the adults, and are ready to feed almost as soon as they emerge from the egg; in fact, they usually die in less than 24 hours if not allowed to feed, though the adults can survive as much as five days of starving. According to Sikora, the rapidity of the development of lice is dependent on temperature and on amount of food. At a temperature of $95^{\circ} \mathrm{F}$. and with as many daily feeds as would willingly be taken, namely six, the lice pass through their first moult in three days, the second in five or six days, and the third, which brings them to maturity, in eight or nine days. Reduction of daily feeds to two increased the period of development to nine or ten days, whereas reduction of temperature to $75^{\circ} \mathrm{F}$. by day and $95^{\circ} \mathrm{F}$. by night, with two daily feeds, prolonged the development to from 13 to 15 days. 
According to observations by Sikora, copulation may take place within ten hours after the last moult has been passed, and Bacot also observed cases in which copulation took place on the day of reaching maturity. Egg-laying begins in from one to four days after the final moult and continues at the rate described on the preceding page until the death of the insect. The average length of life for the females is about 35 or 40 days, and probably a little less for the males.

According to Bacot, hungry lice do not show a tendency to wander on the skin, but proceed to pierce the skin and suck blood at once. Nor do they shift to make another stab, as fleas frequently do, if the first stab does not immediately furnish blood. They apparently place great reliance on the power of the salivary secretion, which is poured into the wound, to dilate the capillaries by its irritation and thus cause a flow of blood. Sometimes blood is not drawn for several minutes after the puncture is made. Bacot states that lice fill their crops in from two to 15 minutes, but Sikora observed that adult lice, if fed only twice daily, sucked for an hour to an hour and a half, and, if left in contact with the skin for several hours, have a tendency to pump blood intermittently with short pauses, meanwhile voiding excrement containing undigested blood corpuscles. Sikora also observed that hungry lice placed on the well-shaved skin of a puppy made repeated attempts to draw blood without success, and also that dog lice, Hæmatopinus ventricosus, tried in vain to draw blood from the human skin. He concludes therefrom that not only is it necessary for lice to penetrate the skin with their piercing apparatus, but that they must also produce an irritation by means of a salivary secretion, apparently specific in its action for certain kinds of blood, in order to cause blood to flow from the tiny puncture. Apparently the salivary secretion deteriorates in unfed lice, for though starved lice may still be able to drive their piercing apparatus into the skin, it takes them three times as long to draw blood.

A fact of far-reaching significance, if found to be commonly true, has recently been reported by Hall in Texas. This author found that a female body louse taken from a Mexican baby, when placed in a bottle with a head louse taken from the same baby, devoured the head louse. Two head lice were then fed to the body louse daily for three days, and the same louse was 
induced to eat crab lice, small black ants, bedbugs, and raw beef. When body lice were placed in a bottle with head lice, bedbugs, and a piece of beef, they ate first the head lice, then the bedbugs then the beef, and finally became cannibals to the extent of the survival of the fittest! This would readily explain such facts as that body lice (according to Hall) can be found in empty box cars used to transport Mexican troops weeks before, and it would account for louse-borne diseases lying dormant in isolated places. A freight car once infected with typhus would be a source of danger for a longer period than the few days a louse can live without food. However, before insectivorousness can be admitted as a usual habit of lice in the absence of normal food, further investigation is necessary.

Digestion is very rapid. An entire two-hour feed may be digested in from eight to ten hours at $95^{\circ} \mathrm{F}$., but digestion is slower at lower temperatures and the stomach contents remain unchanged for ten hours or more at $45^{\circ} \mathrm{F}$. or below. At temperatures above $95^{\circ} \mathrm{F}$. digestion is even more rapid, but there is a high mortality.

It is evident from Sikora's experiments that $95^{\circ} \mathrm{F}$. is the optimum temperature for the development and reproduction of lice. The absence of lice from hot countries - observable in Mexico, for instance, where they are abundant on the central plateau above 5000 to 6000 feet, but absent from the hot coastal strips - is apparently not due to the high temperature but probably to the disastrous effect of profuse perspiration and consequent excessive humidity between the clothes and skin.

The bites of the body louse produce itching red pimples which become covered by a brownish crust, the results of the action of the toxic salivary juices. Scratching produces characteristic white scars, surrounded by brownish pigment; in fact, large areas of the skin may take on a mottled bronze color. The coloring of the skin is said to be due to the stimulation of pigment formation in the skin by toxins secreted by the louse. Many individuals develop an insensibility to the bites of lice, a fact which probably explains the indifference of some communities to them as, for instance, the people of Russian Poland.

Head Louse. The head louse, $P$. humanus capitis, is very closely related to the body louse, and is, in fact, thought by some workers to be a mere variety of the latter. Aside from its different 
habits, however, it differs very slightly from its relative. It is commonly smaller in size, and is more distinctly festooned along the sides, due to constrictions at the joints between the segments. Nuttall, however, has shown that all of the supposed morphological and biological differences between these two kinds of lice are inconstant, and they should, therefore, be considered as races of a single species. The head louse assumes all the characteristics of the body louse when reared under conditions suitable for the latter.

The head louse although usually preferring the fine hair of the head as a habitat occasionally wanders to other parts of the body as well. It is found in every part of the world. Different varieties are said to occur on the different human races and to vary in color with the color of the skin on which they live. The lice which live on the white race are pale gray with a dark line along each side of the abdomen, those on negroes are blackish or brown, on Hindoos smoky brown, on Japanese and Chinese yellowish, and on American Indians dark reddish brown. What a wonderful case of protective coloration, except that, as in so many other cases of so-called protective coloration, there is no practical protection. A negro is as likely to scratch out a black louse as a white one!

As in the case of the body louse reproduction is very rapid but the egg production is lower, due to the smaller capacity of the body, even taking into consideration the slightly smaller size of the eggs. The course of development is practically the same in both species. The average number of eggs, according to Bacot's observations, is from 80 to 100 . Only one mature egg can be developed in the louse's body at a time, but the succession of them is so rapid that eight or ten may be laid in a day. Each egg or "nit" is glued by the lower end to a hair (Fig. 175A), the favorite " nests" being the vicinity of the ears. The young lice hatch in ten or 12 days and reach maturity in two or three weeks, and are then ready to reproduce again. At this rate of reproduction, allowing only a 50 per cent hatch, a single pair of lice theoretically could produce over three-quarters of a million offspring in the fourth generation, and in the course of less than three months!

Although the bites of this species are not quite so irritating as are those of the body louse, yet the frequent piercing of the skin for a gory meal results in much scratching. Often the bites swell into pimples which may bleed when scratched or which 
form a little pus, sufficient in very negligent individuals to make the hair mat together. According to Stiles, if this is allowed to run on, a regular carapace may form, called trichoma, in which fungous growths may develop, and under which the lice abound, and the head may exude a fotid odor.

Crab Louse. - The crab louse, Phthirius pubis (Fig. 176), is quite distinct from the other two species of human lice. It has a very broad short body with long, clawed legs, presenting the general appearance of a tiny crab, from which it derives its name. The first pair of legs are smaller than the others and do not

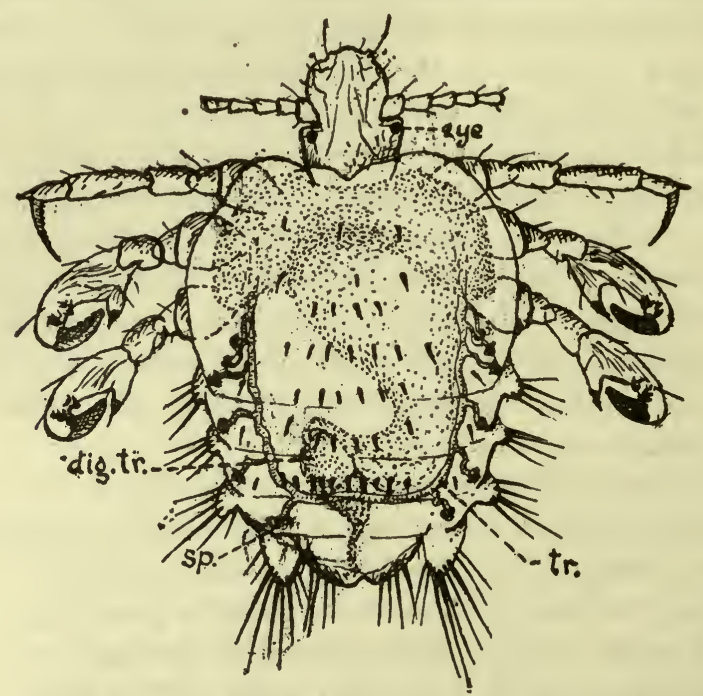

Fig. 176. Crab louse, Phthirius pubis, $\& . \quad \times 35$.

possess a "thumb" in apposition to the curved claw. The abdomen is composed of six segments, and is markedly festooned along the sides. This louse is grayish white in color, with dark shoulder patches and slightly reddish legs. The females are about $\frac{1}{16}$ of an inch in length, the males somewhat smaller. The favorite haunts are the pubic regions and other parts of the body where coarse hair grows, as in the armpits and in the beard and eyebrows. Unlike the other human lice this species is almost exclusively confined to the Caucasian race.

The females produce from ten to 15 eggs and glue them, one at a time, to the coarse hairs among which they live. A number 
of eggs may be glued to a single hair, and often at some distance from the skin. The eggs hatch in six or seven days, and the young become sexually mature in about 15 days. This species, even under favorable conditions, will live apart from its host only ten or 12 hours. The eggs are said not to develop except at temperatures between $68^{\circ} \mathrm{F}$. and $86^{\circ} \mathrm{F}$., which are approximately the temperatures to which eggs attached to hairs beneath the clothing would be exposed.

\section{Lice and Disease}

The rôle of lice in the spread of disease has long been suspected in an indefinite and uncertain way. Only recently, and at the cost of the lives of several great investigators, has the whole portentous truth regarding the transmission by them of typhus and relapsing fever (North African and European types) been brought to light. Foremost among the investigators of louse-borne diseases stands the name of Nicolle and his associates, who in 1909 proved that typhus fever could be transmitted by the body louse, and in 1913 that the Algerian type of relapsing fever could be transmitted likewise. Two American investigators, Ricketts and Wilder, working independently of the French workers, proved in 1910 that the body louse was instrumental in transmitting typhus (tarbardillo) in Mexico, and in 1912 Anderson and Goldberger showed that the head louse could also transmit it. Opinions differ as to whether the infection can be transmitted through the eggs to the lice of the succeeding generation. In 1918 an American commission under Dr. R. P. Strong, and a British committee under Sir David Bruce and Major Byam, working under field conditions on the war front in France, demonstrated that trench fever is a louse borne disease.

There is every reason to belive that typhus fever is normally transmitted exclusively by lice. Wherever the hording together of promiscuous crowds of people becomes necessary and when scrupulous cleanliness, either of necessity or of choice, is not enforced, there the lice will thrive and sooner or later the dread disease will break out. The organism which is believed to be the cause of typhus fever, Rickettsia prowazeki, is discussed on p. 186. Nicolle and his fellow workers have shown that lice which are fed on infected patients do not become infective until the eighth and usually the ninth or tenth day afterward. The same 
results were obtained both in experiments with crushed lice and with the excrement of the lice.

Epidemics of typhus usually occur in winter and in cold countries, due to the huddling together of people in warm, poorly ventilated houses where lice thrive, and where the unhygienic conditions lower the vitality of the people. Typhus has followed in the wake of nearly every army which has ever been assembled. During the present great European war typhus has been largely absent from the armies and population of Britain, France and Germany, due solely to the intensive anti-louse measures which have been enforced by these countries. The less scientific and less cleanly nations have suffered enormous losses. An epidemic began in Serbia in January, 1915, among some Austrian prisoners who were allowed to disperse all over the country. The disease spread with them, and for a time raged almost at will in that war-stricken country. The majority of the small number of Serbian doctors were affected, no sanitary measures for the suppression of lice were understood or enforced, and no adequate accommodations for the sick could be provided. The epidemic continued to rise, and reached its height in April, when there were estimated to be 9000 deaths per day. It was largely through the heroic efforts of the American Red Cross expedition that the epidemic was finally checked, after having destroyed over 150,000 people. In December, 1916, another epidemic was reported to be raging in Syria with over 1000 deaths per day. Milder epidemics have occurred in Austria, Bulgaria and Russia, all countries where science and cleanliness have not been worshipped as they have in the greater nations of Europe. Mexico has suffered also; in December, 1915, 11,000 cases of typhus were reported in Mexico City and its environs.

The rôle of lice in the transmission of the European and North African form of relapsing fever has long been suspected but was not proved until 1913, when Nicolle and his fellow-workers scientifically demonstrated it in Tunis and Algeria. Noting that the louse was the only constant factor affecting the occurrence of the disease, these French workers undertook extensive experiments which resulted in proving that the body louse, and probably also the head louse, serves as a medium for the development of the spirochætes of relapsing fever, and that these insects transmit the disease not by biting but by inoculation of the 
wounds which they make with the infected contents of their bodies when crushed.

Nicolle and his associates also showed that sometimes, at least, the spirochætes, probably in the granule stage, are hereditarily transmitted through the eggs to the young of the next generation, as is the case with the African relapsing fever parasites in the tick. Experiments on the transmission of the relapsing fever of Algeria with other parasites such as bedbugs, fleas, biting flies and ticks were negative. Some observers, however, believe that in Europe other insects also, notably bedbugs, may be instrumental in transmitting relapsing fever. The evidence furnished by the epidemiology of the disease is, however, very strongly in favor of lice as the normal transmitters. The Indian form of relapsing fever is also probably transmitted by lice. Further details of the development of the spirochrtes in the lice are given in Chapter IV, p. 44.

Being transmitted by lice, relapsing fever shows the same peculiarities of occurrence as does typhus; epidemics always rage fiercest in winter, and usually break out during war times. Serbia, which was so stricken by typhus, was held in the grip of an epidemic of relapsing fever earlier in the war.

The demonstration of the relation of lice to the transmission of trench fever in 1918 by American and British Commissions working under war conditions was a very brilliant piece of medical research carried out successfully in spite of adverse conditions. For the two years preceeding the beginning of the investigation concerning the transmission of the disease, trench fever is said to have occasioned more sickness among the troops in France than any other infectious disease. The work of the Commissions showed that trench fever is naturally transmitted by the body louse, and that this is the important and common means of transmission. The louse may transmit the disease by its bite alone, or by infection of abraded sink by the excrement. A discussion of Rickettsia quintana, the probable cause of trench fever, and its occurrence in infected lice will be found on p. 187 .

Lice may also serve as mechanical transmitters of still other diseases. The bacilli of bubonic plague have been found alive in both body lice and head lice taken from victims of the disease, and both this species and the body louse have been experimentally proved to be able to transmit plague from rodent to rodent in Java. De Raadt in Java infected rodents with plague by in- 
jecting them with ground bodies of head lice taken from plague patients. The practice among some natives of killing lice by mashing them against the head of the host, accompanied by the frequent scratching due to irritation from bites, may well be a frequent cause of plague infection if there has been any opportunity for the lice to migrate from an infected to a healthy person.

There is no reason why syphilis could not be transmitted in a similar manner, especially during the second stage of the disease, when the spirochætes are present throughout the blood. The readiness with which spirochætes of other kinds will live in insect or tick bodies makes it reasonable to believe that the spirochætes of syphilis might live in the bodies of human lice, at least long enough to be conveyed from person to person.

Prevention and Remedies. - The prevention of lousiness consists primarily in personal cleanliness. However, no amount of personal hygiene and cleanliness will prevent temporary lousiness if there is association with unclean and careless companions. Lousiness and human wretchedness and degradation have always been companions, but this does not imply that lice have any inherent abhorrence of a clean body if they can get access to it. From the nature of their habitats the common modes of infection of the three different species of human lice vary somewhat. Any of them will spread by contact or close association, but each has its own special means of finding new hosts. The head louse depends largely for distribution on a promiscuous use of combs and brushes or borrowed hats and caps, and on the free-for-all trying on of head gear in haberdasheries and millinery shops. The body louse is dispersed by clothing and bed linen and finds fresh hunting grounds by night migrations from one pile of clothes to another. The crab louse frequently utilizes public toilets for dissemination. Where men are crowded together in prisons or war camps lousiness is almost sure to develop unless particularly guarded against, since some uncleanly persons are nearly always in the aggregation, and conditions are such that the infestation is given every opportunity to spread. There are, however, many ways in which lice may be dispersed among clean people in ordinary life. Stiles reports a case where a large number of girls in a fashionable boarding house in eastern United States developed lousiness shortly after traveling from Chicago to New York in a Pullman sleeper. 
In Washington and other cities where negresses do much of the laundering the family wash is a common source of infestation. Closely packed street cars, school cloak rooms, unclean rooming houses - all these and many other means may serve to start a new colony of lice.

Perfect cleanliness will usually result in their quick elimination. A shampoo with warm water and soap, frequent baths, clean underclothes, pressed suits, and other items of personal care are inimical to the welfare of the unwelcome visitors. Certain remedies are, however, useful in the quick destruction of these pests. Head lice can best be destroyed by a thorough washing of the head with a two per cent carbolic acid solution or a kerosene emulsion (equal parts kerosene and olive oil). When one of these remedies has been thoroughly rubbed into the hair the head should be covered with a cloth. After several hours the ointment is washed off in warm water and soap and the dead lice removed with a fine-tooth comb. In long hair this treatment is applied by having the patient lie down with the hair hanging over the edge of a bed into a pan of the carbolic solution or kerosene emulsion, the hair being sluiced backward and forward for ten minutes until thoroughly saturated. The treatment may have to be repeated after about ten days to destroy lice which have hatched in the meantime, but usually the eggs are destroyed as well as the adult lice. Crab lice can be destroyed best by the use of mercurial ointment applied to the infected parts, accompanied by washing with soft soap and warm water. A close clipping of the hair in the infested regions is the safest and quickest method of getting rid of the nits.

Eradication of body lice is in some respects simpler than that of other lice, since it is the clothes instead of the body which are to be treated. Much work has been done since the outbreak of the war in Europe on testing the effect of various chemicals and methods of treatment on lice. This problem is recognized as one of the most important minor considerations in war.

The methods usually employed for getting rid of body lice are to sterilize the clothes, either by steam, by fumes of carbon bisulphide or sulphur dioxide (if no wool is present), by dry heat of $160^{\circ} \mathrm{F}$., or by treatment with volatile odorous substances, such as kerosene, naphthaline, ether, anise oil, oil of turpentine, oil of eucalyptus or anisol (methylphenylether). The last is a new 
remedy reported by Fränkel, and is said to stun lice in four minutes and to kill them in ten minutes. Soaking for one hour in a $1 \frac{1}{4}$ per cent cresol solution is said to destroy all lice on clothing. The eggs are not so easily destroyed as are the adults, but they succumb to heating, to exposure to carbon bisulphide (100 grams per cubic meter), or to immersion in any of the oils mentioned above. Ammonia gas destroys the eggs on clothing in a closed receptacle. On the Mexican border of the United States a mixture of vinegar and kerosene is used for dipping louse-infested clothing. The French soldiers are said to have kept largely free of lice by the simple expedient of having a hot iron run along the seams of the underwear when laundered, to kill nits.

Preventive measures against lice, simple as they are under ordinary conditions, often constitute a very difficult problem, especially in army camps. Common methods employed are the treatment of the clothes with odorous or poisonous substances, the use of underclothes with smooth inner surface, such as silk or oil cloth, to which lice cannot attach their eggs, or the dusting of naphthaline powder into the shoes, stockings and underwear. A substance which has been found most efficient by the British, and has been used extensively on the western front in France is the now famous NCI, a powder consisting of 96 per cent commercial naphthaline with two per cent creosote added to increase the toxicity and to give lasting qualities and two per cent iodoform to increase the adhesiveness of the powder when dusted on the inside of the clothing. The shepherd people of the Carpathians are said to protect themselves against lice by saturating their underclothes in melted butter which prevents the lice from fastening their eggs to the fibers of the clothes, and probably the fatty acids of rancid butter are also directly deleterious to the pests.

When louse prevention is undertaken on a large scale, as it has been as never before in the present war, enormous difficulties are encountered, largely due to the fact that the soldiers, especially of the less enlightened nations, do not coöperate with the officials. Germany, menaced by louse-borne diseases more, perhaps, than any others of the principal warring nations, due to the constant contact of her troops with the less efficiently caredfor troops of Russia and of the Baltic nations, has largely solved the problem by the erection of "disinfection stations." In 
October, 1915, there were eight of these on the Polish front, and more were being built. Through these stations men are passed as clothes might be passed through a laundry. Entering at the "unclean side," dirty, lousy and unhygienic, they emerge from the "clean side" fresh, clean and free from vermin. Each institution consists of eight separate buildings, grouped around a central power house in which 200 tons of coal are burned daily to supply steam for disinfection, light, power, etc. Laundries, kitchens and administrative quarters are also provided. Each of the eight buildings consists of a clean and an unclean part, with a chief surgeon in charge, and each has a capacity of 500 men every eight hours, a total of 12,000 per 24 hours for the entire institution. At the entrance on the unclean side each man receives a net for whatever apparel he may have, such as boots, helmets, etc., which must be sterilized by dry heat, and a smaller net to receive his valuables, such as notebooks, tobacco, etc. A check number is hung about his neck, and a similar number placed on his belongings. $\mathrm{He}$ is now given a pair of slippers, and enters a large waiting room where he disrobes, placing his clothes in another net which has been given him, to be sterilized by steam. If in need of it he is given a hair-cut and is then subjected to a fifteen minute shower bath with soap, after which he is presented with a towel, clean slippers and clean underwear. He is then allowed to pass to the clean side of the building where he is given his own disinfected clothing, given a meal and conducted to disinfected railroad coaches. The greatest disadvantage is the non-coöperation of Russian prisoners, who by all sorts of subterfuges try to avoid being "laundered." However, Germany has, by this method, practically converted her whole eastern front into a huge filter to guard against lice and lice-borne diseases. Without such radical measures Germany could never have kept herself as free as she has from the diseases of war. 


\section{CHAPTER XXIV}

\section{FLEAS}

David Harum says, "A reasonable amount of fleas is good for a dog. They keep him from broodin' on bein' a dog." A goodly supply of fleas might likewise keep man from brooding over anything deeper than the presence of these fleas, but in many cases this in itself is a rather serious thing to brood over. Not only are fleas very annoying pests and a common cause of insomnia, but they may also serve as the disseminators of a number of serious human diseases, among which the terrible bubonic plague stands foremost.

General Structure. - Fleas are insects which are more or less distantly related to the Diptera or two-winged flies, but which have become so specialized by their particular mode of life as external parasites as to necessitate their segregation into a distinct order of their own, the Siphonaptera. Their bodies are ordinarily much compressed to facilitate gliding between the hairs or feathers of their hosts. The head is broadly joined to the thorax, which is relatively small. The abdomen is large and much compressed from side to side; it consists of ten segments, the first seven of which are simple rings, each protected by two horny plates, a dorsal "tergum" and a ventral "sternum" (Fig. 177). The last three segments are modified differently in the male and female in connection with the sexual organs. In both sexes the "tergum" of the ninth segment has a pitted area covered with little bristles which is called the pygidium, and is probably sensory in function. All parts of the body are furnished with backward-projecting bristles and spines which aid the flea in forcing his way between dense hairs and in preventing him from slipping backward. The efficiency of these spines is apparent when one attempts to hold a flea between his fingers. Many fleas have specially developed, thick, heavy spines arranged in rows suggestive of the teeth of combs and therefore known as ctenidia or " combs" (Fig. 179). Such a comb may be present 
either along the ventral margin of the head or along the hind edge of the pronotum (the dorsal plate covering the first segment of the thorax) or in both places. The presence or absence of these combs and the number of teeth in them is of considerable use in identification of species.

The legs of fleas are very long and powerful, and at first glance seem to possess one more segment than do the legs of other in-

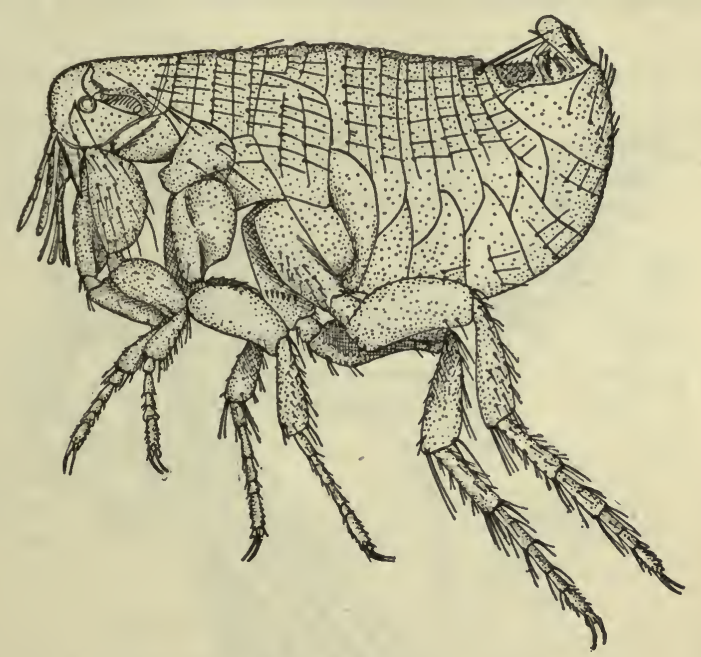

FIg. 177. The Indian rat flea, Xenopsylla cheopis, male. $\times 50$. (After Jordan and Rothschild.)

sects. They really consist of the usual number of segments, however, but are peculiar in the enormous development of the first segments of the legs (coxæ), which in most insects are quite insignificant (Fig. 179). The shape of the sternal plate to which the coxæ are attached is suggestive of still another segment. The great development of the coxæ as well as of the other segments of the leg gives unusual springiness and consequently enormous jumping power. The human flea, Pulex irritans, has been observed by Mitzmain to jump 13 inches horizontally and seven and three-fourths inches vertically. An equivalent jump for a man of average height would be over 450 feet horizontally and over 275 feet vertically! The jumping power must overcome to some extent the disadvantage of winglessness and render migration from host to host comparatively easy. All the legs 
are furnished with rows of stout spines and are armed at the tip with a pair of large stout claws.

Eyes are present in some species of fleas but not in others. The antennæ are short and club-shaped, and when not in use are folded back into special grooves for them on the sides of the head (Fig. 178, ant. gr.). The mouthparts (Fig. 178) are fitted for piercing and sucking. In the normal resting position they appear to consist of a long jointed proboscis, blunt at the tip, with

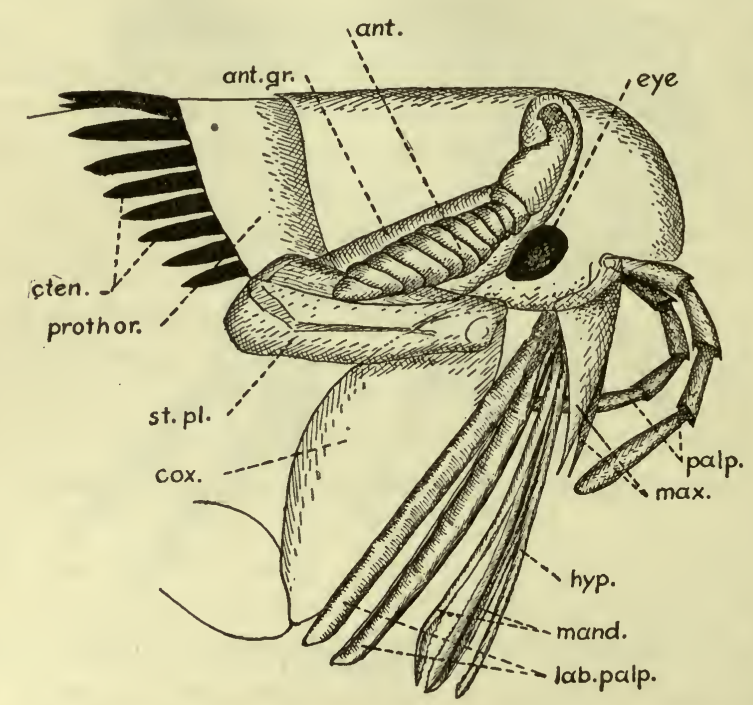

FIG. 178. Head and mouthparts of a flea (squirrel flea, Ceratophyllus fasciatus); ant., antenna; ant. gr., antennal groove; cox., coxa of 1st leg; cten., ctenidium; hyp., hypopharynx; lab. palp., labial palpi, which together form a tube for protecting the lancets; mand., mandibles; max., maxilla; palp., maxillary palpi; prothor., prothorax; st. pl., sternal plate of skeleton with which leg is articulated.

a pair of stout triangular flaps at either side at the base. The ,triangular parts are the maxillæ and each is provided with a stout four-segmented palpus, which might easily be mistaken for an antenna. The proboscis really consists of a pair of segmented gouge-shaped structures, the labial palpi, which fit together to form a more or less perfect tube, in which lie three piercing organs. The latter consist of a pair of thin bladelike mandibles serrated on each edge, curved at the tip, and provided with a longitudinal groove, and a single bristle-like organ, the epipharynx. In piercing the skin of the host the epipharynx first bores 
a tiny puncture, and then the serrated mandibles enlarge the hole by an up and down sawing motion. As these organs are sunk into the flesh of the host the labial palpi bend back like a bow under the flea's head. The two grooved mandibles, placed in apposition, form a tube for the outflow of saliva, while the epipharynx, which is also grooved, forms a tube with the mandibles for the inflow of blood. The digestive tract is provided with a pharynx which acts like a suction pump, and a very large and distensible stomach.

Classification. - Several hundred species of fleas have already been described and it is probable that many more species will be found. Although some authors split the fleas into a considerable number of families, it is more usual to recognize only two

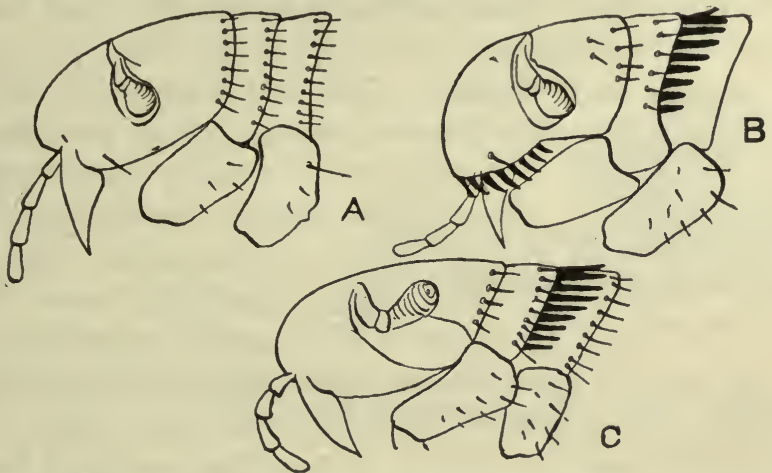

Fig. 179. Heads of common fleas, showing distribution of ctenidia or "combs"; $A$, human flea, Pulex irritans, without combs; B, dog flea, Ctenocephalus canis, with combs on both head and pronotum; C, rat flea, Ceratophyllus fasciatus, with only pronotal combs.

well-defined families or groups - the Pulicidæ and the Sarcopsyllidæ. The former family includes all the "ordinary" fleas, whereas the Sarcopsyllidæ is a very specialized group of fleas with a much shortened thorax, which appears as if mashed between the head and abdomen, with slender anterior and middle legs, and with feeble labial palpi of only three segments. Whereas all of the Pulicidæ lay their eggs singly, or in small groups, and development of the embryos occurs after the eggs are deposited, in some of the Sarcopsyllidæ the eggs, during their early development, are retained in the abdomen of the female, which swells up to such a size that the head and thorax appear as a small appendage at one end of it. 
The exact identification of fleas, especially if the host is unknown, is difficult, being based largely on such minute characteristics as relative lengths of different segments of the legs, number and distribution of spines, etc. Most species of fleas, however, are quite closely confined to their respective hosts, only a few species being able to thrive on a number of different hosts. Some of the commoner species of the fleas which are of most importance to man can be fairly closely identified, if the host and geographic locality is known, by the presence or absence of the "combs". on the head and thorax. The common human flea, Pulex irritans (Fig. 179A), and the Indian rat flea, Xenopsylla cheopis (Fig. 177), have no combs, the common rat and squirrel fleas of temperate climates (Figs. 179C and 178) have only the thoracic comb, while the cat and dog fleas (Fig. 179B) have both facial and thoracic combs.

Life History and Habits. - The life history of all fleas is quite similar. Like the Diptera, or flies, they pass through a

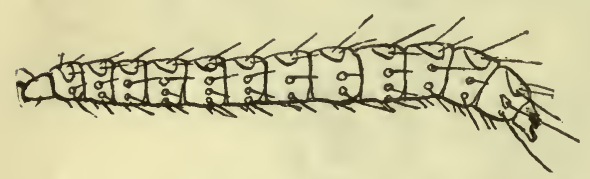

FIG. 180. Larva of Indian rat flea, Xenopsylla cheopis. $\times 12$. (After Bacot and Ridewood.) complete metamorphosis, i.e., undergo a complete reorganization from larval to adult form during a resting pupal stage. The eggs are oval, whitish in color and relatively large, often one-third the length of the parent flea, and are laid singly, except in the chiggers, being dropped at random in the fur of the host or in the lairs or habitations of the hosts. The human flea, for instance, lays its eggs in the dust and débris in cracks in floors, under carpets, etc., whereas the fleas of most mammals lay their eggs loosely in the fur of the host, whence they drop off when the animal shakes himself or prepares to sleep. The time required for the eggs to reach the hatching stage varies with the species and with climatic conditions from two or three days to over two weeks.

The larvæ (Fig. 180) are tiny cylindrical maggot-like creatures with neither legs nor eyes. They have small brown heads and whitish bodies composed of 13 segments, which are provided with rather sparse bristly hairs to aid in crawling. The last segment is terminated by a pair of tiny hooks.

The larvæ squirm about actively in the dirt or débris of the 
lairs or rubbish piles in which they hatched, avoiding light and feeding upon what bits of organic matter they can find, such as mouse pills, crumbs, hairs, epidermal scales from their hosts and the excrement of adult fleas. Some species, if not all, devour their shed skins after moulting. According to Bacot and Ridewood, who have recently made observations on the larvæ of a number of species of fleas, the larvæ become very excited and impatient when disturbed. They sometimes lie quiet, coiled like a watch spring, for repose or concealment, but when about to moult they stretch out at full length. They crawl by alternately expanding and contracting the body like an earthworm, first securing a hold with the hooks at the posterior tip of the body, then with the head which is bent under to hook over some irregularity on the surface. The duration of the larval stage varies with the temperature and humidity and to some extent also with the species. Under favorable conditions, i.e., at relatively low temperatures and high humidity and with plenty of food, the larvæ of some species pass through their two moults and enter the pupal stage in a week, whereas under unfavorable conditions the duration of the larval existence may be drawn out to over three months.

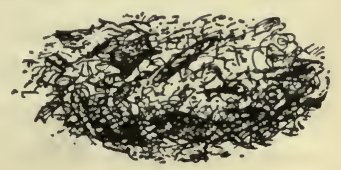

Fig. 181. Cocoon of human flea, Pulex irritans. $\times 12$.

When ready to undergo their transformation into adults, the larvæ spin little silken cocoons which are somewhat viscid, so that particles of dust and lint readily adhere to them and give them a dirty, dingy appearance (Fig. 181). According to Lyon the adult insects may emerge from the cocoons of the cat flea, Ctenocephalus felis, in from two to 14 days, but in most species at least a week is required for the transformation to take place, and this time may be greatly increased by unfavorable climatic conditions. Strickland, in his work on the rat flea of England, Ceratophyllus fasciatus, found that the average pupal existence was 17 days and was extended to four months or more by low temperatures, the fully formed adult insect remaining dormant within the cocoon until exposed to a temperature of about $70^{\circ} \mathrm{F}$. There is much probability that the winter in temperate climates and the hot dry season in tropical climates is tided over by fleas in the cocoon, the emergence of the adults coinciding with the advent of moderately high temperatures and humidity. 
The adult fleas, according to Strickland's work on the rat flea, do not become sexually mature for some days after they escape from the cocoon, and copulation does not occur until this time, nor, in the case of the rat flea, until after a feed of rat's blood, the latter apparently acting as a stimulus to reproduction. Soon after copulation the eggs begin to be laid.

In the dog flea, Ctenocephalus canis, the entire cycle from egg to adult is said to be passed through in a minimum of two weeks, in the human flea, Pulex irritans, in 19 days (in southern Europe) and in the rat fleas in about three weeks. Ordinarily, however, the life cycles occupy a considerably longer time, the average being from one to three months.

The length of life of adult fleas depends largely on food supply, temperature and humidity. Unfed fleas, unless allowed to bury themselves in rubbish, usually die in less than a month, though when buried in débris they may be kept alive many months. Well fed rat fleas kept at low temperatures (about $60^{\circ} \mathrm{F}$.) and high humidity may live for nearly a year and a half, according to Strickland's experiments. The optimum climatic conditions and normal length of life probably vary a great deal with different species.

Unlike most blood-sucking insects, fleas usually feed at frequent intervals, usually at least once a day, and sometimes much oftener than this. The frequent biting is due to the fact that fleas are very easily disturbed while feeding and seldom complete a meal at one bite. Moreover, the capacity of the stomach is not so great as in many other blood-sucking insects. The human flea and some others are mainly nocturnal, visiting their hosts chiefly at night, whereas others, such as the cat and dog fleas, remain in the fur of the host nearly all the time. Some species show a decided preference for certain parts of the body of their host.

Fleas and Disease. - Like most other blood-sucking parasites, fleas are intimately connected with the spread of disease. The most serious charge against them in this connection is the dissemination of bubonic plague, which as a human scourge ranks with such diseases as smallpox and leprosy. In fact, few diseases have ever ravaged the human race with more terrible destructiveness than plague when it breaks forth as an epidemic and becomes rampant. It is estimated that in the epidemic of the 
14th century in Europe one-fourth of the population of that continent, or 25 million people, died of the disease. Superstition and unreasoning terror led to horrible persecution and torture of innocent people who were supposed to cause the plague. At present the disease is practically confined to tropical countries, and is especially prevalent in India, where an average of about one million deaths a year are caused by it. The practical disappearance of plague from Europe is thought by some authors to be associated with a change in the rat fauna of Europe, the domestic and gregarious black rat, Epimys rattus, being replaced by the wilder and more scattered brown rat, Epimys norvegicus. The disease, however, has often been introduced from the tropics into other countries, and there is constant danger of this wherever the strictest preventive regulations are not enforced. In 1900 the disease was introduced into San Francisco, and there is every reason to believe that had knowledge of preventive medicine been at the point where it was 300 years ago, the United States would have been swept as was Europe in the 14th century. Instead, knowing that rats were the chief reservoir of the disease, and that rat fleas were instrumental in transmitting the disease from rat to man, the U. S. Public Health Service took hold of the situation and instituted an anti-rat campaign such as had never been thought of before. Over a million rats were caught, examined and destroyed in the city of San Francisco. The infection spread, however, and became established in the ground squirrels of several counties in California.. From July 1, 1913, to November, 1914, over 20,000,000 ground squirrels were destroyed in infected districts in California. So strenuous were the efforts to stamp out the disease before it could get beyond control that only 187 cases of the disease in man occurred in California, with none since 1914. New Orleans has also had a taste of plague, and infected rats have been taken in the vicinity of the water front in Seattle.

The steps which have made possible an intelligent fight against plague were the discovery of the plague germ, Bacillus pestis, by Yerson in 1894, the establishment of the identity of the disease with that of rats by the same worker, the discovery of the multiplication of the plague germs in the gut of rat fleas, Xenopsylla cheopis, by Liston in 1905, and finally conclusive experimental proof by the British Plague Commission in India in 
1906 that the rat flea was the principal means of transmission of the bubonic form of the disease.

As far as is known the plague bacilli live only in the digestive tract of the fleas and do not infect either the saliva or the body cavity. From this fact it is evident that the germs are inoculated into the wound made by the flea, either with the excrement which is commonly voided while sucking blood or with regurgitated blood. It has been pointed out that a rat flea's stomach will hold about one-half a cubic centimeter of blood and could therefore take 5000 germs with a single feed from an infected animal. These often multiply to such an extent as to form a solid mass of organisms, blocking the digestive tract of the insect (Fig. 182).

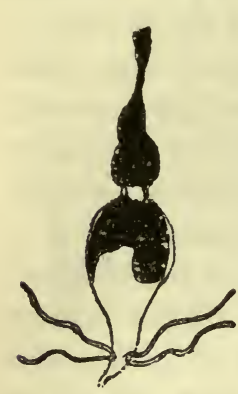

FIG. 182. Digestive tract of flea plugged with solid growth (in black) of plague bacilli. (After Manson.)

It has been stated that when the stomach and intestine of a flea are plugged with plague germs the normal action of the valves of the digestive tract is lost, and the pumping movements of the pharynx result in regurgitating infected material into the wound instead of sucking fresh blood from it. Fleas were found by the British Plague Commission to remain infective for 15 days during the height of an epidemic, though during the non-epidemic season no individual remained infective for more than seven days. In Java the Indian rat fleas have been found to remain infective for 33 days and Bacot has found the European rat flea, Ceratophyllus fasciatus, to remain infective, when kept away from a host, for 47 days.

The Indian rat flea, Xenopsylla cheopis (Fig. 177), is the species most intimately associated with plague transmission. This species has been introduced with rats on ships into all parts of the tropics, and into seaports in many temperate countries, especially such ports as San Francisco, which trade extensively with the Orient. This species, however, is by no means the only one which can serve in the transmission of plague. It is probable that any species which will attack both man and other susceptible animals, such as rats and ground squirrels, may transmit the infection. Thus in India the human flea, Pulex irritans, and the European rat flea, Ceratophyllus fasciatus, have been proved experimentally to be plague carriers, and in California 
the squirrel fleas, Hoplopsyllus anomalus and Ceratophyllus acutus, also have been shown to carry the infection. It is evident also that other animals besides rats and man are susceptible to the disease. Ground squirrels, Citellus beecheyi, guinea-pigs and monkeys have been shown to be susceptible. A marmot or ground hog, Arctomys bobac, common in Manchuria, is thought to have been the chief reservoir of the disease in the Manchurian epidemic in the winter of 1910-11, the flea Ceratophyllus silantiewi being the transmitting agent.

Doubtless any fleas which attack these animals and which also attack man may be instrumental in spreading the plague to human beings in direct proportion to the willingness with which they will bite man, and to their opportunities for doing so. It must not be inferred that only fleas can transmit the disease. The pneumonic form of plague, which is relatively uncommon, is transmitted by particles of sputum or mucus from the mouth or lungs. The bubonic plague may also be transmitted by bedbugs and perhaps by other parasitic insects. A head louse taken from a plague patient was found to be infected. There can be no doubt, however, that the rat fleas are by far the most important spreaders of this terrible disease.

A similar but milder disease of rodents, transmissible to man, occurs in western United States. It is caused by Bacterium tularense and is known as tularemia. Deerflies and certain lice have been shown to be transmitting agents, but the rôle of fleas is only conjectured.

Another disease which is commonly believed to be transmitted by fleas is the Mediterranean or infantile form of kala-azar (see p. 83). This is prevalent in dogs throughout many of the regions bordering the Mediterranean, especially in parts of Italy and North Africa, and is the cause of a high mortality in the numerous cases which occur among children. A number of authors have carried on experiments to prove the instrumentality of the common dog flea, Ctenocephalus canis, and also of the human flea, Pulex irritans, with varied results. (See Chap. V, p. 83.) The rôle of fleas in the transmission of this disease is still uncertain but there is enough evidence against the fleas to warrant their being looked upon with extreme suspicion until definitely proved innocent.

Another instance of the instrumentality of fleas in the trans- 
mission of disease is their relation to the spread of certain species of tapeworms, especially the common dog tapeworm, Dipylidium caninum. The larval stage of this tapeworm is passed in the body cavity of the dog flea, Ctenocephalus canis, the eggs of the parasite, adhering to hair in the vicinity of the anus, being ingested by the flea. Occasional infection of human beings, especially young children, occurs by the accidental swallowing of infected fleas, a thing which could easily happen in cases of too great intimacy between children and their pet dogs. As many as 50 larvæ of Dipylidium have been found in a single flea. The larvæ can also develop in the human flea. The rat flea, Xenopsylla cheopis, has been found to harbor the larval stages of tapeworms of the genus Hymenolepis, as many as nine cysticercoids having been found in a single specimen. These tapeworms are normally parasitic in rats and mice but occasionally parasitize man also.

The relation of fleas to other diseases is suspected. A German writer has put forth the theory that fleas are instrumental in the transmission of typhus. If typhus is purely a bacterial disease, its spread by fleas and other parasites as well as by lice would be quite possible, but if it should be found to be caused by an organism which requires a true intermediate host, it would be doubtful whether such widely different insects as lice and fleas could both function in the same manner. That fleas might act as mechanical transmitters of such diseases as tuberculosis and syphilis is quite possible, though it is doubtful if this often occurs.

\section{Important Species}

Human Flea. - The only species of flea which is known to be a parasite of man primarily, with the exception of the chigger, is the appropriately named human flea, Pulex irritans, though in many places man is annoyed more by certain other species which are primarily parasites of his domestic animals. The human flea is not exclusively a parasite of man. It also attacks badgers, skunks, dogs and other carnivores, occasionally occurs on rats and mice, especially in houses and ships, and has been taken on the blacktail deer, Odocoileus columbianus. ${ }^{1}$ It is now

1 Specimens of fleas taken in considerable numbers on deer in northern California by F. C. Clarke, of the California Fish and Game Commission, were identified by Prof. R. W. Doane of Stanford University as Pulex irritans. On account of the distinctive habits of these deer fleas, Clarke (in litt.) believes that they should be considered a variety of $P$. irritans, for which he proposes the name $P$. irritans cervi. 
cosmopolitan in distribution, probably having originated in Europe, whence it was introduced with Europeans to all parts of the world. This flea is the species which has made California as famous for its fleas as is New Jersey for its mosquitoes. The relatively cool humid summer climate combined with a mild wet winter make the Pacific Coast of the United States an ideal place for this pest. Though more or less of a nuisance throughout the year in mild climates, this flea is less troublesome in winter, due to relative inactivity, to slower reproduction, and to the fact that small mammals are more commonly attacked at this time of year.

The human flea is readily distinguished from most common species in temperate climates by the absence of any combs, either on the head or thorax. From the Indian rat flea, Xenopsylla cheopis (Fig. 177), it is difficult to distinguish, the essential difference being the presence in the rat flea and absence in the human flea of a vertical chitinous thickening of the mesosternum, i.e., the plate to which the middle leg is articulated on either side. The antennæ of the human flea are shorter and more knoblike than are those of Xenopsylla.

The human flea secretes itself in crevices and cracks of houses, in floors, rugs, bedding, etc., coming forth chiefly at night to pierce the flesh and suck the blood of its hosts. The susceptibility of different individuals to flea bites is variable. The irritation that is normally produced, probably chiefly as a result of the injection of the insect's salivary secretions into the wound, causes the formation of a reddish pimple with more or less swelling. Some people, however, are apparently entirely immune to flea bites and feel no pain from them. The writer is one of these fortunate individuals. On his first visit to California he had been fully warned concerning the ravages of the fleas but found to his pleasant surprise that the only discomfort felt from fleas was the tickling occasionally caused by their movements beneath his clothing. A college roommate, however, was attacked to such an extent as to be unable to sleep, and spent a considerable part of many nights in pursuit of the wily fleas and in violent massaging of painful wounds.

As has been noted, the human flea may act as a transmitter of plague, infantile kala-azar and tapeworm (Dipylidium) infection, though it is not the chief villain in the spread of any one of these diseases. 
Dog and Cat Fleas. - Next in importance to the human flea as a parasite of man is the dog flea, Ctenocephalus canis, and the closely allied cat flea, $C$. felis. In the southeastern United States where the flea scourge is as great if not greater than in California, the dog flea is the species usually met with. During the moist hot summers this species becomes exceedingly abundant. Although primarily a parasite of dogs this flea willingly includes man in its bill of fare if opportunity offers, and also attacks cats, rats and other mammals. The usual fleas of cats, however, are now generally considered to be specifically distinct from the dog flea. The cat flea is the only one of the two species found in India, where it is a common parasite of dogs as well as cats. The cat flea has a longer and more slender head than its near relative. Both species can readily be distinguished from any other common species with similar habits by the presence of two conspicuous combs, one along the ventral margin of the head, the other on the pronotum (Fig. 179B).

The eggs of dog and cat fleas are usually laid loosely in the fur of their host, whence they readily fall out when the host shakes himself or is settling himself for a nap. They develop in the dust and dirt of kennels, woodsheds, house floors or other places where infested animals are likely to go. Houses, of course, become infested through the agency of infested animals, and since the fleas, once in houses, encounter man more readily than they do the original hosts, man is very likely to suffer from their attacks. Patton and Cragg found the inside of a hat, in which a kitten had slept overnight, so full of flea eggs that it looked as if it had had sugar sprinkled in it from a sifter. Another author collected a teaspoonful of eggs from the dress of a lady who had held a kitten in her lap for a short time. The writer has been able to find a similar quantity of eggs by dusting a smooth hardwood floor after an infested dog had indulged in one vigorous shake. With these instances in mind one can readily understand how houses into which infested pets are admitted become overrun with fleas.

The dog flea, from its habits, is the species most frequently implicated in the transmission of kala-azar (see p. 83), and is the species usually instrumental in transmitting tapeworm (Dipylidium) infection to children. Since this species will feed on rats there is no reason for doubting that it may act as a trans- 
mitter of bubonic plague, though its preference for dogs or cats would preclude a frequent occurrence of this.

Rat and Squirrel Fleas. - The various species of rat and squirrel fleas are only accidental parasites of man. They readily attack him if opportunity offers but do not remain adherent to him as they do to their normal hosts. If it were not for their enormous importance in the spread of bubonic plague, they would hardly need special consideration.

From its intimate connection with the spread of bubonic plague, the Indian rat flea, Xenopsylla cheopis (Fig. 177), is of prime importance. Though other members of the genus are confined to Africa and Asia, this species has now a world-wide distribution, having accompanied its normal host, the rat, to all warm seaports in both the Old and the New World. It is a rather short, stout flea, resembling the human flea in the absence of combs. Although the normal hosts of Xenopsylla cheopis are rats of various species, the domestic habits of these rodents bring the fleas into close association with man, and they will readily feed upon him if hungry. Furthermore, deRaadt has recently demonstrated that these fleas do not remain constantly in the fur of their normal hosts, but that 80 per cent drop off in the course of 48 hours. This species is not migratory and seldom reaches anyone but the inhabitants of the house in which its host occurs, unless carried by the rats themselves. Swellengrebel states that in Java this flea will willingly bite man on the first day of fasting. In many tropical countries the Indian rat flea is the commonest flea found in houses; in Egypt 96 per cent of fleas caught in plague-infested houses were of this species.

The European rat flea, Ceratophyllus fasciatus, is a species having habits quite similar to those of Xenopsylla cheopis. It replaces the latter species in temperate climates except in seaports, where the Indian rat flea is often more common. The common rat flea of China and Japan is Pygiopsylla ahalce. The larvæ of $C$. fasciatus develop best under cool humid conditions in an abundance of rubbish. Strickland, who has worked out the biology of this flea in detail, found that it would actually attack man in preference to rats, although a feed on the blood of rats seemed to be necessary before any eggs were laid. Another species of the same genus, C. gallince, attacks chickens in Europe, and has been introduced into several parts of the United States. 
It is said to be very annoying to man also. The common squirrel flea in North America, C. acutus, is found on a number of species of wild rodents, and also occasionally on rats and mice. It does not attack man so readily as does $C$. fasciatus, but is nevertheless not averse to human blood. This species has come into great importance in California as the transmitter of plague from rats to ground squirrels. It is probable, however, that other species of this genus and of allied genera may quite as readily transmit plague, depending only on the extent to which their habits bring them in contact with infected animals.

Chiggers. - The chigger, chigoe, jigger or sand flea, Dermatophilus (or Rhynchoprion) penetrans (Fig. 183), as it is vari-

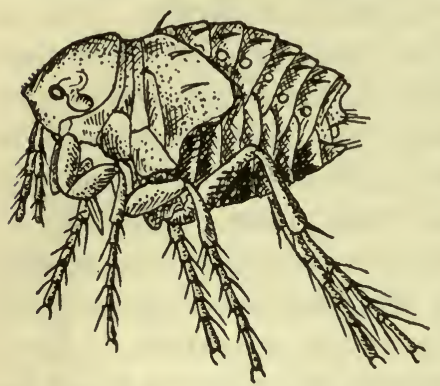

FIg. 183. Chigger or burrowing flea, Dermatophilus penetrans, unimpregated female. $\times 30$. (After Karsten from Riley and Johannsen.) ously called, is one of the most despised pests of tropical countries. It is a very small flea of the family Sarcopsyllidæ, about one $\mathrm{mm}$. in length, with no comblike spines and relatively slender legs. It has a very comical pointed forehead, like a helmet worn with the point forward. The males and virgin females of this species are similar to other fleas in habits, except that they attack a wide range of hosts. Man is the principal host of this particular species, but pigs are also very commonly attacked. Chiggers occur especially in sandy regions where there is much underbrush, and here they lie in ambush on the vegetation, dead leaves or sandy soil, ready to attack any host which may come their way. The particular importance of this flea lies in the fact that the impregnated females have the aggravating habit of burrowing into the skin especially in such tender spots as under the toe nails, where, nourished by the blood of the host, the eggs develop and cause the abdomen to swell into a great round ball as large as a pea, leaving the head and legs as inconspicuous appendages (Fig. 184). Only the two posterior segments of the abdomen do not enlarge; these act as a plug for the hole made in entering the skin. The eggs, up to a hundred in number, mature in about a week and are then expelled by the female through the 
protruding end of the abdomen. Sometimes the entire female is expelled with her eggs by the pressure of the inflamed tissue which surrounds her. The eggs, which fall to the ground, soon hatch into typical flea larvæ (Fig. 185). These, if they happen to fall on sandy soil under conditions suitable for their development, grow to maturity, pupate in a cocoon and emerge as adult insects in the course of ten days or two weeks.

The wounds made by the burrowing female in the skin

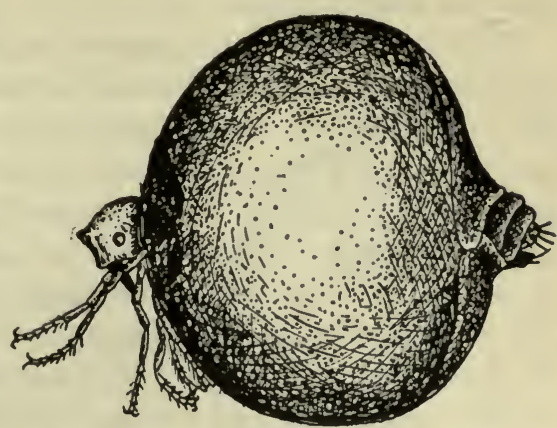

FIg. 184. Chigger or burrowing flea, Dermatophilus penetrans, gravid female. $\times 18$. (After Moniez.)

become much inflamed and very painful. Frequently the distended abdomen of a flea is crushed and the eggs released in the wound. In such cases the inflammation is greatly increased unless the crushed body and eggs are immediately expelled. As soon as the eggs are laid, or even before, the skin surrounding the wound ulcerates and pus is formed. The empty female flea is ex-

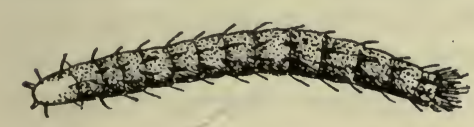

Fig. 185. Larva of chigger, Dermatophilus penetrans. (After Newstead.) pelled. The sore which is left is very liable to infection by bacteria and frequently results in the loss of toes or even whole limbs through blood-poisoning. Quiros has recently pointed out that in Central America, where chigger infection is very common, especially in boys who play barefooted in the streets along which infected hogs are driven to public market, deaths from tetanus and gas gangrene from chigger wounds are very common.

Although usually only a few chiggers are present at a time, there are cases where hundreds infest a person at once, literally honeycombing the skin and making the feet or other parts of the body so sore that the victim is rendered a complete invalid.

This obnoxious flea formerly existed only in the tropical portions of America, especially in the West Indies, but it was introduced to the West Coast of Africa in 1872, and has since become abundant throughout the tropical parts of that continent and 
in the neighboring islands. It has also been introduced by coolies into India, but does not seem to thrive there as it does in tropical America and Africa.

The treatment of chigger wounds formerly consisted in the destruction of the fleas while imbedded in the wounds. This was done by applying various insecticides or pricking with a needle, the dead insect being removed after ulceration. A much better method is to enlarge the entrance hole of the flea with a clean needle and remove the parasite entire. The wound should then be carefully dressed and protected until healed. An ointment recommended by Quiros consists of $21 / 2$ grams salicylic acid and 10 grams ichthyol in 10 grams of yellow vaseline. Bathing of infected parts with kerosene oil is also recommended.

Chiggers can be avoided to a large extent by the use of high boots, or shoes and leggings. Walking barefooted in chiggerinfested regions is almost sure to result in attacks by these pests. Houses, yards, etc., in chigger regions should be kept carefully clean of dust, dirt and débris which might favor the development of the parasites. In Central America Quiros recommends, as one of the best preventive measures, a prohibition against driving hogs affected with chiggers through the streets, along with regulations for treating affected hogs where they are raised. According to Penschke, in German East Africa, attacks by chiggers can be prevented by thoroughly rubbing the feet every two or three days with vaseline to which has been added a few drops of lysol or cresol soap (15 drops to $3 \frac{1}{2}$ oz. of vaseline).

Sticktight Flea. - The "sticktight" flea, Echidnophaga gallinacea (Fig. 186), is another member of the family Sarcopsyllidæ which may be a human pest. It is a small dark-colored flea which very commonly attacks chickens in nearly all tropical and subtropical countries, including the southern United States in America. Although the normal host is the chicken, other poultry, dogs, cats, domestic rabbits, rats and other animals, as well as man, are attacked. This species gets its name from the tenacity with which it adheres to its host. It is gregarious, collecting in dense masses on the heads of poultry (Fig. 186), in the ears of mammals and in other places. It is not averse to attacking man, especially children, but since it is not so active as other fleas it can easily be found and removed. No disease is known to be transmitted by this flea. 
Prevention. - Strict cleanliness in private homes or public buildings prevents fleas from breeding in them. Uncared-for carpets and straw mattings afford excellent breeding grounds for the human flea, as do dusty cracks between floor boards, unswept corners under sinks, and any other place where the eggs and young, undisturbed, may obtain enough moisture to keep them from drying up. The use of bare hardwood floors with rugs which can readily be taken up and swept and thorough sweeping in corners and under pieces of furniture, sinks, etc., do not give fleas an opportunity to breed in the home or in public buildings, and are therefore valuable preventive measures.

One of the best means of ridding an infested house of fleas is to sprinkle

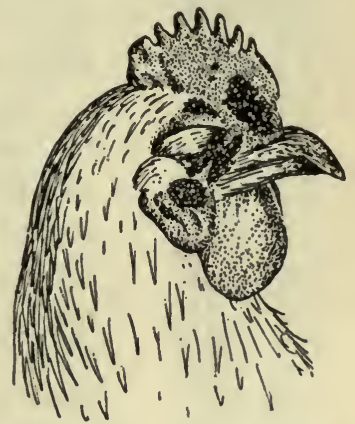

FIG. 186. Head of chicken infested with chicken flea, Echidnophaga gallinacea. (After Bishopp.) the floors with naphthaline and close the rooms for a day or two. This will effectually kill all adult and larval fleas, and the eggs may then be destroyed by washing the floors with hot soapsuds, a five per cent formalin solution or one-tenth per cent solution of corrosive sublimate. It is claimed that alum swept into carpets or a solution of alum soaked into carpet paper prevents fleas from breeding.

Fleas are very susceptible to fumigation with hydrocyanic acid gas. Experiments by the U. S. Public Health Service show that fleas succumb to the amount of gas generated by two and one-half ounces of potassium cyanide in 1000 cubic feet of space. Fumigation with sulphur is also effective. Details of methods of fumigation with these substances will be found on p. 383 . Sodium fluoride in the form of a crystalline powder scattered on floors or blown about by means of a dust-gun will probably prove effective against fleas, as it has against cockroaches and other insects. It is inexpensive and not dangerous to handle.

Various traps for the capture of adult fleas have been devised, one of the simplest and most effective being to clothe the legs in sticky fly paper, and wander about in the infested rooms. A badly infested building in Cornell University was cleared of fleas 
in this manner. Another device, used by the Chinese, is a rod of bamboo, smeared with bird lime, fitted inside of a larger piece

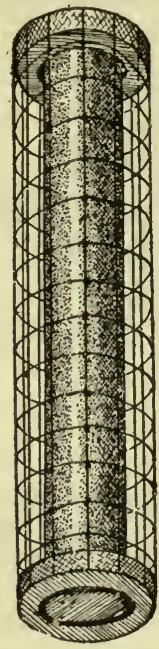

FI 187 . A modification of the Chinese flea trap. Constructed of a broomstick wrapped with sticky fly paper, fitted in a cylinder of wide-meshed wire net. (After Bishopp.) of bamboo which has holes cut in it. A trap of similar type may be constructed by fitting a piece of broomstick wrapped with sticky fly paper inside a wire cylinder (Fig. 187). Such a "flea stick" can be rolled about on floors or in beds and will collect a large proportion of the flea population. Another trap consists of a glass of water with about an inch of oil on the top of it fitted with a little wick in the center of a floating piece of cork. This is placed in the center of a dish of strong soapsuds and lighted at night. The light attracts the fleas, which leap headlong into the soapsuds.

The destruction of fleas, especially cat and dog fleas, on domestic animals is often necessary in order to do away with a flea scourge. Dogs and cats, or other hosts, may be cleared of fleas by washing them in two or three per cent solution of creolin (about one tablespoonful to a quart of warm water), or some other derivative of creosote, or a similar solution of potassium sulphide. According to Bishopp, the solution should be worked into the hair with a brush, and care should be taken to wet the fleas which crowd toward the head of the animal. After about ten minutes the solution should be washed off with warm water and soap, at least in delicate-skinned animals such as cats, to avoid a burning effect. Another method of treatment is to rub powdered moth-balls (naphthaline) into the fur. This causes the fleas to emerge from the fur in a stupefied condition in which they are easily captured and destroyed. Except to sicken cats slightly for a day or two this treatment has no ill effect on the host.

Of temporary value in flea-infested places is the use of repellents, such as oil of pennyroyal, eucalyptus oil, etc., smeared on shoes or clothing, or between bed sheets. Beds may be isolated by elevating them to some distance from the floor, or by sur- 
rounding them with a band of sticky fly paper 12 to 14 inches wide. Where perfect protection from fleas is desired, as in a plague-smitten city, all of these protective measures, as well as fly-paper wrapped legs, and any other means which may come to mind should be made use of. These should be followed up by the more permanent measures leading to the extermination of both larval and adult fleas. 


\section{CHAPTER XXV}

\section{MOSQUITOES}

Importance. - Of all existing insect pests mosquitoes are the greatest enemies of mankind. The mere annoyance which the enormous numbers of them cause by their constant attacks and painful bites is sufficient to have made some parts of the world practically uninhabitable. There are rich pieces of country which have remained absolutely unsettled on account of this pest alone. Some of the choicest hunting and camping grounds in North America, and in other continents also, are practically closed to the camper by the countless millions of mosquitoes which transform a camper's Paradise into an intolerable hell, and drive any bold human invader to frenzy. When travel through such places is necessary no comfort can be hoped for without the presence of a dense smudge or without almost constant application of "mosquito dope," and even then the unceasing "zangs" of the mosquitoes as they threateningly approach is hardly less trying on the nerves than are the actual attacks. Unlike most insect pests the mosquitoes of cold northern countries are if anything more abundant than they are in the tropics. The far northern mosquitoes do not, however, act as carriers of disease; terrible as they are, they wage clean warfare, whereas tropical mosquitoes have their spears poisoned with death-dealing disease germs. The northern mosquitoes bite, suck their fill of blood if they can, and are through; the tropical mosquitoes often leave months or years of suffering and disease, or even death, in their wake. No less than four different diseases are believed to be transmitted by mosquitoes exclusively, namely, malaria, yellow fever, dengue or breakbone fever and filariasis, while a fifth, a form of myiasis in South America, is believed to be transmitted sometimes by mosquitoes. Mosquitoes have been suspected of complicity in the transmission of still other diseases, but their relation to the first four diseases mentioned above is sufficient to brand them as the greatest insect enemies of the human race. 
General Structure. - Mosquitoes are members of the great insect order Diptera, to which so many human pests belong. Their nearest relatives, outside the mosquito family itself, are the midges (Chironomidæ), craneflies (Tipulidæ), sandflies (Phlebotomus), and blackflies or buffalo gnats (Simuliidæ). The members of the mosquito family, Culicidæ, can be distinguished from other Diptera which look more or less like them by the characteristic and quite conspicuous fringe of scales on the hind

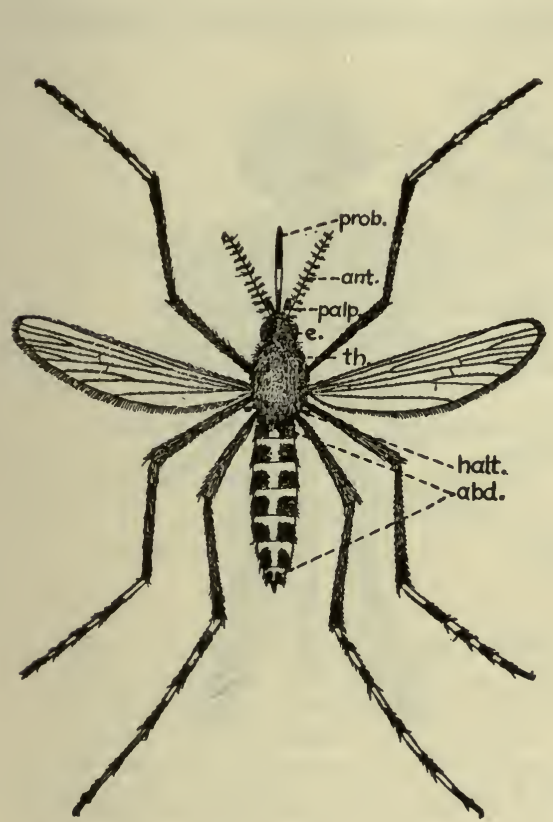

Fig. 188. Diagram of adult female mosquito (Aëdes sollicitans); abd., abdomen; ant., antenna; e., eye; halt., haltere; palp., palpus; prob., proboscis; th., thorax.

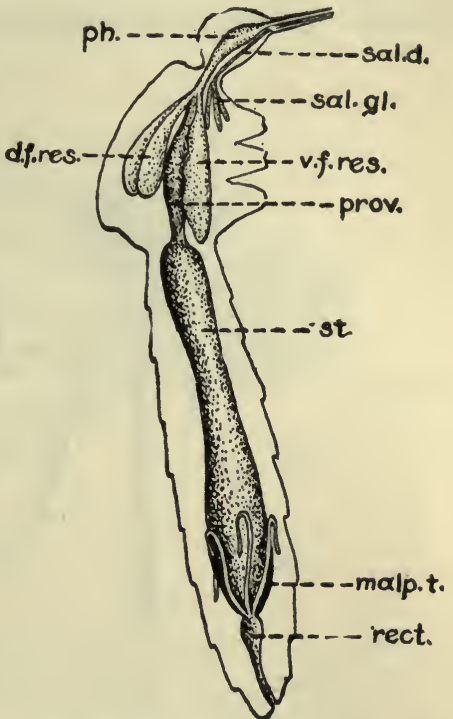

FIG. 189. Digestive tract of a mosquito; d. f. res., dorsal food reservoirs; malp. t., malpighian tubules; ph., pharynx; prov., proventriculus; rect., rectum; sal. d., salivary duct; sal. gl., salivary gland; st., stomach; v. f. res., ventral food reservoir.

margin of the wings. Most of the Culicidæ have a long prominent proboscis containing needle-like organs for piercing and sucking, but in two subfamilies, including the midges of the genus Dixa, and the so-called phantom midges, Corethra (Fig. 192), the adults of which resemble true mosquitoes and are often mistaken for them, there is no long proboscis.

The general appearance of adult mosquitoes is so well known 
as to need no description, but the details of their structure is as little known by most people as are those of the structure of other insects. The diagram on page 425 (Fig. 188) illustrates the details of the parts of a mosquito which are of most use in identifying and classifying. The sexes can be distinguished most readily by the antennæ; in the female (Fig. 190A) they are long and slender with a whorl of short hairs at each joint, whereas in the male (Fig. 190B) they are shortened and have a feathery appearance, due to tufts of long and numerous hairs at the joints. In many mosquitoes the palpi also furnish a means of

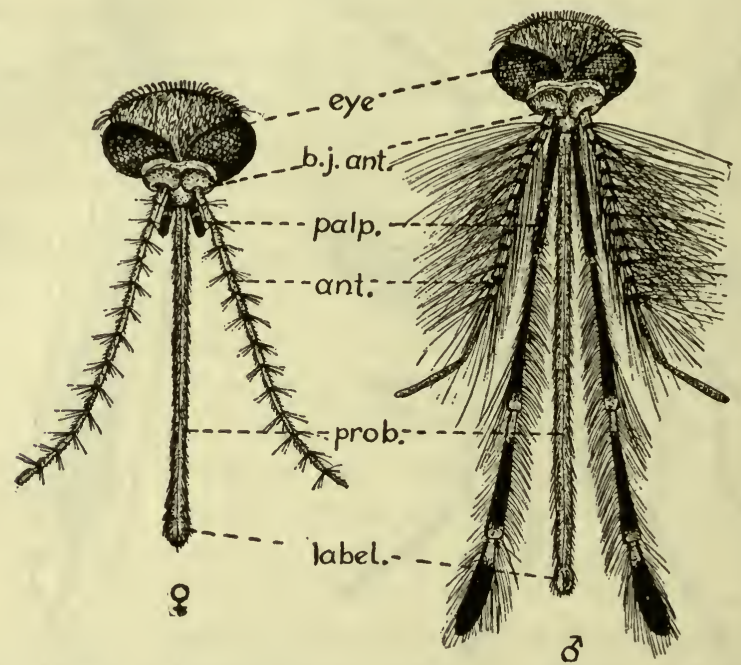

Frg. 190. Heads of female $(\mathcal{Q})$ and male $(\hat{O})$ mosquito, Culiseta incidens: ant., antenna; b. j. ant., basal joint of antenna; label., labellum; palp., palpus; prob., proboscis.

distinguishing the sexes; they are usually long in the males but short in the females, but in Anopheles they are long in both sexes, and in some mosquitoes, e.g., Uranotonia, they are short in both.

The proboscis, which is the most fearful part of a mosquito, also differs in the sexes, and fortunately is so constructed in the male that a mosquito of this sex could not pierce flesh if he would. At first glance the proboscis appears to be a simple bristle, sometimes curved, but when dissected and examined with a microscope it is found to consist of a number of needle-like organs 
lying in a groove in the fleshy lower lip, which was the only part visible before dissection. In the female mosquito there are six of these needle-like organs the nature and names of which are shown in Fig. 191. The "labrum-epipharynx" and "hypopharynx" act together to form a tube for drawing up blood into the mouth. A tiny tube runs down through the hypopharynx, opening at its tip, through which saliva is poured into the wound as through a hypodermic needle to prevent blood from coagulating. The ensheathing lower lip does not itself penetrate the wound, but bows back as the mosquito bites, the flexible tip or

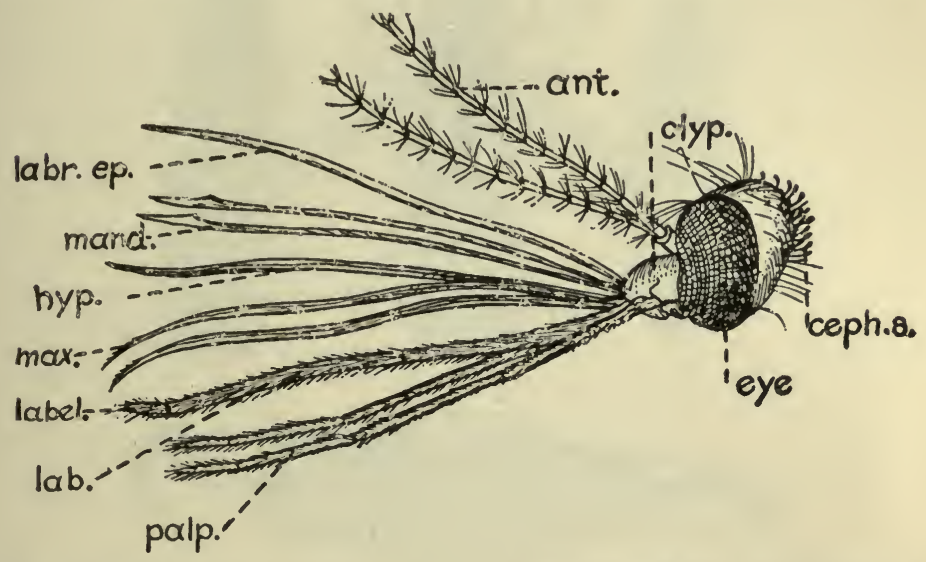

Fig. 191. Side view of head of female Anopheles showing mouthparts; ant., antennæ; clyp., clypeus; ceph. s., cephalic scales; hyp., hypopharynx; lab., labium; label., labellum; labr. ep., labrum-epipharynx; mand.; mandibles; max., maxillæ; palp., maxillary palpi. $\times 20$. (After Nuttall and Shipley.)

"labella" acting as a guide for the piercing organs as they are sunk into the flesh. In male mosquitoes the piercing organs are much degenerated, only the suctorial part of the apparatus being well developed.

Besides the variations of the parts mentioned already, mosquitoes vary as regards the form, distribution, color and other characteristics of the scales which clothe much of the body and the edges and veins of the wings; the details of the male reproductive organs at the tip of the abdomen; the relative length of parts of the leg; and in other respects.

All mosquitoes have good "capacity" as far as the digestive tract is concerned, having three food reservoirs connected with the 
œsophagus, in addition to a large stomach (Fig. 189). Connected with the proboscis is a pair of salivary glands consisting of three lobes each. One of these lobes in each gland differs from the others and instead of secreting ordinary saliva is thought to secrete the poisonous substance which prevents coagulation of blood and produces the inflammation and pain attendant upon a mosquito
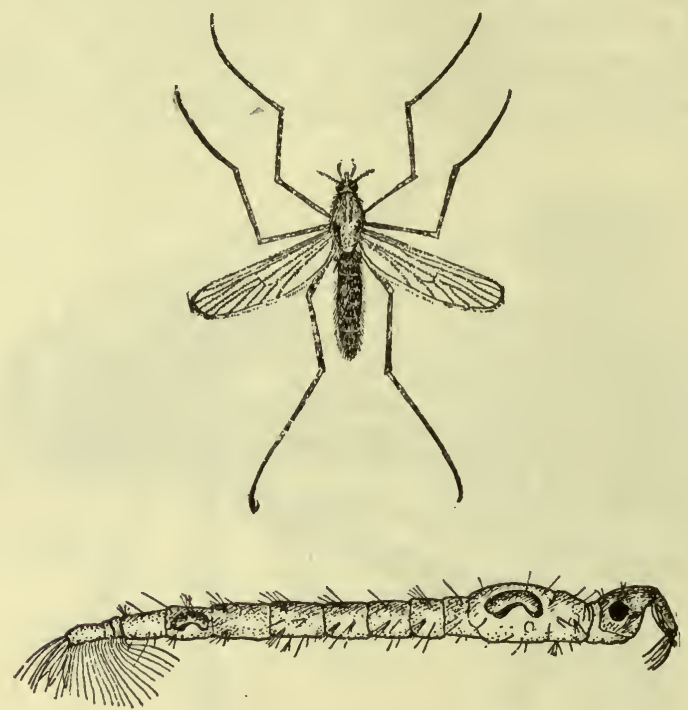

FIg. 192. Larva and adult of Corethra, a member of the Culicidæ which is not a bloodsucker, but is often mistaken for a mosquito. The larvæ prey on mosquito larvæ and other aquatic organisms. Note anterior and posterior "floats" in the larva, and mosquito-like appearance of adult, except for lack of proboscis. (Larva after Howard, Dyar and Knab; adult after Smith.)

bite. Schaudinn, however, has adduced some experimental evidence that it is the contents of food reservoirs which cause the inflammation. It is in the salivary glands that the malaria parasites, and probably the parasites of dengue and yellow fever also, collect, and whence they are poured with the secretions of the glands into the wounds.

Life History. - Mosquitoes, like other Diptera, pass through a complete metamorphosis in the course of their life history, i.e., they undergo a transformation from larval to adult stages during a period of rest. In a general way the life histories of all mosquitoes are much alike, but in details there is much variation 
among them. Without special adaptations in habits and physiology to meet the exigencies of their diverse environment there would be little chance for the mosquitoes of the frozen north or of the parched tropical deserts to meet successfully the struggle for existence. A great store of interesting facts concerning the life history and habits of mosquitoes has been collected by Howard, Dyar and Knab in Part I of their "Monograph of the
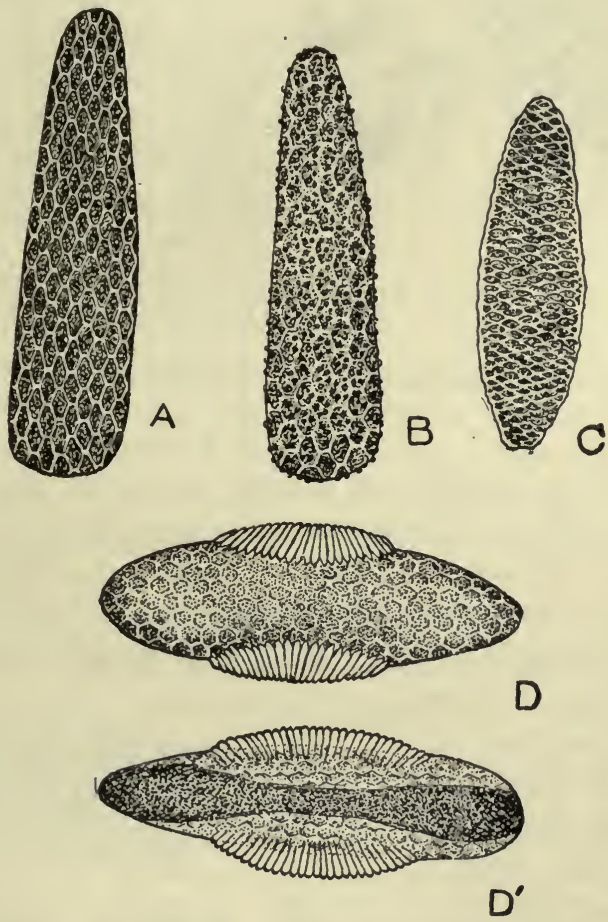

FIG. 193. Eggs of mosquitoes; $A$, Culiseta inornatus; $B$, Mansonia perturbans; $C$, Aëdes calopus; $D$, Anopheles punctipennis, dorsal view; $D^{\prime}$, same, ventral view. $\times$ 75. (After Howard, Dyar and Knab.)

Mosquitoes of North and Central America and the West Indies " and much of the information incorporated into this chapter has been taken from their work.

The eggs of mosquitoes (Fig. 193) are usually oval, with various surface markings, and in Anopheles with a peculiar "float" of air cells. The number of eggs laid by a single female mosquito varies from 40 or 50 to several hundred. Some species 
lay their eggs singly (Fig. 194) while others lay them all at one time in little boat-shaped rafts called egg-boats, the individual eggs standing upright (Fig. 195). The fact that the eggs are a little larger at the lower end makes the whole egg-boat slightly concave, thus making it difficult to overturn. Most of the common mosquitoes of temperate climates lay their eggs on the

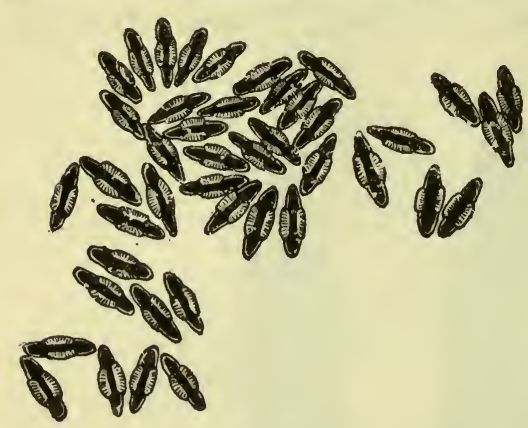

FIG. 194. Eggs of Anopheles quadrimacutatus on surface of water. $\times 13$. (After Howard.)

open surface of water or attach them to some partially submerged object; a few species lay eggs which sink. Many species, however, especially those of the far north and of the tropics, lay their eggs in dry places which are likely subsequently to be covered with water. In most mosquitoes of temperate climates the eggs hatch in a few days, or even within 24 hours. In the species of the far north the eggs probably
never hatch until the following spring, being laid in depressions on the ground which are usually not immersed until the melting of the winter snows. Such hibernating eggs are said not to hatch unless they have been exposed to freezing temperatures. On the other hand the mosquitoes of dry hot countries lay eggs which are highly resistent to desiccation and do not lose their vitality during months of dryness. Such species must almost "live while the rain falls,"

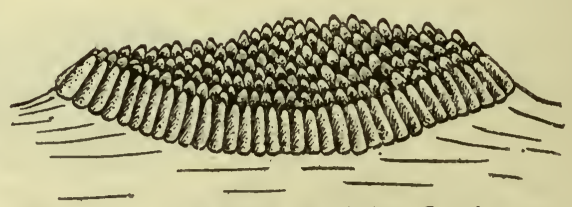

Fig. 195. Egg boat of Culex floating on water. $\times$ about 8 .

and to win in the struggle against an unfavorable climate they must be prepared to utilize the most transitory pools for the completion of their aquatic immature stages. In such cases the embryo within the egg shell develops to the hatching point, so that it is ready to begin the larval existence almost with the first drop of rain. Such mosquitoes further fortify their race against the unkind environment by laying their eggs in a number of small batches instead of in a single mass, as is the habit with 
mosquitoes where water is plentiful. Just as a man runs less risk of ruin if he deposits his money in a number of insecure banks rather than in a single uncertain one, so it is with mosquitoes and the places where they deposit their eggs. The gamble for life in a dry climate would be too risky if all eggs were deposited in one place, and species with this habit have probably long since been weeded out in the struggle for existence. Another remarkable adaptation of dry-climate mosquitoes is the variation in the hatching periods of the eggs in the same batch; not all hatch with the first drops of moisture, but some lie over until subsequent immersions, thus insuring a much better chance that some of them, at least, will not waste their life on the desert air with too little water to enable them to reach maturity.

The eggs of mosquitoes never hatch except in the presence of water. The larvæ, which are always aquatic, are very active wormlike creatures, well known as " wrigglers " or " wriggle-tails" (Fig. 196). When first hatched they are almost microscopic," but they grow rapidly to a length of from a quarter of an inch to almost an inch. The bunches of long bristly hairs on the body take the place of legs, and aid the larva in maintaining a position in the water. The "rotary mouth brush" is a brush of stiff hairs which is used to sweep small objects toward the mouth; in predaceous species these are sometimes modified into rakelike structures or into strong hooked bristles for holding prey. The trumpet-shaped breathing tube (Fig. 196A) is present on all mosquito larvæ except Anopheles (Fig. 196B), in which it is undeveloped. It is used to pierce the surface film of the water to draw air into the air tubes or tracheæ inside the body, for, although aquatic, mosquito larvæ are air breathers, and make frequent trips to the surface to replenish their air supply, remaining suspended by the breathing tube from the surface of the water while breathing. The leaflike "tracheal gills" on the last segment of the abdomen differ from true gills in that air tubes or tracheæ instead of bloodvessels ramify in them. In one species of mosquito, Mansonia, the larvæ absorb air from the aircarrying tissues in the roots of certain aquatic plants, piercing them with the apex of the breathing tube and thus avoiding the necessity of rising to the surface of the water. In well-aërated water the larvæ can live without surface air for a long time by 
using their tracheal gills, but they die within a few hours if shut in water without dissolved air.

Mosquito larvæ, unless suspended from the surface film by means of the breathing tube, have a tendency to sink and they rise again only by an active jerking of the abdomen, using it as
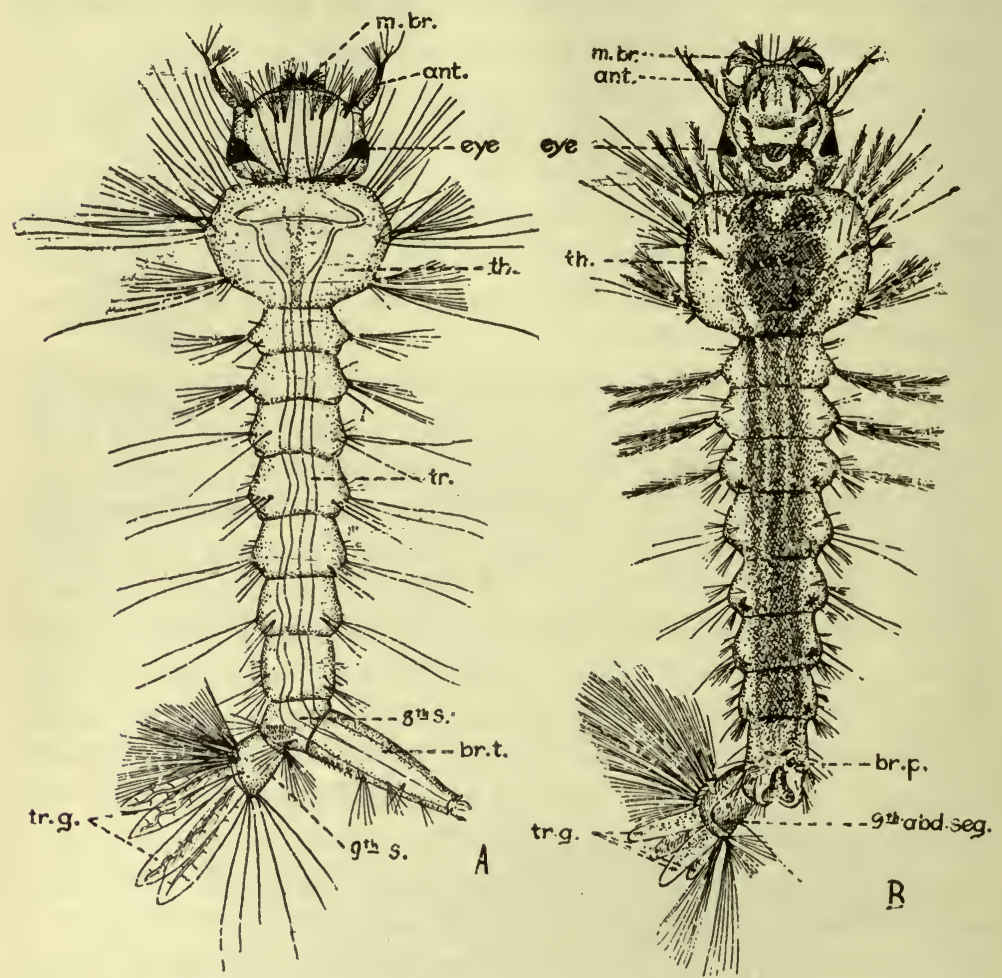

Fig. 196. A, Larva of tropical house mosquito, Culex quinquefasciatus; ant., antennæ; br. t., breathing tube or siphon; m. br., mouth brushes; th., thorax; 8th s., 8th abdominal segment; 9th s., 9th abdominal segment; tr., tracheæ; tr. g., tracheal gills. B, Larva of Anopheles punctipennis; note absence of breathing tube, and starlike groups of scales on abdominal segments; m. br., mouth brushes; br. p., breathing pore; other abbrev. as on Fig. A. $\times 10$. (After Howard, Dyar and Knab.)

a sculling organ. Some species are habitual bottom feeders, others feed at the surface; some live on microscopic organisms, others on dead organic matter, and still others attack and devour other aquatic animals, including young mosquito larvæ of their own and other species.

The larvæ shed their skins four times and then go into the 
resting pupal stage. Mosquitoes of temperate climates usually take from five days to two weeks to complete the larval existence, depending almost entirely on temperature. In the mosquitoes adapted to take advantage of transitory rain-pools the larvæ may transform into pupæ within two days and the pupal stage is a mere matter of hours. On the other hand, some mosquitoes habitually pass the winter as larvæ.

The general form of the pupa can be seen in Fig. 197. Alcock has aptly described this stage of a mosquito as resembling a tiny lobster deprived of appendages and carrying its tail bent. The pair of earlike breathing tubes on the cephalothorax (head and thorax fused) take the place of the trumpet-like tube of the larva and are used in the same manner. Unlike the larva the pupa is lighter than water, and requires muscular effort to sink instead of to rise.

As remarked before, the transformation into the

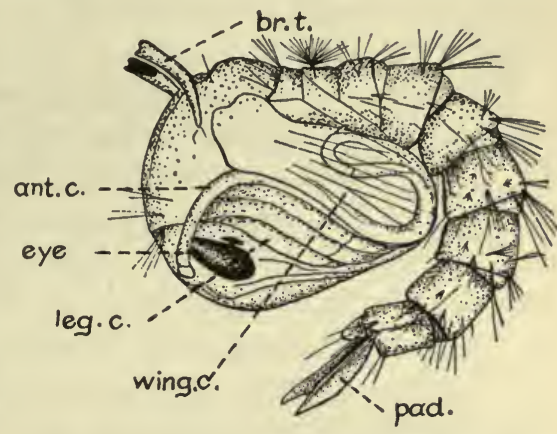

FIg. 197. Pupa of house mosquito, Culex pipiens: ant. c., antennal case; br. t., breathing tubes; leg. c., leg cases; pad., paddles; wing c., wing case. $\times 10$. (After Howard, Dyar and Knab.) adult during the pupal stage may be a matter of a few hours in the case of the dry-climate mosquitoes, but in most species it requires from two days to a week, depending on the temperature. The adult mosquito emerges head first through a longitudinal slit along the back of the thorax. After its exit it rests for a moment on the old pupa skin, stretches and dries its wings, and then takes flight.

Habits of Adults. - Adult mosquitoes vary to a remarkable degree as regards habitats, feeding habits, mode of hibernation, choice of breeding grounds, and other habits. The knowledge, only recently gained, that each species of mosquito has habits and habitats more or less peculiar to itself, is of great economic importance, since it does away with useless expenditure in combating harmless or relatively harmless species, and aids in the fight against particularly noxious ones. The fact, for instance, that one of the commonest summer mosquitoes of northeastern 
United States, Culex territans, does not annoy man does away with the necessity of combating this species, and obviates the necessity of destroying larvæ in certain kinds of marshes and pools where this is practically the only breeder. Again, the fact that mosquitoes breeding in crab holes do not annoy man eliminates the necessity of attempting the almost impossible task of destroying such breeding grounds in order to be free of mosquitoes. The fact that a certain species of Anopheles, A. malefactor, which is a tree-hole breeder, is not a malaria carrier, saved thousands of dollars in the anti-malarial fight in the Canal Zone.

Habitats. - A classification of mosquitoes according to habitats and breeding grounds has been attempted by some authors. Dr. J. B. Smith, for instance, divides the mosquitoes of New Jersey into four ecologic groups, the salt marsh, house, swamp, and woodland mosquitoes. However, almost as many different ecologic groups could be made as there are species of mosquitoes or possible breeding and foraging places. There are species which breed in reedy swamps, woodland pools, eddies of rivers, slow-flowing streams, holes in trees, pools of melted snow, salt marshes, tide pools, crab holes, pitcher plants and other waterbearing plants, or in broken bamboo stems filled with water. There are species which have become "domesticated " and occur almost always in the vicinity of houses, laying their eggs in water troughs, street gutters, rain barrels, water-filled cans in garbage heaps, flower vases, water bottles, and any other collection of water in or about human habitations. Some species show almost no preference as regards breeding places, others, especially those breeding in such specialized places as in waterholding plants, are very closely limited; some species prefer pure clear water, others filthy water, while still others are apparently indifferent.

Migration. - That mosquitoes are seldom found far from their breeding grounds is another fact, only recently recognized, of great economic importance. The evidence points to the fact that most kinds of mosquitoes seldom stray more than from half to three-quarters of a mile from their birthplace, and usually not over a few hundred yards. The supposition that mosquitoes utilize a strong wind to carry them long distances is entirely false, since mosquitoes are so delicate as to be unable to fly at all with a 
strong wind but remain hidden away at times when such wind storms occur. Some mosquitoes are able to resist moderate winds, but nearly always fly against them instead of with them. The salt marsh mosquitoes are apparently an exception to the sedentary nature of mosquitoes, as shown by Smith's work in New Jersey. These mosquitoes commonly migrate for a number of miles and may go as much as 40 miles inland from the salt marshes which bred them. The common salt marsh mosquito, Aëdes sollicitans (Fig. 188), the mosquito that made New Jersey famous, breeds in enormous numbers in the extensive coastal marshes of New Jersey, whence it migrates inland, and sometimes crosses the Hudson River and invades New York City in hordes. The author has seen mosquitoes (not positively identified as this species) literally in clouds on the roofs of buildings in the downtown section of New York, where the day before not a mosquito was to be found. With the exception of a few of the salt marsh species, however, an abundance of mosquitoes can almost always be looked upon as evidence of the existence of breeding places within a mile, and usually within a few hundred yards.

Although most species are not migratory, railroad trains, street cars, ships and other conveyances are efficient means of transfer for mosquitoes. Hawaii is said to have been free of these pests until they were introduced with sailing vessels, in which mosquitoes can usually find plenty of water for breeding. The great number of trains daily running inland in New Jersey from the marsh-studded coast is undoubtedly a factor in keeping more distant suburban towns stocked. Well established cases are on record of places once free of mosquitoes becoming infested after the advent of railroad train or boat service.

Time of Activity. - Although mosquitoes are usually looked upon as strictly nocturnal, and though this is true of most of the common species of temperate climates, it is by no means characteristic of the whole group. Many species, including all Anopheles, are active chiefly at twilight, in the evening, or early morning. Knab found that the mosquitoes of northern prairies, where the nights are too cold for them, are active throughout the day only. A large proportion of forest-living tropical species, at least in America, are said to be diurnal. Some of the mosquitoes of the northern woods are apparently ready to bite when a victim approaches, whether it be day or night. The 
widely distributed yellow fever mosquito, Aëdes calopus (or Stegomyia fasciata) (Fig. 201), feeds by preference in the early morning or late afternoon. Here again a knowledge of the habits of particular species is of importance, since it may aid in the intelligent avoidance of particular disease-carrying forms.

Food Habits. - Heretical as it may sound, mosquitoes feed mainly on plant juices, honey, etc. It is doubtful if the males of any species normally suck blood, and even the females of some species are strict vegetarians. On the other hand, the females of many species have a voracious craving for warm blood. Some species indiscriminately attack any warm-blooded or even coldblooded animal, while others show strong preferences. The yellow fever mosquito normally feeds chiefly on man, and even discriminates against the black race. The other "domestic" mosquitoes apparently have a strong liking for human blood also, and it is not unlikely that their domestic habits are the result of their taste for human blood. Knab found that Aëdes spenceri of the Saskatchewan prairies would fiy toward any large object. On the prairies such an object would usually be a large animal and the mosquitoes would fly toward it instinctively in the hope of satiating the craving for food.

Hibernation. - The method employed by mosquitoes for passing the winter in cold climates, and the dry season in the tropics, varies with the species. Many of the mosquitoes of temperate climates and many in the tropics hibernate or pass the dry season in the adult stage, the females stowing themselves away in hollows in trees, caves, crevices in rocks, cellars, barns, etc., to come forth and lay their eggs in the spring. A few species hibernate in the larval stage, the larvæ of one species, Wyeomyia smithii, becoming enclosed in solid ice in the leaves of the pitcher plant in which they live. Most hibernating larvæ retire to the bottom of their breeding pools during cold weather and do not survive freezing. The majority of temperate- and warmclimate mosquitoes and all of the northern ones pass the unfavorable season in the egg state, and this may be looked upon as the common method of hibernation.

Length of Life. - The length of life of mosquitoes varies with the species and with the sex. Male mosquitoes seldom live more than from one to three weeks; their duty in life is done when they have fertilized the females. The latter usually die shortly after 
they have laid their eggs but some species may live for four months or more. The species which lay all their eggs in a mass at ore time are short lived, and have several generations a year, whereas those in which the eggs are laid in small lots, at intervals, live for several months. Species in which the females hibernate are still longer lived, but since they are not active in winter their effective life is short.

Classification. - Over 500 species of mosquitoes have been described, the majority of which belong in the tropics, although the north is richer in individuals. The task of classifying all of these species into subfamilies and genera is one which has taxed the wits of many scientists. The wide discrepancies in the work of different men as regards mosquito classification is the best possible proof of the difficulties in the way. As in many other groups of animals, intensive study has tended to magnify the value of certain characteristics as criteria of genera or subfamilies, the result being the breaking up of what would ordinarily be looked upon as a single group into a number of poorly defined and intergrading groups. Theobald, who has written a monograph of the mosquitoes of the world, separates the Corethrinæ (forms without a long proboscis) from the mosquitoes, and divides the remainder of the family into ten subfamilies and a very large number of genera based largely on scale characteristics. On the other hand, Howard, Dyar and Knab, whose classification is adopted here, recognize only two subfamilies - the Corethrinæ and the Culicinæ, the latter including all the true mosquitoes. The Culicinæ are further divided into two tribes, the Sabethini, including chiefly forest-dwelling non-blood-sucking forms, and the Culicini. The genera of the latter are arranged in a series from the primitive forms of the genus Anopheles to such highly specialized forms as Megarhinus.

The identification of species of mosquitoes, or even of genera, is often very difficult for anyone but a specialist. Fortunately some of the most important disease-carrying species are so marked that they can quite readily be distinguished even by a novice. Only a few of the disease-bearing species can be separately described here. 


\section{Mosquitoes and Malaria}

As was shown in Chap. IX, malaria is one of the most important and one of the most deadly of human diseases. This being true, the mosquitoes, which are the sole means of transmitting the disease, must be looked upon as among the most important and most deadly enemies of the human race. The rôle of mosquitoes in causing disease, especially malaria, has been suspected by various peoples as far back as any records go. The steps which led to the proof of the relation of mosquitoes to malaria are briefly outlined on pp. 148-149.

Fortunately not all mosquitoes are malaria carriers; in fact, only one genus, Anopheles, com-
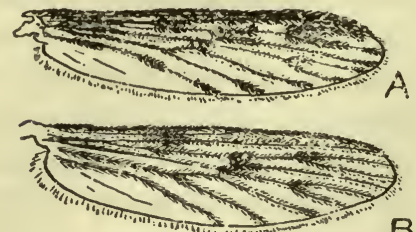

$B$
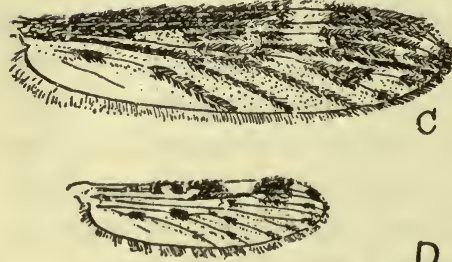

Wings of American Anopheles; A, A. crucians; B, A. quadrimaculatus; $C, A$. punctipennis; $D$, A. albimanus. Drawn to scale. (After Howard, Dyar and Knab.) prising a number of more or less well-defined subgenera which have been considered genera by some workers, is known to be able to transmit the human malarial diseases, and not even all of the species of this genus are incriminated. As will be seen below, some species of Anopheles are able to transmit one type of malaria, but not another. A species of Culex has been shown to be instrumental in transmitting a disease of birds which is allied to malaria.

The rôle of the mosquito in the spread of malaria and the development of the parasites in the mosquito's body have been discussed in Chap. IX, pp. 154-156. Suffice it to repeat here that the sexual phase of the life history of all malaria parasites occurs in the digestive tract of mosquitoes, after which a rapid multiplication of the germs takes place, resulting ultimately in the collection of large numbers of the parasites in the salivary glands of the insect, whence they are poured into the capillaries in the skin of the subsequent victims of the mosquito.

Identification of Anopheles. - The Anopheles mosquitoes, fortunately, are fairly easy to identify in all stages of their development except as pupæ. Thry represent a primitive group 
of mosquitoes, and in many respects are less specialized than other members of the family. The different species of the genus vary a great deal as regards choice of breeding places, habits and appearance, so that it is necessary in any malarial district to determine, if possible, which species are malaria carriers, how they may be identified, where they breed, and what their habits are. The majority of the species have mottled or spotted wings, and the arrangement of the markings is a good means of identification (Fig. 198).

The following comparative table (Fig. 199) shows in a graphic way how Anopheles may ordinarily be distinguished from other common mosquitoes, such as Culex, Aëdes, etc., in their different stages.

Malaria-Carrying Species. - Over a hundred species of Anopheles have been described and they occur all over the temperate and tropical parts of the world. Although not more than about half of these species have been proved to be able to harbor malarial parasites and nurse them to the weaning point, the number of incriminated species is constantly growing, and it is the safest plan to look upon any Anopheles as a potential malaria carrier until proved otherwise. The fact that a given species of Anopheles may transmit one type of malaria but not another complicates the task of determining the rôle of a species, and has caused discrepancies in the results of workers. A. quadrimaculatus (Fig. 200) in North America, for instance, may carry tertian and quartan malaria, but not the more deadly æstivoautumnal type. A. crucians, on the other hand, carries æstivoautumnal malaria but only rarely carries tertian malaria. The third common North American species, A. punctipennis, has recently been proved to be able to nurse and transmit tertian and quartan malaria, but not nearly so readily as does $A$. quadrimaculatus. The situation among these American species fairly illustrates what is found elsewhere - considerable differences among the species of Anopheles as regards their ability to nurse the several types of malaria and the readiness with which they may do so. In most countries, though there may be several species which transmit malaria, there is usually one species which is especially responsible for the disease. In North America it is A. quadrimaculatus and in the southern states $A$. crucians and quadrimaculatus; in tropical parts of America, A. albimanus and 


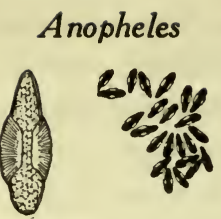

Culex, $A \ddot{e} d e s$, etc.

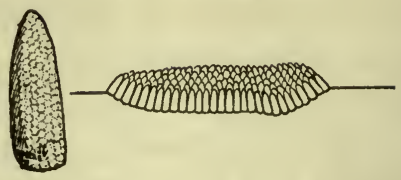

EGGS

Eggs laid singly on surface of water; provided with a partial envelope, more or less inflated, acting as a "float."

Egg 3 laid in rafts or egg-boats, or singly on or near water, or where water may accumulate; never provided with a "float."

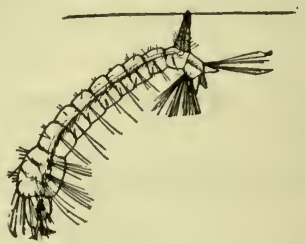

LARV

Larvæ have no long breathing tube or siphon; rest just under surface of water and lie parallel with it.

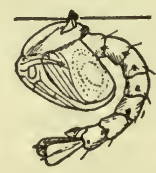

Pupæ have short breathing trumpets; usually do not hang straight down from surface of water.

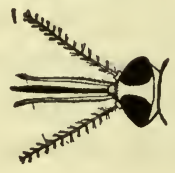

Larvæ have distinct breathing tube or siphon on 8th segment of abdomen; hang from surface film by this siphon, except in Mansonia, which obtains air from aquatic plants.

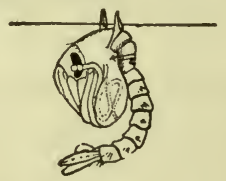

PUPAE

Pupæ have breathing trumpets of various length; often hang nearly straight down from surface of water.

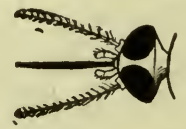

\section{HEADS OF ADULTS}

Palpi of both male and female long and jointed, equaling or exceeding the proboscis in both sexes.

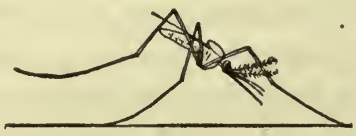

RESTING POSITION OF ADULT

Adult rests with body more or less at angle with surface, the proboscis held in straight line with body.
Palpi of female always much shorter than proboscis, those of male usually long, but sometimes short.

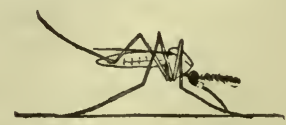

Adult usually rests with body parallel to surface, though sometimes at an angle. Proboscis not held in straight line with body, giving "hump-backed" appearance.

Fig. 199. 
argyrotarsus; in Europe, A. maculipennis; in Africa, A. costalis and funesta; in India, A. culicifacies, stephensi and listoni; in Malay countries, A. umbrosus and willmori; in China and Japan, A. sinensis and listoni; in the East Indies, A. ludlowi; and in Australia, $A$. bancrofti. These species are only a few of the most widely distributed and commonest of the malaria carriers. Many other species may be locally more important.

Habits of Anopheles. - Besides the ability to nurse the parasites of malaria, an efficient malaria spreader must have habits which will insure the use of such ability. The important malaria carriers are, therefore, species which readily attack man, and especially those which are more or less "domesticated." Nearly all species of Anopheles are active only at twilight, and forage out-

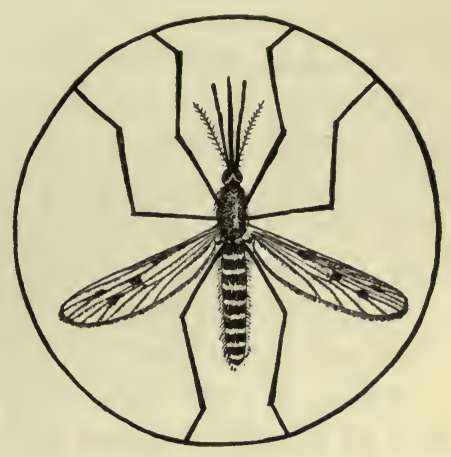

FIG. 200. The common North American malarial mosquito, Anopheles quadrimaculatus.

doors neither in bright daylight nor in the darkness of night, though such species may bite at any hour of the day inside houses. Different species are known to come forth at different times in the evening, some with the first shade of the late afternoon, others not until almost dark. A few species, e.g., A. braziliensis, are diurnal, and many forest species will readily bite in the daytime if disturbed. Nearly all Anopheles hibernate as adults, but a few, notably $A$. bifurcatus of Europe, hibernate as larvæ.

Anopheles may breed in almost any standing water providing it contains microscopic organisms on which to feed. Dr. Smith, of New Jersey, says he has found no pool so insignificant and no stream so rapid that Anopheles could not breed in it somewhere. He says " no other mosquito has as wide a range of breeding places as have the species of Anopheles." Nevertheless, it is apparently true that each species has its favorite breeding grounds and some species are quite particular. A. willmori of the hilly parts of Malay, for instance, will breed only in swiftrunning streams, the banks of which are cleared, whereas $A$. umbrosus of the coastal plains of the same country breeds only in jungle-edged streams; $A$. eiseni of Central America breeds 
only in tree holes; A. cruzi of Brazil breeds in accumulations of water in the leaves of certain tropical plants. A number of species of Anopheles will breed in brackish water, and some in pure or even concentrated sea water. A. ludlowi of the Philippines is believed to breed only in sea water. Some of the coral islands of the East Indies are practically uninhabitable for newcomers on account of the prevalence of malaria which is carried by Anopheles that breed in quiet pools within the coral reefs.

The larvæ of Anopheles are chiefly surface feeders. Some feed upon anything floating on the surface of the water which is small enough to enter the mouth. Others, however, reject many things after they have been swept into the mouth by the mouth brushes, and some feed exclusively on vegetable matter. Only a few species are predaceous.

Apparently neither the eggs nor larvæ of Anopheles are resistant to drying, though they may live on moist mud for some time. Eggs of Anopheles laid in such mud develop to the hatching point but do not hatch until immersed, and die if the mud dries to the extent of losing its glistening surface.

Anopheles are not rapid in development as compared with some mosquitoes. At Washington, D. C., in early summer, $A$. quadrimaculatus was determined by Dr. Howard to develop in a minimum of 24 days - three for the eggs, 16 for the larvæ, and five for the pupæ. In some species the development may be more rapid, but about two weeks is probably a minimum. According to observations by Kulagin near Moscow, Russia, in 1906, there was but one generation of Anopheles in a year, the females always resting over winter before depositing their eggs. This point of the number of generations of Anopheles deserves further local study everywhere.

It is important to note that Anopheles mosquitoes are very sedentary in habit, and seldom fly more than a few hundred yards from their birthplace, and usually not this far. As a group, the insects of this genus are physically incapable of as long flight as are most other mosquitoes. It is frequently reported that Anopheles is found several miles from its nearest breeding places, but the difficulty of knowing with certainty that no water-filled hoofprint or tin can exists in the intervening area is obvious. That an Anopheles may occasionally wander half a mile or more from its breeding ground is unquestionable, but not 
enough individuals do this to make it necessary to look for breeding places more than three or four hundred yards from the infested locality. The conveyance of mosquitoes in trains, boats, etc., must, however, be taken into account.

The effect of anti-Anopheles campaigns on the prevalence of malaria is discussed in Chap. IX, pp. 165-167.

\section{Mosquitoes and Yellow Fever}

Following upon the heels of the discovery of the relation of mosquitoes to malaria, and second only to it in importance, came the discovery of a similar relation to yellow fever, in 1900. As in the case of malaria, some physicians suspected the instrumentality of mosquitoes in the dissemination of this disease before there was any proof of it. The proof came as the result of the illustrious work of the American Army Yellow Fever Commission, composed of Doctors Reed, Carroll, Lazear, and Agramonte, at the cost, indirectly, of the lives of three of them. What is known of the nature of yellow fever, and of the rôle of the mosquito in transmitting it, is discussed in Chap. X, pp. 182-185. It should be repeated here, however, that the "germ" of the disease is still unknown, though believed to be a protozoan. The blood of a patient can infect a mosquito only during the first three days of illness, and the mosquito cannot

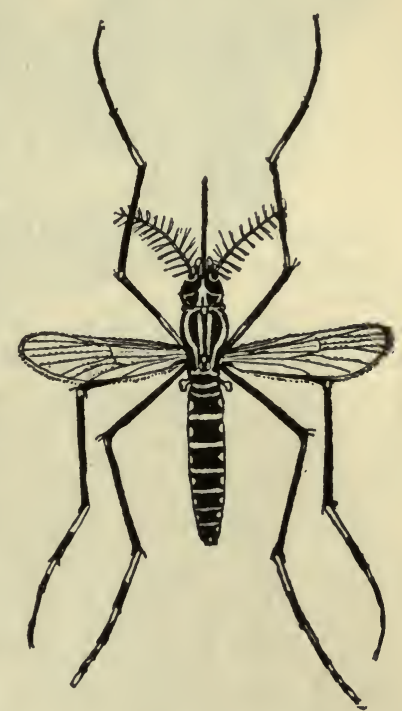

Fig. 201. Yellow fever mosquito, Aëdes calopus, female. (After Doane.) transmit the disease in less than 12 days later. In one case, hereditary transmission of yellow fever from an infected mosquito to its offspring has been shown to occur.

The Transmitting Species, Aëdes calopus. Unlike the condition as regards malaria, yellow fever can be transmitted by only one species of mosquito, Aëdes calopus (or Stegomyia fasciatus) (Fig. 201). This is a small black mosquito, conspicuously marked by white bands on the legs and abdomen, and a white 
lyre-shaped design on the thorax. The female, which, of course, is the only sex connected with the transmission of disease, since the males do not suck blood, has very short palpi which are white at the tip. The wings are clear and somewhat iridescent.

Habits of Aëdes calopus. - The yellow fever mosquito is the most thoroughly "domesticated" species known. It is seldom found except in the vicinity of houses and shows a decided pre-

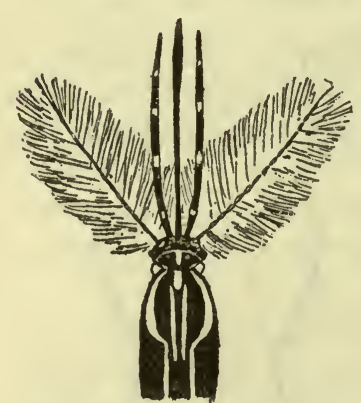

Frg. 202. Head of Aëdes calopus, male. (After Goldberger.) ference for human blood. As a rule it seldom leaves the rooms of houses except to find a suitable place to lay its eggs. Long familiarity with man has made this mosquito one of the most elusive and well-adapted pests, of the human race which nature has ever evolved. Its stealthy attack from behind; its habit of crawling up under the clothing to bite in preference to attacking the exposed ankles; the suppression of the characteristic mosquito "song," so that its bite comes silently and without warning; its habit of concealing itself in pockets, folds, etc., of garments; its hiding behind pictures, under chairs, etc.; the wariness of its larvæ; - all these are the result of lessons learned from long and close association with man.

Aëdes calopus is principally a diurnal mosquito, and becomes particularly hungry in the early morning and during the afternoon. It will bite in lighted rooms, but will never bite in the dark. The French Yellow Fever Commission in Rio de Janeiro stated that Aëdes calopus is nocturnal. The evidence for this conclusion, which is at variance with the observations of other workers, has been shown by Howard, Dyar and Knab to be very inadequate and faulty. The danger of sleeping in an infected place, and the comparative freedom from danger enjoyed by persons who visit infected places only in the daytime, is thought to be due to the fact that most of the mosquitoes obtain a meal very early in the morning, just after daybreak.

Breeding. - Aëdes calopus never lays eggs until it has had a meal of blood and when water or moist surfaces are available. According to recent experiments by Bacot a single male mosquito may fertilize a number of females. Fertile eggs are usually 
laid from four to seven days after a blood meal. The nearest allies of this species are tree-hole breeders, but the yellow fever mosquito has become domesticated to such an extent as to much prefer a rain barrel or water-filled tin can in a garbage heap, or, even better, a water-pitcher or flower-vase indoors. Churches in Central America are usually well supplied with yellow fever mosquitoes which breed in the holy-water fonts.

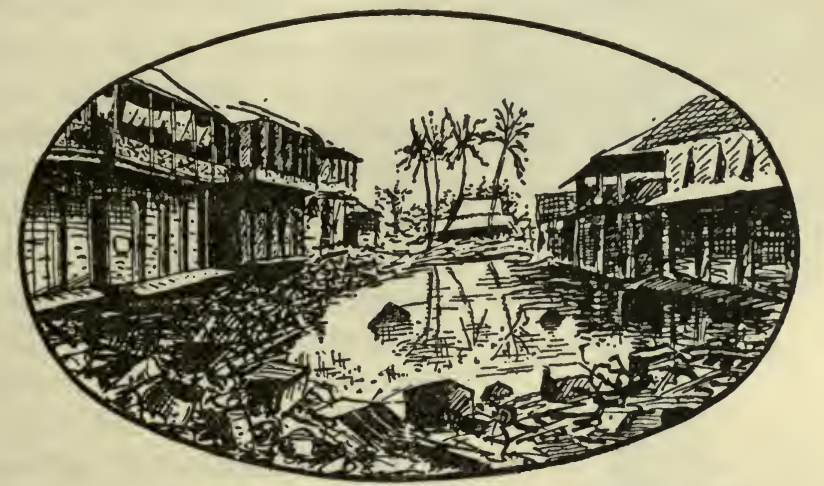

FIg. 203. A yellow fever center in Panama in the pre-American days. (Drawn from photo from Thompson.)

The eggs (Fig. 193C), up to 150 in number, are laid in several lots at intervals of a few days, either on the surface of the water, or, as is more common, on the edges of the container, or on a partially submerged object, wherever a moist surface is presented and where a slight elevation of the water will submerge them. The female mosquitoes die a short time after the last batch of eggs is laid. According to Bacot's experiments the promptness of hatching depends on temperature and on whether the eggs have been kept under moist or dry conditions. The eggs of this species retain their vitality for several months when kept absolutely dry, but they hatch more readily and with less mortality if kept moist. When the eggs are laid directly on the surface of water they mature less rapidly than when laid above the surface, probably on account of the cooling effect of the water. Eggs laid on the surface hatch in a minimum of two days, while those above it, if later submerged, may hatch in less than 12 hours. According to recent work by Atkin and Bacot, eggs will not hatch in sterile water, but will hatch within a few hours after the introduction of living bacteria. The larvæ (Fig. 204) thrive in either clean or foul 
water and even in brackish water, provided food material, in the form of dead organic matter and the accompanying bacteria, is present. Atkin and Bacot have recently shown that the food consists almost, if not quite, exclusively of bacteria, and that

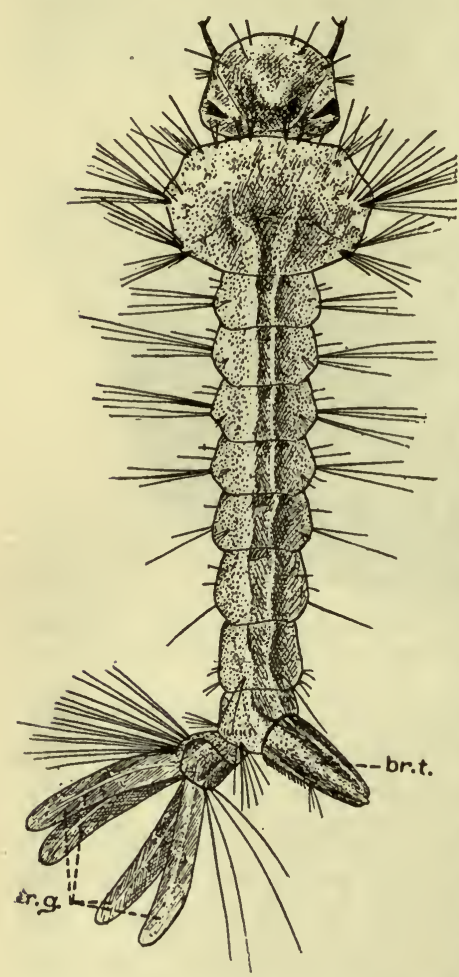

FIG. 204. Larva of yellow fever mosquito, Aëdes calopus. $\times 10$. (After Howard, Dyar and Knab.) when the larvæ are present in large numbers they exert a considerable influence in the purification of water. Often the larvæ are overlooked, since they immediately wriggle to the bottom of their dwelling place when approached, and hug the bottom so closely that even if a barrel containing thousands of them is turned over on its side, about 80 per cent will stay in the little remaining water. The larvæ feed exclusively on the bottom and can often be seen nibbling away at a dead insect or bit of decaying vegetation. With plenty of food and at favorable temperatures the larval existence may be completed in four days, according to Bacot, though it usually requires a longer time than this, and may be drawn out to two months or more. The larvæ are not resistant to drying, and die in a few hours in a dry place, though capable of living nearly two weeks on moist ground.

The pupæ (Fig. 205) transform, under normal conditions, in a day and a half or two days. The entire cycle from egg to adult seldom takes place in less than nine or ten days, and probably 12 or 15 days is more usual under ordinary conditions. As has been shown above, the period of development may be drawn out over several months by unfavorable conditions. The adult mosquitoes may live for a considerable time, and apparently are able to transmit yellow fever any time from 12 days after infection to the end of their lives. Male mosquitoes ordinarily 
will not live beyond 50 days, but the females frequently live under laboratory conditions for four months or more. Kind of food, dryness of climate and facilities for laying eggs are among the chief factors determining the length of life of these mosquitoes, and, strange as it appears at first, the length of life is shortest under the most favorable conditions, namely, plenty of blood for food, plenty of moisture, and suitable places for egg-laying.

The flight of the yellow fever mosquito is strong but, like most other mosquitoes, it seldom flies long distances, usually not more than a few hundred feet. Vessels lying half a mile from shore rarely if ever are visited by these mosquitoes unless the latter are carried from shore by lighters or boats.

Owing to its domestic habits and its ability to "stow away"

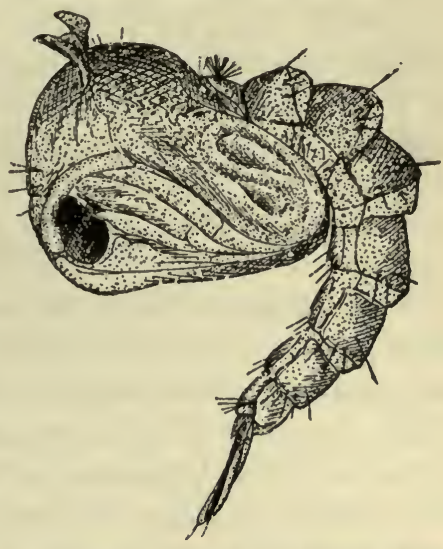

Fig. 205. Pupa of yellow fever mosquito, Aëdes calopus. $\times 10$. (After Howard, Dyar and Knab.)

the yellow fever mosquito has been, and annually is, widely distributed over the world. As pointed out by Howard, Dyar and Knab, its original home was very probably tropical America, since the evidence points to the origin of yellow fever in the West Indies and neighboring mainland, and it is inconceivable that the parasite of this disease would have developed in any other region than the original home of its obligatory host. The permanent home of this mosquito is now almost the entire warm portion of the world, wherever a temperature of $80^{\circ}$ or more is maintained for any length of time, and where freezing does not occur. The once common occurrence and breeding of this mosquito during summer months in cities of the Atlantic Coast of the United States and in other ports outside the frostless zones was due to its importation on ships from such infested cities as New Orleans, Havana and Rio de Janeiro. The cool nights and low summer temperatures on the Pacific Coast of the United States prevents its thriving there, in spite of the fact that it is still sometimes carried there on ships. Since the practical extermination of this mosquito in most of the ports where it was once abundant 
its importation to other places has become a rare occurrence. Since Aëdes calopus has a much wider range than has yellow fever there is constant danger of the introduction of the disease into places where it has not previously been known and where, due to the non-immune condition of the people, it would become a terrible scourge if once successfully introduced. For this reason the yellow fever mosquito is fought as a public menace in India, Australia and many of the South Sea Islands, where it is frequently the most abundant mosquito.

\section{Mosquitoes and Dengue}

The relation of mosquitoes to dengue or breakbone fever was first pointed out by Graham, of Beirut, in 1902, who performed experiments which showed that this disease was not caught by close association with patients in the absence of mosquitoes, whereas isolated men subjected to bites from mosquitoes which had bitten dengue patients readily contracted the disease. Other workers have adduced evidence in favor of the mosquito transmission of the disease, and Ashburn and Craig in the Philippines have shown that laboratory-bred mosquitoes, fed on dengue patients, could transmit the disease three days after the infective meal. The nature of the disease and development of it in mosquitoes and man is discussed in Chap. X, pp. 186-187. It is a disease which resembles a mild form of yellow fever, is seldom fatal, and occurs in very sweeping and rapidly traveling epidemics.

Transmitting Species. The tropical house mosquito, Culex quinquefasciatus (fatigans) and Aëdes calopus have been shown to be the principal transmitters of dengue. Circumstantial evidence, such as distribution and epidemiology of the disease, habits of the mosquitoes, etc., all point to $C$. quinquefasciatus as being the most important species concerned. Aëdes calopus has repeatedly been suspected of transmitting the disease, especially in Australia, but conclusive evidence of this has been brought forth only recently. A species closely related to Aëdes calopus, Desvoidea obturbans, has been incriminated in Formosa.

C. quinquefasciatus is the common house mosquito of the tropics, and very closely resembles the house mosquito of temperate climates, $C$. pipiens, in both appearance and habits. It is brown in color with a broad whitish band on each abdominal 
segment. The thorax and legs are plain brown except for a pale area at the bases of the legs.

This species is very common in houses in all thickly populated parts of tropical and subtropical portions of the world, though not so thoroughly "domestic" as Aëdes calopus. In America it becomes abundant in summer as far north as Washington and St. Louis. It is strictly nocturnal and will bite in complete darkness, therefore its work supplements that of the yellow fever mosquito, the latter taking the day shift, the former the night shift. The house mosquito does not pursue man with as much devilish cunning and perseverance as does Aëdes calopus, and, indeed, shows a very inferior grade of intelligence as compared with it. There is reason to believe that it is primarily a persecutor of birds and poultry, and attacks man only as a second choice. C. quinquefasciatus breeds in almost any standing water but apparently prefers artificial receptacles and is partial to filthy water. The eggs, about 200 to 300 in number, are laid in rafts as is the case with other members of the genus. The larvæ (Fig. 196A), which hatch in from one to three days, have long breathing tubes, and feed chiefly on microscopic organisms. The length of time required for the mosquitoes to reach the adult stage from the time the eggs are laid depends very largely on temperature, food conditions, etc. The minimum period is probably about five or six days.

Alcock remarks about this mosquito: "Apart from its practical importance, Culex fatigans (or Culex quinquefasciatus) has a peculiar interest as being the living document of two discoveries of the first magnitude in the history of medicine, namely, Sir Patrick Manson's discovery ... of the part played by mosquitoes in the life cycle of certain filarial blood-parasites, and Sir Ronald Ross's discovery ... of the necessary connection between mosquitoes and certain Protozoön blood-parasites. The first discovery laid open a new world to Pathology; the second, which is the outcome of the first, will affect the destiny of the human race."

\section{Mosquitoes and Filaria}

As intimated in the last paragraph above, the discovery by Sir Patrick Manson in 1879 of the function of the mosquito as an intermediate host of filarial worms, the larvæ of which live 
in the blood, marked the beginning of a new era in medical science; it was the first evidence of the development of germs of human disease in the bodies of insects. An account of the life cycle of filarial worms, including the development in the bodies of mosquitoes, the means by which the worms are returned to their primary hosts, and the effect of filarial infection on man, will be found in Chap. XVII, pp. 299-306.

Not all species of filarial worms utilize mosquitoes as intermediate hosts, a notable exception being the loa worm of Africa. Four human species, Filaria bancrofti, F. philippinensis, $F$. perstans and $F$. juncea (demarquayi) are known, or thought, to be nursed by mosquitoes. The last two named are not known to have any pathogenic effects, but $F$. bancrofti is connected either directly or indirectly with a number of human ailments (see p. 306). $F$. philippinensis is closely allied to $F$. bancrofti, but differs in that it appears in the peripheral blood diurnally as well as nocturnally, a habit which is supposed to be associated with the diurnal habits of its usual intermediate host, Aëdes pseudoscutellaris.

Although the successful development of filarial worms is not limited to one particular species of mosquito or even to any particular group of species, the development is not completed equally well in all species. The tropical house mosquito, Culex quinquefasciatus, is the species in which the worms apparently develop most frequently with least fatality to either worms or mosquitoes. In Fiji the development of the worms is more regular and more rapid in Aëdes pseudoscutellaris than in any other mosquito. In Aëdes calopus, however, the development of the worms is very slow, and they eventually degenerate in the thoracic muscles without reaching maturity. Many species of Anopheles serve as suitable hosts, as do also some species of Mansonia and other genera. In many species of mosquitoes the filarial larvæ are digested, or else die in the course of their development. On the other hand, there are some mosquitoes which are very susceptible to the injury done by the worms, especially in case of heavy infestation, and the mortality may amount to a large per cent. Apparently the most critical time for the mosquitoes is when the larvæ have penetrated into the proboscis. This is a striling example of the almost universal truth in parasitology, that the host in which the asexual cycle of a 
parasite is passed is less perfectly immune to the parasite, and the parasite less perfectly adapted to the host, than is the case between a parasite and the host in which it goes through the mature sexual phase of its life history. In the case in hand man may be looked upon as the disseminator of a deadly disease among mosquitoes in much the same way that the mosquito may be considered the disseminator of deadly human diseases in the case of malaria and yellow fever.

\section{Mosquitoes and Dermatobia}

In many parts of tropical America where the man-infesting botfly, Dermatobia hominis (see Chap. XXVII, pp. 513-515), is found there has long been a belief among the natives that the maggots of this fly, which develop under the skin of man and of many other animals, are in some way the result of mosquito bites. Inhabitants of some endemic regions, e.g., Trinidad, point to mosquitoes as the cause of the skin maggots, and in some places the larvæ are known as "mosquito-worms." Until recently the scientific world looked upon these beliefs as mere superstition, and gave them no further thought. In 1911, however, Dr. Morales, of Guatemala, received a specimen of a mosquito sent him as a mosquito " carrier" of Dermatobia, with eight relatively large elliptical eggs glued by their posterior ends to its abdomen. A few days later a larva emerged from one of the eggs, and was induced to enter an abrasion in the skin of an attendant, where it thrived so well that for the patient's sake it was removed after a little over six weeks and transplanted to the back of a rabbit. Here it continued its development and escaped, probably just before pupation, exactly two months after the eggs were first received. Dr. Morales was quite certain that the larva was really a Dermatobia. In the same year Dr. Tovar of Caracas, Venezuela, made similar discoveries, and is said to have caused typical Dermatobia tumors by allowing an egg-laden mosquito to bite a susceptible animal. From these tumors the larvæ were obtained at the end of 11 days and from these larvæ the adult flies were reared. Dr. Surcouf of Paris, Dr. Knab of the United States, and Dr. Sambon of England have published observations of their own bearing on the rôle of the mosquito in transmitting Dermatobia infection. From these observations one would be inclined to believe that, as expressed by Dr. Rin- 
cones of Caracas, the mosquito is utilized by the Dermatobia fly as an aeroplane for transporting her eggs or larvæ to a suitable host for development, and we would have here, if true, one of the strangest interrelations of animals in the whole realm of nature, comparable, perhaps, with the manner in which certain mites of the family Tyroglyphidæ assume a special traveling garb and adhere to the appendages of flies to obtain transportation to new feeding grounds (see pp. 339-340).

Dr. Neiva, of the Instituto Oswaldo Cruz, at first did not believe in the mosquito theory. He pointed out that in various parts of tropical America not only mosquitoes, but also craneflies, ichneumon-flies, certain large hairy flies and other insects are accused of being Dermatobia carriers, though they could not possibly serve in this capacity. It is known now, however, that various species of flies as well as the mosquitoes are used by Dermatobia, and it would be expected that at times a fly, impelled by the urgent desire to oviposit, might be content to deposit her eggs on insects which could not very well serve the purpose of transporting the eggs to a suitable host. Neiva, when he objected to the mosquito theory, had not found any egg-carrying mosquitoes in Brazil, where Dermatobia is common, but in a later publication he records not only Janthinosoma lutzi, the mosquito incriminated in Venezuela and Central America, as an egg-carrier, but also another species of Janthinosoma, J. posticata, and several kinds of muscid flies (see p. 514). He also found on dissection of female Dermatobia flies, that the eggs were in various stages of development and thought this argued against the utilization of mosquitoes as carriers, but it has subsequently been shown by Neiva himself that the eggs are laid in small groups on captured insects, and by no means all at one time. Dermatobia is frequently found pestering cattle and horses, and egg-containing females sometimes persistently follow human beings. It is not certain that the fly does not sometimes deposit its eggs directly on the skin of the host in which the larvæ are to develop, but its presence near large animals may be due to its pursuit of other insects which swarm around them.

There is no doubt any longer but that the widespread popular belief in the part played by the mosquito is founded on fact. Insects carrying eggs of the fly have repeatedly been found, and 
the females have been observed holding other flies between their legs, and in captivity have been observed to deposit their eggs on various species of flies. As remarked by Sambon, this fly may have several ways of disposing of its eggs, but the utilization of the mosquito and other insects as transports for them is probably the usual method.

The mosquito involved is most frequently found to be a species of Janthinosoma. In Central America $J$. lutzi alone has been incriminated, but in Săo Paulo, Brazil, this species and $J$. posticata, and also other mosquitoes, have been found to transport Dermatobia eggs. J. lutzi (Fig. 206) is a large and beautifully colored mosquito, with flashes of metallic violet and sky blue on its thorax and abdomen. It is said by Knab to be one Frg. 206. Mosquito, Janthinosoma lutzi, with of the most blood- to abdomen. (After Sambon.)

thirsty of American

mosquitoes and is found throughout tropical America. The larvæ breed almost exclusively in rain puddles, the eggs being laid in dry depressions on the forest floor which will become basins of water after a tropical downpour of rain. The eggs hatch almost with the first drop of rain, and mature so rapidly that adult insects may emerge in four or five days. The larvæ feed on particles of organic matter, and are themselves fed upon by the larvæ of the closely allied genus of mosquitoes, Psorophora, which breed in the same rain pools.

\section{Mosquito Bites and Remedies for Them}

As has been remarked before, the pain and irritation produced by a mosquito bite is usually believed to be due to the injection 
of a bit of poisonous saliva into the wound made by the piercing mouthparts of the insect. The susceptibility of some people to the effect of mosquito poison is much greater than that of others. The author has seen individuals on whom mosquito bites swelled up like bee stings and were even more painful, whereas the author himself has frequently been unaware of the fact that a mosquito was biting him unless the insect was seen by him or was pointed out by a less indifferent companion. Moreover, the effect of the bites of different species of mosquitoes varies, so that while some species may produce very little irritation others may prove unbearably annoying. Dr. Smith, of New Jersey, became practically immune to the bites of some of the salt marsh mosquitoes, but was troubled by the house mosquito, Culex pipiens, and still more so by Anopheles. The author has had similar experience, and has found himself driven almost to frenzy by some species and hardly annoyed at all by others. It is quite probable that the complaints which are heard from visitors to the ocean resorts of the New Jersey coast are due to the fact that these visitors are fully susceptible to the poison of the salt marsh mosquitoes whereas they may have become more or less immune to the inland mosquitoes of their own districts. These facts clearly indicate that there is a specific difference in the poison of different kinds of mosquitoes, and Dr. Smith's experiences show that acquired immunity to one mosquito may give little or no relief from another.

There is a popular belief that if a mosquito is allowed to draw his fill of blood, the bite is less painful and becomes less swollen than if she is killed or driven away. This belief is to a large extent true, the probable reason being that when the insect is allowed to finish her meal, the droplet of poisonous saliva injected into the wound is drawn back into the stomach of the mosquito with the blood on which it acts.

Many different remedies have been recommended for mosquito bites. Ammonia, alcohol, glycerine, indigo, iodine, ether, camphor, naphthaline (moth balls), cresol preparations, a $2 \frac{1}{2}$ per cent carbolic solution - all these and others have had their adherents amongst entomologists, hunters, travelers and housewives. All of them probably have some alleviating effect, and it is not unlikely that their effects may vary with different species of mosquitoes and perhaps even with individuals. Dr. 
Howard found that moist soap rubbed on the bites was the most satisfactory remedy in his own personal experience.

Probably no remedy or disinfectant, no matter how quickly applied after an infected mosquito has been sucking blood, would be effective in preventing infection with malaria, yellow fever or dengue. Filaria and Dermatobia infections, however, could probably be prevented in this manner, since it takes an appreciable time for the larvæ to enter the skin in the vicinity of the wound.

\section{Control and Extermination}

The control of mosquitoes may be undertaken in the following ways, in order of permanent usefulness: (1) personal protection by the use of repellents on or near the person, or of protective clothing; (2) the elimination and exclusion of mosquitoes from dwellings; (3) the local destruction of larvæ by the use of temporary "larvicides"; (4) the prevention of breeding by obliterating breeding places or making them uninhabitable.

Personal Protection. - This method of dealing with mosquitoes has no permanent value whatever, and does nothing to lessen the number of mosquitoes, but it is indispensible to the hunter or visitor in mosquito-infested places. Concerning the use of protective clothing, little need be said; the value of gloves, veils, high boots, leggings, etc., is obvious.

The use of "mosquito dope," or ointments repellent to mosquitoes, on the exposed skin is a popular but usually disappointing safeguard against attacks by these insects. The number of popular repellents for mosquitoes is as great, if not greater, than the number of popular applications for the bites. Nearly all of these are unquestionably effective while they last, but they all have the disadvantage of losing their power by evaporation in a short time, and therefore have to be renewed at frequent intervals. Spirits of camphor, oil of pennyroyal, oil of peppermint, lemon juice, vinegar, anise oil and oil of citronella are all effective protectors while they last. Oil of citronella has been most widely used in America. This mixed with an equal amount by weight of spirits of camphor and half as much oil of cedar is a mixture recommended by Dr. Howard, and one which the author has used with good results. A few drops of this mixture poured on a bath towel at the head of a bed, and a little 
rubbed on the face and hands if the mosquitoes are very persistent, was found by Dr. Howard to last long enough through the night to be effective against all mosquitoes except the yellow fever species, Aëdes calopus, which begins its attacks at daybreak.

Elimination and Exclusion from Buildings. - The second means of controlling mosquitoes, by eliminating and excluding them from dwellings, is of more permanent value than the first, and should never be omitted while the process of mosquito extermination is under way.

One of the best methods of ridding houses of mosquitoes after they are once in is fumigation, and this is also an indispensable method of destroying hibernating mosquitoes in cellars, attics, barns, etc. The substance used for fumigation must depend on the kind of place to be fumigated, and on the conditions under which it is done. The most thorough and certain method of fumigation, when the place to be fumigated can be vacated, is by the generation of hydrocyanic acid gas. A less dangerous and equally effective method, but one which is injurious to metals and house furnishings is by the use of fumes of burning sulphur. These methods of fumigation are described in Chap. XXII, pp. 383-386.

Fumigants which are not dangerous to human beings can be used effectively against mosquitoes since these insects do not require such penetrating fumes as are necessary to destroy hiding parasites, as bedbugs and lice. Pyrethrum or Persian insect powder, manufactured out of the dried flower heads of certain species of chrysanthemums, is an effective fumigant of this type; it can either be dusted into corners, blown into the air of a room, or burned. Powdered jimson weed, Datura stramonium, is recommended by Dr. Smith, eight ounces, mixed with one-third its weight of niter or saltpeter to make it burn more readily, being burned per 1000 cubic feet. "Mimm's Culicide" is a volatile liquid made of carbolic acid crystals and gum camphor in equal parts by weight, which is effective against mosquitoes, four ounces being volatilized by heating for every 1000 cubic feet of space. A fumigant which has come into great favor in the last few years is cresyl; 75 grains to 35 cubic feet is sufficient to kill all mosquitoes, and in this dilution it is not injurious to man or other higher animals. It is not injurious to metals or to household goods. 
In camps which are not mosquito proof, the only effective means of obtaining comfort is by the use of smudges as described for blackflies (p. 484).

Protection of houses against mosquitoes is almost a necessity in many places. To a certain extent the construction of a house affects the number of mosquitoes attracted to it. Light, airy rooms with white walls are much less infested with mosquitoes than are dark, damp houses. Ross says that houses decorated with curtains, pictures, stuffed chairs and similar "barbarous" furnishings are entirely inappropriate for the tropics, and he deplores especially the use of curtains since they " check the breeze which is so cooling to the inmates and so unpleasant for mosquitoes."

The careful screening of houses or rooms is highly valuable, especially in places where mosquito-borne diseases are prevalent. Mosquito net or screen should never be less than 18 meshes to the inch. Cloth net is more effective than wire, since mosquitoes cannot as readily force their way through, but nets with thin threads should be used and should be stretched tightly in order not to exclude the breeze in hot weather. ' The use of tight canopies over beds is extensively practiced in southern United States, especially in malarial districts, and these are very commendable when kept in good repair. Most firms dealing in camp outfits place on the market light folding frames covered with mosquito netting for use when resting or sleeping out of doors in mosquito-infested places.

Usually a few mosquitoes find their way into screened rooms in spite of the screens, through unnoticed crevices, opening of doors and the like. These can usually be discovered and destroyed with a fly spanker, or, what is just as effective in case spotting the walls with blood is to be avoided, by holding a cup of kerosene directly under them. The mosquitoes are stunned by the vapor and fall into the cup in a few seconds. Mosquito traps have been found useful in some places, these contrivances consisting merely of a box, dark colored inside, placed where it will readily be found by mosquitoes and utilized as a hiding place. The box is arranged so that the insects do not readily find their way out and so that it can be fumigated easily.

Larvicides. - Far more effective and satisfactory in every way as a method of coping with mosquitoes is their actual ex- 
termination, not necessarily in a whole continent or a whole country, but in local places. Only comparatively recently has the local extermination or even reduction of mosquitoes ceased to be looked upon as too vast an operation to be undertaken. Because ponds or marshes were known to exist, perhaps miles away, the value of destruction of such breeding places as rain barrels, tin cans full of water, cesspools and troughs was looked upon as a mere drop in the bucket. Knowing as we do now that in most cases every annoying mosquito which attacks us was born and bred within 200 yards of where we meet her, the local extermination of mosquitoes has taken on a very different aspect. It is difficult for the uninitiated to realize that the mosquitoes which make life miserable for him did not travel from distant marshes and ponds but were probably bred in his own backyard or in his own living room.

Wonderful results have been obtained by the destruction of larvæ in their breeding places. This is accomplished either by pouring into the water some substance which will form an emulsion, and will destroy the larvæ when very dilute, or by pouring or spraying some oil on the water which will spread out and form a thin film over the whole surface. When the larvæ rise to obtain air through their breathing tubes or pores, the latter become plugged by a tiny bit of oil, and the larvæ drown. It has recently been pointed out by Lima, in Brazil, that the drowning is hastened by the coating of the body of the larvæ by the oil, especially in Anopheles, so that air cannot be absorbed through the body wall.

The oil film is the method most commonly employed, especially for use on a small scale. Except for wind-swept bodies of water, ordinary petroleum is as cheap and efficient as any oil that can be obtained. The oil film is so thin and light, however, that it is blown aside by a high wind, and a considerable portion of the water left uncovered. Different grades of oil can be used, varying with conditions. The thick heavy grades do not readily form a uniform film, especially if obstructed by water weeds, whereas the very thin oils evaporate rapidly, and the film is easily broken. Howard, Dyar and Knab recommend a grade known as "light fuel oil" for ordinary use. These authors state that about one ounce of petroleum to 15 square feet of water surface gives satisfactory results, and produces a film which lasts for 
ten days. Films of heavier oils or heavy and light oils mixed last longer, and need be renewed only once in two, three or four weeks, according to conditions. Thin oil will spread into a film if simply poured on the surface, but heavier oils are best sprayed on. In Africa mops made by tying kerosene-soaked cloths on long sticks are used for spreading the oil and in Panama waste cloth soaked in oil is placed where a slow flowing stream will constantly take a thin film from it.

In the tropics the use of petroleum has often been found impracticable on account of the rapid evaporation, continued heavy rains, and the interference made by the luxuriant and rapid growth of water plants and algæ and the formation of an interfering scum from a combination of the oil and dead algæ. For this reason substances which are actively poisonous to the larvæ and which form an emulsion in the water are used instead. An almost ideal larvicide of this type is now made at Ancon, C. Z., in enormous quantities. It is made of crude carbolic acid, powdered resin and caustic soda, heated together to make a black liquid resin soap which readily forms a milky emulsion with water. It destroys Anopheles larvæ in 16 minutes in an emulsion of one part in 5000. It also kills larvæ in mud, and destroys grass, algæ and water weeds in which larvæ ordinarily hide. Recently cresol has been advocated as a larvicide, dilutions as low as one part per million being said to be fatal in a short time. Powdered paraform sprinkled on water is said by Roubaud to be specifically fatal to mosquito larvæ.

Prevention of Breeding, and Natural Enemies. - The most valuable method of reducing mosquitoes, where practicable, is to obliterate breeding places or to make them uninhabitable for the larvæ. The first step in reducing mosquitoes is to see that there are no flower-vases or other water receptacles serving as aquaria for the larvæ, that there are no water-filled tin cans in the garbage heap or that the roof or street gutters do not hold standing water. Any rain barrels, cisterns, cesspools or small reservoirs which cannot be disposed of can be made harmless by screening. Pieces of low ground, temporary pools, etc., can usually be eliminated by draining.

The natural enemies of mosquito larvæ can often be exploited successfully for destroying them. Dr. Smith found that one of the most potent factors in the reduction of mosquitoes in the 
great tidal salt marshes of the New Jersey coast were the various species of killifish. These fish abound wherever the marshes are constantly flooded and push into places where there is barely enough water to cover them, and are so active in destroying mos-

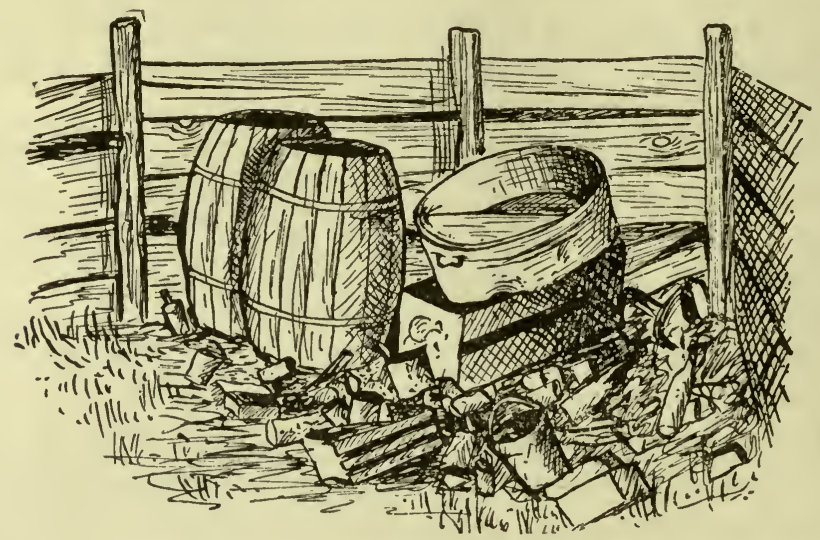

FIG. 207. One of the first places to clean up in a mosquito campaign. A favorite breeding place for such annoying or dangerous species as the yellow fever mosquito, Aëdes calopus, the house mosquitoes, Culex pipiens and C. quinquefasciatus, Anopheles quadrimaculatus, and others.

quito larvæ that the latter can exist only in high-lying or shut-in portions of the marsh over which the tide only occasionally sweeps and to which the "killies" do not penetrate. Knowing the value of killifish as destroyers of larvæ, the problem of preventing the marshes from producing countless mosquitoes resolves itself into so draining that the water on it either will be drawn off at every low tide or will be constantly stocked with fish. A number of workers have recently remarked on the folly of oiling pools which could be stocked with fish, since the oil kills the natural enemies of the larvæ and is not permanent. Instead it is urged that fish be propagated in such pools. The water weeds, however, should be removed and overhanging plants cut back so that the fish can operate freely in their pursuit of larvæ. In the case of swamps it is suggested that a permanent pond be constructed at the lowest level and stocked with fish, and the swamp drained into the pond.

A fresh-water fish of the same family as the killifish (Cyprinodontidæ) known as "millions" (Girardinus pœciloides) has been 
found very efficient as a destroyer of mosquito larvæ and has been extensively introduced into various parts of the tropics from its home in Barbados and other West Indian Islands. Except where other fish are present to prey upon it, this tiny
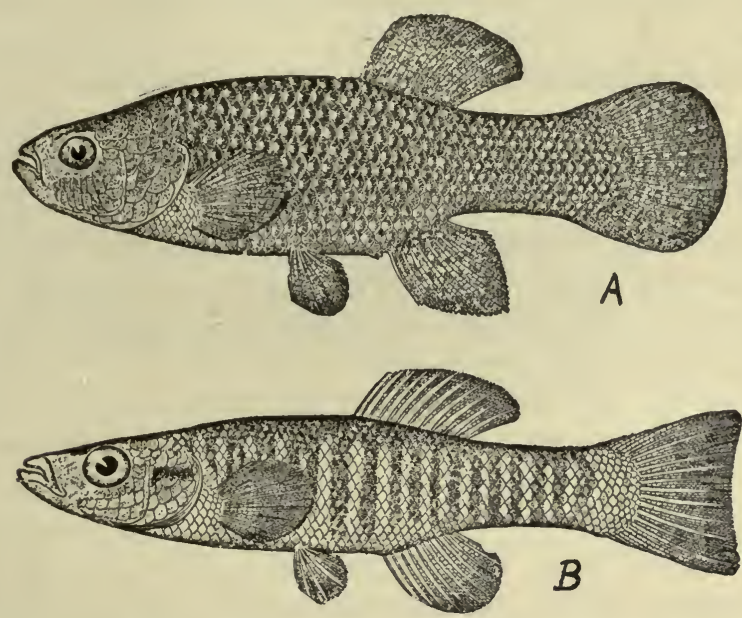

Fig. 208. Some good natural enemies of mosquitoes; $A$, common killifish, Fundulus heteroclitus, of great value in brackish marshes; $B$, fresh-water killifish, Fundulus diaphanus, valuable in fresh-water streams and ponds. $\frac{3}{4}$ nat. size. (After Jordan and Evermann.)

fish usually thrives wherever introduced, and carries with it a noticeable diminution in mosquitoes. Other species of the same family occur in various parts of the world and are almost invariably deadly enemies of mosquito larvæ.

Other natural enemies of the larvæ besides fish might well be encouraged in ponds or reservoirs. The western newt or waterdog, Notophthalmus (or Diemyctylus) torosus, which is abundant all along the Pacific Coast of the United States, has been observed to feed very largely on larvæ. In Oregon the author has observed grassy pools, which were otherwise ideal breeding places for mosquitoes but which contained numerous water-dogs, absolutely free of larvæ, whereas other pools not a quarter of a mile distant in which no newts were found were swarming with larvæ and pupæ. Recent experiments by the author have demonstrated conclusively that this salamander can be utilized successfully to keep mosquito larvæ out of such receptacles as rain barrels, troughs, etc. 
Other efficient enemies are whirligig beetles (Gyrinidæ), predaceous diving beetles and various aquatic predaceous larvæ, including some species of mosquito larvæ. Among birds, ducks have been quoted as efficient destroyers of mosquitoes and Dixon of Pennsylvania recently demonstrated their ability to keep ponds free of larvæ; he believes the mallard duck surpasses any other creature in the number of mosquito larvæ and pupæ which it can destroy. As destroyers of adults the value of such birds as swifts, nighthawks, swallows, etc., is well known. Bats, also, have been exploited as mosquito destroyers. The erection of "bat roosts" for propagation of these animals has been tried in Texas, and was found to reduce markedly the numbers of mosquitoes, and was financially profitable on account of the guano which could be collected. 


\section{CHAPTER XXVI}

\section{OTHER BLOOD-SUCKING FLIES}

Importance. - Although the mosquitoes hold the center of the stage as regards importance as human parasites, there are many other members of the order Diptera which affect the welfare of the human race. From a medical point of view the Diptera are far more important than all other arthropods put together. Besides the mosquitoes, which we have seen are the transmitters of at least four and probably five diseases, two of which are of prime importance, the Diptera include the Phlebotomus flies, which are known to be the sole disseminators of phlebotomus or three-day fever, and are believed to be the transmitters of verruga in Peru and of oriental sore in North Africa and possibly other places; the tsetse flies, which are transmitters and intermediate hosts for the trypanosomes of sleeping sickness; the stable-fly and other biting allies of the housefly, which may carry the bacteria of anthrax and other diseases from dead or dying animals to human beings; the gadflies or horseflies, one species of which is incriminated as the transmitter of the African loa worm, and all of which may act in the same capacity as stable-flies, to transmit bacteria mechanically from the blood of a diseased animal to a healthy animal or person; and the blackflies (Simuliidæ) and "no-see-ums" (Chironomidæ), which are sometimes terrible pests though not known to be disease carriers. Besides these blood-sucking species, the Diptera include also all the insects which live in the human body as maggots, and also the housefly and allied species which, though not properly to be considered parasites, are nevertheless of incalculable importance as mechanical spreaders of disease germs.

General Structure of Diptera. - To understand the relations of these numerous important insects and their classification, we must make a brief survey of the characteristics and classification of the order Diptera. The whole order can usually be 463 
distinguished readily from other insects by the fact that there is only one pair of membranous wings, the second pair of wings being represented only by an insignificant pair of knobbed rodlike appendages known as halteres (Fig. 191, halt.). The head is joined to the thorax by a very slender flexible neck. The thorax itself consists of one mass on account of the fusion of its three component parts, and the abdomen consists of from four to nine visible segments and is terminated by the ovipositors or egg-laying organs in the female, and by the copulatory organs in the male. The head is provided with a pair of antennæ, a pair of maxillary palpi and a proboscis composed of or containing the mouthparts. The antennæ and also the palpi are of considerable use in classification; the extent of the variations in the antennæ may be gathered from Fig. 211. The mouthparts are profoundly modified in accordance with the habits of the flies. In the botflies, in which the adults live only long enough to reproduce their kind, the mouthparts and even the mouth are much degenerated; in the non-blood-sucking forms, such as the common housefly, the mouthparts are more or less fused into a fleshy proboscis which is used for lapping up dissolved foods; in the blood-suckers, which are the forms that particularly interest us here, the mouthparts are developed into an efficient sucking and piercing apparatus. In some, e.g., mosquitoes (Fig. 190) and horseflies (Fig. 225), the lower lip acts as a sheath for the other parts which are fitted for piercing and sucking; in others, e.g., the stable-fly, Stomoxys (Fig. 240), and the tsetse flies, Glossina (Fig. 229), the lower lip itself forms a piercing organ, and the epipharynx and hypopharynx form a sucking tube, the mandibles and maxillæ being absent.

Life Histories. - All of the Diptera have a complete metamorphosis (see p. 329), and sometimes undergo a most profound remodeling of the entire body during the usually short pupal stage. The life history, beyond the fact that a complete metamorphosis occurs, varies within very wide limits. Most flies lay eggs, but some, e.g., the screw-worm fly, Cochliomyia (or Chrysomyia), and allied species, produce newly hatched larvæ or eggs which are just at the point of hatching, while still others, e.g., the tsetse flies, Glossina, do not deposit their offspring until it has undergone its whole larval development and is ready to pupate. 
The larvæ of Diptera may be simple maggots with minute heads and no appendages and capable of only limited squirming movements, e.g., the screw-worms (Fig. 250), or they may be quite highly developed, active creatures, e.g., the larvæ of mosquitoes, midges, etc. Many are aquatic, many others terrestrial; usually the eggs are laid in situations where the larvæ will find conditions suitable for their development, and the flies often show such highly developed instincts in this respect that it is hard not to credit them with actual forethought. The pupæ of the Diptera also vary widely. In one great suborder, Orthorrhapha, the pupa is protected only by its own hardened
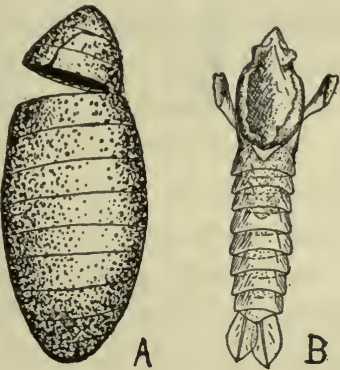

Frg. 209. Types of pupal cases, showing manner of emergence of adults. $A$, empty case of blowfly, typical co-arctate pupa of Cyclorrhapha; $B$, empty case of mosquito, typical obtected pupa of Orthorrhapha.

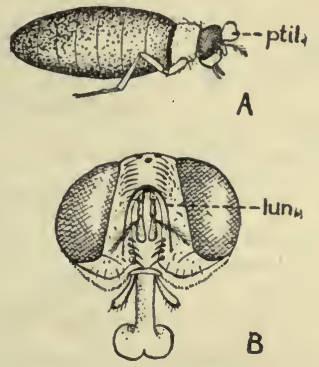

Fig. 210. $A$, fly emerging from pupal case, showing bladder-like ptilinium (ptil.) by means of which the end of the case is pushed off; $B$, face of fly showing scar or lunule (lun.) left by drying up of ptilinium. (After Alcock.)

cuticle, and is often capable of considerable activity; from this "obtected" type of pupa (Fig. 209B) the adult insect emerges through a longitudinal slit along the back. In the other suborder, Cyclorrhapha, the pupa retains the hardened skin of the larva as a protective covering or "puparium," and is usually capable of very slight movement; from this "co-arctate" type of pupa (Fig. 209A) the adult escapes by pushing off the anterior end of the puparium with a hernia-like outgrowth on the front of the head. This outgrowth, called the "ptilinium " (Fig. 210A), shrinks after the fly has emerged, but leaves a permanent crescent-shaped mark on the head known as the "frontal lunule" (Fig. 210B) which embraces the bases of the antennæ, and gives 
a dependable clue to the early life of the insect. Adult flies are usually not long lived, and often live only a few days, just long enough to copulate and lay their eggs. Some species, however, e.g., mosquitoes, may live for several months.

The order Diptera, as already indicated, is

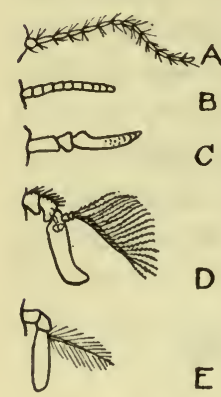

Fig. 211. Types of antennæ of Diptera; $A$, mosquito, female; $B$, blackfly: $C$, gadfly (tabanid); $D$, tsetse fly; $E$, stable-fly.

that they deserve separate consideration and have been discussed in a chapter by themselves (Chap. XXV).

\section{Phlebotomus Flies}

General Description. - Phlebotomus flies, otherwise known as "sandflies" or "owl-midges," are minute mothlike midges which are found in nearly all warm and tropical climates of the world, with the exception of Australia and the East Indies. In Australia (Queensland) they are represented by an allied fly of the same family, Pericoma townsvillensis, which is said to be a very severe biter, producing swellings which may last three weeks. They belong to the family Psychodidæ, which includes a large number of species of flies found all over the world, nearly all of which resemble tiny moths on account of their very hairy bodies and mothlike pose. The latter characteristic, however, is not shared by the genus Phlebotomus. The latter is the only genus, except Pericoma, containing habitual blood-suckers with a long proboscis; in all other members of the family the proboscis is short and inconspicuous. 
The phlebotomus flies (Fig. 212D) are small dull-colored insects, usually yellowish or buff, slender in build, with hairy. body and very long and lanky legs. The hairy-veined wings are narrow, somewhat the shape of mosquito wings, and are held erect over the body when the insect is in repose. The wings are quite remarkable for the inconspicuousness of the crossveins which gives them the appearance of having nine or ten nearly parallel longitudinal veins. The antennæ are long, consisting of a series of beadlike segments with whorls of hairs at the joints. The
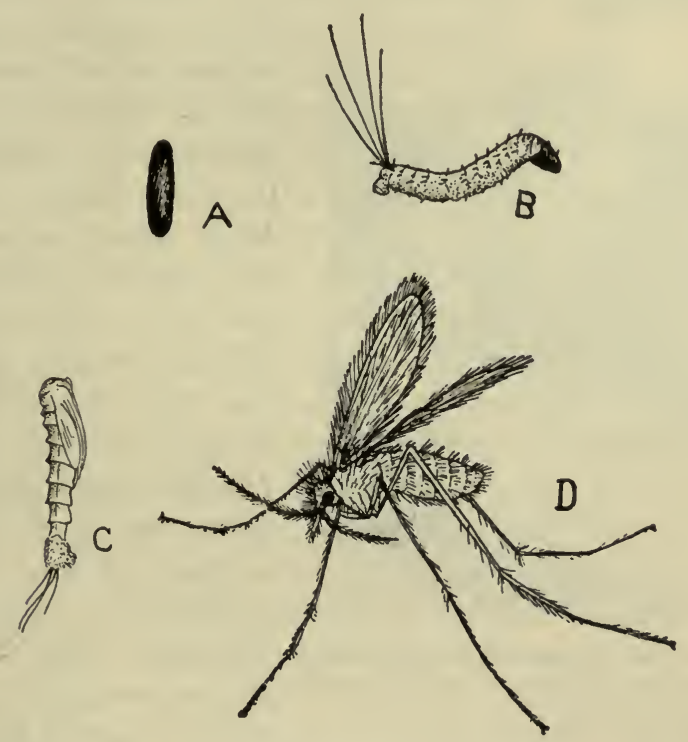

FIg. 212. Life history of phlebotomus fly, Phlebotomus papatasii; A, egg; $B$, larva; $C$, pupa; $D$, adult. $A, \times 80 ; B, C$ and $D, \times 8$. (After Newstead.)

relatively long proboscis is made up in practically the same way as is that of a mosquito (see p. 426), except that the needle-like organs project beyond the tip of the sheath made for them by the labium or lower lip. These insects are usually less than one-fifth of an inch in length and often not over one-eighth of an inch; they can easily crawl through the meshes of an ordinary mosquito net, and are therefore hard to avoid. Their bites are very annoying and cause an amount of irritation which seems quite out of proportion to the size of the insects. In most cases it is only the female which sucks blood, but in some species the 
male has a proboscis equally well fitted for piercing skin and sucking blood, and the male of at least one African species is known to bite as well as the female. Most if not all of the species are nocturnal or become active at twilight only. In Corsica, for instance, it is said to be very difficult to capture these midges except for about one hour after sunset. During the daytime they remain hidden away in dark corners, cellars, crevices of rocks, etc.

Life History. (Fig. 212.) - Most species of Phlebotomus lay their eggs in crevices of rocks, in damp cracks in shaded soil, on

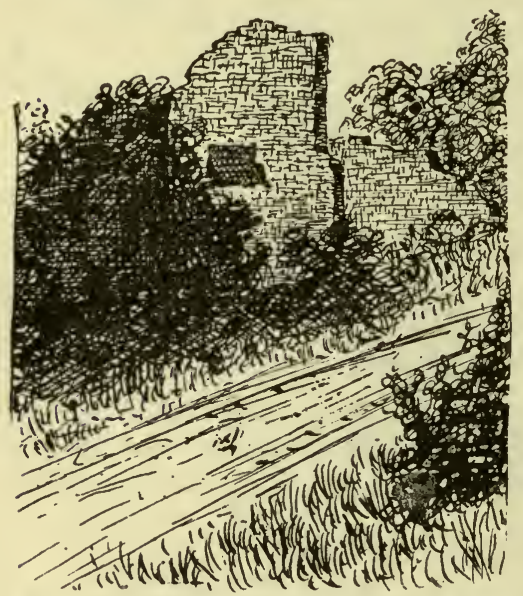

FIG. 213. An earthquake ruin in Sicily, affording favorite breeding places for phlebotomus flies. moist rubbish, in crannies or chinks in cement of dark cellars, between boards in privies and cesspools, and in other similar situations. Most species seem to show a decided preference for crevices in rocks, and find ideal situations in ruins of old stone buildings, crumbling rock fences, etc. In Malta Captain Marett found these insects breeding only in such places. In Peru, according to Townsend, the universal type of fence, a structure of rubble and loose rock, provides ideal breeding places for the species found there, whereas in Italy and Sicily the earthquake ruins furnish equally ideal breeding places for them (Fig. 213). The sandflies which occur in certain parts of Egypt are believed to breed in damp cracks in the sandy soil, since there seem to be no other suitable places.

The eggs are about 40 to 50 in number and are usually all laid at approximately one time, being literally shot out by the female to a distance several times the length of the abdomen. The eggs are viscid and adhere to the surfaces with which they come in contact; it would seem that the peculiar method of ejecting the eggs is a protective adaptation, facilitating their deposition in the farthest reach of a crevice where even the tiny insect itself 
could not penetrate. The eggs are elongate and are of a dark, shiny brown color, with fine surface markings which vary in different species (Fig. 214).

The incubation in the case of the common Old World $P$. papatasii requires from six to nine days under favorable conditions, but the eggs are very susceptible to external conditions, and die quickly if exposed to sunlight or if not kept damp. The larvæ (Fig. 212B) are tiny caterpillar-like creatures with a relatively large head with heavy jaws (Fig. 215), and with two pairs of bristles on the last segment of the abdomen, one pair of which are sometimes nearly as long as the body and are held erect and spread out fanlike; in the newly hatched larvæ there is only one pair of bristles. The body is provided with numerous tíothed spines which give it a rough appearance. These spines have recently been shown by

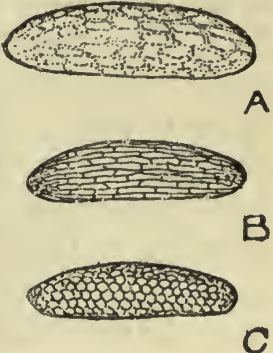

Fig. 214. Eggs of phlebotomus flies; $A, P$. papatasii; $B, P$. argentipes; $C, P$. minutus. $X$ about 200. (After Howlett.) Howlett to differ in different species and, together with the relative length of the caudal bristles, to form good identification marks. The whole length of the larva of $P$. papatasii when full grown is less than one-fifth of an inch, and is therefore not so

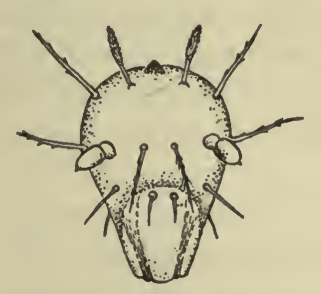

FIG. 215. Front view of head of Phlebotomus minutus larva. Much enlarged. (After Howlett.) large as an ordinary rice grain. It is quite active in spite of the fact that it has neither legs nor eyes; it progresses in the manner of a caterpillar, holding to a rock or board with the tip of the abdomen while stretching the body, then hiding with the doubled-under head while drawing up the body again. It feeds on decaying vegetable matter, and probably also on moulds, etc. When exposed to light the larva of $P$. papatasii has the peculiar habit of flicking itself off the surface on which it has been resting. On approach of danger, Phlebotomus larvæ often "play 'possum" and feign death.

The full development of the larvæ requires from three weeks to two months or more, depending almost entirely on the temperature. Larvæ which hatch at the beginning of cold weather 
do not pupate until the following spring. When, after several moults, they go into the resting pupal stage the last larval skin with its caudal bristles remains adhering to the posterior end. The pupa (Fig. 212C) is characterized by a very rough cuticle over the thorax, but can be identified best by the adhering larval skin. It is colored so much like its surroundings, and looks so much like a tiny bit of amorphous matter, that it is very difficult to find. In warm weather the adult insect emerges after from six to ten days, but this is much prolonged by low temperatures. The entire life cycle from the laying of the eggis to the emergence of the adults may be passed through in a month in hot weather, according to Howlett's observations on an Indian species, though it takes two months or more in cool weather. In Malta, according to Newstead, the cycle takes about three months.

Phlebotomus and Disease. Phlebotomus Fever. - Although sandflies have been accused of transmitting a number of human diseases in various parts of the world, in most cases their actual rôle has not been determined beyond doubt. The most important relation of sandflies to disease is in connection with a relatively mild febrile disease sometimes known as three-days, fever, but more commonly known as phlebotomus fever or papataci fever from the name of the transmitter, Phlebotomus papatasii. The nature of the disease and the rôle of the sandfly in carrying it is discussed in Chap. X, p. 188. As in the case of many other insect-borne diseases, the relation of the insects to the disease was suspected for a long time before the scientific proof of it was made. It was not until 1908 that Doerr demonstrated the part played by sandflies.

The principal species concerned in the transmission of phlebotomus fever is $P$. papatasii, but it is possible that other species, especially $P$. perniciosus and $P$. minutus, both of Mediterranean countries, may also be involved, though as far as is known the disease does not occur outside the range of the first-named species except at Aden.

$P$. papatasii (Fig. 212D) is of medium size, reaching about oneeighth of an inch in length, pale yellowish gray in color with a dull red-brown stripe down the middle of the thorax and a spot of the same color at either side. It is found in many parts of southern Europe, North Africa and in southern Asia. It has the typical habits of the genus, preferring to lay its eggs in 
crevices in damp cellars, in caves, cracks in broken walls, etc. In Malta the life cycle of this species has been observed to take about three months, but under ideal conditions it would probably be shorter.

The adult fly, as observed in Malta, where it has been most extensively studied, chooses caves, catacombs and other similar places as its favorite localities. On still, warm nights it is common in houses, but rarely appears when there is a cool fresh breeze. Some houses were found to be much more infested than others, possibly due to the proximity of suitable breeding places and to the lack of breezes. Newstead found that dark rooms on the sheltered side of the first floor of a house were most likely to be infested; only one individual was found on the second floor. The distance which the adults travel is thought to be very short, but they may be carried by public conveyances, and infection has been known to be transplanted long distances by flies carried on coasting vessels.

Phlebotomus and Other Diseases. - Sandflies have frequently been suspected of complicity in the spread of the parasites of oriental sore, though no definite proof of this has ever been brought out. Wenyon, from his study of oriental sore at Bagdad, believed that these flies, as well as certain other insects, might easily be concerned in the spread of the infection, but he did not have an opportunity to test his belief. Recently a number of French workers in North Africa, including Laveran and the Sergents, have advanced the theory that $P$. minutus var. africanus is the carrier of the infection, and that certain lizards or geckos of the region, Tarentola mauritanica, serve as a reservoir for the disease. Parasites, closely resembling Leishman bodies which cause oriental sore, have been found in the blood of geckos taken near Tunis, and it is well known that reptiles are an important if not the prime source of food for the various species of Phlebotomus, and P. minutus especially harasses the North African gecko. Roubaud found a lizard in West Africa which was covered with gorged females of this species and in India $P$. minutus is said to prefer geckos to man as a source of food. It is interesting to note in this connection that the forest workers in Paraguay, where the more serious American type of leishmaniasis is found, believe the infection to be caused by the bite of blood-sucking arthropods which have fed on snakes. 
Phlebotomus minutus is a buff-colored sandfly. It is small, even for a Phlebotomus; the female measures only about $\frac{1}{12}$ of an inch in length and the male considerably less than this.

Other diseases with which Phlebotomus has been connected are two which occur together in certain regions of the Peruvian Andes, namely, Oroya fever and verruga peruviana (see Chap. $\mathrm{X}, \mathrm{p} .178$ ). These diseases, as pointed out elsewhere, have long been confused, and even yet are held by some investigators to be different phases of the same disease. Townsend, of the U. S. Department of Agriculture, spent two years in Peru investigating the diseases (which he considers identical) and came to the conclusion that Phlebotomus verrucarum is the transmitter, basing his conclusions on the distribution and habits of the insect, and on certain experiments which he undertook. The sandfly in question, which was discovered and named by Townsend, is the only nocturnal insect which is closely limited in its distribution to approximately the same localities as is Oroya fever and verruga, and it seems to be well established that the disease is contracted at night. Townsend believes that he obtained proof of the transmission of verruga, and obtained a typical breaking out, by injecting into a dog the macerated bodies of insects which had fed on a verruga patient, but his results have not been widely accepted. If, as is now more generally believed, Oroya fever and verruga are really distinct, then it is possible that $P$. verrucarum may be the carrier of both diseases, or of either one or the other. If this insect acts as a carrier for both diseases, which would be a very unusual situation, this fact would explain the close limitation of the two diseases to nearly the same zones, and would also explain the frequency with which the two infections occur simultaneously or following each other. That oroya fever is an insect-borne disease is almost certain, and it is quite likely that the sandfly discovered by Townsend will be found to be the carrier of it. Verruga, however, is a smallpox-like disease and may be contagious rather than infective.

Phlebotomus verrucarum is a species of sandfly which breeds principally in the damp recesses of the loose rubble fences which are so universally used in Peru, and probably feeds largely on a species of lizard, Tropidurus peruvianus, which inhabits the same rock fences. According to Townsend it requires for its life cycle a fairly high total of summer heat and much moisture, with an 
absence of night fogs and of low winter temperatures. The adults will not live where there are continuous strong air currents. These conditions limit the species closely to the deep-cut canyons or "quebradas" (Fig. 53), between 3000 and $8000 \mathrm{ft}$. elevation, on the west face of the Andes. There is certainly a remarkable agreement between this distribution and that of Oroya fever.

Control. - Sandflies are very difficult insects to deal with, both on account of the small size of the adults and of the nature of the breeding places.

The only precaution that can be employed to keep the adults out of houses during warm weather is the use of repellents. Spraying mosquito netting with some repelling substance, such as odorous oils, e.g., anise oil, eucalyptus oil, etc., or with a weak solution of formalin, or, in fact, with any of the repelling substances mentioned in connection with mosquitoes, serves to keep the insects out as long as the odor lasts. The insects are attracted toward a light, and are therefore usually very abundant in lighted rooms on warm still nights. A gentle breeze or a current of air from electric fans placed near the windows prevents their entrance and, as has already been mentioned, upstairs rooms are practically immune. Personal protection can be obtained by applications of repellents. Townsend recommends equal parts anise oil, eucalyptus oil and oil of turpentine in a boric acid ointment.

It is almost impossible to destroy sandflies in their early stages. Townsend thinks that the elimination of rubble fences in Peru would reduce their numbers, at least locally, but it would be far from a simple problem to destroy all possible breeding places, even within a very small radius. In Europe, where stone and cement are more extensively used than in America, the problem is still greater. The earthquake ruins of Sicily, as has been mentioned before, give unlimited breeding places. The large numbers of these insects in parts of Egypt where such places are not available indicate that damp cracks in soil may be utilized as breeding places, and it would be obviously impossible to eliminate these or to treat them thoroughly.

\section{True Midges (Chironomidæ)}

General Account. - The family Chironomidæ comprises a large number of species of small flies, sometimes almost microscopic, found all over the world. The larger ones quite closely 
resemble mosquitoes except for the absence of the long proboscis, and the dancing flocks of these insects which can be seen over pools or swamps on any summer day are usually taken for mosquitoes without question. As expressed by Riley and Johannsen, " these midges, especially in spring or autumn, are often seen in immense swarms arising like smoke over swamps, and producing a humming noise which can be heard for a considerable distance." In such swamps the larvæ, most of which are aquatic and live in the mud or amid aquatic vegetation, may be scooped up, literally by the shovelful. Fortunately the great majority of these insects are quite harmless, in fact, inasmuch as the larvæ are an important food for young fishes, they are distinctly beneficial. The blood-sucking species belong to the subfamily

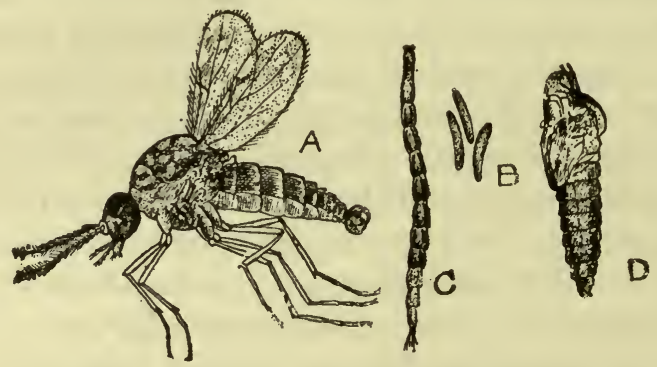

Fig. 216. Life history of blood-sucking midge, Culicoides; $A$, adult male $(C$. reticulatus), $\times 5 ; B$, eggs $(C$. marium $), \times 18 ; C$, larva $(C$. reticulatus $), \times 5 ; D$, pupa (C. marium), $\times 10$. (After Lutz.)

Ceratopogoninæ and are very small; only the females are known to suck blood. They are well known to hunters and anglers and other frequenters of the woods in most parts of the world. In America they are usually called "gnats" or "punkies" and in the West are known as "no-see-ums," on account of their very small size.

These insects (Fig. 216) can usually be distinguished from allied insects by the peculiar venation of the wings, the first two veins being very heavy while the others are indistinct. Though the bodies, and sometimes to a slight degree the wings, are more or less hairy the scales so characteristic of mosquitoes are absent. The proboscis is never long even in the blood-suckers, and one is led to marvel at the irritation which can be inflicted by such a small insect with such a small organ. Usually midges 
rest with the front legs elevated, though not all species have this habit. In most Chironomidæ the thorax of the adult insect projects like a hood over the head, but in the subfamily Ceratopogoninæ, which alone interests us here, this is not the case, and this negative characteristic is the best distinguishing mark of the subfamily.

There are a number of genera and many species included in this group of blood-suckers, but they fall naturally into two groups according to the habits and structure of the larvæ. In one, of which the principal genera are Ceratopogon and Forcipomyia, the larvæ differ from all other Chironomidæ in being terrestrial, living in damp places under bark, stones, moss, etc., and in being covered with spines (Fig. 217). In the other group, of which the principal genus is Culicoides, the larvæ are orthodox in being aquatic and unspined (Fig. 216C); a few species are marine. Most

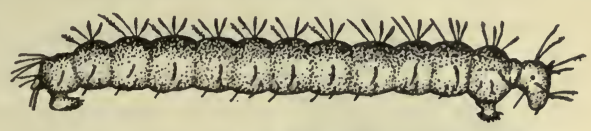

FIG. 217. Larva of Forcipomyia specularis. $\times$ 15. (After Malloch.)

of the blood-sucking midges become active at dusk, but if disturbed they will bite in the shade even on bright sunny days.

Life History. - The eggs of aquatic midges (Fig. 216B), several hundred in number, are laid in water, either floating free or moored to some object. Each one is covered with a gelatinous envelope, and the eggs adhere in chains or in little masses, thus resembling very diminutive bunches of frog or toad eggs. In about six days, more in case of low temperature, the eggs hatch into almost microscopic larvæ (Fig. 216C). The latter are wormlike creatures practically without hairs or spines in the aquatic species, but with conspicuous bristles in the terrestrial forms. Usually the only hairs present are in a pair of tufts on the last segment. In most midge larvæ there is a footlike outgrowth on the first and last segments of the abdomen. The larvæ have inconspicuous blood-gills for breathing in water, and therefore do not need air as do mosquito larvæ. Most midge larvæ are free-swimming, but some excavate tubes in mud and line them with a salivary secretion which hardens on contact with water. The food consists of microscopic plant and animal life. The pupa (Fig. 216D) rather resembles that of a mosquito, except that the abdomen is kept extended instead of curled under and 
the pupa floats in a vertical position, breathing through tufts of threadlike filaments which correspond to the breathing trumpets of mosquitoes. In the terrestrial forms the pupa retains the last larval skin hanging to its posterior end. The aquatic species of the subfamily Ceratopogoninæ are peculiar in that the pupæ must reach a dry surface before the adult will emerge. Little is known about the length of time required for the development from egg to adult, but it is probably comparable with that required by mosquitoes - two weeks or less to a month or more, according to temperature.

Annoyance. - The amount of annoyance which may be caused by midges is sometimes very great. The writer will never for-

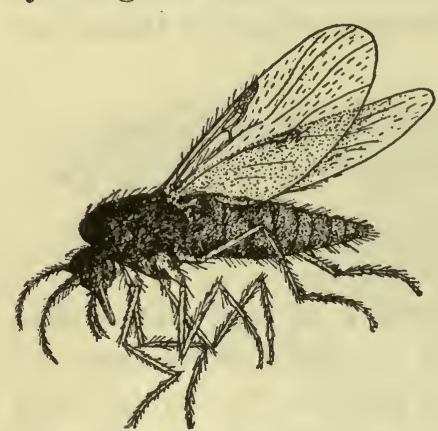

Fig. 218. A "punky" or "nosee-um," Culicoides, which is a scourge of fishermen and campers in the Cascade Mountains of Oregon. $\times 20$. get his experiences with them in a collecting and fishing trip in the Cascade Mountains of Oregon. The midge which proved itself troublesome, a species of Culicoides (Fig. 218), was very local in distribution, and always standing pools of shallow water were found in the near vicinity. The proximity of such pools was invariably proclaimed, towards evening, by the collection of great numbers of these insects on all exposed parts of the body, each one so minute as to be hardly visible, but in the aggregate sometimes giving the arm or shirt sleeve a dark gray color. Each one is presently the cause of an intensely itching spot. That the insects are attracted by animal smells is evident from the following experience. The writer had shot a rabbit and was skinning it. Almost immediately after the animal was cut open and the smell of the warm bowels exposed to the air the writer found himself attacked by myriads of these insects, and was bitten to such an extent as to be driven almost to a complete frenzy, until he discovered that only a few yards from the opened animal he was not attacked at all. The skinning of the rabbit was completed in the welcome protection of a dense smoke.

Midges as Disease Carriers. - Only in one instance have midges been accused of carrying disease. Two species of land- 
breeding midges, Forcipomyia ute and $F$. townsendi, have been incriminated by Townsend as the carriers and intermediate hosts of the protozoan parasite causing "uta" in Peru. Uta (see Chap. V, p. 86) is a form of leishmaniasis occurring on the western face of the Andes. According to Townsend, Leishman bodies are found in abundance in the digestive tract of these midges, and injection into laboratory animals of serum containing the ground bodies of captured insects resulted in the formation of sores which Townsend regarded as uta, and from which he obtained a few Leishman bodies. Two cases are cited, also, in which uta sores developed following the bites of the midges, and supposedly due to them. According to Townsend the infection is evidently transmitted by contamination of the wound made by the proboscis with infected excrement. That these insects are really the transmitters of uta in man cannot be considered as proved, but it must be regarded as a possibility. It should be recalled that many insects have been accused of carrying Oriental sore and allied diseases, among which are blackflies (Simulium), sandflies (Phlebotomus), gadflies (Tabanidæ) and others, and it is open to question whether any insect which harbors a $\mathrm{Her}$ petomonas in its gut may not be able to infect vertebrates if the germs reach the blood. If so, these midges must be regarded as conveyors of a Leishmania infection.

Little is known about these species of Forcipomyia, but it is probable that their habits are similar to those of better known species. In the North American species, the larvæ (Fig. 217) are slender whitish worms about one-eighth of an inch in length which live in damp places in moss and under bark, stones, etc. The pupæ are pale yellowish, later becoming brown.

Control. - The control of the aquatic biting midges is not difficult, and can be accomplished in the same manner as can the control of swamp-breeding mosquitoes, by draining, stocking with natural enemies or oiling. It is improbable that these midges breed to any extent in transient pools, for most of them, at least, prefer pools of standing water, abundant in organic débris and microscopic organisms. The terrestrial-breeding forms of Forcipomyia and Ceratopogon, like the sandflies, are practically impossible to exterminate.

Much protection from the adults can be obtained by the use of repellents as advised for mosquitoes and sandflies (see p. 455). 


\section{Blackflies or Buffalo Gnats}

General Account. - The blackflies, as annoyers of domestic animals and man, are among the most important of insect pests.

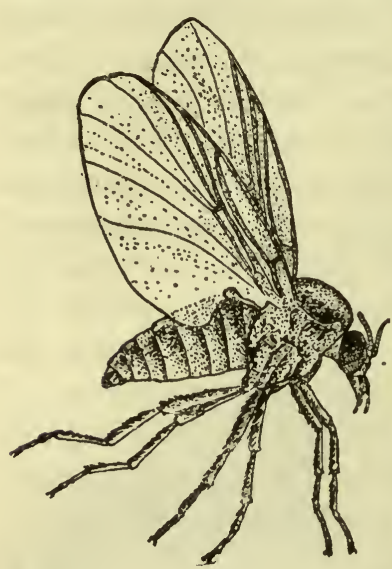

Fig. 219. Blackfly, Simulium pecuarum. $\times 7$. (After Riley.)

The females are most insatiable bloodsuckers, and have been known to attack cattle in such swarms as to kill them; a Himalayan species, according to Alcock, has been said to kill even human beings in the same way. These small insects, which constitute the family Simuliidæ, are quite unlike the other flies of the group to which they belong. Instead of the usual slender, long-legged, midgelike flies of this group we have in the blackflies small, robust, humpbacked creatures with short legs and broad wings, rather resembling, in a general way, miniature houseflies (Fig. 219). The antennæ are composed of 11 segments, but they are short and stocky, and have no hairs at the joints. The proboscis in the female is short but heavy and powerful, while in the male it is poorly developed. The mouthparts are made up of the same parts as in mosquitoes, but are dagger-like instead of needlelike (see Fig. 220). Most of the northern species are black in color, whence their name, but some of the species are reddish brown or yellowish, and they may be variously striped and marked. The wings are

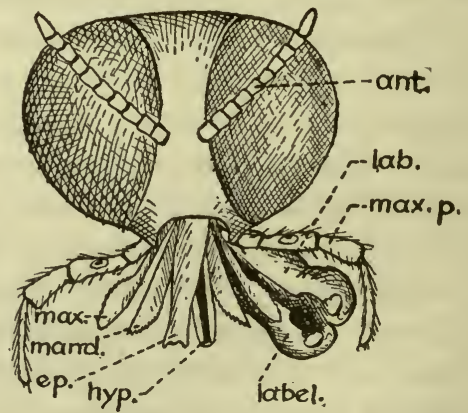

Fig. 220. Mouthparts of blackfly, Simulium; ant., antenna; ep., epipharynx; hyp., hypopharynx; lab., labium; label., labellum; mand., mandible; max., maxilla; max. p., maxillary palpus. (After Alcock.) either clear or of a grayish or yellowish color with the few heavy veins near the anterior margin often distinctively

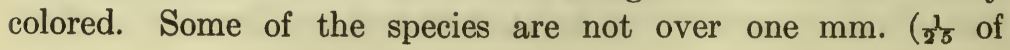


an inch) in length and the largest of them scarcely exceed onefifth of an inch.

Life History. - Unlike the mosquitoes and midges, blackflies breed in running water and few streams flow too swiftly for them. The eggs are laid in large masses, up to many thousands in number, by a number of females. The eggs (Fig. 221A), which are elliptical and yellowish and have a peculiar slimy coating, are deposited by some species on leaves or blades of grass which are occasionally licked by running water, the weight of the eggs sufficing to submerge. them; other species dart into the water and deposit directly on the slimy surfaces of submerged stones or twigs. The author found a favorite breeding place of the blackflies in the woods of Northern Ontario (species undetermined) to be on the slimy boards of old lumber chutes over which water was constantly flowing. It requires at least a week for the eggs to hatch.

The larva (Fig. 221B) as soon as hatched attaches itself by a sucker at the posterior end of the body to a stone or other submerged object. As expressed by Alcock, "one of the most characteristic attitudes of the

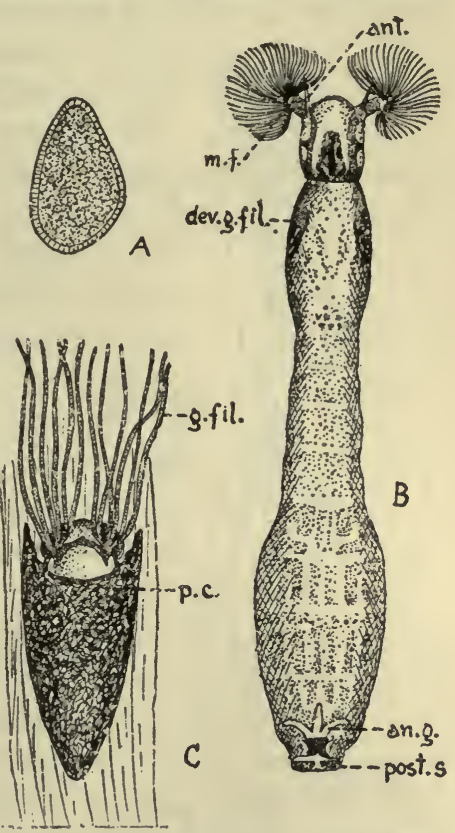

Frg. 221. Developmental stages of blackflies. $A$, egg of Simulium venustum; $B$, larva of S. bracteatum; $C$, pupa (in pupal case) of $S$. venustum; all much enlarged, not drawn to same scale; an. g., anal gills; ant., antenna; dev. g. fil., developing gill filaments of pupa; g. fil., gill filaments; m. f., mouth fans; p. c., wallpocket-like pupal case; post. s., posterior sucker. ( $A$, after Mecznikow from Jobbins-Pomeroy, others after Jobbins-Pomeroy.)

larva is to sit upright on the end of its tail, - to use the language of the poets of the daily press, - with its mouth fans standing out from its head like a pair of shaggy ears." The "mouth fans," which are very delicate and elegant, are used for sweeping microscopic particles into the mouth as they are brought by the running water. The stump of a leg on the 
first segment (Fig. 222 prol.) is used for creeping, in conjunction with the posterior sucker, the larva looping along like a " measuring worm"; it is also of use in constructing the silken cocoon from the secretions of the salivary glands. This single little leg has a crown of tiny hooklets which make it possible for the possessor to hold its ground even in a torrent of water. The

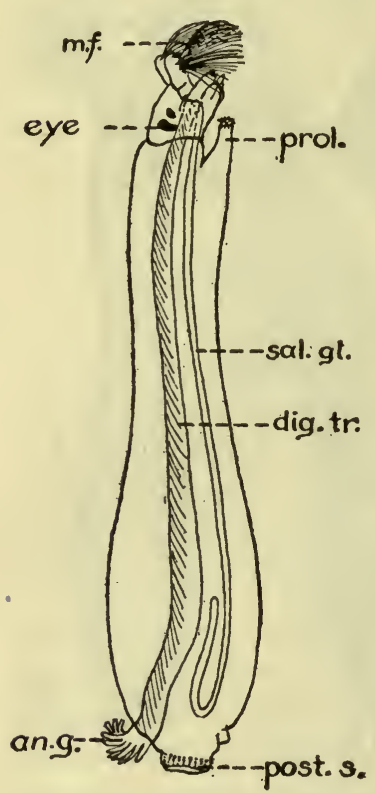

Fig. 222. Larva of blackfly, Simulium venustum, side view showing some of internal anatomy; an. g., anal gills; dig. tr., digestive tract; $m$. f., mouth fans; prol., proleg with sucker and hooks; post. s., posterior sucker; sal. gl., salivary and spinning gland. salivary glands referred to are quite unlike those of other insects, in that they extend clear back to the posterior end of the body (Fig. 222, sal. gl.). The fluid secreted hardens to silk at once on exposure to water, and is used not only in spinning the cocoon, but also in spinning anchoring threads and life-lines. According to Malloch, the larva when disturbed releases its hold and floats downstream, holding by the stumpy leg to a silken thread which is being spun out, and by means of which the insect later regains its former position. The larvæ breathe by means of tiny gills which can be projected through a slit in the last segment of the abdomen (Figs. 221 and 222, an. g.). The larvæ are never found solitary, as would be expected from the manner of laying eggs; the author has seen the boards on the bottom of a log chute completely covered with mosslike patches of these larvæ for areas of a square yard or more.

After four or five weeks, in summer, the larvæ prepare to go into the resting pupal stage, and spin for themselves a partial cocoon which is variously shaped like a jelly glass, slipper, wall pocket, etc., open at the upper end for the extrusion of the branching gill filaments which are used as breathing organs (Fig. 221C). Some species. simply spin a snarl of threads, the work of a whole community, in the meshes of which the pupæ exist in a fair state of protection. The general form of the pupæ can be 
seen in Fig. 223. The breathing filaments vary greatly in different species and may have from four to 60 branches.

The adults escape from the pupæ after from one to three weeks through a slit in the back, and are carried safely to the surface by a bubble of air which has been collecting inside the old pupal skin. The adults are short lived and lay their eggs soon after emergence. The whole life of a generation from egg to egg may be passed in from six weeks to two months or more. Some spe-. cies have several generations a year but the majority produce but a single brood a year. The Canadian species already referred to is seen only for a few weeks in May and early June, during which time it is locally excessively abundant. Most species are diurnal, but the author found the Ontario species to be most active from late afternoon until dark, and again early in the morning. This species will also bite readily at night in the presence of artificial light.

The species of blackflies are numerous, but are all included in the single genus Simulium, with several subgenera which some workers elevate to the rank of true genera. Some species do not attack man but viciously attack various domestic animals. While on a collecting trip in the Cascade Mountains of Oregon the author found it necessary to keep the pack

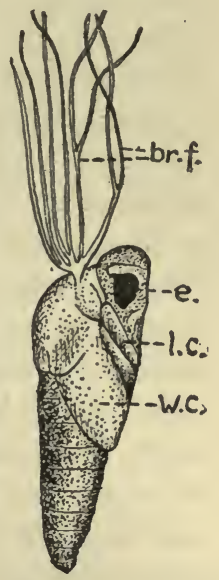

Fig. 223. Pupa of blackfly, Simulium jenningsi, removed from case; e., eye; 1.c., leg cases; br. f., breathing filaments or gills; w. c., wing case. (After Jobbins-Pomeroy.) animal picketed in the smoke of the camp fire constantly to protect the poor creature from the blackflies which congregated in large numbers about his eyes and nose, yet neither the author nor his companion was ever bitten by one of these flies. One of the most troublesome species in the United States is S. pecuarum, the famous buffalo gnat of the south central portion of the country. This species was formerly more abundant than now, and was a terrible scourge to mules and cattle. S. venustum is one of the most important molesters of man. It occurs over the greater part of the eastern portion of North America.

Annoyance. - In the estimation of the author, no insect scourge he has ever experienced is more terrible than an attack 
of blackflies as he encountered them in Canada. From accounts of other authors they must be equally terrible in other places. King, for instance, states that in parts of Sudan (Dongola) a species known as the nimetti, Simulium griseicollis, renders life a burden during the winter months. The famous Columbacz fly, S. columbaczense, of southern Europe is said to be a terrible pest, and there are instances of children having been killed by it. My own experiences occurred in the woods of Northern Ontario early in June. Upon arriving there I did not recognize Dr. Munford of Cornell University, with whom I had been quite intimate, until he spoke. He had been in the region about a fortnight. His face, neck and arms were so swollen from blackfly bites as to completely alter his appearance. The wrists were swollen until no constriction between hand and forearm was present. That evening, having been told of the manner in which deer came and stood in the water near the outlet of the lake, a mile or so from camp, I went in a canoe to watch them, being warned to tie my trouser legs tightly around my shoes and my coat sleeves to my gloves, and to fit a veil stretched from a broad-brimmed hat tightly around my neck. No repellents were at hand. With some impatience (having been bred among the mosquitoes of New Jersey) I submitted to these precautions, though I was careless in carrying them out, and made the trip to the outlet which is an old log chute, and the breeding place of the flies. In spite of the precautions taken, the blackflies, alighting on the veil in such numbers as to make it difficult to see through it, managed to find vulnerable spots in my armor. Unlike mosquitoes they alight and crawl; they found their way up under the veil, between the buttons of shirt and trousers, and through the cords at my wrists. In a few minutes I was driven almost frantic and could hardly restrain myself from diving into the lake to avoid the attacking flies, as did the deer. Each bite, and before I got to the safe haven of a dense smudge at camp I had hundreds of them, was only slightly painful; the flies drilled a tiny hole which bled a drop or two, so that the attacked parts of the body became completely smeared with blood. But this was not the end. The bites next morning were swollen, and itched somewhat; the swelling and irritation grew constantly worse until the third night, when each bite became the site of an oozing pimple. By this time the itching 
was so intense that I was in agony all night and could not sleep. Accompanying this there was a feeling of general "ennui" and despondence with some fever, due, no doubt, to the action of the poison injected by the numerous insects. Subsequent attacks by the flies, though always far from pleasant, were not so severe in their effects, a certain amount of immunity apparently having been built up. On account of the slow development of the symptoms it was my belief that possibly they were due to the injection of a living organism. Stokes, however, has shown that the effects of blackfly bites, essentially as described above, can be reproduced by the injection of material from preserved flies. An interesting suggestion is made by Stokes that possibly the first bites of the flies sensitize the body to the particular poison injected so that it reacts rather violently to subsequent injections of it. This phenomenon, which is known to occur in connection with many poisonous substances, is a form of anaphylaxis (see p. 24). Possibly the rashes produced by mites, lice, etc., may also be due to such a reaction.

As yet blackflies are not known to be the carriers of any diseases. A theory was rampant a few years ago that pellagra was due to a protozoan transmitted by blackflies, but it is now generally held that this disease is due to an imperfect diet, or rather to lack of the necessary assortment of substances in the diet, and so is in no way connected with blackflies or other insects.

Control. - Since blackflies breed in running water the methods to be employed in their extermination are quite different from those ordinarily used in the extermination of mosquitoes. One of the measures most widely used is the treatment of breeding streams with phinotas oil, a poisonous oil which forms an emulsion in the water and slowly soaks through it. In concentrations sufficient to destroy the larvæ, however, this oil is also destructive to fish. Often the breeding grounds of blackflies may be locally destroyed or reduced by damming the stream at intervals, leaving falls between, or in the case of small brooks by the construction of underground channels or of a drain-pipe line. The clearing away of roots and fallen logs from streams is often of value, in that it removes surfaces on which the eggs are laid, and obliterates the numerous small falls which are ideal for the larvæ. In larger streams the cultivation of fishes, such as trout, young bass, darters, etc., greatly reduces the number of black- 
flies if it does not eliminate them entirely. In such cases care should be taken that there are no small trickling streams which are not readily reached by fish. In the author's experience streams which harbor large numbers of caddis worms, dragon-fly larvæ and other carnivorous aquatic insects do not breed blackflies to any extent.

A considerable degree of protection from blackflies can be obtained by the use of repellents such as are used for mosquitoes, but their efficiency seems to be lost more quickly than in the case of mosquitoes. Moreover the crawling habits of the flies must be taken into account, and other parts of the body than those which are directly exposed must be treated. Blackflies may be driven from houses by fumigation with pyrethrum powder or by any other fumigation method. In camp life the use of smudges is indispensable. An efficient smudge which will last all night can be made in an old bucket with a few holes punched near the bottom. A small fire is started in this and then the bucket is filled with partly wet, punky, decayed wood which will smoulder slowly and produce a dense yellow smoke. Sleeping in the presence of such. a smoke is at first almost as unpleasant as are attacks by mosquitoes and blackflies (the latter becoming active only toward dawn) but one soon becomes accustomed to it, and it has none of the terrible after-effects of an attack by the flies.

\section{Gadflies (Tabanidæ)}

General Account. - Although primarily of importance as blood-thirsty pests of domestic animals, the gadflies or horseflies (Tabanidæ) cannot be ignored as biters of human beings, especially as they have been shown to be implicated in the spread of certain human diseases. The bites are painful, and sometimes cause annoyance for several hours; not infrequently these bites, which may bleed, subsequently become infected and give rise to troublesome sores. The females alone are bloodsuckers, the males living chiefly on plant juices. These flies, of which over 2500 species have been recorded, occur in every part of the world, and in every sort of habitat where water or damp places are available for breeding purposes.

The gadflies are of large size and heavy build (Fig. 224A). They are often beautifully colored in black, brown and orange 
tones, sometimes with brilliant green or green-marked eyes, though in most species of temperate climates the huge eyes are brown or black. The head is large, and in the male is almost entirely occupied by the eyes, which meet across the crown of the head (Fig. 224B), though in the females a narrow space is

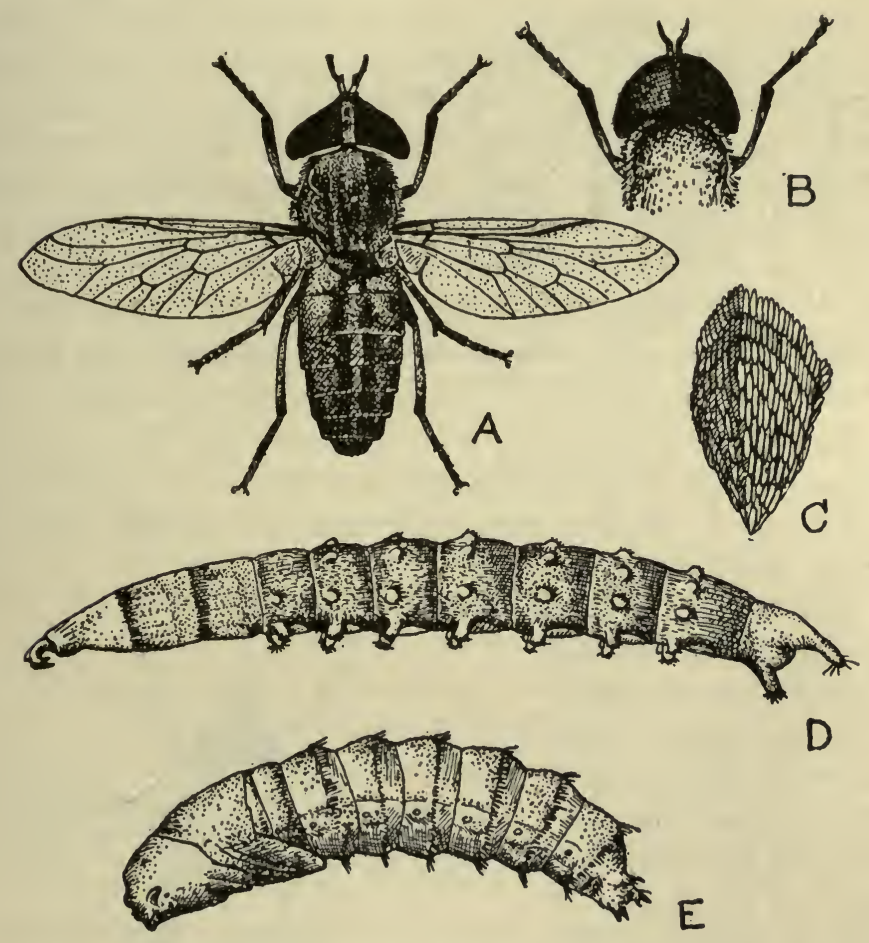

Fig. 224. Life history of a Tabanid, Tabanus kingi, $a$ "seroot" of Sudan. A, adult female, $\times 3 ; B$, head of adult male, $\times 3 ; C$, egg mass, laid in crevices of rock, $\times 5 ; D$, larva, $\times 2 \frac{1}{2} ; E$, pupa, $\times 2 \frac{1}{2}$. (After King.)

left between them. The antennæ are of characteristic shape (Fig. 211C) varying somewhat in the different genera. The mouthparts (Fig. 225) are almost exactly like those of the blackflies on a large scale. The stabbing and cutting parts are usually short; heavy and powerful, though in one genus, Pangonia, the proboscis is very long, enabling the fly to pierce flesh and suck blood while hovering in the air and to pierce even through thick clothing. Most of the species are very 
deliberate and persistent in their feeding and are not easily disturbed when they have begun to suck blood. The thorax is relatively long, and the wings are large and expansive and usually held at a broad angle to the body, as shown in Fig. 227. The markings of the wings usually give the easiest means of identi-

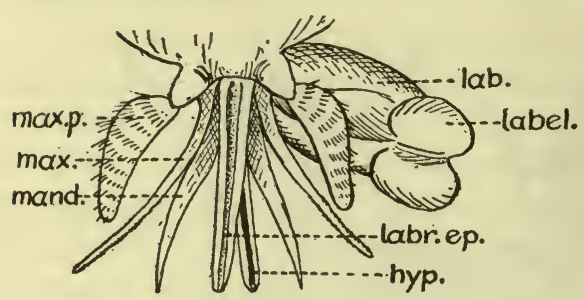

FIG. 225. Mouthparts of a tabanid; hyp., hypopharynx; lab., labium; label., labellum; labr. ep., labrum-epipharynx; mand., mandible; max., maxilla; max. p., maxillary palpus.

fication of the genera. Of the four most important genera as human pests, Tabanus (Fig. 224) is of large size and has clear or smoky wings, with no spots or a few small scattered ones; Pangonia (Fig. 226) also has clear or smoky wings but can be distinguished by the long proboscis; Homatopota is of moderate size and has wings with profuse scroll-like markings; and Chrysops, the species of which are often small, even smaller than a housefly, has a conspicuous black band on the wing (Fig. 227).

Life History. - All the tabanids breed in water or in damp places. The eggs (Fig. 224C), several hundred in number, are laid in definitely shaped masses on the leaves of marsh or water plants, on the leaves or twigs of trees overhanging water, or in crevices of rocks along the sides of streams. The eggs are

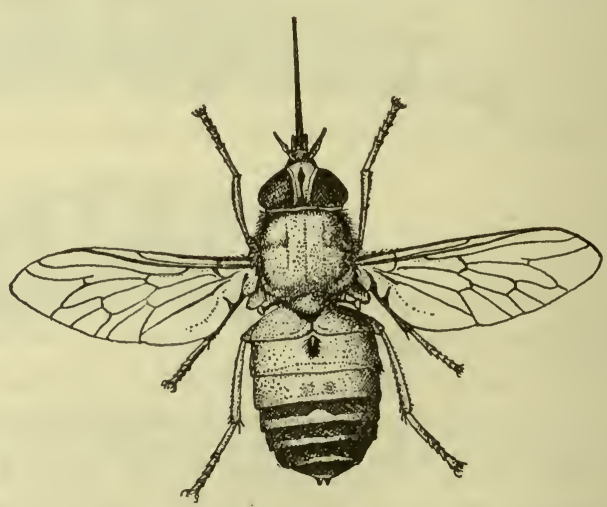

Frg. 226. A long-beaked tabanid, Pangonia ruppellii, of eastern Africa. $\times 2 . \quad$ (After Castellani and Chalmers.)

white when laid, but soon turn dark. They are deposited during the summer and under favorable circumstances hatch in from five to seven days. The newly hatched larvæ fall into the water or to wet ground or decaying vegetation such as occurs around the edges of marshes, in sphagnum bogs, in decaying logs, etc. The larvæ (Fig. 224D) are cylindrical legless creatures, pointed at 
each end, and with a number of spines or warts on the body. They are voracious feeders and prey upon various soft-bodied animals which they find in the water or mud in which they live, and are not averse to the practice of cannibalism if food is scarce. The larvæ grow rapidly during the remainder of the summer, but remain inactive and with little or no growth during the winter. In the spring they complete their development and creep out to drier ground to pupate. The pupa (Fig. 224E) often resembles the chrysalis of a butterfly in form. The adults of the species of temperate climates emerge after two or three weeks, but King states that tabanids in the Sudan exist as pupæ only six to eight days. The whole life history of species of temperate climates therefore occupies about a year, but it is shorter in tropical species, in which there are probably several broods a year.

The adult flies are strictly diurnal, and are often active in the clear sunlight of a summer day, though many forest-dwelling forms, e.g., the deerflies, Chrysops, prefer shade. They do not go in swarms as do many other biting insects but are usually solitary in habit. On account of their powerful wings they are sometimes found at considerable distances from their breeding places. As remarked before, only the females are blood-suckers; the males, and very probably the females to some extent also, feed on plant juices, the dew of leaves which hold a little organic matter in solution, excretions of insects, etc. Gadflies collect near pools and skim over the surface of the water, the under side of the body often touching the water. Portchinsky, in Russia, has devised a means of trapping the flies, based on this habit (see p. 489).

Tabanids and Disease. - Although tabanids are not known to serve as the intermediate hosts of any disease-causing protozoans, they have been shown to be efficient as mechanical disseminators of various disease germs, being especially dangerous in this respect on account of their intermittent feeding. It is quite common for them, having been disturbed while feeding on one animal, to continue their meal on another.

Surra, an important disease of horses in southeastern Asia and Madagascar, caused by a trypanosome, is transmitted in this manner, and also $\mathrm{El}$ debab, a trypanosome disease of camels. Other trypanosome diseases of animals, normally transmitted by tsetse flies, can be transmitted experimentally by tabanids, 
but only immediately after the infective feed. Human trypanosome diseases have been suspected of being transmitted likewise, but there is yet no proof that this takes place.

The most important disease disseminated by tabanids is anthrax. This is a bacterial disease to which nearly all herbivorous animals and man are susceptible, and which is very destructive, sometimes killing over 75 per cent of its victims. The bacilli which cause the disease gain entrance to the body either through abrasions of the skin to the blood, through spores in the air to the lungs, or through contaminated food to the intestine. The bacilli have been found in the alimentary canal of tabanids which have fed on dying or dead victims, and animals inoculated with these bacilli died of anthrax. That these flies could transmit the disease not only when crushed so that the contents of the digestive tract could contaminate the wound, but also by their bites, has been stated many times, and has recently been observed in China under conditions which placed it beyond doubt. The method of transmission is purely mechanical and probably occurs only when a fly which has been feeding on a diseased animal finishes its meal on a healthy animal or on a human being, the disease germs adhering to the mouthparts long enough to be transferred to the new animal. The stable-flies, Stomoxys, and other biting flies which will attack two or more animals in quick succession are equally as dangerous as anthrax carriers.

Tabanids have often been accused of causing diseases similar to, if not identical with, oriental sore. In the intestines of various tabanids there exist flagellate parasites belonging to the genus Herpetomonas, and it is believed that if these should accidentally gain entrance to the flesh of a human being by contamination of the puncture made by the host fly, they might assume the form of Leishman bodies and multiply to a sufficient extent to cause a local sore. Obviously such implanted parasites would be permanently side-tracked, and would stand little chance of ever being released by a fly of the species in which they normally live. Such a theory is proposed to explain the sporadic cases of leishmaniasis of the skin which occur in Panama and other places, and which are usually reported to develop at the site of a horsefly bite.. In São Paulo, Brazil, a form of leishmaniasis is very common among forest workers, even in wild uninhabited regions. The fact that the disease is contracted 
only by men who spend the day in the forest, and is most prevalent in May and June, a time corresponding to the appearance of many tabanids, points strongly to these insects as the carriers of the infection, since they are the only diurnal insects exclusively found in forest regions. The forest leishmaniasis of Paraguay may also be due to tabanids.

In one other case a tabanid is implicated in the spread of a disease. In the tropical jungles of Africa certain species of Chrysops locally known as mangrove flies, serve as intermediate hosts for filarial worms. Leiper and other investigators have found that the larvæ of the loa worm, Loa loa, which swarm in the peripheral blood of the host in the daytime only, undergo rapid development in several Chrysops, especially

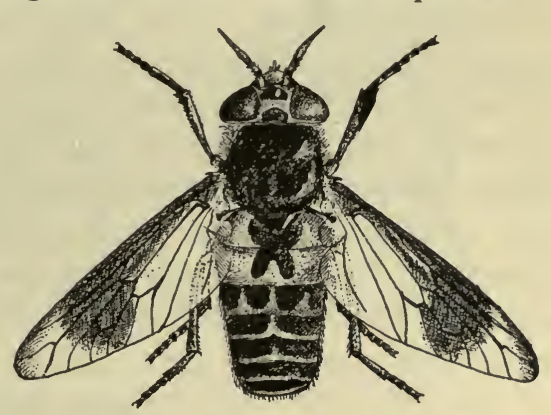

Fig. 227. A deerfly, Chrysops callidus. $\times 4$.

C. dimidiata and $C$. silacea (see p. 309).

It has recently been shown by Francis that tularemia, a rodent disease of western United States, caused by Bacterium tularense, and transmissible to man, may be transmitted, and probably commonly is, by the deerfly, Chrysops discalis. These flies are infective from a few seconds to at least fourteen days after biting an infective rabbit.

Control. - Prevention of bites from tabanids, especially during an epidemic of anthrax, or where diseases believed to be transmitted by tabanids are prevalent, is important. Practically the only means that can be employed is the use of repellents, as for other insect pests (see p. 455). According to Herms, repellents efficient against tabanids usually contain fish oil.

In a recent publication Portchinsky, a Russian entomologist, having found that tabanids have the peculiar habit of skimming over pools, touching the lower side of their bodies to the surface, advised the conversion of such pools into traps by pouring oil on them to produce a surface film, so that the insects would be caught in it, and the spiracles (openings of the tracheæ through which air is absorbed) closed up. In an experiment which he 
performed in a pool with a surface of a little over a square yard, he caught in five days 1260 male and 258 female Tabanus, and 416 male and 33 female Chrysops. This "pool of death" was literally studded with "floating islands of dead tabanids." The flies are said to visit the pools even after sucking blood. Portchinsky suggests the construction of traps of this nature in pastures where tabanids are troublesome, fencing them in, of course, to prevent the stock from getting access to them.

From the solitary nature of the flies, and the great variety of breeding places which may be selected, it is obviously impossible, in most cases, to exterminate tabanids during their early stages. Natural enemies probably do much to limit their numbers; fishes and large carnivorous aquatic insects prey upon the larvæ, and birds and hornets on the adults. Hine describes seeing bald-faced hornets, Vespa maculata, capture and cut to pieces horseflies which were too large for them to carry.

\section{Tsetse Flies}

Next to the mosquitoes the tsetse flies are the most important of the biting flies. The history and destiny of the African continent has been and will be very largely controlled by these insects. As far as their own biting power is concerned, tsetse flies are of little importance; their bites are less painful than are those of many other biting flies of similar size. It is in the rôle of carriers of trypanosome diseases that they gain their importance. Not only the two or possibly three forms of human sleeping sickness, but also a large number of deadly trypanosome diseases of animals are transmitted by these insects. The native wild animals of Africa are largely immune to these diseases and serve as a reservoir for them, but domestic animals and man succumb in large numbers, in fact to such an extent that some parts of Africa are uninhabitable, and in other parts it is impossible to keep domestic animals of any kind. The abundant and varied wild game of Africa, particularly the numerous species of antelopes, are the chief natural source of food for tsetse flies, and since the flies serve as intermediate hosts for the trypanosomes harbored by the wild game, it is obvious that when man or domestic animals are bitten by these flies they are in great danger of being inoculated with one or more species of trypanosomes. 
General Form. - The tsetse flies (Fig. 228) are elongate, dark brown or yellowish brown flies, some species no larger than an ordinary housefly, others larger than blowflies. They are usually included as an aberrant group of the housefly family, Muscidæ, but from other members of the family they differ in a number of striking ways, especially in the manner of reproduction, and in form of the larva. They constitute the genus Glossina which contains 15 species and has no very close allies; some species are of very wide distribution, while others are local or very rare. Tsetses can most easily be distinguished from other flies by their position when at rest (Fig. 228); their wings

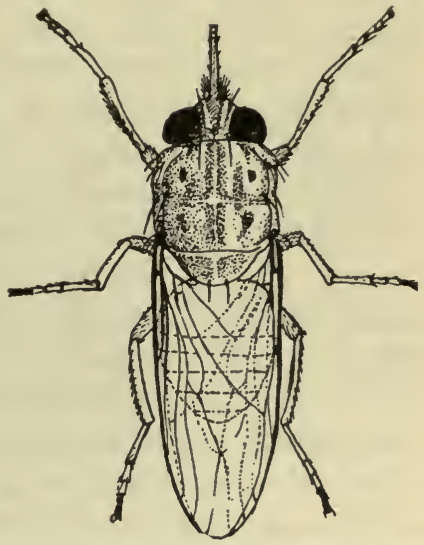

Fig. 228. Tsetse fly in resting position. $\times 4$. (After Austen.) are folded flat, one directly over the other, straight down the back, like the blades of a pair of scissors, while the proboscis

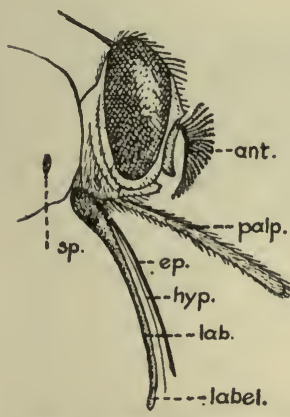

Fig. 229. Head and mouthparts of tsetse fly; ant., antenna; ep., epipharynx; hyp., hypopharynx; palp., palpus; lab., labium; label., labellum; sp., spiracle. (After Alcock.) of which, the hypopharynx, contains a delicate tube for carrying the salivary juices. The proboscis proper is ensheathed in the maxillary palpi which are so grooved as to conceal entirely the projects horizontally in front of the head. Beyond these characteristics there is nothing strikingly distinctive about a tsetse fly, and it is therefore difficult for anyone who is not thoroughly familiar with it to identify it on the wing. The darting manner of flight and buzzing sound are said to be quite diagnostic when one is once familiar with them. When the flies are caught and examined, however, there are a number of good identification marks. Most characteristic, perhaps, is the arrangement of the mouthparts and antennæ (Fig. 229). The proboscis consists of a bulblike base which is continued as a slender shaft, composed of a grooved lower lip with two needle-like puncturing organs within it, one 
mouthparts when the latter are not in use, and it is thus the palpi alone that are seen when the long blunt-tipped proboscis is observed. The characteristic form of the antennæ is shown in

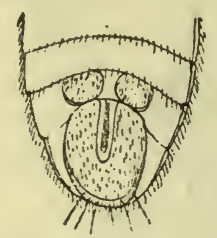

Fig. 230. Hypopygium of $\mathrm{male}$ tsetse fly. (After Alcock.)

Fig. 229. The thorax is relatively large and quadrangular, with a characteristic pattern which is, however, inconspicuous in some species. The abdomen may be nearly uniform dark brown, or pale brown banded with a dusky color. The male has a large oval swelling on the under side of the last segment of the abdomen, the "hypopygium" (Fig. 230), which forms a good distinguishing mark between the sexes.

Distribution, Habits, etc. - Tsetse flies, fortunately, are limited in their distribution to the middle portion of the African continent from south of the Sahara Desert to the northern borders of British South Africa (Fig. $231, \equiv)$. One species occurs in the southwestern corner of Arabia. Tsetses are by no means evenly distributed over this great area, but are limited locally to "fly-belts," chiefly along rivers and at the edges of lakes. All the factors which cause the "patchy" distribution of tsetses are not known; there are cases where close limitation to certain areas cannot be explained by any known requirements of the flies. Different species vary in their choice of habitats; Glossina palpalis (Fig. 236),

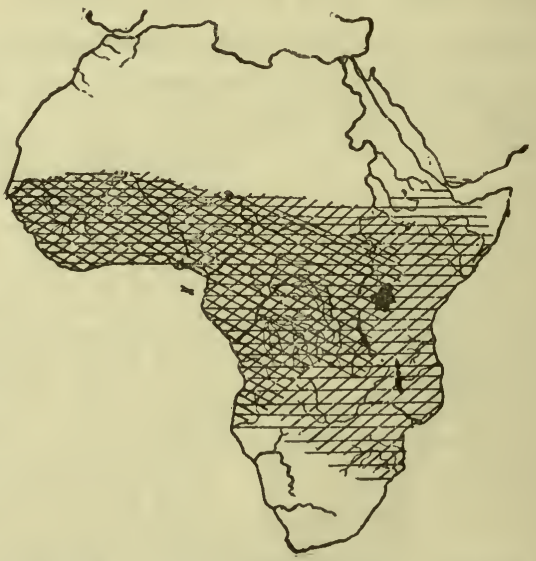

FIG. 231. Approximate ranges of tsetse flies. (Compiled from Austen.)

$$
\begin{aligned}
& \equiv \ldots \text { range of entire genus glossina } \\
& \text { III } \ldots \text { range of } g \text {. morsitans } \\
& / / / . \text { range of } g \text {. palpalis }
\end{aligned}
$$

the carrier of Gambian and Nigerian sleeping sickness, is seldom found more than 30 yards from the edge of water where a sandy bottom and overhanging vegetation is abundant, though it follows animals and man for a few hundred yards from such positions. This species is found only in shady 
places and where there is great humidity. Glossina morsitans (Fig. 237), the fly which is particularly well known to biggame hunters in Africa and is the carrier of Rhodesian sleeping sickness, is less dependent on water, and in fact prefers a rather hot and fairly dry climate. It is confined to open brushy country with scattered trees, where there is a moderate amount of shade for cover. It is never found either in dense forest or in open grass land. Most other species of tsetses resemble one of these two species in choice of habitats, though few if any are as independent of water as is $G$. morsitans.

Tsetses are diurnal in habits, but the time of activity varies with the species. G. palpalis is most active during the middle part of the day on bright days; G. tachinoides, on the other hand, is especially hungry on dull days and early in the morning; $G$. morsitans is active in the morning and afternoon, but usually disappears at midday; $G$. brevipalpis and $G$. longipennis bite in the early morning from sunrise until about 8 A.M. and in the afternoon from 4 P.M. until some time after dark. Both the lastnamed species are attracted by lights at night, and enter lighted railroad coaches passing through the "fly-belts." G. palpalis, and probably other species, also, seldom rise more than a few feet above the ground.

It has been the universal experience of collectors of tsetse flies that the males outnumber the females, often to the extent of ten or more to one. Yet it is a remarkable fact that when bred in the laboratory, males and females are obtained in equal proportions. Many different explanations for these apparently centradictory facts have been proposed, but the most probable is the one recently brought out by Lamborn, based on his observations on $G$. morsitans in Nyasaland. Lamborn has observed that copulation takes place after a rough capture, and that, in captivity at least, females even in an advanced state of gestation are not exempt from the attacks of the males, although this often results in abortion. In nature, therefore, the pregnant females would necessarily have to hide to avoid the males, and so would be less likely to be caught by a casual collector.

Tsetses show marked preference for certain colors, being especially attracted to blacks or browns, and repelled by white. The dark skin of negroes is selected in preference to pale skin to such an extent that a white man is seldom troubled when ac- 
companied by natives. Black or dark clothes are preferred to light ones; khaki color, however, appears to be particularly attractive to them. Moving objects seem to attract the flies, and they are said to follow launches when moving, though they leave them alone when quiet.

When biting, these flies spread apart their front legs, lower the proboscis into the skin and begin to gorge. The abdomen of
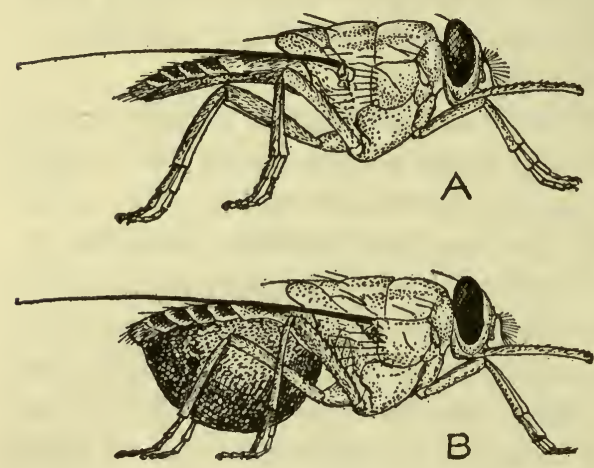

Fig. 232. Glossina morsitans before $(A)$ and after $(B)$ feeding. $\times 4$. (After Austen.)

an unfed tsetse is very flat (Fig. 232A) but after 30 or 40 seconds of feeding it becomes distended like a balloon, sometimes containing over twice the weight of the fly in blood (Fig. 232B). The flies do not feed exclusively on blood, but also suck plant juices and show definite, selective taste for various fluids presented to them under a membrane, according to experiments by Yorke and Blacklock. Both warm- and cold-blooded animals are sucked, but flies fed only on a cold-blooded animal (crocodile) never produce offspring. It has been thought that perhaps water fowl constitute an important article of diet for tsetses, but in the case of Glossina morsitans, at least, birds' blood proved rather indigestible for them, and often produced a clot in the digestive tract, resulting in abortion in female flies. In the case of such species as $G$. palpalis, however, bird blood may be more easily digested, and the diurnal habits and close adherence to the vicinity of water would argue in favor of subsistence on water animals, in part at least. On the other hand, the habit of many species of frequenting places where game animals come to drink or browse and of feeding early in the morning and at evening is apparently an adaptation to the habits of such hosts as wild game animals. Examination of the stomach contents of wild flies usually shows a preponderance of mammal blood, but Carpenter, studying G. palpalis in Uganda, often found that a large proportion of some collections of flies had fed on reptiles, 
especially on certain large lizards. Lloyd thinks that small mammals and birds may be important sources of food for tsetses, for, though these animals are usually able to avoid attacks by the flies during their time of activity, many of the nocturnal species hide during the day in the same places frequented by the flies and would then be easy prey for them.

Life History. - Tsetse flies differ from all others of their family in their remarkable manner of reproduction. Not only do they not lay eggs, but the single developing larva is retained within the body, being nourished by special glands on the walls of the uterus. The larva is full grown and occupies practically the entire swollen abdomen of the mother before it is born. The process of giving birth to the larva is very rapid, occupying only a very few minutes. As soon as born another larva begins its development, etc.

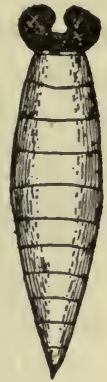

Fig. 233. Newly born larva of tsetse fly, Glossina palpalis. $\times$ 5. (After Roubaud.) In Glossina palpalis the first larva is born three or four weeks after mating, immediately after emergence from the pupal case, and another is born every nine or ten days providing the temperature

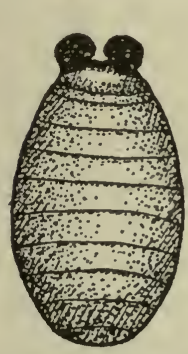

Fig. 234. Pupa of tsetse fly, Glossina palpalis. $\times$ 5. (Partly after Austen.)

\section{Austen)} is around $75^{\circ}$ or $80^{\circ} \mathrm{F}$. and food is abundant. There is little data on the total number of young produced, but in one captive fly eight larvæ were produced in 13 weeks and only one egg was found left in the body. Pregnant flies often abort when disturbed and cases are known in which the larvæ pupated within the abdomen of the mother, to the destruction of both of them.

The larva (Fig. 233) is a yellowish white creature, about one-third of an inch in length, with a pair of dark knoblike protuberances at the posterior end of the body between which are the respiratory openings. It immediately hides itself in loose soil or under dead leaves in the place where it was deposited by the mother, and transforms to a pupa (Fig. 234). The pupation takes place in the course of less than half an hour in soft dry ground, and in an hour to an hour and a half in hard or damp ground. After pupation the color begins to turn dark and in four hours the pupa is a dark purplish brown color. It is shaped 
more or less like a small olive, and has at the tip of the body the blackish knobs which are so characteristic of the larval stage also. The shape and size of the knobs and of the notch between them are good distinguishing marks between species. The duration of the pupal stage depends on the dryness of the soil, temperature, exposure to sunlight, etc., and may occupy from 17 days to nearly three months. In experiments made by Lloyd with Glossina morsitans the pupal stage ranged from 23 days at $85^{\circ} \mathrm{F}$. to 81 days at $70^{\circ}$. Few adults emerged at temperatures below $70^{\circ}$ or above $86^{\circ}$. Little is known about the reproductive season, but it is probable that reproduction occurs only in the warm part of the dry season in cool climates, but may occur to a varying degree throughout the year in hot climates.

The places selected for depositing the eggs vary somewhat with the species, but all species select dry, loose soil in shaded, protected spots, preferably in places where a little sunlight will penetrate for a short time each day and where scratching birds cannot easily reach them. G. palpalis deposits under tree trunks and at the foot of various species of trees, especially where a dense thicket gives a protected spot. In Sierra Leone, Yorke and Blacklock found numerous pupal cases at the foot of oilpalms where the dense foliage of the lower limbs makes approach difficult. G. morsitans is partial to cavities in trees or stumps, or under logs or branches lying a few inches above the ground (Fig. 235). The length of life of tsetses is probably less than a year. Specimens have been kept in the laboratory for over eight months.

Tsetse Flies and Disease. - As remarked before, the enormous importance of tsetse flies lies in their rôle as carriers of trypanosomes. The effect of trypanosome diseases on domestic animals in Africa has practically excluded these aids to development and civilization from some parts of that continent. The importance of trypanosomes to man in Africa is discussed in Chap. VI. It is sufficient here to repeat that sleeping sickness, which is the final stage of trypanosome disease, is one of the most deadly, if not the most deadly, disease known. Several types of the disease are recognized; the most widespread Gambian disease is caused by Trypanosoma gambiense and in nature is transmitted chiefly if not exclusively by Glossina palpalis. The mild Nigerian form of the disease is believed to be a mere variety of the Gambian 
disease and is likewise transmitted by G. palpalis. Rhodesian sleeping sickness, however, is transmitted by $G$. morsitans. It is the belief of some workers that the Rhodesian parasite is a
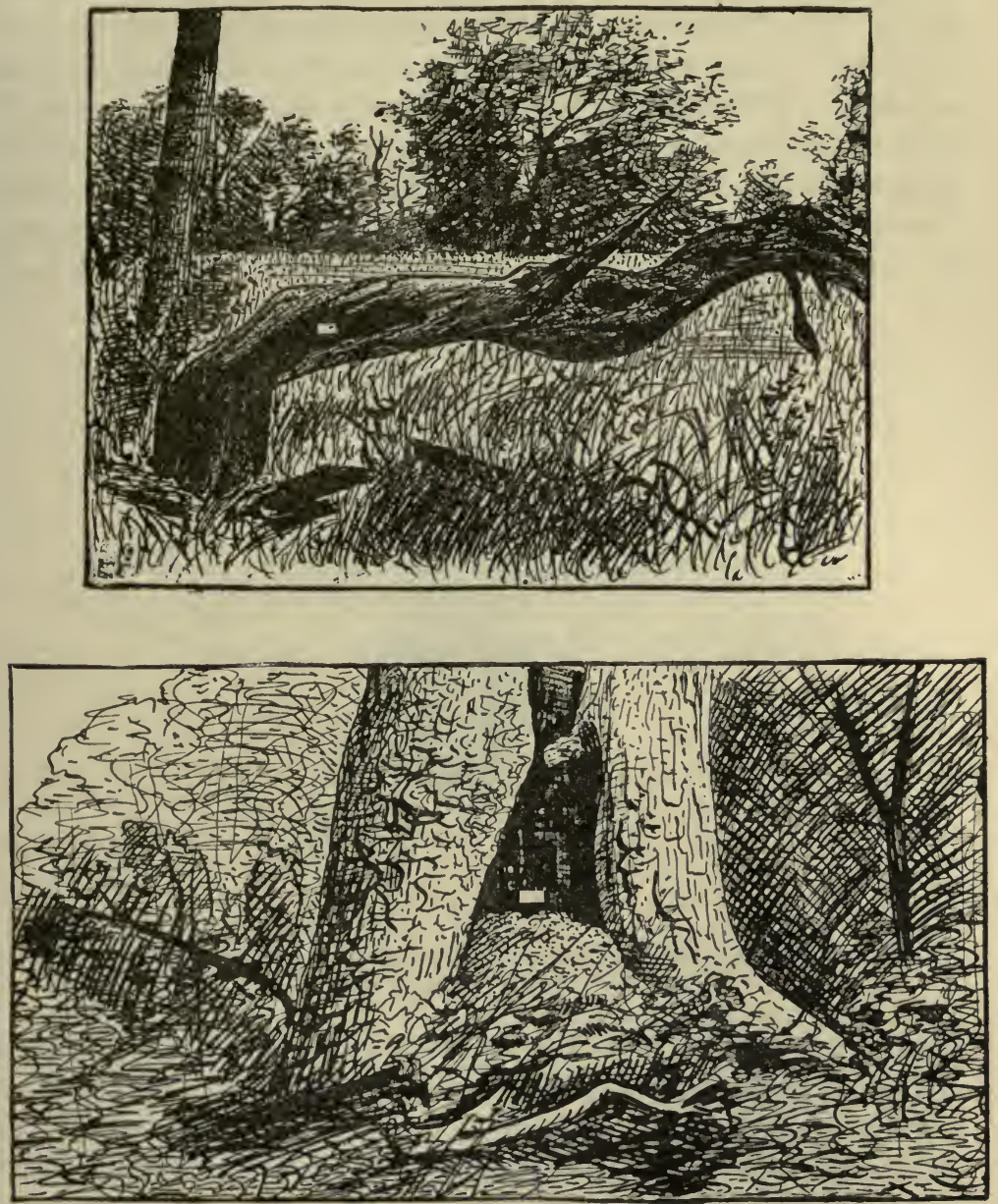

FIg. 235. Typical breeding places of Glossina morsitans in Rhodesia. (From photographs from Kinghorn and Yorke.)

mere strain of the trypanosome, $T$. brucei, which causes nagana in animals and which also is transmitted by $G$. morsitans. The development of the trypanosome in the flies and the mode of transmission is discussed on p. 99. 
Glossina palpalis (Fig. 236) is a large dark species with blackish brown abdomen and with gray thorax having indistinct brown markings. This species is found over the whole of West Africa, from the Senegal River to Angola, and east to the upper valley of the Nile and the eastern shores of the central lakes (Fig. 231, III). Its range is thus nearly coincident with that of Gambian sleeping sickness. This species, more than any other except possibly G. tachinoides, which occurs around the southern border of the Sahara Desert, is dependent on the presence of water. Its natural range is said seldom to exceed 30 yards from the edge of water, and the distance that it will follow animals or man is not more

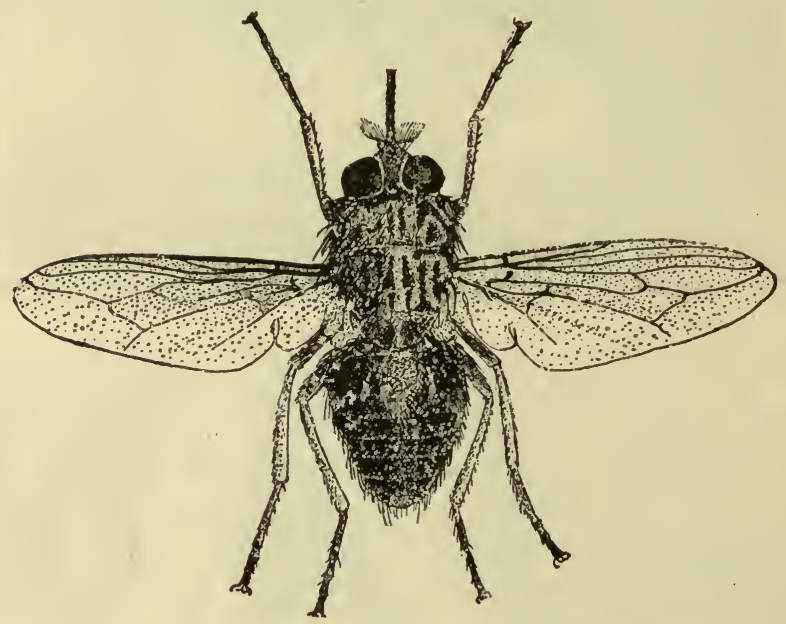

FIG. 236. Glossina palpalis, carrier of Gambian and Nigerian sleeping sickness. $\times 4$. (After Austen.)

than a few hundred yards. Muddy, reedy sloughs or swamps are not frequented by this fly, but rather sandy- or gravellybanked streams with abundant overhanging vegetation. In the rainy season the flies extend their range to headwaters which are dry during the remainder of the year and retreat again with the drying up of the water. It is feared that this species may sometime bridge the short gape between the headwaters of the Congo and the Zambesi, and become established along the latter river and its tributaries, carrying sleeping sickness with it.

This fly probably feeds naturally on a number of different animals. Wild game, especially the Situtunga antelope, is 
utilized to a large extent, and the habitats of the flies imply that they feed considerably on water animals. According to Fiske the crocodile is the host of first choice, Varanus, a lizard, second, and the Situtunga antelope third. Water fowl are believed to be attacked also. Goats are remarkably unattractive to the flies. Data concerning the life history has already been given.

Glossina morsitans (Fig. 237), carrier of many trypanosome diseases of animals and of the newly arisen and still narrowly limited Rhodesian sleeping sickness, is the most widely distributed species of tsetse fly, occurring all the way across Africa

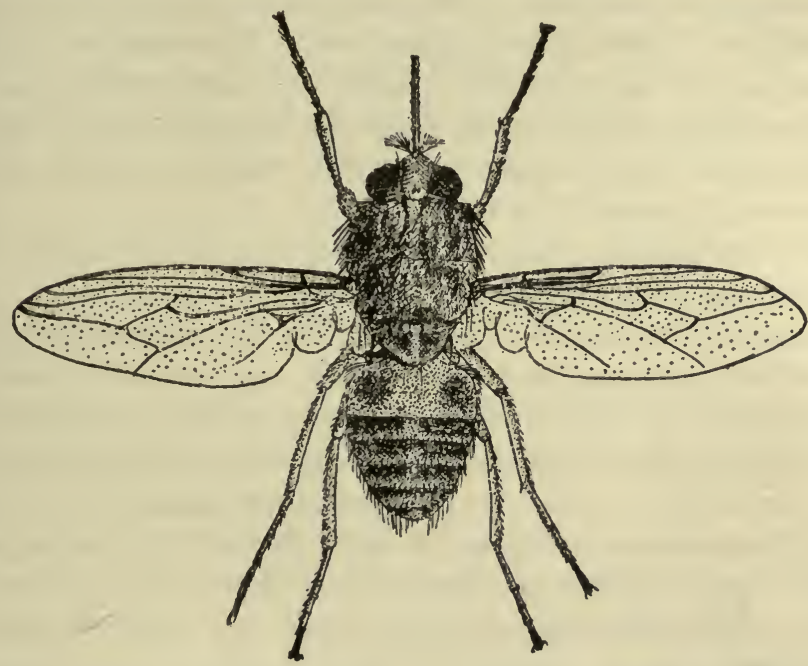

FIG. 237. Glossina morsitans, carrier of Rhodesian sleeping sickness. $\times 4$. (After Austen.)

from Senegal to southern Sudan and Abyssinia on the north, to northeastern Transvaal and Zululand on the south (Fig. 231, III). This is also the best known species, and is the one which has attracted to itself the attention of big-game hunters in Africa for many years. It is slightly smaller than $G$. palpalis and much lighter colored, with very inconspicuous markings on the gray thorax, and with more or less distinct dark bands, not continuous across the middle line, on the buff colored abdomen. As remarked elsewhere, G. morsitans is not confined to the immediate vicinity of water, but prefers hot dry country, covered with bush or scattered trees. In some places it is found at an 
altitude of over 5000 feet, but usually occurs at much lower levels. It feeds on the blood of almost any large mammal which comes its way. It was long supposed that the fly was especially dependent on the Cape buffalo, Bubalis caffer, as it undoubtedly was before this animal was almost exterminated by rinderpest, but the fly is certainly able to exist in the absence of the buffalo, though often in less numbers than when the abundant food supply was at hand. Baboons are said to be relished by the fly in some parts of Africa.

Glossina morsitans, though most active in the morning and late afternoon, sometimes bites at midday and even after dark, especially on warm moonlight nights. The habit of following moving objects is especially marked in this species, and some observers state that flies have followed them several miles, frequently alighting on the ground to rest, or on the person pursued, often without attempting to bite.

The reproduction and choice of breeding places of this species have already been mentioned.

Although $G$. palpalis is undoubtedly the normal transmitter of Gambian sleeping sickness and $G$. morsitans of Rhodesian sleeping sickness, they are not the only species which have been found capable of transmitting these diseases, at least under laboratory conditions. $G$. morsitans has been found to be able to nurse Trypanosoma gambiense in some districts but not in others. $G$. pallidipes, which resembles $G$. morsitans but is larger, and confined to southeastern Africa, can be experimentally infected also.

G. tachinoides is suspected of carrying sleeping sickness in parts of Nigeria and Togoland. This is one of the smallest species, being about the size of a housefly. It has very distinct bands on the abdomen, and is browner and darker than G. morsitans. It is found around the southern edges of the Sahara Desert and in southwestern Arabia. Its habitats are practically the same as those of $G$ : palpalis but it is active on dull days and early in the morning when the latter species is quiet. It frequently bites after dark, also, and in some places is said to be more troublesome than mosquitoes.

Another species experimentally able to transmit human trypanosomes, T. gambiense, is G. brevipalpis, of South Central and East Africa. This is a large species found in abundant 
shade, in bush mixed with creepers and young trees near water courses. Its counterpart in the more northern parts of East Africa is Glossina longipennis, a large warm-brown species with indistinct markings. G. brevipalpis is said to be desirous of feeding only before 8.00 A.M. and after 4.00 P.M. In the middle of the day it hides under leaves or grass blades near the ground, so that its presence would never be suspected.

To sum up it may be said that while there is much variation in the susceptibility of different species of tsetses to different trypanosome infections, so that one or a few species come to serve as the usual transmitters of any particular trypanosome, yet other species cannot be definitely excluded as carriers without extended experimentation. Even in the case of natural carriers of a particular trypanosome, a very small per cent of flies are found naturally infected, and not more than a few per cent can be infected experimentally. Moreover it is evident that a single species of fly shows marked differences in receptivity to infection in different parts of the range. The refractory nature of some West African races of $G$. palpalis probably accounts for the absence of sleeping sickness in Dahomey and neighboring states. It is probable that climatic conditions and food habits play a leading part in determining susceptibility of flies to trypanosome infections.

Control. - Attacks of tsetse flies can be avoided to some extent by the use of the usual insect repellents (see p. 455), by fly-proof clothing or veils, and by wearing white clothes. When it is necessary to travel through fly-infested places where sleeping sickness occurs, all of such measures should be adopted, or, better still, the fly-belts should be passed through in the darkness of night when the insects are inactive. Railroad trains and steamboats passing through fly-belts should be protected by fly-proof screens; this expedient is adopted in many parts of Africa at the present time.

Extermination of tsetses on a large scale is a very difficult matter, but locally it is quite feasible. There are probably factors influencing the distribution of the flies which are still unknown, and which may be turned to account in destroying them.

Clearing away of brush along fly-infested streams in the case of such species as $G$. palpalis and $G$. tachinoides, which are closely 
confined to patches of brush along water courses, is the most valuable measure in connection with their local destruction. As said before, these-flies seldom go over 50 yards from such brushy borders of streams except when following prey, in which case they may go several hundred yards. If brush is cleared away and low branches of trees cut out for a distance of 30 yards from the edge of water in the vicinity of fords, villages, washing places, etc., the flies quickly disappear, and do not reappear as long as the cleared area is kept clear. The effectiveness of this method of extermination has been demonstrated especially well by the Portuguese Sleeping Sickness Commission on the Island of Principe where tsetse flies were almost, though not entirely, exterminated in a four years' campaign. In addition to clearing margins of bodies of water, the beds of the water courses were straightened and leveled to make the clearing easier, and forests were completely cleared away on a large scale where they seemed to harbor tsetses. In addition some of the men employed in these operations wore on their backs black cloths smeared with sticky bird-lime, thus being converted into active traps for capturing flies. Nearly half a million flies were thus caught, and the number caught daily gave a good index to the effectiveness of the preventive measures being used, and must of itself have been a supplementary means of destruction which was of value. The eradication of Glossina morsitans is a much more difficult problem, since its habitats, though sharply confined to "belts," are not so closely limited to the edge of water, and are therefore more difficult to clear. Since, however, the areas occupied are usually not over a few square miles at the most, complete deforestation of such areas when near villages or highways would often be feasible.

The destruction of pupæ of tsetse flies by natural enemies undoubtedly aids in limiting their numbers, but the instinct which leads tsetses to deposit their offspring where birds cannot scratch gives the pupæ a high degree of immunity to this class of natural enemies and to artificial means of destruction. The newly deposited larvæ are covered by a slimy secretion which apparently protects them against the attacks of the ants which almost always abound in the tsetse breeding places. The pupæ are attacked by parasitic insects (Fig. 238), but apparently not to a sufficient extent to seriously reduce their numbers. 
However, five species of Hymenoptera and two of Diptera are known to parasitize the pupæ of tsetse flies. It is possible that some of these insects could be successfully exploited. The adults of $G$. morsitans are attacked, according to Lamborn, by a species of dragon-fly, Orthetrum chrysostigma, which persistently pursues them and diligently searches the vicinity of men and animals for them. Elimination of breeding places is the only feasible method for exterminating tsetses in their early stages.

Constructive measures should follow the destructive ones, such measures, for instance, as the cultivation of unfavorable plants and encouragement of natural enemies. Following are summarized briefly the methods of fighting tsetses advised by Bagshawe:

(a) clearing of fly-infested brush, and its re-

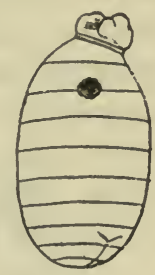

Fig. 238. Pupal case of Glossina morsitans, showing hole of emergenco of a small chalcid parasite. $\times 4$. (After Waterston.) placement by citronella grass or other plants noxious, or at least not favorable, to the flies;

(b) filling up, straightening out and draining of pools and water courses where possible;

(c) destruction of main food animals, if found feasible and possible. (The wholesale destruction of wild game is not advised by Bagshawe.)

$(d)$ encouragement and introduction of natural enemies, and investigation of food habits of possible enemies among birds and bats. The black drongo, Dicrurus ater, and the small bee eater, Melittophagus meridionalis, are known to feed on the adult flies.

(e) collection and destruction of pupæ or adult flies. This can be facilitated by creating artificial sites for depositing larvæ to which the flies will be attracted. Natives in Sudan are said to use gourds filled with blood for capturing flies to be turned loose to torture the stock of enemy tribes. Other traps have been devised also, among which should be mentioned the black bird-lime cloths already described as being used on the Island of Principe.

Some workers have advocated the wholesale destruction of wild game animals in parts of Africa where deadly trypanosome diseases occur, in the hope that in this way the natural reservoirs 
of the disease could be destroyed, and that the tsetse flies would disappear if their main source of food were cut off.

Domestic animals are, however, quite as suitable for tsetse flies to feed upon as are wild game and there is ample reason to believe that the flies would be able to subsist on small forest mammals, birds, crocodiles, etc., in the absence of other food. Even if all the wild game were destroyed, and domestic animals excluded for many years, enough flies would survive to reëstablish the scourge with the subsequent introduction of domestic animals. The destruction of the rich and varied, and indeed unique, wild life of Africa is a measure so radical, so contrary to our present growing determination to save the irreplaceable handiworks of nature, and, to be sure, so inhuman, that it cannot be advocated or even tolerated until absolutely proved to be an effective, and the only effective measure.

\section{Stable-Flies (Stomoxys) and Their Allies}

Belonging to the family Muscidæ in company with the houseflies, blowflies and tsetse flies, are a number of other biting flies, most important of which are the stable-flies, Stomoxys, especially

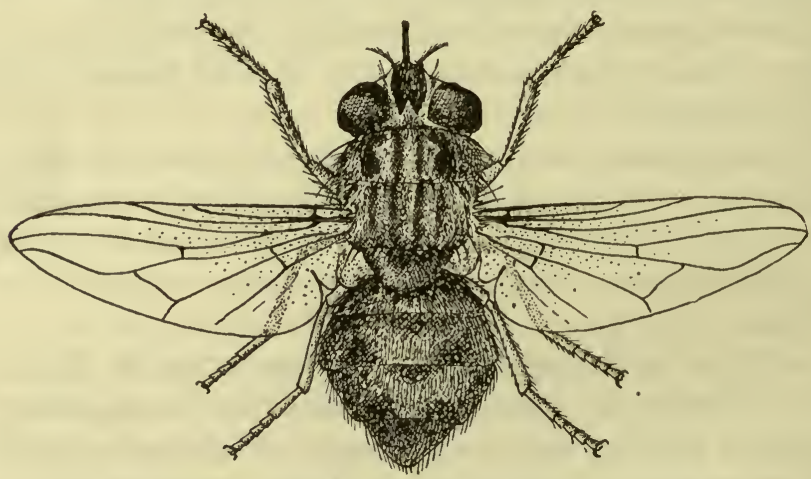

FIg. 239. Stable-fly, Stomoxys calcitrans. $\quad \times 5$.

the common species, S. calcitrans (Fig. 239), which makes itself annoying and often dangerous in nearly every part of the world. It is chiefly a persecutor of domestic animals, but is very willing to attack man when opportunity is offered.

The stable-fly in general appearance so closely resembles the housefly, Musca domestica, as often to be mistaken for it, whence 
the frequent statement that houseflies sometimes bite. They differ, however, in several ways. The stable-fly is more robust, browner in color, rests with the wings spread at a broader angle, and has a narrow, pointed shining-black proboscis (Fig. 240) which is quite different from the blunt fleshy proboscis of the housefly.

The mouthparts (Fig. 240) differ from those of many other biting flies in that the lower lip, which usually merely forms a sheath for the piercing mouthparts, is itself a piercing organ. It is bent at nearly right angles under the head so that it projects straight forward, being, therefore, fixed to the head like a bayonet to a rifle. The short basal segment is movable and muscular, and is used to manipulate the proboscis itself. The latter has at its tip rasplike spines which aid in perforating the skin of the host. Inside the groove in the lower lip is the labrum and hypopharynx which together form a sucking tube. The maxillary palpi, which form enclosing

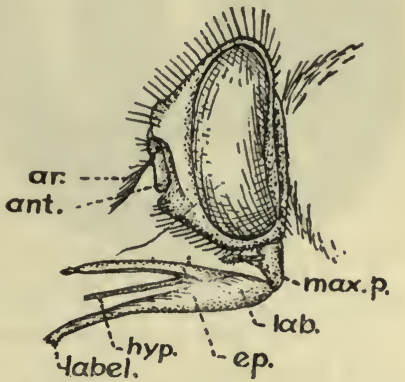

Fig. 240. Head and mouthparts of stable-fly, Stomoxys calcitrans; ant., antenna; ar., arista of antenna; ep., epipharynx; hyp., hypopharynx; lab., labium; label., labellum: max. p., maxillary palpus. (After Herms.) sheaths for the proboscis in tsetse flies, are less than half the length of the proboscis in Stomoxys.

The stable-fly is commonly believed to breed in manure, and gains its name from the frequency with which it is found about stables, presumably having been bred in manure. As a matter of fact, the presence of stable-flies about stables is due to the presence there of animals - horses, cattle, etc., - on which they feed. The breeding place which is most preferred is moist, decaying straw or rotting vegetable matter. According to Herms, the very best breeding places are afforded by the left-over hay, alfalfa or grain in the bottoms of, or underneath, out-of-door feed troughs in connection with dairies. In this soggy, fermented material practically pure cultures of Stomoxys larvæ may be obtained.

The eggs of Stomoxys (Fig. 241) are banana-shaped white objects about one $\mathrm{mm}$. in length, curved on one side and flat on the other, with a groove on the flat side. They are de- 
posited, sometimes deep in the decaying material selected, in small batches of from two to half a dozen, until from 25 to 50 or more are laid; there are a number of such depositions made by a single fly during her life. The eggs hatch in from two to five

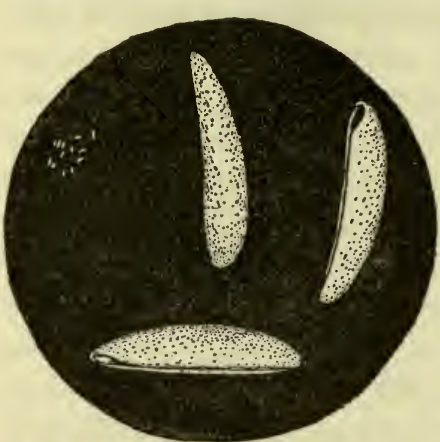

Fig. 241. Eggs of stable-fly, Stomoxys calcitrans. $\times 20$. Note eggs natural size in upper corner. (After Newstead.) days, usually three, into whitish, almost transparent footless maggots (Fig. 242A) very similar to those of the housefly, but easily distinguishable by the position of the posterior stigmal plates (see Fig. 243). The larvæ mature in a minimum of from 12 days to over two months, usually in about 15 to 20 days, and crawl into drier portions of the breeding material to pupate. The pupæ (Fig. 242B) are olive-shaped, chestnut-colored objects, one-fourth of an inch in

length. With favorable temperatures the adult fly emerges in from six to ten days, but this period may be much prolonged by cold weather. The shortest time in which a stable-fly may develop from the time of egg-laying is about three weeks, and this is extended under conditions which are not ideal. According to Herms' experiments, the average length of life of stable-flies is about 20 days. They sometimes live several months, however.

There are several other genera and species of the family Muscidæ which sometimes bite man, but none of them are habitual feeders on human blood, and they are hardly worthy of special consideration. They all resemble Stomoxys in general appearance, though some, notably the common hornfly,

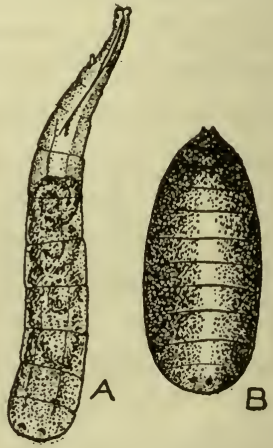

FIG. 242. Larva $(A)$ and pupa $(B)$ of stablefly, Stomoxys calcitrans $\times 4$. (After Newstead.) Hæmatobia serrata (or Lyperosia irritans), are much smaller. Their life histories are in general like that of Stomoxys, though there is some variation as regards choice of breeding places. Manure of various kinds is selected by some species, as it is by the housefly, much more than in the case of the stable-flies. 
Stomoxys and Disease. - Like the tabanids, the stable-flies are intermittent feeders, i.e., they frequently leave one animal in the course of a meal if disturbed, to finish feeding on another. For this reason they are of importance in mechanically transmitting blood diseases.

It has been shown that the trypanosome of sleeping sickness, $T$. gambiense, can be transmitted by interrupted feeding, and a few years ago Macfie showed that the Nigerian strain of the parasite could go through at least part of its development in the gut of the black stable-fly, Stomoxys nigra (see p. 98).

More serious than this is the relation of stable-flies to anthrax (see p. 488). This fatal disease of domestic animals and man is caused by bacteria which live long enough on or in the proboscis of stable-flies to be readily transmitted by them within an hour or two after an infective feed. The biting flies of this or other species which congregate to feed on sick or dying animals must be looked upon as a serious source of danger. Other diseases, such as foot-and-mouth disease, to which both animals and man are susceptible, may presumably be transmitted in like manner by these flies, though no proof of it has yet appeared.

In 1912 and 1913 several American workers, among them Dr. M. J. Rosenau, of the U. S. Public Health Service, adduced the theory that the stable-fly, Stomoxys calcitrans, was responsible for the transmission of infantile paralysis, and the theory was apparently supported by some facts in the epidemiology of the disease (though contradicted by others), and by carefully conducted experiments. In subsequent experiments, however, by the same and other workers, the results have been uniformly negative, and in the meantime much data has been collected to show that this terrible disease, which reached unprecedented proportions in New York City and vicinity during the past year and terrorized the entire United States, is transmitted by contagion, and not through the agency of any particular insects. It cannot be said that the disease is never transmitted by biting flies, or by ordinary houseflies, but that insects are not the main or even important factors in the spread of the disease is now a fairly well-established fact.

Control. - Control of the stable-flies and of allied species of biting flies depends almost entirely on the elimination of their favorite breeding places. In the case of Stomoxys, which is the 
most important of this group of biting flies, preventive measures are fairly easy. The drying out, burning, or burying of waste vegetable matter, such as piles of weeds, wet hay, lawn clippings, waste vegetable matter in garbage heaps, etc., eliminate the main breeding places. Poorly constructed hay stacks, around which there is a good deal of loose hay which becomes soggy and decays, are breeding centers for the flies. Stacks, when needed, should be constructed with evenly rounded top and vertical sides; but a better way, when possible, is to bale hay or straw and store it in dry places. Manure especially when mixed with straw is utilized by stable-flies in lieu of better breeding places, but the principal manure-breeder is the housefly, Musca domestica. According to recent work by the U. S. Department of Agriculture, manure can be treated in such a way as to destroy the young stages of stable-flies and houseflies without injuring its fertilizing value. A mixture of ten oz. of borax and $12 \mathrm{oz}$. of crude calcium borate. (colemanite) is applied to ten cubic feet (eight bushels) of manure, the manure being then sprinkled with two or three gallons of water. A still better substance to apply is hellebore powder, one-half $\mathrm{lb}$. in ten gallons of water to eight bushels of manure. An excessive quantity of the powder has no injurious action on the fertilizing power of the manure, as has an excess of borax. 


\section{CHAPTER XXVII}

\section{FLY MAGGOTS AND MYIASIS}

General Account. - Disgusting as it may seem, the human body is attacked not only by the numerous adult flies discussed in the last chapter, but is subject to attacks or invasion by the maggots or larval stages of some species of flies. Such an infestation by fly maggots is commonly known as myiasis, intestinal myiasis being the presence of fly larvæ in the intestine, cutaneous myiasis in the skin, etc.

All of the maggots which habitually or occasionally parasitize man belong to the order Diptera, and to the suborder Orthorrhapha, in which the larvæ have very small and indistinct heads, and the pupæ are inactive oval bodies from which the adults emerge by pushing off one end, like a cap (see p. 465 and Fig. 209A).

Most cases of myiasis are caused by flies quite closely allied to houseflies, and this famous transporter of germs and filth is itself occasionally guilty. The identification of maggots is often a difficult matter and is sometimes impossible without rearing the adult insect. Larvæ of the botfly family, Estridæ, are of various shapes, but seldom taper evenly from the posterior to the anterior end; the body has a leathery covering and is armed with girdles of thornlike spines. Larvæ of the genus Fannia (Fig. 253) are flattened, and have very characteristic fleshy processes along their sides. Nearly all other maggots causing myiasis are cylindrical, whitish, footless creatures, tapering from the broad posterior end to the small head, and are difficult to identify. The chief characteristics used for distinguishing them are the number and form of the mouth hooks (see Fig. 251), and the nature of the respiratory openings at the posterior end of the abdomen. These openings consist of two "stigmal plates," hardened, yellowish, eyelike spots, in which are three slits or openings, with sometimes a button-like mark at their base. The relative position of the stigmal plates to each other and to the surface of the larva, and the form of the slits, whether straight, curved or wavy, and whether vertical or oblique, are some of 
the characters used in distinguishing genera and species of fly maggots. A few typical forms are shown in Fig. 243.

It is more convenient to consider the different types of myiasis according to the way in which the larvæ attack the body or according to parts affected than according to the families and genera to which the flies belong. We may divide the various flies
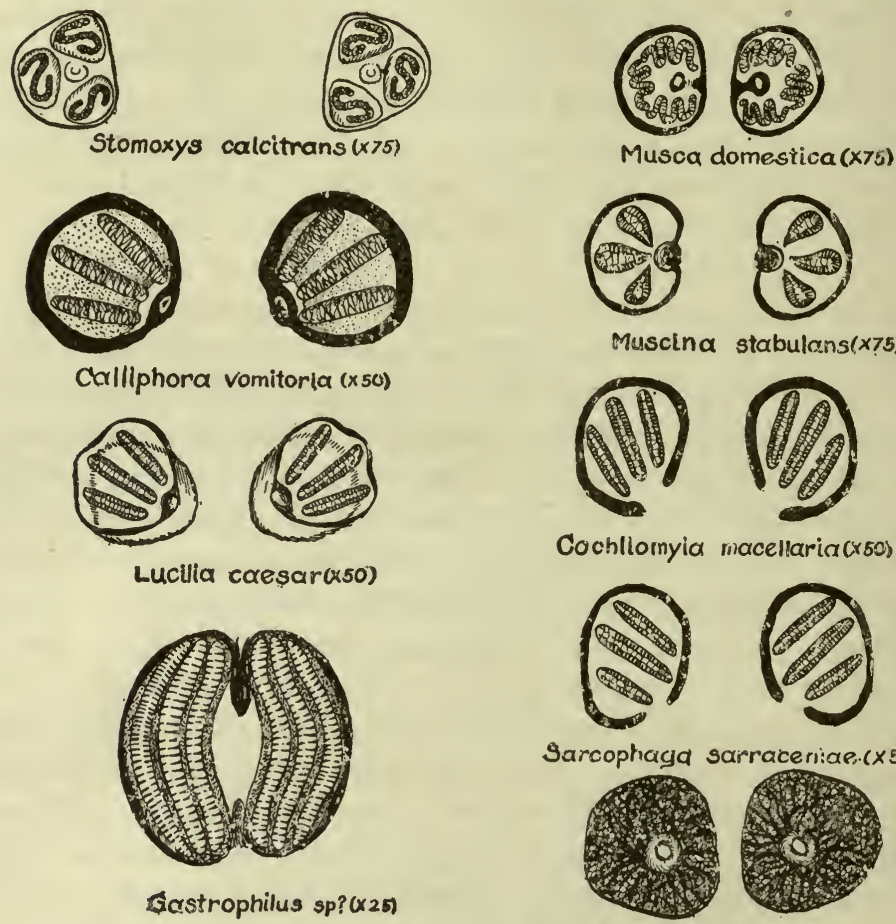

Muscina stabulans $(x 75)$
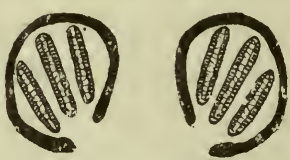

Cochllomyia macellariacx50)
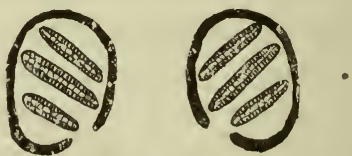

Sarcophaga sapraceritae.(x 50 :

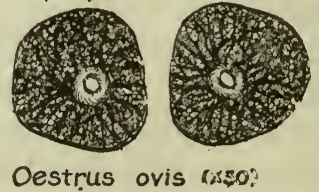

Frg. 243. Posterior stigmata and breathing pores of various maggots. Note distance apart of opposite stigmal plates, form and position of spiracles, presence or absence of button, etc.

causing myiasis into four groups: (a) those in which the larvæ live outside the body and suck blood by puncturing the skin, (b) those in which the larvæ develop under the skin; (c) those in which the eggs or young larvæ are deposited in wounds or in natural cavities of the body, such as the nose, ears and vagina; and $(d)$ those which live in or pass through the intestine or urinary passages. 


\section{Blood-Sucking Maggots}

A number of species of flies allied to the blowflies are known to deposit their offspring in the nests of birds, where the maggots attach themselves to the nestlings and suck blood. The only species of fly in which the larva sucks blood by puncturing the skin of man, however, is the Congo floor maggot, Aucheromyia luteola (Fig. 244), found throughout tropical Africa south of the Sahara Desert. Its range closely coincides with that of the
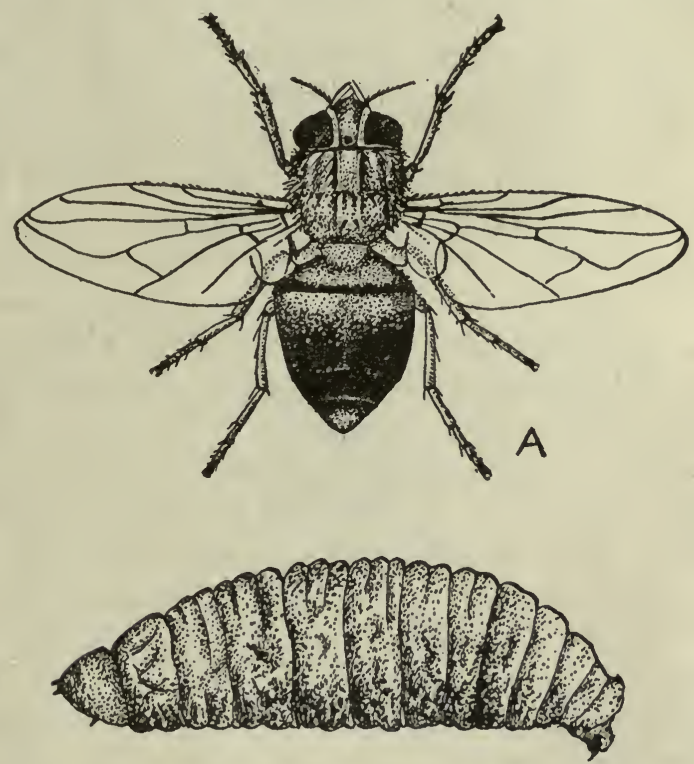

B

Fig. 244. Congo floor maggot and adult female fly, Aucheromyia luteola. $A, \times 3 ; B, \times 4$. (After Manson.)

Negro and Bantu races of men; it does not occur in countries inhabited by Arabs and Berbers.

The adult fly (Fig. 244A) resembles the blowfly, to which it is nearly related. The color, however, is different, being a dirty yellowish brown with the tip of the abdomen rusty black. This fly can usually be observed in shady places about human habitations, preferring the vicinity of latrines; it feeds principally on rotting fruits and on excrement. The female lays her eggs during the daytime in dust or débris in shady places, especially 
on the floors of native huts. The fly is said by Roubaud to make a furrow in the dust with her abdomen while running on the ground, feeling for breaks or cracks in which to deposit her eggs. Having found such a spot she forces her abdomen into it and deposits usually a single egg, then seeks a new crack, deposits another egg, etc., until the whole number of from 30 to 80 eggs has been disposed of. The eggs, the development of which is favored by dry surroundings, hatch in a few days. Within four or five hours after emergence the larvæ are ready to suck blood if opportunity presents itself, but they are able to live nearly a month without food, remaining buried an inch or so in the dust of floors. They can always be collected by digging with the point of a knife in cracks in the earth under sleeping mats. Roubaud collected 100 larvæ in half an hour, many of them filled with blood, in a hut where a dozen children slept.

The maggots (Fig. 244B) are dirty-white creatures, much wrinkled in appearance, but otherwise quite like the larvæ of houseflies. The tapering anterior end of the body is provided with a pair of black hooks to aid in piercing the skin of the host, and has retractile sucking mouthparts. The thick leathery skin and the position in a crack in the ground protects the larva from injury when stepped on by the bare feet of the natives. The body is beset with rings of spines which aid in the wriggling method of locomotion. The maggots are inactive in the daytime, but come forth at night to suck the blood of sleepers, biting them usually on the side of the body next to the ground. The bites are less irritating than those of mosquitoes, and according to Roubaud the bites of 20 larvæ at once produced no inflammation or itching.

Under ideal conditions the larvæ pass through two moults and go into the pupal stage in 15 days, but this may be extended to about two and one-half months under unfavorable conditions, such as low temperature and irregular food supply. The pupal stage lasts about 11 days. The adults do not begin laying eggs until about two weeks after emergence. The whole life cycle, therefore, from egg to egg, is about one and one-half months under favorable conditions.

The Congo floor maggot is not known to attack any animals but man in nature, though a closely allied maggot, Choromyia, lives in the burrows of the wart hog and other hairless mam- 
mals. Its bite is more painful to man than is that of the normal human parasite.

The attacks of the floor maggot can very easily be avoided by sleeping on mats or beds raised just a few inches from the ground.

\section{Maggots Under the Skin}

There are several species of flies in which the larvæ develop under the human skin, like "warbles" in cattle, but they are found only in Africa and in tropical America. The African species are closely related to the blowflies and fleshflies, whereas the American species, of which there is usually believed to be but a single one, is a true botfly, closely allied to the ox warble.

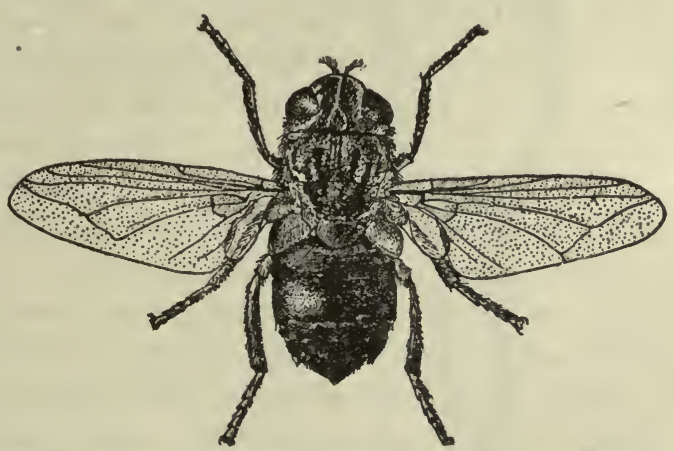

Fig. 245. Adult of South American skin maggot, Dermatobia hominis. $\times 2$. (After Castellani and Chalmers.)

Dermatobia. - The American species, sometimes called the human botfly, Dermatobia hominis (Fig. 245), is found throughout tropical America from Mexico to northern Argentina. Its larvæ develop not only in man but also in many other animals, as dogs, cattle, mules, hogs, etc. In certain parts of South America the hides of cattle become so riddled with the perforations made by these bots that they are rendered quite worthless. The infestation in man is contracted chiefly in forest regions, and apparently very seldom in houses, a fact which possibly accounts for the greater degree to which dogs are parasitized by it than are cats, and men than women or young children.

The adult fly (Fig. 245) is about the size of a blowfly (half an inch in length) with face and legs yellowish, thorax bluish black with a grayish bloom, and the abdomen a beautiful metallic 
violet blue. The mouthparts are not fitted for piercing flesh, and there is no "stinger" at the posterior end of the body to drill a hole for depositing the eggs. Evidently, therefore, the . many accounts which one can find of the fly's biting or stinging at the time the eggs are deposited are faulty.

The manner in which the larvæ gain access to the skin of their hosts is one of the most remarkable and unusual adaptations known in nature. When ready to oviposit, the female fly captures various species of insects, among them large mosquitoes, particularly Janthinosoma lutzi (see pp. 451-453), various flies of the
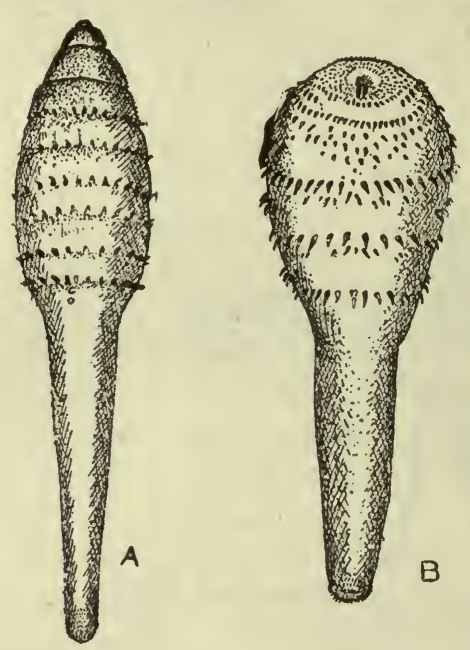
family Muscidæ including the house fly and stable fly, tabanids, and possibly ticks, and glues her eggs by means of an adhesive, quick-drying cement to the under side of the abdomen of these insects. When the latter insects alight upon the skin of warmblooded animals the maggots emerge, penetrate the skin of the host, several minutes to an hour being required for this process, and begin their development. According to Neiva and Gomes, captive females laid from 16 to 54 eggs on individual

Frg. 246. South American skin mag- flies, and two specimens laid a got, Dermatobia hominis; $A$, dorsal
view, extended; $B$, ventral view. $\times$ total of nearly 400 eggs. Eggs about 3. (After Neiva.)

frequently found on leaves are said to be deposited there by females that have not succeeded in holding flies they have tried to capture, and are compelled to oviposit. It is possible that the flies may also deposit their eggs directly in the skin of the host. The eggs require about six days' incubation, after which the larvæ are ready to emerge when a suitable occasion is presented. The larvæ may partially emerge from the egg shells and then draw back again if they do not succeed in reaching the warm skin of a host.

The time required for the larvæ to reach maturity in the host's skin varies from five to ten weeks. They ultimately reach a length of half or three-quarters of an inch (Fig. 246). The anterior end of the larva is broad and is provided with double rows 
of thorn-shaped spines; the posterior end is constricted, especially in fully-developed larvæ, and does not possess spines. As the larva develops, a sort of boil or cyst forms about it, opening to the surface of the skin by a little pore. This is plugged by the posterior end of the maggot, and used for obtaining air. At intervals these warble-like boils give rise to the most excruciating pain, due, no doubt, to a turning over or moving about of the spiny larva in its close quarters. When mature the larvæ voluntarily leave their host and fall to the ground to pupate. They transform into the adult form in the course of several weeks.

The swellings under the skin occupied by human botflies, as remarked before, are very painful at intervals, while at other times they are entirely painless. As the larva matures, a puslike material exudes from the open end of the "boil," containing, no doubt, the excretions of the maggot. After the worm has evacuated its cyst or has been removed the wounds sometimes become infected, and may even result in blood poisoning and death.

The method usually employed to remove the maggots is to apply tobacco juice or tobacco ashes to the infested spots, thus killing the worms and making their extraction easy. Another method used by natives in some parts of South America is to tie a piece of fat tightly over the entrance to the boil. The larva, deprived of air, works its way out into the fat, being thus induced to extract itself. A much more satisfactory method of dealing with the worms is to kill them with an injection of weak carbolic acid, mercuric bichloride, or some other poisonous substance, then enlarge the entrance to the cyst with a sharp clean knife and remove the body of the worm. A washing of the wound with a weak carbolic or lysol solution, followed by an antiseptic dressing, obviates any danger of subsequent infection. The wound heals quickly but leaves a scar.

Other Bots. - Other botflies occasionally infest man and cause cutaneous myiasis. The common warble-flies of cattle, Hypoderma lineata and $H$. bovis (Fig. 247), have been recorded as occurring in the skin or flesh of human beings and there is one fatal case on record where an ox warble caused an ulceration in the back part of the lower jaw of a boy six years old. Ox warbles usually gain access to the tissue under the skin of cattle in an indirect way, the hairy bee-like flies depositing their eggs on hairs of cattle where they will be licked off. As soon as licked the 
eggs hatch, and the larvæ burrow out through the wall of the œsophagus, migrate through whatever tissues they may find in their path, and ultimately reach a position just under the skin, usually on the back, where they finish their development. Occasionally the larvæ penetrate the skin directly, but the indirect
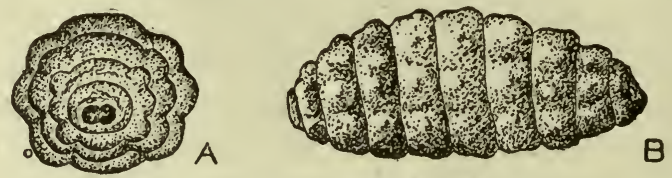

Fig. 247. Larva of Hypoderma bovis; $A$, posterior view; $B$, lateral view. $\times 2$.

method is the usual one. Recent investigations indicate that the two species differ somewhat in this respect. In Russia the horse botfly, Gastrophilus hoemorrhoidalis, which normally develops in the stomach of the horse, occasionally lives under the human skin.

African Skin Maggots. - The commonest species of maggot which develops in the human skin in Africa is the "ver du Cayor,"

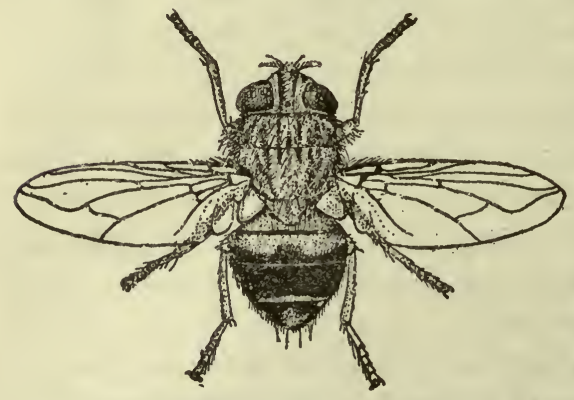

Fro. 248 . Adult female of African skin the vicissitudes of Frg. 248. Adult female of African skin the vicissitudes of life in

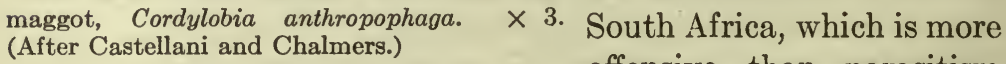
Cordylobia anthropophaga. This fly belongs to the same family as blowflies and houseflies. It is widespread throughout Africa, from Senegal and Khartoum to the Transvaal. To quote from Fuller, "There is no ill the flesh is heir to among the larva of the tumbu fly, offensive than parasitism by (this insect)." Man is not the main host of the larvæ of this fly, but he suffers in common with a large number of wild and domesticated animals, especially domestic dogs.

The adult fly (Fig. 248) is about the size of a blowfly (half an inch long), and is brown in color. The thorax is rusty to yellowish brown with indistinct dusky stripes, the abdomen pale brown, a little darker toward its tip, and with two dusky bands. Ex- 
actly where the fly deposits its eggs or newly hatched maggots is not quite certain. According to some observations the living larvæ are deposited directly on the skin and immediately bore their way in, while according to others the eggs or young larvæ are laid on the hair, on clothing which has been hung out, on soiled bed-clothes of children, etc. There is good reason to believe that the fly when about to lay eggs is attracted by fresh animal smells, such as perspiration, fresh excrement, etc., when these occur on the skin or on fabrics. The heads of infants, especially if not kept perfectly clean, are favorite places for the flies to deposit their offspring, and cases are on record in which 20 or 30 maggots were taken from the scalp of a child under six months old. Woolen clothing, if smelling of perspiration, is almost sure to become infested with the maggots when hung out in an exposed place, and it is dangerous to put on such clothing where the fly is abundant. Roubaud, in experiments with this fly, induced a specimen to lay 150 eggs on the walls of a glass vessel and on rotten fruit, and obtained infestation of a guinea-pig with 15 larvæ hatched apart from the host. That in some cases, at least, the eggs hatch before being deposited is evident from the fact that living maggots can sometimes be squeezed out of the bodies of the flies, and it is quite probable that the fly normally produces living young. The maggots are usually most abundant in the southern summer (January to

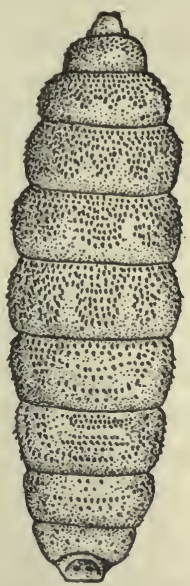

Fig. 249. African skin maggot, Cordylobia anthropophaga, dorsal view. $\times 6$. (After Brumpt.) March), especially in March. It is probable that there are not more than two or three generations a year, all of them during the summer, the rest of the year being spent in the adult stage.

The maggots (Fig. 249) are said to bore into the skin rapidly with active flapping movements and without causing any pain. As pointed out by Fuller it would endanger the life of the species if in entering the skin it excited its victim to dislodge it. Even if, as is probably often the case, the larva enters the skin during sleep, unless quite painless the host would probably wake and scratch it out, especially in the case of wild animals which must always sleep with an ear and an eye open, so to speak. As many 
as 300 maggots have been taken from the skin of a puppy, and it is not unusual for 20 or more to be present at once in a human being. They come to rest just under the surface of the skin, where they give rise in a few days to an inflamed boil, the inflammation being due to the movements of the spiny worm, and to the presence of toxic excretions. As in the case of Dermatobia, an opening is left to the surface of the skin from which the larva obtains air through the spiracles at the posterior end of the body. In some cases very little discomfort is felt from the maggots, but in other cases an intense pain is caused at intervals.

The larva is a fat, creamy-white maggot which reaches a length of half an inch when full grown. It is bluntly pointed at the anterior end but broad at the posterior end. The body is thickly covered with minute dark brown spines, each one recurved like a rose thorn.

Maturity is reached in about two weeks or less from the time the infestation occurs, though usually the time is underestimated, due to the fact that the larva is not noticed during the early part of its existence in the skin. When fully developed the larva voluntarily leaves its host and falls to the ground to pupate. The pupa is of the usual barrel-shaped form characteristic of the group of flies to which this species belongs. It is a little less than half an inch in length, at first of a light rusty color, later turning dark purplish brown. The adult insect emerges from the pupal case in about two weeks.

Preventive measures against the fly consist to some extent in personal cleanliness, since it is doubtful if the flies will deposit their offspring except on surfaces smelling of perspiration or other body excretions. Infants seem to be especially subject to attack, and should, therefore, be kept scrupulously clean. Since the larva of the fly lives readily in many domestic and wild animals, its extermination is hardly possible. In some parts of Africa, notably in Natal, the worm becomes abundant for several seasons, and then disappears for a number of years. The reason for this is not understood.

A closely allied fly, C. rodhaini, occurs in the damp equatorial forests of Africa, attacking thin-skinned animals such as antelopes and rodents, and occasionally man. Dogs, cattle and other thick-skinned animals are immune. The female of this species may deposit over 500 eggs, which hatch rin from two to 
four days. The mature larva, which closely resembles that of $C$. anthropophaga, leaves the host in from 12 to 15 days, buries itself to a depth of several inches in the ground, and pupates. If a little moisture is present, the transformation into the adult occurs in a little over three weeks. About two months is required for the whole life cycle of this fly.

\section{Myiasis of Wounds and of Natural Cavities of the Body}

A large number of flies, all of them related to the blowflies and houseflies, occasionally deposit their eggs or newly hatched larvæ in neglected wounds when offensive discharges are exuding from them. In severe cases infestations with maggots of these flies may lead to a most horrible and loathesome death.

The instinct of the female flies of all the species implicated is to deposit offspring in places from which the odor of meat or of decaying animal matter is emanating, regardless of where the place may be. This instinct is, of course, of the highest value to the species, since the larvæ live upon the substances from which such smells arise. It is an instinct analogous to that which causes a mosquito to lay its eggs in water, or a horsefly to oviposit in objects overhanging water - an unknowing but accurate intuition on the part of the parent to provide for the welfare of its young.

Screw-worm. - One of the most important species in this connection is the American screw-worm fly, Cochliomyia (or Chrysomyia) macellaria, which occurs throughout America from Canada to Patagonia, though abundant only in warm countries.

The adult fly (Fig. 250A) is a handsome insect, slightly larger than a housefly, of a metallic blue-green color with three dark stripes on the thorax. It belongs to the family Muscidæ, and to the same section as the ordinary blowflies. The adults congregate about carcasses of dead animals on which they ordinarily deposit their offspring and on which the larvæ feed. Records differ as to whether the eggs hatch within the body of the parent or after being deposited, and it is probable that during the early part of the season and in cool climates the eggs are deposited, while under other circumstances the living maggots are born. The number produced by a single fly may be several hundreds, but they are deposited with amazing rapidity. The maggots 
(Fig. 250B) are white, footless creatures, provided with a pair of stout hooks near the mouth, and with bands of minute spines which give them a screwlike appearance, whence they derive their name. Eating away at flesh and even bone, they develop rapidly to a length of about half an inch, and maturity may be
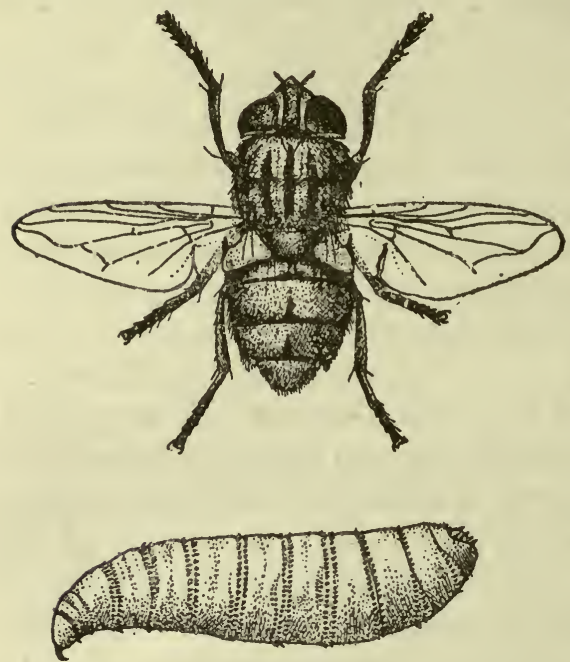

Fig. 250. Screw-worm fly, Cochliomyia (or Chrysomyia) macellaria, adult and maggot. $\times 3$. (Adult after Castellani and Chalmers, larva after Blanchard.) reached in three days, though four or five days is usually required. When fully developed the larva leaves its feeding grounds and buries itself in loose earth nearby, where it pupates in two or three days. The pupæ are brown in color, and shaped somewhat like olives. After four days or more in the pupal case the adult insect emerges, climbs up on nearby herbage and rests in a characteristic position with the head down. The whole life cycle occupies from nine days to two weeks or more.

As remarked before, the female screw-worm fly, about to reproduce, is attracted to any animal smell and frequently finds a suitable place for egg-laying in exposed wounds, or in the nose or ears of people sleeping out doors, especially in case of foul-smelling catarrh. Sometimes the flies select recently vacated Dermatobia nests, boils, sores, etc., for the young to develop in. As soon as hatched the maggots begin eating their way into the tissues with which they are in contact, using their strong mandibles as nippers for cutting flesh and even bone. From the ear they may make their way into the inner ear, completely destroying the auditory apparatus. From the nose they penetrate to the pharynx, frontal sinus, the eye-ball, and even the brain, occasionally doing such extensive damage as to cause death. Usually an abundant discharge of pus and scraps of tissue, intense pain, and delirium accompany the infestation. A severe 
case which occurred in Kansas, reported by Professor Snow, was substantially as follows: The victim had been suffering from nasal catarrh and was subject to offensive discharges. On August 22 he complained of a peculiar sensation at the base of the nose, followed by violent sneezing, and later by excruciating pain in the region of the forehead back of the nose. On the 24th there was a profuse discharge of offensive matter from nose and mouth with a subsidence of pain, the discharge continuing three days and amounting to 16 ounces, becoming almost pure pus with particles of bone, blood, etc., in it. The odor was very offensive, and coughing and fever developed, together with difficulty in speech and swallowing. At this time a maggot dropped from the nose, giving the first inkling of what the trouble was. The worms continued to drop from the nostrils and mouth, burrowing from under the soft palate and covering of the hard palate. The palate was completely honeycombed, and in places patches as large as a dime were entirely destroyed. The estimated number of maggots which escaped during 48 hours was over 300 . The whole of the soft palate was destroyed by this time, and the patient died four days after the emergence of the last worm.

Other Species. - Although the screw-worm is the species most thoroughly addicted to breeding in wounds and natural cavities of the human body, it is by no means alone in this nefarious habit. The beautifully colored green-bottle fly, Lucilia coesar, and other species of Lucilia have this habit, and the common blowflies, Calliphora vomitoria and C. erythrocephala, are sometimes implicated. These ubiquitous pests are said to have been a great torment to wounded soldiers in the Civil War. A closely related species of screw-worm, Chrysomyia bezziana, widely distributed in southern Asia, is, according to Patton, a very frequent parasite of man and animals in India. From observations on habits and structure, and from experimentation, Patton thinks this species deposits its eggs only in living animals. Of the fleshflies, which are related to the Muscidæ, but are placed in a separate family, Sarcophagidæ, many, and possibly all, will at least occasionally breed in wounds or natural cavities of living bodies.

A particularly troublesome species in Europe, especially in Russia, where it is almost as much of a scourge as is the screwworm in America, is the fleshfly, Wohlfartia magnifica. In Russia during hot weather this fly attacks the nose, ears, mouth, 
sores, wounds of any kind, or even the eyes, of human beings. In one case 70 maggots were extracted from one eye after about this many had already escaped and been thrown away. This fly, unlike most of its allies, is said to attack only living animals. The larvæ are unusually resistant to substances which readily kill other insects; they will survive two hours in 95 per cent

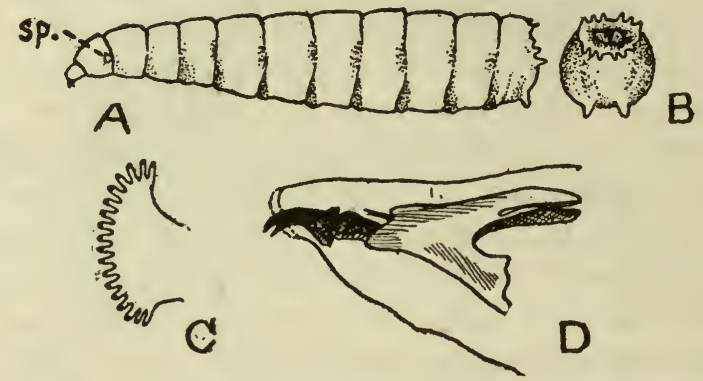

FIG. 251. Larva of fleshfly, Sarcophaga; $A$, side view of larva; $B$, posterior view showing posterior spiracles in depression; $C$, anterior spiracle, marked "sp." in Fig. $A ; D$, skeleton of pharynx, with mouth hooks. (After Riley and Johannsen.)

alcohol, and ten minutes in turpentine or pure hydrochloric acid. This species is said to be a great pest in war, where it causes myiasis in the wounds of soldiers. In France it is said to add much to the sufferings of wounded men.

Other fleshflies occasionally deposit their eggs on living animals or human beings. Sarcophaga carnaria is particularly likely to deposit eggs or larvæ in the vagina when it has access to it. As in the case of the flies mentioned above, this species will readily attack the nose or ears, especially if there is a foul-smelling catarrhal discharge flowing from it, and will infest inflamed or diseased eyes, sometimes nesting in large numbers under the eyelids and eating away the cornea.

The fleshflies are mainly gray in color, with longitudinal dark stripes on the thorax and a checkered abdomen which is changeable in varying lights. In summer the smell of decaying flesh will invariably attract them. The checkered abdomen and the broad angle at which the wings are held serve to distinguish them from other gray flies. Their life history is essentially the same as that of the screw-worm fly.

Another fly which must be mentioned in this connection is the sheep head-maggot, Estrus ovis, a species of botfly. It 
normally lays its eggs in the nostrils of sheep, from which place the maggots burrow into various parts of the head. In Algeria it is said to lay its eggs while flying without alighting, upon the eyes, nostrils and lips of - shepherds, especially those whose breath smells of fresh sheep or goat cheese. It somewhat resembles a housefly, but is larger and of a warmer brown color. Its mouthparts are deficient to such an extent that the fly is incapable of feeding, its only instincts being those connected with the reproduction of its kind.

Treatment. - The danger arising from attacks of screwworms and flies of similar habits is that the infestation is often not discovered until too late. Even when one is aware of an attack by the fly, it is not always possible to drive it away soon enough to prevent the eggs or maggots from being deposited. The larvæ should be removed as speedily as possible since they may do a great deal of damage in a very short time. Usually the maggots may be induced to release their hold and to fall out by douching the infested part of the body with a 20 per cent solution of chloroform in sweet milk, or with a carbolic or lysol wash. Even salt water is often effective in removing the maggots and should be used if no better wash is at hand. Maggots in the ear, if outside the ear drum, should be removed by means of water or milk saturated with chloroform, but if they have already pierced the ear drum, surgery will probably be necessary. Often where infections are two or three days old surgery must be resorted to and the larvæ removed by means of curved forceps. Frequent antiseptic washes prevent the injuries made by the maggots from becoming infected with bacteria.

\section{Myiasis of the Intestine}

There are a number of species of fly maggots which may accidentally be taken into the intestine of man and cause trouble there. To quote from Banks, "When we consider that these dipterous larvæ occur in decaying fruits and vegetables and in fresh and cooked meats; that the blowfly, for example, will deposit on meats in a pantry; that other maggots occur in cheese, oleomargarine, etc., and that pies and puddings in restaurants are accessible and suitable to them, it can readily be seen that a great number of maggots must be swallowed by persons each year, 
and mostly without any serious consequences." Banks gives the following quotation from Walsh, - "Taking everything into consideration, we doubt whether, out of 10,000 cases where the larvæ of two-winged flies have existed in considerable numbers in the human intestines, more than one single case has been recorded in print by competent entomological authority for the edification of the world."

Botflies. - There are some flies of the botfly family, Estride, which as larvæ habitually parasitize the digestive tracts of horses and other domesticated animals, and are especially adapted in habits and structure for such a larval life.

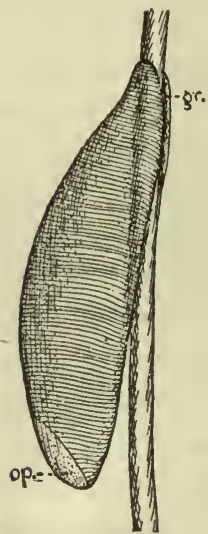

FIG. 252. Egg of horse botfly, Gastrophilus equi, attached to hair; gr., groove for cementing to hair; op., operculum. (After Collinge.) They occasionally, though rarely, occur in man. The horse botfly, Gastrophilus equi, for instance, lays its eggs (Fig. 252) on the hairs of horses in spots where they are likely to be licked. The moisture and rubbing of the horse's tongue cause the eggs to hatch at once, and the new larvæ, adhering to the tongue, make their way to the stomach and intestine where they attach themselves and develop to full-grown spiny larvæ, three-quarters of an inch in length. In the following spring the larvæ let go their hold, pass out with the fæces of their host and pupate in the ground. Obviously it could be only by a series of unusual circumstances that these larvæ could gain access to the human stomach, yet a number of cases have been recorded.

Fannia Larvæ. - A much more common occurrence in man is infection of the intestine with larvæ of various species of housefrequenting flies, especially the lesser housefly, Fannia canicularis, and the latrine fly, $F$. scalaris. The former species is very common in houses both in Europe and America. It closely resembles the housefly but is smaller, and appears earlier in the spring. The peculiar manner of flight, a sudden dart followed by a hovering, is very characteristic and a good means of identification. This fly is frequently observed hovering about chandeliers hanging near the center of rooms. The eggs are oval, white objects and are laid in decaying vegetable and animal matter 
and sometimes in excrement. The occasional presence of eggs in partially decayed vegetables, as in decayed lettuce leaves, rotten fruit, etc., probably accounts for the not uncommon appearance of the larvæ in the human intestine, although the egge may also be laid in or near the anus of people using unsanitary privies, whence the larvæ work their way up into the large intestine. The larvæ (Fig. 253) are very different from those of houseflies and blowflies, being broad and flattened, about one-

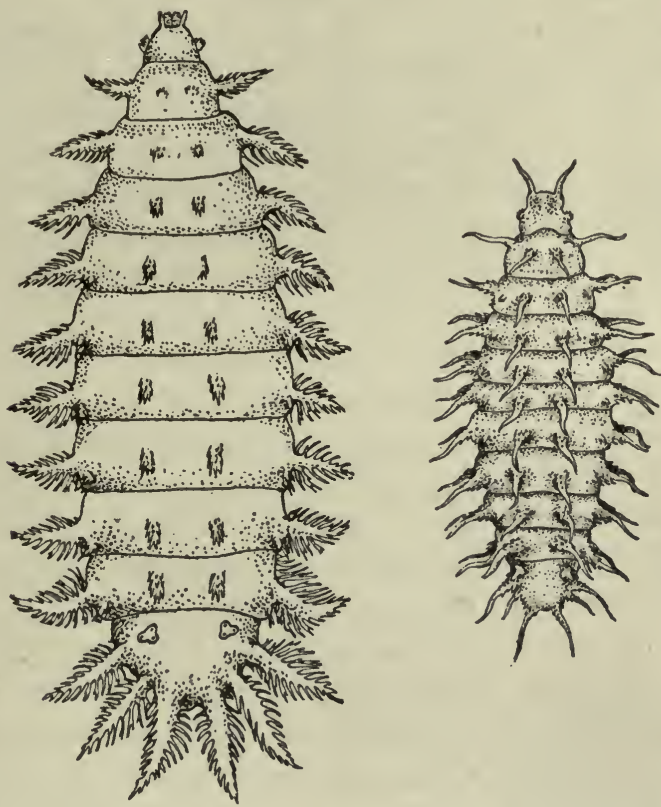

Fig. 253. Larvæ of Fannia scalaris (left) and Fannia canicularis (right), $\quad \times 8$. (After Hewitt.)

fourth of an inch in length when full grown, brown in color, with rows of spiny processes to which adhere particles of dirt and filth. The latrine fly, $F$. scalaris, is very similar to the species described above, but is larger and differs in minor details of form and habits. It prefers excrement, especially human excrement, on which to deposit its eggs, and has gained its common name from its frequent presence about privies and latrines. The author has found larvæ of this species very abundant in chicken manure. The adult has the same darting and hovering 
manner of flight as its close relative, $F$. canicularis. The larvæ (Fig. 253) differ from those of the latter species in the form and arrangement of spines. Several cases are on record in which Fannia larvæ were passed in the fæces intermittently for a number of years, often accompanied by a chronic disorder of the intestine. It is probable in these cases that repeated reinfections occur, though it may be conceived that the complete life history of the fly could be passed within the intestine of the host. The probability of this seems rather remote.

Other Species. - Another common cause of intestinal myiasis is the larvæ of the cheesefly, Piophila casei, popularly called

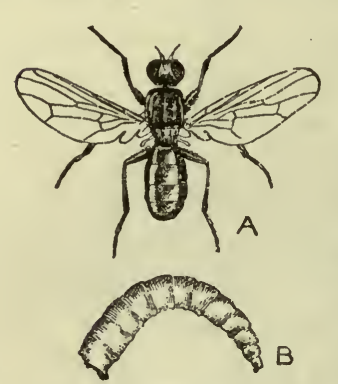

FIg. 254. Cheese-skipper and adult, Piophila casei. $\times$ 3. (After GrahamSmith from Riley and Johannsen.) which may become infected and ultimately lead to ulceration. Severe pain in the abdomen, headache and vertigo have been known to be caused by these larvæ in the intestine.

There is one case on record of the infection of a Chinaman with the fleshfly, Sarcophaga fuscicauda. He passed about 50 larvæ in each stool for eight days. Occasional infection of the intestine with maggots of other species of flies has been recorded, but the instances are so rare as to be of interest only as abnormal occurrences.

The powerful resistance of fly maggots to substances which would quickly destroy other animals makes it possible for many species to pass through the stomach safely if accidentally swallowed either as eggs or young worms. As said before experiments show that the larvæ of the fleshfly, Wohlfartia magnifica, can survive two hours in 95 per cent alcohol, and ten minutes in 
pure hydrochloric acid or turpentine. It is a little wonder, then, that fly maggots are not destroyed by the 0.2 per cent hydrochloric acid of the stomach or by the other digestive juices.

Effects. - The effects of fly larvæ in the intestine are extremely variable, depending on the heaviness of the infection, the species of flies, and on individual susceptibility. There are many cases where the presence of the larvæ in freshly passed stools is the first indication of their having existed in the intestine, and it is practically certain that the majority of infections are never known or suspected.

On the other hand more or less serious symptoms may be caused by intestinal myiasis. The presence of Fannia larvæ or of cheese-skippers in the digestive tract often gives rise to temporary intestinal disturbances, such as loss of appetite, vomiting, general malaise, abdominal pains, diarrhea, constipation and intestinal bleeding. Sometimes headache and vertigo indicate the absorption of toxic substances secreted by the maggots or their entrance to the blood circulation through the wounds. Four cases of death from intestinal myiasis have been recorded, and it is probable that appendicitis may sometimes be caused through injury to the walls of the appendix by fly larvæ which start sores leading to ulceration. Those maggots which pass directly through the digestive tract, feeding only on food substances with which they come in contact en route, do little or no harm to the temporary host. Those larvæ, however, which attack the living tissues lining the stomach and intestine are the cause of the symptoms named above. Even the maggots of the housefly, Musca domestica, have been known to damage the walls of the digestive tract. In a case which occurred in the Philippines, the walls of the stomach were extensively eaten away by larvæ of this common fly, and 20 or 30 maggots were obtained by means of a stomach pump. A liver abscess which was not due to the usual amebic infection accompanied this case, but whether due directly or indirectly to the myiasis can only be conjectured.

Fly maggots can usually be expelled readily by means of purges and doses of the drugs which are used for intestinal worms (see p. 237). The chief danger from infection, as in other forms of myiasis, lies in the fact that the presence of the maggots is usually not even suspected until much of their damage has been done. Prevention, of course, consists principally in care as to 
what is eaten, especially in regard to such foods as raw vegetables and partly decayed fruits.

Myiasis of Urinary Passages. - Myiasis of the urinary passages, both urethra and bladder, is a rare but occasional occurrence. The flies implicated are usually the lesser housefly, Fannia canicularis, and the closely allied latrine fly, F. scalaris, which have already been described in connection with intestinal myiasis. In most cases infection occurs from eggs laid near the external opening of the urethra, the larvæ working their way up into this tube and even into the bladder; apparently they need very little oxygen. Contamination is favored by sleeping without covers in hot weather, so that flies have free access to the anal and genital region. The larvæ, when escaping, are said to be able to project themselves with a flicking motion to a distance of from 12 to 20 inches. 


\section{SOURCES OF INFORMATION}

The following list of "sources of information" includes only those periodicals which are at least partly devoted to parasitology and preventive medicine, or which frequently contain important articles on these subjects, and those books which cover the entire subject or parts of it in a comprehensive manner. Books which are out of date and have been superceded by newer ones are not included. Most of the books listed contain more or less extensive bibliographies which should be of great assistance to anyone who desires to pursue any phase of the subject of human parasitology beyond the hallway to which this book may lead him.

\section{PERIODICALS}

\section{United States and Canada}

Amer. Journ. Publ. Health, New York, 1911-

Amer. Journ. Trop. Diseases and Prev. Med., New Orleans, 1913-1915. (Merged with New Orleans Med. and Surg. Journ.).

Amer. Journ. Trop. Med., Baltimore, 1921-

Exper. Sta. Bull. (Contains synopsis of interest in sections on "Economic Zoölogy and Entomology " and "Veterinary Medicine.")

Harvard School Trop. Med., Rep. (one issued), 1913-

Index Medicus, Washington, 1879-

Journ. Amer. Med. Assoc., Chicago, 1883- (Contains references to all current medical literature, and reviews of much of it.)

Journ. Canad. Med. Assoc., Toronto, 1911-

Journ. Cutaneous Diseases, Boston, 1882- (Continuation of "Journ. Cutaneous and Venereal Diseases.")

Journ. Econ. Ent., Concord, 1908-

Journ. Exper. Med., New York, 1896-

Journ. Inf. Diseases, Chicago.

Journ. Med. Research, Boston, 1901-

Journ. Parasitology, Urbana, 1914-

New Orleans Med. and Surg. Journ., 1844-

Publ. Internat. Health Comm., Rockefeller Foundation, New York.

Publ. Rockefeller San. Comm. for Eradication Hookworm Disease. Washington.

U. S. Bur. Animal Industry, Bull., Washington.

U. S. Bur. Ent., Bull., Washington.

U. S. Dep't Agr., Bull., Washington.

U. S. Naval Med. Bull., Washington.

U. S. War Dep't Bull., Washington. 


\section{South America}

Brazil Medico, Rio de Janiero, Brazil, 1887Cronica Medica, Lima, Peru, 1884-

Mem. do Inst. Oswaldo Cruz, Maguinhos, Rio de Janiero, Brazil, 1909-

\section{Great Britain}

Ann. Trop. Med. and Parasitology, Liverpool, 1907-

Brit. Med. Journ., London, 1857-

Bull. Entom. Research, London, 1910-

Journ. Econ. Biology, London, 1906-

Journ. Hyg., Cambridge, 1901-

Journ. London School Trop. Med., London, 1911-1913

Journ. Royal Army Med. Corps, London, 1903-

Journ. Trop. Med. and Hyg., London, 1898-

Lancet, London, 1823-

Memoirs, Liverpool School Trop. Med.

Parasitology, Cambridge, 1908-

Quarterly Journ. Micr. Science, London.

Rep. Sleeping Sickness Comm. Roy. Soc., London, 1903-

Review Applied Entom., Ser. B (Med. and Vet.), London, 1913-

(Contains reviews of all important work on medical and veterinary entomology.)

Sleeping Sickness Bull., London, 1908-1912

Trans. Soc. Trop. Med. and Hyg., London, 1907-

Trop. Diseases Bull., London, 1913-

(Contains reviews of all important work on tropical diseases, including nearly all work on protozoan parasites and on helminthology.)

\section{France}

Ann. d'hyg. et de med., Paris, 1898-

Ann. de l'institute Pasteur, Paris, 1887-

Arch. de parasitologie, Paris, 1898-

Bull. de la soc. de path. exotique, Paris, 1908-

Bull. Sci. de la France et de la Belgique, 1888-

Comp.-Rend. de la soc. de biol., Paris, 1849-

Comp.-Rend. des seances de l'acad. des sci. Paris, 1835-

Revue de med. et d'Hygiène tropicales, Paris, 1904-

\section{Germany and Austria}

Arch. für Protistenkunde, Jena, 1902-

Arch. für Schiffs- und Tropen-Hyg., Leipzig, 1897-

Bibliographica Zoologica.

Centralblatt für Bakt. und Parasitologie, 1 abt., Orig. und Ref.,. Jena, 1887(Ref. contains references and reviews of many articles dealing with infectious diseases.) 
Deutsche Med. Wochenschrift, Berlin, 1875-

Wiener Klinische Wochenschrift, Vienna, 1888-

Zeitschr. für Hyg. und Infektionskrank., Leipzig, 1886-

\section{Italy}

Annali d'Igiene, Rome, 1895-

Malaria e Malattie dei Paesi Caldi, Rome, 1910-

Malariologia, Rome, 1908-

Policlinico, Rome, 1893-

Pediatria, Naples, 1893-

\section{Portugal}

Arch. de hyg. e path. exot., Lisbon, 1905-

\section{Asia}

China Med. Journ., Shanghai, 1887- (Contains bimonthly, beginning 1916, "Japanese Medical Literature," a review in English of current Japanese medical work, also issued separately.)

Ind. Journ. Med. Research, Calcutta, 1913-

Ind. Med. Gazette, Calcutta.

Philip. Journ. Sci., Ser. B (Trop. Med.), Manila, 1906-

Proc. All India San. Conferences.

Sci. Mem. by Officers Med. and San. Dep't of Gov't of India, Calcutta.

\section{Africa}

Arch. de l'inst. Pasteur, Tunis, 1906-

Nyasaland Sleeping Sickness Diary, Zomba, 1908-

Rep. Wellcome Research Lab., Khartoum, 1906-

\section{Australia}

Australian Inst. Trop. Med., Collected Papers, Townsville, 1914-

\section{BOOKS}

\section{General}

Bolduan, C. F., and Koopman, J. Immune Sera, 5th ed., 206 pp., 9 figs., New York, 1917.

Braun, M., AND LüHe, M. Handbook of Practical Parasitology. (translated from German by L. Forster), vii + 208 pp., ill., 1910.

Braun, M. Die Tierischen Parasiten des Menschen, 5th ed. Part 1, Naturgeschichte, 560 pp., 407 figs., Wurzburg, 1915. Part 2 by Siebert, O., to appear later.

Breinl, Anton. The Distribution and Spread of Disease in the East; Protozoa and Disease; The Influence of Climate, Disease and Surroundings on the White Race Living in the Tropics (Stewart Lectures of Univ. of Melbourne) 31 pp., Melbourne, 1914. 
Brumpt, E. Précis de parasitologie, xxviii + 1011 pp., Paris, 1913.

Castellani, A., and Chalmers, A. J. A Manual of Tropical Medicine, 3rd ed., $\mathrm{x}+2436$ pp., 909 figs. 16 pls., London and New York, 1919.

Fantham, H. B., Stephens, J. W. W., and Theobald, F. V. The Animal Parasites of Man (partly adapted from Braun's "Die Tierischen Parasiten des Menschen), xxvii + 900 pp., London, 1916.

Hegner, R. W., and Cort, W. W. Diagnosis of Protozoa and Worms Parasitic in Man. 72 pp., 8 pls., Baltimore, 1921.

Laloy, L. Parasitisme et mutualisme dans la nature, 284 pp., 82 figs., Paris, 1906.

Leuckart, R. Die Parasiten des Menschen und die von ihnen herruhrenden Krankheiten, 2nd ed., (also English translation), Leipzig and Heidelberg, 1886-1889.

Manson. Tropical Diseases, ed. by Manson-Bahr, P. H., 7th ed., xvi +960 pp., 404 figs., 27 pls., 31 charts, London and New York, 1921.

Mense. Handbuch der Tropenkrankheiten, Band I, 1905.

Neumann, R. O., AND Mayer, M. Atlas und Lehrbuch wichtiger tierischer Parasiten und ihrer Ueberträger. Vol. IX of Lehman's Medizinische Atlanten, vi + 580 pp., 45 pls., 237 figs., Munich, 1914.

Neveu-Lemaire, M. Precis de parasitologie humaine, parasites vegetaux et animaux, 4th ed., Paris, 1911.

Rivas, D. Human Parasitology, 715 pp., 422 figs., 18 pls., Philadelphia, 1920. Shipley, A. E. The Minor Horrors of War. 184 pp., London, 1915.

Stiтt, E. R. Practical Bacteriology, Blood Work and Animal Parasitology, 6th ed., xi + 633 pp., 1 pl. 177 figs., Philadelphia, 1921.

Zinssner, H. Infection and Resistance, xiii + 546 pp., ill., New York, 1914.

\section{Protozoölogy and Helminthology}

Boyce, R. Yellow Fever and Its Prevention, 396 pp., London, 1911.

Brahmachari, U. Kala-azar and Its Treatment, 2nd ed., vii $+258+8$ pp., 16 pls., 24 charts, Calcutta, 1920.

Bruto da Costa, B. F., Santa Anna, J. F., dos Santos, A. C., and de Aranjo Alvares, M. G. Sleeping Sickness, a Record of Four Years' War Against it in the Island of Principe (Trans. from Portuguese by Wyllie, J. A.), xii + 261 pp., ill., London, 1916.

Calkins, Gary N. Protozoölogy, ix + 349 pp., 125 ill., New York, 1909.

Clark, J. J. Protozoa and Disease, 4 vols., London and New York, 19031916.

Craig, C. F. The Malarial Fevers, Hæmoglobinuric Fever and the Blood Protozoa of Man, New York, 1909.

Dobell, C. The Amœbæ Living in Man, vii + 155 pp., 5 pls. London, 1919. Dobell, C., and O'Connor, F. W. The Intestinal Protozoa of Man, ix + 211 pp., 8 pl., 2 figs., London, 1921.

Doflein, F. Lehrbuch der Protozoenkunde, 3rd ed., xii + 1043 pp., 951 figs., Jena, 1911.

Fantham, H. B., And Porter, A. Some Minute Animal Parasites, 319 pp., 56 figs., Jena, 1911. 
Hazen, H. H. Syphilis, 650 pp., 160 figs., 16 pls., St. Louis, 1919.

Kolle, W., and Wasserman, E. von. Handbuch der Pathogenen Mikroorganismen, 2nd ed., Band VII (Protozoa), 1039 pp., 121 figs., 20 pls., and Band VIII (Worms and Obscure Organisms), 1109 pp., 372 figs., 22 pls., 1913.

Laveran, A., And Mesnil, F. Trypanosomes et Trypanosomiases (1904 ed. trans. to English by D. N. Nabarro), Paris and Chicago, 1912.

Looss, A. The Anatomy and Life History of Ankylostoma duodenale, 451 pp., 19 pls., Cairo, 1908.

MacNeal, W. J. Pathogenic Micro-organisms, xxi + 462 pp., 213 ill., Philadelphia, 1914.

Minchin, A. E. An Introduction to the Study of the Protozoa, xi +517 pp., London, 1912.

Phillips, L. P. Amœbiasis and the Dysenteries, xi + 147 pp., London, 1915.

Prowazek, S. von. Taschenbuch der Mikroscopischen Technik der Protisten-untersuchungen; Leipzig, 1909.

Handbuch der Pathogenen Protozoen, in 6 Lief., in all 878 pp., 24 pls., 310 figs., Leipzig, 1912-1914.

Ross, R. The Prevention of Malaria, xx + 669 pp., London, 1910.

Staübl, C. Trichinosis, Wiesbaden, 1909.

Stephens, J. W., And Christophers, S. R. The Practical Study of Malaria and Other Blood Parasites, ix + $414+$ xiv pp., 6 pls., ill., London, 1908.

Thim , C. A. Bibliography of Trypanosomiasis. Issued under the Direction of the Honorary Managing Committee of the Sleeping Sickness Bureau, London, 1909.

\section{Medical Entomology}

Alcock, A. Entomology for Medical Offices, 2nd ed., xv + 380, 197 figs., London, 1920.

Austen, E. African Blood-sucking Flies, British Mus. Publ., London, 1909. A handbook of the Tsetse Flies, British Mus. Publ., $x+110$ pp., 24 figs., 10 pls., London, 1911.

Boyce, R. W. Mosquito or Man? The Conquest of the Tropical World, xvi +267 pp., 44 pls., New York, 1909.

Doane, R. W. Insects and Disease, xiv + 227 pp., New York, 1910.

Graham Smith, G. S. Flies in Relation to Disease (Non-Blood-Sucking Flies), xiv + 292 pp., Cambridge, 1913.

Hegh, E. Notice sur les glossines ou téstsés, 148 pp., 29 figs., London, 1915.

Herms, W. B. Medical and Veterinary Entomology, xii + 393 pp., 228 figs., New York, 1915.

Hindle, E. Flies and Disease (Blood-Sucking Flies), 414 pp., ill., Cambridge, 1914.

Howard, L. O., Dyar, I., And Knab, F. The Mosquitoes of North and Central America and the West Indies, Carnegie Inst. Publ., 4 vols., $520+1064$ pp., $14+150$ pls., Washington, 1913-1917.

Larrousse, F. Étude Systématique et Médicale des Phlébotomes. 106 pp., 20 figs., Paris, 1921. 
LePrince, J. A., And Orienstein, A. J. Mosquito Control in Panama, with Introduction by L. O. Howard, xvii + 335 pp., 100 ill., New York, 1916. Nuttall, G. H. F., Warburton, C., Cooper, W. F., and Robinson, L. E. Ticks; a Monograph of the Ixodoidea, Parts 1 to 3, Cambridge, 1908-1915 Patton, W. S., and Cragg, F. W. A Textbook of Medical Entomology, 764 pp., London, 1913.

Puerce, W. D. Sanitary Entomology, xxvi + 518 pp., 88 figs., Boston, 1921. Riley, W. A., ANd Johannsen, O. A. A Handbook of Medical Entomology, ix +348 pp., 174 figs., Ithaca, 1915.

Russell, H. The Flea, 125 pp., 9 figs., Cambridge, 1913. 


\section{INDEX}

Abyssinia, relapsing fever, 44 ; tapeworm infections, 240; Ornithodorus savignyi, 361, 368, 369.

Acanthaspis sulcipes, and endemic goitre in Africa, 382.

Acanthocephala, 199; 283-285.

Acanthocheilonema perstans, see $-\mathrm{F} i$ laria perstans.

Acarina, 324; 331-333; see also mites.

Acid, resistance of maggots to, 522 .

Acne, relation of Demodex to, 347.

Adaptations, of parasites and hosts, 14-15.

Aden, phlebotomus fever outside range of Phlebotomus papatasii, 470.

Aëdes, intermediate host of Filaria bancrofti, 301.

calopus, and yellow fever, 70, 72, 443; 443-448; and dengue, 183, 448; time of activity, 436 , 444; food preferences, 436; description, 443-444; habits, 444; breeding, 444-447; habits of larvæ, 446; flight and distribution, 447-448.

pseudoscutellaris, intermediate host of Filaria, 301, 450.

sollicitans, migrations, 435.

spenceri, habits, 436 .

Africa, relapsing fever, 42, 43; yaws, 63 ; yellow fever, 69 ; spirochrtal bronchitis, 73B; kalaazàr, 77; importance of sleeping sickness, 93; distribution of Trypanosoma gambiense and $T$. rhodesiense, 98; malaria, 147-148; blackwater fever, 161; Schistosoma homatobium, 213,
Schistosoma mansoni, 217 ; Gastrodiscoides in horses, 229; Watsonius watsoni, 229; Hymenolepis nana, 242; Trenia africana, 245; Diphyllobothrium latus, 246; Sparganum mansoni, 252; Necator americanus, 255; Physaloptera mordens, 282; Ternidens deminutus, 283; Esophagostomum apiostomum, 283; $E$. stephanostonum, 283; Filaria perstans, 307-308; Loa loa, 308, 489; Chrysops with larval filariæ, 310 489; Onchocerca volvulus, 310 ; Dracunculus medinensis, 311; aquatic leeches, 317; tick paralysis, 358-359; Ornithodorus moubata, 360; Argas reflexus, 364; Otiobius mégnini, 365; Amblyomma hebraum, 367; Cimex hemipterus, 373; Triatoma rubrofasciatus, 381; Acanthaspis sulcipes, 382 ; Xenopsylla, 417; chigger, 419; malariacarrying Anopheles, 441; oil films for mosquito larvæ, 459; Phlebotomus papatasii, 470, 471; tsetse flies, 490, 492; Glossina morsitans, 493, 499; Glossina palpalis, 498; other species of Glossina, 500-501; destruction of wild game, 503-504; bloodsucking maggots, 511-513; skin maggots, 513, 516-519.

African relapsing fever, relation of ticks to, 8, 43-46; importance, 42; transmission, 43-44, 360361; in Persia, 44; severity, 47. 
African skin maggot, see Cordylobia anthropophaga.

Agglutination, 21; of trypanosomes, 102-103.

Agramonte, C. A., 70, 443.

Akamushi, see Leptus akamushi.

Aкаshi, 244.

Alcock, A., 433, 449, 478.

Alcohol, in prevention of filarial infection, 307; for red-bug rash, 336 ; resistance of maggots to, 522,526 .

Alcresta ipecac, for Balantidium infections, 127; for amebic dysentery, 138.

Algeria, relapsing fever transmission, 44, 398; infantile kala-azar, 82; sheep head-maggot, 522-523.

Alum, for fleas, 421.

Amblyomma, 366.

cajennense, 367.

hebraum, 367.

Amebæ, cultivation, 9; encysted, in Egypt, 34; 128-146в; classification, 128-132; and dysentery, 135-140; effect of emetin on, 137-138; of mouth, 142-146B.

Amebic dysentery, in United States, 6; 135-140; importance, 135; parasites of, 135-136; course of, 136-137; carriers, 136; treatment, 137-139; prevention, 139-140.

America, relapsing fever, 42, 43; origin of syphilis, 48; importation of yaws, 63 ; yellow fever, $69-70,72$; possibility of kalaazar, 77; introduction of sleeping sickness, 93; Schistosoma mansoni, 217; Hymenolepis nana, 242; introduction of Necator americanus, 255; hosts of trichina, 288; Demodex folliculorum, 347; Amblyomma cajennense, 367; original home of Aëdes calopus, 447; Culex quinquefasciatus, 449; Janthinosoma lutzi, 453; oil of citronella as repellent for mosquitoes, 455; Chironomidæ, 474; skin maggots, 513-516; screw-worm, 519; Fannia, 524. See also various geographic subdivisions.

American hookworm, see Necator americanus.

American Hookworm Commission, use of thymol, 263; work of, 268.

American leishmaniasis, see Espundia.

American Red Cross, work in Serbia, 378, 398; work on trench fever, 187, 397, 399.

American Yellow Fever Commission, 7-8, 70, 443.

Ammonia, for red-bug rash, 335; for body lice, 402 .

Amoeba, 131; see also amebæ.

Anaphylatoxin, 23-25.

Anaphylaxis, 23-25; specific, 24; treatment, 25 .

Ancon, manufacture of larvicide, 459.

Ancylostoma duodenale, distribution, 255; description, 255-257. ceylanicum, 255.

Anderson, J. F., 8, 397.

Andes, uta, 86; Oroya fever, 178, 472; Phlebotomus verrucarum, 473.

Animal experimentation, 10-11.

Anise oil, for body lice, 401; for mosquitoes, 455; for phlebotomus flies, 473 .

Anisol, for body lice, 401 .

Annelida, 199-200; 215; relation to arthropods, 323 ; see also Leeches.

Anopheles, malaria-carrying species, 157-158, 438, 439-441; and malaria in winter, 156; cessation of breeding and subtropical malaria, 162; number necessary to propagate malaria, 165; intermediate host of Filaria bancrofti, 301, 450; 
palpi, 426; eggs, 429; larvæ, 431,442 ; time of activity, 435,437 ; identification, 438439; habits, 441-443; variable ability of species to transmit different kinds of malaria, 439; effect of oil on larvæ, 458; effect of larvicide, 459.

albimanus, and malaria in tropical America, 439.

argyrotarsus, and malaria in tropical America, 441.

bancrofti, and malaria in Australia, 441.

bifurcatus, hibernation, 441.

braziliensis; habits, 441.

costalis, and malaria in Africa, 441. crucians, relation to various types of malaria, 439 .

culicifacies, and malaria in India, 441.

cruzi, habits, 442.

eiseni, habits, 441-442.

funesta, and malaria in Africa, 441.

listoni, and malaria in India, 441; in China and Japan, 441.

ludlowi, and malaria in East Indies, 441; habits, 442.

maculipennis, and malaria in Europe, 441.

malefactor, not a malaria carrier, $158,434$.

punctipennis, development of

Leishmania donovani in, 78 ; relation to certain types of malaria, 439.

quadrimaculatus, resistance of Plasmodium vivax to low temperatures in, 156; carrier of certain types of malaria, 439 ; development, 442 .

sinensis, and malaria in China and Japan, 441.

stephensi, and malaria in India, 441.

umbrosus, and malaria in Malay countries, 441; habits, 441.

willmori, and malaria in Malay countries, 441; habits, 441.

Anoplura, 388; see also lice.

Antelope, host of Trenia saginata, 240 ; host of tsetse flies, 490 ; host of Cordylobia rodhaini, 518 .

Anthelmintics, 270.

Anthrax, and tabanids, 488; and stable-flies, 488, 507.

Antibodies, 21; duration, 22.

Antigen, 22.

Antimony, metallic, in kala-azar, 81; compounds in trypanosomiasis, 104-105; see also Tartar emetic.

Antitoxin, 21.

Anti-vivisectionists, 10-11.

Apes, hosts of Trypanosoma cruzi, 112; relation of intestinal worms to typhoid in, 204.

A phthomonas infestans, and foot-andmouth disease, 76, 193.

Aphthous fever, see Foot-and-mouth disease.

Aponomma, 366.

Appendicitis, relation of intestinal worms to, 204; and intestinal myiasis, 527.

Arabia, oriental sore, 85; Ornithodorus savignyi, 361; tsetse flies, $492,500$.

Arachnida, 324.

Aradidæ, 383.

Aragão, H. DeB., 73D, 130.

Archi-annelida, 199.

Arctomys bobac, and plague in Manchuria, 413.

Argas miniatus, see $A$. persicus. persicus, and relapsing fever, 45 , 361 ; importance, 364 ; control, 369.

reflexus, 364.

Argasidæ, egg-laying habits, 355; general characteristics, 356357; important species, 364366.

Argentina, trypanosocen an Tratoma, 108, 112, 381; dengue, 182; Tetranychus molestissimus, 341. 
Armadillo, host of Trypanosoma cruzi, 112; and Triatoma geniculata, 380-381.

Arsenic, in trypanosomiasis, 104-105; see also atoxyl and tryparsamide.

Arsenical dip, to remove ticks from domestic animals, 368 .

Arsenobenzol, for guinea-worms, 314.

Arthropoda, 322-330; importance, 322 ; rôle in disseminating disease, 7-8, 322-323; relationships, 323-324; classification, 324-325.

Ascaris, nutriment absorbed, 202; toxins, 202-203; 273-276; description, 273; life history, 274275 ; symptoms, 276 ; treatment 276.

lumbricoides, 274.

marginata, see Toxascaris limbata. mystax, see Belascaris cati.

suilla, 274.

Ascaridæ, 282.

Ashburn, P. M., 301, 448.

Asia, oriental sore, 84; blackwater fever, 161; dengue,. 182; kedani, 190; Schistosoma hamatobium, 213; Clonorchis sinensis, 224; Yokagawa yokagawa, 228; Heterophyes heterophyes, 228; Fasciolopsis buski, 229; Hymenolepis nana, 242; Necator americanus, 255; Filaria bancrofti, 299; Dracunculus medinensis, 311; Porocephalus moniliformis, 351; Cimex hemipterus, 373; Triatoma rubrofasciata, 381; Xenopsylla, 417; Phlebotomus, 470; surra, 487; Chrysomyia bezziana, 521 .

Asopia farinalis, intermediate host of Hymenolepis diminuta, 244.

Assam, eradication of kala-azar, 82. Astacus japonicus, intermediate host of lung fluke in Korea, 222.

Atkin, E. E., 445, 446.

Atoxyl, discovery, 8; for trypanosomes, 105.
Aucheromyia luteola, 511-513; description, 511; life history, 511512; maggots, 512; avoidance of, 513 .

Australia, Aëdes calopus carrier of dengue, 448; hydatids, 248, 250; Filaria bancrofti, 299; land leeches, 319-320; tick paralysis, 358-359; malaria-carrying Anopheles, 441; Aëdes calopus, 448; transmission of dengue, 448; Pericoma townsvillensis, 466 .

Austria, relapsing fever, 43; typhus, 398.

Auto-salvarsanized serum, for syphilis of nervous system, 57; for sleeping sickness, 106.

Axopodia, 31.

Axostyle, 32.

Baboon, host of Trichostronglyus instabilis, 282; fed upon by tsetse flies, 500 .

Bacillus coli, 204. pestis, discovery, 411.

Васот, А. W., 375, 376, 391, 393, 395, 409, 412, 444, 445, 446.

Bacteria, distinguished from Protozoa, 27; relation to trachoma, 194; and intestinal worms, 204; relation to filarial diseases, 305306; food of Aëdes calopus, 446. Bacterium tularense, transmitted by fleas, 413; by deer flies, 489 .

Badger, host of Pulex irritans, 414. Bagdad, oriental sore, 85, 88, 471.

Bagshawe, A. G., 503.

BAHR, P. H., 303.

Baking soda, for mites, 335, 339.

Balanitis, cause of, $73 \mathrm{~A}$; treatment, $73 \mathrm{~B}$.

Balantidial dysentery, 129.

Balantidium coli, discovery, 7, 37; 115; 126-127; description, 126; pathogenicity, treatment and prevention, 127.

Balkans relapsing fever, 43,45 . 
Balsam of Peru, for itch, 346.

Baltic countries, Diphyllobothrium latus in, 246.

Bancroft, Th., 7.

BANKs, N., 333, 339, 523, 524.

Barbados, home of "millions," 461.

Barbeiro, see Triatoma megista.

Barber, M. A., 206.

BARLOW, N., 117, 132, 281.

BarRetT, M. T., 142.

Barton, 179.

Bartonella bacilliformis, 168; 179 $181 ; 360$.

Basal granule, 30 .

Basile, C., 84.

Bass, C. C., 9, 149, 164.

Bats, Cimex in, 372, 375; trypanosome disease of, carried by Cimex pipistrelli, 378; natural enemies of mosquitoes, 462; natural enemies of tsetse flies, 503.

Bayon, H., 378.

Beauperthuy, L. D., 322.

Bedbugs, and relapsing fever, 45, 46; 378; and kala-azar, 77-78, 377; and Leishmania infantum, 83; and oriental sore, 86, 377-378; and Trypanosoma cruzi, 112, 370, 378; Rickettsia in, 185; 371-379; general structure, 371 ; odor, 371-372; species, 372-373; habits, 373-375; effect of bites, 374; hosts, 374-375; life history, 375-376; and disease, 376-379; and bubonic plague, 378-379; remedies and prevention, 383; fumigation of, 385-386.

Bee eater, natural enemy of tsetse flies, 503.

Beef tapeworm, see Tania saginata.

Beetles, hosts of Gigantorhynchus hirudinaceus, 284; natural enemies of mosquitoes, 462 .

Belascaris cati, 282.

Belgium, Tydeus molestus, 341.

Bello Herizonte, eradication of Chagas' disease, 114.
Beta-naphthol, for Giardia infections, 125; for hookworm infections, 264; ointment for itch, 346.

Bête rouge, 335, 336.

Bi-flagellate Protozoa, of intestine, 117-118.

Bilharz, Th., 7.

Biliary fever, of horses, 168.

Binucleata, 30.

Bird lice, see Mallophaga.

Birds, hosts of Cimex, 372; chief prey of Culex quinquefasciatus, 449; as food for tsetse flies, 494, 495; natural enemies of tsetse flies, 503; blood-sucking maggots in nests of, 511 .

Bishopp, F. C., 422.

Bismuth salicylate, for Giardia infections, 125.

Bismuth subnitrate, for amebic dysentery, 137.

Biting flies, see Flies, blood-sucking.

Black corsair, see Melanolestes picipes.

Black drongo, natural enemy of tsetse flies, 503.

Blackflies, and espundia, 92; and Onchocercacacutiens, 311; mouthparts, 327, 478; 478-484; description, 478-479; life history, 479-481; annoyance, 481-483; and disease, 483; control, 483484.

Blackheads, relation of Demodex to, 347.

BlaCKLOCK, B., 494, 496.

Black sickness, see Kala-azar.

Black vomit, in yellow fever, 71.

Blackwater fever, 161-162.

Bladderworms, types of, 235; damage done by, 236-237.

beef bladderworms, see Cysticercus bovis.

pork bladderworms, see Cysticercus cellulosœ.

Blanfordia, host of Schistosoma japonicum, 219. 
Blepharoplast, 29; see also Parabasal body.

Blood, immunity reactions, 20-22; relation to anaphylaxis, 23-24.

Blood corpuscles, white, see Leucocytes.

Blood flukes, discovery, 7, 8; 213220; relation of sexes, 213; possibility of introduction into United States, 219-220. See also Schistosoma.

Bloodsuckers, see Leeches.

Blowflies, maggots parasitic on birds, 511.

Bodo, 115; 117-118.

Bolivia, oriental sore, 87.

Bombay, relapsing fever, 43.

Bont tick, see Amblyomma hebroum.

Borax, and calcium borate for treating manure, 508.

Botflies, mouth parts, 464; in human skin, 513-516; and intestinal mýiasis, 524.

Bozzolo, C., 8.

Braun, M., 36.

Brazil, yaws, 63; kala-azar, 77; espundia, 90, 488; trypanosomiasis, 94, 108-114; bug-proof houses, 114; Trichomonas pathogenic, 121; Enteromonas hominis, 122; hookworm disease, 255; Esophagostomum stephanostomum thomasi, 283; Filaria magalhãesi, 308; Triatoma, 380-381; Anopheles cruzi, habits, 442; Dermatobia and mosquitaes, $452,453$.

Breakbone fever, see Dengue.

Breinl, A., 73D.

British Columbia, tick paralysis, 358-359; Dermacentor venustus, 363.

British Guiana, Sparganum mansoni, 252; hookworm disease, 262; filarial infections, 308 .

British Isles, see Great Britain.

British Plague Commission, 411, 412.

British Royal Commission on Vene- real Diseases, report, 50,58 , $59-60$.

British Trench Fever Commission, 187, 397, 399.

Bronchitis, caused by spirochætes, 71.

Bruce, D., 7, 98, 108, 397.

BrUмpт, E., 83.

Buba braziliensis, 89 .

Bubalis caffer, host of Glossina morsitans, 500.

Bubonic plague, see Plague.

Buenaventura, yellow fever in, 70. Buffalo, host of Glossina morsitans.

Buffalo gnats, see Blackflies.

Bugs, see Hemiptera.

Bulgaria, typhus in, 398.

Bullinus contortus and B. dybowskii, intermediate hosts of Schistosoma in Egypt, 215, 216, 217.

Bursa, of hookworms, 256.

Butter, on clothing to prevent lousiness, 402.

Bуам, Maj. W., 397.

Bythinia striatula, var japonica, inter-mediate host of Clonorchis, 226.

Cachexia, malarial, 161, 162; treatment, 164.

Cairo, prevention of Schistosoma infections, 216в.

Calabar swellings, 309 .

Calandruccio, S., 284.

Calcium borate, and borax for treating manure, 508.

California, hookworm in mines, 262; hookworm introduced by Hindus, 268; Dermacentor occidentalis, 358, 363; Ornithodorus coriaceus, 364-365; plague in ground squirrels, 411; Pulex irritans, 415; Ceratophyllus acutus, 418.

Calkins, G. N., 33.

Calliphora vomitoria, cause of myiasis, 521.

erythrocephala, cause of myiasis, 521. 
Calomel, for Giardia infections, 125.

Camel, and oriental sore, 86 ; host of Trichostrongylus instabilis, 282; host of Ornithodorus savignyi, 361; el debab, 487.

Camphor, for red-bug rash, 336; repellent for mosquitoes, 455 .

Canada, blackflies, 479, 481, 482.

Capitulum, of ticks, 354 .

Carapatos, see Ornithodorus moubata and $O$. turicata.

Carbolic acid, for head lice, 401; to remove Dermatobia from skin, 515.

Carbon bisulphide, fumigation, 386; for fumigation of body lice, 401 , 402.

Carpenter, G. D. H., 494.

Carriers, definition, 19,21 ; in amebic dysentery, 136.

Carrion, D., 178.

Carrion's fever, see Oroya fever.

Carroll, J., 70, 443.

Castellani, A., 121, 307, 340.

Castor oil, for Giardia infections, 125 ; in treatment of hookworm infections, 264.

Cats, and infantile kala-azar, 82; and Giardia, 125; hosts of Opisthorchis, 225; Dipylidium caninum, 245; fewer parasites than dogs, 266; trichina, 288;

Notodres cati, 343; and bedbugs, 375; 'fleas, 416-417; Echidnophaga gallinacea, 420; destruction of fleas on, 422-423.

Cattle, hosts of spotted fever tick, 189; Paramphistomum cervi, 229; Trenia saginata, 240; hydatids, 248, 250; hosts of Dermacentor venustus, 363; hosts of stable-flies, 505; Dermatobia in, 513; warble-flies, 515-516.

Central America, relapsing fever, 46; checking of yellow fever epidemic, 72; mal-de-boca, 73A; trypanosomes in Triatoma, 108, $112,380-381$; use of shoes, 265 ; work of Hookworm Commission, 268; bête rouge, 335; Ornithodorus, 361; Triatoma, $379,380,381$; other Reduviidæ, 382 ; chiggers, 418, 419; Anopheles eiseni, habits, 441; yellow fever mosquitoes breeding in holy-water fonts, 445; Dermatobia carriers, $452,453$.

Centrosome, in Protozoa, see Basal granule.

Ceratophyllus, cysticercoids in, 243; and plague, 412.

acutus, and plague, 413, 418.

fasciatus, life history, 409; haoits, etc., 417-418

gallinœ, 417.

silantiewi, and plague, 413.

Ceratopogon, 475, 477.

Ceratopogoninæ, 474, 475, 476.

Cercaria, 211.

Cercomonas, 115, 117-118.

Cerebrospinal fluid, spirochætes in, 49, 57; trypanosomes in, 104; trichina in, 290.

Cerebrospinal meningitis, animal experimentation with, 10 .

Cerodon rupestris, host of Triatoma chagasi, 381.

Cestoda, 198; see also Tapeworms. Ceylon, Necator americanus, 255; beta-naphthol used for hookworm infections, 264; landleeches, 319; copra itch, 340 .

Chætopoda, 199.

Chagas, C., 8, 94, 110, 111, 112.

Chagas' disease, 108-114; course of, 113-114; treatment and prevention, 114 .

Chancre, 54.

Chandler, A. C., 212, 245, 285, 461.

Chaparro amargosa, for intestinal amebæ, 138.

Chatton, E., 140.

Cheesefly, see Piophila casei.

Cheese-skipper, see Piophila casei.

Cheliceræ, of Acarina, 331; of ticks, 354. 
Chenopodium, oil of, for amebic dysentery, 138-139; for intestinal flukes, 230; for tapeworms, 237; for hookworms, 263-264; specific action, 270; for Ascaris, 270, 276; for whipworms, 277; for pinworms, 279 .

Chicken mite, 341.

Chickens, and bedbugs, 375; and Triatoma, 379; Ceratophyllus gallinoe, 417; Echidnophaga gallinacea, 420 .

Chigger, see Dermatophilus penetrans and Harvest mites.

Chigoe, see Dermatophilus penetrans.

Chilomastix, 117.

mesnili, 122-123.

China, relapsing fever, 43; syphilis, 50; kala-azar, 77; Embadomonas sinensis, 118; Trichomonas pathogenic, 121; Schistosoma japonicum, 215; Schistosoma mansoni, 218; lung flukes, 220; human liver flukes, 224; Yokagawa yokagawa, 228; Fasciolopsis buski, 229; use of shoes, 265; rat fleas, 417; malaria-carrying Anopheles, 441; transmission of anthrax by tabanids, 488 .

Chinese fluke, see Clonorchis sinensis.

Chironomidæ, and uta, 86, 477;

- 473-477; description, 473-475; habits, 475; life history, 475476 ; annoyance, 476 ; as disease carriers, 476-477; control, 477.

Chlamydophrys stercorea, 132-133.

Chlamydozoa, 170, 191-195.

Chloroform, for hookworm infections, 264; as an anthelmintic, $270-272$; in milk for myiasis of nose, ear, etc., 523 .

Chlorosis, 255.

Choromyia, 512-513.

Christiansen, E., 124.

Christopherson, J. B., 216A.
Chromidia, 28.

Chrysomyia bezziana, 521. macellaria, see Cochliomyia.

Chrysops, and Loa loa, 309-310, 486, 487, 489; trap for, 490 . dimidiata, 489. discalis, 489. silacea, 489.

Chyluria, filarial, 305.

Cilia, 30.

Ciliata, cilia in, 30 ; 35 ; 36 ; human parasites, 126.

Cimex, 371, 372; see also Bedbugs. boueti, 373 .

hemipterus, and kala-azar, 77-78, 377; characteristics, 372-373. lectularius, characteristics, 372373; hosts, 374-375.

pipistrelli, 378.

rotundatus, see $C$. hemipterus.

Cimicidæ, characteristics, 371.

Cinchona, see Quinine.

Cinchonization, see Quininization.

Cirri, 30.

Cirrus pouch, 232.

Citellus beecheyi, and plague, 413.

Citronella, oil of, repellent for mosquitoes, 455 .

Civet cats, hosts of Ancylostoma ceylanicum, 255.

Civil War, fly maggots in, 521 .

Clarke, F. C., 414.

Clonorchis endemicus, $=$ sinensis, 225 . sinensis, discovery, 7; 211, 224225; life history, 226.

Cnidosporidia, 36.

Cocaine, for leeches, 318.

Coccidians, 168; 170-173; life history, 170-172; in man, 172-173.

Coccidium seeberi, 174.

Coccoid bodies in spirochætes, 39 .

Cochliomyia macellaria, in espundia sores, 90; egg-laying, 464, 519; 519-521; description, 519; effects, 520-521; treatment, 523.

Cockle bur, mites on, 341 .

Cockroach, and Davainea madagascariensis, 244; and Monili- 
formis moniliformis, 284.

Conurus, 235.

Cold storage, effect on bladder worms, 238; on trichina, 295; on Clonorchis larvæ, 227.

Colemanite, and borax for treating manure, 508.

Colombia, relapsing fever, 46; spirochætal bronchitis, 73в; Balantidium infections, 127; yellow fever, 70; hookworm disease, 254.

Columbacz fly, 482.

Combs, on fleas, 404, 408.

Compulsory notification, of venereal disease, 60.

Cone-nose, see Triatoma.

Congo, rubber industry and sleeping sickness, 107; Porocephalus, 351.

Congo floor maggot, see Aucheromyia luteola.

Contractile vacuole, 31 .

Copepods, intermediate hosts of $\mathrm{Di}$ phyllobothrium, 246-247.

Copper sulphate, for destruction of snails, 212.

Copra itch, 340.

Cordylobia anthropophaga, 516-518; deposition of eggs, 517; development of maggot and life history, 518; prevention, 518. rodhaini, 518-519.

Corethra, 425.

Corethrinæ, 437.

Cornwall, J. W., 377, 378.

Corrosive sublimate, see Mercuric chloride.

Corsica, phlebotomus flies, 468.

Councilmania, 129.

lafleuri, 129, 131,132, 140, 141.

Coyote, host of Opisthorchis pseudofelineus, 225.

Crabs, relation to lung flukes, 8, 222-223.

Cragg, F. W., 374, 416.

Craig, C. F., 132, 143, 145, 301, 448.

Craigia, hominis, 132.

migrans, 132.
Craneflies, allied to mosquitoes, 425 .

Crawley, H., 176.

Crayfish, possible host of lung flukes, 223.

Creolin, for removal of ticks, 367; for fumigation, 386 ; to destroy fleas, 422-423.

Cresol, for killing Schistosoma cercariæ in water, 217; for body lice, 402; for chiggers, 420 ; to destroy mosquito larvæ, 459.

Cresyl, for fumigation, 386; for fumigation of mosquitoes, 456 .

Crithidia, 75; stage of trypanosome, 95-96.

Crocodiles, fed on by tsetse flies, 494, 499.

Cruickshank, J. A., 306.

Crustacea, first intermediate host of Diphyllobothrium latus, 246; 324; see also Crabs, Shrimp, Cyclops.

Ctenidia, 404, 408.

Ctenocephalus canis, and infantile kala-azar, 83, 413; life cycle, 410; and Dipylidium caninum, 414; habits, etc., 416-417.

felis, life cycle, 409; habits, etc., 416-417.

Culex, intermediate host of Filaria bancrofti, 301; and bird malaria, 438.

fatigans, see C. quinquefasciatus. pipiens, resemblance of $C$. quinquefasciatus to, 448.

quinquefasciatus, and dengue, 183, 448; and Filaria bancrofti, 301; description, etc., 448-449.

territans, 434.

Culicidæ, characteristics, 425 .

Culicinæ, 437.

Culicini, 437.

Culicoides, habits of larvæ, 475; annoyance, 476.

Cultivation, of animal parasites, 9.

Cyclasterion scarlatince, 191.

Cyclops, and guinea-worm, intermediate host of Diphylloboth- 
rium, 246-247; 312, 313-314.

Cyclorrhapha, 465, 466.

Cyprinodontidæ, natural enemies of mosquitoes, 460 .

Cysticercoid, nature of, 235.

Cysticercus, nature of, 235.

bovis, thermal death point, 237238; effect of cold storage, 238; description, 240.

cellulose, thermal death point, 237-238; effect of cold storage, 238; description, 241; hosts, 241-242; in man, 251.

Cytopyge, 31.

Cytoryctes variolox, 170; see-also guarneiri bodies.

Cytostome, 31.

Dahomey, absence of sleeping sickness in, 501.

Da Matta, A., 92.

Darling, S., 129, 175.

Darwin, Chas., 4, 381.

Dasypus novemcinctus, host of Trypanosoma cruzi, 112.

Datura stramonium, for fumigation of mosquitoes, 456 .

Davainea madagascariensis, 244. formosana, 244.

Deer, host of Pulex irritans, 414.

Deerfly, see Chrysops.

DeKruif, P. H., 23-25.

Demarquay, J. N., 7.

Demodecidæ, 333, 346.

Demodex folliculorum, 346-348; life history, 347; and leprosy, 347.

Dengue, relation of mosquitoes to, 8 , $183,448-449$; parasites of, $182-$ 183; 182-184; prevention, 184.

De RaAdt, O. L. E., 399, 417.

Dermacentor, 366; Rickettsia in, $185,186$.

andersoni, see $D$. venustus.

occidentalis, effects of bite, 358-

359, 366; possible transmitter

of spotted fever, 363, 366-367. variabilis, 367.

venustus, transmission of spotted fever, 188-189; and tick paralysis, 358; description, 361-363.

Dermacentroxenus rickettsi, 188, 191.

Dermanyssus gallinæe, 341.

Dermatobia hominis, and mosquitoes, 451-453, 514; transmitting mosquitoes, 453 ; 513-515; description, 513-514; manner of reaching host, 514; effects, 514-515.

Dermatophilus penetrans, 418-420.

Deschiens, R., 125.

De Silva, P., 83.

Desvoidea obturbans, and dengue, 183, 448.

Diaptomus, intermediate host of $\mathrm{Di}$ - phyllobothrium, 246-247.

Dibothriocephalidæ, larvæ of, 235; characteristics, 239; important species of, 245-247; Sparganum larva of, 251.

Dibothriocephalus, see Diphyllobothrium.

Dicrurus ater, natural enemy of tsetse flies, 503.

Diemyctylus torosus, natural enemy of mosquitoes, 461.

Dientamoba, 132.

fragilis, 132, 142.

Dioctophyme renale, 200.

Diphyllobothrium latus, 246-247. cordatus, 247.

Diplocercomonas, 122.

Diplogonoporus grandis, 247.

Diplozoa, 26.

Diptera, 326; characteristics, 330; 425; used by Dermatobia, 452, 514; 463-464; importance, 463; general structure, 463464; life histories, 464-466; classification, 466; parasites on tsetse fly pupæ, 503; and myiasis, 509.

Dipylidium caninum, 245; and fleas, 414, 415, 417.

Dirt-eating, in hookworm disease, 262.

Diseases, conquest of, 2 ; ignorance of, 4 ; causation by germ, 6 ; 
transmission by arthropods, $7-8$, 322-323.

Disinfection, of mosquito bites, 307; of tick bites, 367 ; to eradicate lousiness, 402-403.

Dixa, 425 .

Dixon, S. G., 462.

Doane, R. W., 414.

Dobell, C., 130, 131, 132, 133, 134, 140, 142, 146A, 172.

Doerr, R., 470.

Dog flea, see Ctenocephalus canis.

Dogs, and infantile kala-azar, 82,83 ; and oriental sore, 86; Trypanosoma gambiense in, 108; host of Trypanosoma cruzi, 112; susceptible to lung fluke infections, 220; host of Clonorchis sinensis, 224; host of Opisthorchis, 225; Dipylidium caninum, 245; host of Diphyllobothrium cordatus, 247; Echinococcus granulosus, 248, 250-251; hosts of Ancylostoma ceylanicum, 255; more parasites than cats, 266; trichina, 288; Demodex, 347;

Yinguatula rhinaria, 349-350; Dermacentor variabilis, 367 ; and bedbugs, 375 ; inability of human lice to draw blood from, 393; host of Pulex irritans, 414; fleas, 416-417; Echidnophaga gallinacea, 420; destruction of fleas on, 422; Dermatobia in, 513; Cordylobia anthropophaga in, 518.

Dog ticks, see Dermacentor variabilis and Ixodes ricinus.

Dongola, blackflies in, 482 .

Donovan, C., 7, 74, 377.

Dracunculus medinensis, 311-314; distribution, 311; life history, 312-313; extraction and prevention, 314

Dragon-flies, natural enemy of tsetse flies, 503.

Dubini, A., 7.

Ducks, natural enemies of mosqui- toes, 462 .

Dumdum fever, see Kala-azar.

Dutcher, B. H., 305.

Dutron, J. E., 7, 8, 359.

Dwarf tapeworm, see Hymenolepis nana.

Dyar, I., 429, 437, 444, 447, 458.

Dysentery, types of, 135 ; rôle of amebæ, 135-136.

Dysentery ameba, see Endamoba histolytica.

Dysodius lunatus, 382-383.

Ear tick, see Otiobius mégnini.

East Coast fever, of cattle, 168.

East Indies, gangosa, 64; blackwater fever, 161; land-leeches, 319; Porocephalus moniliformis, 351; transmission of malaria, 441; Anopheles in coral reef pools, 442 .

Echidnophaga gallinacea, 420.

Echinorhynchus hominis, 284; see also Moniliformis.

Echinococcus granulosus, 236; 240 251; distribution, 248; adult and life history, 248; development of hydatids, 248-249; other species of Echinococcus, 250 ; prevention, 250-251.

Echinostomum ilocanum, 228-229. malayanum, 229.

Ectoplasm, 29.

Ecuador, hookworm disease, 262.

Education, present need, 3-4; concerning sex hygiene, 62 ; conerning sanitation, 268-269.

Eelworms, see Ascaris.

Egypt, Embadomonas intestinalis, 118; amebx in sand, 128; Schistosoma hamatobium, 213$214,215,216 \mathrm{~B}$; Heterophyes heterophyes, 228; Paramphistomum cervi, 229; Sparganum mansoni, 252; hookworm disease, 255; work of Hookworm Commission, 268; Trichostrongylus instabilis, 282; Xenop- 
sylla cheopis, 417; breeding places of Phlebotomus, 468, 473.

ЕнrLich, P., 8, 47, 49, 56.

Eimeria, in man, 172-173.

oxyspora, 172.

snijderi, 172.

stied $\propto$, in man, 172. wenyoni, 172.

El debab, 487.

Elephantiasis, 304-305; relation of bacteria to, 305-306; treatment, 306; and Onchocerca volvulus in Congo, 311.

Elephantoid fever, 305.

Elk, host of Dermacentor venustus, 363.

Elurs, A. W. M., 57.

El Marg, Schistosoma hæmatobium, $214,215$.

Embadomonas, 115, 117.

intestinalis, 118.

sinensis, 118.

Emetin, discovery, 8; not effective for Giardia, 125; for Balantidium infections, 127; nature of, 137; in amebic dysentery, 137138; effects on other amebæ, 141 ; in pyorrhea, 145, 146A.

Emrich, W., 138, 139.

Encapsulation, 20-21; of trichina, 291.

Encystment, 34.

Endamœba, in jaw lesion, 121; decription of genus, 129.

buccalis, see $E$. gingivalis.

coli, 132; compared with $E$. histolytica, 133; 135; description, 140-141.

gingivalis, $142-146 \mathrm{~B}$; relation to pyorrhea, 142, 144-146A; description, 143 ; relation to tonsilitis and goitre, 146; effect of emetin on, 146A.

histolytica, 115, 129; description and life history, 133-134; compared with $E$. coli, 133; and disease, 135-136; effect of emetin on, 137-138. muris, 140.

nana, see Endolimax nana.

Endolimax, description of genus, 129.

nana, 130, 132, 135, 141.

Endomixis, 33-34.

Endoplasm, 29.

Endotoxins, 24.

Entamœba, see Endamœba.

Enteromonas, 117. hominis, 122.

Eosinophiles, increase with worm infections, 203.

Epimys norvegicus, and plague in Europe, 411.

rattus, and plague in Europe, 411.

Epipharynx, 326.

Epsom salts, see Magnesium sulphate.

Erdmann, R., 175.

Eriocheir japonicus, intermediate host of lung fluke, 222.

Escomel, P., 117, 121, 125.

Espundia, 89-92; distribution, 89; parasites, 89; skin sores, 89; mucous membrane ulcerations, 90 ; treatment, 91-92; prevention, 92 .

Ether, for body lice, 401.

Eucalyptus, oil of, for hookworm infections, 264; for body lice, 401; for fleas, 422 ; for phlebotomus flies, 473.

Eupodidæ, 333, 341.

Europe, plague in, 2, 411; relapsing fever, 42, 44, 378; introduction of syphilis, 48 ; infectious jaundice, 65; amebic dysentery, 134; blackwater fever, 161; dengue, 182; trench fever, 187, 399; Opisthorchis felineus, 225; Hymenolepis nana, 242; hookworm disease, 255; Ancylostoma duodenale, $\cdot 255$; hookworm in miners, 265; trichina, 287, 288; Filaria bancrofti, 299; Hamopis, 317; red-bugs, 336; 
Norwegian itch 343; Demodex folliculorum, 347; Linguatula rhinaria, 350; Argas reflexus, 364 ; Ixodes ricinus, 367; typhus, 186, 398; origin of Pulex irritans, 414; Ceratophyllus sp., 417, malaria-carrying Anopheles, 441; Phlebotomus papatasii, 470; control of Phlebotomus, 473; Columbacz fly, 482; Wohlfartia magnifica, 521522; Fannia, 524. See also geographic subdivisions.

European War, typhus in, 2, 398; infectious jaundice, 68; amebic dysentery, 131.

Eustrongylus gigas, see Dioctophyme renale.

Evans, J. S., 144.

Ewrng, H. E., 332.

Fæces, search for parasite eggs in, 206, 272.

Fannia, characteristics of larvæ, 509; and intestinal myiasis, 524-526; effects of myiasis caused by, 527.

canicularis, and intestinal myiasis, 524-526; and myiasis of urinary passages, 528 .

scalaris, and intestinal myiasis, 524-526; and myiasis of urinary passages, 528 .

Fantham, H. B., 39, 102, 174.

Farmers responsibility for trichiniasis, 296.

Fasciola hepatica, discovery of life cycle, 7; life history, 209-211; in man, 224.

Fasciolopsis buski, 229-230.

FAUST, E. C., 118, 119.

Fibrolysin, in clephantiasis, 307.

Fiji Islands, yaws in, 63; Filaria bancrofti, 301.

Filaria, discovery, 7, 298; relation of mosquitoes to, 7, 301-303, 449-451; 298-314; prevalence, 298-299. bancrofti, 299-307; distribution, 299; life history, 299-303; periodicity, 300-301; cycle in mosquitoes, 301-303, 450; transmission, 303; pathogenic effects, 303-306; treatment for, 306307 ; prevention, 307.

demarquaii, sce $F$. juncea.

juncea, 308, 450.

loa, see Loa loa.

magalhãesi, 308.

perstans, 301, 450.

philippinensis, 307-308, 450.

Filarial diseases, 303-306; elephantiasis, 304-305; chyluria, 305; relation of bacteria to, $305-$ 306 ; treatment, $306-307$; prevention, 307.

Finocchiaro, F., 73c.

Fish, intermediate hosts of Clonorchis sinensis, 226; of Opisthorchis, 226-227; of Dibothriocephàlido, 245; of Diphyllobothrium latus, 247; relation to Sparganum mansoni, 252; relation to Sparganum proliferum, 253.

Fish oil, repellent for tabanids, 489 .

Fish tapeworm, see Diphyllobothrium latus.

Flagellata, flagella in, 29, 35, 36.

Flagellates, chlorophyll-bearing, 27; of blood-sucking insects in vertebrates, 74,75 .

Flagellum, 29.

Flame cells, 197.

Flatworms, 196-198.

Fleas, and infantile kala-azar, 83, 413; Rickettsia in, 185; intermediate hosts of Dipylidium caninum, 245, 414; fumigation, 386, 421; general structure, 404-407; classification, 407; identification, 408; life history, 408-410; habits, 410; and disease, 410-414; and plague, 410-413; and typhus, 414; human flea, 414-415; dog 
and cat fleas, 83, 416-417; rat and squirrel fleas, 417-418; chig'gers, 418-420; sticktight flea, 420-421; prevention, 421-423; traps, 421.

Fleshflies, and Sarcosporidia, 175; and myiasis of wounds, 521522 ; description, 522 ; and intestinal myiasis, 526-527.

Flexner, S., 10.

Flies, see also Diptera.

blood-sucking, mouthparts, 327, 464; importance, 463; see also various groups and species.

non-blood-sucking, and oriental sore, 86; and espundia, 92; rôle in transmission of Giardia, 125; and tapeworm eggs, 240; mouthparts, 327, 464 .

Flood fever, see Kedani.

Florida, prevention of malaria by screening, 167; Sparganum proliferum, 252-253.

Flukes, 207-230; general anàtomy, 207-208; reproduction, 208; life history, 208-211; parasitic habitats, 212; control, 212; see also Blood flukes, Lung flukes,

- Liver flukes, Intestinal flukes.

FluRY, F., 202, 290, 293.

Fly-belts, 106, 492-493.

Fonseca, O. O.R. da.

Foot-and-mouth disease, parasite of, $76,169,193$.

Forcipomyia, and uta, 86; habits, $475,477$.

townsendi, and uta, 86, 477.

utce, and uta, 86, 477.

Forde, R. M., 7.

Formaldehyde, for fumigation, 386; to destroy flea eggs, 421; to repel phlebotomus flies, 473 .

Formalin, see Formaldehyde.

Formosa, transmitter of dengue, 183, 448; lung flukes, 220, 222; Y̌okagawa yokagawa, 228; Davainea formosana, 244; aquatic leeches, 317.
Foster, W. D., 263, 264, 270, 272, $274,275,276$.

Fournier, 50.

Fowl tick, see Argas persicus.

Fracastorio, G., 6.

Frambesia, see Yaws.

France, amebic dysentery, 135; trench fever among troops, 399; Wohlfartia magnifica, 522

Francis, E., 489.

FRÄNKEL, S., 402.

French Guiana, Onchocerca, 310.

French Yellow Fever Commission, 444.

Fricks, L. D., 190.

Frontal lunule, 465.

Fuller, C., 516, 517.

Fumigation, for ticks, 369; hydrocyanic acid, 383-386; sulphur, 386; carbon bisulphide, 386 ; cresyl, 386; formaldehyde, 386 ; for fleas, 421; for mosquitoes, 456.

FUTAKI, K., 73.

Gadflies, see Tabanidæ.

Gallipoli, Giardia, in soldiers from, 123, 125; Coccidian infections, 172.

Galyl, substitute for salvarsan, 65 .

Gamasidæ, see Parasitidæ.

Gangosa, 64.

Gasoline, for bugs, 383.

Gastrodiscoides hominis, 229.

Gastrophilus equi, 524.

hæmorrhoidalis, 516.

Gates, Dr. H., 253.

Gecko, Algerian, and oriental sore, $86,471$.

Geese, host of Holothyrus coccinella, 341.

Geographic distribution, of parasites, 18-19.

Gerlach, A. C., 345.

Germany, multilocular hydatids, 250; trichina, 286; Linguatula rhinaria in man, 350 ; louse prevention, 402-403. 
Giardia, 115, 123-125; description, 123; multiplication, 123-124; pathogenicity and treatment, 125; rôle of flies in transmission, 125.

intestinalis, 123-125.

muris, $124 ; 125$.

Gibraltar, relapsing fever, 47.

Gigantorhynchus hirudinaceus, 284. gigas, 284.

Gill, A. A., 162.

Giraffe, host of Tænia saginata, 240.

Girardinus pœciloides, natural enemy of mosquitoes, 460-461.

Girault, A. A., 376.

Glossina, 491-492; see also Tsetse flies.

brevipalpus, time of activity, 493, 501; and human trypanosomes, 500.

longipennis, time of activity, 493, 501.

morsitans, and Trypanosoma rhodesiense, 98, 101, 497; distribution, 98; fly-belts, 106, 492-493; 499-500; time of activity, 493, 500; habits, 493; food, 494, 500 ; duration of pupal period, 496; breeding places, 496; and nagana, 497; distribution, 499; description, 499; and Trypanosoma gambiense, 500; control, 501; attached by dragon-flies, 503.

pallidipes, and Trypanosoma gambiense, 500 .

palpalis, and Trypanosoma gambiense, 98-101, 496-497, 501; fly-belts, 106, 492-493, 498; time of activity, 493; food, 494; 498-499; life history, 495; breeding places, 496; distribution, 498; description, 498; control, 501.

tachinoides, time of activity, 493, 498; and sleeping sickness, 500; habitats, 500; control, 501.

Glyciphagus, 340. buski, 340 .

Gnats, see Chironomidæ.

Goats, hosts of Linguatula rhinaria, 349 , unattractive to tsetse flies, 499.

Goddard, F. W., 229, 230.

Goitre, caused by Trypanosoma cruzi, 114; caused by Endamoba gingivalis, 144-145; transmitted by Acanthaspis sulcipes, 382.

Goldberger, J., 8, 339, 397.

Gomes, J. F., 514.

Gonder, R., 34.

Gonzales, E., 217.

Goodey, T., 119.

Gorgas, W. C., 166.

Gorilla, host of Necator americanus, 255; host of Esophagostomum stephanostomum, 283.

Graham, H., 8, 448.

Grain mites, see Tyroglyphidœ.

Grassi, B., 284.

Grayback, see Pediculus humanus.

Great Britain, syphilis in, 50; amebic dysentery, 134.

Greece, downfall due to malaria, 147.

Greenland, Diphyllobothrium cordatus, 247.

Grocers' itch, 340.

Groll, 293.

Ground itch, 259, 260.

Guam, gangosa, 64.

Guarnieri bodies, in smallpox, 191.

Guatemala, Onchocerca coecutiens in, 311.

Guiana, Cimex boueti, 373.

Guinea-pigs, for experimentation, 10; immunization against infectious jaundice, 68; host of Trypanosoma cruzi, 112, 378, 381 ; susceptible to trichina, 288; and plague, 413.

Guinea-worm, see Dracunculus medinensis.

Gumma, 54.

Gyrinidæ, and mosquitoes, 462. 
Haeckel, E., 27.

Homadipsa ceylonica, 319. japonica, 320.

Hamaphysalis, 366.

Homatobia serrata, 506.

Homatopinus ventricosus, inability to draw human blood, 393 .

Hæmatopota, 486.

Hæmoflagellata, 75.

Homopis, 316, 317.

HAGLER, 378.

HaLL, M. C., 263, 264, 270-272, 276.

Hall, H. C., 393, 394.

Haltere, 464.

Haplosporidia, Rhinosporidium member of, 173 .

Harvard School of Tropical Medicine, South American expedition, on uta, 86; on Oroya fever, $178,179,180,181$.

Harvest mites, 333-337; life history, 334; annoyance, 335; species, 336; and kedani, 191, 336-337.

Haughwout, F. G., 121.

Havana, reduction of malaria, 166; reduction of yellow fever, 70 , 72.

Hawaii, introduction of mosquitoes, 435.

Hayashi, N., 190.

Head-maggot, of sheep, see EEstrus ovis.

Hellebore, for treating manure, 508.

Hemiptera, mouthparts, 326; digestive tract, 327-328; characteristics, 330,370 .

Herms, W. B., 364, 365, 489, 505, 506.

Herrick, W. W., 293.

Нerrick, G. W., 335, 384, 385, 387.

Herpetomonas, stage of Leishmania, 74 ; species, 75 ; relationships, 75 ; in blood in leishmaniasis, 75; developed from Leishmania donovani in bedbugs, 78; in Anopheles punctipennis, 78; stage of trypanosomes, 95-96; in tabanids, 488 . ctenocephali, and infantile kalaazar, 83.

Heterophyes heterophyes, 228.

Heteroptera, 370.

Hindle, E., 187, 188.

Hine, J. S., 490.

Hippobosca canina, and leishmaniasis of dogs, 86 .

Hirudinea, 199-200; see also Leeches.

Hirudo, 316.

History of parasitology, 6-13.

Hogs, Trypanosoma gambiense in, 108; and human intestinal Protozoa, 116; and Balantidium coli, 127; Paragonimus kellicotti, 220; Gastrodiscoides hominis, 229; Fasciolopsis buski, 229; Tania solium, 240-241; Ascaris, 274, 275; trichina, 286, 287, 288, 292, 296; Ornithodorus turicata, 361; Dermatophilus penetrans, 418; Dermatobia in, 513.

Hogue, M. J., 118.

Holothyrus coccinella, 341.

Honduras, craigiasis, 137; Strongyloides, 281.

Нооке, 387.

Hookworms, economic importance of, $3,254,262-263$; in immigrants, 5; discovery, 7 ; detection of ova by various methods, 206; toxins, 203, 261; 254-269; history, 254-255; local names, 254-255; distribution, 255; description of species, 255-257; life history, 257-260; eggs, 258; mode of infection, 259-260; disease, 260-263; dirt-eating, 262 ; ' treatment, 263-264; preyention, 264-265; sanitation, 265-269.

American, see Necator americanus. Old World, see Ancylostoma duodenale.

Hoplopsyllus anomalus, and plague, 413.

Horseflies, see Tabanidæ. 
Horsehair snakes, popular supersti. tion, 4; see Nematomorpha.

Horse leech, 316.

Horses, and oriental sore, 86; spotted fever tick, 189, 363; Gastrodiscoides, 229; leeches, 317, 319; Ornithodorus savignyi, 361; Otiobius mégnini, 365; surra, 487; hosts of stable-flies, 505 ; hosts of Gastrophilus hamorrhoidalis, 516.

Housefly, sec Musca domestica.

House mosquito, of tropics, see Culex quinquefasciatus; of temperate zones, see Culex pipiens.

How ARD, I. O., 148, 429, 437, 442, $444,447,455,458$.

Howlett, F. M., 469, 470.

Hume, 81.

Hyalomma, 366.

Hydatids, nature of, 235, 248-251; distribution, 248; life history, of Echinococcus, 248; development, 248-249; multilocular, 250 ; effects on host, 250; prevention, 250-251.

Hydrocyanic acid, for fumigation, of bedbugs, 383, 385; method, 383-385; effectiveness, 385386 ; for fleas, 421.

Hydrogen peroxide, for balanitis, 73в.

Hydrophobia, see Rabies.

Hymenolepis, prevention, 238; cysticercoids in fleas, 414 .

diminuta, 244.

murina, 242.

nana, discovery, 7 ; oil of chenopodium for, 237; prevention, 238; 242-244.

nana fraterna, 242.

Hymenoptera, parasites of tsetse fly pupx, 503.

Hypoderma bovis, 515-516.

lineata, 515-516.

Hypopharynx, 326.

Hypopus, 339-340.
Hypopygium, 492.

Hypostome, 354.

Iceland, hydatids, 248, 250.

Ichneumon flies, and Dermatobia, 452.

Ichthyol, for microfilariæ, 306; in ointment for chiggers, 420 .

Idaho, spotted fever, 189.

IDo, Y., 65.

IJima, J., 252, 253.

Illinois, Moniliformis clarki, 285. Immunity, natural, 19-22; artificial, 22-23; passive, 22; of Protozoa to drugs, 34-35; in relapsing fever, 47; in infantile kala-azar, 84; in oriental sore, 87; in Rhodesian sleeping sickness, 94; reactions among trypanosomes, 96-97; in malaria, 162-163; in yellow fever, 71, 72; in dengue, 183, in phlebotomus fever, 185; in trichiniasis, 294-295.

Immunization, history, $8-9$; in relapsing fever, 47 ; in infectious jaundice, 68; in yellow fever, 72 ; in oriental sore, 88 ; against trypanosomes, 106.

Immunology, development of, 9 .

INADA, R., 65.

India, plague in, 3, 411; relapsing fever, 42, 43, 44; kala-azar, 77; oriental sore, 85; amebic dysentery, 135; malaria, 147; fulminant malaria, 163; Rhinosporidium, 173; dengue, 182; phlebotomus fever, 184; Clonorchis sinensis, 224; Gastrodiscoides hominis, 229; Trnia saginata and dung-eating habits of cattle, 240; hookworm disease, 262 ; use of shoes, 265; landleeches, 319-320; Rhizoglyphus buski, 340; Ornithodorus savignyi, 361 ; bedbugs and kalaazar, 377; cat flea, 416; chigger, 420; malaria-carrying Anopheles, 441; Aëdes calopus, 448; 
Phlebotomus minutus, 471; blackflies, 478; Chrysomyia bezziana, 521 .

Indian bedbug, see Cimex hemipterus Infantile kala-azar, 82-84; distribution, 82 ; transmission, 82-83, 413,417 ; course of, 84 ; treatment, 84 ; prevention, 84 .

Infantile paralysis, 195; and stableflies, 507.

Infectious jaundice, 65; course of, 65-66; mode of infection, 67; in rats, 67-68; treatment, 68; prevention, 68-69.

Infusoria, see Ciliata.

Insanity, relation of syphilis to, 53, 54.

Insects, 325-330; general characteristics, 325; mouthparts, 325327 ; general anatomy, 327-329; life history, 329-330; classification, 330 .

Intestinal flukes, 228-230; life history, 230.

Intestinal Protozoa, 115-127; of man and animals, 115, 117; encystment, 115-116; specific hosts, 116; geographic distribution, 116; pathogenic effects, 116-117; prevalence, 116; biflagellate species, 117-118; multiflagellate species, 118-125, ciliates, 126, 127; effects on progress of school children, 266267 ; see also amebæ.

Intestinal worms, entrance and exit from host, 201; effects on host, 201-204; nutriment absorbed by, 202; toxic effects, 202-203; infection of wounds made by, 203-204; effects on progress of school children, 266-267; round worms, 270-272; selection of drug for treatment, 270; search for eggs, 272; prevention, 266269,272 ; see also various species.

Iodamoba, 129, 132. bütschlii, 132, 142.

Iodine, for Trichomonas infections, 122.

Iodine or I. cysts, 142 .

Ipecac, and amebic dysentery, 137.

Island of Principe, extermination of sleeping sickness, 108, 502.

Ismailia, reduction of malaria, 165 .

Isospora, in man, 172, 173.

hominis, 172.

Italy, ińfantile kala-azar, 84; fulminant malaria, 163; reduction of malaria, 165; phlebotomus fever, 184; Hymenolepis nana, 242; Moniliformis moniliformis, 284; Pediculoides, 338; breeding places of phlebotomus flies, 468 .

Itch, 342, 344-345; Norwegian, 343; treatment, 345-346; prevention, 346.

Itch mites, 342-346; description, 342-343; life history, 343-344; disease caused by, 344-345; treatment, 345-346; prevention, 346.

ITURBe, J., 217.

Ixodes, habits, 356; characteristics, 366.

holocyclus, and tick paralysis, 359 . pilosus, and tick paralysis, 359 . ricinus, 367.

Ixodidæ, egg-laying habits, 355; general characteristics, 356-357; important species, 366-367; key to genera, 366 .

JANICKI, C., 246.

Janthinosoma lutzi, carrier of Dermatobia, 452, 453

posticata, 452, 453 .

Japan, relapsing fever, 43 ; infectious jaundice, 65, 67; rat-bite fever, 73; seven-day fever, 73D; kedani, 190; Schistosoma japonicum, 218, 219; lung flukes, 220, 223; human liver flukes, 224, 225, 227; Yokagawa yoka- 
gawa, 228; Heterophyes heterophyes, 228; Davainea formosana, 244; Diphyllobothrium latus, 246; Diplogonoporus grandis, 247; .Sparganum mansoni, 252; Sparganum proliferum, 252-253; Trichostrongylus orientalis, 282; land-leeches, 319-320; kedani mite, 336-337; rat flea, 417; malaria-carrying Anopheles, 441.

Japanese flood fever, see Kedani.

Japanese seven-day fever, 73D.

Java, malaria in children and adults, 162; lice and plague, 399-400; Xenopsylla, 417.

JeNNer, E., 4, 9.

JEPPS, M., 142.

Jigger, see Dermatophilus penetrans. Jimson weed, for mosquitoes, 456.

Johannsen, C. A., 339, 474

Johns, F. M., 9, 149.

Johnson (Mrs.), see Lawson, Mary

Kabure, relation to Schistosoma japonicum, 218.

Kala-azar, 77-82; distribution, 77; transmission, $\quad 77-79, \quad 377$; human cycle of parasite, 79; course of, 80 ; mortality, 81 ; treatment, 81 ; prevention, $81-$ 82; see also Infantile kala-azar.

KANEKO, R., 73.

Kansas, screw-worm, 521 .

Katajama, see Blanfordia.

Kedani, 168, 169, 186, 190-191.

Kedani mite, see Leptus akamushi.

Keen, W. W., 10.

KellogG, V. L., 389.

Kerosene, see Petroleum.

Killifish, natural enemies of mosquitoes, 460-461.

Kinetonucleus, see Parabasal body.

KING, A. F. A., 149.

KING, H. H., 482, 487.

KING, W. V., 156.

King, W. W., 368.

Kinghorn, A., 100.
Kissing, and syphilis, 51; and amebic infections of mouth, 146.

Kissing bugs, 382 .

Kleine, F. K., 310.

KNAB, F., 429, 435, 436, 437, 444, $447,451,453,458$.

КовауаSнi, H., 226, 227.

Косн, R., 8, 9, 162.

Koford, C. A., 112, 118, 120, 124, 132, 134, 141, 206, 381.

Korea, lung flukes, 222; Clonorchis sinensis, 224, 225; Yokagawa yokagawa, 228.

Kornhauser, S. J., 118, 134.

Kulagin, N. M., 442.

Labella, of mosquitoes, 427.

Labial palpi, 325.

Labium, 325.

Labrum, 325.

Labrum-epipharynx, 326.

Lamblia, see Giardia.

Lamborn, W. A., 493, 503.

Land-leeches, 319-321.

LANING, R. H., 218.

Larvicides, 457-459.

Laveran, A., 7, 148, 471.

Laverania malariœ, see Plasmodium falciparum.

Lawson, Mary R., 150.

Lazear, J. W., 70, 443.

Leeches, 315-321; general anatomy, 315-316; importance as parasites, 315, 316-317; intermediate hosts of trypanosomes, 317 ; in nose and throat, 317-318; land-leeches, 319-321.

LeeUWenhoek, A. van, 6, 37, 391.

LeIPER, R. T., 8, 211, 215, 216, 216в, 217, 219, 224, 228, 230, $267,310$.

Leishman, W. B., 7, 43, 74.

Leishman bodies, see Leishmania.

Leishmania, 74-92; discovery, 7, 74; transformations in insects, 74 ; species, 75, 76; relationships, 75 ; diseases, 76; and kala-azar, 77-82, 377; and infantile kala- 
azar, 82-84; and oriental sore, $84-88,377-378$; of uta, 86 ; stage of trypanosomes, 95 .

americana, 76, 89; and espundia, 89-92.

braziliensis, see L. americana.

donovani, discovery, 7,74 ; cultivation, $9,76,78$; development in bedbugs, 77-78; in Anopheles punctipennis, 78; human cycle, 79; distribution in body, 80 .

infantum, 76; infantile kala-azar, 82-84.

tropica, 76 ; in oriental sores, 85 ; transmission, 85-86.

Leishmaniasis, in Panama, 74-75; origin from insect flagellates, 75 ; see also Kala-azar, Oriental Sore and Espundia.

Lemon juice, for land-leeches, 319; repellent for mosquitoes, 455 .

Leprosy, spread by bedbugs, 379 . Leptomonas, see Herpetomonas.

Leptospira, 67; possibly parasite of dengue and phlebotomus fever, 182.

hebdomadis, 73D.

icterohamorrhagia, discovery, 65; description, 66-67; effect of salvarsan on, 68 . icteroides, 70.

Leptus, 336; see also Harvest mites. akamushi, and kedani, 190, 333. americanus, 336. autumnalis, 336. irritans, 336.

LEUCKART, R., 7, 202.

Leucocytes or white blood corpuscles, prey on parasites, 20 .

LEwIS, T. R., 298.

Lice, intermediate hosts of Dipylidium caninum, 245; fumigation, 386, 387-403; importance, 387; general structure, 388-389; human species, 389 ; specificity of action of salivary juice, 393; lice and disease, 397-400; and typhus, 8, 378, 397-398; and relapsing fever 44-46, 378, 398399 ; and trench fever, 187-188, 399 ; and bubonic plague, 399400; and syphjlis, 400; dispersal, 400; prevention, 400-403; control in war, 402-403; body louse, see Pediculus humanus corporis; head louse, see Pediculus humanus capitis; crablouse, see Phthirius pubis.

Life histories of parasites, discoveries of, 7 .

Lima, A. C., 458.

Limnoea, host of Fasciola hepatica, 209; host of Schistosoma japonicum, 219; occurrence in United States, 220.

Limnatis nilotica, 316 ; in nose and mouth, 317-318.

Linguatula rhinaria, 349-350.

Linguatulina, 333, 348-351.

Linstow, O. F., von, 245.

Liston, W. G., 411.

Liver abscess, sequel of amebic dysentery, 134, 137; in a case of myiasis, 527.

Liver flukes, of sheep, goats, etc., 209-211, 224; human, 224-228; symptoms, 227; prevention, 227-228.

Livingston, D., 360.

Llama, host of Trenia saginata, 240.

LLOYD, L., 495, 496.

Loa loa, 308-310; and Chrysops, 489.

London, copra itch, 340 .

Looss, A., 258.

Lösch, F., 7.

Louse-mite, see Pediculoides ventricosus.

Lucilia, 521. cossar, 521.

Luetin test, for syphilis, 55 .

Lung flukes, relation of crabs to, 8; 220-224; distribution, 220; relation to host, 220-221; life history, 211, 221-223; mode of infection, 223; prevention, 223-224. 
Lunule, frontal, 465.

LUTZ, A., 217.

Lymphangitis, in filarial disease, 305.

LyNCH, K. M., 117, 120, 140.

Lyon, H., 409.

Lyperosia irritans, see Homatobia serrata.

Lysol, in prevention of filarial infections, 307; for chiggers, 420 .

MacAdaM, W., 135.

MacConnell, J. F. P., 7.

McCulloch, Miss I., 112, 381.

MACFIE; J. W. ScotT, 98, 507.

MACGREGor, W., 7.

Mackie, F. P., 81.

McNadghton, J. G., 306.

MacNeal, W. J., 9, 10.

Macronucleus, 28.

Macrostoma, see Chilomastix.

Madagascar, Triatoma rubrofasciata, 381 ; surra, 487.

Maggots, in espundia sores, 90; and myiasis, 509-528; characteristics, 509-510; blood-sucking, 511-513; under skin, 513-519; in wounds and natural cavities of body, 519-523; in intestine, 523-528; in urinary passages, 528 ; resistance to reagents, 522 , 526-527.

Magnesium sulphate, in treatment of amebic dysentery, 139 .

Malaria, in Panama, 2; importance of, $5,147-148$; relation of mosquitoes to, $7,149,157-159$; 147-167; prevalence of, 148; history, 148-149; parasites of, 149-150; occurrence of attacks of ague, 153; quotidian, 153; numbers of parasites, 153; over-wintering, 156; benign vs. malignant, 156; propagation, 157-159; latent, 158; effect of weakening of host, 158-159; course of, tertian and quartan, 159-161; æstivo-au- tumnal, 161; immunity, 162163; tropical vs. sułtropical, 162 ; carriers, 162, 165, 438443; fulminant, 163; treatment, 163-164; prevention, 164-167; number of mosquitoes necessary to propagate, 165.

Malarial parasites, see Piasmodium. Malay bug, see Triatoma rubrofasciata.

Malay States, kedani or pseudotyphus, 190; Echinostomum, 228; malaria-carrying Anopheles, 441; habits of Anopheles, 441.

Malcœur, 254.

Mal de boca, cause of, 73A-73в.

Mal d'estomac, 254.

Male fern, for tapeworms, 237; for hookworms, 264; for pinworms, 279.

Malloch, J. R., 480.

Mallophaga, Rickettsia in, 185; compared with Anoplura, 388.

Malmsten, P. H., 7, 37.

Malpighian tubules, 328.

Malta, phlebotomus flies, 468, 470, 471.

Manaos, reduction of yellow fever, 70,72 .

Manchuria, plague, 413.

Mandibles, of insects, 325 .

Mange, 342 .

Mangrove fly, see Chrysops.

Manila, Balantidium infections, 127; amebic dysentery, 130; Echinostomum ilocanum, 229; Tcenia philippina, 245.

Manson, Sir P., 7, 47, 80, 162, 298, $301,305,309,322,449$.

Mansonia, habits of larvæ, 431; host of Filaria, 450.

Manure, treatment to prevent flybreeding, 508.

MARETT, P. J., 468.

Margaropus annulatus, 12; life history, 356, 366; extermination, 368 . 
MARLATt, C. L., 373, 375, 376.

Marmot, host of Moniliformis moniliformis, 284; and plague in Manchuria, 413.

Marriage, syphilis and, 60-61.

Masterman, E. W. G., 317, 318.

Mastigamœba, 35.

Mastigophora, see Flagellata.

Mauritius, Holothyrus coccinella, 341; trypanosomes in Triatoma rubrofasciata, 381 .

Maxilla, 325.

Maxillary palpi, 325.

Mayflies, life history, 329.

Mayne, M. B., 156.

Mealworm, intermediate host of $\mathrm{Hy}$ - menolepis diminuta, 244.

Measles, human, 169, 193; beef, 240; pork, 241.

Meat, fitness for food when diseased, 296-297.

Meat inspection, 286; relation to trichiniasis, 295-296.

Medical entomology, beginning of, 7; summary, 322-323.

Mediterranean countries, infantile kala-azar, 82; oriental sore, 84, 85; dengue, 182; phlebotomus fever, 184; Schistosoma homatobium, 213; Limnatis nilotica, 317; phlebotomus flies, 470.

Megarhinus, 437.

Melania, host of lung fluke, 221; not of Clonorchis sinensis, 226; of Yokagawa, 228.

libertina, and Paragonimus ringeri, 221; and Clonorchis sinensis, 226; and Yokagawa, 228.

Melanolestes, 382. picipes, 382.

Melittophagus meridionalis, and tsëtse flies, 503.

Melophagus, Rickettsia in, 185.

Membranelles, 30.

Mercurial ointment, for crab lice, 401.

Mercuric chloride (corrosive sublimate), for syphilis, 56 ; for rat- bite fever, $73 \mathrm{~A}$; for guineaworm, 314; for destroying flea eggs, 421 ; to remove Dermatobia from skin, 515.

Merida, yellow fever in, 70 .

Mesozoa, 27.

Metagonimus, see Yokagawa.

Metamorphoses, of insects, 329.

Metastrongylus apri, 200.

Metazoa vs. Protozoa, 26-27.

Methylene blue, for Trichomonas infections, 121; for Balantidium infections, 127.

Mexico, relapsing fever, 46; amebic dysentery, 130, 136; tlalsahuate, 335; Ornithodorus, 361, 365; Otiobius mégnini, 365; distribution of lice, 394; lice and typhus, 186, 396, 397.

Miana tick or bug, see Argus persicus.

Mice, and infantile kala-azar, 82; hosts of human intestinal Protozoa, 116; Giardia of, 125; spread of Sarcosporidia among, 175; Sarcocystis muris, 176; and kedani mite, 190; experimental infection with Schistosoma, 217, 219; Hymenolepis nana and diminuta, 242-244; and bedbugs, 375; Moniliformis moniliformis, 284; trichina, 288, 296; occasional hosts of Pulex irritans, 414.

Microfilaria, discovery, 7, 299-300; periodicity, 301; effect of drugs on, 306.

bancrofti, 299-300; periodicity, 301 ; effect of drugs on, 306 ; comparison with $m f$. loa, 309.

juncea, 308.

loa, 309.

perstans, 308.

volvulus, 311 .

Micronucleus, 28.

Microtus montebelli, and Japanese seven-day fever, 73D; and kedani, 190, 336. 
Mice, and Giardia, 125.

Middleton, W. S., 144.

Midges, see Chironomidæ.

Migliano, L., 73c.

Miller's itch, caused by Pediculoides, 338.

"Millions," natural enemy of mosquitoes, 460-461.

Mimm's culicide, 456.

Minas Geraës, Triatoma megista, 380.

Miner's itch, see Hookworm.

Mines, hookworm in, 262, 265.

Miracidium, 209.

Mites, and kedani, 190; general account, 331-332; life history, 332; parasitism, 332-333; families containing parasites, 333; toxic effects of salivary juices, 337; see also various species.

Mitzuain, M. B., see Mayne, M. B. Miyairi, K., 218, 219, 222.

Moco, host of Triatoma chagasi, 381 . Mongols, possibly result of syphilis, 53.

Moniliformis moniliformis, 284-285. clarki, 285.

Monkeys, for experimentation, 10; relapsing fever, 43,47 ; and infantile kala-azar, 82; and espundia, 89; susceptible to Schistosoma infections, 216A; Trichuris trichiura, 277; hosts of Ternidens deminutus, 283; Esophagostomum apiostomum, 283; probable host of Esophagostomun stephanostomum thomasi, 283 ; and plague, 413.

Montana, spotted fever, 188, 189; Porocephalus in man, 351.

Morales, R., 451.

Moscow, transmission of relapsing fever, 378; habits of Anopheles, 442.

Mosquitoes, and espundia, 92; and Oroya fever, 181; mouthparts, 327, 426-427; fumigation, 386, 456; 424-462; importance, 424; general structure, 425-428; dis- eases carried by, 424; relationships, 425; sexes distinguished, 426; life history, 428-433; habits of adults, 433-434; habitats, 434; migration, 434-435; time of activity, 435-436; food habits, 436; hibernation, 436; length of life, 436-437; classification, 437; effect of bites, 453; remedies for bites, 454455; personal protection, 455456; elimination and exclusion from buildings, 456-457; larvicides, 457-459; prevention of breeding, 459; natural enemies, 459-462.

and malaria, discovery, 7 ; development of Plasmodium falciparum in, 154-156; malaria carriers, 157-159, 438-441; number necessary to propagate malaria, 165; habits of Anopheles, 441-443; and yellow fever, discovery, 7,443 ; transmitting species, 443-448; and Filaria, discovery, 7, 298, 449; development of Filaria bancrofti in, 301-303, 450-451, as transmitting species, $450-$ 451 ;

and dengue, discovery, 8, 448; 183 ; transmitting species, 448449 ;

and Dermatobia, 451-453, 514; objections to mosquito transmission theory, 452; transmitting species, 453.

Mosquito-worm, 451.

Mouchet, R., 351.

Mould, cause of kedani, 192.

Mouth, spirochrtes in, 70; Trichomonas in, 119; amebæ in, 142-146B.

Mouth ameba, see Endamœba gingivalis.

Mouthparts of insects, 325-327.

Mule, host of Dermatobia, 513.

Murray, C. H., 371, 372, 374, 375.

Musca domestica, changed attitude 
towards, 3 ; stable-flies mistaken for, 504; breeds in manure, 508; and intestinal myiasis, 527.

Muscidæ, includes tsetse flies, 491; stable-flies, 504; other bloodsuckers, 506 ; captured by Dermatobia, 514; 'screw-worm, 519; other species causing myiasis of wounds, 521.

Musgrave, W. E., 221.

Muto, M., 226.

Myiasis, 509-528; definition, 509; flies causing, 509; classification, 510 ; by blood-sucking maggots, 511-513; of skin, 513-519; of wounds and natural cavities of body, 519-523; of intestine, 523-528; of urinary passages, 528.

Myoneme, 31.

Myriapoda, 324-325.

Nagana, 108, 497.

NAGAYo, M., 192, 337.

Nakagawa, K., 8, 221, 222, 223.

Naphthaline, for intestinal fluke infections, 230; for body lice, 401 , 402; to eliminate fleas, 421, $422-423$.

Nasal polypus, 173-174.

Natal, hookworm quarantine, 268; Cordylobia anthropophaga, 518.

N C I, for lice, 402.

Nebraska, Tania confusa, 245.

Necator americanus, distribution, 255; description, 255-257; see also Hookworms.

Negri bodies, 170, 191-193.

Negroes, syphilis among, 51; immunity to malaria, 163.

Neiva, A., 381, 452; 514.

Nematoda, 198; intestinal, 270-272, 282 ; see also various species.

Nematomorpha, 199.

Nemathelminthes, 198-199; intestinal forms, 270-272; see also various species.

Nemertinea, 198.
Neosalvarsan, for syphilis, 57; to prevent trypanosome infection, 107.

Nephridia, 199.

Neuroryctes hydrophobia, 170, 191193.

New Jersey, ecologic groups of mosquitoes, 434; migrations of salt marsh mosquitoes, 435; control of salt marsh mosquitoes, 459460.

New Orleans, plague in, 2, 411; yellow fever, 69-70.

Newstead, R., 470, 471.

Newt, natural enemy of mosquitoes, 461.

New York, amebæ in school children, 143; invasion by mosquitoes, 435 .

Nichols, H. J., 54.

NicolL, W., 267.

Nicolle, C. N., 8, 44, 397, 398, 399.

Nigeria, sleeping sickness, 98, 102, 104; transmission of sleeping sickness, 500 .

Nighthawk, natural enemy of mos-quitoes, 462 .

Night-soil, use in oriental countries, 227-228, 267.

Nimetti, 482.

Nit, 391.

NoGuchi, H., 9, 67, 70, 71, 72, 182.

Noma, cause of, $73 \mathrm{~A}$; treatment, 73в.

North America, infectious jaundice, 65; tick paralysis, 358; Dermacentor variabilis, 367; Reduviidæ, 382; typhus, 186, 398; mosquito scourge, 424; malaria-carrying Anopheles, 439; blackflies, 481 .

No-see-um, 474.

Notoedres cati, 343.

Notophthalmus torosus, natural enemy of mosquitoes, 461.

Novy, F. G., 9, 23-25.

Nuttall, G. H. F., 34, 395.

Nymph, 329, 332. 
Obermeier, O., 7, 43.

O'Connor, F. W., 118, 121.

Odocoileus columbianus, and Pulex irritans, 414.

Estridæ, characteristics of larvæ, 509; and intestinal myiasis, 524 .

Estrus ovis, and myiasis, 522-523.

Esophagostomum apiostomum, 283. brumpti, see $E$. apiostomum. stephanostomum thomasi, 283.

Ogata, M., 192.

Oil, poured in ears to remove Otiobius mégnini, 366; for removal of ticks, 367; film to destroy mosquito larvæe, 458, 460; film to trap tabanids, $489-490$.

OKUDA, K., 73.

Onchocerca volvulus, 310-311. coecutiens, 311.

Ontario, blackflies, 479, 481, 482.

Onychophora, 324.

Opilagço, 255.

Opisthorchis felineus, 225.

noverca, 225.

pseudofelineus, 225.

Opsonin, 20.

Oregon, trichina, 292; tick paralysis, 358-359; Dermacentor occidentalis, 363; Notophthalmus torosus and mosquitoes, 461; Culicoides, 476; blackflies, 481.

Oregon State Board of Health, correspondence concerning venereal diseases, 59 .

Organelles, 29-32.

Oriental sore, 84-88; distribution, 84-85; transmission, 85-86, 377-378, 477, 488; susceptible animals, 86 ; course of, $86-88$; treatment, 88 ; prevention, 88 .

Ornithodorus, effect of bites, 361 , 364.

coriaceus, effect of bites, 364365.

mégnini, see Otiobius mégnini.

moubata and relapsing fever, 43-44, 360-361; control, 369.

savignyi, and relapsing fever, 44,
361 ; control, 369.

talaje, and relapsing fever, 46 , 361 ; habits, 361 ; control, 369 . tholosani, and relapsing fever, 44, 361.

turicata, and relapsing fever, 46, 361 ; severity of attacks, 361 ; control, 369.

Oroya fever, 168, 176-181; history, 176; distribution, 176; confusion with other diseases, 177; course of, 179; parasite of, 179181; transmission, 181, 360.

Orthetrum chrysostigma, preys on tsetse flies, 503.

Orthorrhapha, pupæ, 465, 466; and myiasis, 509.

Osler, Sir W., 49.

Osumi, S., 73.

Otiobius mégnini, 365-366.

Owl midges, see Phlebotomus flies.

Oxyuris vermicularis, nutriment absorbed, $202 ;$ 277-279.

Pajaroëllo, 364 .

Palestine, leeches in nose and mouth, 317-318.

Palpi, of insects, 325 ; of acarina, 331.

Panama, French failure and American success, 2 ; yellow fever during French operations, 71; Leishmania sores, 74-75, 488; malaria and the Canal, 149; non-malarial Anopheles, 158; reduction of malaria, 166; malaria-proof houses, 166; reduction of yellow fever, 71; dengue, 182, oil films for mosquitoes, 459 .

Pangonia, 485, 486.

Papataci fever, see Phlebotomus fever.

Parabasal body, 29-30.

Paraform, larvicide, 459.

Paragonimus, 220-224; see also Lung flukes.

kellicotti, 220, 223. 
ringeri, 220-224.

westermani, see $P$. ringeri.

Paraguay, rattlesnakes and espundia, $92,471$.

Paralysis, general, result of syphilis, 53, 54; from tick bites, 358-359.

Paramocium, old age, 33.

Paramphistomum cervi, 229.

Paraplasma flavigenum, and yellow fever, 184.

Parasites, discoveries of, 7 ; life histories discovered, 8; definition, 12; kinds of, 12-13; effects of parasitism on, 14; effects on hosts, 15-17; modes of infection and transmission, 17; geographic distribution, 18-19; effects of temperature, 19; immunity to, 19-22; introduction to virgin territory, 20; relation to intermediate and to final hosts, 450-451.

Parasitic diseases, history of treatment of, 8 .

Parasitidæ, 333, 341.

Parasitism, degrees of, 12; effects on parasites, 14; origin among mites, 332-333.

Parasitology, importance of, 5; history of, 6-11.

Paratyphoid, confused with Oroya fever, 178.

Paris, syphilis in, 50.

Pasteur, L., 7, 9, 20.

Patagonia, Filaria bancrofti, 299.

Patton, W. S., 77, 377, 416; 521.

Peacock, A. D., 7.

Pediculoides ventricosus, 337-339.

Pediculus, hosts, 389, Rickettsia in, 185-187.

capitis, see $P$. humanus capitis.

corporis, see $P$. humanus corporis.

humanus capitis, 389, compared with corporis, 389-390, 394-395; 394-396; habitat, 395; life history, 395; effects of bites, 395-396; and typhus, 397; and relapsing fever, 398; and plague,
399-400; dispersal, 400; remedies, 401.

humanus corporis, 389-394; compared with capitis, 389-390, 394-395; and disease, 390, 397400; habitat, 390; life history, 391-393; habits, 393-394; effects of bites, 394; and typhus, 397-398, 186; and relapsing fever, 398-399, 44-46; and trench fever, 399, 187-188; and plague, 399-400; dispersal, 400; eradication, 401-403.

vestimenti see humanus corporis.

Pedipalpi, 331.

Pellagra, and blackflies, 483.

Pelletririne, for tapeworm infections, 237.

Pennyroyal, oil of, repellent for fleas, 422 ; for mosquitoes, 455.

Penschke, 420.

Peppermint, oil of, repellent for mosquitoes, 455 .

Pericoma townsvillensis, 466.

Peripatus, 324.

Persia, African relapsing fever, 44; oriental sore, 85,86 ; Ornithodorus tholosani, 361; Argas persicus, 364.

Persian insect powder, see Pyrethrum insect powder.

Peru uta, 86; oriental sore, 87; Trichomonas pathogenic, 121; Oroya fever, 176, 181, 472; lung fluke, 224; breeding places of phlebotomus flies, 468; Phlebotomus verrucarum, 472, 473.

Petroleum, for removal of ticks, 367; for bugs, 383; emulsion for head lice, 401; for body lice, 401, 402; for chigger wounds, 420 ; for mosquitoes, 457; for mosquito larvæ, 458.

Phalangomyia debilis, and Oroya fever, 181.

Philamon, 320.

Philippine Islands, dengue, 182; kedani, 190; Schistosoma jap- 
onicum, 218; lung flukes, 220 , 221; human liver flukes, 224; Echinostomum ilocanum, 228; Tania solium, 241; Esophagostomum apiostomum, 283; Anopheles ludlowi, habits, 442; Musca domestica and intestinal myiasis, 527.

Phinotas oil, for blackflies, 483.

Phlebotomus, and oriental sore, 86, 471 ; and Oroya fever, 181, 472473; and phlebotomus fever, $184-185,470-471 ; 463$; 466473; general description, 466468; life history, 468-470; and diseases, 470-473; control, 473. perniciosus, and phlebotomus fever, 470 .

minutus, and oriental sore, 86,471 ; and phlebotomus fever, 470; habits, 471; description, 472.

minutus, var. africanus, and oriental sore, $86,471$.

papatasii, and phlebotomus fever, 184, 185, 470; life history, 469; description, habits, etc., 470-471. verrucarum, and Oroya fever and verruga peruviana, 181, 472473.

Phlebotomus fever, parasites of, $182 ; 184-185$.

Phthirius, hosts, 389.

inguinalis, see $P$. pubis.

pubis, 389, 396-397; dispersal, 400; remedies, 401.

Physaloptera mordens, 282.

Physopsis africana intermediate host of Schistosoma homatobium in South Africa, 216.

Pigeon, Argas reflexus, 364 .

Pinworm, see Oxyuris vermicularis.

Piophila casei, and intestinal myiasis, $526,527$.

Piroplasmata, 27, 168; Bartonella a member of, 180-181; and spotted fever, 168; and kedani, 190-191; transmission by ticks, $168,181,360$.
Pito bug, 382 .

Plague, in Europe, 2, 411; in United States, 2, 411; in India, 3, 411; and bedbugs, 378; and lice, 399-400; and fleas, 410 413; and rats, 411.

Planorbis, occurrence in United States, 220.

boissyi, intermediate host of Schistosoma mansoni in Egypt, 215, 216, 217.

guadelupensis, intermediate host of Schistosoma mansoni in Venezuela, 217.

olivaceus, intermediate host of Schistosoma mansoni in Brazil, 217.

Plasmodium, discovery, 7; cultivation, 9 ; 35; 149-159; species, 149-150; life history, human cycle, 150-154; relation to red blood corpuscles, 150-151; mosquito cycle, 154-156; effects of temperature on development in mosquito, 156.

falciparum, 150-156; life history, human cycle, 150-154; clogging of capillaries, 152 ; sporulation, 152-153; numbers in blood, 153; crescents, 154; mosquito cycle, 154-156; resistance to low temperatures, 156.

malarice, 150; description, 157.

vivax, 150; resistance to low temperatures, 156; description, 156.

Plate, J. T., 118, 134.

Platyhelminthes, 196-198.

Plenciz, M. A., 6.

Plerocercoid, nature of, 235.

Pliny, 372.

Poliomyelitis, acute, anterior, see Infantile paralysis.

Pork, measly, 241; trichina in, 286, 287, 292; killing of trichina in, 295; inspection, 295-296; fitness for food, 296-297.

Pork tapeworm, see Tania solium. 
Porocephalus armillatus, 350-351. crotali, 351.

moniliformis, 351.

Portchinsky, I. A., 487, 489, 490.

Porter, A., 123.

Porto Rico, hookworm disease, 262; unsanitary conditions, 266.

Portuguese Sleeping Sickness Commission, extermination of sleeping sickness on Island of Prin-

- cipe, 108; eradication of tsetse flies, 502.

Potamon dehaanii, intermediate host of lung flukes, 222.

obtusipes, intermediate host of lung fluke, 222.

Potassium cyanide, for hydrocyanic acid fumigation, 384 .

Potassium permanganate, for tropical ulcer, 73c.

Potassium sulphide, to destroy fleas, 422.

Precipitins, 21.

PRICE, J. D., 377.

Priestley, H., 73D.

Privies, lack of, in warm countries, 266.

Procercoids, 246-247.

Proglottid, 232.

Prostitution, and syphilis, 61; municipal control of, 61-62.

Protista, 27.

Protozoa, vs. Metazoa, 26-27; vs. bacteria, 27; structure, 28-29; organelles, 29-32; nutrition, 32 ; reproduction, 32-34; life cycle, 33-34; immunity to drugs, 3435 ; classification, 35-36; importance, 37 ; discovery, 37 .

Protozoölogy, importance of, 37 .

Prowazekella, 118.

Prowazek's bodies, 193.

Pseudopodia, 29.

Pseudotyphus, 190.

Psorophora, larvæ prey on Janthinosoma larvæ, 453.

Psychodidæ, 466.

Ptilinium, 465.
Pulex irritans, jumping power, 405; identification, 408; egg-laying habits, 408; life cycle, 410; and plague, 412 ; and infantile kalaazar, 413; and Dipylidium caninum, 414; habits, etc., 414415.

Pulicidæ, 407.

Punkies, see Chironomidæ.

Puparium, 465.

Pygidium, 404.

Pygiopsylla ahalo, 417.

Pyorrhea, 142-146в; importance, 142 ; relation of amebæ to, 144 146; relation of bacteria to, 145; prevention and treatment, $146 \mathrm{~A}-146 \mathrm{~B}$.

Pyrethrum insect powder, for ticks, 369 ; for mosquitoes, 456.

Python, host of Porocephalus, 350, 351.

Quack doctors, 4; and syphilis, 56. Queensland, hookworm disease, 262. Quinine, discovery and history, 8; for malaria, 163-164, 167.

Quininization, and prevention of malaria, 165, 166.

Quiros, D., 419, 420.

Rabbits, Eimeria stiedoe of, in man, 172; susceptible to trichina, 288; Linguatula rhinaria, 349; and bedbugs, 375; Echidnophaga gallinacea, 420 .

Rabies, 169; parasite of, $170,191-$ 193.

Ransom, B. H., 238, 243, 274, 275, 286, 287, 292, 294, 295.

Rasahus, 382.

Rat-bite fever, 73-73A.

Rats, relapsing fever immunization, 47 ; reservoir of infectious jaundice, 67-68; and rat-bite fever, 73; Giardia of, 125; and infantile kala-azar, 82; development of Trypanosoma rhodesiense in, 97; hosts of human 
intestinal Protozoa, 116; and amebic dysentery, 140; and Hymenolepis nana, 242-244; Hymenolepis diminuta, 244; development of Ascaris in, 274-275; Moniliformis moniliformis, 284; relation to trichiniasis, 287, 288, 296; and bedbugs, 375; occasional hosts of Pulex irritans, 414; fleas, 417-418; Echidnophaga gallinacea, 420 .

Rattlesnakes, Porocephalus crotali, 351.

Redbugs, see Harvest mites.

Redi, F., 6.

Redia, 209, 211.

Red spider, 340 .

Reduviidæ, 379.

Reduvius, 382.

Reed, W., 70, 443.

Relapsing fever, 42-48; distribution, 42 ; spirochætes of, 42,46 ; transmission, 43-46, 378, 398-399; nature of, 46-47; mortality, 47; treatment, 47; prevention, 47-48; development in lice, 399.

Repellents, for fleas, 423; for mosquitoes, 455; for phlebotomus flies, 473; for chironomids, 477; for tabanids, 489 ; for tsetse flies, 501.

Reptiles, reservoirs of Leishmanian diseases, 471; fed on by tsetse flies, 494.

Réunion, trypanosomes in Triatoma rubrofasciata, 381.

Rhinosporidium, 168; 173-174.

kinealyi, 173. .

Rhipicephalus, 366.

Rhizoglyphus parasiticus, 340 .

Rhizopoda, see Sarcodina.

Rhodesia, sleeping sickness, 94 .

Rhodnius prolixus, 382.

Rhynchoprion, see Dermatophilus.

Rhynchota, see Hemiptera.

Ricketтs, H. T., 8, 186, 188, 397.
Rickettsia-like organisms, 168, 169, 170, 185-191; 194.

Rickettsia, 181; in insects, 185-186; in human diseases, 186; 191. pediculi, in lice, 187. prowazeki, and typhus, 186-187. quintana, and trench fever, 187-188.

RIDEWood, W. G., 409.

Rigg's disease, see Pyorrhea.

Riley, W. V., 339, 474.

Rincones, G., 451-452.

Rio de Janeiro, reduction of yellow fever, 70, 72; Triatoma vitticeps, 381.

Robertson, Miss, 99.

Robles, R., 311.

Rocha-Lima, H., 186.

Rockefeller, J. D., 268.

Rockefeller Institute, 10, 72, 105.

Rocky Mountain spotted fever, see Spotted fever.

Rodents, hosts of Trypanosoma cruzi, 112, 114; and spotted fever, 189, 369; susceptible to Schistosoma infections, 216A; hosts of immature stages of Dermacentor venustus, 362-363; and Triatoma, 380-381; plague transmitted by lice, 399 ; hosts of Cordylobia anthropophaga, 518 .

Rogers, L., 8, 9, 81, 377.

Rosen, F., 246.

Rosenau, M. J., 48, 507.

Ross, Sir R., 7, 147, 148, 149, 156, $158,164,165,449,457$.

Roubaud, E., 156, 459 471, 512, 517.

Rougets, 336.

Roundworms, see Nemathelminthes. Russia, relapsing fever, 43, 45; Diphyllobothrium latus, 246; Gigantorynchus. hirudinaceus in man, 284; typhus, 398; Gastrophilus hamorrhoidalis in man, 516; Wohlfartia magnifica, 521-522.

Sabethini, 437.

Salicylic acid, in ointment for chig- 
gers, 420 .

Salt, enema for amebic dysentery, 138; to destroy hookworm larvæ, 267.

Salt water, for myiasis of nose, ears, etc., 523 .

Salvarsan, discovery, 8,49 ; for relapsing fever, 47; for syphilis, $56-57$; for yaws, 64 ; for infectious jaundice, 68 ; for rat-bite fever, 73A; for Vincent's angina and noma, 73B; for tropical ulcer, 73c; for trypanosomes, 105, 107; for Balantidium infections, 127.

Salvarsan copper, to prevent trypanosome infections, 107.

Salzman, F., 294.

Sambon, L. W., 350, 351, 451, 452, 453.

Samoa, periodicity of Filaria bancrofti, 301.

Sand flea, see Dermatophilus penetrans.

Sandflies, see Phlebotomus flies.

San Francisco, plague in, 2; anti-rat campaign, 411.

Sanitation, and syphilis, 61; and prevention of amebic dysentery, 139; relation to intestinal parasites, 265-269; effect on school children's progress, 266267; necessity for practical demonstrations, 268-269.

Santonin, for intestinal fluke infections, 230; for Ascaris infections, 276.

São Paulo, Triatoma sordida, 381; Dermatobia transmitters, 453.

Sarcocystin, toxin from Sarcosporidia spores, 175 .

Sarcocystis muris, sexual phenomena, 176.

tenella bubalis, in Indian buffaloes, 176.

Sarcodina, pseudopodia in, 29, 36, 129.

Sarcophaga fuscicauda and intestinal myiasis, 526 .

magnifica, see Wohlfartia magnifica.

Sarcophagidæ, 521.

Sarcopsyllidæ, 407, 418, 420.

Sarcoptes, 342-346.

scabiei, 342-346.

scabiei crustosø, 343, 345.

Sarcoptidæ, 333, 342.

Sarcosporidia, 168, 174-176; in man, 176.

Savarelli, 184.

Scabies, 342.

Scarlet fever, 169, 191.

Schaddinn, F., 7, 49, 428.

Schistosoma, discovery, 7; life history discovered, 8,213 ; see also Blood flukes.

hamatobium, 213-217; distribution, 213 ; relation to host, 214 ; pathogenic effects, 214; life history, 215-216; treatment, 216A; mode of infection, 216A; prevention, $216 \mathrm{~A}-216 \mathrm{~B}$.

japonicum, 215, 218-219; symptoms of infection, 218; life history, 219.

mansoni, 216, 217-218.

Schroeder, O., 6.

SchuefFNer, W., 190.

Schulze, 6.

SchWANN, 6.

Scolex, 231

Screening, for mosquitoes, 457.

Screw-worm, see Cochliomyia macellaria.

Scutum, of ticks, 354 .

Seal, host of Diphyllobothrium cordatus, 247.

Seattle, plague in, 411.

Seed ticks, 355.

Seidelin, H., 184.

Sellards, A. W., 136.

Serbia, relapsing fever, $45,378,399$; typhus, 398.

Sergent, E., 471.

Sesarma dehaani, intermediate host of lung flukes, 222. 
Seven-day fever, Japanese, 73D.

Seven-days' fever, see Dengue.

Shanghai, Clonorchis sinensis, 225.

Shaohing, Fasciolopsis infections, 229-230.

Sheep, liver fluke, 209-211; Paramphistomum cervi, 229; hydatids, 248; host of Trichostrongylus instabilis, 282; Linguatula rhinaria, 349; tick paralysis, 358359 ; grazing to destroy ticks, 369; head maggot, 523.

Shipley, A. E., 204.

Shrimp, host of Fasciolopsis buski, 229.

Siberia, Opisthorchis felineus in man, 225.

Sicily, breeding places of phlebotomus flies, 468, 473.

SikorA, H., 391, 392, 393, 394.

Silver, organic compounds of, for Balantidium infections, 127.

Silver nitrate, for Vincent's angina and noma, 73в.

Simuliidæ, 478-484.

Simulium, 481; relation to Onchocerca cacutiens, 311.

columbaczense, 482 .

griseicollis, 482 .

pecuarum, 481.

venustum, 481.

Siphonaptera, characteristics, 330 , 404; see also fleas.

Siphunculata, 330, 388.

Situtunga antelope, reservoir for sleeping sickness trypanosomes, 107; host of Glossina palpalis, $498,499$.

Skunk, host of Pulex irritans, 414.

Sleeping sickness, importance, 93; Rhodesian, 94, 97, 103; Gambian, 97, 103; Nigerian, 98, 103, 104; 98-108; transmission, 9899, 490, 496-501 ; course of, 103104; treatment, 104-106; prevention, 106-108; animal reservoirs, 107, 503-504.

Smallpox, 169; parasite of, 170, 191.
Sмith, A. J., 142.

Sмith, J. B., 434, 435, 441, 456, 459.

Smudge, for mosquitoes, 457; for blackflies, 484 .

Snails, intermediate hosts of flukes, 209-210; destruction of, 212; of Schistosoma, 214, 215, 216, 217, 219-220.

Snakes, possible reservoirs of Leishmania, 92, 471.

SniJders, E. P., 173.

SNow, F. H., 521.

SNow, W. F., 58.

Soap, in treatment of itch, 345 .

Sodium fluoride, for fleas, 421-422.

Somaliland, relapsing fever, 44 .

Sources of Information, periodicals, 529-531; books, 531-533.

South Africa, blood-fluke infections in British soldiers, 214-215; ihtermediate host of Schistosoma homatobium, 216.

South America, oriental sore, 85; uta, 86; espundia, 89; trypanosomiasis (Chagas' disease), 94, 108; Coccidium seeberi, 174; dengue, 182; use of shoes, 265; Filaria perstans, 308; Filaria juncea, 308; land-leeches, 319; Cimex hemipterus, 373; Triatoma, 379-381; Rhodnius prolixus, 382; Dysodius lunatus, 382383; Dermatobia in cattle, 513.

South Sea Islands, dengue, 182; Filaria bancrofti, 301; prevalence of elephantiasis, 305; Aëdes calopus, 448.

Spagnolio, G., 83.

Spallanzani, A., 6.

Sparganum, 247; 251-253.

mansoni, 252.

proliferum, 252-253.

Spinose ear tick, see Otiobius mégnini.

Spiny-headed worms, see Acanthocephala.

Spiracles, of insects, 328; of ticks, 354. 
Spirochceta, see Spirochætes.

balanitidis, 41.

bronchialis, transmission, 40, 41; cause of bronchitis, $73 \mathrm{~B}$.

buccalis, 40 ; pathogenicity, $73_{\mathrm{A}-}$ 73в.

carteri, 42; in bedbugs, 378 .

dentium, 40.

duttoni, 42.

hebdomadis, 73D.

icterohœmorrhagioe, 41; see also under Leptospira.

icteroides, see under Leptospira.

morsus muris, 73.

nodosa, see Leptospira icterohomorrhagiœ.

novyi, 42 .

obermeieri, see Sp. recurrentis.

orientalis, 41.

pallida, discovery, 7, 41, 49, 52.

pertenuis, 41, 63.

recurrentis, discovery, 7,43 ; description, 52; distribution in body, 52 .

refringens, 52 .

schaudinni, 41, 73c.

vincenti, 41.

Spirochætes, cultivation, 9; 38-73; relationships, 38; multiplication, 39; granule shedding, 39; and disease, 40-42, 73; localization, 41; and relapsing fever, 42-48; and syphilis, 48-62; and yaws, 63-65; and infectious jaundice, 65-69; and rat-bite fever, 73-73A; and Vincent's angina, $73 \mathrm{~A}$; and noma, $73 \mathrm{~A}$; and balanitis, $73 \mathrm{~A}$; and mal-deboca, 73A; and bronchitis, 73в; and tropical ulcer, $73 \mathrm{c}$; and ulcerating granuloma, 73c-73D; transmission by ticks, 360 .

Spleen rate, and prevalence of malaria, 161.

Sporocyst, of coccidian, 172; of flukes, 209; 211.

Sporozoa, 35, 36; and human disease, 149, 168.
Spotted fever, relation of ticks to, 8, 188-189, 361-363; and Piroplasmata, 168; 188-189; distribution, 188; parasite of, 188; reservoirs, 189.

Spotted fever tick, see Dermacentor venustus.

Squirrels, hosts of Moniliformis clarki, 285; hosts of immature stages of Dermacentor venustus, 362 ; and plague, 411,413 ; fleas, 418.

Stable-flies, see Stomoxys.

Stanley, and spread of sleeping sickness, 93.

Staten Island, reduction of malaria, 166.

Stä̈Bl, C., 290.

Stadffacher, H., 76, 193.

Stegomyia, see Aëdes.

fasciatus, see Aëdes calopus.

Stephens, J. W. W., 282.

Stephensport, quininization, 164165.

Sterilization, of blood against trypanosomes, 107.

Stewart, F. H., 274, 275.

Sticktight flea, see Echidnophaga gallinacea, 420 .

Stigmal plates, of maggots, 509-510.

Stiles, C. W., 116, 125, 242, 252, 254, 266, 296, 297, 400 .

STOKES, J. H., 483.

Stomoxys, transmission of Trypanosoma gambiense, 98, 507; relation to Onchocerca volvulus, 311 ; mouthparts, 327, 464, 505; transmission of anthrax, 488, 507; 504-508; general description, 504-505; life history, 505506; and disease, 507; control, 507-508.

calcitrans, 504; and infantile paralysis, 507 .

nigra, and Trypanosoma gambiense, 98, 507.

Streptothrix, and rat-bite fever, 73A. Strickland, C., 409, 410, 417. 
StronG, R. P., 86, 178, 179, 180, 397.

Strongyloides stercoralis, 279-282; life history, 280-281; symptoms, 281-282.

Strongylus, see Trichostrongylus.

Sudan, spirochætal bronchitis, 73в; kala-azar, 77; Simulium, 482; tabanids, 487 ; methods of capturing tsetse flies, 503.

Sulphur, for mites, 335, 339, 341, 346 , 348; for fumigation, 383 , 386; for lice, 401.

Sulphur ointment, for itch, 346 .

Sulphuric acid, for hydrocyanic acid fumigation, 384 .

Sumatra, Eimeria snijderi, 172; pseudo-typhus or kedani, 190; land-leeches, 319, 320.

Surcone, M. J., 451.

Surra, 99, 487.

SUzUKI, M., 219.

Swallows, bugs on, 374; natural enemies of mosquitoes, 462.

Swellengrebel, N. H., 417.

SwEzy, O., 120, 132, 141.

SwiFT, H. F., 57.

Swift-Ellis treatment, for syphilis of nervous system, 57 .

Swifts, natural enemies of mosquitoes, 462.

Switzerland, Diphyllobothrium latus, 246.

Syphilis, importance of, 3; history, 48-49; prevalence, 49-51; transmission, 51-52; spirochætes of, 52 ; course of, $53-55$; congenital, 53; malignant, 55; diagnosis, 55-56; treatment, $56-58$; standard of cure, 5758; prevention, 58-63; exclusion from hospitals, 58; free diagnosis and treatment, 59; compulsory notification, 60; relation to yaws, 63; possible spread by bedbugs, 379 ; by lice, 400 .
Syria, oriental sore, 85; typhus epidemic, 398.

Tabanidæ, and leishmaniasis, 75, 488; and espundia, 92, 488-489; mouthparts, 327, 485; 484-490; general account, 484-486; life history, 486-487; and disease, 487-489; transmission of surra and el debab, 487; and human trypanosomiasis, 488; and anthrax, 488; and loa worms, 489; control, 489-490.

Tabanus, 486; trap for, 490 .

Tabardillo, see Typhus fever.

Tania africana, 245.

bremneri, 245.

confusa, 245.

philippina, 245.

saginata, discovery of life history, 7 ; nutrition absorbed, 202; description, 239-240; life history, 240.

solium, 240-242; cysticerus in man, 251.

Tæniidæ, 238; important species of, 239-245.

TAKAKI, F., 73.

Tampan, see Ornithodorus moubata.

Taniguchi, T., 73.

Tapeworms, 231-253; general structure, 231-233; reproduction, 233-234; life history, 234-235; damage to host, 236-237; treatment, 237; prevention, 237238; important species, Tæniidæ, 239-245; Dibothriocephalidx, 245-247; larval tapeworms of man, 247-253.

African, see Tonia africana. beef, see Tænia saginata. dog, see Dipylidium caninum. dwarf, see Hymenolepis nana. fish, see Diphyllobothrium latus. pork, see Tonia solium.

Tarentola mauritanica, and oriental sore, $86,471$. 
Tarsonemidæ, 333; 337.

Tartar emetic, discovery, 8; for ulcerating granuloma, $73 \mathrm{D}$; for kala-azar, 81 ; for infantile kalaazar, 84; for oriental sore, 88; for espundia, 91-92; for trypanosomes of sleeping sickness, 105; for Chagas' disease, 114.

TAute, M., 107.

Teeth, pyorrhea cause of loss of, 142; amebæ among, 142-144.

Temperature, limiting factor in distribution of parasites, 19.

Tennent, J. E., 319.

Ternidens deminutus, 283.

Tetramitus, see Chilomastix.

Tetranychidæ, 333; 340.

Tetranychus molestissimus, 341. telarius, 341.

Texas, Tænia confusa, 245, Sparganum mansoni, 252; Moniliformis, 285; propagation of bats to destroy mosquitoes, 462 .

Texas fever, caused by Piroplasmata, 168, 180, 360.

Texas fever tick, see Margáropus annulatus.

Theileria tsutsugamushi, 191.

Theobald, F. V., 437.

ThÉzÉ, J., 310.

Thomas, W., 8.

Three-days' fever, see Phlebotomus fever.

Thymol, discovery, 8; for intestinal flukes, 230; for tapeworms, 237; danger from, 237; for hookworms, 263; for pinworms, 279; for microfilariæ, 306.

Thysanura, direct life history, 329 .

Tick-bite fever, 367.

Tick fever, see African relapsing fever.

Tick paralysis, 358-359.

Ticks, and espundia, 92; transmitters of Piroplasmata, 168, 181, 360; and kedani, 190, 360; 352-369; importance, 352; general anatomy, 352-354; habits,
354; life history, 355-357; effects of bites, 357-358; tick paralysis, 358-359; and disease, 359-360; and relapsing fever, discovery, 8; 43-44; 352 ; transmitting species, 360 361 ; and spotted fever, discovery, 8; transmission, 188189; 352 ; transmitting species, 361-363; troublesome Argasidæ 364-366; troublesome Ixodidæ, 366-367; treatment of bites, 367 ; removal, 367 ; prevention, 368-369.

Tipulidæ, mosquitoes allied to, 425 . Tlalsahuate, 335 .

Tobacco, for leeches, 318, 319; to remove Dermatobia from skin, 515.

TodD, J. L., 8, 359.

TOEPFER, 187.

Togoland, transmitter of sleeping sickness in, 500.

Tongue-worms, 348-351; general account, 348; life history, 348349 ; species found in man, 349 351.

Tonkin, relapsing fever epidemic, 47.

Tonsilitis, relation of Endamøba gingivalis to, 146.

Torres, M., 111, 380.

Tovar, N., 451.

Townsend, C. H. T., 86, 181, 468, $472,473$.

Toxascaris limbata, 282.

Toxins, 16-17; from spores of Sarcosporidia, 175; from intestinal worms, 202-203; from tapeworms, 236; from Diphyllobothrium latus, 247; from hookworms, 261; from maggots in intestine, 527.

Tracheæ, of Arachnida, 324; of insects, 325, 328; of Acarina, 332.

Trachoma, 169; 193.

Trematoda, 197-198; see also Flukes. 
Trench diarrhea, 135 .

Trench fever, 168, 169, 186, 187-188; relation of lice to, 399 .

Treponema pallida, see Spirochata pallida.

Triatoma, relation to Trypanosoma cruzi, 8, 108, 110-112, 380-381; houses proof against, 114, 370; 379-382; habits and life history, 379.

chagasi, infected with trypanosomes, 381 .

dimidiata, infected with trypanosomes, 381 .

geniculata, and Trypanosoma cruzi, 112, 380-381.

infestans, infected with trypanosomes, 381.

megista, and Trypanosoma cruzi, 110-112; habits and life history, 380 .

protracta, and Trypanosoma triatomæe, 112, 379, 381.

rubrofasciata, and Trypanosoma cruzi, 112, 381; and kala-azar, 377 , 382; possible carrier of trypanosome disease in Mauritius and Réunion, 381.

sanguisuga, 110, 379.

sordida, infected with trypanosomes, 381.

vitticeps, infected with trypanosomes, 381.

Tricercomonas, 122.

Trichina worms, see Trichinella spiralis.

Trichinella spiralis, discovery, 7; 286-297; history, 286; prevalence, 286-288; life history, 288-292; hosts, 288; reproduction, 289; distribution in body of host, 290; formation of cysts, 291; trichiniasis, 292294; treatment, 294-295; prevention, 295-297; effects of cold storage and heat, 295; meat inspection for, 295-296.

Trichiniasis, prevalence, 286-288; course of, 292-294; treatment, 294-295; prevention, 295-297. Trichinosis, see Trichiniasis.

Trichoma, 396.

Trichomonas, 115; 118-122; in vagina, 119; in mouth, 119; description, 119; multiplication and encystment, 120; pathogenicity, 121; treatment of infections, 121.

buccalis, 119.

hominis, 118-122.

vaginalis, 119.

Trichostrongylus, 282-283.

instabilis, 282.

orientalis, 282.

subtilis, see $T$. instabilis.

Trichuris trichiura, 276-277.

Trinidad, mosquitoes thought to transmit Dermatobia, 451.

Triodontophorus, see Ternidens.

Trombidiidæ, see Harvest mites.

Trombidium, and kedani, 190.

akamushi, 336.

holosericeum, 336.

Tropical sloughing phagedæna, 73c.

Tropical ulcer. 73c.

Tropidurus peruvianus, host of Phlebotomus verrucarum, 472 .

Trypanoplasma, 117.

Trypanosoma, relation of tsetse flies to, 7, 490, 496-497, 500-501; cultivation, 9; immunity to drugs, 34, 105-106; developmental stages, 75, 96-97; 93114; importance, 93-94; description of, 94-95; hosts, 96; identification of species, 9697; species pathogenic to man, 97; and sleeping sickness, 98108; spores, 102; granule-shedding 103 agglutination, 103; and Chagas disease, 108-114; and leeches 317: earried by Cimex pipistrelli, 378; possible cause of disease in Mauritius and Réunion, 381-382.

brucei, relation to Rhodesian sleep- 
ing sickness, 108, 497.

cruzi, relation of Triatoma to, 8, 108, 380-381; and Chagas' disease, 108-114; distribution, 108; human cycle, 109-110; life cycle in Triatoma, 110-113; other intermediate hosts, 112, 378; vertebrate hosts, 112.

gambiense, discovery, 7 ; direct transmission, 34, 97; 98-108; transmission, 98-99, 496; distribution, 98 ; life cycle in fly, 99-101; life cycle in man, 101103 ; spores, 102 ; granule-shedding, 103; agglutination, 103; and drugs, 105-106; transmitting species, 496-501; relation of stable-flies to, 98,507 .

lewisi, immunity to drugs, 105.

nigeriense, 98; and stable-flies, 98, 507.

rhodesiense, 97; distribution, 98; and sleeping sickness, 98-108; pathogenicity and relation to T. brucei, 107-108; and drugs, 106; transmitting species, 98, 497, 499-501.

triatome, 112, 381.

Trypanosome fever, 103-104.

Tryparsamide, for trypanosomiasis, 105.

Tsetse flies, relation to trypanosomes, 7, 98-101, 496-497, 500-501; relation to Onchocerca volvulus, 311; mouthparts, 327, 464, 491; reproduction, 464, 495; 490-504; importance, 490; general account, 491-492; distribution, 492; habits, 493495; life history, 495-496; and disease, 496-501; control, 501504.

Tsutsugamushi, see Leptus akamushi and Kedani.

Tuberculosis, possible spread by bedbugs, 379 .

Tularemia, 413, 489.
Tumbu fly, see Cordylobia anthropophaga.

Tunis, Leishmania in gecko, 86.

Tunnel disease, see Hookworm.

Tunnicliff, R., 73A.

Tuntun, see Hookworm:

Turbellaria, 197.

Turpentine, to keep away ticks, 368; for bugs, 383; oil of, for body lice, 401; resistance of maggots to, 522 .

Tydeus molestus, 341.

Typhoid, relation of intestinal worms to, in apes, 204.

Typhus, 168; organisms of, 169, 186-187; nature of, 186; discovery of louse transmission, 397; epidemiology, 397-398.

in European War, 2, 398; relation of lice to, 8, 397-399; cause of, $73,169,195$, 397, epidemics, 398399 ; and fleas, 414.

Tyroglyphidæ, 333; 339-340.

Tyroglyphus, 340.

longior, 340.

longior castellanii, 340 .

Uganda, sleeping sickness, 93; fishing industry and sleeping sickness, 106-107; Filaria perstans, 308.

Ulcerating granuloma, 73C-73D.

Undulating membrane, 30.

United States, plague in, 2, 411; syphilis in, . 3; hookworm in immigrants, 5, 268; amebic dysentery, 6, 134 ; relapsing fever, 43; syphilis, 50; yellow fever, 69-70; rat-bite fever, 73; possibility of kala-azar, 77; prevalence of intestinal Protozoa in South, 116; Embadomonas, 118; Trichomonas pathogenic, 121; amebic dysentery, 134; malaria, 147-148, 163; blackwater fever, 161; swamp land and malaria, 166; dengue, 
182; spotted fever, 188-189; possibility of introduction of blood flukes, 220; Paragonimus kellicotti, 220, 223; Opisthorchis pseudofelineus, 225; Paramphistomum in cattle, 229; Tonia solium, 240; Hymenolepis nana, 242; Hymenolepis diminuta, 244; Diphyllobothrium latus, 246; hydatids, 248; hookworm, 254, 255, 262, 263, 268; privies, 266; Moniliformis, 285; prevalence of trichina in hogs, 286, 287; prevalence of trichina in man, 287; Filaria bancrofti, 299; red-bugs, 336; Pediculoides, 338; Norwegian itch, 343; economic importance of ticks, 352; tick paralysis, 358; Dermacentor venustus, 363; Otiobius mégnini, 365; Triatoma, 379, 381 ; kissing bugs, 382 ; plaguelike disease transmitted by fleas, 413; Pulex irritans, 415; Ctenocephalus canis, 416; Ceratophyllus, 418; Echidnophaga gallinacea, 420; Aëdes calopus, 447; Culex quinquefasciatus, 449; mosquito canopies, 457; Notophthalmus torosus natural enemy of mosquitoes, 461 ; blackflies, 481; tularemia, 489; infantile paralysis, 507.

United States Army, syphilis in, 50.

United States Bureau of Animal Industry, experiments with trichina, 295.

United States Bureau of Entomology, 508.

Uranotonia, palpi, 426.

Uruguay, dengue, 182; Tetranychus molestissimus, 341.

Uta, $86 ; 87 ; 88 ; 477$.

Vaccination, 4; broad meaning of, 22 ; in yellow fever, 72 ; in treatment of hookworm disease, 263.
Vaccine or cowpox, 191.

Vahlkampfia, 132.

Van den Branden, F., 107.

VAUllegeard, A., 202.

VEDder, E. B., 8, 50.

Venezuela, Schistosoma mansoni, 217; Rhodnius prolixus, 382; Dermatobia carrier, 452.

Vera Cruz, amebic dysentery, 139; malaria, 166.

Ver-du-cayor, see Cordylobia anthropophaga.

Vermes, 196.

Verruga peruviana, 169, 193; relation to Oroya fever, 178, 195; and Phlebotomus verrucarum, 472.

Vespa maculata, natural enemy of tabanids, 490 .

VianNa, G., 8, 73D, 81, 109.

Vincent's angina, cause of, $73 \mathrm{~A}$; treatment, 73в.

Vinchuca, see Triatoma infestans.

Vinegar, effect on Clonorchis cercariæ, 227; and kerosene for lice, 402 ; repellent for mosquitoes, 455 .

Volhynian fever, 187.

Von Dusch, 6.

Von Ezdorf, H., 148.

Von Linstow, see Linstow, von.

WALKer, E. L., 136, 138, 139.

WALLACE, A., 320.

WALSH, B. D., 524.

Warbles, see Hypoderma.

WARD, H. B., 245, 285.

Wart hog, host of Chœromyia, 512 .

Washington, D. C., dispersal of lice in family wash, 401; development of Anopheles quadrimaculatus, 442.

Waskia, see Embadomonas.

Wassermann, A. von, 49.

Wassermann reaction 49 ; 50; 55-56.

Water-dogs, natural enemies of mosquitoes, 461.

Watsonius watsoni, 229. 
Weil's Disease, see Infectious jaundice.

Weinberg, M., 204.

Weinland, D. F., 7.

WenYon, C. M., 85, 118, 121, 123 , 124, 125, 156, 172, 173, 471.

Western Australia, compulsory notification of syphilis, 60 .

West Indies, dengue, 182; Schistosoma mansoni, 217; hookworm disease, 264; Necator americanus, 255; work of Hookworm Commission, 268; Filaria perstans, 308; bête rouge, 335; Cimex hemipterus, 373; chigger, 419, origin of yellow fever, 447; home of "millions," 461.

West Point, syphilis at, 51.

Whipworm, see Trichuris trichiura.

Whitmarsh, P. L., 305.

Wilder, R. M., 8, 397.

Wild game, extermination to eradicate sleeping sickness, 107; and control of spotted fever in United States, 189; hosts of Dermacentor venustus, 363; hosts of tsetse flies; 490, 498.

Williams, Anna, 143, 193.

Wohlfartia magnifica, 521-522.

Wolbach, S. B., 188.

Woodrats, host of Triatoma protracta, 112.

Woodtick, see Dermacentor.

Worcester, D. C., 320.

Worms, 196-205; classification, 196; flatworms in general, 196-198; roundworms in general, 198-199; annelids in general, 199-200; parasitic habitats, 200; life history and modes of infection, 200-201; effects of parasitism, 201-204; nutriment absorbed, 202 ; toxic effects, 202; infection of wounds made by, 204; relation to appendicitis, 204; diagnosis, 204-206; eggs of, 205.
See also Intestinal worms and various species.

Wrigglers, 431.

Wright, R. E., 306.

Wyeomyia smithii, hibernation, 436.

Xanthium macrocarpum, mites on leaves of, 341.

Xenopsylla cheopis, identification, 408; and plague, 411, 412; intermediate host of Hymeno. lepis, 414; distinguished fron Pulex irritans, 415; habits, etc. 417.

X-ray, for ulcerating granuloma, 73D

YAKIMOFF, W. L., 377.

Yaws, 63-65; distribution, 63; spirc chætes of, 63 ; course of, 6 : treatment, 64-65; preventior 65.

Yellow fever, 69-72; in Panama, : relation of mosquitoes to, $7,44 \%$ parasite of, 70, 169; distribu. tion, 69-70; course of, 71; treatment and prevention, 7172 ; transmitting mosquito, 443-448.

Yellow fever group of parasites, 169, 182.

Yellow fever mosquito, see Aëdes calopus.

Yersin, A., 411.

Yokagawa yokagawa (Metagonimus), intermediate host, 226; 228.

YoRke, W., 100, 494, 496.

Yoshida, S., 222, 223.

Yquitos, reduction of yellow fever, 72.

Yucatan, yellow fever in, 70.

Zambezi, danger of spread of Glossina palpalis to, 498.

Zebu, possible intermediate host of Tænia africana, 245.

ZENKer, F. A. von, 7. 



THIS BOOK IS DUE ON THE LAST DATE STAMPED BELOW

\section{RENEWED BOOKS ARE SUBJECT TO IMMEDIATE} RECALL

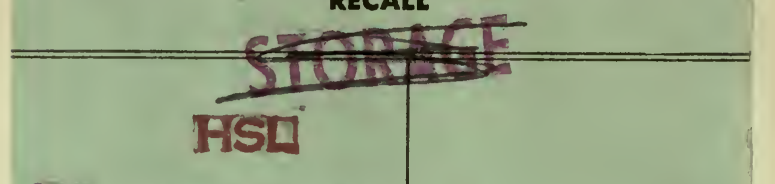

\section{IUE JUN 291982}

RER'ERELLODTH 14989

AUG is 1983

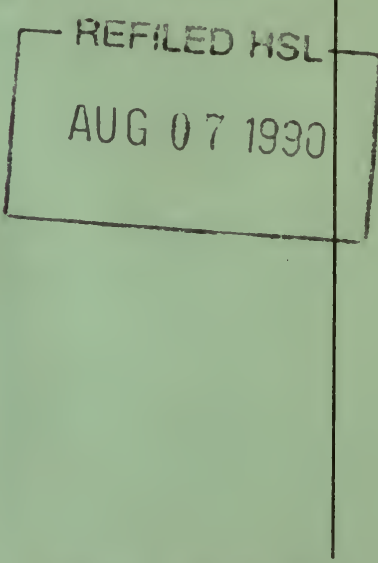

LIBRARY, UNIVERSITY OF CALIFORNIA, DAVIS 
31175006270998

DEPARTMENT BOOK CARD

$$
623
$$

Chandler; Asa Crawford 445

Animal parasites and 1922

$$
623
$$

Chandler.

$Q \times 4$
$C 45$

Animal parasites

1922

HEALTH

SCIENCES

LIBRARY 
सx

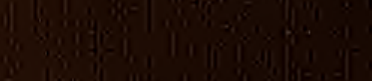

10

in 